\title{
Argonne
}

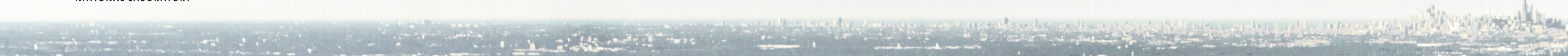

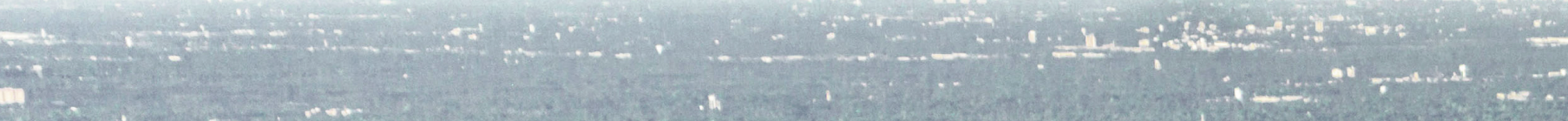

Advanced Photon Source

Argonne National Laboratory

9700 S. Cass Ave.
Argonne, IL 60439 USA

www.anl.gov

www.aps.anl.gov

\section{APS SCIENCE 2014}

ReSEARCH AND ENGINEERING HIGHLIGHTS fROM the

AdVAnced Photon Source at

ANL-15/03

Argonne National Laboratory

ISSN 1931-5007

May 2015

-

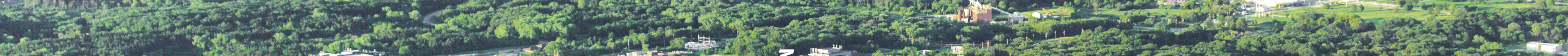

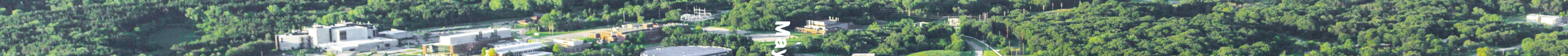

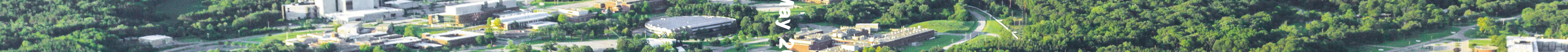

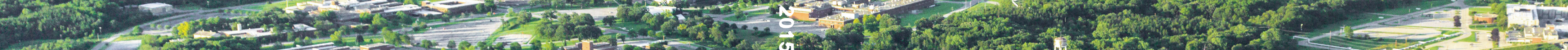

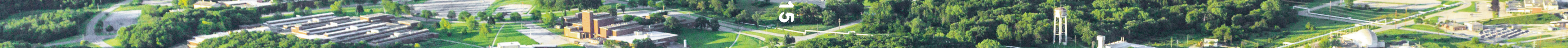

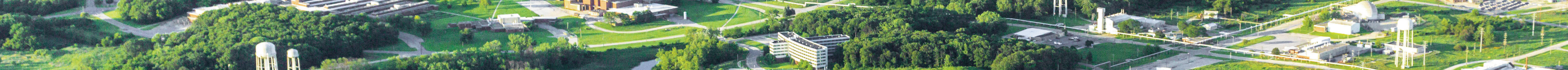

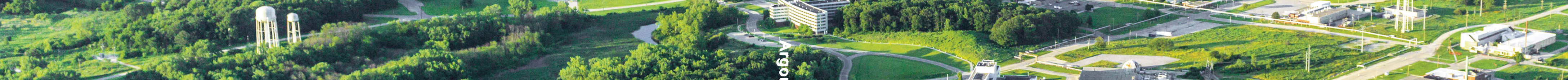
1.

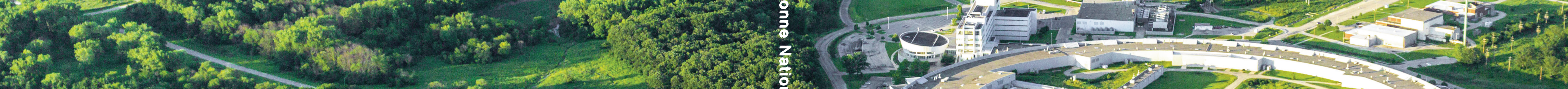
$40+3+3+25-2$

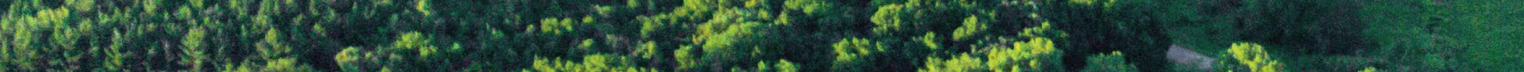

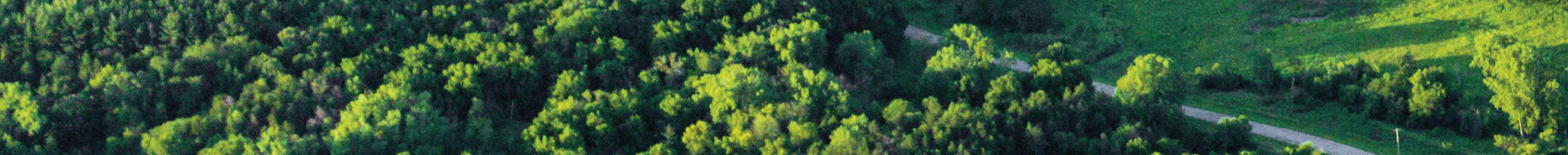

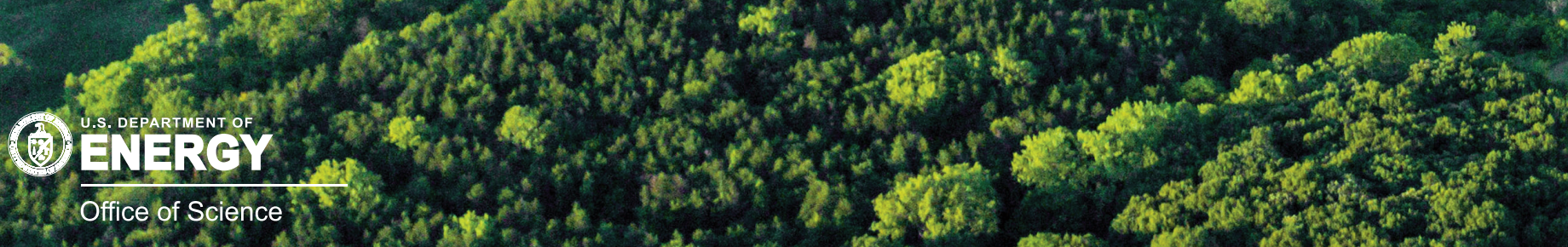

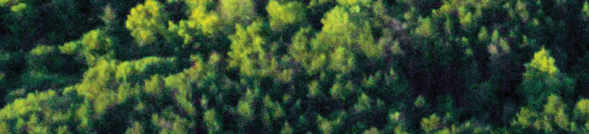
(2) $=-5$
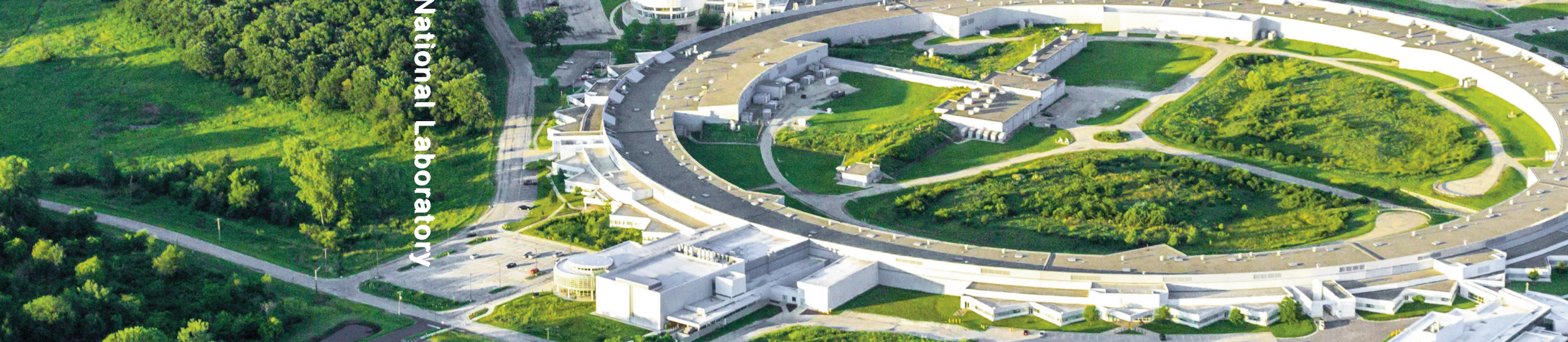

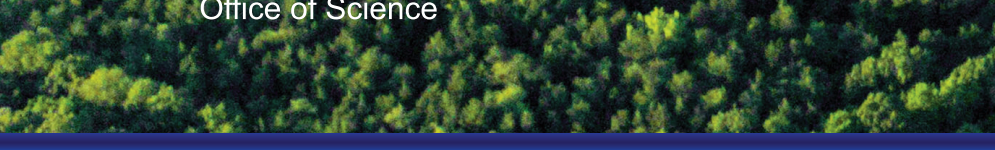
b.

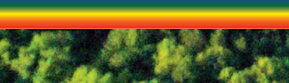


ANL-15/03 - SSN 1931-5007・ May 2015

The Advanced Photon Source is a U.S. Department of Energy (DOE) Office of Science User Facility operated for the DOE Office of Science by Argonne National Laboratory under Contract No. DE-AC02-06CH11357.

About Argonne National Laboratory

Argonne is a U.S. Department of Energy laboratory managed by UChicago Argonne, LLC under contract DE-AC02-06CH11357. The Laboratory's main facility is outside Chicago, at 9700 South Cass Avenue, Argonne, Illinois 60439. For information about Argonne and its pioneering science and technology programs, see www.anl.gov.

\section{DOCUMENT AVAILABILITY}

Online Access: U.S. Department of Energy (DOE) reports produced after 1991 and a growing number of pre-1991 documents are available free via DOE's SciTech Connect (http://www.osti.gov/scitech/)

Reports not in digital format may be purchased by the public from the National Technical Information Service (NTIS):

\section{U.S. Department of Commerce}

National Technical Information Service

5301 Shawnee Rd

Alexandria, VA 22312

www.ntis.gov

Phone: (800) 553-NTIS (6847) or (703) 605-6000

Fax: (703) 605-6900

Email: orders@ntis.gov

Reports not in digital format are available to DOE and DOE contractors from the Office of Scientific and Technical Information (OSTI):

\section{U.S. Department of Energy}

Office of Scientific and Technical Information

\section{P.O. Box 62}

Oak Ridge, TN 37831-0062

www.osti.gov

Phone: (865) 576-8401

Fax: (865) $576-5728$

Email: reports@osti.gov

\section{Disclaimer}

This report was prepared as an account of work sponsored by an agency of the United States Government. Neither the United States Government nor any agency thereof, nor UChicago Argonne, LLC, nor any of their employees or officers, makes any warranty, express or implied, or assumes any legal liability or responsibility for the accuracy, completeness, or usefulness of any information, apparatus, product, or process disclosed, or represents that its use would not infringe privately owned rights. Reference herein to any specific commercial product, process, or service by trade name, trademark, manufacturer, or otherwise, does not necessarily constitute or imply its endorsement, recommendation, or favoring by the United States Government or any agency thereof. The views and opinions of document authors expressed herein do not necessarily state or reflect those of the United States Government or any agency thereof, Argonne National Laboratory, or UChicago Argonne, LLC. 
ANL-15/03

ISSN 1931-5007

May 2015

\section{APS SCIENCE}

Research and Engineering Highlights

from the Advanced Photon Source

at Argonne National laboratory

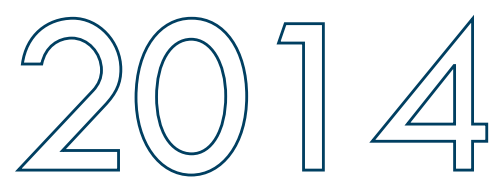

Argonne

Argonne is a U.S. Department of Energy (DOE) laboratory managed by UChicago Argonne, LLC.

The Advanced Photon Source is a DOE Office of Science User Facility

operated for the DOE Office of Science by Argonne National Laboratory under Contract No. DE-AC02-06CH1 1357. 


\section{TABLE OF CONTENTS}

III APS ORganization ChaRT

1 The Advanced Photon Source Facility at Argonne National Laboratory

2 WELCOME

4 The Advanced Photon Source Upgrade Project

6 Access to BeAm TIME AT THE APS

7 Argonne national laboratory 400-Area Facilities

9 Electronic \& Magnetic Materials

10 Stressing Out Copper TSVs With Temperature

12 Earth-Crushing Pressure? This Electron Spin Doesn't Care

14 TO Understand What Makes a TOPOLOGICAL INSULATOR, ZOOM OUT

16 How Magnetic Dimers Interact to Create Long-Range Order

18 Squeezing Out the Hidden Lives of Electrons

20 X-ray Scattering Studies of the Spin Disorder and Magnetic Coupling in Chromium Spinels

22 Tuning the Magnetic Energy Scales of Strontium Iridate Thin Films

24 Interfacial Octahedral Rotations and Magnetism in OXide Superlattices

26 High-Performance Piezoelectrics - Coming Soon in Unleaded

28 Ultrafast InHOMOgeneous Strain IN ROOM-TEMPERATURE MultifERroics

29 When an Exciton Acts LiKe a Hole

30 Metals on the Couch: Does Deformed Aluminum Retain Internal Stress?

31 Pinning Down New Fe-Based Superconductors

33 Engineering Materials \& Applications

34 Keep Them Turning: Protective Ceramic Coatings for Engine Blades

36 StRETCHING THE LIMITS OF SYNTHETIC SPIDER SiLK

38 A Framework for Next-Gen Optical Sensors and LeDs

40 In Certain Polymer Solar Cells, Disorder Can Be a Delight

42 How Atomic Vibrations Stabilize Metallic Vanadium Dioxide

44 Cracking the Puzzle of Material Failure

46 BUILDING LoNGER-LASTING LITHIUM-SULFUR BATTERIES

48 IMAGING BATTERY STRAIN

50 How lons Cycle at High Rates Inside of Lithium-Ion Batteries

51 Writing SMALler for Bigger Data

53 Designing Better See-Through Contacts for Displays and Solar Power

54 Probing nanostructures in Steel Alloys

56 AROUnd the APS: KeEping the LN 2 Flowing to APS Users

57 SOFT MATERIALS \& LIQUIDS

58 Probing the Geometry of Wetting Ridges in Soft Solids

60 Tracking the Strain of Deforming Gels

62 Wet Granules are Like Glass

64 Around the APS: A Photo-Cathode Rf Gun for Brighter Beams at the APS

65 Chemical Science

66 Acid Ions are More Than Spectators

68 Acid and IRon Make the Most Sugar

70 Peering Deep Inside of Nongraphitic Anodes with Synchrotron Microtomography

72 Hydrogen Adsorption on Shape-Shifting Platinum Nanoparticles

74 Atomic-Level Clustering Responses of Metallic Alloy Liquids to Changing Temperatures

75 Graphene Goes Organic

76 Metals and Oxides Work Together to Make Better Catalysts

78 WHO SHRUNK THE CATALYST?

80 Investigating the Ties That Bind: Catalysts with Paired-Up Metals

82 Single-Atom Gold Catalysts for Low-Temperature, Low-Cost Hydrogen Production

84 JUST SAY NO TO IRON PORPHYRINS

86 Stability vs. Activity: Characterizing Strontium Ruthenate Crystals for Electrochemical Applications

87 Peeling Back the Layers of Thin-Film Structure and Chemistry

89 LIFE SCIENCE

90 A novel Mode of Action for an Osteoporosis Drug

92 Protein-Lipid Interactions Dictate Cellular Fates

94 COMBATING AN INFECTIOUS INVADER

96 UnRAVELING PROTEIN Folding

98 A Molecular Basis for Protease's Role in Preventing Alzheimer's Disease 
99 Structural Biology

101 On the Path toward Designer Proteins

102 Playing Hide and SEeK With HIV

104 Synthetic Peptides Use the Force to Influence Cell Survival

106 How Ebola Silences Those Who Would Sound the Alarm

108 Lights, Conformational Change...Action!

110 A Tale of Two Glutamate Receptors

112 How to Remove TANgles IN Your DNA

114 A Novel Mechanism of DNA DEMEThyLATION

116 Generating More Efficient and Stable Bispecific Antibodies

118 The Viral Secrets of Replication and IMmune System EVAsion

120 A Common Form of Protein Modification that is Abundant in Bacteria

122 Targeting Certain Cardiovascular Diseases

124 An Engineered Protein for Extracting Uranium from Seawater

126 Finding Novel ANTIBIOTIC TARgets

127 NolR-Mediated Regulation of Symbiotic Root Nodule-Dependent Genes

128 A New Perspective on Cancer Treatment

129 A Structural Basis for the Evolution of Bacterial Antibiotic Resistance

130 HiJacking the Sulfur-Delivery Machinery for Bacterial ThIOSUgar Formation

131 Environmental, Geological \& Planetary Science

132 STARDUST ON EARTH

134 SMOOTH Flow In MOLTEN ROCK

136 Respiration of Bacteria in Anoxic Aquifers

138 The Earth's Deep Lower Mantle is Less Stable than We Thought

140 A Meteorite Tells Tales of the Earth's Deep Mantle

142 Hidden Carbon In the EARTh'S CoRe?

144 Testing Crystal Candidates for Earth's Inner Core

145 NANOSCIENCE

146 Self-Assembly of Layered Membranes

148 Polymer Nanostructures Grow Bigger When Agitated

150 BuILding NanOCRYstals to ORder With DNA

151 Laying Out Nanoparticles According to Plan

152 ImPROVING NANOSCALE ElECTRONICS

153 Novel X-Ray Techniques \& Instrumentation

154 New Light for Fluorescence Microscopy at the APS

156 Ptychography: Difficult To Pronounce, But Easy to Use for Beautiful Images

157 Data Exchange: a Common Data Format for Tomographic Data Sets

158 AN IMPULSE FOR DYNAMIC COMPRESSION SCIENCE

160 A Narrower Spectrum for a Wider VieW of Matter

162 Two Good Things That Are Better Together: STM and X-Ray Synchrotron Microscopy

164 Fabricating, Testing, and Installing a KirkPatrick-Baez Mirror System at the APS

166 ID'ing Cells With the Help of a Computer

167 Wave Hello to Faster X-Ray Pulses

168 X-RAY Ptychography: IMAging Vibrations Without the BluR

169 Defining the Thermal Fatigue Limits of GlidCop® High-Heat-Load X-Ray Absorbers

171 SONICC AT GM/CA-XSD

172 High-Performance Computing Software to Improve Beamline and Accelerator Efficiency

174 HYBRID: Simulating Partially Coherent X-Ray Wavefronts Down Non-Ideal Beamline Components

175 Seeing the Light at 29-ID With a New Transition-Edge Superconducting Detector

176 Taming the Tsunami: A New Approach for Tomographic Data

178 StATE-OF-THE-ARt MX Detectors at the APS

179 A New tXM Instrument to Address Future Challenges in Nanoscience at the APS

180 A ReVolver Undulator for SECtOR 35 of THE APS

181 AROUND THE APS: HONORS \& AWARDS

182 AROUND THE APS: CONFERENCES \& WORKSHOPS

184 DATA

186 Typical APS Machine Parameters

186 APS SOURCE PARAMETERS

188 ACKNOWLEDGMENTS 


\section{APS ORGANIZATION ChaRT}

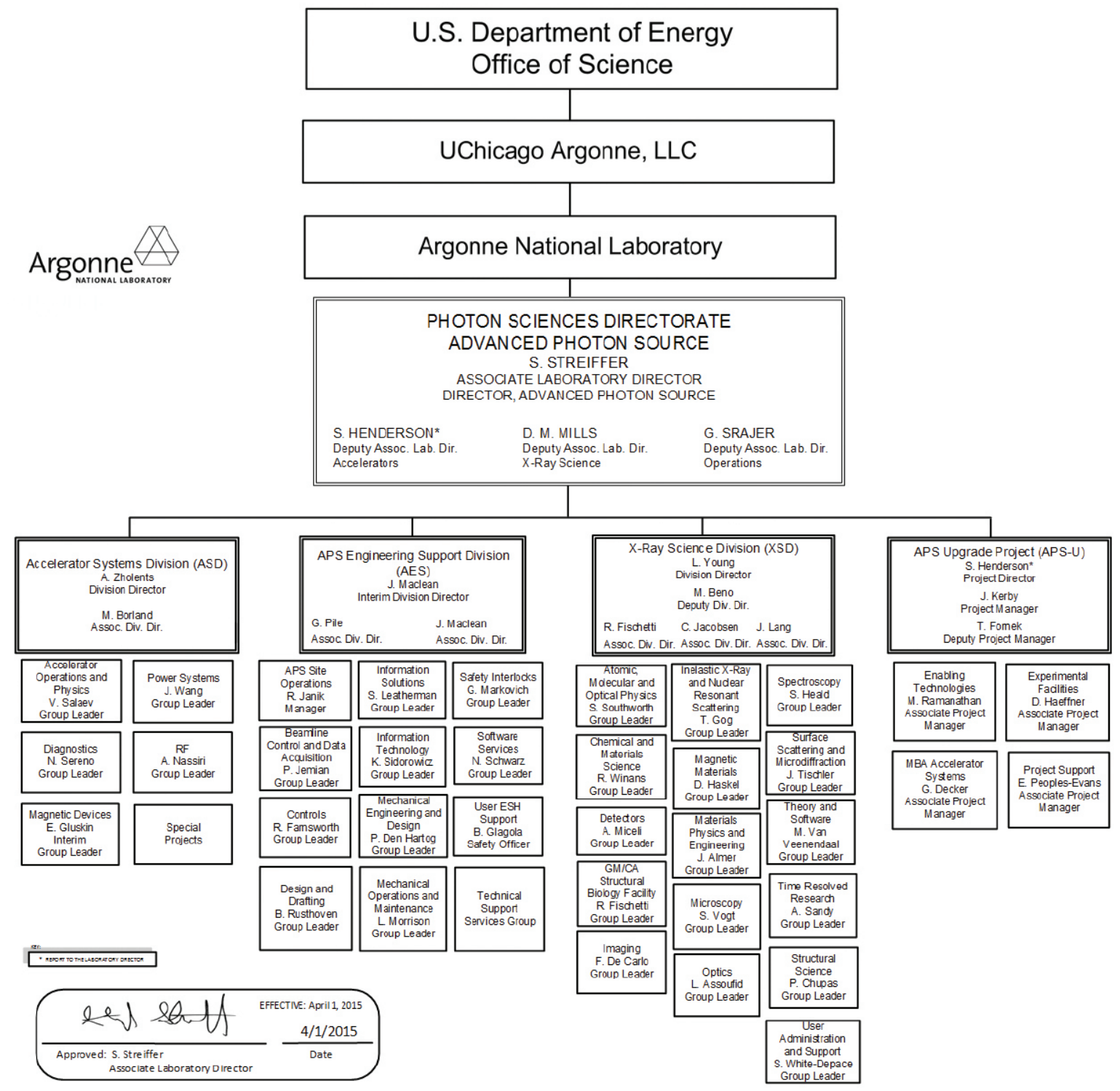

ACRONYMS FOR ARGONNE DIVISIONS USED IN THIS BOOK

AES - APS Engineering Support Division

ASD - Accelerator Systems Division

CEP - Communications, Education, and Public Affairs Division

XSD - X-ray Science Division 


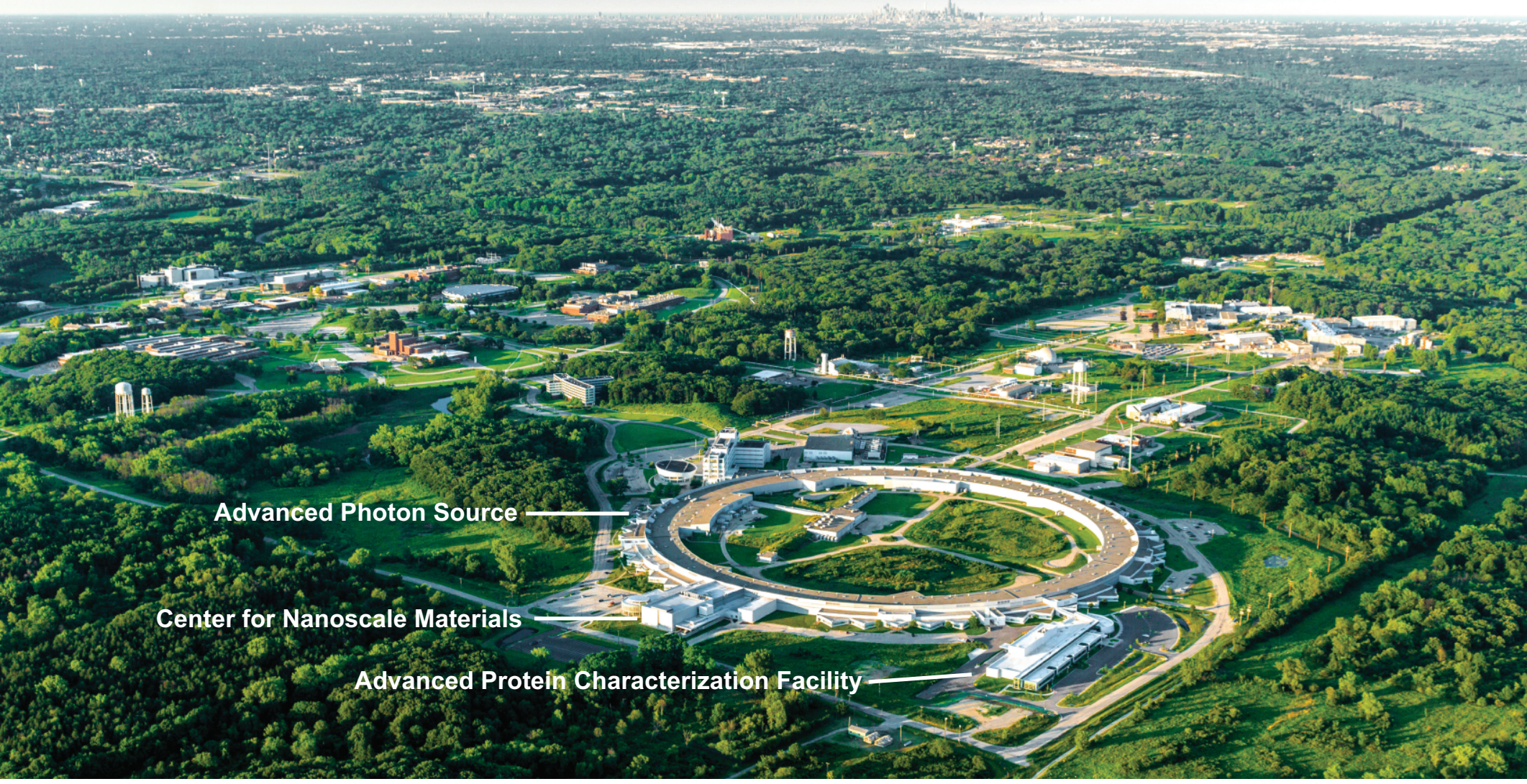

The Advanced Photon Source Facility at Argonne National laboratory

The Advanced Photon Source (APS) occupies an 80-acre site on the Argonne National Laboratory campus, about 25 miles from downtown Chicago, Illinois.

It shares the site with the Center for Nanoscale Materials and the Advanced Protein Characterization Facility

For directions to Argonne, see http://www.anl.gov/directions-and-visitor-information.

The APS, a national synchrotron radiation research facility operated by Argonne for the U.S. Department of Energy (DOE) Office of Science, provides this nation's brightest high-energy x-ray beams for science. Research by APS users extends from the center of the Earth to outer space, from new information on combustion engines and microcircuits to new drugs and nanotechnologies whose scale is measured in billionths of a meter. The APS helps researchers illuminate answers to the challenges of our high-tech world, from developing new forms of energy, to sustaining our nation's technological and economic competitiveness, to pushing back against the ravages of disease. Research at the APS promises to have far-reaching impact on our technology, our economy, our health, and fundamental knowledge of the materials that make up our world.

\section{CONTACT US}

For more information about the APS or to order additional copies of this, or previous, issues of APS Science, send an email to apsinfo@aps.anl.gov, or write to APS Info, Bldg. 401, Rm. A4115, Argonne National Laboratory, 9700 S. Cass Ave., Argonne, IL 60439, or go to http://www.aps.anl.gov/Science/Reports/ to download PDF versions.

Visit the APS on the Web at www.aps.anl.gov 


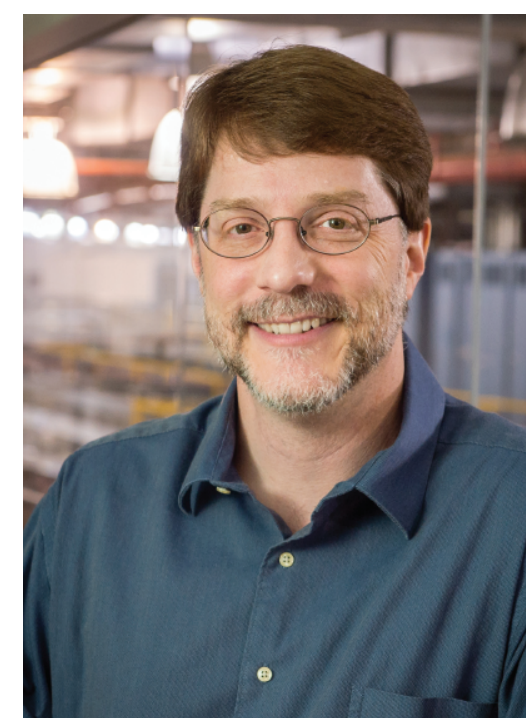

Stephen K. Streiffer

\section{May 2015}

On March 26, 2015, we paused to celebrate the 20th anniversary of first light from our facility, the U.S. Department of Energy Office of Science (DOESC) Advanced Photon Source (APS) at Argonne. Those first APS x-rays arrived by design 100 years (although not to the day) from Wilhelm Conrad Röntgen's discovery of the $x$-ray. One remarkable aspect of Röntgen's monumental achievement was the speed with which the x-ray was adapted to a vast array of uses, most scientific, a few not.

The evolution and proliferation of synchrotron $\mathrm{x}$-ray user facilities has been equally remarkable. According to a recent Nature Photonics editorial ("Synchrotron sources accelerate," Nat. Photonics 9, 281 [2015]), "there are 47 synchrotron radiation research facilities based on electron storage rings (some with more than one ring) in 23 countries currently in operation, under construction or being planned." As Fig. 1 shows, industrialized nations have long realized that membership in the light-source community is a necessity. An effort is now under way to bring an $\mathrm{x}$-ray light source to Africa, which is, as Nature Photonics points out, "the only habitable continent that is without a synchrotron source."

The U.S. is a charter member of that light-source community. The first sighting of synchrotron radiation oc- curred in 1947 at the General Electric Research Laboratory in Schenectady, New York. The first synchrotron radiation experiment with an insertion device was carried out at Stanford University in 1953.

Today, on behalf of our nation, the DOE-SC Basic Energy Sciences (BES) Scientific User Facilities Division maintains a leadership role in synchrotron $\mathrm{x}$-ray science by developing and supporting five complementary synchrotron radiation light source user facilities. The APS is one of those five, alongside the Advanced Light Source, the Linac Coherent Light Source, the Stanford Synchrotron Radiation Laboratory, and the new National Synchrotron Light Source-II.

Some examples of the frontier science produced by our users and the critical technological advances achieved by our staff follow this "Welcome." Regrettably, space in this book does not allow for complete coverage of even a significant fraction of the excellent results APS users produce in a year, but more highlights can be found on our Web site at https://www1.aps. anl.gov.

Five new APS beamlines are in the final stages of commissioning and are readying to start accepting proposals for research from the General User contingent of our user community:

- At the higher-energy diffraction (COMPRES) beamline at 6-BM, operated by the X-ray Science Division (XSD) Materials Physics \& Engineering Group, users can choose between a large-volume press for high-pressure energydispersive diffraction (EDD) experiments or general EDD with spatially resolved $x$-ray diffraction.

- The resonant inelastic $x$-ray scattering (RIXS) beamline at 27-ID-B, operated by the XSD Inelastic Xray and Nuclear Scattering (IXNS) Group, will consolidate the RIXS program at the APS on one dedicated, state-of-the art insertion device beamline, offering superb en- ergy resolution (20 meV to 30 $\mathrm{meV}$ ) combined with advanced beam focusing, high photon flux, and a selection of extreme sample environments (high-P/low- $\mathrm{T}$ ) for meaningful in situ experimentation.

- The intermediate energy $x$-ray beamline at 29-ID, operated by the XSD Magnetic Materials Group, is optimized to study emergent phenomena in correlated electron systems using angle-resolved photoemission spectroscopy and resonant soft x-ray spectroscopy.

- The Dynamic Compression Sector (DCS) at 35-ID, funded by the DOE National Nuclear Security Administration, is a first-of-its-kind facility designed to enable mechanistic understanding of condensed matter phenomena at extremes, dynamic loading conditions, and time-resolved multi-scale measurements in single-event experiments.

Other notable improvements occurred on the x-ray source side of our facility:

- Next-generation fluorescence and Compton x-ray beam position monitors were installed and tested at beamlines 27-ID and 35ID. Early results from tests at 27ID were very good and further refinement of the designs are under way to simplify and enhance beam stability.

- The APS superconducting undulator (SCU1), which contains a longer magnet than the SCU0 prototype but in the same kind of cryostat, was installed at Sector 1. The SCU0, installed last year at Sector 6, immediately began enabling excellent results for users of that beamline - see for instance, "Making Aperiodic Structure Crystal Clear," APS Science 2013, pg. 32. (A revolver undulator prototype was completed for installation at DCS in January 2015 . See page 180 of this book.) 


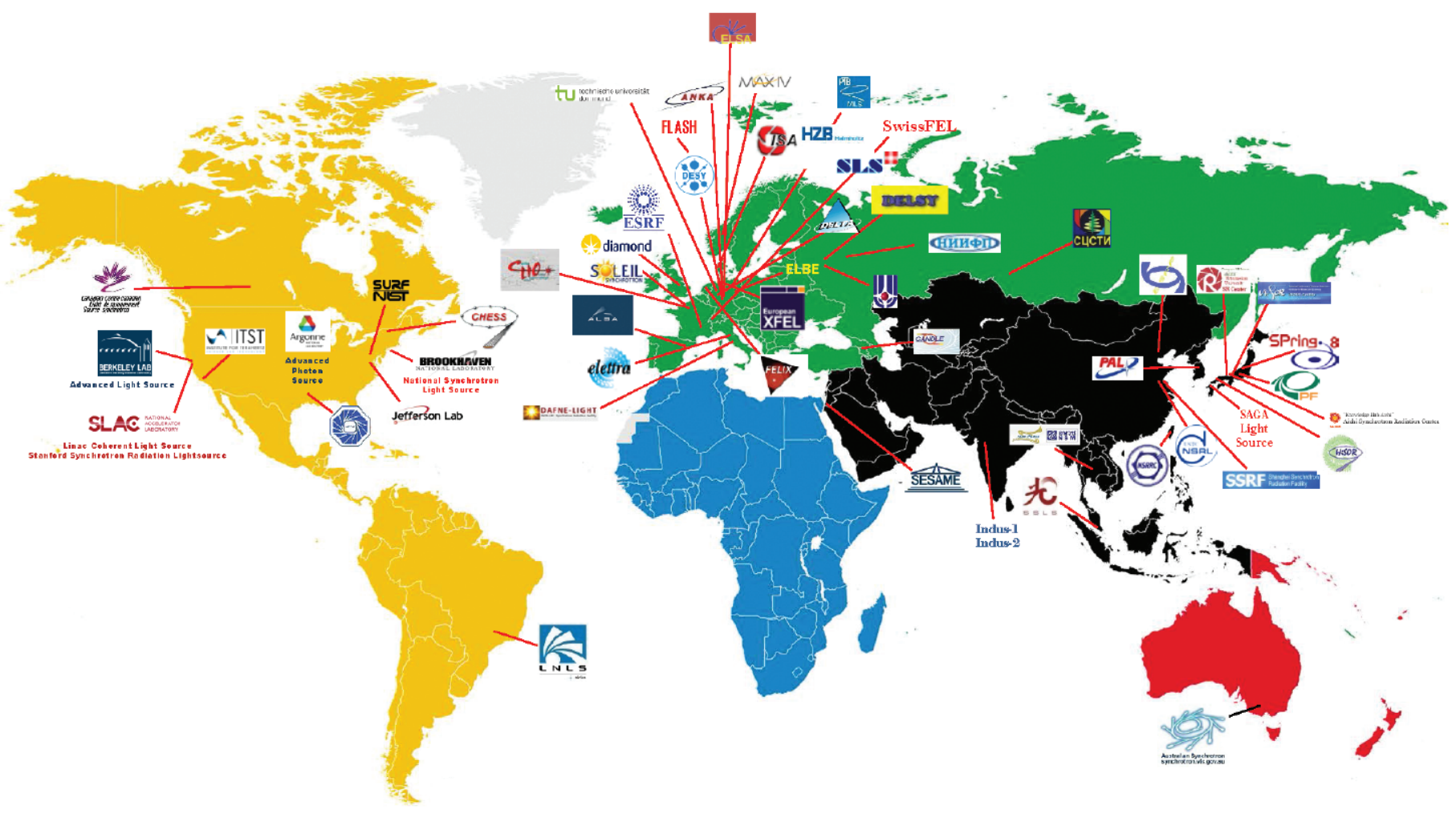

Fig. 1. Storage ring and free-electron laser $x$-ray light sources of the world.

- Beamline 30-ID-B,C, also operated by the XSD IXS Group, received new short-period, 1.72cm-period insertion devices that double the incident flux, and substantially improve flux density, beam divergence, and stability for the high-energy resolution inelastic $x$-ray scattering instrument. This is having an immediate, significant impact on user operations at 30-ID; doubling the incident flux enables users to obtain publishable data sets within as little as one visit to the APS, instead of multiple visits.

- At GSECARS Sector 13, one of three sectors at the APS managed by the Consortium for Advanced Radiation Sources at The University of Chicago, the bending magnet beamline $13-\mathrm{BM}-\mathrm{C}$ is receiving a new diamond anvil cell to upgrade an already worldleading high-pressure research program.

Each year, the APS produces considerable software for data reduction, analysis, and simulation for $\mathrm{x}$-ray imaging, scattering, and spectroscopy. An on-line catalog of both nascent and ma- ture active software development efforts contains more than 20 on-going software development projects that have been documented through discussion of the scope of each code, how it is funded and distributed, and its impact and future development directions; additional projects are still being added.

2014 was also the year for the triennial review of the APS program by our sponsors in the BES Scientific User Facilities Division. The reviewers noted that, overall, the APS is doing well ("the accelerator Research and Development program...exhibits high quality and is well managed...There are a number of well-defined optics and detector development projects...the number of users and publications continues to grow every year...," and accelerator performance was "stellar"). There were, of course, areas where we can, and will, improve in order to be efficient and effective in all aspects of our operation, and thus maximize both our users' experience at the APS and the return on taxpayer funding of our program. High on the BES request list was submission of a strategic plan. That document, "The Advanced Photon Source Five-Year Facility Plan," is now online at www1.aps.
anl.gov/The-Advanced-Photon-SourceFive-Year-Facility-Plan.

When we talk about the future of the APS, topic "A" is the proposed upgrade of our storage ring to a multibend achromat (MBA) lattice. Stuart Henderson, who leads the APS Upgrade Project, has more to say on this subject on the pages immediately following, so I will just note that this revolutionary accelerator technology can provide our users with an x-ray source of increased brightness that could reach three full orders of magnitude beyond that of the current APS. An MBA lattice can deliver a powerful, versatile, nextgeneration facility for users of highbrightness, high-energy x-rays.

The purpose of these APS Science highlight books is to look back at the past year's achievements by our users and staff. But we always keep an eye on future research possibilities, just as we celebrated the first-light anniversary as a prelude to new opportunities on the horizon.

Stephen K. Streiffer Argonne National Laboratory Associate Laboratory Director, Photon Sciences;

and Director, Advanced Photon Source 


\section{The Advanced Photon Source UPGRADE PROJECT}

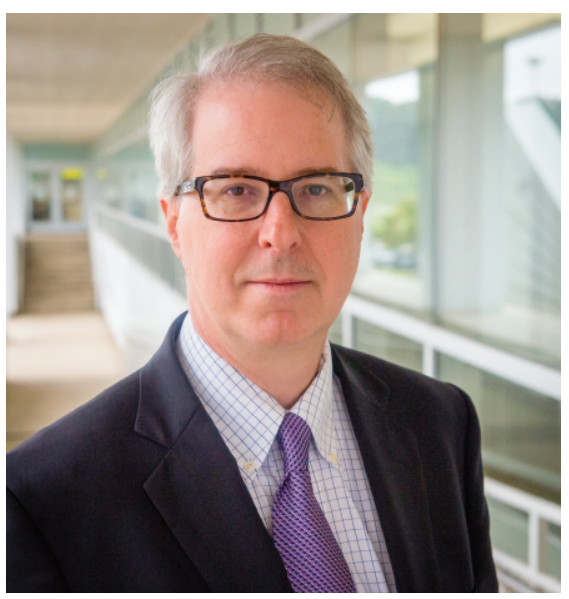

Stuart D. Henderson

May 2015

This has been an exciting and productive year for the APS Upgrade Project. In the 11 months since I joined the Project, we have made tremendous progress toward our goal of building the ultimate 3-D x-ray microscope, opening new research frontiers for our users, and assuring continued U.S. leadership in the x-ray sciences.

This Project is critically important to the future of American science. The development of a new storage ring magnet lattice design - the multi-bend achromat (MBA) - will make it possible to transform the APS into a fourth-generation storage ring source that will provide orders-of-magnitude increases in brightness and coherence. The exceptional capabilities of the upgraded APS will offer researchers access to a new frontier of x-ray science, making it possible to map matter and dynamics across length scales - in real time, in 3$D$, in macroscopic systems, in a single experiment.

The Upgrade will put the APS on the path to continued world leadership in hard x-ray science, creating a revolutionary scientific tool that will provide unparalleled brightness and spatial coherence at high energies. The potential impacts of these new capabilities will include the ability to observe and characterize individual structures as small as single atoms inside materials, making it possible to solve important problems in chemical catalysis, cell biology, environmental science, structural materials, and functional materials. The unprecedented coherent flux at high energies enabled by the MBA lattice will facilitate exciting new research techniques, such as coherent diffractive imaging with resolution approaching the x-ray wavelength, and photon correlation spectroscopy that promises to open new frontiers in dynamic studies. This new technology also will provide researchers with the highest possible spatial resolution over a broad temporal range, and with x-ray sensitivity to atomic structure and strain, elemental composition and chemical states, and electronic and magnetic structure.

It is no small task to undertake a Project of this magnitude. But over the past year, the APS-U team has done an amazing job of moving forward on all three vital and interconnected components of this Project: A credible technical concept, a robust and well-defined Project plan, and - most importantly - a visionary science case that will fully convey the transformative future impacts of the APS-U.

On the technical side, we've done some great work on the nuts and bolts of the Project. We have completed a draft of the Conceptual Design Report (CDR), a 440-page document that describes a full, coherent technical concept to meet the APS Upgrade Mission Need. The CDR includes all technical aspects of the project: Accelerator, front-ends and insertion devices, beamlines, storage ring removal and installa- tion, utilities, and issues related to environment, safety, health \& quality (ESH\&Q.) Our new Machine Advisory Committee, chaired by Bob Hettel at SLAC, came to Argonne to review the CDR in February - despite a major snowstorm. They were extremely positive in their comments on the accelerator concept, and they agreed that our CDR is well advanced for a project at this stage.

As we move ahead on our technical plan, we are fortunate that we can draw on the expertise of colleagues around the world. In November, the 4th Diffraction Limited Storage Ring (DLSR) Workshop was held here at Argonne. The Workshop featured presentations by distinguished scientists at other synchrotron facilities around the world that are adopting multi-bend achromat (MBA) lattice technology, which will push the performance of high-energy storage rings towards the diffraction limit in the hard $x$-ray regime. MAX IV (Sweden) is well along in construction, SIRIUS (Brazil) is in the final design stages, and several other major facilities in Europe and Asia are at various stages of design. At the Workshop, it was instructive to hear our international and U.S. colleagues talk about their own approaches to the challenges we all are facing in the design, construction, commissioning and operation of MBA storage rings.

Our Project team has made great strides in refining APS-U's scope, cost and schedule over the past months. The team is getting strong support from 
a thoughtful collaboration that brings together lead personnel from Argonne's programmatic and mission support staff, alumni of similar projects in the National Laboratory complex, DOE Argonne Site Office staff, and our DOESC sponsors. To provide additional guidance as we move forward, Laboratory Director Peter Littlewood has convened a group of tremendously qualified leaders to serve on the APS-U's Project Management Advisory Committee (PMAC) and provide expert guidance as we work together to launch the Upgrade, and execute the Project within budget and on schedule.

Our good efforts on the technical and Project fronts have been more than matched by the progress we are making with our science case, which is the heart of this Project. Our science team has put together a tremendous set of examples of ambitious science that will be enabled by the Upgrade's high energy, brightness and coherence, and that demonstrate how crucial the Upgrade is to our nation's scientific and economic competitiveness.

The Upgrade's science case has benefited greatly from the work of Stefan Vogt, Group Leader for the Microscopy X-ray Science Division, who has become the APS' Principal Science Advisor to the Upgrade Project, and Paul Evans, Professor of Materials Science and Engineering at University of Wisconsin, who is serving as a senior consultant on the Project. Stefan, Paul, and APS Director Stephen Streiffer have been engaging with leaders across the community to identify and develop some of the user community's most exciting research ideas. Over the next year, they will be working with the full user community to expand and deepen our vision of the Upgrade's revolutionary multidisciplinary impacts.

At this writing, we are working to organize a series of Science Planning workshops to identify some of the highest-impact early science opportunities that will be enabled by the Upgrade. The workshops are designed to focus on the wide range of science that the APS Upgrade will make possible. We expect these workshops to lead to a report, Early Science at an Upgraded Advanced Photon Source, that will help us document the Upgrade's most exciting scientific opportunities and map out the high-level technical requirements necessary to pursue them.

To keep our user community up-todate on our plans and progress, we have established the APS Upgrade Forum, a regular meeting to provide information and promote discussion around Upgrade topics of mutual interest to beamline scientists, accelerator and Project staff, and the larger scientific community. Associate Project Manager Dean Haeffner and Stefan Vogt are organizing these forums, which have become a useful mechanism for communication and discussion between the Upgrade team and our resident scientific community at the APS. The Forums have provided some good opportunities to connect with users, talk about our plans, and discuss their questions and concerns, and we look forward to continuing those conversations in the months to come.

Ultimately the success of the APS lies in the excellence and creativity of the researchers who have made it one of the most productive and successful scientific tools in the world. As we take the next steps in this important Project, our top goal is to work even more closely with our users to articulate and implement a clear, compelling longterm vision of the APS Upgrade and the revolutionary science it will make possible.

I encourage every member of our user community and staff to contribute energetically and creatively to this process, sharing your thoughts, concerns, expertise and ideas as we work together to achieve this vital Upgrade and keep the APS at the forefront of the world's hard $\mathrm{x}$-ray lightsources. As this Project moves forward, we are counting on you to bring your best ideas and your most creative solutions to this shared enterprise.

Thank you.

Stuart D. Henderson

Argonne National Laboratory

Deputy Associate Laboratory Director,

Facility Development, Photon Sciences;

and Director,

Advanced Photon Source Upgrade Project

The APS Upgrade Project is funded by the U.S. Department of Energy Office of Science under Contract No. DE-AC02-06CH11 


\section{Access to Beam Time at the APS}

Five types of proposals are used at the APS: General User, Partner or Project User, Collaborative Access Team (CAT) member, CAT staff, and APS staff. All beam time at the APS must be requested each cycle through the web-based Beam Time Request System. Each beam time request (BTR) must be associated with one of the proposals mentioned above.

\section{General User PROPOSALS AND BTRS}

Proposals are peer reviewed and scored by a General User Proposal Review Panel, and time is allocated on the basis of scores and feasibility. A new BTR must be submitted each cycle; or each cycle, allocation is competitive. Proposals expire in two years or when the number of shifts recommended in the peer review has been utilized, whichever comes first.

\section{Partner or Project User proposals and BTRs}

Proposals are peer reviewed by a General User Proposal Review Panel and reviewed further by a subcommittee of the APS Scientific Advisory Committee and by APS senior management. Although a new BTR must be submitted each cycle, a specific amount of beam time is guaranteed for up to three years.

\section{CAT MEMBER PROPOSALS}

Proposals from CAT members are typically much shorter and are reviewed by processes developed by individual CATs. Allocation/scheduling is determined by the CAT management.

\section{CAT AND APS STAFF MEMBER PROPOSALS AND BTRS}

These proposals are also very short and are reviewed through processes developed by either the CAT or the APS. Each CAT/beamline determines how beam time is allocated/scheduled. Collaborative Access Team and/or APS staff may submit General User proposals, in which case the rules for General User proposals and BTRs are followed.

In addition to the above, the APS has developed a pilot industrial Measurement Access Mode (MAM) program to provide a way for industrial users to gain rapid access for one-time measurements to investigate specific problems. A MAM proposal expires after one visit.

The "APS User Information" page (https://www1.aps.anl.gov/Users-Informationl) provides access to comprehensive information for prospective and current APS users. 


\section{ARGONNE NATIONAL LABORATORY 400-AREA FACILITIES AdVANCED PHOTON SOURCE \\ (Beamlines, Disciplines, and Source Configuration)}

\section{ADVANCED PROTEIN CHARACTERIZATION FACILITY CENTER FOR NANOSCALE MATERIALS}

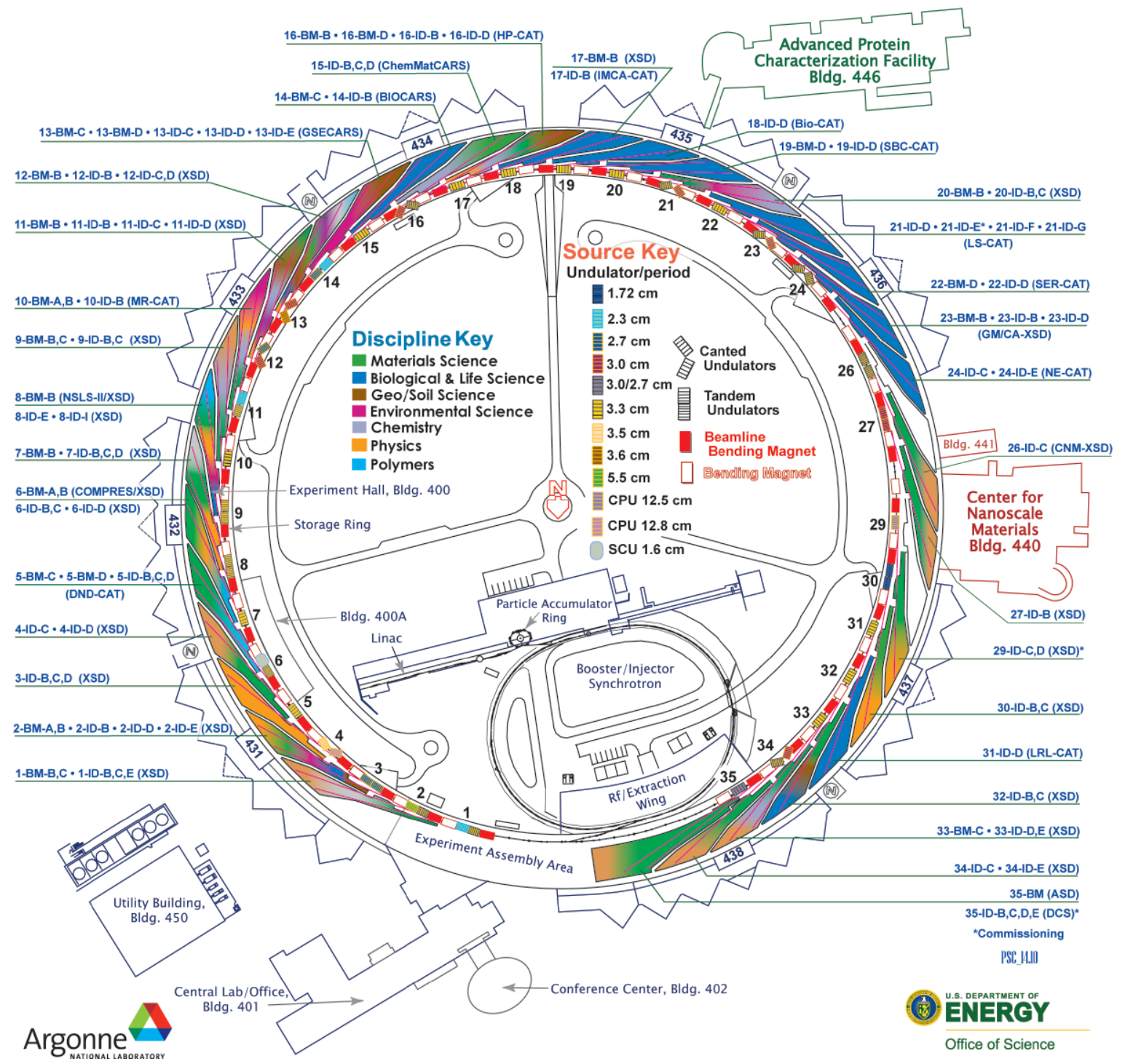

APS SECTORS: At the APS, a "sector" comprises the radiation sources (one bending magnet and nominally one insertion device, although the number of insertion devices in the straight sections of the storage ring can vary), and the beamlines, enclosures, and instrumentation that are associated with a particular storage ring sector. The APS has 35 sectors dedicated to user science and experimental apparatus. X-ray Science Division (XSD) sectors comprise those beamlines operated by the APS. Collaborative access team (CAT) sectors comprise beamlines operated by independent groups made up of scientists from universities, industry, and/or research laboratories.

Key to the beamline descriptions that accompany each science highlight: Beamline designation - Sector operator • Disciplines $\bullet$ Techniques $•$ Radiation source energy • User access mode(s) • General-user status $\bullet$ 


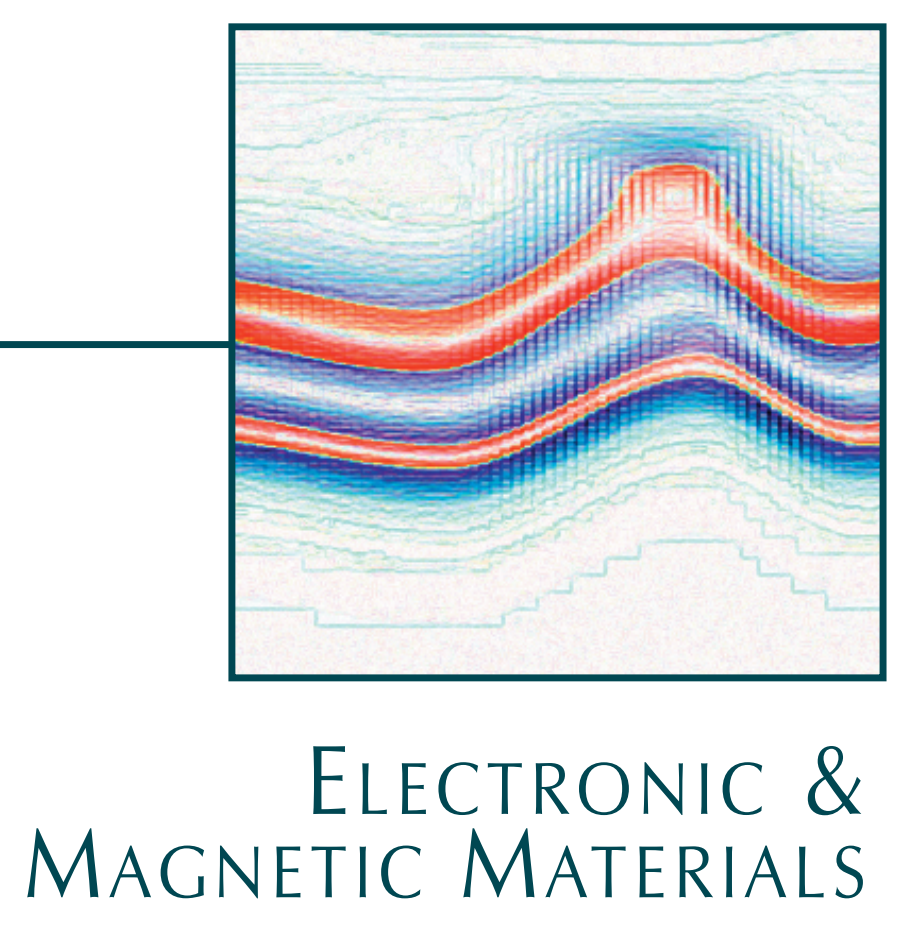




\section{Stressing Out Copper TSVs with Temperature}

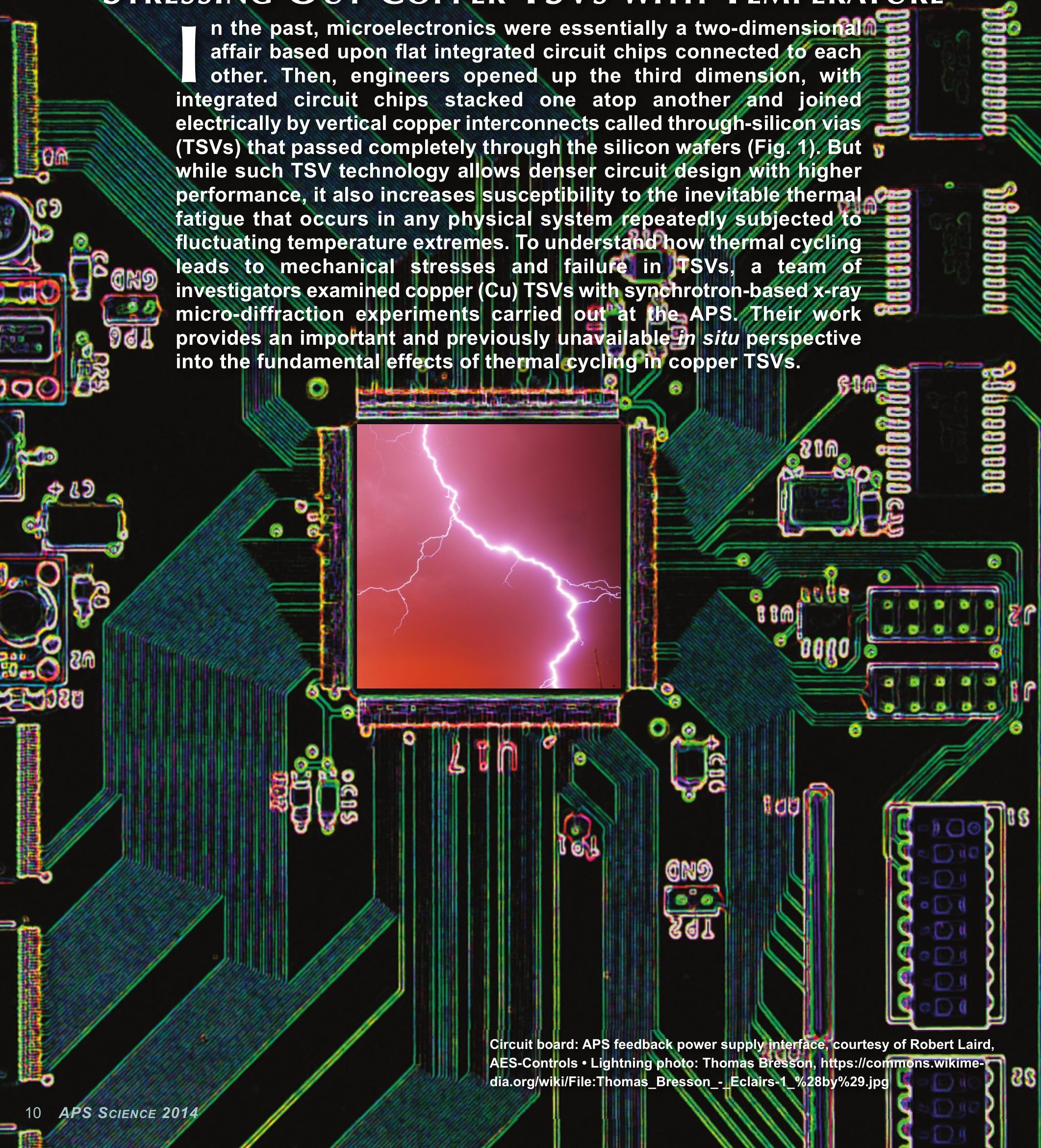


Based on these experiments, the investigators from the $\mathrm{Na}$ tional Institute of Standards and Technology, Argonne, and SEMAT$\mathrm{ECH}$ suggest that currently employed "keepout zone" design rules should be modified to account for the observed evolution of these thermally-induced stresses over time, instead of merely accounting for only the static stresses present in newly-fabricated $\mathrm{Cu}$ TSVs.

While some previous studies have examined the reliability of Cu TSV interconnects after thermal cycling, no work had been published that experimentally related the built-up stresses (which are the underlying cause of failure in $\mathrm{Cu}$ TSVs) with the witnessed number of thermal cycles.

In this experiment, the researchers examined and compared $\mathrm{Cu}$ TSVs in an "as received" state (new and unused, without any thermal cycling except during the manufacturing process) with identical TSVs subjected to 1000 thermal cycles.

Utilizing their previously demonstrated measurement procedure, which includes a depth-profiler and three optimally positioned area detectors, the investigators achieved in situ determination of the full strain and stress tensors as a function of TSV depth.

Samples were studied at the XSD 34-ID-E beamline at the APS. The team subjected one TSV to thermal cycling that involved repeated heating and cooling from $30^{\circ} \mathrm{C}$ to $150^{\circ} \mathrm{C}$. The $\mathrm{x}$-ray micro-diffraction instrument on the 34ID-E beamline provided depth-dependent data for stress characterization, along with information on lattice and crystallographic structure.

The stresses in the as-received $\mathrm{Cu}$ TSV sample were quite low, although they fluctuated somewhat along the
TSV depth. The mean absolute hydrostatic stress in the sample was measured at $16 \pm 44 \mathrm{MPa}$, a value attributed to stress relaxation resulting from storage at room temperature for nine months.

The TSV subjected to 1000 thermal cycles also displayed significant fluctuations in stress along its depth. However, the stresses were significantly greater, measuring an average of eight times higher at $123 \pm 37 \mathrm{MPa}$. Significant differences were also measured in shear stresses between the asreceived sample and the cycled TSV at different depths.

The as-received TSV showed a mean absolute shear stress of $28 \pm 20$ $\mathrm{MPa}$ with a maximum of $73 \pm 19 \mathrm{MPa}$ at a 32.5- $\mu \mathrm{m}$ depth, while the cycled TSV demonstrated a maximum shear stress of $-118 \pm 18 \mathrm{MPa}$ at $22.5-\mu \mathrm{m}$ depth, and a mean absolute shear stress of approximately $34 \mathrm{MPa}$. Differences in grain orientation coupled with the variation in the confinement of the TSV resulted in the depth-dependent stress fluctuations measured in both the as-received and cycled TSV samples.

The researchers cite strain hardening resulting from the entanglement of dislocations as the cause of the much higher stresses observed in the 1000-cycled TSV sample, which measured $353 \pm 21 \mathrm{MPa}$ at their maximum value in the center of the TSV.

The continued increase in dislocation density and entanglement caused by repeated thermal cycling will eventually lead to the formation of defects such as microscopic cracks and voids, which can result in structural weakness and failure as damage continues to accumulate. These researchers note that such repeated thermal cycling and damage in Cu TSVs will adversely affect the performance and lifespan of neighboring front-endof-line devices. - Mark Wolverton

See: Chukwudi Okoro ${ }^{1}$, Lyle E. Levine $^{1}$, Ruqing $\mathrm{Xu}^{2}$, Klaus Hummler $^{3}$, and Yaw Obeng ${ }^{1}$, "Synchrotron-based measurement of the impact of thermal cycling on the evolution of stresses in Cu throughsilicon vias," J. Appl. Phys. 115, 243509 (2014). DOI:

10.1063/1.4885461]

Author affiliations: ${ }^{1}$ National Institute of Standards and Technology,

${ }^{2}$ Argonne National Laboratory, ${ }^{3}$ SEMATECH

Correspondence:

*chukwudi.okoro@nist.gov

This research used resources of the Advanced Photon Source, a U.S. Department of Energy Office of Science User Facility operated for the DOE Office of Science by Argonne National Laboratory under Contract No. DE-AC02-06CH11357.

34-ID-E $\cdot X S D \cdot$ Materials science, physics, environmental science, geoscience $\cdot \mathrm{Mi}$ crodiffraction, Laue crystallography, microbeam $\cdot 7-30 \mathrm{keV} \cdot$ On-site $\cdot$ Accepting general users • 


\section{Earth-Crushing Pressure? This Electron Spin Doesn't Care}

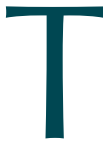

o fully understand something, it is often instructive to view it at its extremes. How do materials behave when their bits are forced much closer together than is comfortable? How do electrons accommodate proximity? What normal behaviors break down? The highest pressure a nuclear submarine can safely experience is around $850 \mathrm{lbs}$ per sq in. (psi), or nearly 6 million Pascals $(\mathrm{Pa})$. Pressure washers spew out water at roughly five times this pressure, or 30 million $\mathrm{Pa}$. Three-hundred miles below the surface of the Earth, in a part of the mantle called the transition zone, minerals are squeezed at an immense 15-billion $\mathrm{Pa}$ - enough to turn carbon into diamonds. This is the kind of intense environment that researchers working at the APS applied to GdSi, a compound of gadolinium $(\mathrm{Gd})$ and silicon ( $\mathrm{Si}$ ) with the potential to be well-suited for computer memory and magnetic sensors. These results provide new information about the interplay between electron spin, charge, and atomic arrangement, and how a material's magnetic properties change under extreme conditions.

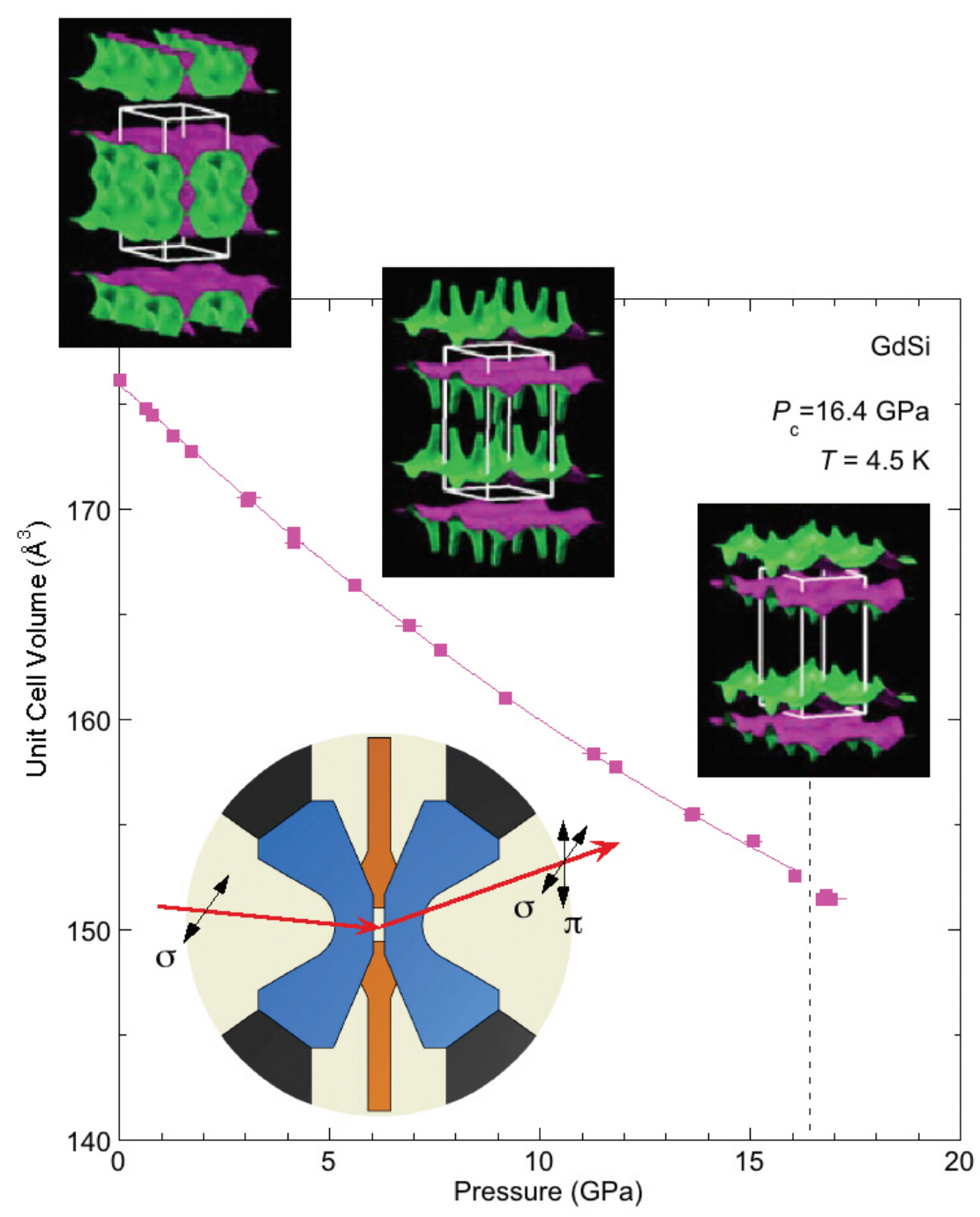


While a diamond anvil cell bore down on the crystalline fleck, $x$-rays penetrated within, bouncing off of, ejecting, and energizing the material's component parts, and relaying back to the researchers information about the locations and states of its atoms and electrons.

Despite shrinking by one-seventh of its volume, the team discovered that GdSi's magnetism remained robust. This behavior was surprising. High pressure will often quench a magnet. But here, the electrons responded to the harsh conditions by forming a resilient superstructure -ideal behavior for digital memory that needs to withstand abuse.

Some computer memory is based on a concept called "giant magnetoresistance." These devices consist of thin, alternating layers of magnetic and non-magnetic materials that interact such that changes in magnetic attraction and repulsion of one layer affect the electrical resistance in the next. Small magnetic fluctuations can create large electronic variations. In this way, digital information can be stored: electric signals change the characteristics of the magnetic material and when the user wants that information back, the magnetic material changes electron flow in the adjacent non-magnetic layer and the digital information is read back.

< Fig. 1. X-rays from a synchrotron-based source are so brilliant that it is now possible to perform magnetic diffraction in a specially-designed, high-pressure diamond anvil cell at pressures above $15 \mathrm{GPa}$ (circular inset). A sample's magnetic nature is reflected in the modified polarization of the x-rays in both transverse directions. When applied to GdSi, the researchers were able to directly trace its antiferromagnetism over a large volume reduction of up to $1 / 7$ th of the original size (main figure). The magnetism was found to remain constant, while the pressure's effect is reflected in the gradual change in the material's band structure. The Fermi surface, which supports the formation of itinerant antiferromagnetism at ambient pressure, grows increasingly one dimensional (to a sheet shape) under pressure (three inset panels). However, as long as it survives, magnetism does as well.
One of the challenges with memory storage is stability. Sometimes the thin layers of material do not match up, and this causes great internal stress. Heat adds another layer of instability. If these stresses change the magnetic material, information is lost. A material like GdSi, which retains its magnetism despite great stress, could make for very stable memory storage.

But what causes this behavior? It can be traced, in part, to a cooperative interaction between mobile electrons and localized spins. With high-sensitivity x-ray diffraction, the researchers examined both the atomic structure and the magnetic state of the GdSi crystal as it was squeezed from all sides (see the figure). Until recently, such direct magnetic tracking was nearly impossible and in fact, this is the first successful use of x-ray magnetic diffraction at such high pressures.

Previously, researchers had to rely on more indirect methods. Now, utilizing the high-brightness $x$-rays from the 4-ID-D x-ray beamline at the APS, the team could "watch" how the magnetic states evolved and compare their observations with theoretical calculations. Previous work had shown that these interactions should stabilize GdSi's magnetism, but now the researchers were able to prove that these effects remained under high pressure.

With experimentally sourced lattice information, the researchers in this study from Argonne; The University of Chicago; Los Alamos National Laboratory; the National Science Foundation; the University of Tennessee, Knoxville; and Oak Ridge National Laboratory then calculated the structure of GdSi's electronic energy bands - the ranges of energy that an electron within the material may assume. These energy bands depend on many factors, including the location of atomic species, as well as the long-range atomic patterns (Fig. 1).

The researchers found that through the entire pressure range one dominant band flattened yet still persisted. This continuity likely benefited from the material's stable atomic structure - Gd atoms in hexagonal sheets, layered to form tubes surrounding paired Si atoms, a linear arrangement that translated into robust electronic bands. Each is connected: the lattice created a stable electron band, which together with stable local spins helped to maintain the material's robust magnetism despite immense pressure.

The interplay between electron spin, charge, and atomic arrangement is complex and not always fully understood. GdSi clearly has potential as a material well-suited to computer memory and magnetic sensors but beyond this, the ability to observe how a material's magnetic properties change under extreme conditions should promote more discoveries. Researchers now have a new window into the complex lives of electrons under stress.

$$
\text { - Jenny Morber }
$$

See: Yejun Feng ${ }^{1,2 *}$, Jiyang Wang ${ }^{2}, A$. Palmer $^{2}$, J.A. Aguiar ${ }^{3}$, B. Mihaila ${ }^{4}$, J.-Q. Yan $^{5,6}$, P.B. Littlewood ${ }^{2,1}$, and T.F. Rosenbaum ${ }^{2 * *}$, "Hidden one-dimensional spin modulation in a three-dimensional metal," Nat. Commun. 5, 4218 (2014).

DOI: $10.1038 /$ ncomms5218

Author affiliations: ${ }^{1}$ Argonne National Laboratory; ${ }^{2}$ The University of Chicago; ${ }^{3}$ Los Alamos National Laboratory; ${ }^{4} \mathrm{Na}-$ tional Science Foundation; ${ }^{5}$ University of Tennessee, Knoxville; ${ }^{6}$ Oak Ridge National Laboratory

Correspondence: * yejun@aps.anl.gov, ** t-rosenbaum@uchicago.edu

P.B.L. was supported by the Materials Sciences and Engineering Division, Basic Energy Sciences, U.S. Department of Energy (DOE). B.M. was supported in part by the DOE under the LANL-LDRD program. A.P was supported in part by the DOE Office of Science Graduate Fellowship under Contract No. DEAC05-06OR23100. under a Material Transfer Agreement. This research used resources of the Advanced Photon Source, a DOE Office of Science User Facility operated for the DOE Office of Science by Argonne National Laboratory under Contract No. DE-AC02-06CH11357.

4-ID-D - XSD - Physics, materials science • Anomalous and resonant scattering (hard $x$ ray), magnetic $x$-ray scattering (hard $x$-ray), magnetic circular dichroism $\bullet 2.7-40 \mathrm{keV} \cdot$ Onsite $\cdot$ Accepting general users $•$ 


\section{TO UNDERSTAND WHAT MAKES A TOPOLOGICAL INSULATOR, ZOOM OUT}

$\mathrm{T}$

en years ago, a student in an undergraduate physics course would likely learn electron behavior in three basic material types: conductors, insulators, and semiconductors. But about five years ago, an exciting newcomer hit the physics spotlight - topological insulators. Topological insulators act like insulators on the interior, but conduct on the surface. To better understand these unique materials, an international team of researchers carried out experiments at the APS seeking answers to questions such as: Which compounds can exhibit topological states? And what, exactly, are the necessary conditions? A better understanding of the electron interactions that lead to topological states may help advance spintronics devices and practical quantum computing. Perhaps more importantly, studies like these give scientists another puzzle piece to a phenomenon many regard as one of the most exciting physics developments of this decade.

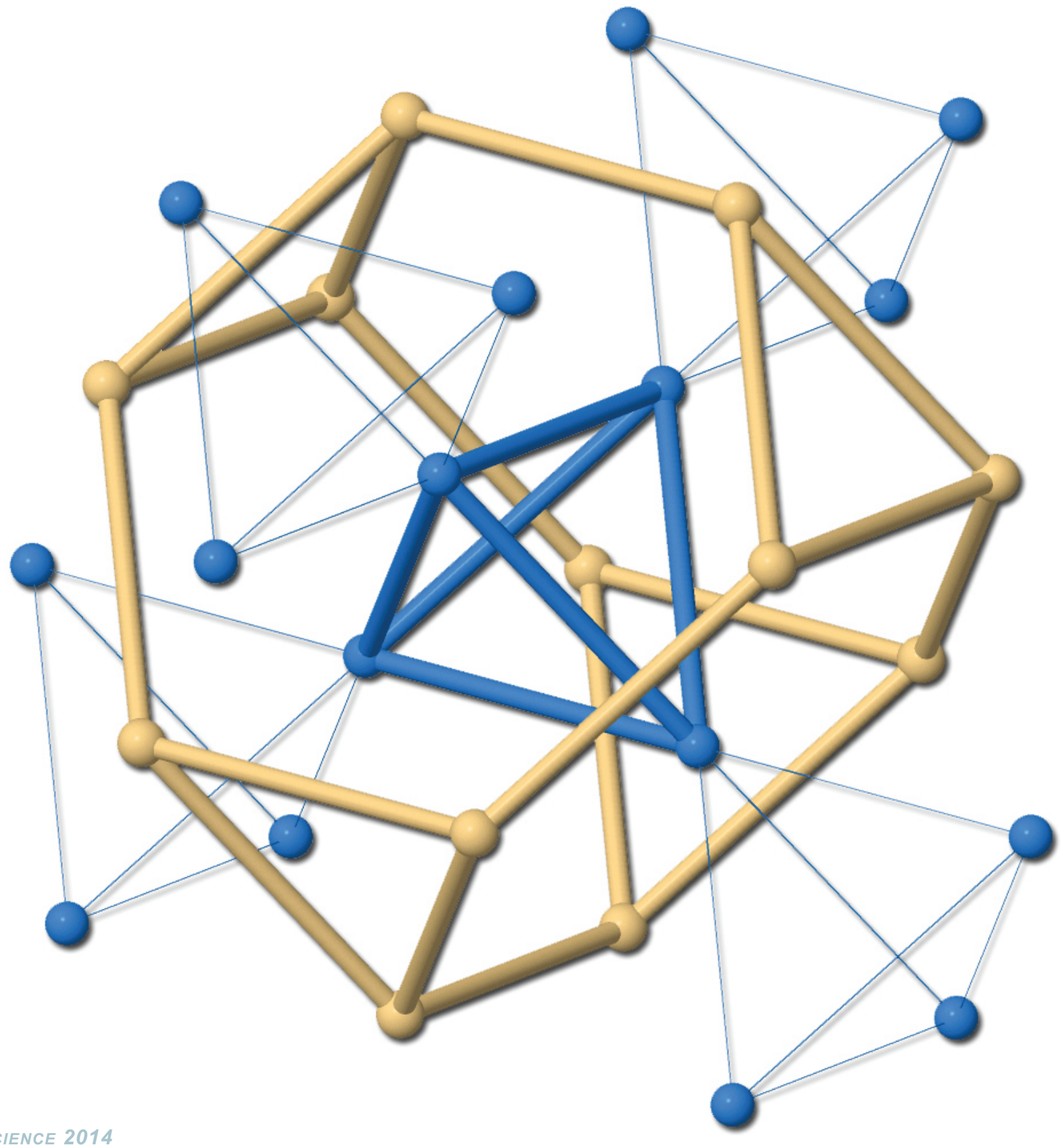


Unlike most materials, which are insulators or conductors through and through, topological insulators conduct only on the surface. These materials behave as if there is a strong magnetic field holding otherwise conducting electrons in place on the inside whose influence breaks up at the edges. Except there is no external magnetic field - the effect comes about from interactions between electrons' orbital movements around the nucleus and their spin. It's a little like a waltz. If electron spins match up just right with their orbital motion, they can "dance" around the outside, while electrons in the middle only spin and never move around.

To probe electron behavior, the researchers from IFW Dresden (Germany), the University of Toronto (Canada), Seoul National University (South Korea), the Max-Planck-Institut für Physik komplexer Systeme (Germany), the Joint Institute for Nuclear Research (Russia), POSTECH (South Korea), Argonne, and the Technical University Dresden (Germany) utilized inelastic $x$-ray scattering at XSD beamline 9-ID-B,C at the APS. (Supplementary high-resolution measurements using the MERIX spectrometer were carried out on XSD beamline 30-ID-B,C). The research team employed the inelastic $x$ ray scattering technique, in which energy in the form of an x-ray photon kicks an electron up to a higher energy level, leaving a lower-level hole. A nearby electron falls down to fill the hole, and releases its own photon. By carefully recording the energy of the photons going into a material, and the energy of the photons coming out, researchers can develop an understanding of how the material's electrons are arranged.

The team studied how electrons

< Fig. 1. Three-dimensional network of cornersharing Iridium (Ir) tetrahedra (blue) in $\mathrm{A}_{2} \mathrm{Ir}_{2} \mathrm{O}_{7}$ iridates, and the hexagonal rings of $A$ ions (yellow) around four of the Ir sites. Each hexagon is part of a planar Kagome net. (A Kagome net is a two-dimensional pattern of edge-sharing hexagons and triangles.) The smaller-volume $\mathrm{O}$-ligand cages around each cation, distorted $\mathrm{O}_{6}$ octahedra around $\mathrm{Ir}$, and distorted $\mathrm{O}_{8}$ cubes around the $A$ sites, are not shown. in compounds that may form topological insulators fill their energy levels, and how these energy levels change as key elements change. Here the investigated compounds were of the form $\mathrm{A}_{2} \mathrm{Ir}_{2} \mathrm{O}_{7}$, where $A$ can be any of the lanthanide metal elements samarium (Sm), europium (Eu), lutetium (Lu), or transition metal yttrium (Y; Ir is iridium and $O$ is oxygen.)

Topological states had been predicted in these compounds, but whether the materials actually exhibit the unique conducting behavior was unclear. In these materials, the strength of spin-orbit interactions matches well with that of the electron-electron interactions. The former give rise to topological states, while the latter are detrimental to them. It is a competition, and it was felt that the outcome hinged on distortions around small groups of atoms.

In $\mathrm{A}_{2} \mathrm{Ir}_{2} \mathrm{O}_{7}$ compounds, the $\mathrm{A}$ atoms frame a hexagonal ring around each $\mathrm{IrO}_{6}$ octahedron. Above and below are three additional $\mathrm{IrO}_{6}$ octahedra (Fig. 1). Theory predicted that jostling and distortions of and among the $\mathrm{O}_{6}$ ligand cages would determine topological phase behavior in such $\mathrm{A}_{2} \mathrm{Ir}_{2} \mathrm{O}_{7}$ materials. Instead, the x-ray measurements and electronic-structure calculations showed that the influence from interactions across the extended three-dimensional networks of both $A$ and Ir sites is much stronger (Fig. 2). It turns out that local ligand distortions don't matter nearly as much as longer-range lowsymmetry fields.

These results are not just relevant for the few compounds investigated here. The authors suggest that researchers may have underestimated longer-range electrostatics in numerous transition-metal series compounds. Failure to accurately predict topological states indicates that we do not yet fully understand them. Work such as this draws us closer to understanding and exploiting this exciting new topological state of matter. - Jenny Morber

See: L. Hozoi ${ }^{1 *}$, H. Gretarsson², J.P. Clancy ${ }^{2}$, B.-G. Jeon ${ }^{3}$, B. Lee ${ }^{3}$, K.H. Kim ${ }^{3}$, V. Yushankhai ${ }^{4,5}$, Peter Fulde $^{4,6}$, D. Casa ${ }^{7}$, T. Gog${ }^{7}$, Jungho $\mathrm{Kim}^{7}$, A.H. Said ${ }^{7}$, M.H. Upton ${ }^{7}$, YoungJune $\mathrm{Kim}^{2}$, and Jeroen van den Brink $^{1,8}$, "Longer-range lattice

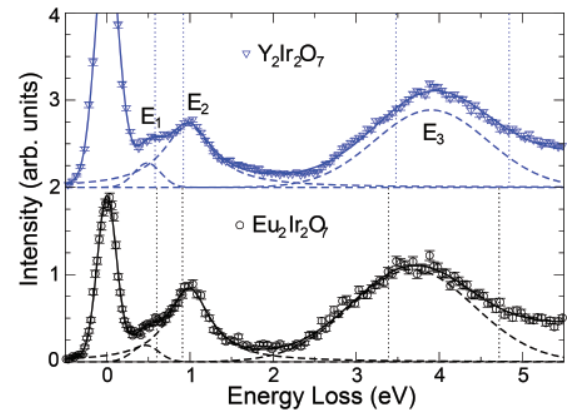

Fig. 2. Resonant inelastic $x$-ray scattering spectra of $\mathrm{Y}_{2} \mathrm{Ir}_{2} \mathrm{O}_{7}$ and $\mathrm{Eu}_{2} \mathrm{Ir}_{2} \mathrm{O}_{7}$ at the $\mathrm{Ir}$ L-edge. (The edge name depends on the core electron excited by the x-ray. Here, L-edge indicates $2 p$ electrons.) Dashed curves are the result of fitting; vertical dashed lines show the results of electronic-structure calculations for the $\operatorname{Ir} 5 d$ level excitations. Two distinct spectral features, $E_{1}$ and $E_{2}$, in the range of $0.5-1 \mathrm{eV}$ indicates significant deviations from cubic symmetry.

anisotropy strongly competing with spin-orbit interactions in pyrochlore iridates," Phys. Rev. B 89, 115111 (2014). DOI: 10.1103/PhysRevB.89.115111 Author affiliations: ${ }^{1} \mathrm{IFW}$ Dresden, ${ }^{2}$ University of Toronto, ${ }^{3}$ Seoul National University, ${ }^{4}$ Max-Planck-Institut für Physik komplexer Systeme, ${ }^{5}$ Joint Institute for Nuclear Research, ${ }^{6} \mathrm{POSTECH},{ }^{7} \mathrm{Ar}-$ gonne National Laboratory, ${ }^{8}$ Technical University Dresden Correspondence:

\section{*I.hozoi@ifw-dresden.de}

Research at the University of Toronto was supported by the Natural Sciences and Engineering Research Council of Canada, the Canada Foundation for Innovation, and the Organic Materials Review Institute. Work at Seoul National University was supported by the National CRI (2010-0018300) program. L.H. acknowledges financial support from the German Research Foundation (Deutsche Forschungsgemeinschaft, DFG). This research used resources of the Advanced Photon Source, a U.S. Department of Energy Office of Science User Facility operated for the DOE Office of Science by Argonne National Laboratory under Contract No. DE-AC02-06CH11357.

9-ID-B,C $\cdot$ XSD • Chemistry, materials science - Liquid surface diffraction, ultra-small-angle $x$ ray scattering $\bullet$ 4.5-30 keV $\bullet$ On-site $\cdot$ Accepting general users

30-ID-B,C • XSD • Physics, materials science - Inelastic x-ray scattering • 5-30 keV, 23.7$23.9 \mathrm{keV} \cdot$ On-site Accepting general users • 


\section{HOW MAGNeTIC Dimers INTERACT to Create long-Range Order}

$\mathrm{R}$

esearchers carrying out experiments at the APS and at the Spallation Neutron Source at Oak Ridge National Laboratory have shed new light on how

magnetic long-range order forms and remains stable despite the presence of disruptive quantum fluctuations. Since the material under study, $\mathrm{SrCu}_{2}\left(\mathrm{BO}_{3}\right)_{2}(\mathrm{SCBO})$, bears important similarities to layered $\mathrm{Cu}-\mathrm{O}$ compounds that exhibit high-temperature superconductivity, the research may provide insights into this form of quantum magnets as well, which has eluded thorough understanding for nearly 30 years.

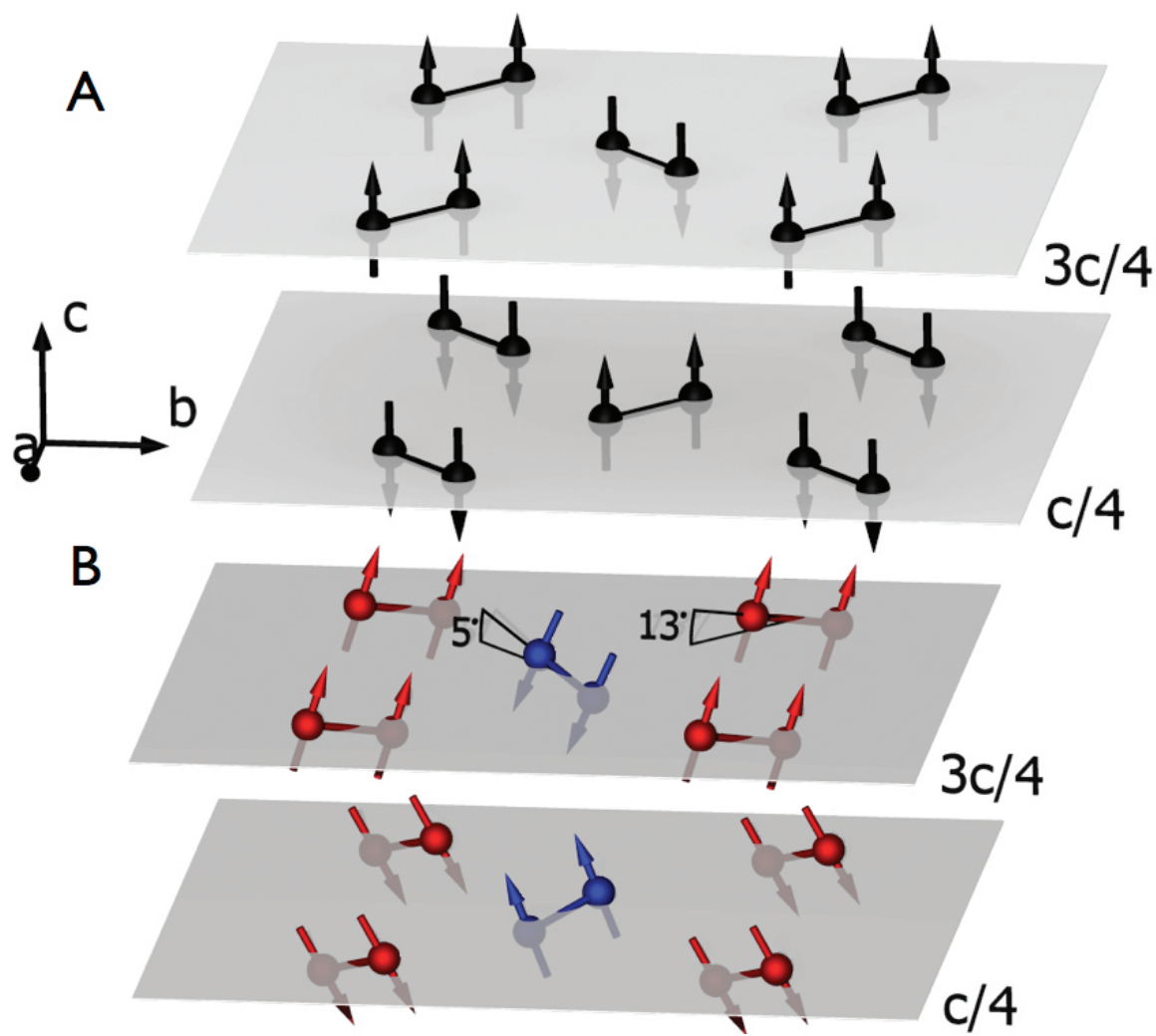

Fig. 1. Schematic of local dimer rearrangements at the onset of long-range order. (A) Magnetic structure of SCBO based on the theoretical model's prediction of an antiferromagnetic ground state. The solid lines show the intra-dimer $\mathrm{Cu}^{2+}$ couplings. (B) Proposed magnetic structure of SCBO extracted from diffraction data collected at a pressure of $5.5 \mathrm{GPa}$ and temperatures below about $122 \mathrm{~K}$. The no longer equivalent red and blue $\mathrm{Cu}-\mathrm{Cu}$ dimers are elongated and compressed, respectively, and tilt out of the crystallographic a-b plane.
SCBO contains magnetic spins that derive from $\mathrm{Cu}^{2+}$ ions sitting at the corners of square lattices on stacked, wellseparated, two-dimensional sheets. The result is a network of $\mathrm{Cu}-\mathrm{Cu}$ couplings of various strengths. Copper ions that are closest to one another on each atomic sheet are strongly bound together, forming dimers through exchange interactions. These $\mathrm{Cu}$ ion dimers experience inter-dimer couplings with nearest-neighbor dimers on the same sheet and with nearby dimers on adjacent sheets. The dimers give rise to a non-magnetic state at ambient pressure; however, it has been theoretically predicted that SCBO can be driven to an antiferromagnetic state by applying sufficient pressure.

SCBO is an experimental realization of an important theoretical model of how sheets of magnetic spins interact. Although the antiferromagnetically ordered phase was predicted, it had not been directly measured nor was there any insight into how a two-dimensional system could accommodate long-range order. The researchers in this study, who are based at The University of Chicago, Argonne, the University of Bristol, Oak Ridge National Laboratory, and McMaster University, were able to observe the long-anticipated ordered phase using a new high-pressure single-crystal neutron scattering technique at the SNAP beamline of the U.S. Department of Energy's Spallation Neutron Source at Oak Ridge National Laboratory.

The team performed a battery of experiments using $x$ ray and neutron diffraction techniques at pressures up to 6 $\mathrm{GPa}$. These measurements revealed the complex interplay of structural distortions and spin alignments that permit longrange magnetic order to emerge. 


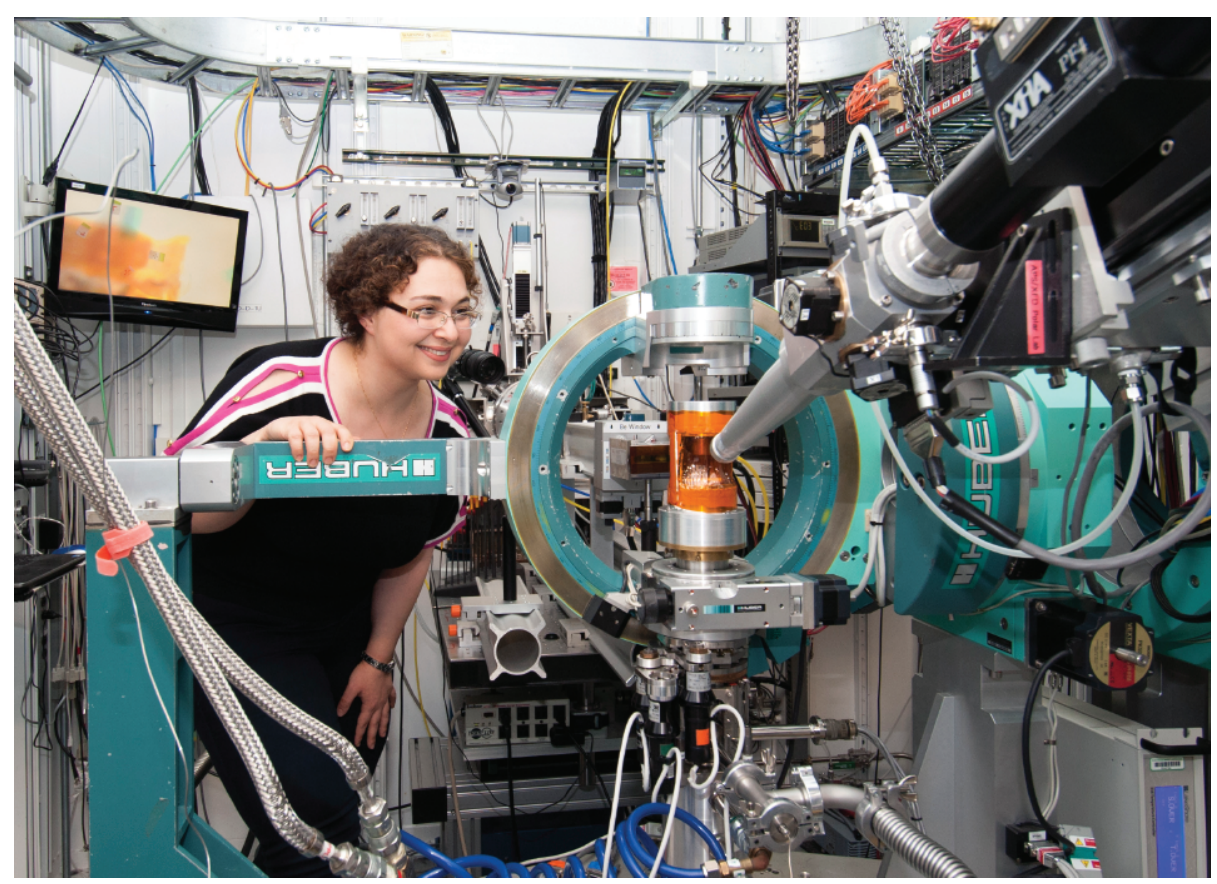

Sara Haravifard with the six-circle diffraction stage in the 4-ID-D experiment station.

The data showed that the antiferromagnetic state of SCBO becomes stabilized through subtle dimer contractions, elongations, and tilts, which impact the relative strengths of the intra-dimer and inter-dimer couplings.

The results of $x$-ray scattering measurements at XSD beamline 4-ID-D of the APS were particularly valuable in showing that couplings between the ordered, tilted dimers on consecutive sheets contribute importantly to the long-range order that arises, and thus that the antiferromagnetic phase emerges from inherently three-dimensional magnetic and structural distortions. The x-ray data also enabled the researchers to pinpoint the phase transition temperature as being $122 \mathrm{~K}$.

Complementary high-resolution powder neutron diffraction measurements conducted at the SNAP beamline added more detail to this picture by determining the atomic positions of the copper ions as they shifted with temperature at a pressure of $5.5 \mathrm{GPa}$. Above $122 \mathrm{~K}$, all of the dimers were found to be equivalent in that they were of equal length and remained within the plane of their respective atomic sheet.

At $122 \mathrm{~K}$ and below, these previously equivalent dimers separated into two interleaved sets of dimers of different lengths. One set consisted of elongated dimers, while the other set contained contracted dimers. Both sets of dimers became tilted as well, with the elongated dimers showing a larger slant $\left(13^{\circ}\right)$ than the subtle tilt $\left(5^{\circ}\right)$ evidenced by the contracted dimers (Fig. 1). These results provided additional evidence that the antiferromagnetic state in SCBO is due to three-dimensional magnetic and structural distortions.

At a pressure of $5.5 \mathrm{GPa}$, the researchers observed that the system went into the antiferromagnetic phase at temperatures below $122 \mathrm{~K}$. The results illuminate the microscopic mechanism needed to stabilize long-range order, revealing that the very subtle expansions, contractions, and tilts of the dimers permit the long-range order to emerge.

So although the system has a dimensional crossover from two dimensions to three dimensions, it is barely three dimensional, and the combination of $\mathrm{x}$-ray and neutron diffraction results indicate that this subtle emergence in the third dimension is driven by the anisotropic weak spin-orbit magnetic interactions between the $\mathrm{Cu}$ dimer layers. This underscores the importance of such interactions to the formation of exotic magnetic ground states.

$$
\text { - Vic Comello }
$$

See: S. Haravifard ${ }^{1,2}$, A. Banerjee ${ }^{1,3}$, J. van Wezel ${ }^{4}$, D.M. Silevitch ${ }^{1}$, A.M. dos Santos $^{3}$, J.C. Lang 2 , E. Kermarrec ${ }^{5}$, G. Srajer $^{2}$, B.D. Gaulin ${ }^{5}$, J.J. Molaison ${ }^{4}$,
H.A. Dabkowska ${ }^{5}$, and T.F. Rosenbaum ${ }^{1, *}$, "Emergence of Long-Range Order in Sheets of Magnetic Dimers", Proc. Natl. Acad. Sci. USA 111, 14372 (2014). DOI: 10.1073/pnas.1413318111 Author affiliations: ${ }^{1}$ The University of Chicago, ${ }^{2}$ Argonne National Laboratory, ${ }^{3}$ The University of Bristol, ${ }^{4}$ Oak Ridge National Laboratory, ${ }^{5}$ McMaster University ҒPresent address: California Institute of Technology

Correspondence: * tfr@caltech.edu

The work at The University of Chicago was supported by National Science Foundation Grant No. DMR-1206519. The work performed at the Advanced Photon Source was supported by the U.S. Department of Energy (DOE) Office of Science-Basic Energy Sciences under contract No. DE-AC02$06 \mathrm{CH} 11357$, and that at the Spallation Neutron Source was supported by the U.S. DOE Office of Science-Basic Energy Sciences. This research used resources of the DOE Office of Science User Facility operated for the DOE Office of Science by Argonne National Laboratory under Contract No. DE-AC02-06CH11357.

4-ID-D - XSD • Physics, materials science Anomalous and resonant scattering (hard $\mathrm{x}$ ray), magnetic $x$-ray scattering, magnetic circular dichroism (hard x-ray) $\cdot 2.7-40 \mathrm{keV}$ • On-site $\cdot$ Accepting general users $•$ 


\section{Squeezing Out the Hidden Lives of Electrons}

n our daily lives we tend to think of electrical conductivity as largely static: Copper is a good choice for conduction; clay is not. But heat up that copper wire, and electron conduction slows. Give a flake of that ceramic a good squeeze, and conduction may perk up. Conductivity is determined by much more than simple chemistry. Metal-to-insulator transitions have excited and perplexed researchers for over a century, and they continue to provide fodder for research today. The key to understanding what causes changes in material conductivity lies in teasing out contributions from structural atomic arrangements and electron interactions. Researchers using high-energy $\mathrm{x}$ rays from the APS have managed to disentangle these components in vanadium sesquioxide $\left(\mathrm{V}_{2} \mathrm{O}_{3}\right)$, an extensively studied model solid. By decoupling the effects of spin, charge, and lattice variables in $\mathrm{V}_{2} \mathrm{O}_{3}$, the team is uncovering a mechanism that has eluded researchers for six decades.

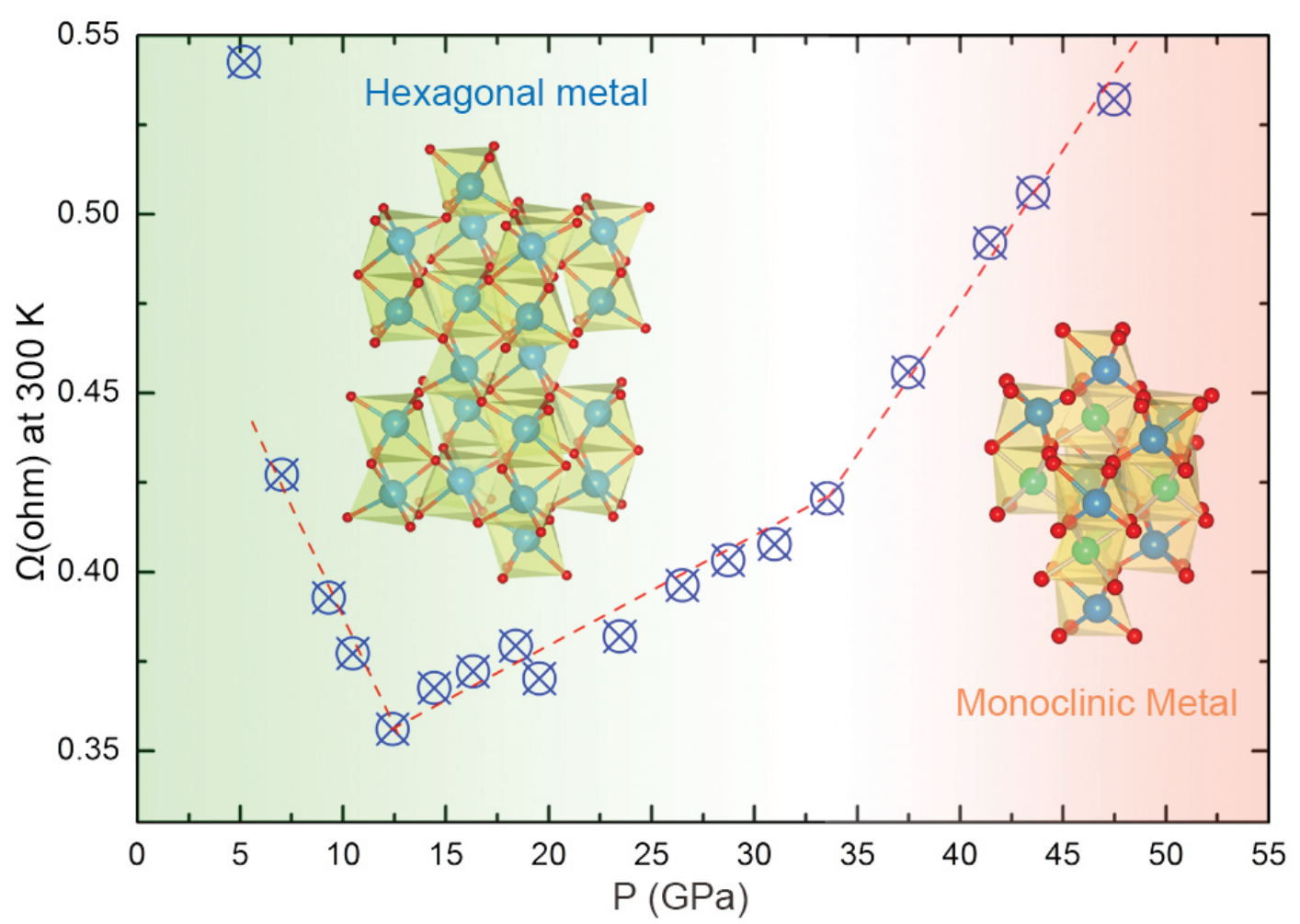

Fig. 1. Electrical resistance as a function of pressure in $\mathrm{V}_{2} \mathrm{O}_{3}$. As pressure increases from $5 \mathrm{GPa}$, resistance decreases as expected. At 12.5 GPa the sharp increase in resistance is an unexpected result of electron-lattice interactions. At $\sim 33 \mathrm{GPa}$, the material's corundum hexagonal structure changes to monoclinic, and resistance rises more sharply due to electron-electron interactions. Here the material is on the cusp of a metal-to-insulator transition. 
With measurements performed at the LERIX instrument at XSD beamline 20-ID-B,C, and at the HP-CAT beamline 16-BM-D, both at the APS, and calculations from the XSD Theory Group, the researchers have identified a structural phase change in $\mathrm{V}_{2} \mathrm{O}_{3}$ that occurs under great pressure, but without the usual metal-to-insulator transition. The interplay between crystal structure and electronic properties underlies almost every modern device, from pressure sensors to superconducting high speed trains.

Under normal conditions, $\mathrm{V}_{2} \mathrm{O}_{3}$ is a black metallic solid with a corundum crystal structure, like that of rubies and sapphires. With changes in temperature it undergoes spectacular metal-toinsulator transitions, often with changes in magnetic behavior as well. These unusual properties make $\mathrm{V}_{2} \mathrm{O}_{3}$ a material of choice in devices that include temperature sensors and current regulators.

Researchers had previously reported interesting behavior in $\mathrm{V}_{2} \mathrm{O}_{3}$ as temperature changed and pressure remained constant. Here the team tested the opposite condition, monitoring the material's resistance while increasing the pressure at a constant temperature.

At first everything seemed normal: as the pressure increased the material's resistance also decreased. But around $12.5 \mathrm{GPa}$ the resistance began to rise. This result was unexpected. Even more unusual, at greater pressures near $33 \mathrm{GPa}$, the material's structure changed from corundum to a more compact monoclinic arrangement of atoms, but this change was not accompanied by a corresponding spike in resistance (Fig. 1). The material remained metallic. Previously, all corundum to monoclinic changes in structure had been accompanied by a simultaneous transition from metallic to insulating behavior.

To understand what was happening, the researchers from Argonne; Stanford University; the Korea Advanced Institute of Science and Technology; the Pacific Northwest Consortium Synchrotron Radiation Facility; the
Carnegie Institution of Washington; Jilin University (China); the University of $\mathrm{Ne}$ vada, Las Vegas; the Center for High Pressure Science and Technology Advanced Research (China); and Northern Illinois University performed inelastic x-ray scattering measurements and compared the results with theoretical simulations. Because inelastic x-ray spectroscopy measures the unoccupied vanadium electron valence states, these measurements provide a more detailed picture of electron screening interactions.

While the resistivity measurements clearly showed changes at $12.5 \mathrm{GPa}$, the inelastic $x$-ray spectra showed no differences up to the phase change pressure of $33 \mathrm{GPa}$. This means that the early changes in resistance were due not to changes in electron correlations, but to interactions between electrons and the lattice (or phonons).

At high pressure the electronic structure changed drastically in the inelastic $x$-ray spectra, suggesting an increase in electron correlations, but not quite enough to tip the material into the category of an insulator. At such high pressure, $\mathrm{V}_{2} \mathrm{O}_{3}$ is on the verge of becoming an insulator, but can't quite make the change due to competing effects from the lattice.

This work adds another clue to our understanding of how long-range atomic arrangement and local electron interactions work competitively to manifest metal-to-insulator transitions in solids.

The next step will be to explore electron correlations in $\mathrm{V}_{2} \mathrm{O}_{3}$ by using more advanced techniques, such as the resonant $x$-ray inelastic scattering method with temperature, as another parameter to extend the unique phase diagram of $\mathrm{V}_{2} \mathrm{O}_{3}$. - Jenny Morber

See: Yang Ding ${ }^{1 *}$, Cheng-Chien Chen ${ }^{1}$, Qiaoshi Zeng ${ }^{2}$, Heung-Sik Kim³ ${ }^{3}$, Myung Joon $\mathrm{Han}^{3}$, Mahalingam Balasubramanian $^{1}$, Robert Gordon ${ }^{1,4}$, Fangfei $\mathrm{Li}^{5,6}$, Ligang Bai ${ }^{7}$, Dimitry Popov ${ }^{5}$, Steve M. Heald ${ }^{1}$, Thomas Gog ${ }^{1}$, Hokwang $\mathrm{MaO}^{5,8}$, and Michel van Veenendaal ${ }^{1,9}$, "Novel high-pressure monoclinic metallic phase of $\mathrm{V}_{2} \mathrm{O}_{3}$," Phys. Rev. Lett. 112, 056401 (2014). DOI: 10.1103/PhysRevLett.112.056401 Author affiliations: ${ }^{1}$ Argonne National Laboratory, ${ }^{2}$ Stanford University, ${ }^{3}$ Korea Advanced Institute of Science and Technology, ${ }^{4}$ Pacific Northwest Consortium Synchrotron Radiation Facility, ${ }^{5}$ Carnegie Institution of Washington, ${ }^{6}$ Jilin University, ${ }^{7}$ University of Nevada Las Vegas, ${ }^{8}$ Center for High Pressure Science and Technology Advanced Research, ${ }^{9}$ Northern Illinois University

Correspondence:

* yangding@aps.anl.gov

This research was supported in part by EFree, an Energy Frontier Research Center funded by the U.S. Department of Energy (DOE) Office of Science, Basic Energy Sciences (BES) under Award No.

DESC0001057. C.C.C. was supported by the Aneesur Rahman Postdoctoral Fellowship at ANL. M.v.V was supported by DOEBES under Award No. DE-FG02-03ER46097 and the NIU Institute for Nanoscience, Engineering and Technology. High Pressure Collaborative Access Team operations were supported by the DOE National Nuclear Security Administration under Award No. DENA0001974, and DOE-BES under Award No. DE-FG02-99ER45775, with partial instrumentation funding by the National Science Foundation. APS sector 20, which is managed by XSD in partnership with the Canadian Light Source (CLS), is funded by the U.S. Department of Energy Office of Science, and by the Natural Sciences and Engineering Research Council of Canada and the University of Washington via the CLS. This research used resources of the Advanced Photon Source, a U.S. DOE Office of Science User Facility operated for the DOE Office of Science by Argonne National Laboratory under Contract No. DE-AC02-

16-BM-D • HP-CAT • Materials science, geoscience, chemistry, physics $\bullet$ Powder angular dispersive $\mathrm{x}$-ray diffraction, $\mathrm{x}$-ray absorption near-edge structure, single-crystal diffraction, high-pressure diamond anvil cell $\cdot 6-70 \mathrm{keV} \cdot$

20-ID-B,C $\cdot X S D \cdot$ Materials science, environmental science, chemistry $\cdot X$-ray absorption fine structure, $x$-ray Raman scattering, micro $x$-ray absorption fine structure, microfluorescence (hard $x$-ray), time-resolved $x$-ray absorption fine structure, $x$-ray emission spectroscopy • 4.3-27 keV, 7-52 keV • On-site -Accepting general users 


\section{X-Ray Scattering Studies of the Spin Disorder and Magnetic Coupling in Chromium Spinels}

W

hile magnesium chromite $\left(\mathrm{MgCr}_{2} \mathrm{O}_{4}\right)$ and zinc chromite $\left(\mathrm{ZnCr}_{2} \mathrm{O}_{4}\right)$ spinels have been extensively studied, the effects of spin and lattice disorder on the structural ground states of these frustrated materials had not been investigated until recently. Employing variable-temperature high-resolution x-ray diffraction, performed on the XSD 11-BM-B powder diffraction beamline at the APS, and time-of-flight neutron scattering, performed at the Neutron Powder Diffractometer (NPD) at Los Alamos National Laboratory, researchers from the University of California, Santa Barbara; Argonne; the University of Illinois at Urbana-Champaign; and Los Alamos National Laboratory have shown that even slight spin disorder on the nonmagnetic $\mathrm{Mg}^{2+}$ and $\mathrm{Zn}^{2+}$ sites can significantly alter the structural ground states of these systems.

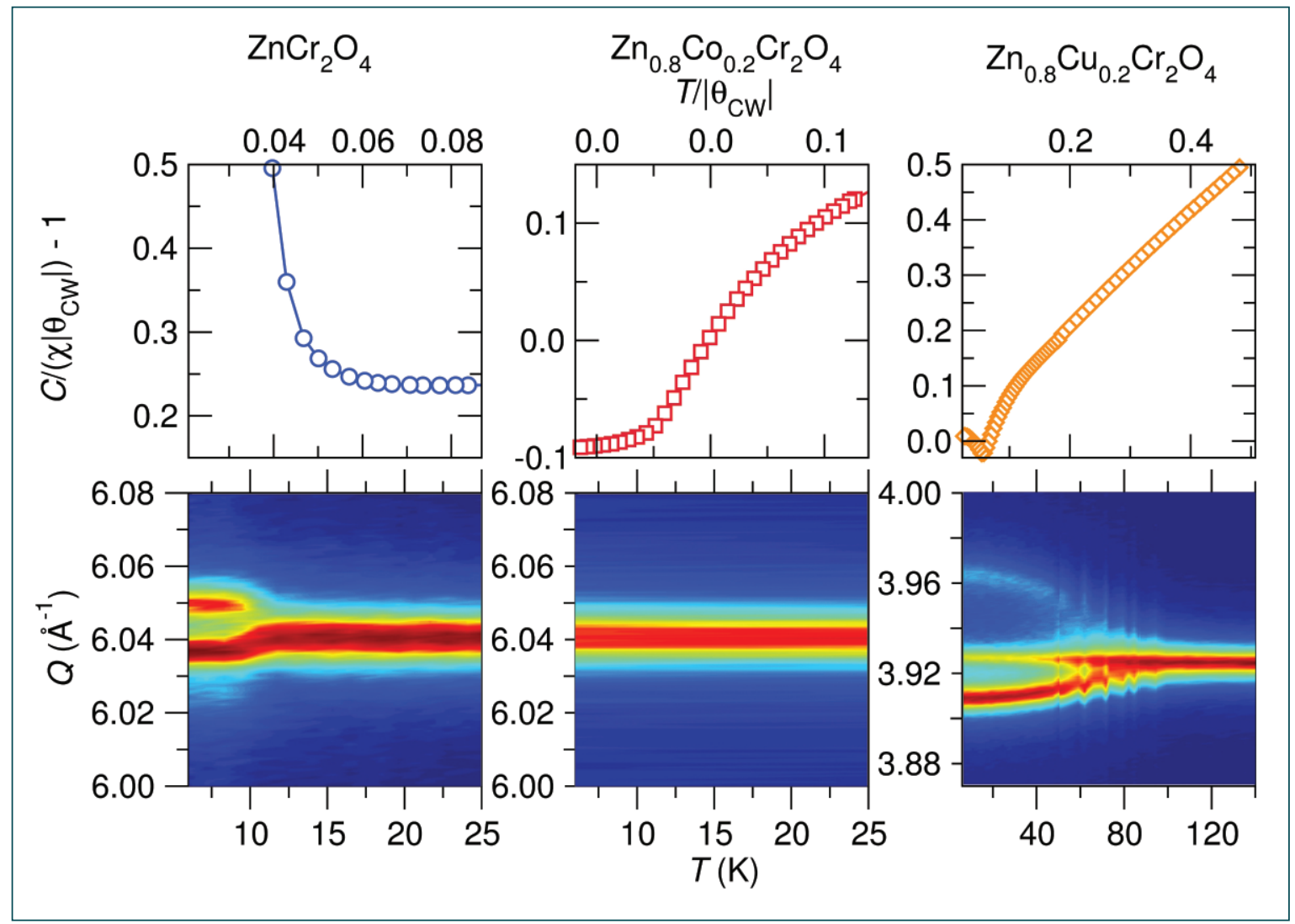


Chromium-rich spinels $\left(\mathrm{ACr}_{2} \mathrm{O}_{4}\right.$, where $A=\mathrm{Mg}, \mathrm{Zn}$ ) have attracted intense interest in recent years due to their multifaceted and often surprising magnetic ground state configurations. These ground states arise from a complex network of strong magnetic and structural interactions involving cations on two sublattices; $A^{2+}$ cations occupy tetrahedral sites on one sublattice and $\mathrm{Cr}^{3+}$ cations with antiferromagnetically interacting spins populate octahedral sites on the other sublattice. The geometric arrangement of $\mathrm{Cr}^{3+}$ cations on a pyrocholore network of corner sharing tetrahedra inhibits antiferromagnetic coupling, thus resulting in "geometric frustration." Spin frustration suppresses the onset of magnetic order to low temperatures and leads to structural distortions occurring concomitantly with antiferromagnetic ordering, an effect known as spin-Jahn-Teller ordering. Spinels exhibit a vast array of magnetic phenomena due to the presence of two cation sites, which accommodate significant compositional diversity along with the high degree of interconnectivity in this structure.

The researchers introduced small amounts of magnetic cations on the nonmagnetic $A$ sites of $\mathrm{MgCr}_{2} \mathrm{O}_{4}$ and $\mathrm{ZnCr}_{2} \mathrm{O}_{4}$. Specifically, they examined the effects of these dilute $A$-site spins on the structural changes that accompany mag-

$<$ Fig. 1. The evolution of magnetism (top panels) and structure (bottom panels) in the spinels $\mathrm{ZnCr}_{2} \mathrm{O}_{4}, \mathrm{Zn}_{0.8} \mathrm{Co}_{0.2} \mathrm{Cr}_{2} \mathrm{O}_{4}$, and $\mathrm{Zn}_{0.8} \mathrm{Cu}_{0.2} \mathrm{Cr}_{2} \mathrm{O}_{4}$. All of these spinels show frustrated magnetic interactions with magnetic ordering occurring at $12.3 \mathrm{~K}, 11 \mathrm{~K}$, and $16 \mathrm{~K}$ in $\mathrm{ZnCr}_{2} \mathrm{O}_{4}$, $\mathrm{Zn}_{0.8} \mathrm{Co}_{0.2} \mathrm{Cr}_{2} \mathrm{O}_{4}$, and $\mathrm{Zn}_{0.8} \mathrm{Cu}_{0.2} \mathrm{Cr}_{2} \mathrm{O}_{4}$, respectively. Magnetism evolves from antiferromagnetism in $\mathrm{ZnCr}_{2} \mathrm{O}_{4}$ to ferrimagnetism in $\mathrm{Zn}_{0.8} \mathrm{Co}_{0.2} \mathrm{Cr}_{2} \mathrm{O}_{4}$ and $\mathrm{Zn}_{0.8} \mathrm{Cu}_{0.2} \mathrm{Cr}_{2} \mathrm{O}_{4}$. The bottom panels show the changes in the high-temperature cubic (800) reflections with temperature. While the onset of antiferromagnetic order in $\mathrm{ZnCr}_{2} \mathrm{O}_{4}$ is accompanied by a structural distortion, substitution of $\mathrm{Co}^{2+}$ for $\mathrm{Zn}^{2+}$ completely suppresses the spin-Jahn-Teller distortion of $\mathrm{Zn}_{0.8} \mathrm{Co}_{0.2} \mathrm{Cr}_{2} \mathrm{O}_{4}$. In contrast, the substitution of $\mathrm{Cu}^{2+}$ for $\mathrm{Zn}^{2+}$ introduces lattice distortions above the magnetic ordering temperature of $\mathrm{Zn}_{0.8} \mathrm{Cu}_{0.2} \mathrm{Cr}_{2} \mathrm{O}_{4}$. Despite the high-temperature average structure distortion in $\mathrm{Zn}_{0.8} \mathrm{Cu}_{0.2} \mathrm{Cr}_{2} \mathrm{O}_{4}$, this system remains geometrically frustrated. netic ordering in these spinels. The magnetic substituents $\mathrm{Co}^{2+}$ and $\mathrm{Cu}^{2+}$ were selected, resulting in the powder samples of $\mathrm{Zn}_{1-\mathrm{x}} \mathrm{Co}_{\mathrm{x}} \mathrm{Cr}_{2} \mathrm{O}_{4}, \mathrm{Mg}_{1-\mathrm{x}} \mathrm{Cu}_{\mathrm{x}} \mathrm{Cr}_{2} \mathrm{O}_{4}$, and $\mathrm{Zn}_{1-\mathrm{x}} \mathrm{Cu}_{\mathrm{x}} \mathrm{Cr}_{2} \mathrm{O}_{4}$ (in all cases, $x \leq 0.2$ ). The similarity in ionic radii between the $A$-site cations in these compositions minimized lattice distortions due to cation size discrepancies while allowing the researchers to probe the effect of dilute $A$-site spins on the structural ground states of these spinels. While $\mathrm{Co}^{2+}$ and $\mathrm{Cu}^{2+}$ substitutions both introduce spin interactions on the nonmagnetic $A$ sites of $\mathrm{MgCr}_{2} \mathrm{O}_{4}$ and $\mathrm{ZnCr}_{2} \mathrm{O}_{4}$, $\mathrm{Cu}^{2+}$ cations have degenerate electronic ground states, and therefore they also introduce structural distortions around the $\mathrm{Cu}^{2+}$ cation in order to achieve a unique electronic ground state. The researchers showed that both $\mathrm{Co}^{2+}$ and $\mathrm{Cu}^{2+}$ substitutions alter the spin-Jahn-Teller distortions of $\mathrm{MgCr}_{2} \mathrm{O}_{4}$ and $\mathrm{ZnCr}_{2} \mathrm{O}_{4}$; however, these cations influence the structural ground states of $\mathrm{MgCr}_{2} \mathrm{O}_{4}$ and $\mathrm{ZnCr}_{2} \mathrm{O}_{4}$ differently.

The substitution of magnetic $\mathrm{Co}^{2+}$ on the nonmagnetic $\mathrm{Zn}^{2+}$ site in $\mathrm{Zn}_{1-\mathrm{x}} \mathrm{Co}_{\mathrm{x}} \mathrm{Cr}_{2} \mathrm{O}_{4}$ completely suppressed the spin-Jahn-Teller distortion of $\mathrm{ZnCr}_{2} \mathrm{O}_{4}$, although $\mathrm{Zn}_{1-\mathrm{x}} \mathrm{Co}_{\mathrm{x}} \mathrm{Cr}_{2} \mathrm{O}_{4}$ remained frustrated with magnetic ordering transitions occurring at temperatures below $20 \mathrm{~K}$. In contrast, spin and lattice disorder due to the substitution of $\mathrm{Cu}^{2+}$ in $\mathrm{Mg}_{1-\mathrm{x}} \mathrm{Cu}_{\mathrm{x}} \mathrm{Cr}_{2} \mathrm{O}_{4}$ and $\mathrm{Zn}_{1-\mathrm{x}} \mathrm{Cu}_{\mathrm{x}} \mathrm{Cr}_{2} \mathrm{O}_{4}$ induced so-called JahnTeller distortions above the magnetic ordering temperatures of these compounds. Despite the Jahn-Teller structural distortions in $\mathrm{Mg}_{1-\mathrm{x}} \mathrm{Cu}_{\mathrm{x}} \mathrm{Cr}_{2} \mathrm{O}_{4}$ and $\mathrm{Zn}_{1-\mathrm{x}} \mathrm{Cu}_{\mathrm{x}} \mathrm{Cr}_{2} \mathrm{O}_{4}$, antiferromagnetic interactions remained frustrated with longrange magnetic ordering occurring below $20 \mathrm{~K}$ with no accompanying structural transformations. In other words, the Jahn-Teller-active $\mathrm{Cu}^{2+}$ cations decoupled structural and magnetic ordering in $\mathrm{MgCr}_{2} \mathrm{O}_{4}$ and $\mathrm{ZnCr}_{2} \mathrm{O}_{4}$, even when substituted in only small amounts. The Jahn-Teller-distorted solid solutions $\mathrm{Mg}_{1-\mathrm{x}} \mathrm{Cu}_{\mathrm{x}} \mathrm{Cr}_{2} \mathrm{O}_{4}$ and $\mathrm{Zn}_{1-x} \mathrm{Cu}_{\mathrm{x}} \mathrm{Cr}_{2} \mathrm{O}_{4}$ adopted the orthorhombic structure of ferrimagnetic $\mathrm{CuCr}_{2} \mathrm{O}_{4}$, with analysis of distortions in $\mathrm{Zn}_{0.8} \mathrm{Cu}_{0.2} \mathrm{Cr}_{2} \mathrm{O}_{4}$ indicating a flattening of $\mathrm{AO}_{4}$ tetrahedra in the orthorhombic phase. Total neutron scattering studies of $\mathrm{Zn}_{1-\mathrm{x}} \mathrm{Cu}_{\mathrm{x}} \mathrm{Cr}_{2} \mathrm{O}_{4}$ suggested that there were local $A_{4}$ distortions in these $\mathrm{Cu}^{2+}$-containing solid solutions at room temperature and that these distortions became cooperative at the Jahn-Tellerdistortion temperature at which average structural distortions occur (Fig. 1).

Magnetism in these frustrated compounds evolves from compensated antiferromagnetism in $\mathrm{MgCr}_{2} \mathrm{O}_{4}$ and $\mathrm{ZnCr}_{2} \mathrm{O}_{4}$ to uncompensated antiferromagnetism when magnetic cations are substituted on the nonmagnetic sites. This finding shows that spin-Jahn-Teller ordering is extremely sensitive to spin disorder, while Jahn-Teller ordering is robust, occurring even when only a few Jahn-Teller-active cations are substituted into the spinel structure.

\section{- Vic Comello}

See: Moureen C. Kemei ${ }^{1}$, Stephanie L. Moffitt $^{1}$, Lucy E. Darago ${ }^{1}$, Ram Seshadri $^{1 *}$, Matthew R. Suchomel ${ }^{2}$, Daniel P. Shoemaker ${ }^{3}$, Katharine Page ${ }^{4}$, and Joan Siewenie 4 , "Structural ground states of $\left(A, A^{\prime}\right) \mathrm{Cr}_{2} \mathrm{O}_{4}\left(A=\mathrm{Mg}, \mathrm{Zn} ; A^{\prime}=\right.$ $\mathrm{Co}, \mathrm{Cu})$ spinel solid solutions: SpinJahn-Teller and Jahn-Teller effects," Phys. Rev. B 89, 174410 (2014). DOI: 10.1103/PhysRevB.89.174410 Author affiliations: ${ }^{1}$ University of California, Santa Barbara; ${ }^{2}$ Argonne National Laboratory; ${ }^{3}$ University of Illinois at Urbana-Champaign; ${ }^{4}$ Los Alamos $\mathrm{Na}$ tional Laboratory

Correspondence:

*seshadri@mrl.ucsb.edu

This project was supported by the National Science Foundation (NSF) through Grant No. DMR 1105301. M.C.K. was supported by the Schlumberger Foundation Faculty for the Future fellowship. The work benefited from the use of NPDF at the Los Alamos Neutron Scattering Center at Los Alamos National Laboratory (LANL), funded by the U.S. Department of Energy Office of Basic Energy Sciences. This research used resources of the Advanced Photon Source, a U.S. Department of Energy (DOE) Office of Science User Facility operated for the DOE Office of Science by Argonne National Laboratory under Contract

11-BM-B - XSD • Chemistry, materials science, physics, geoscience $\cdot$ Powder diffraction $\cdot 25-35 \mathrm{keV} \cdot$ On-site, mail-in • Accepting general users • 


\section{Tuning the Magnetic Energy SCales of STRONTIUM IRIDATE THIN FILMS}

7 here is a great deal of interest in strontium iridate $\left(\mathrm{Sr}_{2}\left(\mathrm{rO}_{4}\right)\right.$ because its novel magnetic properties are strikingly similar to those of the parent compounds of cuprate superconductors, raising the possibility that unconventional superconductivity could be realized in $\mathrm{Sr}_{2} \mid \mathrm{IO}_{4}$ by tuning elements of its crystal structure, such as its Ir-related bond angles and bond lengths. Strain engineering accomplishes structural tuning by growing a $\mathrm{Sr}_{2} \mathrm{IrO}_{4}$ thin film on a substrate whose crystal lattice differs slightly in size, thereby inducing strain-related structural distortions in the interfacial layers of the film. To date, most research has focused on the structural and electronic properties of strained $\mathrm{Sr}_{2} \mathrm{IrO}_{4}$ thin films, making recent experiments at the APS among the first to explore the effects of strain on their magnetic properties.

(a)

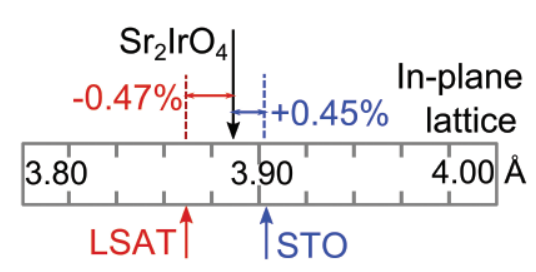

(b)

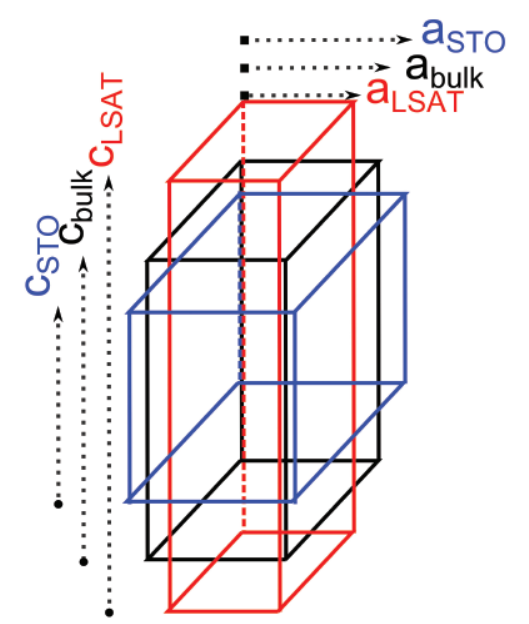

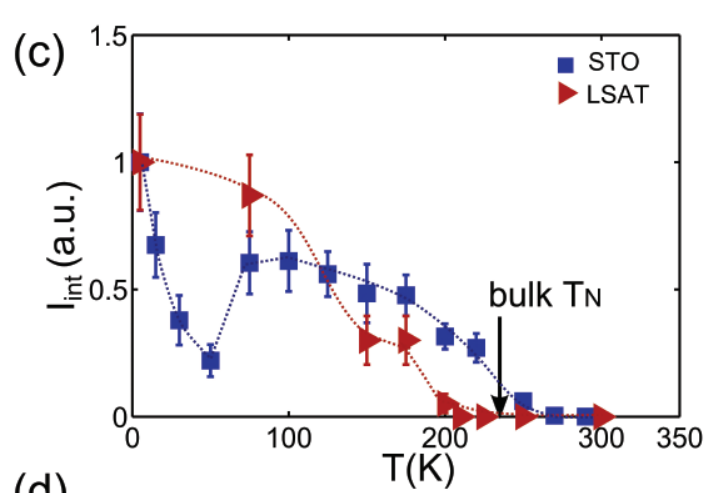

(d)

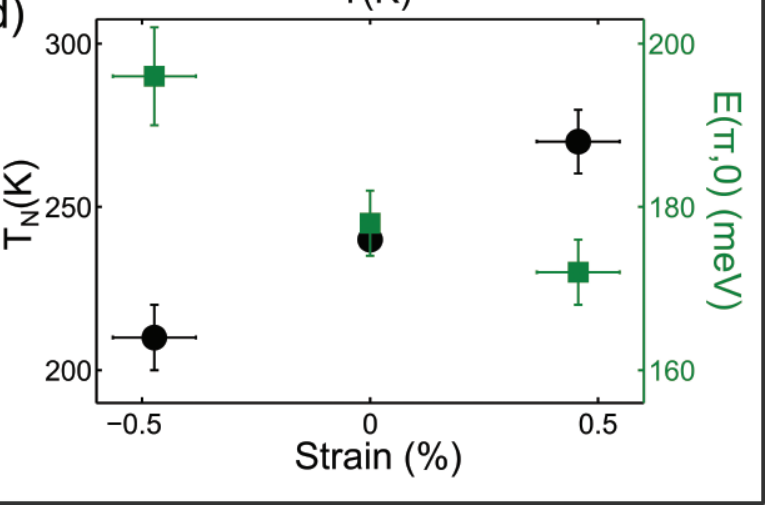

Fig. 1. (a) Comparison of the pseudo-cubic, in-plane lattice parameter of $\mathrm{Sr}_{2} \mid \mathrm{IO}_{4}$ with the lattice parameters of the $\left(\mathrm{LaAlO}_{3}\right)_{0.3}\left(\mathrm{Sr}_{2} \mathrm{AlTaO}_{6}\right)_{0.7}(\mathrm{LSAT})$ and $\mathrm{SrTiO}_{3}$ (STO) substrates. (b) Schematic diagram depicting the change in lattice parameters with strain (proportions are exaggerated for clarity). (c) The change in integrated intensity with temperature for the $(0,1,14)$ reflection, for the $\mathrm{Sr}_{2} \mid \mathrm{IO}_{4}(\mathrm{SIO})$ film on the LSAT substrate (red triangles) and the STO substrate (blue squares). The black arrow indicates the magnetic transition temperature of bulk SIO, and the red and blue dotted lines are provided as a guide to the eye. (d) The magnetic transition temperature, $\mathrm{TN}$, and the energy of the zone boundary magnon, $E(\pi, 0)$, as a function of strain. 
These results suggest that the most significant structural change taking place in the films was a tuning of the Ir-O bond length. This and related studies suggest that epitaxial strain can be an excellent means of studying the magnetic properties of iridates.

The researchers from the University of Toronto (Canada), the University of Kentucky, Argonne, IFW Dresden (Germany), and the Universität Stuttgart (Germany) performed complementary investigations using $\mathrm{x}$-ray resonant magnetic scattering (XRMS) and resonant inelastic $\mathrm{X}$-ray scattering (RIXS) to study $\mathrm{Sr}_{2} \mid \mathrm{IO}_{4}$ thin-film samples that were epitaxially grown on $\mathrm{SrTiO}_{3}$ and $\left(\mathrm{LaAlO}_{3}\right)_{0.3}\left(\mathrm{Sr}_{2} \mathrm{AlTaO}_{6}\right)_{0.7}$ substrates, which were chosen to provide slight amounts of tensile and compressive strain, respectively.

The XRMS measurements were conducted using XSD beamline 6-ID$B, C$, while the RIXS measurements were carried out on XSD beamline 30ID-B,C. Both sets of measurements involved the Ir $\mathrm{L}_{3}$ absorption edge.

Although the strained $\mathrm{Sr}_{2} \mathrm{IrO}_{4}$ thin films developed magnetic structures that were reminiscent of bulk $\mathrm{Sr}_{2} \mathrm{IrO}_{4}$, the magnetic correlation lengths were found to be extremely anisotropic, with correlation lengths in the interfacial plane being significantly longer (300 $\AA$ to $400 \AA$ ) than the out-of-plane correlation lengths ( $10 \AA$ to $20 \AA$ ).

The most surprising finding was that the magnetic ordering temperature (Néel temperature) was suppressed $(210 \mathrm{~K})$ relative to the Néel temperature of bulk $\mathrm{Sr}_{2} \mathrm{IrO}_{4}(240 \mathrm{~K})$ in samples under compressive strain, and elevated $(270 \mathrm{~K})$ in samples under tensile strain. This was surprising because the magnetic exchange interactions determined from the RIXS measurements increased in energy under compressive strain and decreased under tensile strain (Fig. 1).

Because precise structural details about how the $\mathrm{Sr}_{2} \mathrm{IrO}_{4}$ crystal lattices changed under stress were experimentally difficult to access in the films, the researchers performed $a b$ initio multireference configurationinteraction simulations using two different structural models.

In the first set of calculations, the Ir-O-Ir bond angles were kept the same as those in bulk $\mathrm{Sr}_{2} \mathrm{IrO}_{4}$ and only the Ir$\mathrm{O} / \mathrm{Ir}$-Ir interatomic distances were allowed to change with strain (model I).

At the other extreme, the researchers considered a structural model (model II) in which the in-plane Ir $\mathrm{O}$ bond lengths were fixed to the values measured in bulk $\mathrm{Sr}_{2} \mathrm{IrO}_{4}$ and the $\mathrm{Ir}-\mathrm{O}-\mathrm{Ir}$ angles could be modified by epitaxial strain. The model I results were closer to the experimental data, suggesting that the structural changes occurring in the epitaxial thin films concerned the tuning of the Ir-Ir and Ir-O bond lengths.

All of these results pointed to the following picture of what was going on.

Application of compressive epitaxial strain reduced the bond lengths in the interfacial plane while enlarging the out-of-plane bond length. Because the magnetic exchange interactions between neighboring Ir ions are very sensitive to bond lengths, compressive strain strengthened the inplane interactions and weakened the out-of-plane interactions.

Since the energy scale associated with magnetic excitations is primarily set by the strength of the in-plane exchange interactions, compressive strain led to an increase in the energy of the magnetic interactions. In contrast, the energy scale associated with magnetic ordering is set by the strength of the interactions between neighboring Ir-O layers, which decreased under compressive strain, leading to a reduction in the Néel temperature.

Because tensile epitaxial strain enlarged the in-plane bond lengths while reducing the out-of-plane bond length, similar, but opposite, consequences occurred when tensile strain was applied: stretching the inplane bond lengths reduced the in plane interactions and lowered the energy of the interactions, while shrinking the out-of-plane bond length enhanced the out-of-plane interactions, thus increasing the Néel temperature.

Overall, the results suggest that the most significant structural change taking place in the films was a tuning of the Ir-O bond length. This result and related studies suggest that epitaxial strain can be an excellent means of studying the magnetic properties of iridates. - Vic Comello

See: A. Lupascuํ, J.P. Clancy¹, H. Gretarsson ${ }^{1}$, Zixin $\mathrm{Nie}^{1}$, J. Nichols ${ }^{2}$, J. Terzic ${ }^{2}$, G. Cao ${ }^{2}$, S.S.A. Seo ${ }^{2}$, Z. Islam ${ }^{3}, \mathrm{M} . \mathrm{H}$. Upton ${ }^{3}$, Jungho $\mathrm{Kim}^{3}$, D. $\mathrm{Casa}^{3}$, T. Gog ${ }^{3}$, A.H. Said ${ }^{3}$, Vamshi M. Katukuri ${ }^{4}$, H. Stoll ${ }^{5}$, L. Hozoi ${ }^{4}$, J. van den Brink ${ }^{4}$, and Young-June $\mathrm{Kim}^{1 *}$, "Tuning Magnetic Coupling in $\mathrm{Sr}_{2} \mathrm{IrO}_{4}$ Thin Films with Epitaxial Strain," Phys Rev Lett 112, 147201 (2014). DOI: 10.1103/PhysRevLett.112.147201. Author affiliations: ${ }^{1}$ University of Toronto, ${ }^{2}$ University of Kentucky, ${ }^{3}$ Argonne National Laboratory, ${ }^{4}$ IFW Dresden, ${ }^{5}$ Universität Stuttgart Correspondence:

*yjkim@physics.utoronto.ca

Research at the University of Toronto was supported by the Natural Sciences and Engineering Research Council of Canada, Canada Foundation for Innovation, and Ontario Ministry of Research \& Innovation. This research used resources of the Advanced Photon Source, a U.S. Department of Energy (DOE) Office of Science User Facility operated for the DOE Office of Science by Argonne National Laboratory under Contract No. DE-AC02-06CH11357.

6-ID-B,C $\cdot$ XSD $\cdot$ Physics, materials science Magnetic $x$-ray scattering, anomalous and resonant scattering (hard $x$-ray), general diffraction, grazing incidence diffraction $\cdot 3.2-38 \mathrm{keV}$ - On-site $\cdot$ Accepting general users •

30-ID-B,C $\cdot$ XSD • Physics, materials science - Inelastic x-ray scattering • 5-30 keV, 23.7$23.9 \mathrm{keV} \cdot$ On-site $\cdot$ Accepting general users $•$ 


\section{INTERFACIAL OCTAHEDRAL ROTATIONS AND MAGNETISM IN OXIDE SUPERLATTICES}

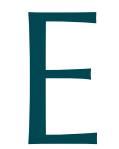

lectric interfaces in oxide materials are the new frontier for use in thin-film devices. Although experimental evidence has thus far been scarce, lattice distortion due to oxygen octahedral rotations has been believed to be useful for mediating oxide magnetism. This study based on research carried out at the APS and the Beijing Synchrotron Radiation Facility demonstrates that octahedral rotation is closely correlated to strongly modified ferromagnetism in oxide superlattices. The fact that new interfacial states and associated novel properties can be created in oxide heterostructures with variant interfacial reconstruction mechanisms opens a new path for engineering functional magnetic materials. Knowledge of the varieties of interfacial mechanisms is crucial for the design of future-generation electronic devices.
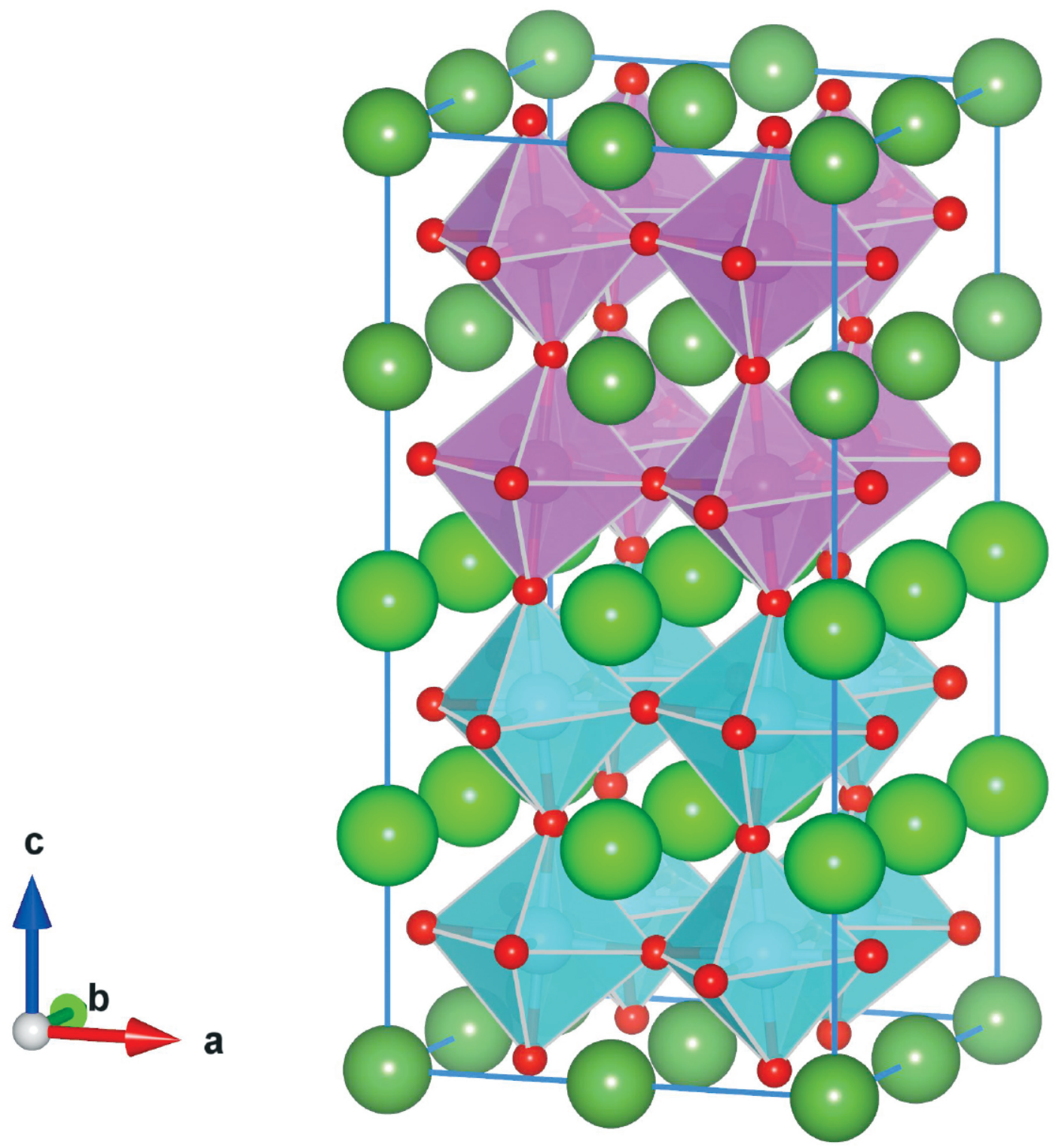
The revolution in oxide materials at electric interfaces parallels the success that thin semiconductor films have had in photonic and electronic applications for more than four decades. The important electronic and ionic characteristics of oxide materials include magnetism, superconductivity, ionic condition and ferroelectricity. As in semiconductor films, interactions between oxide layers can be controlled to regulate and increase the properties that are beneficial for use in batteries, fuel cells, and information storage, among other things. Oxide crystals are better than conventional semiconductors in these applications because the polar character of the bonds between ions enhances their interactions. Rapid breakthroughs in this technology in the past decade have resulted from the realization of new functionality at oxide interfaces.

To advance the technological uses of oxide materials, scientists need to better understand the electronic interactions of these complex materials. For example, samples grown in different crystallographic orientations may reveal new useful properties. The addition of diverse chemical elements into the oxide crystal structure can increase the variety of applications for the oxide compounds. To be valuable for use in thin films, these characteristics must be present at room temperature and need to be very strong; in addition, expensive or rare elements should not be used.

In this study, the researchers from the University of Science and Technology of China, Argonne, Southeast University (China), the Chinese Academy of Sciences, and the University of IIIinois at Urbana-Champaign looked at the intimate correlation between oxy-

\footnotetext{
< Fig. 1. A three-dimensional atomic structure model showing the fitted octahedral rotations. Green, purple, and red-filled circles indicate La, Mn, and $O$ atoms, respectively.
}

gen octahedral rotations (OOR) and magnetic properties in $\left(\mathrm{LaMnO}_{3+} \delta\right)$ and $\left(\mathrm{SrTiO}_{3}\right) N(N=2,3,4,6,8,16)$ superlattices to investigate the impact of interface OOR misfit.

By utilizing state-of-the-art research advances in synchrotron $x$-ray diffraction (XRD), the researchers were able to track the OOR in thin films, heterostructures, and artificial superlattices, which are the major work horses in oxides for generating novel electronic and magnetic functions.

XRDx-ray diffraction measurements were carried out at the XSD 33BM-C beamline of the APS. The researchers also conducted synchrotron $\mathrm{x}$-ray absorption fine structure experiments at the 1W1B beamline of the Beijing Synchrotron Radiation Facility to identify the valence change of the superlattice samples upon the change of the layer period.

These experimental results together provide strong support for the idea that the maximized ferromagnetic moment found in the $N=6$ superlattice has a metastable structure that is related to minimal octahedral rotations. On the other hand, the almost quenched ferromagnetism for $N<4$ superlattices is intimately correlated to a substantially enhanced c-axis rotation. The authors used Monte-Carlo simulation to qualitatively reproduce the experimental results, and suggest that the OOR tips the balance between two important magnetic interactions, i.e., double exchange and super exchange interaction, and causes the magnetism to change.

Interconnecting conventional semiconductors and oxides promises to combine semiconductor electronics with correlated electron systems. Scientists are beginning to understand how to design and control emergent phenomena in these material systems. This work demonstrates that engineering superlattices with controllable interfacial structures will be a new path to- ward developing functional magnetic materials. - Dana Desonie

See: Xiaofang Zhai ${ }^{1 *}$, Long Cheng ${ }^{1}$, Yang Liu ${ }^{2 * *}$, Christian M. Schlepütz ${ }^{2}$, Shuai Dong ${ }^{3 * * *}$, Hui $\mathrm{Li}^{1}$, Xiaoqiang Zhang ${ }^{1}$, Shengqi Chu ${ }^{4}$, Lirong Zheng ${ }^{4}$, Jing Zhang ${ }^{4}$, Aidi Zhao ${ }^{1}$, Hawoong Hong $^{2}$, Anand Bhattacharya ${ }^{2}$, James N. Eckstein ${ }^{5}$, and Changgan Zeng ${ }^{1}$, "Correlating interfacial octahedral rotations with magnetism in $\left(\mathrm{LaMnO}_{3+} \delta\right) \mathrm{N} /$ $\left(\mathrm{SrTiO}_{3}\right) N$ superlattices," Nat. Comm. 5, 4283 (2014).

DOI: $10.1038 /$ ncomms5283

Author affiliations: ${ }^{1}$ University of Science and Technology of China, ${ }^{2} \mathrm{Ar}$ gonne National Laboratory, ${ }^{3}$ Southeast University, ${ }^{4}$ Chinese Academy of Sciences, ${ }^{5}$ University of Illinois at UrbanaChampaign

Correspondence: *xfzhai@ustc.edu.cn, **yangliu3@aps.anl.gov, *** sdong@seu.edu.cn

This work was supported by the National Basic Research Program of China (2012CB 922000, 2014CB921102, 2011CB922101), the National Natural Science Foundation of China (grants no. 11104258, 11034006, 11374279,11274060 , and 51322206), the Chinese Academy of Sciences (XDB0102 0000, KJCX2-EWJ02), the Specialized Research Fund for the Doctoral Program of Higher Education (20113402110046), and Fundamental Research Funds for the Central Universities (WK2340000035). A.B. acknowledges support of the Materials Science and Engineering Division, U.S. Department of Energy (DOE) Office of Science-Basic Energy Sciences. This research used resources of the Advanced Photon Source, a U.S. DOE Office of Science User Facility operated for the DOE Office of Science by Argonne National Laboratory under Contract No. DE-AC02-06CH11357.

33-BM-C - XSD • Materials science, physics, chemistry - Diffuse x-ray scattering, general diffraction, powder diffraction, $x$-ray reflectivity, grazing incidence diffraction, anomalous and resonant scattering (hard x-ray) $\cdot 5-35$ $\mathrm{keV} \cdot$ On-site $\cdot$ Accepting general users $\bullet$ 


\section{High-Performance Piezoelectrics COMING SOON IN UnLEADED}

$\mathrm{P}$

iezoelectricity can seem a little like magic. Give the piezoelectric material a squeeze and it produces an electric charge. Zap it with a little electricity and it will stretch and move. Amazing! The "trick" is a disturbance in the material's structural symmetry, so that positive charges get squeezed a bit one way, and negative charges the other. Only certain structures will react to pressure in this way, so only certain materials and phases are piezoelectric. Researchers from labs and universities in the U.S., China, and the Ukraine used cutting edge optical microscopy, and x-ray diffraction microscopy at the APS to peek inside classical ferroelectric (and piezoelectric) materials barium titanate $\left(\mathrm{BaTiO}_{3}\right)$ and potassium niobate $\left(\mathrm{KNbO}_{3}\right)$. The researchers sought to identify why certain areas within these materials exhibit much stronger piezoelectricity (and different optical properties, too) than their surroundings. The work ends a longstanding debate about the source of strong piezoelectric domains in lead-free ferroelectrics, and indicates that these materials can be engineered to demonstrate high-performance piezoelectric properties - a boon for green technologies.

The term piezoelectric is derived from the Greek "piezo," meaning press or squeeze. The material's ability to transfer mechanical deformation into electric energy and vice-versa make piezoelectric materials valuable in numerous applications, from transducers to sensors to tiny generators. Microphones, clocks, stove lighters, and scanning probe microscopes all include piezoelectric materials as crucial components.

Many materials exhibit a piezoelectric effect, including silk, collagen, viral proteins, quartz, and others, but some of the best-performing piezoelectric materials, and therefore some of the most widely used, are lead-based ferroelectrics. The problem with leadbased ferroelectrics is lead. The combination of lead's biological and environmental toxicity and its low melting point have pushed researchers to seek robust piezoelectric performance in other, more benign materials.

Scientists have known that certain areas in $\mathrm{BaTiO}_{3}$ and $\mathrm{KNbO}_{3}$ demonstrated strong piezoelectric behavior in response to stress from external electric fields, but they disagreed on its origin. Were these areas the result of an intrinsic intermediate phase within the material that could be reproduced and exploited, or the summation of a bunch of tiny bits of the original phase?

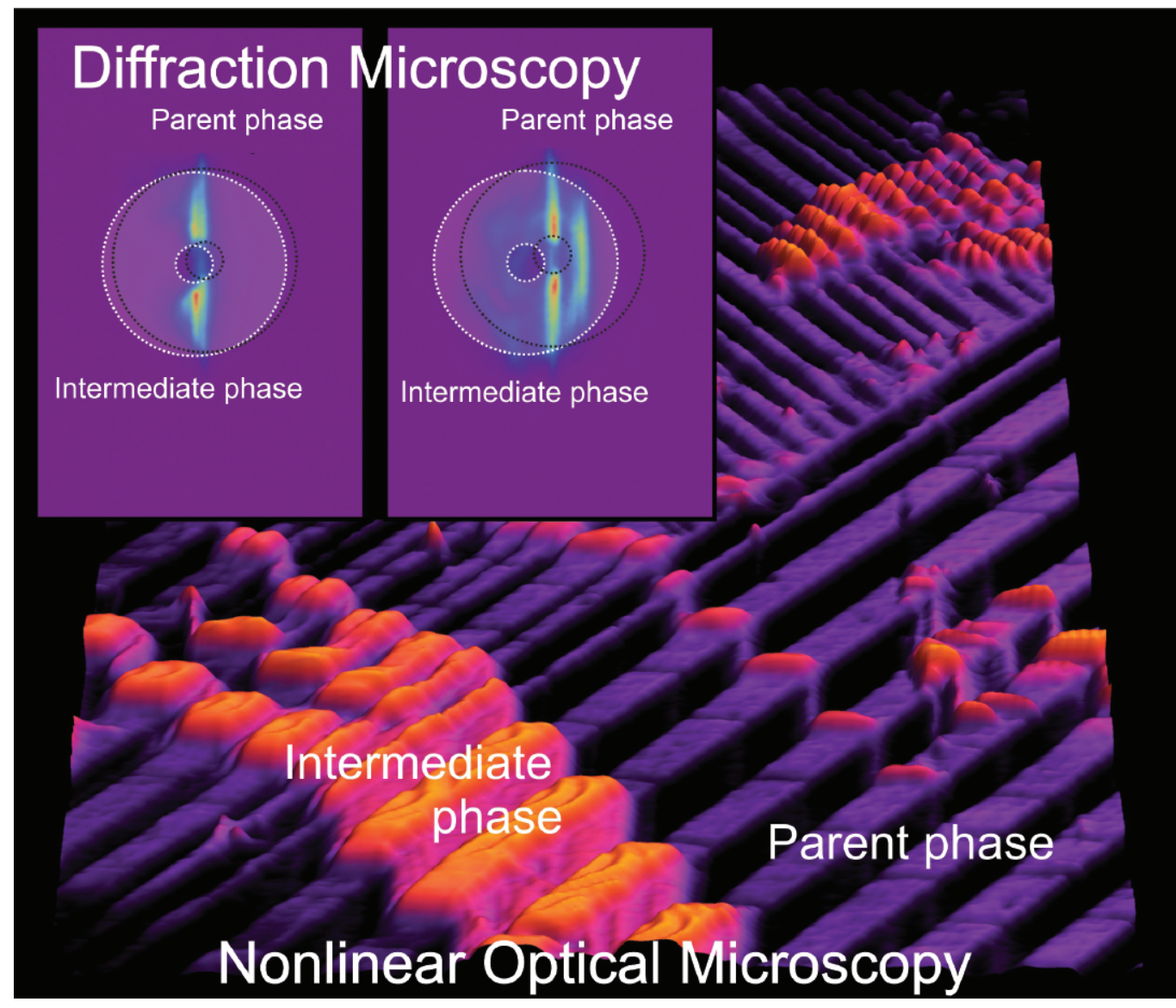

Fig. 1. Optical second harmonic generation imaging of domains in a thermotropic $\mathrm{BaTiO}_{3} \mathrm{crystal}$ Dark and purple colors correspond to conventional, parent phase domains, and bright orange/yellow domains depict the large property enhancements in the newly discovered intermediate phase. Insets depict the nanoscale variation in the structural characteristics of this distinct intermediate phase, as measured at the Hard X-ray Nanoprobe at the APS. Two sets of diffraction peaks are shown, corresponding to two different positions on the sample. These structural variations are shown to directly correlate to the observed property enhancements in the lead-free ferroelectric material. 


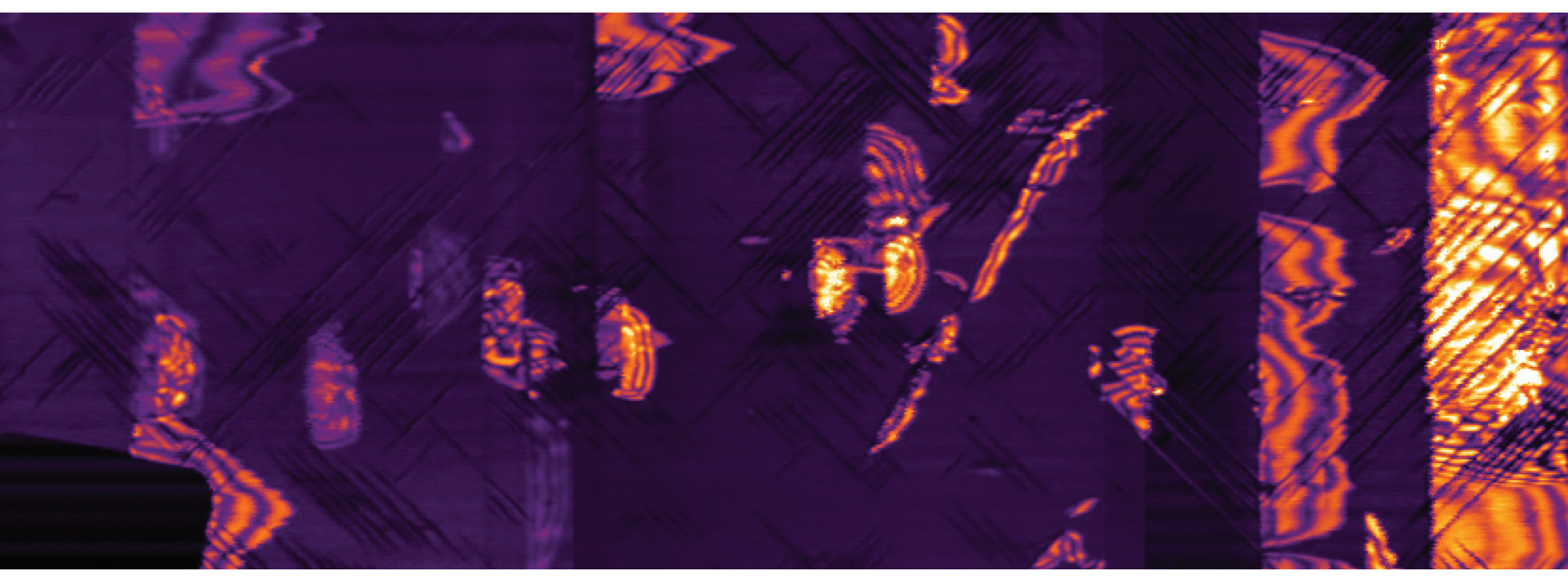

Fig. 2. Optical second harmonic generation imaging of domains in a $\mathrm{KNbO}_{3}$ crystal. The purple regions are conventional orthorhombic phases, while the bright yellow regions are the newly discovered intermediate phase that exhibit large property enhancements.

Previous reports had relied on conventional x-ray techniques, in which the large spread of the beam probed many ferroelectric domains at once. There was no way to resolve individual structural contributions. To solve the debate, the researchers in this study from The Pennsylvania State University, the University of Science and Technology Beijing (China), Oak Ridge National Laboratory, Argonne, and the National Academy of Sciences of Ukraine needed a smaller pixel.

They turned to the Hard X-ray Nanoprobe at the CNM/XSD 26-ID-C beamline of the APS to perform a stateof-the-art focused beam diffraction technique. Coupled with detailed optical microscopy and other experiments, including band-excitation piezoresponse force microscopy at Oak Ridge National Laboratory, the results showed that as the materials were heated through a phase boundary, intermediate piezoelectric phase domains appeared and remained (Figs. 1 and 2). These areas were not just piezoelectric, but strongly so, exceeding values in both the parent phases by up to $440 \%$.

The results are the first direct experimental evidence of such thermal ferroelectric phase transitions in these materials, which have been studied for over 60 years. The authors note similarities to compositional phase boundaries in lead-based ferroelectrics and the temperature-dependent order of liquid crystals.

And there is no reason why this behavior should be unique to $\mathrm{BaTiO}_{3}$ and $\mathrm{KNbO}_{3}$ - any non-triclinic ferroelectric crystal system should demonstrate similar intermediate phases with potentially enhanced properties. It doesn't even take much energy: Small strains can produce relatively large property enhancements. The trick is to manipulate the symmetry of the material to create new phases.

These are fundamental insights, but they point to exciting new avenues for property enhancement, tuning, and optimization in the future. There is potential, the authors say, for creating high-performance materials through "domain-engineering-by-design." Leadbased piezoelectric materials may have some tough new competition.

\section{- Jenny Morber}

See: Tom T.A. Lummen ${ }^{1}$, Yijia Gu ${ }^{1}$, Jianjun Wang ${ }^{1,2}$, Shiming Lei ${ }^{1}$, Fei Xue $^{1}$, Amit Kumar ${ }^{1,3}$, Andrew T.

Barnes $^{1}$, Eftihia Barnes ${ }^{1}$, Sava Denev ${ }^{1}$, Alex Belianinov ${ }^{3}$, Martin Holt ${ }^{4}$, Anna N. Morozovska $^{5}$, Sergei V. Kalinin ${ }^{3}$, LongQing Chen ${ }^{1}$, and Venkatraman Gopalan 1*, "Thermotropic phase boundaries in classic ferroelectrics," Nat. Commun. 5, 3172 (20 January 2014). DOI: $10.1038 /$ ncomms 4172 Author affiliations: ${ }^{1}$ The Pennsylvania
State University, ${ }^{2}$ University of Science and Technology Beijing, ${ }^{3}$ Oak Ridge National Laboratory, ${ }^{4}$ Argonne National Laboratory, ${ }^{5}$ National Academy of Sciences of Ukraine

Correspondence: *vxg8@psu.edu

This research was supported by the U.S. National Science Foundation (NSF) through Penn State MRSEC grant DMR-0820404 (for T.T.A.L., Y.G., J.W., F.X., A.K., A.T.B., E.B., S.D., L.-Q.C. and V.G.), and grants DMR-1210588 (for A.N.M., L.-Q.C. and V.G.), DMR-0908718 (for A.N.M., L.-Q.C. and V.G.) and DMR-1006541 (for L.-Q.C.). A.N.M. acknowledges the State Fund of Fundamental Research of Ukraine, SFFRNSF project UU48/002. The Center for Nanoscale Materials was supported by the U. S. Department of Energy (DOE) Office of Science-Basic Energy Sciences, under Contract No. DE-AC02-06CH11357. This research used resources of the Advanced Photon Source, a U.S. DOE Office of Science User Facility operated for the DOE Office of Science by Argonne National Laboratory under Contract No. DE-AC02$06 \mathrm{CH} 11357$.

26-ID-C • CNM/XSD • Physics, materials science $\cdot$ Nanofluorescence imaging, microdiffraction, nanotomography $\bullet 8-12 \mathrm{keV} \cdot$ On-site - Accepting general users • 


\section{Ultrafast InHOMOGENEOUS StRain IN ROOM-TEMPERATURE MULTIFERROICS}

M ultiferroics are materials that exhibit a ferroelectric and magnetic order simultaneously. These orders have been the building blocks for technologically and economically important applications such as ferroelectric memory and spintronics. The ferroelectric polarization couples strongly to ultraviolet light by exciting electron-hole pairs. The charge separation under the influence of remnant polarization generates a large electric field across the multiferroic film. These novel properties arising from lightmatter interaction open opportunities for efficient electric-field control of magnetism on ultrafast time scales. To facilitate the application of multiferroics in new technologies, a microscopic picture needs to be developed to illustrate the process that starts from light-matter interaction and carries through to the relaxation of the photoinduced effects so that we can fully understand how these new properties come about and how best to harness them. Scientists using the APS discovered a strong and inhomogeneous lattice deformation in a few-nanometer-thick film of $\mathrm{BiFeO}_{3}$ after photoexcitation due to the generation of localized excited charge carriers. The deeper understanding of the transient processes will allow for much faster and even more efficient devices in future technology.

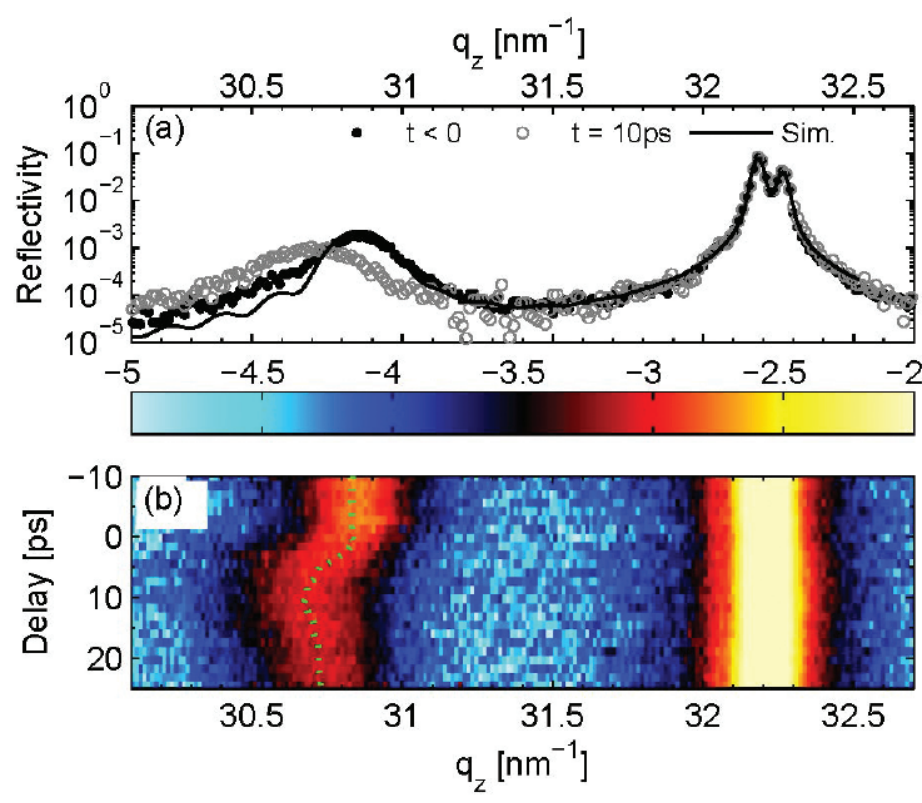

Fig. 1. The transient $\theta / 2 \theta$ scans reveal a maximum shift of the $\mathrm{BiFeO}_{3} \mathrm{OO}_{2}$ Bragg peak around $30.8 \mathrm{~nm}^{-1}$ to smaller values of the scattering vector $\mathrm{qz}$ within 10 ps while the substrate $\mathrm{SrTiO}_{3}$ peak at $32.2 \mathrm{~nm}^{-1}$ remains unchanged. (a) The black dots and gray circles correspond to slices of (b) at $t<0$ ps and $t=10$ ps, respectively.

Optical pump x-ray probe experiments were carried out at the Plasma $X$-ray Source (PXS) at the University of Potsdam (Germany) and at the XSD 7ID-B,C,D time-resolved beamline at the APS. The combined analysis of the transient diffraction data from both the
PXS and the APS allowed the researchers to follow the amplitude and spatial profile of photoinduced structural dynamics in $\mathrm{BiFeO}_{3}$ on multiple time scales, from femtosecond up to microsecond delays under comparable excitation conditions (Fig. 1). As a re- sult, a new model of the dynamics and lattice coupling of photoexcited charge carriers in $\mathrm{BiFeO}_{3}$ was developed.

The researchers from the Universität Potsdam (Germany), Fritz-HaberInstitute der Max-Planck- Gesellschaft (Germany), Helmholtz-Zentrum Berlin (Germany), Argonne, the University of Wisconsin-Madison, Cornell University, Stanford University, and Kavli Institute at Cornell for Nanoscale Science showed that the force driving the ultrafast lattice expansion in $\mathrm{BiFeO}_{3}$ must be quasi-instantaneous ( $<100$-fs rise time), possibly due to the inverse piezoelectric effect caused by photoexcited charge carriers. Further, a strongly inhomogeneous deformation of the $\mathrm{BiFeO}_{3}$ film from the picosecond up to the microsecond indicates a fast trapping and localization of these charge carriers.

Complete analysis of the diffraction data gives very detailed information on the material's excitation and decay dynamics over several orders of time scales. This technique can be applied for a broad range of functional materials with a strong coupling of electronic degrees of freedom to the crystal lattice.

The success of this project exemplifies the synergetic collaboration of time-resolved research utilizing unique capabilities around the world to gain significant insight on the challenging problems that cannot be solved with the limited resources of any one institute. - Haidan Wen

See: Daniel Schick ${ }^{1}$, Marc Herzog ${ }^{1,2}$, Haidan Wen ${ }^{3}$, Pice Chen ${ }^{4}$, Carolina Adamo $^{5,6}$, Peter Gaal ${ }^{7}$, Darrell G. Schlom ${ }^{5,8}$, Paul G. Evans ${ }^{4}$, Yuelin $\mathrm{Li}^{3}$, and Matias Bargheer ${ }^{1,7 *}$, "Localized Excited Charge Carriers Generate Ultrafast Inhomogeneous Strain in the Multiferroic $\mathrm{BiFeO}_{3}$," Phys. Rev. Lett. 112, 097602 (2014).

DOI: 10.1103/PhysRevLett.112.097602

Author affiliations: ${ }^{1}$ Universität Potsdam, ${ }^{2}$ Fritz-Haber-Institut der MaxPlanck- Gesellschaft, ${ }^{3}$ Argonne "Ultrafast" cont'd on page 32 


\section{When An EXCiton Acts Like a Hole}

W

hen is an electron hole like a quasiparticle (QP)? More specifically, what happens when a single electron hole is doped into a two-dimensional quantum antiferromagnet? Quasiparticle phenomena in such a system are predicted by theory, but have eluded observation, complicating the understanding of electron behavior in high-temperature superconducting cuprates. A team of experimenters working at the APS have taken a different approach to the problem with their recent observation of an excitonic quasiparticle in strontium iridate $\left(\mathrm{Sr}_{2}\left(\mathrm{rO}_{4}\right)\right.$, a quasi-two-dimensional, spin-1/2, antiferromagnetic Mott insulator. Their work sheds new light on a classic problem in condensed matter physics and opens a new pathway for the study of high-temperature superconductors.

(a) RIXS
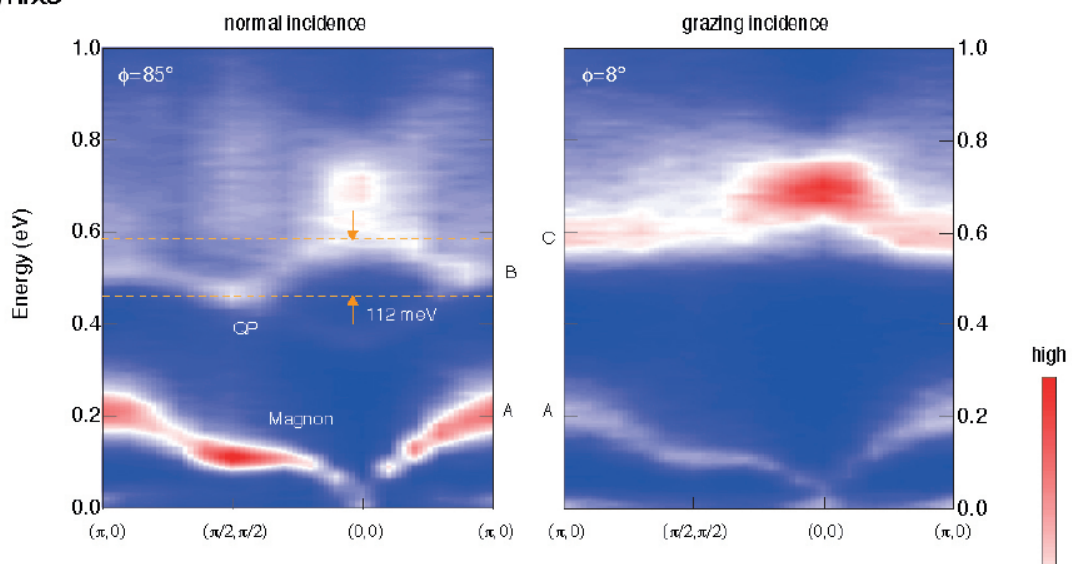

(b) SCBA calculation
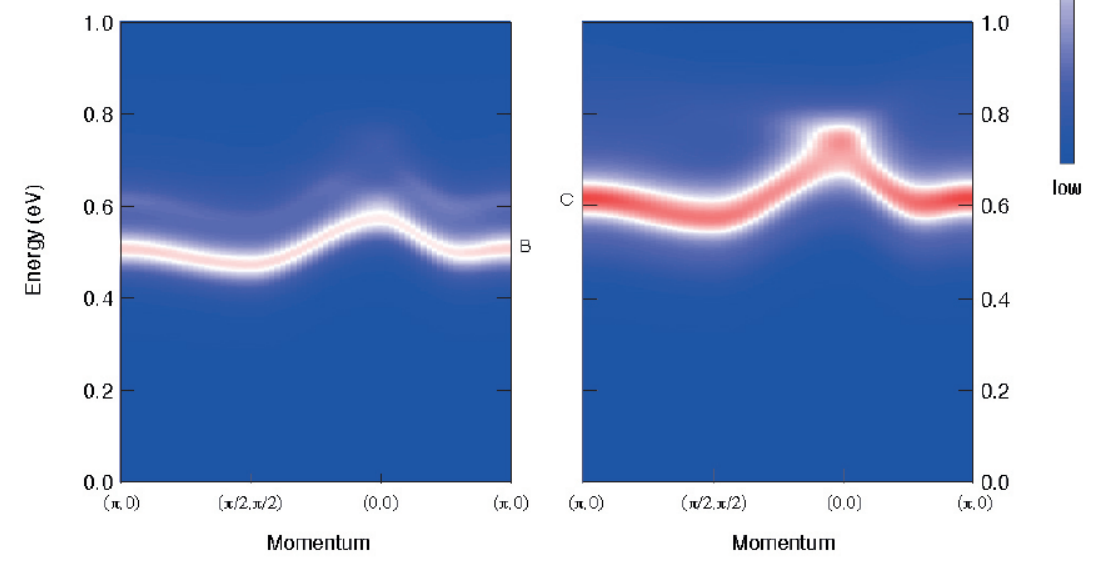

Fig. 1. Selective mapping of the two-exciton modes and their comparison to SCBA calculations. (a) Image plot of RIXS spectra measured along high-symmetry lines in the normal and grazing incidence geometry. (b) SCBA calculations.

Strontium iridate is a newly-discovered pseudospin-1/2 Heisenberg antiferromagnet in which superconductivity has also been predicted but not yet observed. The experimenters from Argonne, the Institute for Theoretical Solid State Physics, and the Max Planck Institute for Solid State Re- search (Germany) used resonant inelastic $x$-ray scattering (RIXS) at the XSD 30-ID-B,C beamline of the APS. They carried out their studies utilizing the MERIX spectrometer, a mediumenergy-resolution diffractometer for non-resonant and resonant inelastic $x$ ray scattering (RIXS) measurements of samples that had been prepared at the Argonne Materials Science Division.

Earlier RIXS studies of $\mathrm{Sr}_{2} \mid \mathrm{IO}_{4}$ showed that the dispersions of an electron hole excited across spin-orbit coupling levels could be related to the QP problem (Fig 1). The investigators attempted to reflect the behavior of an electron hole in $\mathrm{Sr}_{2} \mathrm{IrO}_{4}$ by using an exciton analog.

Using a theoretical model structured around the observation that the propagation of an orbital excitation through a Mott insulator such as $\mathrm{Sr}_{2} \mathrm{IrO}_{4}$ can be mapped on the motion of a single hole, the researchers employed a self-consistent Born approximation (SCBA) for comparison and interpretation of the RIXS data. They found an excellent agreement between the effective $t-J$ model (used to calculate high- $T_{C}$ states) calculated within the SCBA and the experimental spectra. Energy distribution curves showed a sharp exciton peak, which is both narrower than the sharpest peak measured in $\mathrm{Sr}_{2} \mathrm{IrO}_{4}$ by angle-resolved photoemission spectroscopy (ARPES) and smaller than the total bandwidth of about $112 \mathrm{meV}$, which reveals the exciton to be a QP.

While this demonstrates that a coherent particle can propagate through a quantum antiferromagnet, it does not yet explain the lack of a QP with a single-hole excitation. The researchers attribute this to the charge-neutral nature of an exciton compared to the chargedparticle character of a hole. Because a charged hole interacts much more strongly with the lattice structure and the inevitable impurities present in an insulator, it also tends to dampen or completely wash out QP phenomena, particularly in ARPES measurements. On the other hand, a charge-neutral exciton is not subject to these effects and thus can more readily reveal subtle QP dynamics that can elude experimenters.

The work confirms that QPs can definitely be observed in Mott insulators and that these materials can display striking parallels with high-temperature cuprate superconductors. By showing that an exciton in a spin-1/2 antiferro-

"Exciton" cont'd on page 32 


\section{Metals on the COUCH: Does Deformed Aluminum Retain Internal Stress?}

M etalworking is, and for centuries has been, about making tiny crystals. Long ago, blacksmiths heated, cooled, and pounded steels to make them stronger, lengthening the tiny crystallites we call grains, nucleating new grains, and pinning them in place. Today we repeat these processes, helped by machines to do the working and specialized tools to observe and understand the changes. But despite centuries of metalworking and decades of careful scientific study, we are still seeking stronger, lighter metals, and still working to understand the complex processes that transpire to create them. In aluminum, a remaining question is whether large deformations can leave the material with long-range internal stresses - areas of unresolved stress between atoms. External and internal stresses can be additive, so the presence of internal stresses in metals can be beneficial or detrimental, depending on their nature. In a material undergoing fatigue, such as the skin of an airplane, compressive internal stresses may toughen the material, while tensile stresses can hasten cracking. Researchers used high intensity x-rays at the APS to peek inside a bit of aluminum subjected to repeated, severe deformations and probe the nature of long range stresses within the material. This work, which is an extension of an earlier report, suggests that long-range internal stresses only modestly increase with plastic strain.
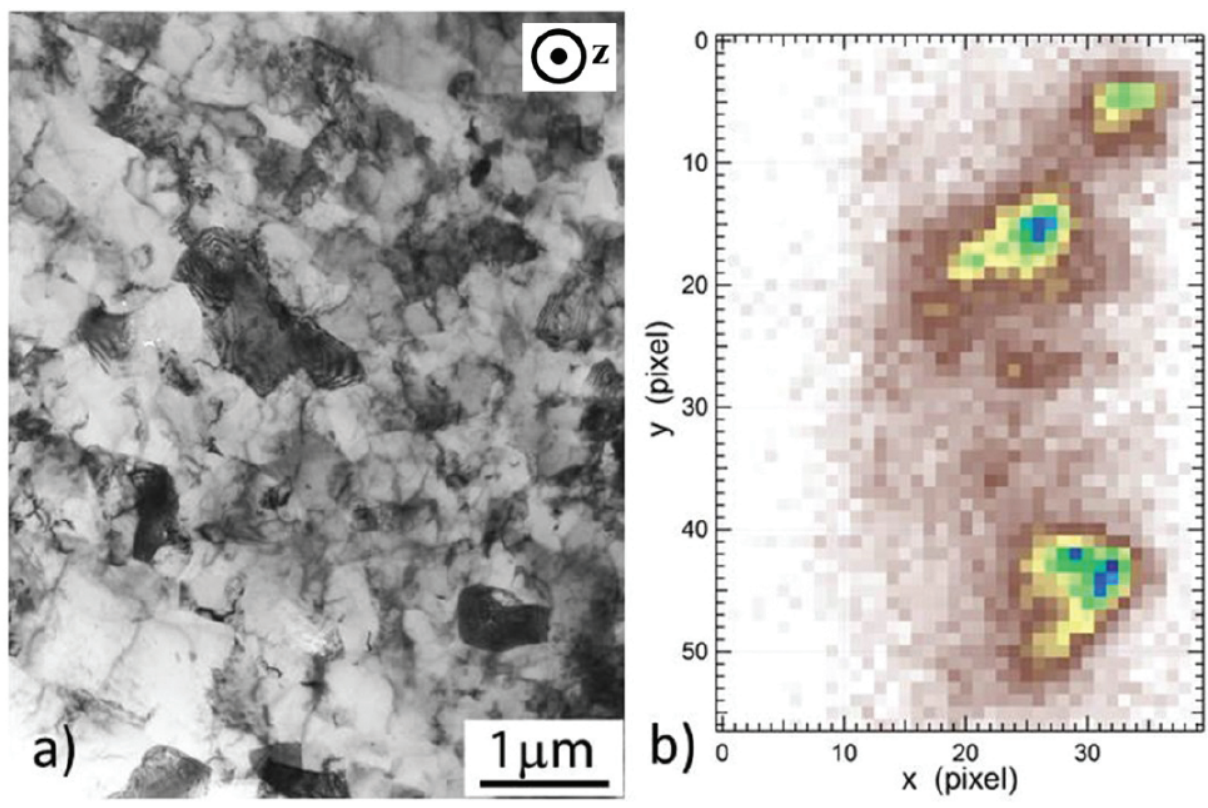

Fig. 1. (a) Transmission electron microscopy image of AA1050 (commercial purity Aluminum) after two ECAP passes (b) False-color image of the energy-integrated diffracted intensity from an individual grain/subgrain in a two-pass sample. The peaks are from low-dislocation density regions and the smeared intensity is from the high dislocation density volumes from walls or cell interiors with a relatively high dislocation density. Image in (b) is taken from earlier work on the same alloy as (a).
Small grains make a metal hard and tough. One way to impart ultrafine grains into a metal is by grossly deforming it, a process called "severe plastic deformation." Plastically deformed materials cannot pop back into their former state, and obtaining very fine grains requires that metals must be really worked over, and uniformly so.

One popular method of metal deformation is a process called ECAP, which stands for "equal-channel angular processing." Much like an oldschool play dough extruder, in ECAP a bit of metal is placed into the top of an L-shaped channel, mashed down, and forced through the connecting perpendicular chute. The metal flows into the horizontal cavity having undergone massive structural changes. In this study, the researchers from the University of Southern California, the National Institute of Standards and Technology, Argonne, the University of Southampton (UK), and the Asian University (Thailand) repeated this process on a sample of commercial purity aluminum. After intervals of 1, 2, 4 , and 8 presses, they took a look inside the metal by sending $x$-rays careening into the sample, and observed how the signals changed.

$X$-ray diffraction is ideal for measuring crystalline structure, atomic defects, and deviations from the mean. But to probe the very small regions required here, the x-rays had to be both intense and highly focused. This capability was provided by microbeam x-ray diffraction at XSD beamline 34-ID-E of the APS, with supporting measurements at XSD beamline 11-BM-B.

The results suggested that the aluminum deformed according to the so-called "composite model," in which a deforming metal contains "hard" areas, dense with atomic dislocations, and "softer" regions that deform more "Metals" cont'd on page 32 


\section{Pinning Down New Fe-Based Superconductors}

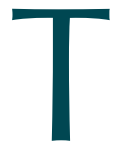

he quest for high-temperature superconducting materials that can be put to new practical uses has been a major preoccupation in solid-state physics for decades, and it is hardly slowing down. One of the most intriguing recent developments is the discovery of new classes of iron ( $\mathrm{Fe})$-based high-temperature superconductors. Among these are the $\mathrm{Ca}_{10}\left(\mathrm{Pt}_{3} \mathrm{As}_{8}\right)\left(\mathrm{Fe}_{2-x} \mathrm{Pt}_{x} \mathrm{As}_{2}\right)_{5}$ and $\mathrm{Ca}_{10}\left(\mathrm{Pt}_{4} \mathrm{As}_{8}\right)\left(\mathrm{Fe}_{2-x} \mathrm{Pt}_{x} \mathrm{As}_{2}\right)_{5}$ compounds, known more simply as the 10-3-8 and 10-4-8 compounds, respectively. These compounds become superconducting with transition temperatures up to $38 \mathrm{~K}$. But knowledge of their precise structure and phase behavior, not to mention the similarities and differences of their properties compared to other known Fe-based superconductors, has remained ambiguous. Now a group of investigators has pinned down some of the nagging unknowns of these 10-3-8 and 10-4-8 materials.
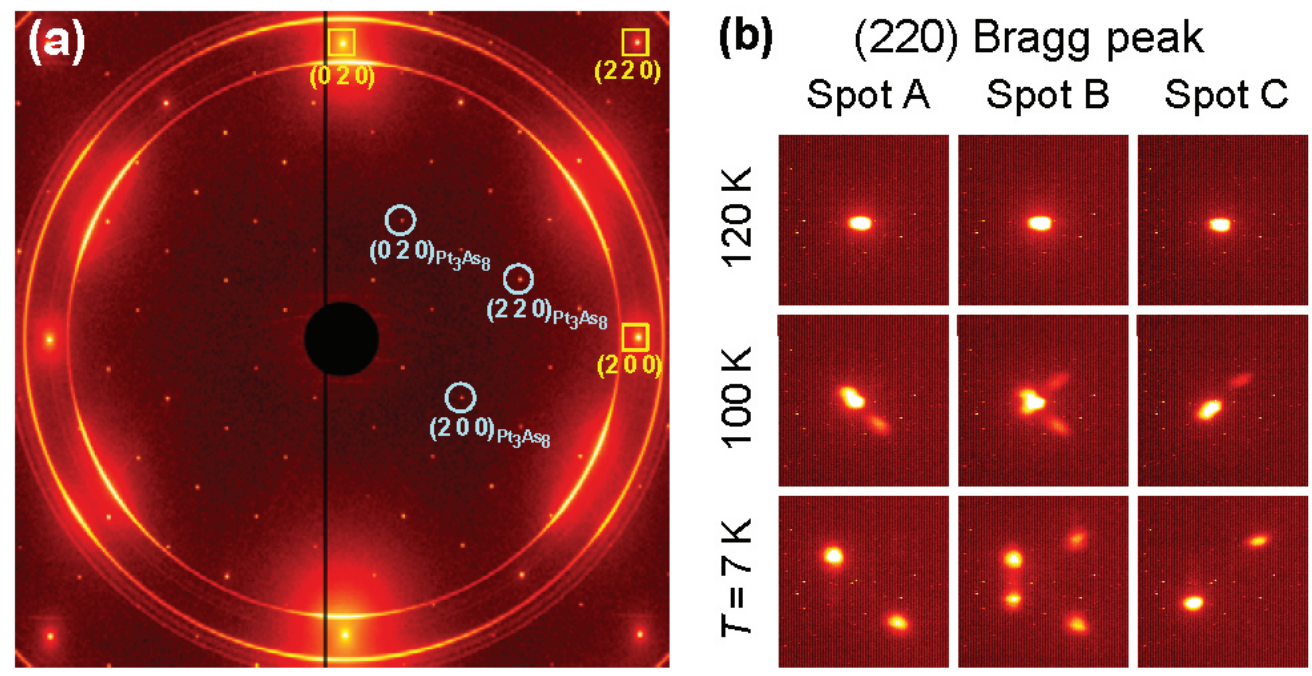

Previous attempts to study these compounds have been hindered by the difficulty in comparing samples prepared with different techniques and the wide variation in properties they display. To overcome this problem, the researchers from Ames Laboratory; lowa State University; Oak Ridge National Laboratory; the University of California, Los Angeles; and Princeton University examined a single homogeneous crystal of $\mathrm{Ca}_{10}\left(\mathrm{Pt}_{3} \mathrm{As}_{8}\right)\left(\mathrm{Fe}_{2} \mathrm{As}_{2}\right)_{5}$, conducting both high-resolution, high-energy $\mathrm{x}$ ray diffraction $(X R D)$ studies at the $X S D$ 6-ID-D beamline of the APS and elastic neutron scattering studies on the same sample at the High Flux Isotope Reactor at Oak Ridge National Laboratory.

In the crystal's $(H K O)$ reciprocal lattice plane at high temperatures, the XRD studies show essentially two groups of Bragg peaks. One group

Fig. 1 (a). High-energy $x$-ray diffraction patterns of the $C a_{10}\left(\mathrm{Pt}_{3} \mathrm{As}_{8}\right)\left(\mathrm{Fe}_{2} \mathrm{As}_{2}\right)_{5}$ single crystal recorded by a MAR345 detector as the sample was rocked about axes perpendicular to the incident beam. The measured reciprocal lattice plane shows strong Bragg peaks related to the body-centered tetragonal base structure built by the Fe-As layers and Ca planes that are marked with squares. Circles depict selected Bragg peaks related to the $\mathrm{Pt}_{3} \mathrm{As}_{8}$ superstructure. The ring-like scattering features are caused by the $\mathrm{Cu}$-sample holder and the Be domes. (b). High-resolution, high-energy x-ray diffraction patterns measured with the ScintX detector at three different spots on the sample. The (220) Bragg peak of the tetragonal body-centered base structure splits due to the orthorhombic distortion of the lattice at low temperatures. Adapted from A. Sapkota et al., Phys. Rev. B 90, 100504(R) (2014)

consists of strong peaks on a large, square-like Fe lattice related to the structure of the Fe-As layers, while the other, weaker group of Bragg peaks on a tilted, smaller square-like lattice arises from a complex $\mathrm{Pt}_{3} \mathrm{As}_{8}$ layer structure. Below $110 \mathrm{~K}$, the high-resolution XRD patterns show splitting or broadening of Bragg peaks consistent with a tetragonal-to-orthorhombic distortion.

The neutron diffraction studies also show a second phase transition in the single crystal involving the Fe magnetic moments. Below $96 \mathrm{~K}$, these adopt a stripe-like antiferromagnetic order along the [110] direction and ferromagnetic arrangements in both perpendicular directions.

Both of these structural and magnetic phase transitions are seen to be continuous. Each is distinct and separate from the other as demonstrated by the lack of an antiferromagnetic signal at the temperatures at which the orthorhombic lattice distortion sets in.

"Pinning" cont'd on page 32 
"Ultrafast" cont'd from page 28

National Laboratory, ${ }^{4}$ University of Wisconsin-Madison, ${ }^{5}$ Cornell University, ${ }^{6}$ Stanford University, ${ }^{7}$ Helmholtz-Zentrum Berlin, ${ }^{8}$ Kavli Institute at Cornell for Nanoscale Science

Correspondence:

*Bargheer@uni-potsdam.de

The work at Argonne was supported by the U.S. Department of Energy (DOE) Office of Science-Basic Energy Sciences under Contract No. DE-AC02-06CH11357. This research used resources of the Advanced Photon Source, a U.S. DOE Office of Science User Facility operated for the DOE Office of Science by Argonne National Laboratory under Contract No. DE-AC02$06 \mathrm{CH} 11357$.

7-ID-B,C,D • XSD • Materials science, atomic physics, chemistry $\cdot$ Time-resolved $x$-ray scattering, time-resolved $x$-ray absorption fine structure, phase contrast imaging $\bullet$ 6-21 keV • On-site $\cdot$ Accepting general users $•$

\section{"Exciton" cont'd from page 29}

magnet can display fundamentally similar dynamics to a charged hole doped into the same kind of system, the researchers have cast new light on a classic problem in condensed matter physics and opened a new pathway for the study of high-temperature superconductors. - Mark Wolverton

See: Jungho Kim¹, M. Daghofer ${ }^{2}$, A.H. Said ${ }^{1}$, T. Gog ${ }^{1}$, J. van den Brink ${ }^{2}$, G. Khaliullin" , and B.J. Kim,1*, "Excitonic quasiparticles in a spin-orbit Mott insulator," Nat. Commun. 5, 4453 (2014). DOI: $10.1038 /$ ncomms5453

Author affiliations: ${ }^{1}$ Argonne National Laboratory, ${ }^{2}$ Institute for Theoretical Solid State Physics, ${ }^{3}$ Max Planck Institute for Solid State Research Correspondence: * bjkim@fkf.mpg.de

Work in the Argonne Materials Science Division was supported by the U.S. Department of Energy (DOE) Office of Science, Basic Energy Sciences, Materials Science and Engineering Division. This research used resources of the Advanced Photon Source, a DOE Office of Science User Facility operated for the DOE Office of Science by Argonne National Laboratory under Contract No. DE-AC02-06CH11357.

30-ID-B,C - XSD - Physics, materials science - Inelastic x-ray scattering • 5-30 keV, 23.7$23.9 \mathrm{keV} \cdot$ On-site $\cdot$ Accepting general users
"Metals" cont'd from page 30

easily (Fig. 1). As the composite is strained, areas of high and low stress form.

The team observed compressive internal elastic strains along the pressing direction for all ECAP passes, increasing slightly after each pass. The authors lament that this study did not allow them to measure the strains in all directions within a given metal grain, and say that this information will be the focus of future efforts. Still, the results are informative. ECAP, it appears, may be ideal for creating stronger, lighter-weight metal structures that can take a beating.

$$
\text { - Jenny Morber }
$$

See: Thien Q. Phan ${ }^{1 *}$, I-Fang Lee ${ }^{1}$, Lyle E. Levine ${ }^{2}$, Jonathan Z. Tischler ${ }^{3}$, Yi Huang ${ }^{4}$, Alan G. Fox ${ }^{5}$, Terence G. Langdon $^{1,4}$, and Michael E. Kassner ${ }^{1}$, "X-ray microbeam measurements of long-range internal stresses in commercial-purity aluminum processed by multiple passes of equal-channel angular pressing," Scripta Mater. 93, 48 (2014).

DOI: 10.1016/j.scriptamat.2014.09.001 Author affiliations: ${ }^{1}$ University of Southern California, ${ }^{2}$ National Institute of Standards and Technology, ${ }^{3} \mathrm{Ar}$ gonne National Laboratory, ${ }^{4}$ University of Southampton, ${ }^{5}$ Asian University Correspondence: * thienqph@usc.edu

Research at the University of Southern California was supported the National Science Foundation through DMR-1401194, and research at the University of Southampton was supported by the European Research Council under ERC Grant Agreement No. 267464-SPDMETALS. This research used resources of the Advanced Photon Source, a U.S. Department of Energy (DOE) Office of Science User Facility operated for the DOE Office of Science by Argonne National Laboratory under Contract No. DE-AC02$06 \mathrm{CH} 11357$.

11-BM-B - XSD - Chemistry, materials science, physics, geoscience $\cdot$ Powder diffraction $\cdot 25-35 \mathrm{keV} \cdot$ On-site, mail-in • Accepting general users .

34-ID-E • XSD • Materials science, physics, environmental science, geoscience $\cdot \mathrm{Mi}$ crodiffraction, Laue crystallography, microbeam • 7-30 keV • On-site - Accepting general users $•$
"Pinning" cont'd from page 31

This work provides strong evidence for the similarity in physical properties of the 10-3-8 class compounds with other Fe-based superconducting substances. Such similarities persist despite some considerable structural differences in the 10-3-8 compounds from the other compounds, including a much more complex structure with lower crystal symmetry, and much greater separation of the Fe-As layers from each other along with strong disorder in the Pt-As layers.

The experiments confirm that the major physical features appear to be quite robust in this class of compounds and that the Fe-As layers chiefly determine their properties. By answering some pesky questions concerning these particular materials, the research team has helped bring them a little closer to their eventual practical application. - Mark Wolverton

See: A. Sapkota ${ }^{1,2}$, G.S. Tucker ${ }^{1,2}$, M. Ramazanoglu' ${ }^{1,2}$, W. Tian ${ }^{3}$, N. Ni ${ }^{4,5}$, R.J. Cava $^{5}$, R.J. McQueeney ${ }^{1,2,3}$, A.I. Gold$\operatorname{man}^{1,2}$, and A. Kreyssig ${ }^{1,2 *}$, "Lattice distortion and stripelike antiferromagnetic order in $\mathrm{Ca}_{10}\left(\mathrm{Pt}_{3} \mathrm{As}_{8}\right)\left(\mathrm{Fe}_{2} \mathrm{As}_{2}\right)_{5}$," Phys.

Rev. B 90, 100504(R) (2014).

DOI: 10.1103/PhysRevB.90.100504 Author affiliations: ${ }^{1}$ Ames Laboratory, ${ }^{2}$ lowa State University, ${ }^{3}$ Oak Ridge National Laboratory, ${ }^{4}$ University of California, Los Angeles, ${ }^{5}$ Princeton University Correspondence:

*kreyssig@ameslab.gov

The research conducted at Argonne National Laboratory and Oak Ridge National Laboratory was sponsored by the U.S. Department of Energy (DOE) Office of Science-Basic Energy Sciences, Scientific User Facilities Division. This research used resources of the Advanced Photon Source, a U.S. Department of Energy (DOE) Office of Science User Facility operated for the DOE Office of Science by Argonne National Laboratory under Contract No. DE-AC02-06CH11357.

6-ID-D • XSD • Physics, materials science • Magnetic x-ray scattering, high-energy $x$-ray diffraction, powder diffraction, pair distribution function • 50-100 keV, 70-130 keV • On-site • Accepting general users • 


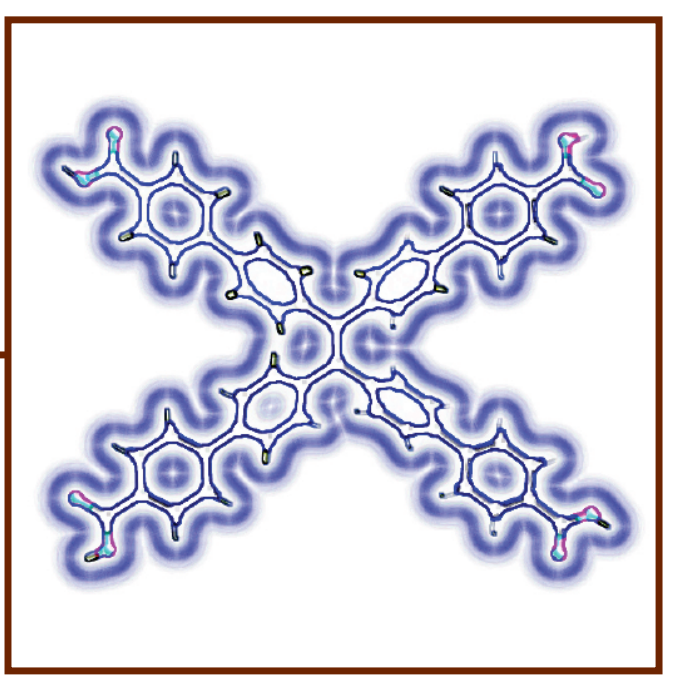

EnGineEring Materials \& APPLICATIONS 
oCrAIY alloy, with a thickness of about a tenth of a millimeter; and a thermally grown oxide (TGO) layer, with a thickness of approximately $300 \mathrm{~nm}$, which developed between the other two layers with exposure. Load and thermal instrumentation analyzed the resulting deformations and temperature changes within the coatings.

Three scenarios were used to simulate the conditions of gas turbine blades found in aircraft engines: single-flight cycles, mechanical tests at various temperatures reaching $1000^{\circ} \mathrm{C}$ (a little over $1800^{\circ} \mathrm{F}$ ), and variations of internal cooling at similarly high temperatures. The researchers measured depth-resolved strains appearing on the coatings as thermal gradients and/or mechanical loads were applied (and varied) at elevated temperatures. Observing interactions between the $x$ rays and the test material, the team determined strain response through the depth of its many layers by analyzing the resulting $x$-ray diffraction patterns.

Through highresolution data (Fig. 2), they found an association between the controlled conditions of internal cooling and external heating and the evolution of local strains. The results showed that such conditions produce strains, with the largest ones found in the TGO and at or near the interfaces between the different layers. The team emphasized that as temperatures decrease across the coating depth, major strain gradients are seen, prompting increased failure risks especially near the interface.

Based on their results, the team looks forward to conducting further research to identify ways to extend the useful lifespans of TBCs by identifying

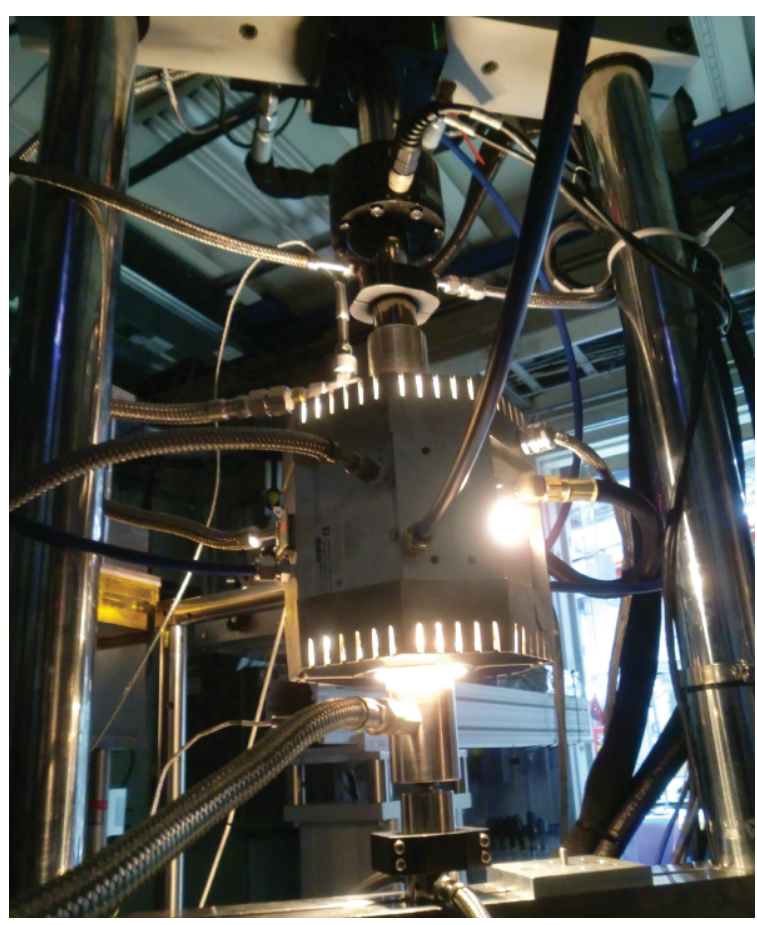

< Fig. 1. The custom-built, compact furnace and cooling system at beamline 1-ID-B,C,E at the APS. It accurately simulates the extreme conditions existing when turbine engines are operating in real time for mechanical stress tests that probe experimental specimens with high-energy $x$-rays.

See: Kevin Knipe ${ }^{1}$, Albert Manero II $^{1}$, Sanna F. Siddiqui ${ }^{1}$, Carla Meid ${ }^{2}$, Janine Wischek ${ }^{2}$, John Okasinski ${ }^{3}$, Jonathan Almer ${ }^{3}$, Anette M. Karlsson $^{4}$, Marion Bartsch ${ }^{2}$, and Seetha Raghaven 1*, "Strain response of thermal barrier coatings captured under extreme engine environments through synchrotron X-ray diffraction," Nat. Commun. 5, 4559 (July 31, 2014). DOI: $10.1038 /$ ncomms5559 Author affiliations: ${ }^{1}$ University of Central Florida, ${ }^{2}$ Deutsches Zentrum für Luft-und Raumfahrt e.V. (DLR), ${ }^{3}$ Argonne National Laboratory, ${ }^{4}$ Cleveland State University Correspondence:

* Seetha.Raghavan@ucf.edu

This material is based upon work supported by the National Science Foundation (NSF) grants OISE 1157619, CMMI 1125696, and by the German Science Foundation (DFG) grant no. SFBTRR103, Project A3. This material is additionally based upon work supported by the NSF Graduate Research Fellowship Program under grant no. 1144246 . This research used resources of the Advanced Photon Source, a U.S. Department of Energy (DOE) Office of Science User Facility operated for the DOE Office of Science by Argonne National Laboratory under Contract No. DE-AC02-06CH11357.

loads and thermal gradients affect the performance, efficiency, and durability of coated turbine blades through full lifecycle use.

Such work will help to advance technologies in other extreme-temperature environments such as in space and hypersonic propulsion and energy conversion. - William A. Atkins
Original turbine photo: http://pixabay.com/p371412/?no_redirect

1-ID-B,C,E - XSD • Materials science, physics, chemistry $\cdot$ High-energy $x$-ray diffraction, tomography, small-angle $x$-ray scattering, fluorescence spectroscopy, pair distribution function, phase contrast imaging $\cdot 50-90 \mathrm{keV}$, 50-150 keV - On-site - Accepting general users • 


\section{Stretching the Limits of Synthetic Spider Silk}

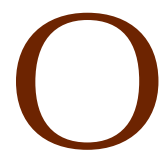

ver the past 400 million years, spider silks have evolved into biomaterials with remarkable mechanical properties, ranging from medical to military. But, because spiders are difficult to raise in captivity, mass production of natural silks is not possible, leaving scientists with the job of developing synthetic spider silk fibers with the same attributes as the native fibers. Synthetic silk's molecular structure and the conditions under which the fibers are spun, and then stretched, determine its strength, elasticity, and stiffness. Although stretching the fibers generally enhanced these properties, researchers failed to observe structural differences between as-spun and stretched fibers. The chemical composition and temperature of the bath solution in which stretching occurred had mixed effects on the properties of synthetic fibers composed of increasing ratios of an amino acid motif thought to confer elasticity. Research at the APS is helping scientists understand the precise impact of the post-spin environment and gain detailed knowledge of these unique interactions, which may make it possible to create designer fibers with tunable mechanical properties for specific applications.

Spider silk proteins are made up of hundreds of specific, highly conserved amino acid motifs, each of which contribute specific mechanical properties. Orb-web weaving spiders use separate glands in their abdomens to spin six functionally different types of silk. For example, flagelliform silk is very elastic in order to absorb the energy of a flying insect without breaking. In contrast, major ampullate silk — also known as dragline silk - is incredibly strong and provides a lifeline for the spider. Dragline silk is made up of two proteins: Major ampullate Spidroin 1 (MaSp1) lends strength to the fiber while Major ampullate Spidroin 2 (MaSp2) lends elasticity through a spring-like $\beta$-spiral structure.

Silk's protein composition is not always predictive of its mechanical properties. The dragline silk of the spider species Argiope aurantia is stronger and stiffer, yet not any more elastic, than that of the spider species Nephila clavipes, although $A$. aurantia contains, proportionately, three times more elasticity-conferring MaSp2 protein.

Post-spin stretching emulates natural processes occurring in spiders' spinning apparatuses and enhances synthetic silk's characteristics. However, the precise impacts of various conditions under which stretching occurs have not been thoroughly exam- ined. Researchers from the University of Wyoming and Arizona State University tested these conditions on three versions of the $A$. aurantia MaSp2 protein designed with increasing elasticityto-strength amino acid motif ratios: $1 \mathrm{E}$, $2 \mathrm{E}$, and $3 \mathrm{E}$. $3 \mathrm{E}$ was hypothesized to form the most elastic and weakest of the three fiber types, while 1E was hypothesized to yield the strongest, least elastic fibers.

To identify structural features underlying stretched silk's improved mechanical properties relative to as-spun silk, the researchers subjected both fiber types to x-ray diffraction and Raman spectroscopy carried out at the BioCARS beamline 14-BM-C at the APS.

Surprisingly, even though stretched silk, regardless of its elasticity ratio, was half the diameter of as-spun silk, few differences were observed between the fibers in the amount of $\beta$-sheet formation or the $\beta$-sheet secondary structure or orientation. Any functional differences, the researchers suspected, must depend on changes in the fibers' molecular confirmation as a result of the stretching process.

Aqueous isopropanol (IPA) was found to be the most effective solution for increasing the extensibility of postspin fibers. Other solutions, such as aqueous ethanol and aqueous methanol, were optimal for increasing tensile strength in some cases, depending on the specific fiber type.

IPA post-spin stretching produced a significant increase in the strain and extensibility values for all three fibers. The increase in extensibility correlated to the elasticity ratio, with $3 \mathrm{E}$ having the greatest increase, followed by $2 \mathrm{E}$ then $1 \mathrm{E}$ fibers. In contrast, although the average strength of $1 \mathrm{E}$ and $3 \mathrm{E}$ fibers was similar, the strongest individual fiber was a $3 E$ fiber.

Heating the IPA bath to $60^{\circ} \mathrm{C}$ also had unpredictable results. For example, heat substantially improved the maximum strain and extensibility values for $2 \mathrm{E}$ and $3 \mathrm{E}$ but not $1 \mathrm{E}$ fibers, while heating increased tensile strength of $1 \mathrm{E}$ and $2 \mathrm{E}$, but not $3 \mathrm{E}$ fibers.

The researchers attributed the fact that the synthetic fibers did not necessarily perform in ways predicted by their elasticity ratio to a combination of factors, including non-linear interactions between water in the IPA solution and the proteins' primary structures and the unpredictable nature of $\beta$-sheet alignment. - Chris Palmer

See: Amy E. Albertson ${ }^{1}$, FlorenceTeulé $^{1 \ddagger}$, Warner Weber ${ }^{2}$, Jeffery L. Yarger $^{2}$, and Randolph V. Lewis ${ }^{1 * \ddagger \ddagger}$, "Effects of different post-spin stretching conditions on the mechanical properties of synthetic spider silk fibers," J. Mech. Behav. Biomed. Mat. 29, 225 (2014). DOI: 10.1016/j.jmbbm.2013.09.002 Author affiliations: ${ }^{1}$ University of Wyoming, ${ }^{2}$ Arizona State University Present address: łUniversity of

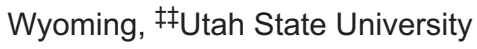
Correspondence: * Silk@uwyo.edu

This work was supported by National Institute of Health (NIH) Award no. EB000490, U.S. Department of Energy (DOE) Award no. DE-SC0004791, and Department of Defense Air Force Office of Scientific Research (AFOSR) Award no. FA9550-09-1-0717. Jeffery Yarger would like to acknowledge support from the Department of Defense AFOSR under Award no.FA9550-10-1-0275 and the U.S. National Science Foundation, Division of Materials Research under Award no. DMR-0805197. 


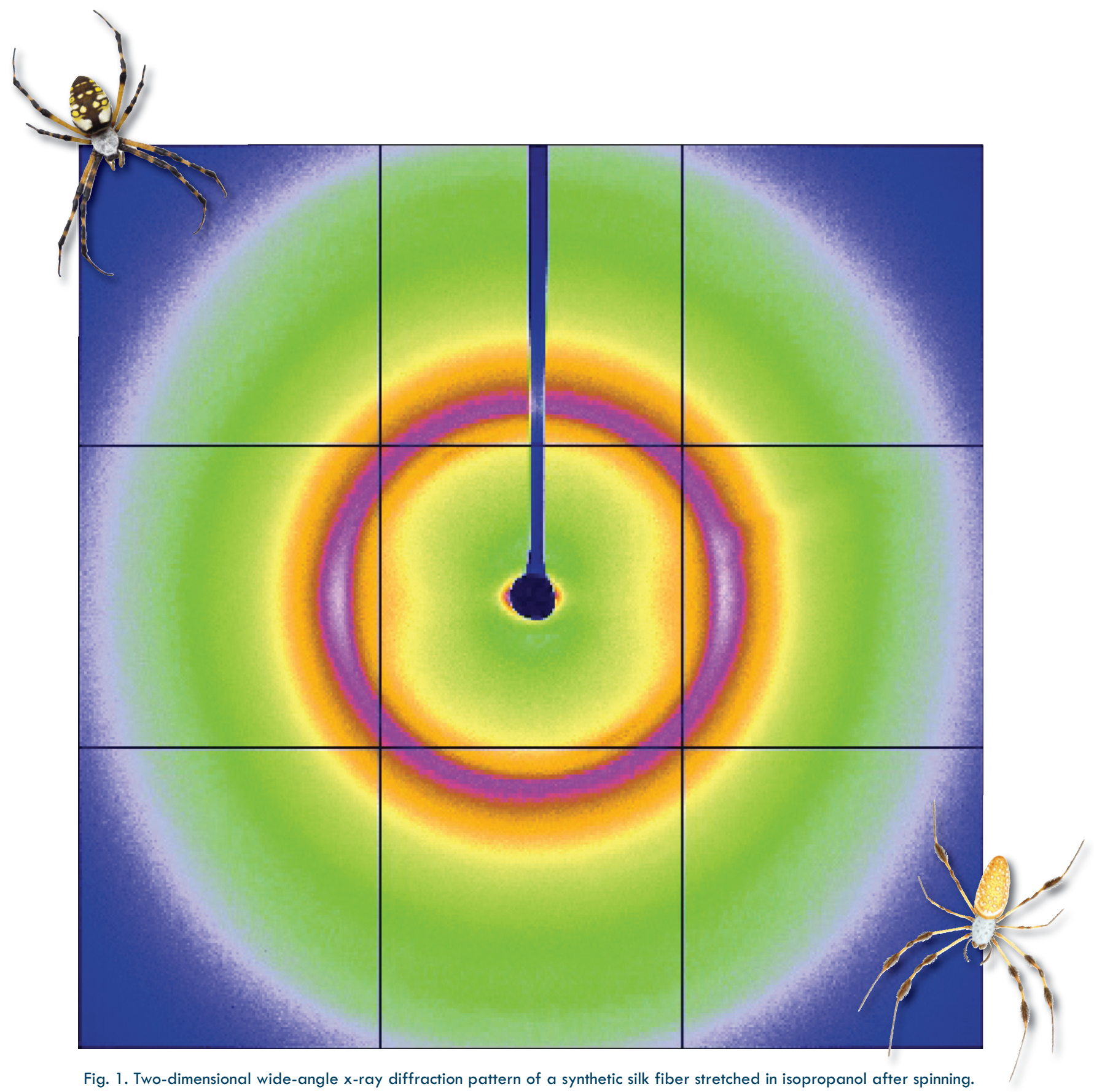

Fig. 1. Two-dimensional wide-angle x-ray diffraction pattern of a synthetic silk fiber stretched in isopropanol after spinning.

The Argiope aurantia (left above, not to scale) is one of the more conspicuous species of orb-weaving spiders. The species name "aurantia" comes from medieval Latin urantium meaning orange (the fruit). The argiope breeds once a year. They are carnivorous predators, attacking aphids, flies, grasshoppers, mosquitoes, wasps and bees that are trapped in the web. The female spider hangs, head down, in the center of its web while waiting, often holding her legs together in pairs so that it looks as if there are only four of them. When an insect hits the web, the spider feels the vibrations and comes running. As is the case for all spiders, A. aurantia has a venomous bite that paralyzes its prey. Source: Galveston County Master Gardners, http://aggie-horticulture.tamu.edu/galveston/beneficials/beneficial24_spider_blackandyellow_argiope.htm

This research used resources of the Advanced Photon Source, a U.S. DOE Office of Science User Facility operated for the DOE Office of Science by Argonne National Laboratory under Contract
In Florida and other southeastern states, the golden silk spider (right above, not to scale), Nephila clavipes (Linnaeus), a large orange and brown spider with feathery tufts on its legs, is particularly despised by hikers and hunters because the large golden webs of this species make a sticky trap for the unwary. As is typical with most spiders, there is little real danger from an encounter; the spider will bite only if held or pinched, and the bite itself will produce only localized pain with a slight redness, which quickly goes away. The webs are made in open woods or edges of dense forest, usually attached to trees and low shrubs, although they may be in the tops of trees or between the wires of utility lines. Source: University of Florida, http://entnemdept. ufl.edu/creatures/misc/golden_silk_spider.htm

14-BM-C • BioCARS • Life sciences • Macromolecular crystallography, fiber diffraction, biohazards at the BSL2/3 level, subatomic $(<0.85 \AA)$ resolution, large unit cell crystallography $\cdot 8-14.9 \mathrm{keV} \cdot$ On-site $\cdot$ Accepting general users • 


\section{A Framework for Next-Gen Optical Sensors AND LEDS}

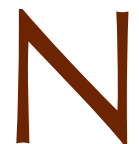

ew, highly efficient organic and inorganic light-emitting diodes (OLEDs and LEDs, respectively) and environmental and medical sensors might be made possible by utilizing porous materials known as metal-organic frameworks (MOFs) that combine metallic elements with a diverse range of fluorescent molecules - usually organic molecules - as ligands. The design and synthesis of rigid, fluorescent materials has been APS - research that could lead to new applications as well as allowing researchers to fine tune the properties of the materials.
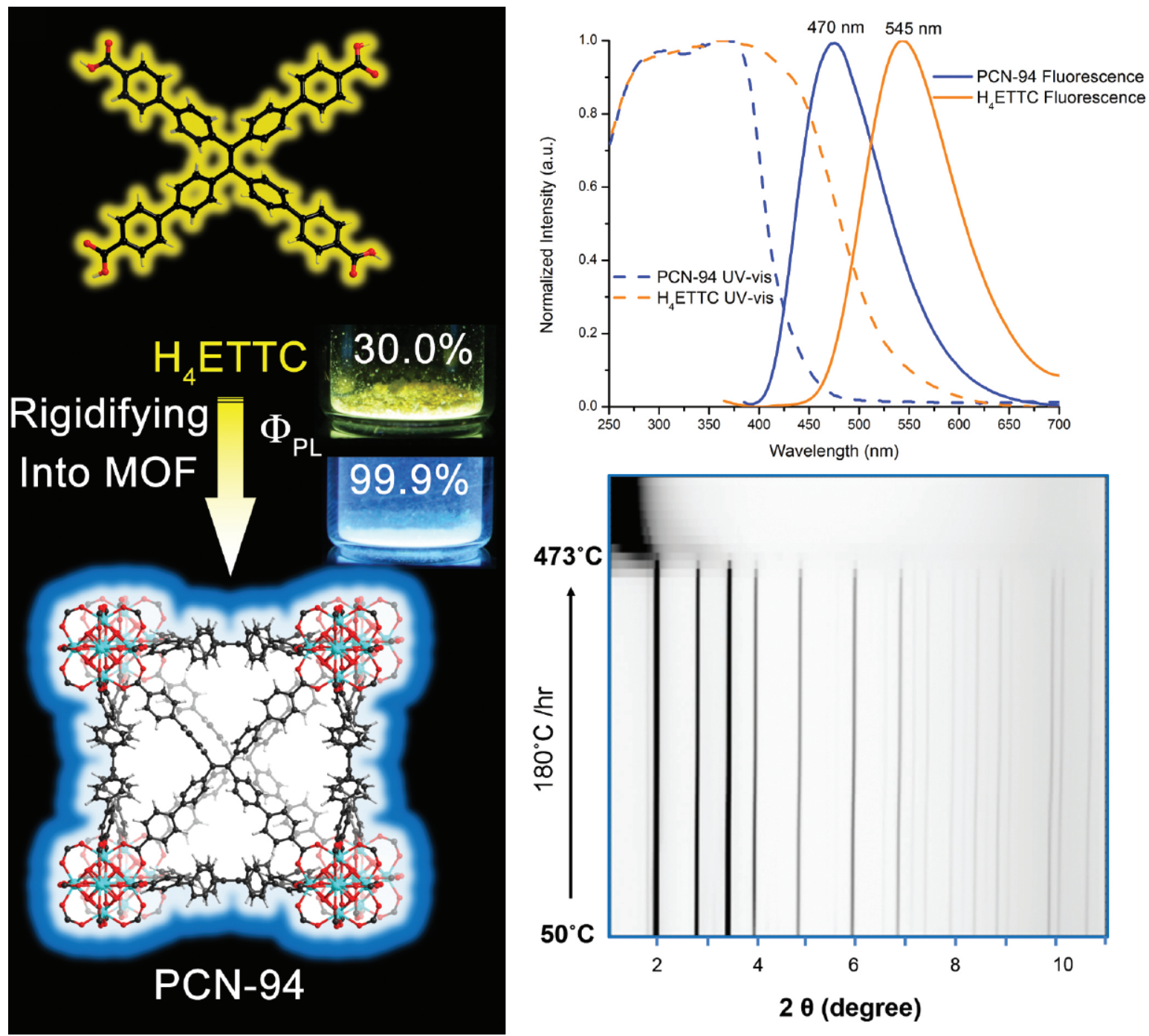

Fig. 1. A new tetraphenylethylene-based zirconium MOF exhibits a deep-blue fluorescent emission at $470 \mathrm{~nm}$ with a unity quantum yield $\left(99.9 \pm 0.5 \%\right.$ ) under $\mathrm{Ar}$, representing ca. $3600 \mathrm{~cm}^{-1}$ blue shift and tripled radiative decay efficiency vs the linker precursor. 
Over the last 50 years, electronic devices have been built around semiconductor elements such as silicon and germanium, or toxic inorganic semiconductors such as gallium arsenide and cadmium sulfide. There are, however, a myriad of fluorescent molecules, known as fluorophores, in the realm of organic chemistry that have interesting optoelectronic properties, which could be exploited as novel glowing sensors and OLEDs/LEDs. Even state-of-the-art molecular materials for such applications usually incorporate elements that are rare in the Earth's crust, such as lanthanide metals, whereas the new MOFs do not contain such rare elements, promoting sustainability and green chemistry technologies.

Unfortunately, incorporating such molecules into a working device is difficult because these molecules tend to quench their own fluorescence and so produce only weak light. Additionally, blue light emitters that are both efficient and stable remain the most coveted active device materials, especially for OLEDs.

Researchers from Texas A\&M University and the University of North Texas now think they have a solution to these problems by using organic components as the fluorophores of the solid frameworks (bright blue-emitting solid MOFs). The team produced rigid fluorescent MOF materials that respond to external stimuli by glowing strongly with no possibility for the fluorophores to dim through quenching, as occurs in solution or even the solid organic molecule without metal coordination.

The team has investigated a newly synthesized MOF based on the organic fluorophore, tetraphenylethylene and clusters of the metal zirconium, present in groups of six. The group carried out studies of the MOF, dubbed PCN-94, using powder $x$-ray diffraction (PXRD) data collected on XSD beamline 17BM-B at the APS to help ascertain its rigid structure that is critical to its fluorescence color and circumvention of quenching.

The new MOF glows with a deepblue fluorescence at a wavelength of $470 \mathrm{~nm}$ as peak maximum (Fig. 1). The team reports that $\mathrm{PCN}-94$ is highly efficient, which is quantified by the quantum yield (QY) of the material. They found that the QY of PCN-94 is $99.9 \pm$ $0.5 \%$ when held under an unreactive atmosphere of argon gas - even at room temperature, which is both remarkable and unprecedented in fluorescent MOFs! Tetraphenylethylene, when coordinated to zirconium metal in the PCN-94, was showing a blue shift with higher efficiency compared to the isolated tetraphenylethylene molecule, which glows a weaker yellow color at a spectral wavelength of $545 \mathrm{~nm}$ as peak maximum.

Moreover, the fluorescence process is over three times as efficient as the isolated tetraphenylethylene (approximately $100 \%$ vs $30 \%$ in QY) under complete deaeration conditions, whereas under ambient air the improvement in radiative decay and $Q Y$ is approximately $2 x$ for the solid MOF vs the free ligand. The team expected efficiency to be greatest at the cryogenic chilling temperature of $110 \mathrm{~K}$ (about $-163^{\circ} \mathrm{C}$ ) because self-quenching of the emission from the fluorophores is inhibited by the lower temperature, i.e., $110 \mathrm{~K}$ vs ambient room temperature (about $293 \mathrm{~K}$ or $20^{\circ} \mathrm{C}$ ). Paradoxically, however, they saw the fluorescence lifetime - how long the glow lasts - as well as the relative intensity of the light increase as they warmed the solid MOF from cryogenic to room temperature, whereas the solid organic fluorophores follows the normal behavior with lower emission intensity upon heating from cryogenic toward ambient temperatures. The team reports that this unusual fluorescence behavior is perhaps due to the fact that the fluorophores are "frozen" in their twisted connections between the metals in the framework at all temperatures while heating provides thermal assistance to a secondary excitation mechanism from a higher-lying excited state that feeds the blue-fluorescent excited state. In the free organic molecule, however, the twisting becomes more important as the temperature rises (which again impedes quenching).

The team suggests that their discovery of an efficiency boost and the "shift" of the fluorescent glow of the organic unit from yellow to the strongly sought-after deep-blue could open a new strategy for making OLED/LED and sensor materials that are based on this and other related MOFs that the two complementary research groups are currently designing on the heels of this pioneering work as a backdrop.

The field of MOF research has grown rather significantly since the turn of the Millennium and - given that there are dozens of different metals that can be used to build frameworks with thousands of different organic fluorophores - numerous possibilities exist toward building MOFs that fluoresce across the entire visible spectrum with similarly-remarkable efficiency as that of PCN-94 for a wide range of molecular electronic device and sensor applications. - David Bradley

See: Zhangwen Wei ${ }^{1}$, Zhi-Yuan $\mathrm{Gu}^{1}$, Ravi K. Arvapally ${ }^{2}$, Ying-Pin Chen ${ }^{1,2}$, Roy N. McDougald, Jr. ${ }^{2}$, Joshua F. Ivy², Andrey A. Yakovenko1, Dawei Feng ${ }^{1}$, Mohammad A. Omary ${ }^{2 * *}$, and Hong-Cai Zhou ${ }^{1,2 *}$, "Rigidifying Fluorescent Linkers by Meta-Organic Framework Formation for Fluorescence Blue Shift and Quantum Yield Enhancement," J. Am. Chem. Soc. 136, 8269 (2014).

DOI: $10.1021 /$ ja5006866

Author affiliations: ${ }^{1}$ Texas A\&M University, ${ }^{2}$ University of North Texas Correspondence:

*zhou@mail.chem.tamu.edu, ** omary@unt.edu

This work was supported as part of the Center for Gas Separations Relevant to Clean Energy Technologies, an Energy Frontier Research Center (EFRC) funded by the U.S. Department of Energy (DOE), Office of Science, Basic Energy Sciences under Award no. DE-SC0001015, and part of the Methane Opportunities for Vehicular Energy (MOVE) Program, an ARPA-E Project under Award no. DE-AR0000249. Z.W. and D.F. were supported by MOVE. Y.-P.C. and Z.-Y.G. were supported by EFRC. M.A.O. acknowledges support of his group's contributions by the National Science Foundation (CHE1413641; CHE-0911690; CMMI-0963509; CHE-0840518) and the Robert A. Welch Foundation (Grant B-1542). This research used resources of the Advanced Photon Source, a U.S. DOE Office of Science User Facility operated for the DOE Office of Science by Argonne National Laboratory under contract no. DE-AC02-06CH11357.

17-BM-B • XSD • Chemistry, materials science - Powder diffraction, high-pressure diamond anvil cell • 15-18 keV • On-site • Accepting general users • 


\section{in Certain Polymer Solar Cells, Disorder Can Be a Delight}

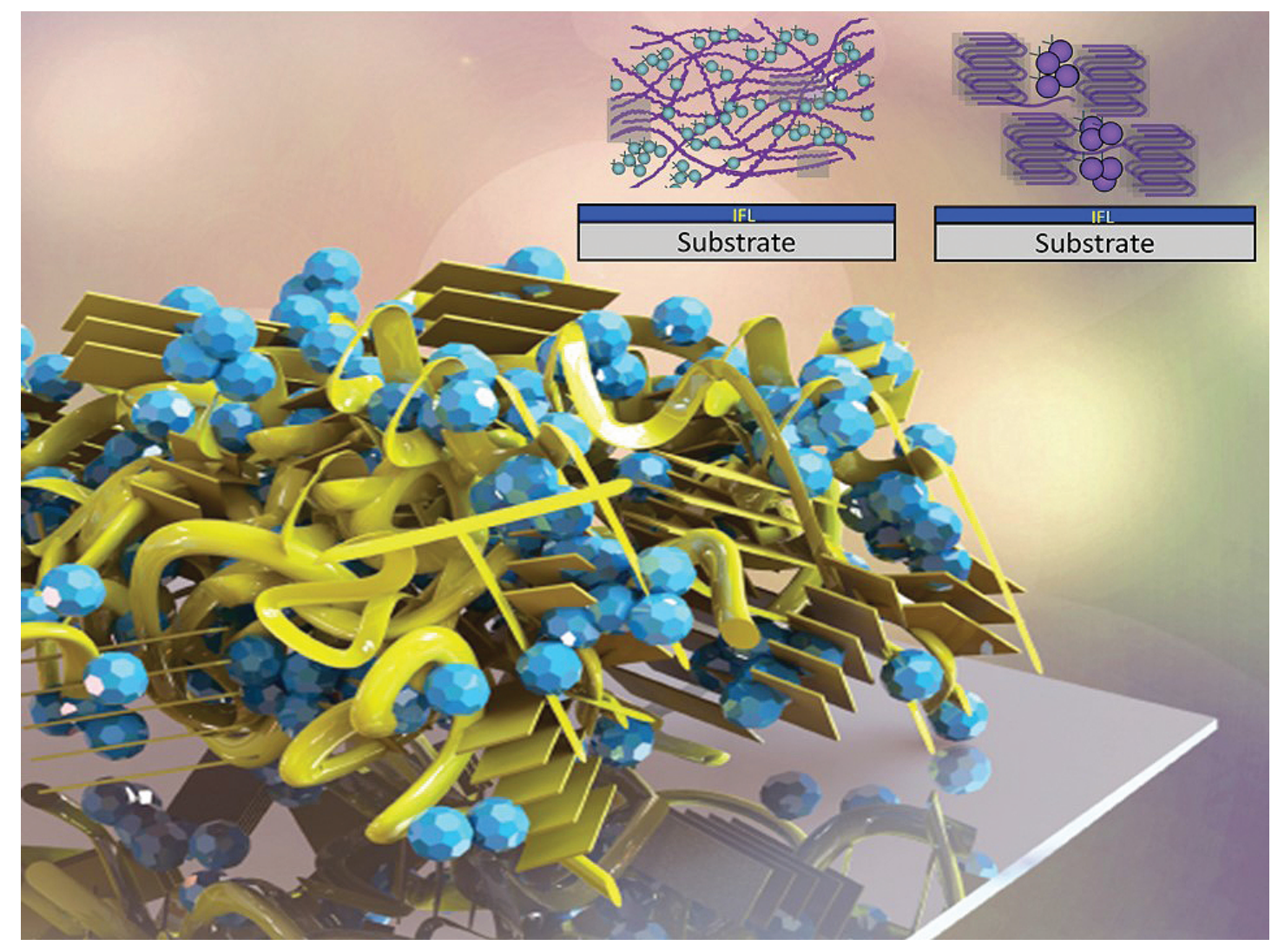

Fig. 1. Visualization of photovoltaic copolymers. In the foreground is a computer-generated three-dimensional image with an electron donating polymer molecules in yellow and an electron accepting fullerene molecules in blue. Exciton separation occurs at the interface between the two materials. Note the alternately tangled and ordered polymer sections. The left inset is a visualization of high-efficiency charge transfer copolymers such as PTB7. This copolymer has small crystalline regions on the order of $2 \mathrm{~nm}$ with large areas of disorder between. Fullerene density is high. Single chains can pass multiple separation sites to enhance charge carrier mobility. The right inset shows photovoltaic polymers like P3HT. Here the crystalline areas are larger, on the order of $10 \mathrm{~nm}$, and fullerene density is reduced. Polymer chains are bunched, with a single chain fitting within a 10-nm-diameter sphere. Here, slower exciton separation means more recombination and less efficient electricity production. Image by Jenny Morber and Lin X. Chen based on J.M. Szarko et al., Adv. Funct. Mater. 24, 10 (2014). 
$\mathrm{F}$ ossil fuels began as sun-powered plants and animals. Wind begins as unevenly heated air. Only recently have we learned how to harvest our electricity directly from sunlight. We do this using the photovoltaic effect - literally "light voltage" — in which solar energy frees charge carriers to move about. A photoelectric device, or solar cell, uses this conversion to generate electrical current. Traditional solar cells are made of stiff semiconductors, but polymer solar cells use long, flexible carbon-based molecules. Polymer solar cells aspire to be everything traditional solar cells are not: lightweight, flexible, simple to produce, environmentally friendly, low-cost, and ubiquitous. Unfortunately, they currently suffer problems with efficiency and stability - enough to all but cancel out their attributes. Recently, a polymer was discovered with excellent solar efficiency, meaning that each bit of sunlight absorbed produces more power than other polymers. Researchers working at the APS went looking for the why. What they found may force others to reevaluate how they select solar cell polymers, and help to create more efficient, market-valuable devices.

The new promising polymer is called PTB7. If this were sports instead of science, PTB7 would be the young upstart and P3HT would be the seasoned champion unceremoniously knocked off the leader board. But how was PTB7 able to perform so well?

To answer their questions, the researchers needed a thorough understanding of both polymers' structures, and intimate knowledge of how their electrons interact and move in and between their molecules. For structural studies, the researchers from Northwestern University and Argonne turned to the XSD 8-ID-E beamline at the APS. There, the researchers from Northwestern University and Argonne performed grazing-incidence small angle x-ray scattering (GISAXS) and wide-angle x-ray scattering (GIWAXS) measurements, which supplied information such as the presence and size of repeating units with the polymers, and how they were arranged. Polymers are notoriously difficult to probe because their long molecules often tangle like a bowl of spaghetti. The high brightness of the APS $x$-rays and the state-of-the-art 8-ID-E x-ray beamline enabled the scientists to find tiny crystalline areas inside the tangles.

Current polymer photovoltaics are usually a film of mixed materials. The mixture contains polymers that donate electrons, and soccer ball-like fullerene derivatives that grab electrons (Fig. 1).

An efficient polymer solar cell must do four things well: harvest sunlight to create highly energetic excitons, move excitons to the boundary of the electron donor/acceptor materials, split excitons into positive and negative charges (holes and electrons), and collect these charges at the electrodes to generate current. The interface between electron donor materials and electron acceptor materials act as exciton separation sites. The researchers found that PTB7 excitons separated into electrons and holes more easily than other polymers.

Structural measurements showed that molecules within PTB7 arranged themselves into a much more disordered arrangement than $\mathrm{P} 3 \mathrm{HT}$, whose molecules formed more crystalline blocks. This meant that exciton separation sites were interspersed throughout PTB7, while separation sites in P3HT tended to pack together at the crystallite edges.

Excitons also separated into charge carriers at junctions within PTB7 molecules. This was strange because exciton separation usually occurs at interfaces between donor and acceptor materials. PTB7 molecules were working as both.

The researchers propose that the structural and chemical characteristics of PTB7 help excitons move along and between molecules and to separation sites more efficiently. An exciton travels along the polymer backbone until it comes to a charge acceptor. This acceptor can be an added material, or it can be a polymer segment within the molecule with different electron affinity. With so many available sites, exciton dissociation happens quickly. This super-fast carrier transport decreases instances in which excitons re-combine, leading to more efficient solar to electrical energy generation.

This work is significant because it had been assumed that charge transfer and separation processes within molecules were unimportant for device performance. Here the researchers found that structural and chemical variations caused stark differences in exciton splitting in the two polymers, with intramolecular charge separation playing an important role.

The researchers suggest that others reevaluate the necessary conditions for exciton splitting in photovoltaic polymers, using PCB7 as a model. Greater efficiency is crucial for polymer solar cells to become the solar energy device of choice, and this information brings polymer photovoltaics closer to the consumer market. — Jenny Morber

See: Jodi M. Szarko ${ }^{1,2}$, Brian S. RolCzynski ${ }^{1,2}$, Sylvia J. Lou ${ }^{1,2}$, Tao Xu1 ${ }^{1}$, Joseph Strzalka ${ }^{2}$, Tobin J. Marks ${ }^{1}$, Luping $\mathrm{Yu}^{1 * *}$, and Lin X. Chen ${ }^{1,2 *}$, "Photovoltaic Function and Exciton/Charge Transfer Dynamics in a Highly Efficient Semiconducting Copolymer," Adv. Funct. Mater. 24, 10 (2014). DOI: 10.1002/adfm.201301820 Author affiliations: ${ }^{1}$ Northwestern University, ${ }^{2}$ Argonne National Laboratory Correspondence: * Ichen@anl.gov, ** lupingyu@uchicago.edu

This research is supported by the ANSER Center, an Energy Frontier Research Center funded by the U.S. Department of Energy (DOE) Office of Science- Basic Energy Sciences, under Award Number DESC0001059. This research used resources of the Advanced Photon Source, a U.S. DOE Office of Science User Facility operated for the DOE Office of Science by Argonne National Laboratory under Contract No. DE-AC02-06CH11357.

8-ID-E $\cdot$ XSD $\cdot$ Materials science, polymer science, physics - Grazing incidence small-angle scattering, x-ray photon correlation spectroscopy $\bullet 7.35-7.35 \mathrm{keV} \cdot$ On-site $\cdot$ Accepting general users • 


\section{How Atomic Vibrations Stabilize Metallic Vanadium Dioxide}

M aterials that undergo metal-to-insulator transitions are of wide practical importance in semiconductor and optoelectronic devices, but their behavior still presents some fundamental challenges for condensed matter theory. Vanadium dioxide, an archetype of such materials, changes from insulator to conductor when heated through $340 \mathrm{~K}$, and the transition is accompanied by a change in lattice structure (Fig. 1). Using both x-ray scattering and spectroscopy measurements at the APS and at the Oak Ridge National Laboratory's (ORNL's) Spallation Neutron Source (SNS), respectively, researchers have shown that the phase transition is driven by a large change in the entropy associated with lattice vibrations. This new evidence, backed up by detailed calculations of the bonding in the metallic phase, brings theoretical insight that will be valuable for predictive design of novel materials.

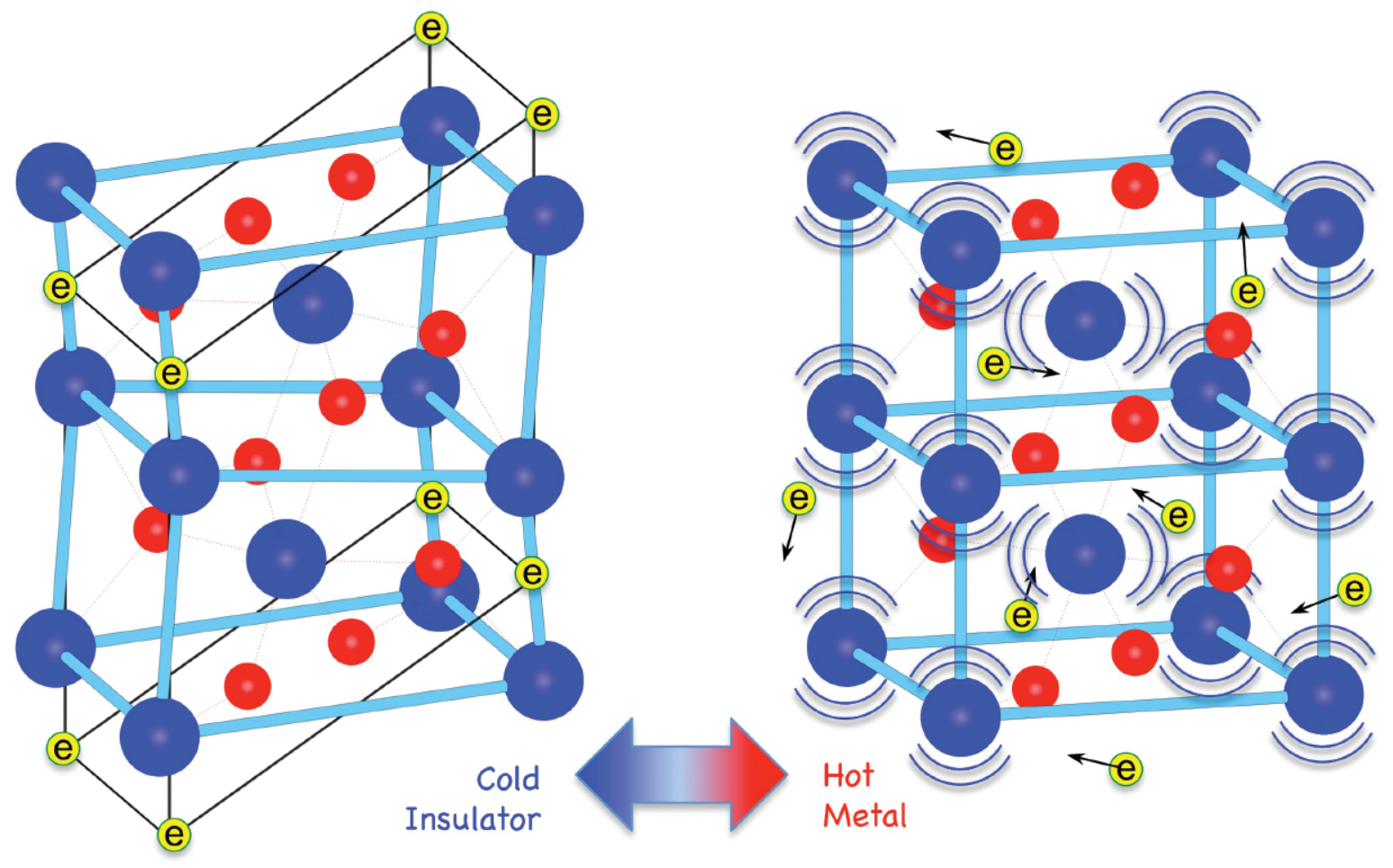

Fig. 1. Left: Monoclinic lattice structure of vanadium dioxide in its insulating phase, with vanadium atoms (blue) paired up in dimers and electrons bound to them. Right: In the conducting phase, large vibrational motions stabilize the tetragonal phase and free up conduction electrons. 
In its metallic phase, vanadium dioxide has a tetragonal lattice in which the vanadium atoms form a regularly spaced rectilinear network. On cooling through the transition temperature, the vanadium atoms pair up and the spacing between them becomes unequal, and they lie on zigzag lines. The lattice is now monoclinic. At the same time, the conduction electrons that were free to move throughout the lattice become localized to the paired vanadiums. In this material, as in others of this sort, the fact that both the lattice and electronic configurations change has made it difficult to know whether this is primarily a Peierls transition (driven by electron-lattice interactions) or a Mott transition (driven by electron-electron interactions).

An additional difficulty is that vanadium scatters neutrons incoherently, so that identifying lattice vibration modes through traditional single-crystal inelastic neutron scattering studies is impossible. So a team of researchers from ORNL and Argonne turned to newly-developed $x$-ray and neutron scattering techniques to shed light on the phonon physics involved in the vanadium dioxide metal-insulator transition.

Using powder-averaged neutron spectroscopy measurements at the SNS, the team found that a high density of soft phonons, with energies around $12 \mathrm{meV}$, appears in the vanadium dioxide lattice as it goes from insulator to metal. Ab initio calculations of lattice vibrations provided good agreement with the measured phonon density of states, provided that a significant degree of anharmonicity was included in the interatomic potential. Through these calculations the researchers were able to conclude that the appearance of low-energy phonons accounts for about two-thirds of the entropy change of the transition.

X-ray thermal diffuse scattering measurements at XSD beamline 33BM-C at the APS identified these phonons as coherent vibrations of the vanadium atoms associated with particular lattice periodicities (wave vectors).
But the team had only an incomplete picture of the phonons: the neutron studies revealed their energy, and the diffuse $\mathrm{x}$-ray scattering their wavevectors, but more work was needed to put those two things together.

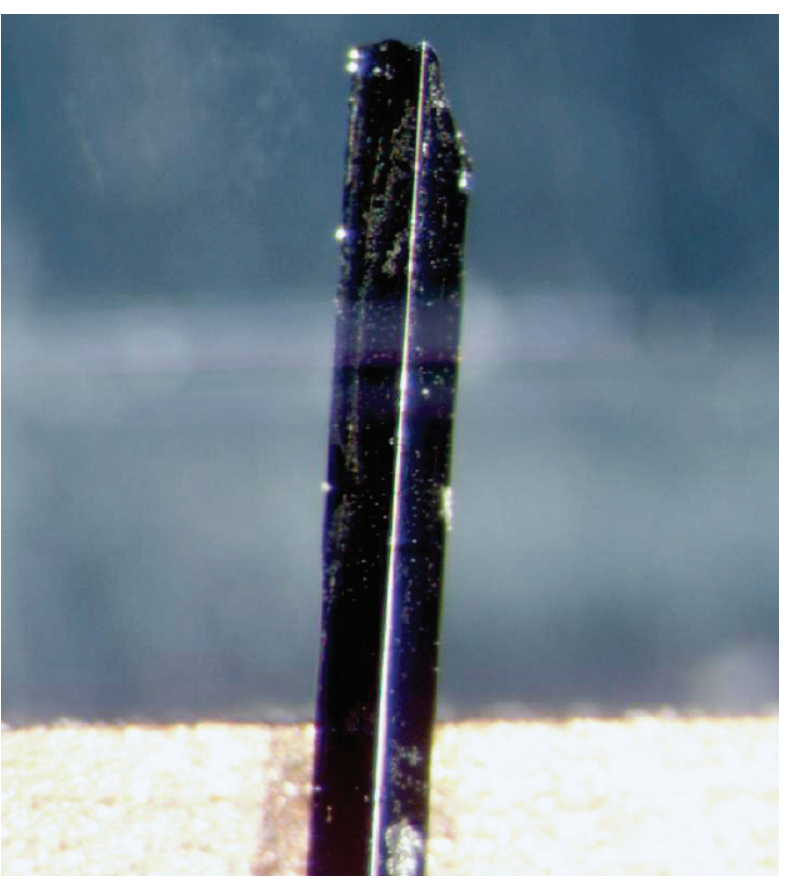

A single crystal of vanadium dioxide.

The team therefore conducted inelastic $x$-ray scattering measurement with the HERIX spectrometer at XSD beamline 30-ID-B,C, where the instrumentation can detect energy changes in kiloelectronvolt $\mathrm{x}$-rays with millielectronvolt resolution while also recording the direction of scattered photons. The energy measurements found that the low-energy photons, associated with motions of the vanadium atoms, had unusually broad and asymmetric peaks, indicating very short phonon lifetimes and strong damping. The phonon dispersion spectrum in metallic vanadium dioxide is similar to that in titanium dioxide (rutile), which has the same tetragonal structure, but is shifted to lower energies. The amplitude of the vanadium atomic vibrations is correspondingly remarkably large, about $0.02 \mathrm{~nm}$, and not much less than the shift in vanadium positions that occurs in the transition from the monoclinic to the tetragonal lattice.

Combining the new experimental data with calculations of lattice motion, the team concludes that large amplitude vibrations of the vanadium atoms stabilize the tetragonal structure above $340 \mathrm{~K}$. Below that transition temperature, by contrast, the lattice is distorted by a Peierls instability that causes conduction electrons to localize to paired vanadium atoms. The researchers say that this detailed understanding of the relationship between changes in electronic and lattice structure will be helpful in efforts to refine the performance of metal oxides in practical applications. - David Lindley

See: John D. Budai ${ }^{1 *}$, Jiawang Hong $^{1}$, Michael E. Manley ${ }^{1}$, Eliot D. Specht ${ }^{1}$, Chen W. Li ${ }^{1}$, Jonathan Z. Tischler ${ }^{2}$, Douglas L. Abernathy ${ }^{1}$, Ayman H. Said², Bogdan M. Leu², Lynn A. Boatner ${ }^{1}$, Robert J. McQueeney ${ }^{1}$, and Olivier Delaire ${ }^{1}$, "Metallization of vanadium dioxide driven by large phonon entropy," Nature 515, 535 (27 November 2014).

DOI: 10.1038/nature13865

Author affiliations: ${ }^{1}$ Oak Ridge National Laboratory, ${ }^{2}$ Argonne National Laboratory

Correspondence: * budaijd@ornl.gov

Research by J.D.B., O.D., M.E.M., E.D.S., L.A.B. and R.J.M. was supported by the U.S. Department of Energy (DOE) Office of Science-Basic Energy Sciences (BES), Materials Sciences and Engineering Division (MSED). Research by J.H. was supported by the Center for Accelerating Materials Modeling, funded by the U.S. DOE-BES, MSED. Experimental work by C.W.L. was sponsored by the Laboratory Directed Research and Development Program of ORNL (Principal Investigator, O.D.). Research by D.L.A. at the Spallation Neutron Source and J.Z.T., A.H.S. and B.M.L. at the APS was supported by the U.S. DOE-BES, Scientific User Facilities Division. This research used resources of the Advanced Photon Source, a U.S. DOE Office of Science User Facility operated for the DOE Office of Science by Argonne National Laboratory under Contract No. DEAC02-06CH11357.

30-ID-B,C $\cdot X S D \cdot$ Physics, materials science - Inelastic x-ray scattering • 5-30 keV, 23.7$23.9 \mathrm{keV} \cdot$ On-site $\cdot$ Accepting general users

33-BM-C - XSD - Materials science, physics, chemistry $•$ Diffuse $x$-ray scattering, general diffraction, powder diffraction, $\mathrm{x}$-ray reflectivity, grazing incidence diffraction, anomalous and resonant scattering (hard x-ray) $\cdot 5-35$ $\mathrm{keV} \cdot$ On-site $\cdot$ Accepting general users $•$ 


\section{Cracking the Puzzle of Material Failure}

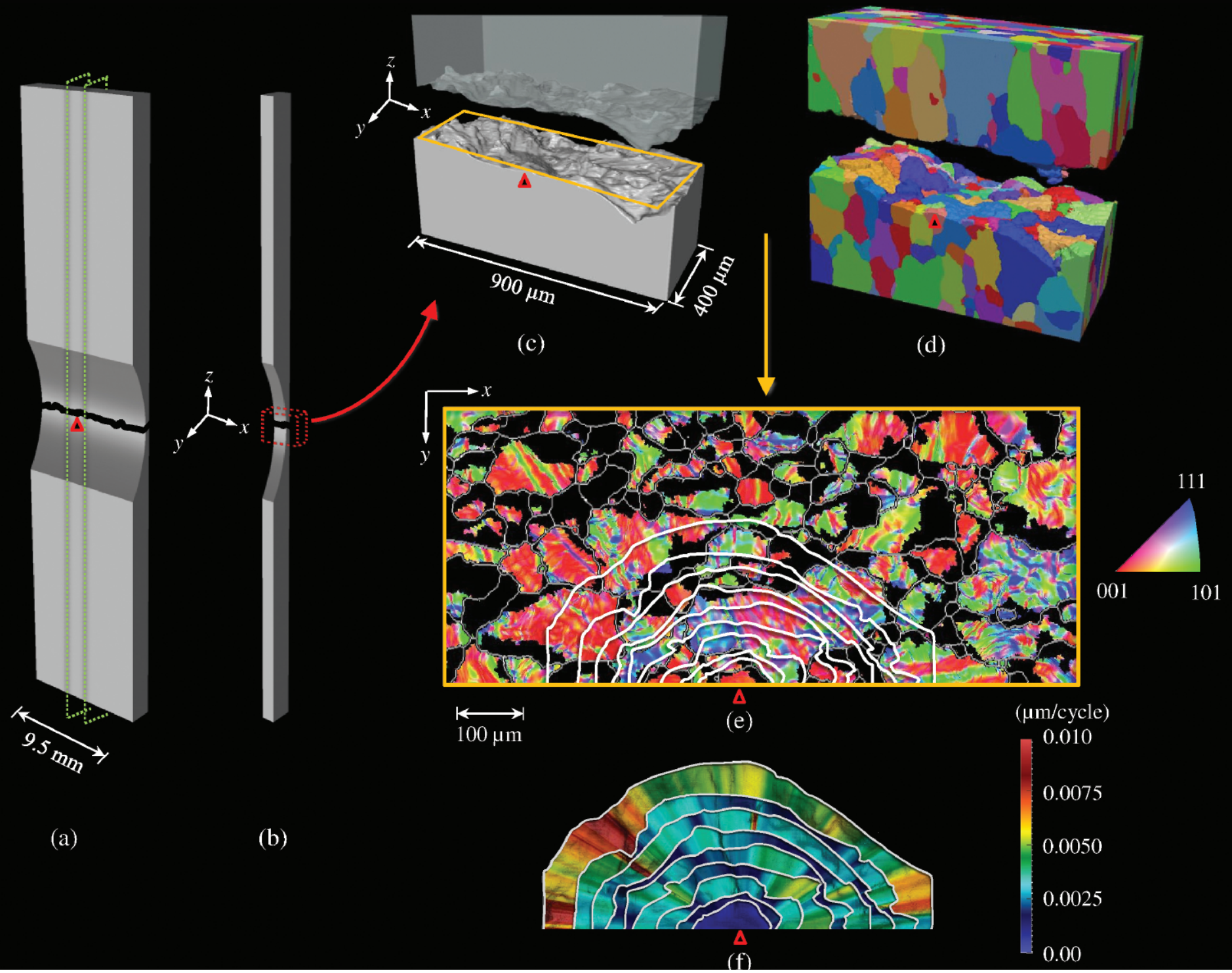


U nderstanding how cracks spread through a material is important for predicting how long a structure might last before breaking, as well as for designing new materials more resistant to failure. Researchers have long been able to watch a crack spread along a surface, but because cracks propagate in three dimensions (3-D), such two-dimensional (2-D) studies missed crucial information. Some 3-D methods use $x$-rays or electron beams to characterize the structure of the cracked crystals that make up the material, but these tend to study only a portion of a crack and typically have no information about its 3-D evolution over time. Researchers using the high-brightness, highly penetrating x-rays from the APS have combined experimental methods to obtain high-resolution, quantitative measurements of the events involved in material cracking, and link the measurements to a history of how a crack evolved.

The cracks in question are microstructurally small, meaning that their dimensions are on the order of the grain size in a crystalline material, and that their growth is sensitive to the crystalline structure; the way local grains respond to forces at the leading edge of the crack will determine the next step in the crack's evolution. The researchers - from Cornell University, Lawrence Livermore National Laboratory, and Carnegie Mellon University, studied an aluminum-magnesium-silicon alloy used as a liner in a container that stores pressurized fluids.

They started by taking a small sample of the alloy, $9.5 \mathrm{~mm} \times 44.5 \mathrm{~mm}$ $x 1.75 \mathrm{~mm}$ with a region in the center milled down to a thickness of $400 \mu \mathrm{m}$, where they hoped a crack would form. They alternately applied and released tension to the sample, generating a series of marker bands that let them calibrate how the crack grew over time. They periodically imaged the sample using a scanning electron microscope (SEM) to see how far the crack had spread on the visible surface. Once the sample broke in two, they used the

\footnotetext{
$<$ Fig. 1. An aluminum-magnesium-silicon sample was mechanically tested until a crack formed and grew to failure (a), then excess material was cut away around the crack-formation site (b) to create a smaller sample for study using x-ray CT (c) and near-field HEDM (d). A top-down view shows crack growth along grain boundaries (black regions) or within grains (colored regions), with white curves representing crack shape over time (e). Also shown is the local variability in rate of crack evolution ( $f$ ).
}

marker bands and SEM images to map the crack history and trace the crack to the site where it originated.

Next, they bonded the two halves back-to-back for ease of imaging and mounted the sample at the XSD 1-ID$B, C, E$ beamline at the APS. They performed x-ray computed tomography (CT), taking approximately 900 tomograms as they rotated the sample $180^{\circ}$, then stacked the 2-D images together to reconstruct 3-D images of the exposed crack surfaces.

The team also used the beamline to perform near-field, high-energy $\mathrm{x}$-ray diffraction microscopy (HEDM). That technique yielded 3-D maps of the grain shapes and orientations, letting the researchers see whether the crack had grown within grains or along boundaries between grains.

Combining the fracture map, the $x$ ray $\mathrm{CT}$, and the HEDM provided an unprecedented view of crack evolution from a "naturally" initiated crack (Fig. 1). The collected data can now be used to run a computer simulation of cracking, which will help scientists learn what factors control a crack's evolution in 3-D. They may discover, for example, how big a crack has to grow before forces acting on the bulk of a material become more important than the local microstructure in directing the fracture.

The researchers would like to validate their findings with other aluminum alloys, as well as with other materials, though in some it may be difficult to create the marker bands. There is still much to learn about microstructurally small cracks, the researchers say, but the fact that the APS beamline enables
3-D studies of materials that are actually used in real engineering applications could advance the science significantly. - Neil Savage

See: Ashley D. Spear ${ }^{1 \neq *}$, Shiu Fai Li ${ }^{2}$, Jonathan F. Lind ${ }^{3}$, Robert M. Suter ${ }^{3}$, and Anthony R. Ingraffea", "Three-dimensional characterization of microstructurally small fatigue-crack evolution using quantitative fractography combined with post-mortem X-ray tomography and high-energy $\mathrm{X}$-ray diffraction microscopy," Acta Mater. 76, 413 (2014).

DOI: 10.1016/j.actamat.2014.05.021 Author affiliations: ${ }^{1}$ Cornell University, 2Lawrence Livermore National Laboratory, ${ }^{3}$ Carnegie Mellon University †Present address: University of Utah Correspondence:

* ashley.spear@utah.edu

This work is supported by the National Science Foundation Graduate Research Fellowship Program under Grant no. DGE-0707428; under the auspices of the U.S. Department of Energy (DOE) by Lawrence Livermore National Laboratory under Contract no. DE-AC52-07NA27344; and by grant DESC0002001 at Carnegie Mellon University. This research used resources of the Advanced Photon Source, a U.S. DOE Office of Science User Facility operated for the DOE Office of Science by Argonne National Laboratory under Contract No. DE-AC02-06CH11357.

1-ID-B,C,E - XSD - Materials science, physics, chemistry $\cdot$ High-energy $x$-ray diffraction, tomography, small-angle $x$-ray scattering, fluorescence spectroscopy, pair distribution function, phase contrast imaging 50-90 keV, 50-150 keV • On-site • Accepting general users . 


\section{BUILDING LONGER-LASTING LITHIUM-SULFUR BATTERIES}
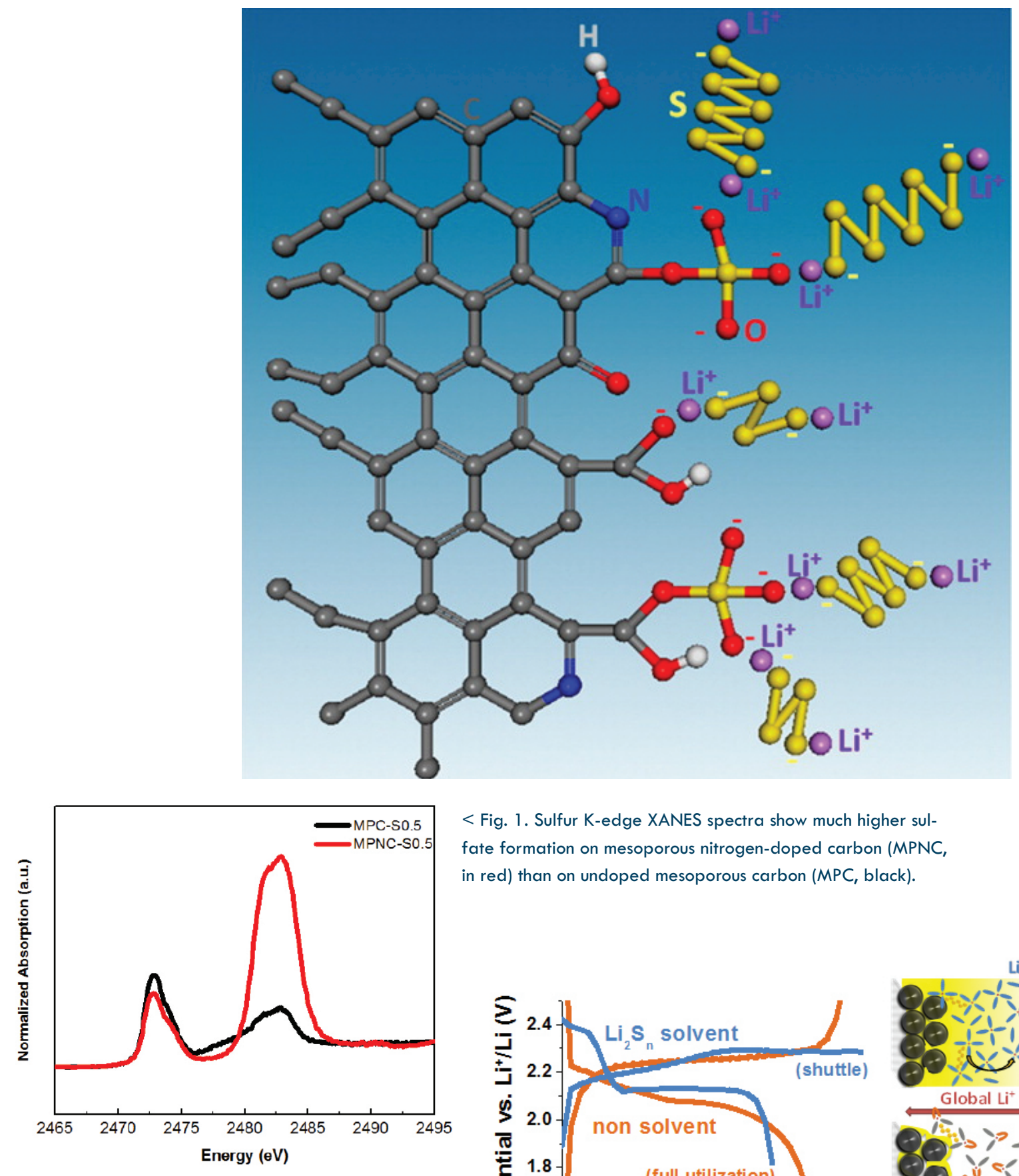

$<$ Fig. 1. Sulfur K-edge XANES spectra show much higher sulfate formation on mesoporous nitrogen-doped carbon (MPNC, in red) than on undoped mesoporous carbon (MPC, black).
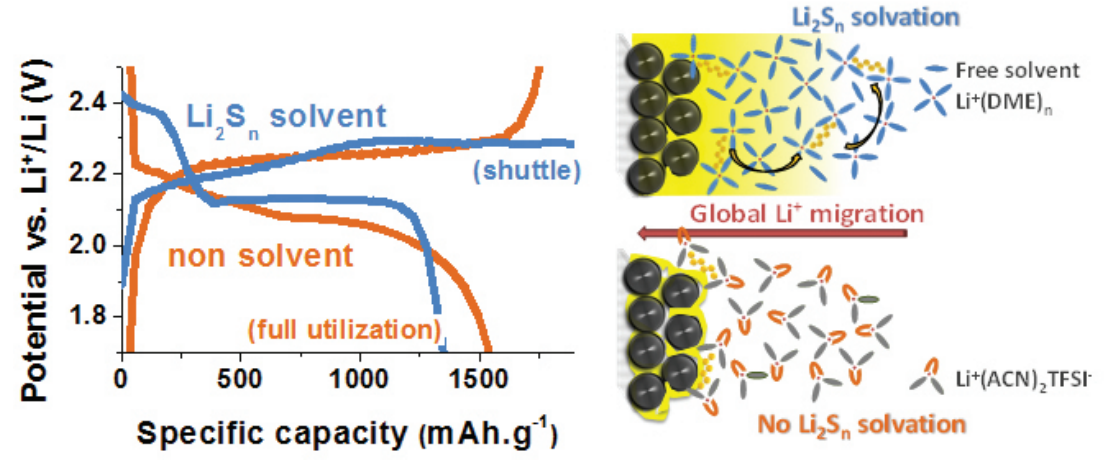

Fig. 2. A graph of voltage profiles (left) shows better discharge (lines descending to right) and charge (ascending lines) for batteries using a non-solvent electrolyte (orange) than a typical electrolyte (blue). In a typical lithium ion 1,2-dimethoxy ethane solvent (top right), sulfur atoms (yellow squiggles) form polysulfides that dissolve (yellow field). In the non-solvent electrolyte (bottom right), the polysulfide remain near the carbon (black circles) of the electrode. 
ithium-sulfur (Li-S) batteries are promising for the next generation of energy storage devices, for potential use in electric vehicles, and to capture renewable energy generated by solar and wind power. They can have three to five times the energy of a comparable lithium-ion battery, and sulfur is lightweight and inexpensive. But the efficiency of Li-S batteries drops quickly, and their lifetimes currently are too short for commercial use. Part of the reason is that sulfur forms compounds, polysufides, which dissolve in the battery's electrolyte, then travel back and forth between the positive and negative electrodes, undergoing chemical reactions that reduce the efficiency of the cell, a phenomenon known as the "shuttle effect." Two teams of researchers have proposed different solutions to these problems, and used user facilities including the APS to test their ideas.

One team of scientists from The Pennsylvania State University, the National Institute of Standards and Technology (NIST), and Argonne developed a cathode material that trapped the new sulfur species and reduced the shuttle effect [1]. They created their cathode out of mesoporous carbon, studded with pores between $2 \mathrm{~nm}$ and $50 \mathrm{~nm}$ in size, which they doped with nitrogen. Sulfur molecules are adsorbed in the pores and immobilized, unable to return to the electrolyte, and during subsequent charge-discharge cycles, the battery retained approximately $99.5 \%$ of its capacity. The researchers wanted to understand why the carbon doped with nitrogen achieved a higher capacity retention than un-doped mesoporous carbon.

The researchers started with doped and un-doped samples of the carbon, then added sulfur to simulate battery performance and performed $x$ ray absorption near-edge structure (XANES) spectroscopy on the samples. They used the NIST U7A beamline at Brookhaven National Laboratory to determine the coordination structures of the carbon, nitrogen, and oxygen elements on the carbon cathode materials before and after sulfur loading. To measure the sulfur, they performed the same procedure with the XSD 9-BM-B$B, C$ beamline at APS, which provided the higher-energy $\mathrm{x}$-rays needed to produce spectra from that element.

The team looked at the chemical environment of the nitrogen before and after the sulfur was adsorbed and saw no change, which showed that the nitrogen was not directly forming chemical bonds with the sulfur to immobilize it. There was, however, a change in the oxygen functional group and an increase in sulfates in the nitrogen-doped sample (Fig 1). The team's theory is that the presence of the nitrogen weakens the double bond between carbon and oxygen, rendering the carbon more reactive with the sulfur.

The second team of scientists from the University of Waterloo (Canada) and BASF SE (Germany), and Argonne attacked the problem with a new class of electrolytes, consisting of a solventsalt complex and a highly fluorinated ether [2]. The electrolyte, they expected, would be less likely to dissolve the polysulfides, thus limiting their ability to participate in the shuttle effect. The trick was to make an electrolyte with low solubility to control the polysulfides, but also low enough viscosity that the lithium ion carrier could move easily between the electrodes. The researchers made several electrolytes with different chemistries, then measured their viscosity and conductance.

They built coin-style batteries with their materials, and brought them to the 9-BM-B,C beamline at the APS, where they performed XANES spectroscopy while the batteries continuously charged and discharged (Fig. 2). The experiment confirmed the mechanism at work: the polysulfides were, in fact, formed.

However, the researchers found that the polysulfides' limited dissolution and mobility in the electrolyte strongly affected their equilibria processes, so that Li-S precipitated in a smoother, more controlled way than in the traditional electrolyte, allowing the battery to reach almost its theoretical capacity.
The non-solvent electrolyte also prevents the polysulfides from migrating away from the positive electrode, eliminating the drop in capacity caused by the shuttle effect.

The knowledge the teams gained from their work will help them to design even better electrolytes, and suggests ways they might change the architecture of the electrodes to handle even more sulfur, thus increasing the energy density of the battery. - Neil Savage

See: [1] Pengyu Zhu ${ }^{1}$,Jiangxuan Song ${ }^{1}$, Dongping $\mathrm{Lv}^{1}$, Donghai Wang ${ }^{1 *}$,

Cherno Jaye ${ }^{2}$, Daniel A. Fischer ${ }^{2}$, Tianpin $\mathrm{Wu}^{3}$, and Yongsheng Chen ${ }^{1 * *}$,

"Mechanism of Enhanced Carbon Cathode Performance by Nitrogen Doping in Lithium-Sulfur Battery: An X-ray Absorption Spectroscopic Study," J. Phys. Chem. C 118, 7765 (2014).

DOI: $10.1021 / \mathrm{jp} 4123634$

Author affiliations: ${ }^{1}$ The Pennsylvania State University, ${ }^{2}$ National Institute of Standards and Technology, ${ }^{3}$ Argonne National Laboratory

Correspondence:

*dwang@psu.edu, **yzc2@psu.edu

and

See: [2] M. Cuisinier ${ }^{1}$, P.-E. Cabelguen $^{1}$, B.D. Adams ${ }^{1}$, A. Garsuch ${ }^{2}$, M. Balasubramanian ${ }^{3}$, and L.F. Nazar ${ }^{1 *}$, "Unique behaviour of nonsolvents for polysulphides in lithium-sulphur batteries," Energ. Environ. Sci. 7, 2697 (2014).

DOI: 10.1039/c4ee00372a

Author affiliations: ${ }^{1}$ University of Waterloo, bBASF SE, 'Argonne National Laboratory

Correspondence:

*Ifnazar@uwaterloo.ca

Supported by the BASF International Scientific Network for Electrochemistry and Batteries. This research used resources of the Advanced Photon Source, a U.S. DOE Office of Science User Facility operated for the DOE Office of Science by Argonne National Laboratory under Contract No. DE-AC0206CH11357.

9-BM-B,C $\cdot$ XSD - Materials science, chemistry, environmental science $\cdot \mathrm{X}$-ray absorption fine structure $\cdot 2.1-24 \mathrm{keV} \cdot$ On-site • Accepting general users • 


\section{IMAGING BATTERY STRAIN}

ithium-ion (Li-ion) batteries provide the juice for many portable electronic devices. These rechargeable batteries offer several advantages, such as a high energy density and low maintenance. But like all batteries, they degrade over time. The constant charging and discharging of a lithium-ion battery causes volume contraction and expansion inside electrode components. This mechanical strain can eventually lead to cracking and is one of the main causes of $\mathrm{Li}$ ion battery failure. To better understand the impact of strain, researchers have produced the first three-dimensional mapping of atom-level strain inside metal oxide nanoparticles that store and release charge. The experiments, performed at the APS, show that strain is not uniform across these battery components. These precise nanoscale images may help in optimizing the shape and size of nanoparticles, so that strain is less of a drain on battery life.

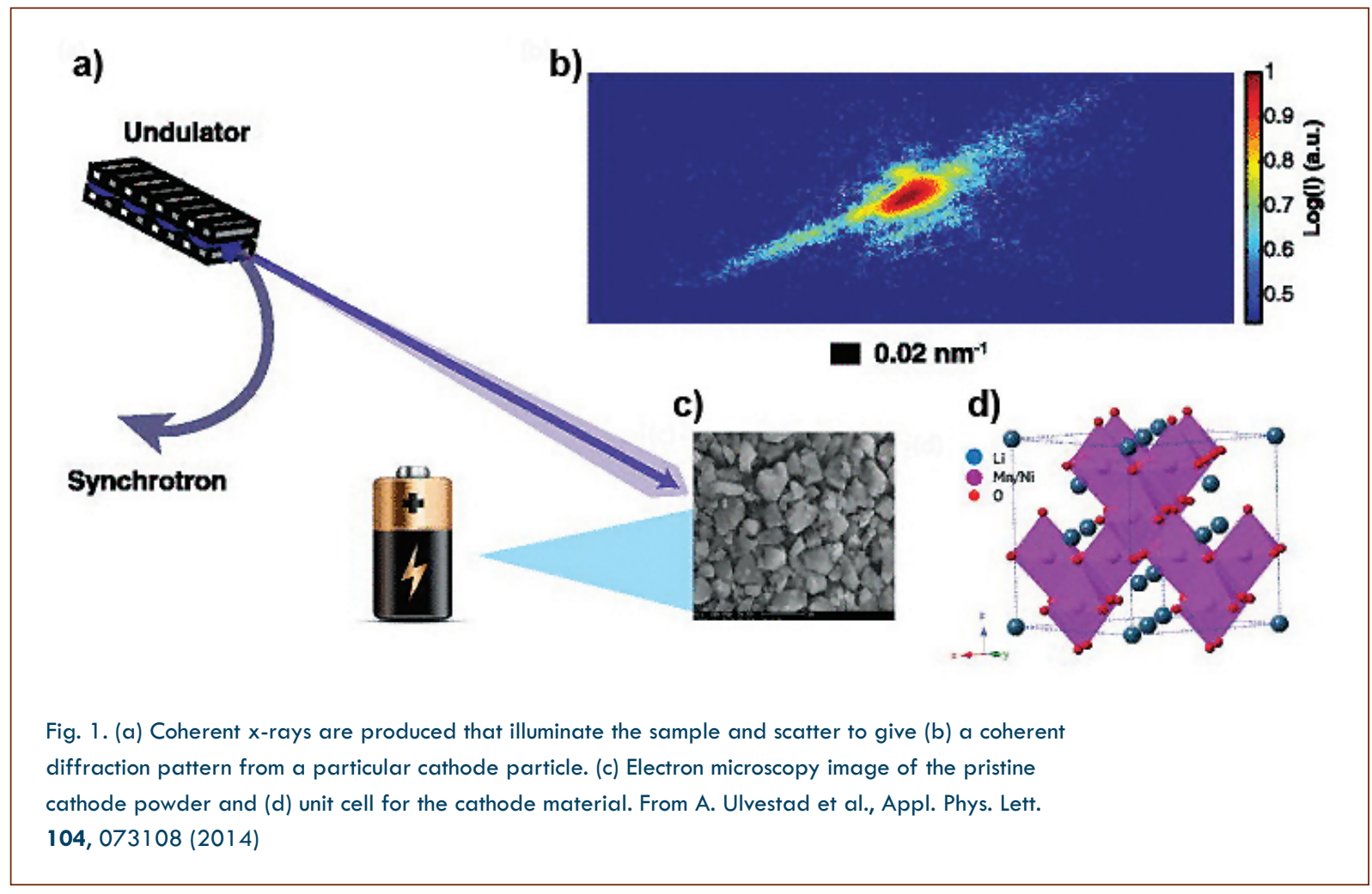


Lithium (from the Greek word for stone, "lithos") was discovered in the mineral petalite by Johann August Arfvedson in 1817. It was first isolated by William Thomas Brande and Sir Humphrey Davy through the electrolysis of lithium oxide. Today, larger amounts of the metal are obtained through the electrolysis of lithium chloride. Lithium is not found free in nature and makes up only $0.0007 \%$ of the Earth's crust. Many uses have been found for lithium and its compounds. Lithium has the highest specific heat of any solid element and is used in heat transfer applications. It is used to make special glasses and ceramics, including the 200-in. mirror in the Hale Telescope at the Palomar Observatory in California. Lithium is the lightest known metal and can be alloyed with aluminum, copper, manganese, and cadmium to make strong, lightweight metals for aircraft. Lithium hydroxide is used to remove carbon dioxide from the atmosphere of spacecraft. Lithium stearate is used as a general purpose and high-temperature lubricant. Lithium carbonate is used as a drug to treat manic depression disorder. Lithium reacts with water, but not as violently as sodium. Source: Jefferson Lab, "It's Elemental," http://education.jlab.org/itselemental/ ele003.html. Hale Telescope photo by Paul Vladuchick, https://www.flickr.com/photos/vladdythephotogeek/3828538652/

Like most batteries, the Li-ion variety consist of a cathode and an anode separated by an electrolyte. The cathode in this case is made of a lithium metal oxide that allows lithium ions which are the battery's charge carriers - to leave and reenter. To facilitate this ion movement, the metal oxide is routinely fabricated as a powder of small particles that are micrometers or nanometers in size. During charging, lithium ions diffuse out of these particles and travel through the electrolyte to the anode, which is often some form of carbon such as graphite. The loss of lithium in the cathode particles results in a contraction of their crystal structure. When the battery starts to discharge, the lithium ions flow back into the particles, causing them to re-expand. The strain from this cycling of ions into and out of the cathode material may induce structural damage. For this reason, battery manufacturers are interested in understanding how and where strain occurs.

To see the strain inside nanoscale particles, researchers from the University of California, San Diego; Argonne; Los Alamos National Laboratory; and New Mexico State University employed coherent $x$-ray diffraction imaging (CXDI) at the XSD 34-ID-C beamline at the APS. This relatively new technique was developed to take images of nanosized objects, like gold nanoparticles and semiconductor quantum dots. The beam used in CXDI is formed by extracting a small fraction of coherent light from the APS x-ray output (see top of Fig. 1). The 34-ID-C beamline is optimized for performing CXDI, with a set of curved Kirkpatrick-Baez mirrors for focusing the synchrotron light on a sample. When the coherent $x$-rays scatter off the sample's atoms, they produce a high-precision diffraction pattern, which can be transformed through a computer algorithm - into a three-dimensional map of the crystal structure in the sample.

For their experiments, the research team chose to look at cathode materials called spinels, which are used in special high-performance batteries. Spinels are a type of oxide with a specific cubic crystal lattice structure. In spinels containing lithium, this cubic structure allows lithium ions to diffuse out in three different directions, as compared to a single direction in other metal oxide materials. This greater ion mobility results in a higher voltage for spinel-based batteries than for the more common lithium cobalt oxide battery. A further advantage of spinels is that they don't require environmentally hazardous materials in their fabrication.

The researchers performed CXDI experiments on nanoparticles made from the spinel material lithium-nickelmanganese-oxide. By examining the asymmetrical shape of Bragg peaks in the diffraction pattern, the team recovered the strain inside single nanoparticles that were isolated from both a prepared powder sample and a coin cell battery (see bottom of the Fig. 1).

The maximum strain was measured to be around one part in 103 , which is about ten times the strain found in gold nanoparticles of similar size. Interestingly, the strain was not uniform across the nanoparticles. The research team assumed this inhomogeneity was due to differing concentrations of lithium ions in the crystal structure. This would imply that lithium

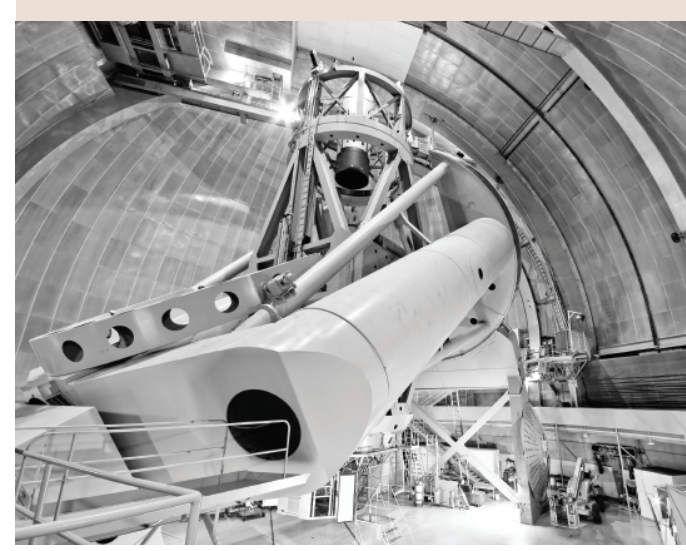

The Hale Telescope

diffuses out of the nanoparticles in a non-uniform way, contradicting certain theoretical models that predict diffusion proceeds uniformly from the surface to the core.

Subsequent work with CXDI techniques has shown how strain evolves over time in these spinel materials as they cycle through charge and discharge. By experimenting with different nanoparticle shapes and sizes, the researchers hope to find ways to limit the effects of strain. - Michael Schirber

See: A. Ulvestad ${ }^{1 *}$, H.M. Cho ${ }^{1}$, R. Harder $^{2}$, J.W. Kim ${ }^{1}$, S.H. Dietze, ${ }^{1}$ E. Fohtung $^{3,4}$, Y.S. Meng ${ }^{1}$, and O.G. Shpyrko", "Nanoscale strain mapping in battery nanostructures," App. Phys.

Lett. 104, 073108 (2014).

DOI: $10.1063 / 1.4866030$

Author affiliations: ${ }^{1}$ University of California, San Diego; ${ }^{2}$ Argonne National Laboratory; ${ }^{3}$ Los Alamos National Laboratory; ${ }^{4}$ New Mexico State University

Correspondence: * aulvesta@ucsd.edu

This work was supported by the U.S. Department of Energy (DOE) Office of ScienceBasic Energy Sciences, under Contract No. DE-SC0001805 and by the University of California, San Diego Chancellor's Interdisciplinary Award. This research used resources of the Advanced Photon Source, a U.S. DOE Office of Science User Facility operated for the DOE Office of Science by Argonne National Laboratory under contract no. DEAC02-06CH11357.

34-ID-C • XSD • Materials science, physics • Coherent $x$-ray scattering $\cdot 5-15 \mathrm{keV}, 7-25$ $\mathrm{keV} \cdot$ On-site $\cdot$ Accepting general users • 


\section{HOW IONS CyCle AT High Rates INSIDE OF LITHIUM-ION BATTERIES}

A lithium-ion battery gets its name from the fact that it charges and discharges by shuttling lithium ions back and forth through an electrolyte in passing between the battery's cathode and anode electrodes. The reversibility of the charge and discharge process ensures the energy received from an external source of electrical power upon charging can be delivered during discharge. Many emerging applications, such as powering electric vehicles, require cathode materials to release lithium ions very quickly while charging and then swiftly reincorporate them when the battery discharges upon use. Nanoparticulate lithium iron phosphate $\left(\mathrm{LiFePO}_{4}\right)$ cathodes are commercially important because they do that with ease, yet until recently scientists did not understand why. That understanding came thanks to work by an international team of researchers utilizing the APS.

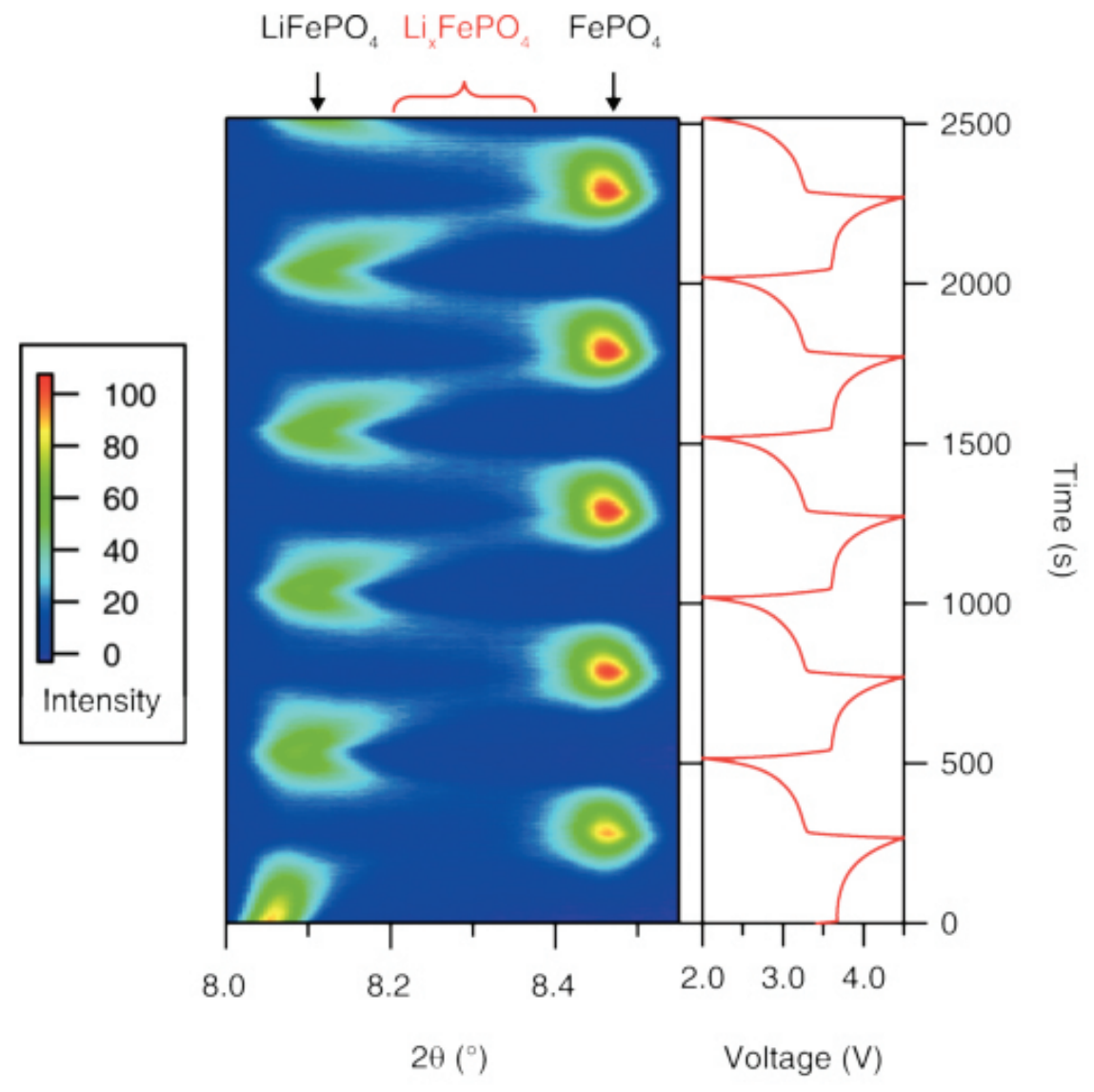

Fig. 1. The in situ XRD pattern clearly shows the intermediate phases as evidenced by the intensities observed in the intermediate $2 \theta$ region (labeled $\mathrm{Li}_{x} \mathrm{FePO}_{4}$ in red).
17-BM-B $\cdot X S D \cdot$ Chemistry, materials science - Powder diffraction, high-pressure diamond anvil cell $\cdot 15-18 \mathrm{keV} \cdot$ On-site $\cdot$ Accepting general users $•$
The difficulty was that, although $\mathrm{LiFePO}_{4}$ nanoparticulate cathodes cycle lithium ions very quickly, the reason this occurred remained unclear, if the results of previous ex situ investigations told the whole story. Those investigations, which studied the cathodes before and after but not while cycling, suggested that cycling induced a $\mathrm{LiFePO}_{4}-\mathrm{FePO}_{4}$ phase transition, implying that a moving phase boundary formed and then swept back and forth across the nanoparticles. Yet such phase transitions usually proceed very slowly, providing no explanation for the cathodes' great cycling speed.

To find out what was going on, the researchers from the University of Cambridge (UK), Argonne, and Stony Brook University utilized XSD beamline 17-BM-B at the APS to perform in situ synchrotron $\mathrm{x}$-ray powder diffraction (XRD) measurements on $\mathrm{LiFePO}_{4}$ cathodes as they were being cycled at high rates while inside $\mathrm{LiFePO}_{4}$-Li battery cells. The XRD patterns showed the expected disappearance of $\mathrm{LiFePO}_{4}$ Bragg reflections on charging and the simultaneous formation of $\mathrm{FePO}_{4}$ reflections.

But they also showed reflections in between indicating that particles with lattice parameters deviating from the equilibrium values of $\mathrm{LiFePO}_{4}$ and $\mathrm{FePO}_{4}$ were being formed (Fig. 1). This phenomenon was even more pronounced at high currents.

The researchers then compared their experimental results with simu"Ions" cont'd on page 52 


\section{Writing Smaller for Bigger Data}

M

agnetic data storage on hard drives is running into a brick wall. After continually improving through the years, the technology has now reached a density limit on the tiny magnetic grains that represent $1 \mathrm{~s}$ and 0s: if they get any smaller they will become thermally unstable, flipping randomly and corrupting data. One potential solution is to use grains with high magnetic anisotropy, which are directionally dependent. The magnetization of such grains is very stable and hard to flip at the temperature of data storage. But writing data onto such stable magnetic grains also becomes a challenge, requiring either a very high magnetic field or heated grains. The heating approach seems to be more practical with existing technology, so a team of researchers used the APS to better understand how heat moves through the layers of metal and ceramic in next-generation high-density hard drives. The results of their research could lead to heat assisted magnetic recording (HAMR) devices with much higher density information storage than is currently available.

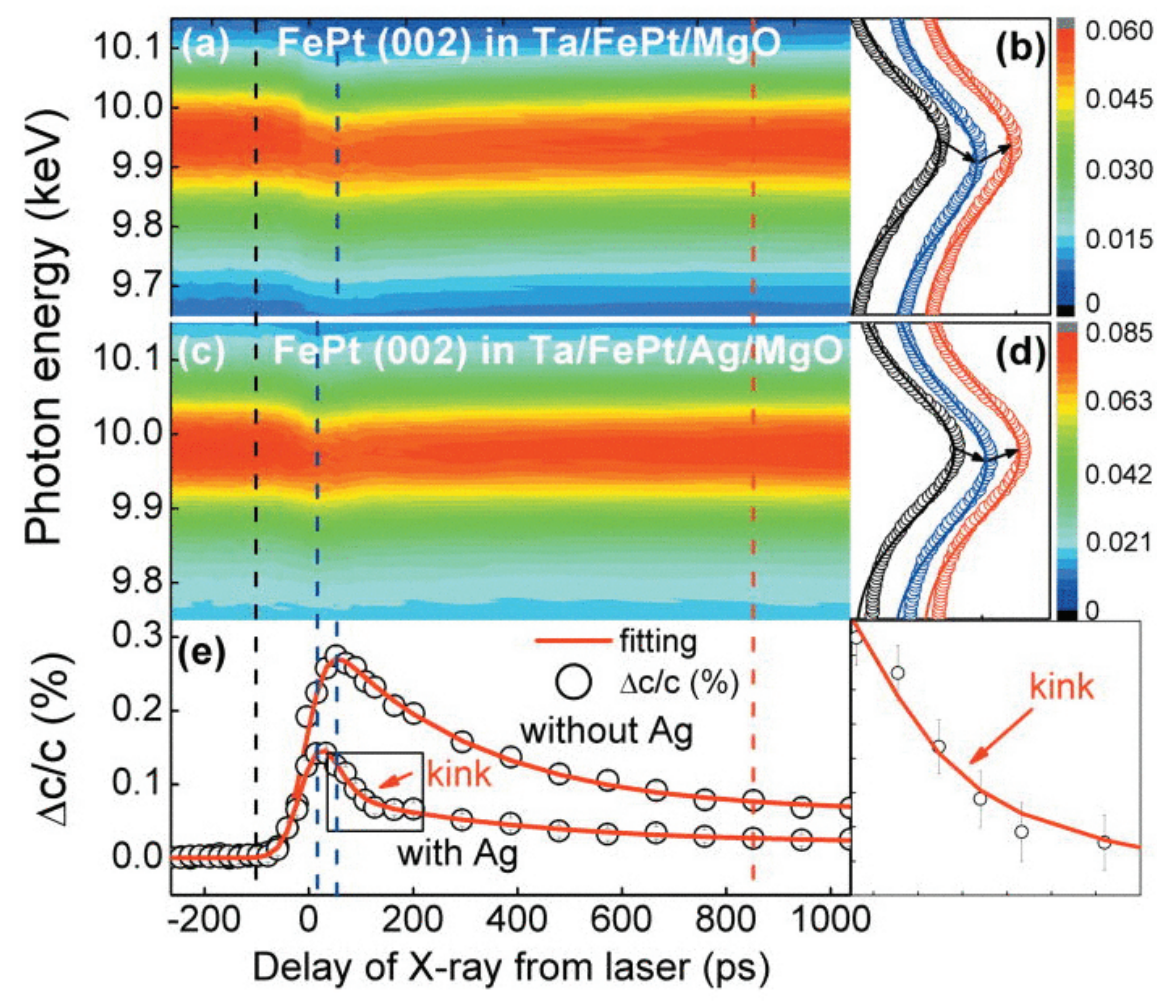

Fig. 1. The contour plots of the FePt diffraction peak as a function of the time delay between $x$ ray and laser pulses from -264 ps to 1038 ps are shown in (a) for $\mathrm{Ta} / \mathrm{FePt} / \mathrm{MgO}$ and (c) for $\mathrm{Ta} / \mathrm{FePt} / \mathrm{Ag} / \mathrm{MgO}$. (b) and (d) show the diffraction curves at three marked time delays with corresponding colors (black, blue, and red), highlighting the Bragg peak's shift in energy when the laser was applied. The diffraction peak shifted to lower energy (arrow from black to blue) indicates the lattice expansion during heating, whereas the peak shifting to higher energy (arrow from blue to red) indicates the contraction of expanded lattice in cooling. From D.B. Xu et al., J. Appl. Phys. 115 (24), 243907 (2014).
One attractive candidate for HAMR devices is iron-platinum (FePt) magnetic thin film. FePt has high magnetic anisotropy and high corrosion resistance, making it both stable and long lasting. To record on such a material, it is heated with a laser to reduce the magnetic field necessary to flip the magnetization direction of the magnetic grains, and data is written into it with a magnetic writing head. Then the FePt must be rapidly cooled to fix the magnetization in place. This can be done by layering a heat sink, such as silver, underneath the FePt. Silver (Ag) is particularly good because its crystallographic structure is compatible with the desirable growth direction of the magnetic FePt layer. Such metal-metal layers need to be supported on a substrate; for this experiment, the researchers supported the FePt and Ag layers on top of a ceramic, magnesium oxide.

Heat moves differently through the interfaces of metal-to-metal and metalto-ceramic. In metals, heat travels primarily via electrons, while phonons (vibrations moving through the atomic structure of a material) conduct heat in ceramics. If heat conducts in unexpected ways at the interface between metal and ceramic, this could complicate the operation of a HAMR device. The researchers in this study from Argonne, the National University of Singapore, and Chonbuk National University (Republic of Korea) probed the FePt-Ag and Ag-MgO interfaces at sub"Writing" cont'd on page 52 
"Ions" cont'd from page 50

lated XRD patterns and found that the lattice parameter variation could not be explained by a moving $\mathrm{LiFePO}_{4}-\mathrm{FePO}_{4}$ interface within the particles. Instead, the results indicated a continuous compositional variation either within or between the particles. This is only plausible if a solid solution was being formed — in this case, only under nonequilibrium conditions.

The researchers finally adapted a whole-pattern fitting method to quantify the compositional variation in the electrodes during cycling. The results demonstrated the formation of a nonequilibrium solid solution phase, $\mathrm{Li}_{x} \mathrm{FePO}_{4}(0<x<1)$, during high-rate cycling, with compositions spanning the entire range between the two thermodynamic phases, $\mathrm{LiFePO}_{4}$ and $\mathrm{FePO}_{4}$. This showed that, at least at high rates, phase transformations in nanoparticulate $\mathrm{LiFePO}_{4}$ proceed via a continuous change in structure rather than a distinct moving phase boundary between $\mathrm{LiFePO}_{4}$ and $\mathrm{FePO}_{4}$. The ability of $\mathrm{LiFePO}_{4}$ to transform via a nonequilibrium single-phase solid solution that avoids major structural rearrangements across a moving interface helps to explain its high-rate performance despite the very limited solubility of lithium in $\mathrm{LiFePO}_{4}$ and $\mathrm{FePO}_{4}$ at room temperature. The existence of nonequilibrium solid solution phases may also underpin the high-rate capability of other materials that nominally operate via twophase reactions. This result additionally suggests that the creation of a low-energy nonequilibrium path by, for example, particle size reduction or cation doping could enable the high-rate capabilities of other phase-transforming electrode materials. - Vic Comello

See: Hao Liu ${ }^{1}$, Fiona C. Strobridge ${ }^{1}$, Olaf J. Borkiewicz², Kamila M. Wiaderek $^{2}$, Karena W. Chapman², Peter J. Chupas ${ }^{2}$, Clare P. Grey ${ }^{1,3 *}$, "Capturing metastable structures during high-rate cycling of $\mathrm{LiFePO}_{4}$ nanoparticle electrodes," Science 344, 1480 (2014)

DOI: $10.1126 /$ science. 1252817

Author affiliations: ${ }^{1}$ University of Cambridge, ${ }^{2}$ Argonne National Laboratory,

${ }^{3}$ Stony Brook University

Correspondence: * cpg27@cam.ac.uk
This work was supported as part of the Northeastern Center for Chemical Energy Storage, an Energy Frontier Research Center funded by the U.S. Department of Energy (DOE), Office of Science, Office of Basic Energy Sciences, under Award no. DE-SC0001294. Work performed at Argonne and use of the Advanced Photon Source, an Office of Science User Facility operated for the DOE Office of Science by Argonne National Laboratory, were supported by the DOE under Contract no. DE-AC02-06CH11357. H.L. received funding from European Union FP7-265368 via the Eurolion Project and the Cambridge Overseas Trust. F.C.S. received funding from the Engineering Physical Science Research Council via a Doctoral Training Partnership Award.

"Writing" cont'd from page 51 nanosecond timescales. The goal was to more precisely understand how electron- and phonon-heat transport couple at the interfaces, and how heat moves through these interfaces over time.

The researchers pulsed a laser beam onto the FePt layer to heat it, and then performed picosecond time-resolved laser pump/x-ray probe diffraction to trace the heat as it moved through the samples (Fig. 1). They used the XSD 20-ID-B,C beamline at the APS because of its excellent set-up both for x-ray scattering and spectroscopy, and because the scientists working on that beamline are always ready to modify the existing setup to explore new experiments.

The team found that the laser transferred heat energy to electrons a few hundred femtoseconds after the pulse. The heat energy was transferred through the entire FePt film within a few picoseconds via electron diffusion.

The researchers believe that two mechanisms of thermal transport are coupling at the metal-nonmetal interface. Electrons and phonons exchange the thermal energy within the metal layer first, and the energy then transfers from the metal to the ceramic through phonon-phonon interactions.

Of the current technologies under consideration for next-generation magnetic recording, HAMR is the most compatible with current hard drive architecture and manufacturing capabilities. HAMR devices could break the current storage wall, allowing data stor- age densities of up to 50 terabits per square inch, about 100 times denser than current commercial technology. The researchers believe that a stochastic model which incorporates ballistic phonons and electrons should be developed to provide a realistic description of heat transfer in such layered metal-ceramic structures. Such a model could provide insights into how to better structure HAMR devices, bringing them closer to commercial feasibility.

- David Bradley

See: D.B. $X^{1,2} \neq$, C.J. Sun ${ }^{2 *}$, D.L.

Brewe $^{1}$, S.-W. Han ${ }^{3}$, P. Ho ${ }^{2}$, J.S. Chen², S.M. Heald ${ }^{1}$, X.Y. Zhang ${ }^{1}$, and G.M. Chow ${ }^{2 * *}$, "Spatiotemporally separating electron and phonon thermal transport in $\mathrm{L}_{1}$ FePt films for heat assisted magnetic recording," J. Appl. Phys. 115

(24), 243907 (2014).

DOI: $10.1063 / 1.4885428$

Author affiliations: ${ }^{1}$ Argonne National Laboratory, ${ }^{2}$ National University of Singapore, ${ }^{3}$ Chonbuk National University łPresent address: Seagate Singapore Correspondence: * cjsun@aps.anl.gov,

** msecgm@nus.edu.sg

Work at the National University of Singapore was supported by the Ministry of Education, Singapore, under Grant no. MOE2012-T2-2031. Work at Chonbuk National University was supported by a National Research Foundation of Korea government grant funded by the Basic Science Research Program (no. 2012R1A1A4A01007669) and the PAL XFEL project. APS Sector 20, which is managed by XSD in partnership with the Canadian Light Source (CLS), is funded by the U.S. Department of Energy (DOE) Office of Science, and by the Natural Sciences and Engineering Research Council of Canada and the University of Washington via the CLS. This research used resources of the Advanced Photon Source, a U.S. DOE Office of Science User Facility operated for the DOE Office of Science by Argonne National Laboratory under Contract No. DE-AC02$06 \mathrm{CH} 11357$.

20-ID-B, C • XSD • Materials science, environmental science, chemistry $\cdot X$-ray absorption fine structure, $x$-ray Raman scattering, micro $x$-ray absorption fine structure, microfluorescence (hard x-ray), time-resolved x-ray absorption fine structure, $x$-ray emission spectroscopy • 4.3-27 keV, 7-52 keV • On-site -Accepting general users • 


\section{Designing Better See-Through Contacts for Displays and SOlar Power}

$\mathrm{N}$

ew transparent materials for use in liquid-crystal displays, light emitting diodes, photovoltaic cells, and other optoelectronic devices are being developed by researchers from the U.S. and South Korea and investigated utilizing high-brightness x-rays from the APS. The researchers' focus on the structure and properties of amorphous thin films made from zinc $(\mathrm{Zn})$ tin $(\mathrm{Sn})$ oxide (a-ZTO) could lead to less costly devices with none of the disposal problems of other experimental devices that use toxic indium and gallium compounds.

Many amorphous metal-oxide semiconductor materials are transparent and have potential in optoelectronic devices in which transparency and the ability to transmit light is just as important as the ability to carry a current, a flow of electrons. The fact that these materials are amorphous, with no crystalline order, also means that they cannot, by definition, have crystal structure defects, which also means greater electron mobility and faster operation with no barriers thrown up by random defects. In addition, amorphous materials can be grown at low temperatures, thereby reducing thermally induced defects during fabrication.

Other researchers have worked with amorphous indium-gallium-zincoxide, but the researchers in this study from the Massachusetts Institute of Technology, Harvard University, Chonnam National University (South Korea), and the Illinois Institute of Technology point out that indium and gallium are much more expensive than other metals with potential such as tin, as well as having toxicity problems. This makes indium and gallium compounds less attractive overall for consumer products from both the end-user safety perspective and in terms of recycling and disposal when the device reaches end of life. The team believes that with appropriate structural control and the right setup tin might provide the necessary electron mobility and transparency characteristics to displace its indium or gallium counterparts.

Indeed, other researchers have investigated methods for making high- quality zinc tin oxide films including sol-gel methods, sputtering, pulsed laser deposition, and the most promising: atomic layer deposition (ALD). The team explains that ALD allows them to control more precisely the exact proportions of each element as they are deposited on the thin layer, which is being grown on a smooth quartz surface at $120^{\circ} \mathrm{C}$ from the organometallic starting materials diethylzinc and a cyclic tin amide compound.

Specifically, controlling the ratio of tin to zinc atoms allows optoelectronic properties to be tuned and optimized for solidstate device applications. The significant impact of the zinc/tin ratio of a-ZTO thin film on device performance suggests a strong correlation between the atomic structure and electronic transport properties of the films. Because amorphous materials can exhibit a continuum of structures, a local probe is required.

The team used synchrotron $x$-ray absorption spectroscopy (XAS) at the MR-CAT 10-ID-B beamline to probe the chemical environment of zinc and tin atoms and investigate how the local atomic structure of an amorphous thin film of a-ZTO affects its electron transport properties (Fig. 1). Both quantitative extended $x$-ray absorption fine structure and qualitative $x$-ray absorption near-edge structure

"Displays" cont'd on page 55

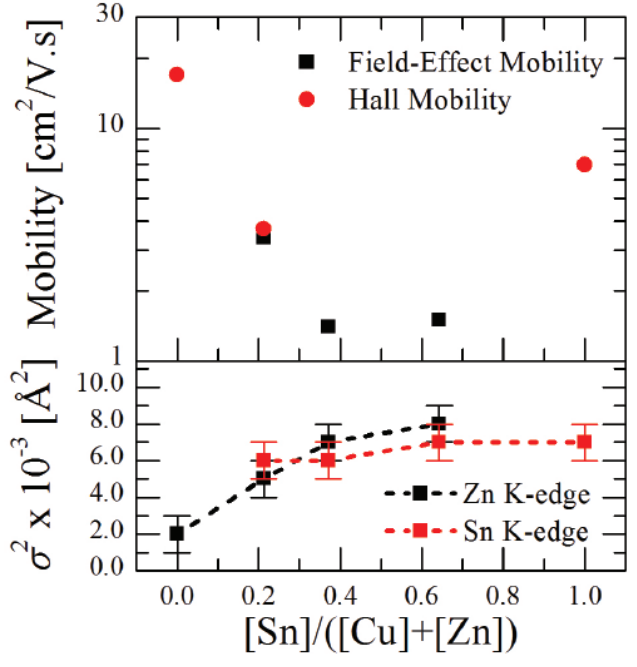

Fig. 1. Field-effect and Hall-effect mobilities and pseudo-Debye-Waller factors are plotted against film composition, $[\mathrm{Sn}] /([\mathrm{Sn}]+[\mathrm{Zn}])$.

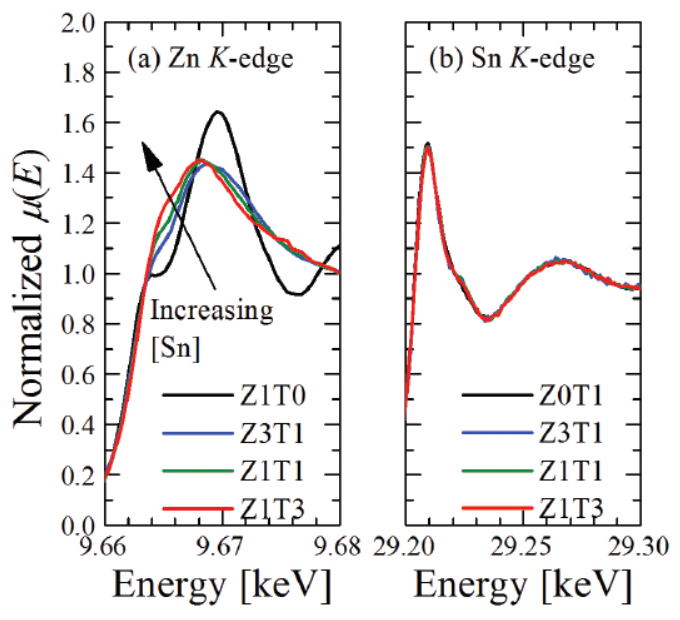

Fig. 2. XANES spectra at (a) Zn and (b) Sn K-edges. 


\section{Probing Nanostructures in Steel Alloys}

$\mathrm{T}$

he designers of nuclear fission reactors would like to use steel that can last longer in the high temperatures and radiation produced in the crucible of nuclear reactions. Scientists know that yttrium, titanium, and oxygen within a steel alloy can form nanoscale features that make the metal less susceptible to cracking and weakening, but the details of those features are not well understood. Now researchers have used the APS to better understand how oxide nanostructures form in steel alloys, which could lead to the creation of even more durable steel.

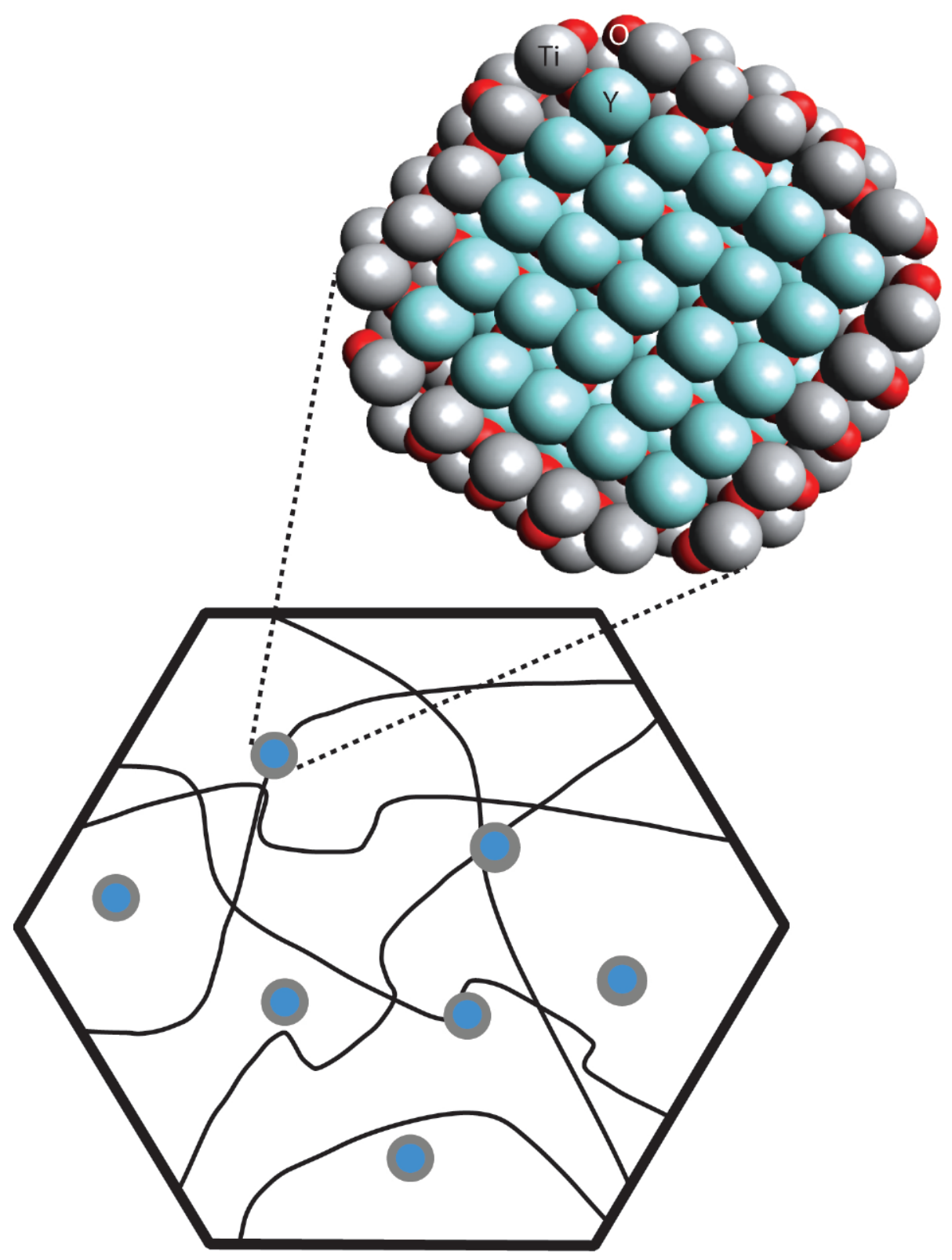

Fig. 1. A schematic representation of bulk steel shows grain boundaries (black lines) and nanofeatures (circles). The researchers in this study postulate that the nanofeatures' structure (above) consists mainly of yttrium oxide surrounded by titanium oxide (blue=yttrium, gray=titanium, red=oxygen). 
Titanium (Ti) within the steel can remain in the metallic steel matrix, or can combine with yttrium $(Y)$ and oxygen $(\mathrm{O})$ into complex oxide compounds, creating tiny structures within the alloy. These nanofeatures block incipient cracks and helium bubbles (which are produced in the steel by the nuclear reaction) from spreading and weakening the metal.

By using $x$-ray absorption spectroscopy, researchers from the Illinois Institute of Technology and the University of California, Santa Barbara, have discovered that titanium atoms migrate from the steel matrix to the surface of oxide nanoparticles during high-temperature processing to form these nanofeatures (Fig. 1).

The researchers began with a powdered alloy, into which they mixed yttrium-trioxide $\left(\mathrm{Y}_{2} \mathrm{O}_{3}\right)$ by ball-milling the powders together for $8 \mathrm{~h}$ in an inert argon atmosphere. The elements involved don't form alloys chemically, but the ball milling dispersed the $\mathrm{Y}_{2} \mathrm{O}_{3}$ throughout the steel and created a mechanical alloy. The powders were annealed at $850^{\circ} \mathrm{C}, 1000^{\circ} \mathrm{C}$, or $1150^{\circ} \mathrm{C}$, as well as hot pressed at $1150^{\circ} \mathrm{C}$ at a pressure of $200 \mathrm{MPa}$.

These samples, along with commercial steel alloys manufactured in Japan, were studied using high-brightness $\mathrm{x}$-rays at the APS. The characteristic absorption energies of $\mathrm{Y}$ and $\mathrm{Ti}$ are very different and required the use of two separate beam lines: the MR-CAT 10-1D beamline to examine the $Y$ in the samples and the XSD 9-BM beamline to study the Ti, with a method called extended $\mathrm{x}$-ray absorption fine-structure spectroscopy. The researchers found that the oxidation state and the local structural environment of Ti changed most during processing.

They found no difference between the characteristics of the annealed and hot-pressed powders. The results showed that nearly all of the Ti in the milled powder started in a metallic state, but was mostly oxidized during the hot-pressing or annealing process. Additionally, powders that were treated at higher temperatures showed somewhat more oxidation of the $\mathrm{Ti}$ than at lower temperatures. In contrast, the $\mathrm{Ti}$ in the commercial alloys was found to be mostly in the metallic steel matrix.

The results were consistent with the possibility that the $\mathrm{Ti}, \mathrm{Y}$, and $\mathrm{O}$ had formed one of several compounds such as $\mathrm{Y}_{2} \mathrm{Ti}_{2} \mathrm{O}_{7}$, but there was also evidence that the titanium had formed a shell around the surface of an yttrium-oxide nanoparticle.

With this new understanding of the differences between these newly prepared alloys and the proprietary commercial steels, it will be possible to evaluate how the structure and composition of the nanofeatures affect the durability and performance of such advanced steels under irradiation.

Eventually, this work may allow engineers to produce steel alloys that are more durable for the nuclear reactors of the future. - Neil Savage

See: S. Liu ${ }^{1}$, G.R. Odette ${ }^{2}$, C.U. Segre $^{1 *}$, "Evidence for core-shell nanoclusters in oxygen dispersion strengthened steels measured using X-ray absorption spectroscopy," J. Nucl. Mat. 445, 50 (2014).

DOI: 10.1016/j.jnucmat.2013.10.042 Author affiliations: ${ }^{1}$ Illinois Institute of Technology, ${ }^{2}$ University of California, Santa Barbara

Correspondence: * segre@iit.edu

MR-CAT operations are supported by the U.S. Department of Energy (DOE) and the MR-CAT member institutions. Work at the XSD 9-BM beamline is supported in part by the U.S. DOE Office of Science-Basic Energy Sciences and by the U.S. National Science Foundation Division of Materials Research. This research used resources of the Advanced Photon Source, a U.S. DOE Office of Science User Facility operated for the DOE Office of Science by Argonne National Laboratory under Contract No. DEAC02-06CH11357.

10-ID-B • MR-CAT • Materials science, environmental science, chemistry $\cdot \mathrm{X}$-ray absorption fine structure, time-resolved $x$-ray absorption fine structure, micro $x$-ray absorption fine structure, microfluorescence (hard $x$ ray) - 4.3-27 keV, 4.3-32 keV, 15-90 keV • On-site $\cdot$ Accepting general users $•$
"Displays" cont'd from page 53 (XANES) analyses showed that in aZTO, structural disorder around the zinc atoms, as indicated by the DebyeWaller factor, increases with the tin content (Fig. 2). Furthermore, increasing the local structural disorder surrounding zinc atoms is correlated with decreasing charge-carrier mobility.

The team hypothesized that in zinc-rich a-ZTO films, the predominant conduction paths are between hybridized $\mathrm{Zn}-4 s$ and $\mathrm{O}-2 p$ orbitals. Therefore, reduced hybridization strength of $\mathrm{Zn}-4 s$ and $\mathrm{O}-2 \mathrm{p}$ orbitals due to structural disorder may reduce mobility. These insights can be used today to tune the material to optimize carrier transport, and might be useful tomorrow when designing the next generation of transparent contact materials.

- David Bradley

See: Sin Cheng Siah ${ }^{1 *}$, Sang Woon Lee $^{2}$, Yun Seog Lee ${ }^{1 \ddagger}$, Jaeyeong $\mathrm{Heo}^{3}$, Tomohiro Shibata ${ }^{4}$, Carlo U. Segre ${ }^{4}$, Roy G. Gordon², and Tonio Buonassisi ${ }^{1 * *}$, "X-ray absorption spectroscopy elucidates the impact of structural disorder on electron mobility in amorphous zinc-tin-oxide thin films," Appl. Phys. Lett. 104, 242113 (2014).

DOI: 10.1063/1.4884115]

Author affiliations: ${ }^{1}$ Massachusetts Institute of Technology, ${ }^{2}$ Harvard University, ${ }^{3}$ Chonnam National University, ${ }^{4}$ Illinois Institute of Technology ‡Present address: Ajou University Correspondence:

* sincheng@alum.mit.edu, **buonassisi@mit.edu

This work was supported by the Office of Naval Research ONR N00014-10-1-0937, the U.S. National Science Foundation (NSF) Award No. CBET-1032955, and NSF CAREER Award No. ECCS-1150878. MR-CAT operations are supported by the U.S. Department of Energy (DOE) and the MR-CAT member institutions. This research used resources of the Advanced Photon Source, a U.S. DOE Office of Science User Facility operated for the DOE Office of Science by Argonne National Laboratory under Contract No. DE-AC02-06CH11357. 


\section{Keeping the LN 2 Flowing to APS Users}

Liquid nitrogen $\left(\mathrm{LN}_{2}\right)$ is an essential commodity at the APS because it is utilized for cooling experimental equipment and samples. To facilitate easy access to $L N_{2}$ for users, a fully automated liquid nitrogen distribution system (LNDS) was installed in 1999.

The original system consists of four separate modules, each of which supplies $\mathrm{LN}_{2}$ to $25 \%$ of the sectors in the APS experiment hall. Each module includes a 3000-gal $\mathrm{LN}_{2}$ storage tank, vacuum jacketed piping, valves, and automatic controls.

Each module maintains a steady supply of $\mathrm{LN}_{2}$ to the beamlines through a manifold system that encircles the inside perimeter of the experiment hall. The modules operate independently of each other but can be connected to adjacent modules through a series of interconnect valves. These connections allow one module to supply $\mathrm{LN}_{2}$ to the beamlines of an adjacent module if the need arises.

The demand for $\mathrm{LN}_{2}$ has substantially increased since the system was originally installed. Prior to recent improvements, some of the 3000-gal tanks required daily filling.

For various reasons (e.g., mechanical problems with delivery trucks) delivery to these tanks was occasionally delayed. To prevent the tanks from running completely empty, they were valved out of the system when they reached a predetermined minimum level. To ensure a steady delivery of $\mathrm{LN}_{2}$ to the affected beamilnes, the interconnect valve from the adjacent module was opened. In each instance, when the interconnect valves were opened, the $\mathrm{LN}_{2}$ supply to several beamlines was affected.

To reduce the likelihood of tanks running low again, larger tanks were installed. The two highest usage tanks were replaced with 9000-gal

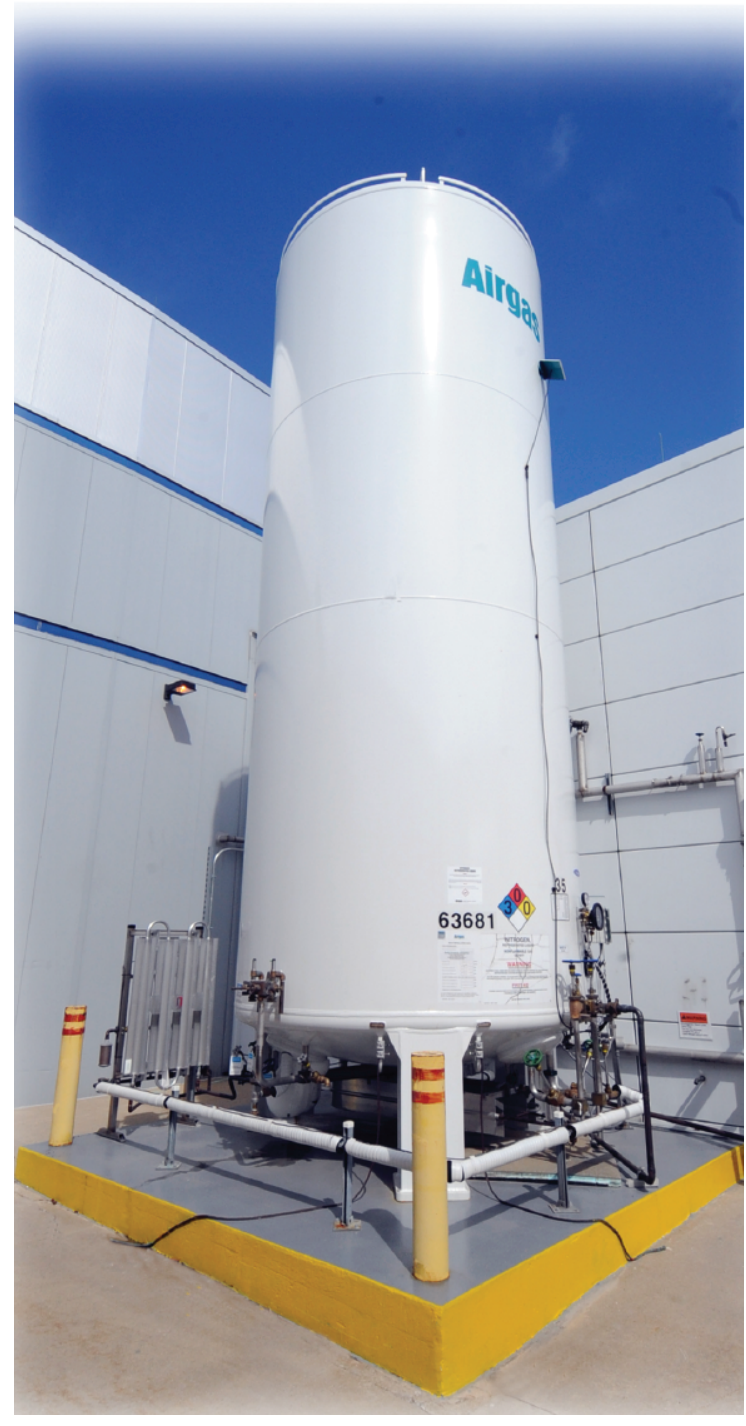

Fig 1. One of the $9000-$ gal $\mathrm{LN}_{2}$ tanks.

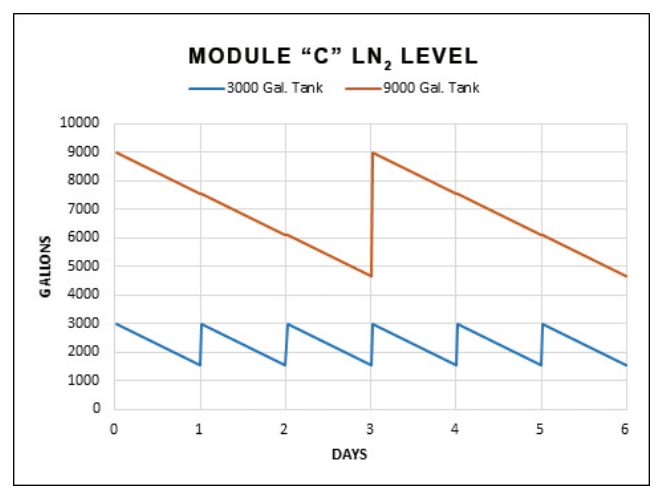

Fig. 2. Typical liquid nitrogen consumption and refill cycle for the " $\mathrm{C}$ " Module tank before and after the larger tank was installed. tanks (Fig. 1) during the 2014 AprilMay maintenance shutdown of the APS electron accelerator. These tanks will now last three days or more before they require a refill.

Figure 2 illustrates the tank level vs. days of use for the 3000-gal and 9000-gal tanks. Under normal conditions, the tanks are not allowed to fall below the 3/8-full level, which allows for a reserve margin. The new tanks provide approximately a two-day reserve at current usage levels. Additionally, to assist the supplier in maintaining proper tank levels, telemetry units were installed on each tank. These devices are solar powered and supply level and pressure data directly to the supplier in real time using cellular technology.

In addition to the tank upgrade, the APS has also completed an upgrade to the LNDS control systems for each module. The original controls were no longer supported by the manufacturer and new spare parts were unavailable. The new control systems are PLC-based and use touch-screen displays and interfaces. These new controls provide a greater level of flexibility to modify or add controls, improve system reliability, and increase the availability of spare parts.

The new system is performing well. Since installation, there have been no low-level $\mathrm{LN}_{2}$ conditions observed. The reorder process has become automatic; the $\mathrm{LN}_{2}$ supplier receives the tank level data via the telemetry units and initiates an order $8 \mathrm{~h}$ in advance of the predicted 3/8full level. This allows ample time for refilling the tanks. In practice, the tanks are usually refilled at about the $50 \%$ of full level. This leaves about a 2.5-day safety margin before the tanks run low.

Contact: Jeffrey Toeller, jtoeller@anl.gov 


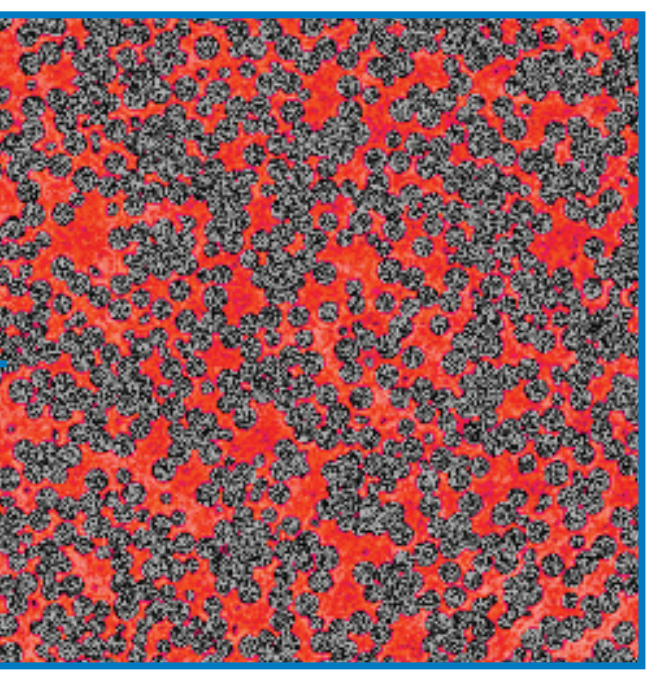

Soft Materials \&
Liquids 


\section{Probing the Geometry of WetTING RidGeS IN SOFt SOLIDS}

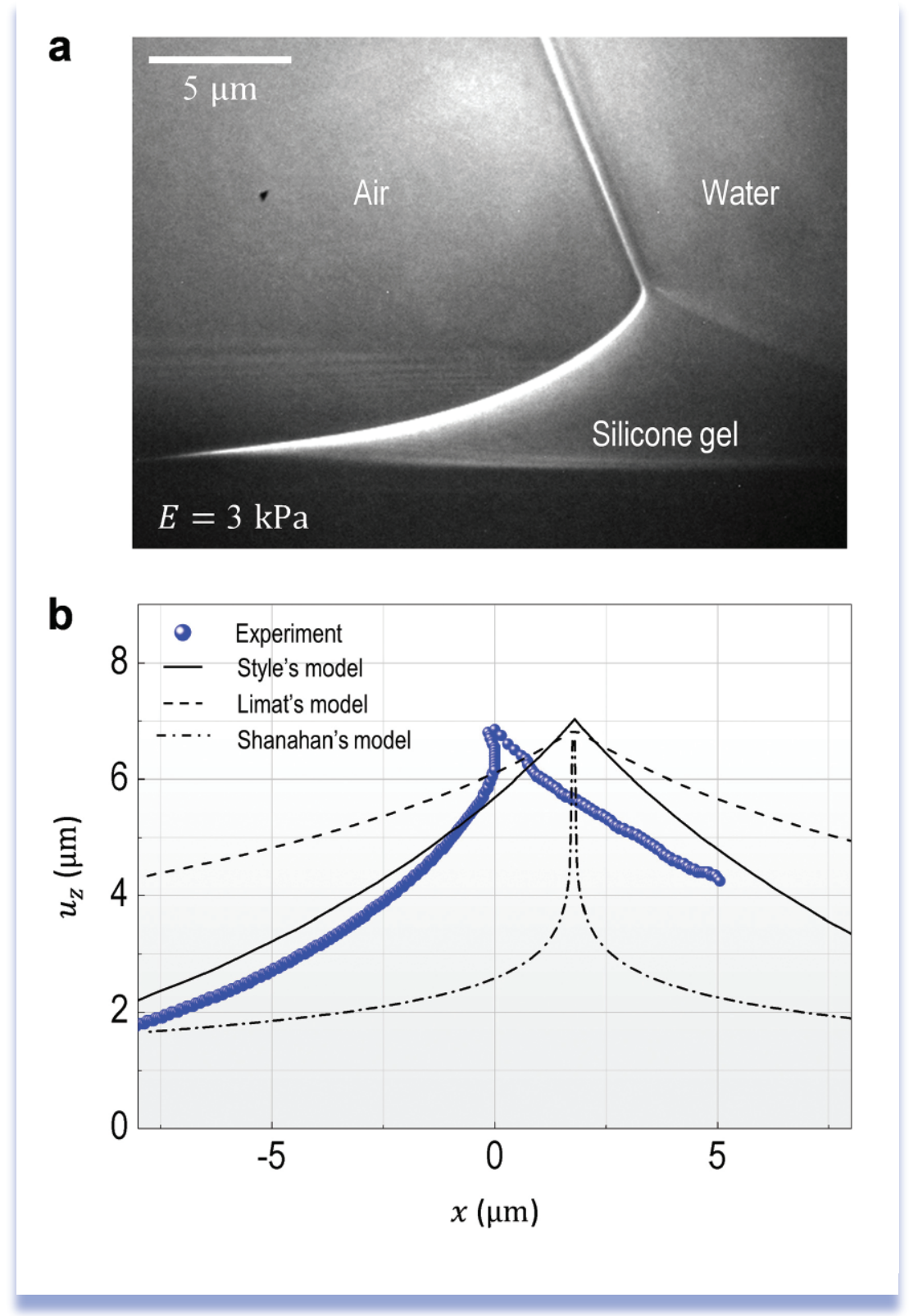


W hen a soft, elastic semisolid natural or synthetic material such as a thin film, fiber, or gel gets wet, interfacial tensions between its surface, the wetting liquid, and the surrounding air can create ripples, wrinkles, and other deformations. These deformations can profoundly affect a material's behavior, causing problems when it is used in applications such as microfluidic devices and inkjet printing. Recent work by a group of researchers utilizing the APS has achieved direct visualization of the previously uncharacterized wetting ridge tip, shedding new light on the miniscule geometry of moisture on soft solid surfaces and revealing the presence of a "wetting ridge" structure at the liquid-surface interface.

Traditionally, Young's modulus, which is a measure of the stiffness of an elastic material and is used to describe the elastic properties of objects like wires, rods or columns when they are stretched or compressed, has been employed quite successfully to explain wetting behavior on solid materials. When it comes to semisoft materials, however, Young's modulus fails to provide the full picture, because it does not take into account the component of vertical surface tension that gives rise to the wetting ridge structure. To allow a more accurate characterization of wetting on soft surfaces, various attempts have been made to directly visualize the wetting ridge through optical microscopy techniques, but light scattering in the interfacial regions inevitably limited the resolution of the images, making the ridge tip impossible to see.

The experimenters in this study from the Pohang University of Science and Technology (South Korea) and the SKKU Advanced Institute of Nanotechnology (South Korea) utilized transmission $x$-ray microscopy (TXM) at the XSD 32-ID-B,C beamline of the APS (see page 179) to overcome these difficulties, allowing the team to precisely measure the geometry of the wetting

Fig. 1. Direct visualization of a wetting ridge by transmission x-ray microscopy. (a): A representative $x$-ray image of a wetting ridge on a silicone gel $(E \approx 3 \mathrm{kPa})$. The wetting ridge forms asymmetrically by a water droplet $(r \approx$ $1 \mathrm{~mm})$. (b): A surface profile extracted from the $x$-ray image in a clearly shows an asymmetric cusp compared with three linear elastic models by Style (solid line), Limat (dashed line), and Shanahan (dash-dotted line). The Shanahan's model is basically invalid in the observed region here because the experimental data are in the plastic deformation region. ridge tip and devise a basic theoretical approach for describing the wetting behavior of soft solids. Because of the high temporal resolution of TXM, they were also able to obtain real-time movies of the formation of wetting ridges during the growth process.

The team focused on drops of water and a $40 \%$ ethylene glycol solution on a silicone gel or polydimethylsiloxane surface. They found that the wetting ridge consisted of a bent cusp with an asymmetric tip rotated toward the liquid-vapor (air) interface. The shape of the ridge tip in various samples was nearly identical despite variations in elasticity $(E)$ or the height of the ridge. Using the surface profiles they were able to determine from the TXM imaging, the researchers attempted to fit them with three different linear elastic models.

Neither the de Gennes/Shanahan model nor the Limat model matched well with the experimental data. The former could not be applied to the cusp regions, while the latter could not adequately account for the asymmetric surface energies observed. The Style and Dufresne model proved to fit most closely with experimental observations, although not with respect to the asymmetric microscopic contact angles of the wetting ridge tips.

To reconcile the differences between theoretical models and their experimental data, the researchers settled on a dual-scale approach that simultaneously links Young's modulus with respect to the macroscopic contact angles and Neuman's law for the microscopic contact angles. This also accounts for the invariant geometry of the asymmetric ridge tips, which seems to result from macroscopic and microscopic force balances.
The team notes that their characterization of the wetting ridge geometry, achieved here for the first time in unprecedented detail and accuracy, can provide a good general framework for a useful new approach to the description of wetting behavior and the measurement of surface stresses on soft solid materials. The experiments also demonstrate the great utility of TXM for further investigation of a variety of wetting phenomena involving soft solids.

Such work promises to be valuable not only for industrial applications such as microfluidics but also in the study of biological microenvironments and biomechanical processes. - Mark Wolverton

See: Su Ji Park ${ }^{1}$, Byung Mook Weon ${ }^{2}$, Ji San Lee ${ }^{1}$, Junho Lee ${ }^{1}$, Jinkyung $\mathrm{Kim}^{1}$, and Jung Ho Je ${ }^{1 *}$, "Visualization of asymmetric wetting ridges on soft solids with X-ray microscopy," Nat. Commun. 5, 4369 (2014).

DOI: $10.1038 / n c o m m s 5369$

Author affiliations: ${ }^{1}$ Pohang University of Science and Technology, ${ }^{2}$ SKKU Advanced Institute of Nanotechnology Correspondence: * jhje@postech.ac.kr

This research was financially supported by the Creative Research Initiatives (Functional X-ray Imaging) of MEST/National Research Foundation. This research used resources of the Advanced Photon Source, a U.S. Department of Energy (DOE) Office of Science User Facility operated for the DOE Office of Science by Argonne National Laboratory under Contract No. DE-AC02-06CH11357.

32-ID-B,C - Materials science, life sciences, geoscience - Phase contrast imaging, radiography, transmission $x$-ray microscopy, tomography • 7-40 keV • On-site • Accepting general users • 


\section{Tracking the Strain of Deforming Gels}

- he failure of a solid object when subjected to a load can have dramatic and even tragic consequences but is often difficult to predict. A solid's strength and its behavior when it fails are directly related to its microscopic structure. Most of our knowledge about materials' microstructures comes from measurements taken when they are at rest, leaving out crucial information about how microstructure changes during non-equilibrium processes such as mechanical deformation and failure. Hoping to learn more about these changes, researchers used $\mathrm{x}$-ray photon correlation spectroscopy (XPCS) at the APS to measure the nanoscale dynamics of a concentrated colloidal gel subjected to oscillatory shear. The gel was found to undergo a sharp transition to irreversible microscopic deformation at a threshold strain amplitude (near 7\%). However, macroscopically strain softening occurred well below this threshold. These XPCS measurements under in situ strain provide clues to the nanoscale roots underlying the observed fundamental properties of bulk materials and show XPCS to be a valuable tool in studying and assessing the structural dynamics associated with yielding in nanostructured soft solids.

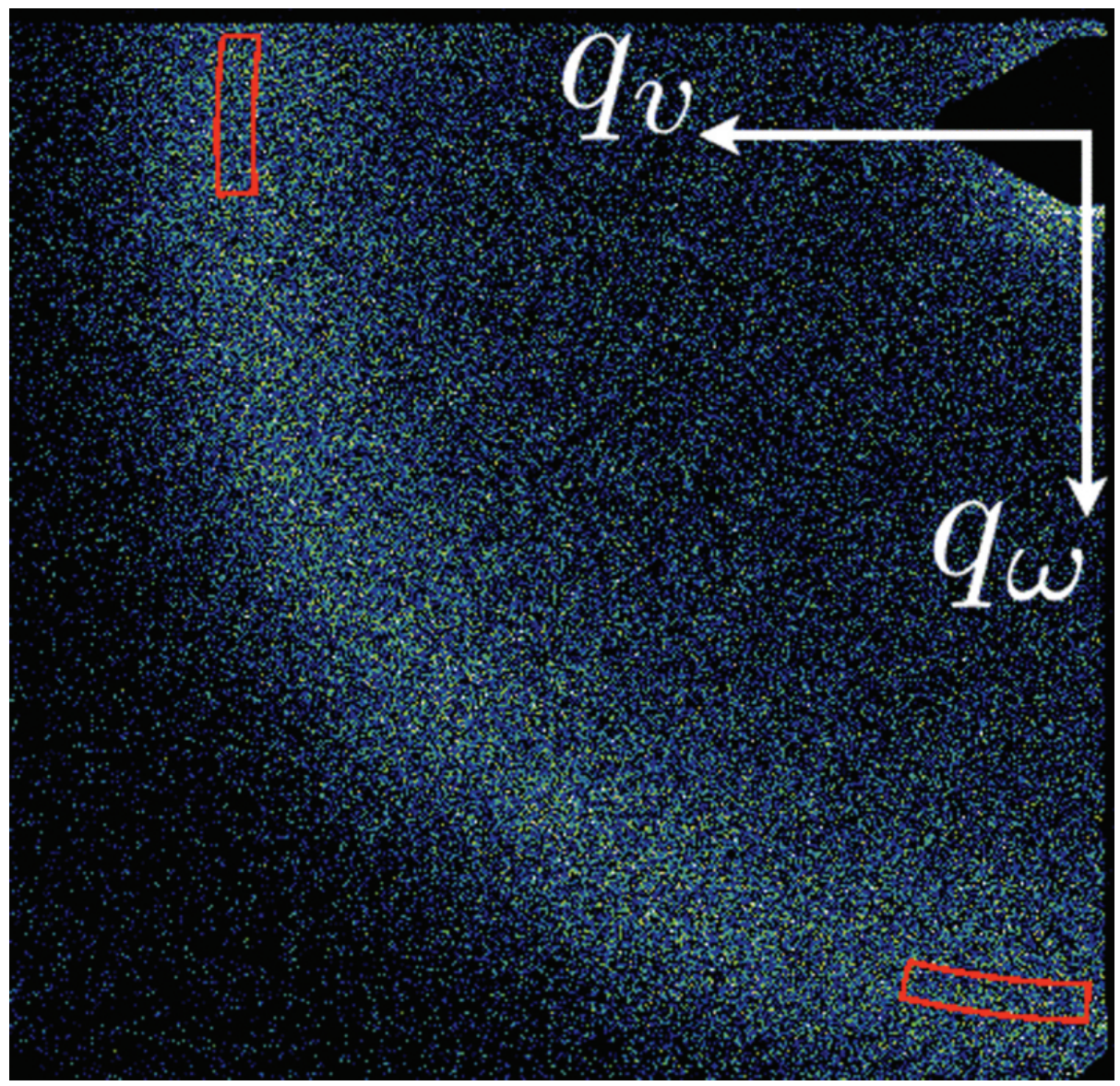

Fig. 1. Image of the coherent $x$-ray scattering intensity from a concentrated nanocolloidal gel under oscillatory shear. The incident beam position, corresponding to $q=0$, is obscured by the shadow of the beam stop in the upper right. The wave-vector directions parallel to the flow direction $q_{V}$ and parallel to the vorticity direction $q_{\omega}$ are indicated by arrows. Regions of approximately fixed wave vector, such as those shown by the red boxes, were analyzed to identify changes in the speckle pattern due to shear-induced irreversible microscopic rearrangements. These two partitions are located at $q=0.19 \mathrm{~nm}^{-1}$. 
Solid objects subjected to stress possess an elastic limit above which the material structure undergoes plastic, or irreversible, changes. The intrinsic disorder of the structure of amorphous solids, such as glasses and gels, makes identifying these changes difficult at best. Microstructural changes can be captured dynamically with XPCS thanks to the high-intensity $x$ ray beam produced by synchrotrons such as the APS. Researchers from the University of Ottawa (Canada), Johns Hopkins University, Argonne, and the Florida A\&M University-Florida State University College of Engineering used XSD beamline 8-ID-I of the APS for their XPCS study of a concentrated nanocolloidal gel subjected to an oscillating shear strain, creating an x-ray speckle pattern captured by a chargecoupled device camera (Fig. 1).

If the deformation of the gel during the shear had been completely reversible, the material's scattering particles would have returned to their original positions after the completion of each shear cycle, causing the x-ray speckle pattern to recover its original configuration. Upon successive applications of shear, the speckle pattern would have displayed "echoes."

But these studies showed that shearing with sufficient amplitude induced some particles to fail to return to their original positions, so the recovery of the speckle pattern was imperfect (Fig. 2), and patterns separated by increasing number of cycles showed more and more differences, indicating nanometer-scale structural irreversibility and the beginning stages of fluid-like flow.

Typical for such materials, the gels were observed to yield - that is, to de- form plastically - at a strain amplitude near $5 \%$, a level of strain normally associated with initial bond-breaking events. The speckle pattern echoes decreased sharply between strain amplitudes of $6 \%$ and $8 \%$, indicating a transition to irreversible deformation at a strain threshold of $7 \%$.

For strains even well below the threshold, the researchers observed softening - the reduction in the apparent strength of the gel to resist deformation. This phenomenon suggests the existence of a range of strains at which the gel structural response to stress is nonlinear, yet the microscopic deformations are still fully reversible.

While this type of behavior has previously been reported in repulsive colloidal glass systems, it can now be generalized to systems with attractive particle interactions, such as the nanocollodial gel under study.

At high shear intensity, the entire gel sample eventually undergoes irreversible rearrangement. That is, when viewed over multiple shear cycles, the irreversible rearrangements were not isolated to a portion of the gel as is seen in shear banding; rather, they were distributed randomly throughout the structure.
The XPCS measurements indicate the size of the speckle pattern echoes also depended on the angle through which the x-rays scattered off the gel, and this dependence reveals a power-law distribution in the size of regions undergoing shearinduced rearrangement, similar to the power-law distribution observed for earthquake magnitudes. - Chris Palmer

See: Michael C. Rogers ${ }^{1 *}$, Kui Chen², Lukasz Andrzejewski ${ }^{1}$, Suresh Narayanan ${ }^{3}$, Subramanian Ramakrishnan ${ }^{4}$, Robert L. Leheny ${ }^{2 * *}$, and James L. Harden $^{1 * * *}$, "Echoes in X-ray speckles track nanometer-scale plastic events in colloidal gels under shear," Phys. Rev. E 90, 062310 (2014).

DOI: 10.1103/PhysRevE.90.062310 Author affiliations: ${ }^{1}$ University of Ottawa, ${ }^{2}$ Johns Hopkins University, ${ }^{3} \mathrm{Ar}$ gonne National Laboratory, ${ }^{4}$ Florida A\&M University-Florida State University College of Engineering

Correspondence:

*michael.rogers@uottawa.ca,
${ }^{* *}$ leheny@jhu.edu,
*** jharden@uottawa.ca

This research was supported by National Science Foundation (NSF) Grant no. CBET1336166, NSF Grant no. HRD-1238524, and the Natural Sciences and Engineering Research Council of Canada Discovery and Research Tools and Instruments programs. This research used resources of the Advanced Photon Source, a U.S. Department of Energy (DOE) Office of Science User Facility operated for the DOE Office of Science by Argonne National Laboratory under Contract No. DE-AC02-06CH11357.

8-ID-I XSD • Polymer science, materials science, physics $-X$-ray photon correlation spectroscopy, intensity fluctuation spectroscopy, small-angle $x$-ray scattering $\cdot 6-12.5 \mathrm{keV}, 7.35$ $7.35 \mathrm{keV}, 7.35 \mathrm{keV} \cdot$ On-site • Accepting general users • 


\section{Wet Granules are Like Glass}
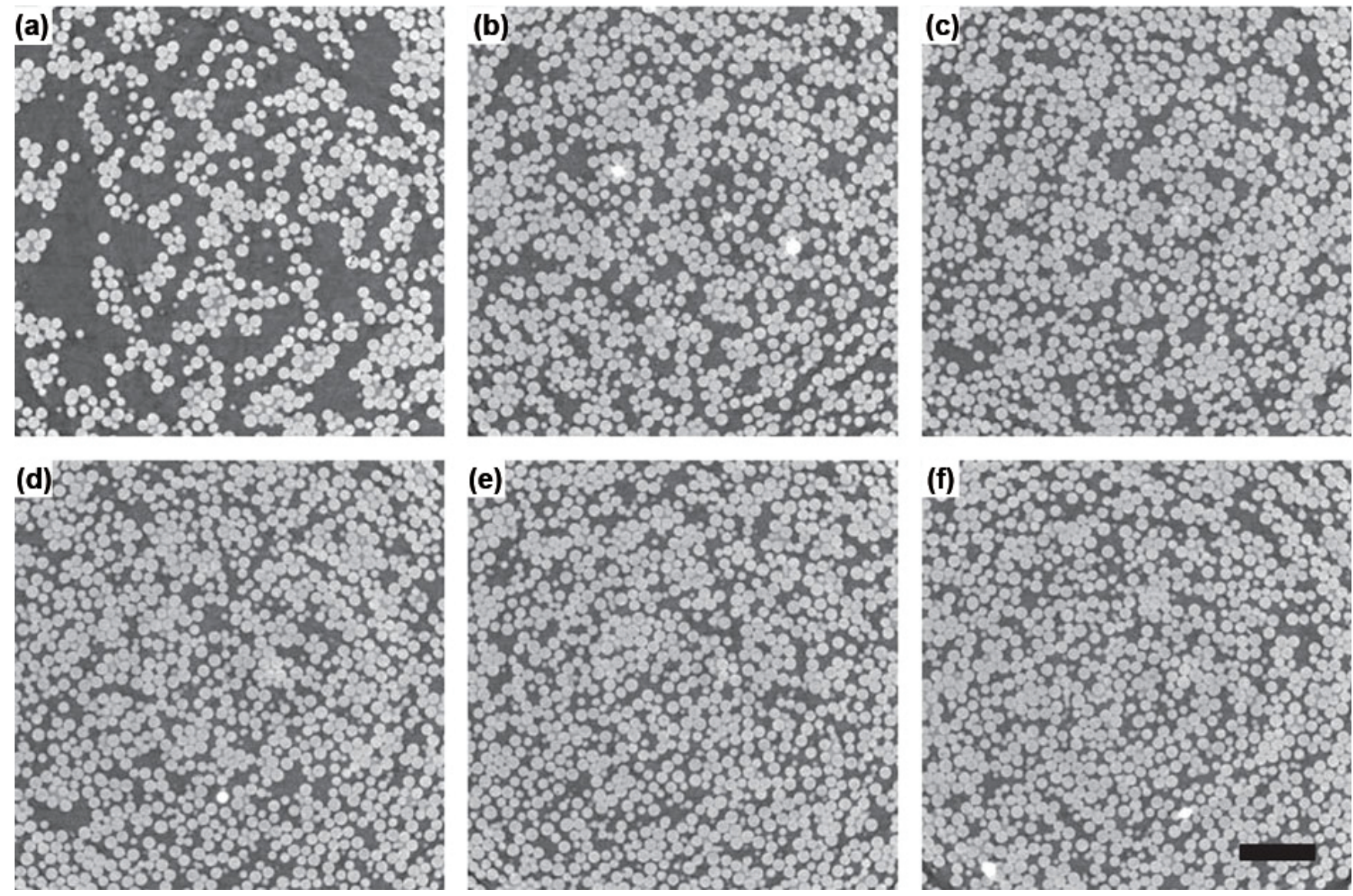

Fig. 1. A sequence of cross-sections of the arrangement of wet glass beads, taken from x-ray microtomography images, shows the evolution of structure as the system is repeatedly tapped. From a to $f_{\text {, }}$ the number of taps is $0,10,40,100,1,000$ and 5,000. Scale bar at bottom right is $1 \mathrm{~mm}$. 
lasses, gels, and collections of granular particles display similarities in both structure and dynamics. The components of all these systems show a variety of short- and long-range ordering, form local structures with various symmetries, and exhibit dynamic frustration mechanisms that prevent the formation of more orderly arrangements. Going back to the 1960s, researchers have studied granular packing of dry materials in order to gain insight into the behavior of liquids and glasses. Tapping such a system causes its structure to evolve as the granules shift around, but that evolution can be blocked when the particles get stuck in clumps that resist further shifting. Geometrical frustration of this sort is also characteristic of glasses. However, glasses typically also have attractive or repulsive intermolecular forces that affect their dynamics, but these forces are absent from dry granules. Researchers utilizing the APS mapped out the detailed structures in a system of wet glass beads. The team found that the stickiness of the beads led to the appearance of small-scale structuring that is not seen in dry granules, and conclude that the system offers a good model for frustration mechanisms in glasses.

The researchers from Shanghai Jiao Tong University (China) and Argonne reasoned that wet granules might offer a more accurate analogy to the behavior of glasses, because capillary effects provide a local attractive force between touching granules. They studied a system of glass beads, 200$\mu \mathrm{m}$ in diameter, moistened with an aqueous solution of potassium iodine. The ratio of liquid volume to that of the beads was 0.033 . Under these conditions, the capillary adhesion between beads was a few hundred times their weight.

After thorough stirring, the wet beads were poured into a container that could be tapped by an electromagnetic mechanism that delivered one cycle of a $30-\mathrm{Hz}$ sine wave, with a peak acceleration 12 times that of gravity. The team used microtomography at XSD beamline 2-BM-A,B at the APS to map out the positions of the particles in the system (Fig 1.). Each map was compiled from 1800 separate images and gave the researchers a full threedimensional picture of the system, with beads, liquid, and air all distinguishable. From these maps the team calculated a number of characteristics of the granular packing and tracked how they changed as the system was repeatedly tapped. The fractional volume of air fell rapidly at first, much faster than in a comparable system of dry particles, because the wet system began with larger open pores in its structure. Consistent with this observation, the average num- ber of beads touching a bead rose quickly as the system became more compact.

More interesting results emerged when the researchers investigated the structures in the system more closely. Pair correlation functions suggested that wet bead systems had more smallscale structures than dry beads. That result was reinforced by analysis of the angular distributions of pairs of beads touching the same third bead, which showed evidence of both equilateral triangles and bipyramids.

To confirm these suggestions, the team looked at a variety of multipole moments of the distribution of particles that were in contact with another particle. The fourth and sixth order moments exhibited no sign of local crystal order in either wet or dry packing, but a modified sixth-order moment (based on Wigner symbols and representing products of spherical harmonics) showed a significant peak in the wet system, corresponding to the presence of local ordered structures with 5 -fold symmetry. These structures were not seen in drybead packing, but have been observed in some colloidal systems.

Finally, the researchers looked directly for local structures by noting the positions of beads in contact with each other. They found tetragonal and pentagonal bipyramids, octahedra, and other shapes, several of which displayed 5 -fold symmetry.

Local structures with 5-fold symmetry have been implicated in theoreti- cal arguments for dynamical arrest of the evolution of glassy systems through geometric frustration. The researchers conclude that such effects are at work in wet-bead but not dry-bead systems, which suggests that short-range capillary attraction is an important ingredient in the dynamics of wet granular packing. The results also suggest that wet granular systems are useful and easily studied models for the structure and dynamics of glasses with short-range attractions. - David Lindley

See: Jindong $\mathrm{Li}^{1}$, Yixin $\mathrm{Cao}^{1}$, Chengjie $\mathrm{Xia}^{1}$, Binquan Kou ${ }^{1}$, Xianghui Xiao ${ }^{2}$, Kamel Fezzaa ${ }^{2}$, and Yujie Wang ${ }^{1 *}$, "Similarity of wet granular packing to gels," Nat. Commun. 5, 5014 (2014). Author affiliations: ${ }^{1}$ Shanghai Jiao Tong University, ${ }^{2}$ Argonne National Laboratory Correspondence:

*yujiewang@sjtu.edu.cn

This work is supported by the Chinese $\mathrm{Na}$ tional Science Foundation Nos. 11175121, National Basic Research Program of China (973 Program, 2010CB834301). This research used resources of the Advanced Photon Source, a U.S. Department of Energy (DOE) Office of Science User Facility operated for the DOE Office of Science by Argonne National Laboratory under Contract No. DE-AC02-06CH11357.

2-BM-A,B - XSD - Physics, life sciences, geoscience, materials science $\cdot$ Tomography, phase contrast imaging $\cdot 10-170 \mathrm{keV}, 11-35 \mathrm{keV} \cdot$ Onsite $\cdot$ Accepting general users $•$ 


\section{A Photo-Cathode Rf Gun for BRIGHTER BEAMS AT THE APS}

As part of the process that produces high-brightness $x$-ray beams at the APS, electrons orbiting in the APS storage ring are extracted from the thermionic cathode of a radio-frequency (rf) gun. In 2013, the APS acquired a state-of-the-art photocathode (PC) rf gun [1], which produces much brighter electron beams compared to the thermionic cathode rf gun that feeds the APS accelerator complex. This new gun will supply high-brightness $\mathrm{x}$-ray beams to experimenters.

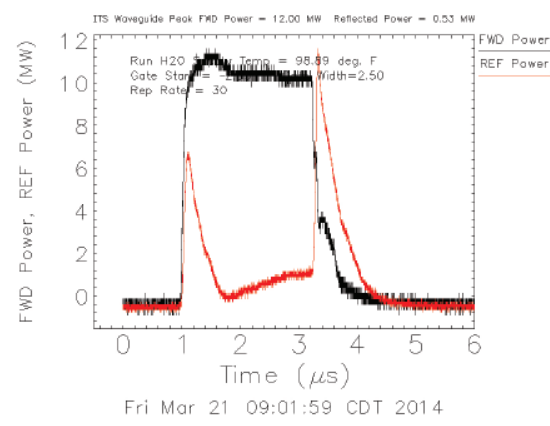

Above: Fig. 1. Forward and reflected rf waveforms at the PC gun waveguide.

Below: Fig. 2. Normalized emittance measured for bunch charge $\sim 25 \mathrm{pC}$.

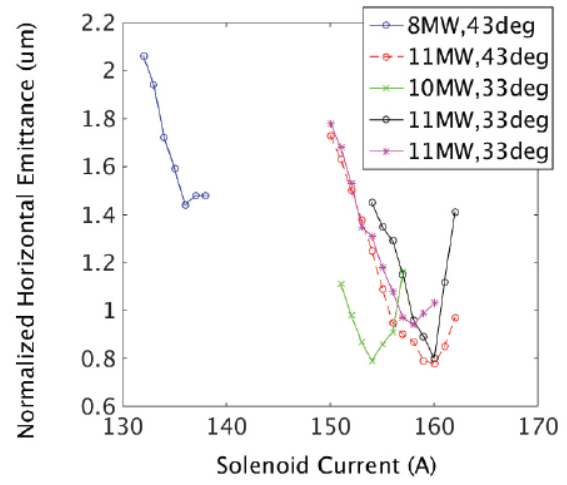

The PC gun consists of a half and a full copper cell, with the length of the half-cell designed to be 0.6 times that of the full cell. The two cells resonate at $2856 \mathrm{MHz}$ in the TM010 m-mode. The polished copper back plate of the halfcell also serves as the photocathode. The repetition rate can be as high as $120 \mathrm{~Hz}$ but is limited to the $30-\mathrm{Hz}$ maximum operating repetition rate of the APS linac. When a class-IV laser of 263-nm wavelength and a couple of pi- cosecond-duration impinges on the cathode, electron bunches with a timestructure similar to the drive-laser are photo-emitted and instantly accelerated by the rf field of the gun. On the cathode surface, the acceleration gradient can be as high as $120 \mathrm{MV} / \mathrm{m}$. Immediately downstream of the PC gun, a main solenoid magnet with integrated dipole and quadrupole correction components provides cylindrically symmetric focusing to the electron beam and is critical in preserving the high brightness of the electron beam.

For high-power rf conditioning, the PC gun was installed on the APS injector test stand. The gun was conditioned to 12-MW forward rf power, 2.5- $\mu$ s rf pulse length, and $30-\mathrm{Hz}$ repetition rate over a two-week period in March 2014 (Fig. 1).

Following the successful rf conditioning, electron beam commissioning started and first photo-electron beams were observed April 10, 2014.

There are several parameters that characterize the quality of the electron beam, including normalized beam emittance, bunch length, average energy, energy spread, and the quantum efficiency of the photocathode. These parameters are affected by the rf field of the gun (particularly the accelerating gradient on the cathode), the photocathode drive-laser transverse and longitudinal profiles, external focusing provided by the solenoid magnets, and the vacuum pressure inside the gun. The photo-electron beams were systematically characterized under various operating conditions [2]. Normalized emittances at different bunch charges and drive-laser spot sizes were measured as functions of the solenoid strength, gun gradient, and phase. At $\sim 25-p C$ bunch charge, a normalized emittance of $\sim 0.8 \mu \mathrm{m}$ was obtained (Fig. 2). A beam energy of $>6 \mathrm{MeV}$ was measured, and the cathode quantum efficiency was found to be in the range of (2 4) $\times 10^{-5}$ during the first beam-commissioning period.

The PC gun and its newly de- signed beamline were installed in the APS linac front end during the September 2014 machine maintenance period (Fig. 3). After rf conditioning and photocathode drive-laser alignment, the first photo-electron beam was extracted at the front end of the APS linac in December 2014 (Fig. 4).

Future plans call for accelerating the high-brightness photo-electron beam through the APS linac in 2015. At the end of the linac, the beam can be either transported to the existing straight-ahead beamline tunnel for experiments that require high-brightness pulsed electron beams, or injected into the particle accumulator ring/booster

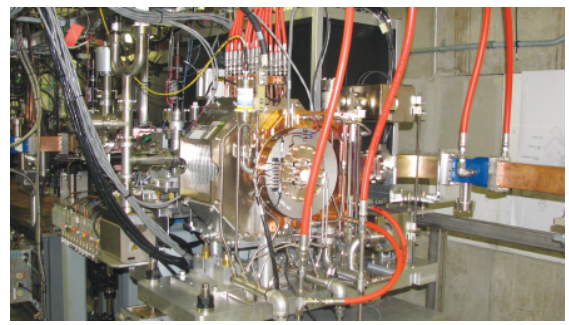

Above: Fig. 3. PC gun and its solenoids as installed in the APS linac front end.

Below: Fig. 4. First photo-electrons $\sim 50 \mathrm{pC}$ on the YAG screen in the PC gun front end installed in the APS linac.

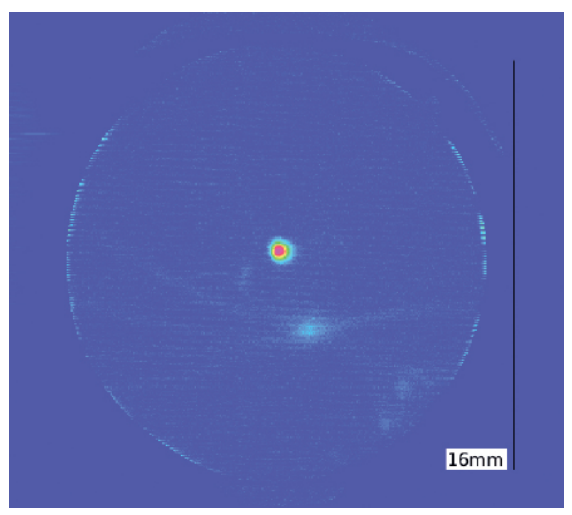

synchrotron and eventually into the storage ring for $\mathrm{x}$-ray generation in support of APS user experiments, provided that sufficient bunch charge can be extracted from the photocathode once the quantum efficiency improves.

Contact: Yine Sun, yinesun@aps.anl.gov

\section{REFERENCES}

[1] D. H. Dowell et al., TUPMS047, Proceedings of PAC07, June 2007, Albuquerque, New Mexico, USA.

2] Y. Sun, et al., THP039, Proceedings of FEL2014, August 2014, Basel, Switzerland. 


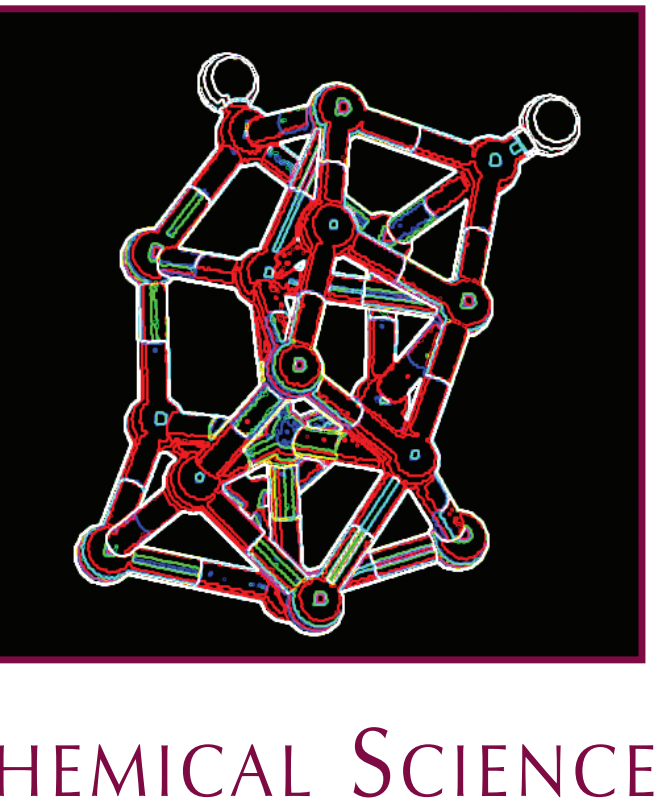




\section{Acid IOnS ARe More Than Spectators}

$X$

-ray absorption fine structure (EXAFS) measurements carried out at the APS, coupled with state-of-the-art density functional theory (DFT) simulations reveal that strong acids, such as hydrochloric acid, form counter-ion pairs in solution across all concentrations, a result that had not been seen in gas-phase studies. The discovery suggests that it is not simply the release of protons - hydrogen ions - that is important for the properties of acids and provides a template to investigate and understand the details of other acids and their chemistries under a variety of different environments including interfaces.

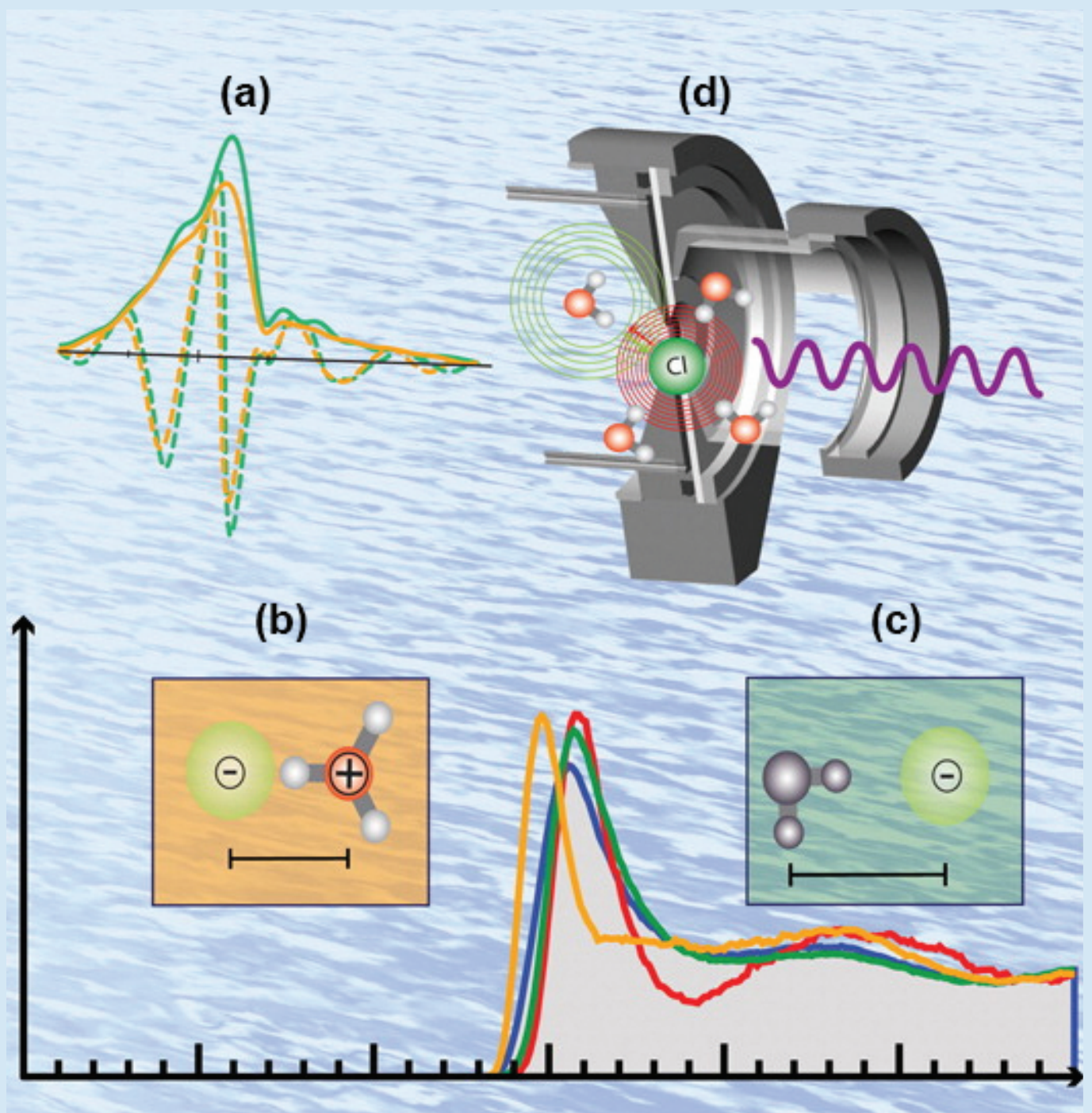


When a strong acid, such as hydrochloric acid, forms a solution in water, the hydrogen ions break away from the compound and give rise to the acidity of the solution. These hydrogen ions can diffuse quickly in the solution and attack other chemicals present, neutralizing basic, or alkaline, substances, or corroding metals and reacting with organic substances. Meanwhile, the counter ion (the chloride in the example of hydrochloric acid) was assumed to form independent, negatively charged solvated fragments, but little was known about the properties of the counter ions or precisely what ion fragments they generated.

Chemists had computer models at their disposal, often based on gasphase studies of the particles involved. However, acids are present in the condensed phase and, thus, the role of the condensed phase environment, as opposed to an isolated molecule in the vapor, should be considered. Moreover, the researchers in this study found that the gas-phase studies are not representative of the more common acidic solution.

Countless chemical reactions hinge on the presence of an excess of protons in the reaction solution, formed by the dissociation of a strong acid into dissolved hydrogen ions and the counter ion. Indeed, these so-called "Brønsted acids" are used in many industrially important chemical reactions, are involved in numerous biological reactions, and play a role in atmospheric chemistry.

Chemists have assumed that the dissociation of a strong acid has two steps. First, the hydrogen-bearing, or protic, acid bonds loosely to a water molecule and the electrical charge is redistributed so that the counter ion becomes more negative while the water molecule gains a positive charge. In the second step, the counter ion is released as a free-floating chloride ion, for instance, and the positively charged water molecule grabs the proton from the acid to form $\mathrm{H}_{3} \mathrm{O}^{+}$, the hydronium ion. The extra proton on this chemical species is free to diffuse through the solution, endowing the solution with its acidic character. These researchers from Pacific Northwest National Laboratory and Argonne have provided a molecular picture of deviations from ideal acid dissociation.

The team carried out extended $\mathrm{x}$ ray absorption fine structure studies at the XSD 20-BM-B beamline at the APS. They combined these data with molecular dynamics and corroborated earlier neutron and x-ray diffraction data.

Specifically, the bond distance between chloride ion and the oxygen in the hydronium ion comprising the contact ion pair significantly shorter than the interaction between chloride and the oxygen of water in the ideal dissociation picture (Fig. 1). This difference in distance and geometry can be measured. Furthermore, the newly discovered molecular moieties can be used to re-interpret in a new light long-standing neutron and $x$ ray diffraction data.

Thus, the contact ion pair between the $\mathrm{H}_{3} \mathrm{O}^{+}$and chloride suggests that the long-misunderstood counter ion of a protic acid is not a mere spectator but is integral to defining the properties of acids across a broad concentration range. Indeed, across the concentration range, the team demonstrates that the contact ion pair is ubiquitous with very few free protons in solution at the highest concentrations. The study provides a template to investigate and understand the details of other acids and their chemistries under a variety of different environments including interfaces. - David Bradley

See: Marcel D. Baer ${ }^{1}$, John L. Fulton ${ }^{1}$, Mahalingam Balasubramanian², Gregory K. Schenter ${ }^{1}$, and Christopher J. Mundy ${ }^{1 *}$, "Persistent lon Pairing in Aqueous Hydrochloric Acid," J. Phys. Chem. B 118, 7211 (2014). DOI: 10.1021/jp501091h Author affiliations: ${ }^{1}$ Pacific Northwest National Laboratory, ${ }^{2}$ Argonne National Laboratory Correspondence:

*chris.mundy@pnnl.gov

This work was supported the U.S. Department of Energy (DOE) Office of ScienceBasic Energy Sciences, Division of Chemical Sciences, Geosciences, and Biosciences. M.D.B. is grateful for the support of the Linus Pauling Distinguished Postdoctoral Fellowship Program at Pacific Northwest National Laboratory. APS Sector 20, which is managed by XSD in partnership with the Canadian Light Source (CLS), is funded by the U.S. DOE Office of Science, and by the Natural Sciences and Engineering Research Council of Canada and the University of Washington via the CLS. This research used resources of the National Energy Research Scientific Computing Center, which is supported by the Office of Science of the U.S. Department of Energy under Contract DE-AC02-05CH11231, and of the Advanced Photon Source, a U.S. DOE Office of Science User Facility operated for the DOE Office of Science by Argonne National Laboratory under Contract No. DE-AC02$06 \mathrm{CH} 11357$.

20-BM-B $\cdot X S D \cdot$ Materials science, environmental science, chemistry $\cdot \mathrm{X}$-ray absorption fine structure, microfluorescence (hard $x$-ray), micro $x$-ray absorption fine structure, diffraction anomalous fine structure $\cdot 2.7-25 \mathrm{keV}$, 2.7-32 keV, 2.7-35 keV • On-site • Accepting general users
$<$ Fig. 1. The experimental prediction and theoretical confirmation of persistent ion pairing between hydronium and chloride provides a unified description of the molecular structure of concentrated hydrogen-chloride solutions. The partial decompositions into $\mathrm{Cl}-\mathrm{O}\left(\mathrm{H}_{3} \mathrm{O}^{+}\right)$and $\mathrm{Cl}-\mathrm{O}\left(\mathrm{H}_{2} \mathrm{O}\right)$ are shown in orange and green, respectively, with the structural portions depicted as insets in (b) and (c). Water hydrogens and oxygens are rendered as small and large gray-scaled spheres, respectively. Charge is indicated with "+" or "-" and the chloride is rendered as a green sphere. (d) is the experimental set-up. 


\section{Acid and Iron Make the Most Sugar}

$\mathrm{T}$

he sugar from corn kernels can be used to make ethanol, an alternative to fossil fuels that can help reduce greenhouse gas emissions. However, since corn kernels are a major source of food for humans and livestock, many efforts are turning to the evaluation of corn stover - the stalk and leaves left over after the sweet ears have been removed - for its viability as a source of ethanol. Experiments at two U.S. Department of Energy $x$-ray light sources, including the APS, provided some answers when researchers characterized corn stover after it was chemically treated in preparation for ethanol production. This treatment is intended to fragment cellulose fibrils - polysaccharide chains in corn stover cells which bond together - into individual chains from which glucose molecules can be obtained. The efficacy of these treatments determines the amount of glucose available for conversion to ethanol. These new results show that treatments that break fibrils into fragments yield higher amounts of sugar, and are preferable to those that only remove non-sugar molecules from the fibrils' surfaces to make the glucose molecules more accessible.

(a)

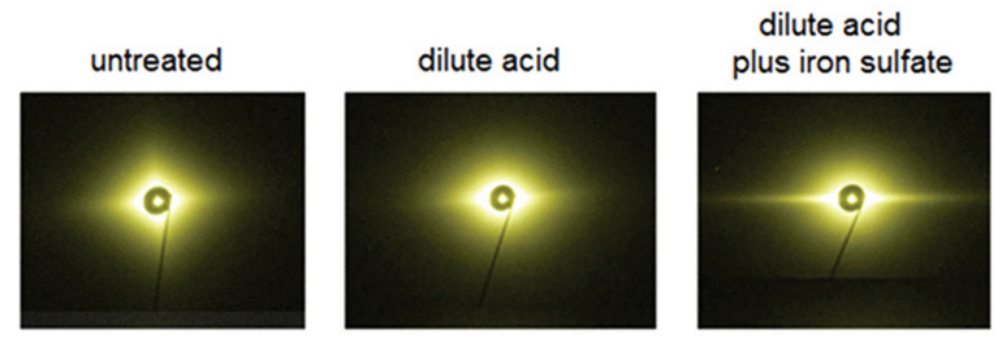

(b)

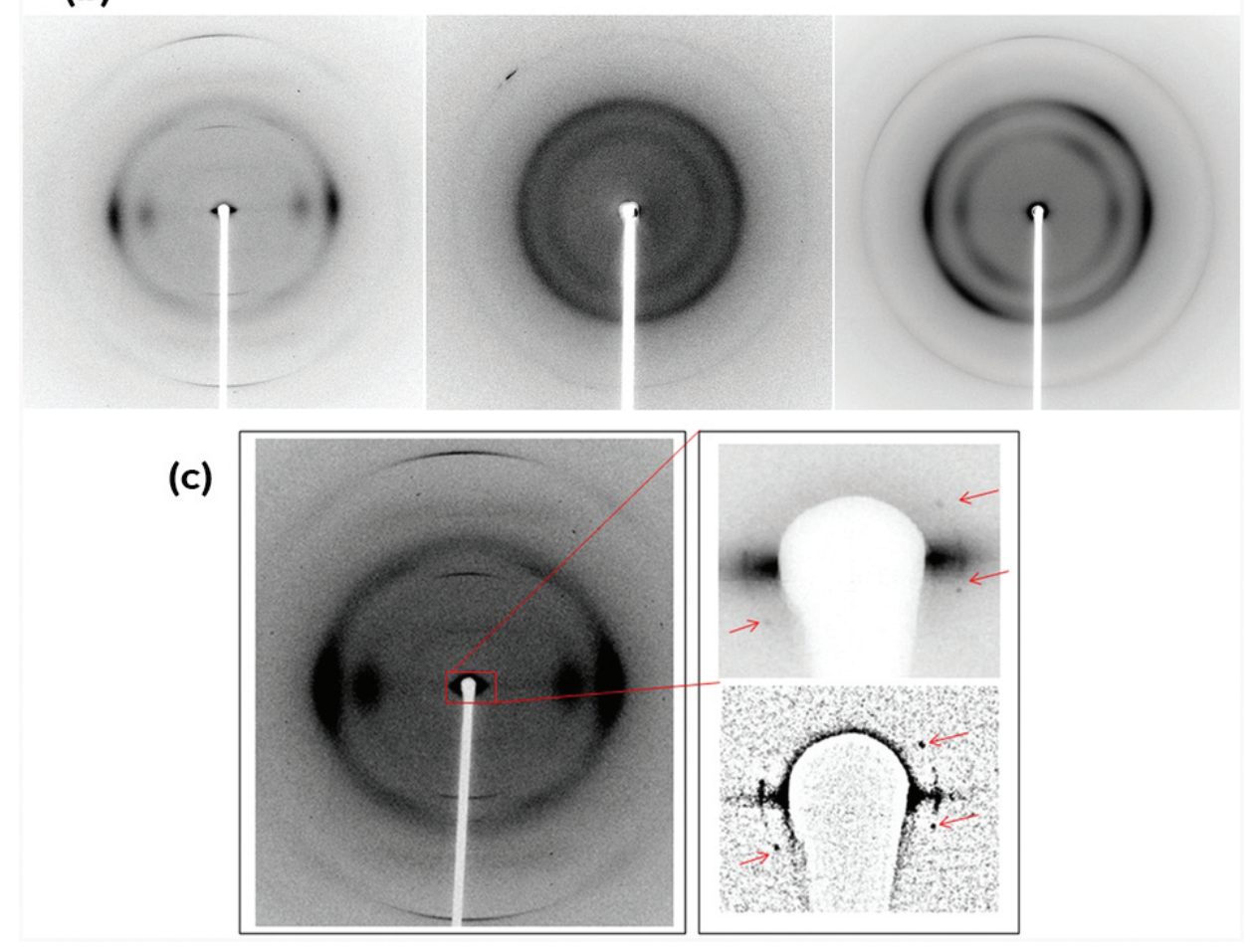


The sugar in corn stover comes from its cellulose, the main structural component forming plant cell walls. Cellulose is a long, linear chain of glucose molecules. Each polysaccharide chain bonds side-to-side with other chains into stiff, stable columns (fibrils). These connected chains form a crystalline lattice that breaks only when the strong hydrogen bonds - which hold the chains together as a fibril — are broken. Fibrils are further grouped into the columns that make up the plant's cell wall, with the orientation of the fibrils parallel to the column they comprise.

The process of producing ethanol begins by treating plant cell walls to weaken the hydrogen bonds between their polysaccharide chains in order to partially free individual glucose molecules. The treatment options include hot water, dilute sulfuric acid, and iron sulfate, both separately and in different combinations. The second step is hydrolysis: breaking the hydrogen bonds between chains. After pretreatment and hydrolysis, the glucose molecules are fermented to produce ethanol and carbon dioxide, which are distilled to separate out the ethanol.

To determine how the cellulose is affected by the pretreatments, the research team from Northeastern University, Brookhaven National Laboratory, Argonne, Rensselaer Polytechnic Institute, Renewable Energy Laboratory, and Northeastern University began by milling, drying, and steam-exploding

< Fig. 1. (a) USAXS patterns (left to right), untreated; DA-pretreated; DA/Fe-pretreated. (b) WAXS patterns (left to right), untreated; DA-pretreated; DA/Fe-pretreated samples. (c) WAXS pattern from a sample exhibiting sharp SAXS reflections observed from very small $\left(<5 \mu^{3}\right)$ scattering volumes of untreated material. Insets show an enlargement of the small angle region (top) and a high-pass filtered version to enhance their visualization (bottom). From H. Inouye et al., Sci. Rep. 4, 3756 (2014). (C) 2015 Macmillan Publishers Limited. the corn stover. They pretreated the corn stover, then measured the saccharine yield of each pretreated sample. The dilute acid with iron pretreatment produced the most sugar, the acid-only treatment the second highest amount, and the hot water-only treatment the lowest amount.

Next, the team characterized the pretreated samples to determine the amount of cellulose degradation that resulted from each pretreatment. Figure 1 shows the extent to which two pretreatments permeated the samples; the iron sulfate remains in the plant cell wall while the dilute acid spreads through the entire cell. Ultra-small angle x-ray scattering (USAXS) at beamline X9 of the U.S. Department of Energy's National Synchrotron Light Source and scanning electron microscope data showed that none of the pretreatments affected the size of the cellulose fibrils but did change their texture and their orientation with respect to that of their wall column. The dilute acid changed the orientations of the fibrils so they no longer ran parallel to the column. Using wide-angle $x$-ray scattering (WAXS) at the GM/CA-XSD 23-ID-B beamline at the APS, the team discovered that the dilute acid pretreatment partially broke down fibrils into groups of cellulose chains. The dilute acid with iron pretreatment degraded the cellulose further: it removed a sufficient amount of non-cellulose molecules to allow the remaining fibrils to coil around themselves, no longer orienting parallel to their column, and broke some fibrils into fragments.

Neither the pre-treatments of dilute acid nor the dilute acid with iron changed the radial extent of the crystalline lattice of cellulose within a fibril. The team concluded that the sugars produced after these pretreatment methods come from fibril fragments rather than the hydrolysis of the crystalline lattice within intact fibrils. They further concluded that the most effective pretreatments would break down the crystalline lattice within the corn stover's cellulose. These pretreatments would do more damage than the dilute acid with iron, ripping the lattice apart - although not exploding it like popcorn - and through that destruction make corn stover a more viable source of ethanol. - Mary Alexandra Agner

See: Hideyo Inouye ${ }^{1}$, Yan Zhang $^{1}$, Lin Yang $^{2}$, Nagarajan Venugopalan ${ }^{3}$, Robert F. Fischetti ${ }^{3}$, S. Charlotte Gleber $^{3}$, Stefan Vogt ${ }^{3}$, W. Fowle ${ }^{1}$, Bryan Makowski $^{4}$, Melvin Tucker $^{5}$, Peter Ciesielski $^{5}$, Bryon Donohoe ${ }^{5}$, James Matthews $^{5}$, Michael E. Himmel ${ }^{5}$, and Lee Makowski 1,6, "Multiscale deconstruction of molecular architecture in corn stover," Sci. Rep. 4, 3756 (2014). DOI: $10.1038 /$ srep03756

Author affiliations: ${ }^{1}$ Northeastern University, ${ }^{2}$ Brookhaven National Laboratory, ${ }^{3}$ Argonne National Laboratory, ${ }^{4}$ Rensselaer Polytechnic Institute, ${ }^{5} \mathrm{Re}$ newable Energy Laboratory, ${ }^{6}$ Northeastern University Correspondence:

*makowski@ece.neu.edu

This work was supported as part of the Center for Direct Catalytic Conversion of Biomass to Biofuels (C3Bio) led by Purdue University, an Energy Frontier Research Center funded by the U.S. Department of Energy (DOE) Office of Science-Basic Energy Sciences under Award No. DESC0000997. Use of GM/CA-XSD has been funded in whole or in part with Federal funds from the National Cancer Institute (ACB12002) and the National Institute of General Medical Sciences (AGM-12006). This research used resources of the Advanced Photon Source, a U.S. DOE Office of Science User Facility operated for the DOE Office of Science by Argonne National Laboratory under Contract No. DE-AC02$06 \mathrm{CH} 11357$.

23-ID-B • GM/CA-XSD • Life sciences • Macromolecular crystallography, microbeam, large unit cell crystallography, subatomic $(<0.85 \AA)$ resolution, multi-wavelength anomalous dispersion, single-wavelength anomalous dispersion • 3.5-20 keV • On-site, remote $\cdot$ Accepting general users $\bullet$ 


\section{Peering Deep Inside of Nongraphitic AnOdes WITH SYNCHROTRON MiCROTOMOGRAPHY}

W

ith so much of our 21st century technology dependent on ever smaller and more efficient rechargeable lithium-ion (Li-ion) batteries, finding new ways to non-destructively peer inside an operating battery is of critical importance, because so many of the physical characteristics of each battery material change during the cycling process. Understanding what those changes are is the first step in choosing and developing new and better battery components, such as moving to metallic electrodes instead of the traditional graphitic carbon materials. X-ray-based microcomputed tomography (MicroCT) can provide a valuable window into Li-ion batteries, although with a somewhat slow collection rate. To overcome that problem, a group of researchers from MIT and Argonne working at the APS has developed a synchrotron-based, full-field MicroCT technique that can capture highresolution tomography images in as fast as $15 \mathrm{sec}$. The research provides the first detailed observations of structural and electrochemical phenomena in candidates for new, high-capacity Li-ion battery anode materials.

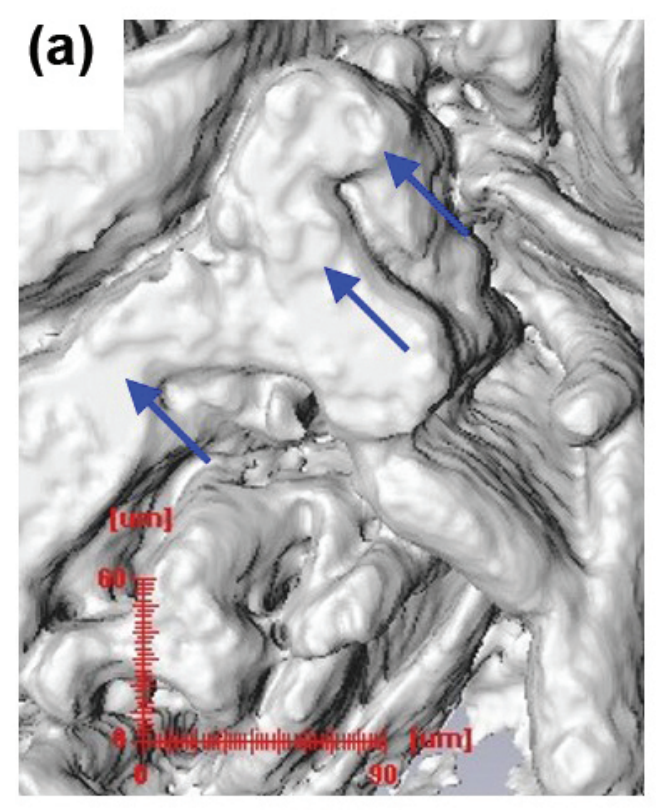

Initial

Fig. 1. (a-c) A series of in situ qualitative microtomographic images of the same $\mathrm{Cu}_{6} \mathrm{Sn}_{5}$ on $\mathrm{Cu}$ foam electrode were taken at various points over the life of the beamline cell. These images show the electrochemically-driven topological changes in the electrode structure. The blue arrows are intended to guide the eye to the more prominent changes from cycle to cycle. The horizontal and vertical scale bars are $90 \mu \mathrm{m}$ and $60 \mu \mathrm{m}$, respectively.

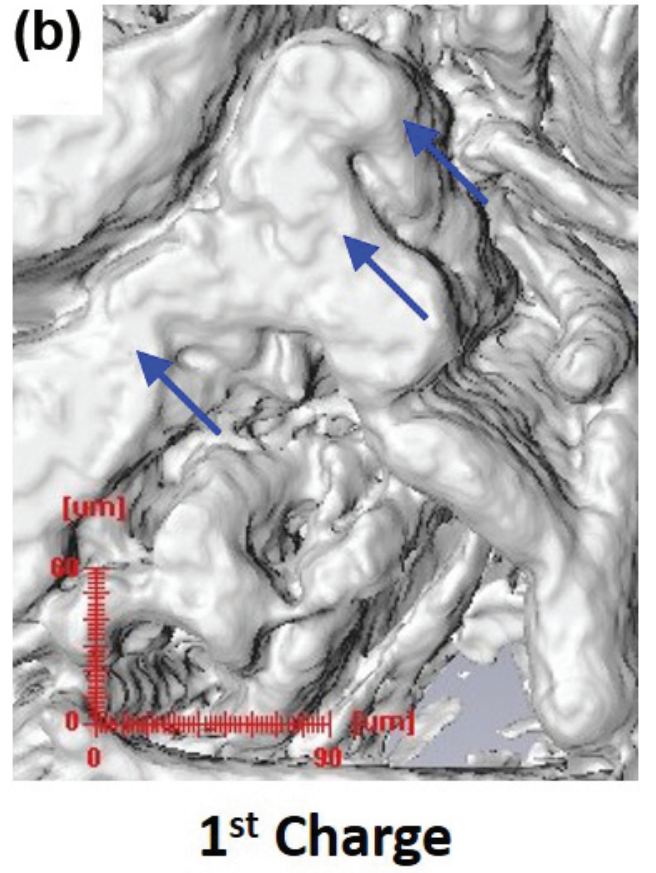


In 1799, Alessandro Volta developed the first electrical battery, known as the Voltaic Cell, which consisted of two plates of different metals immersed in a chemical solution. Volta introduced the theory of electrical current and observed electricity separating water into its component parts of hydrogen and oxygen. Before Volta, it was assumed that electricity was generated by living beings (animal electricity). He discovered that electricity could be generated chemically and made to flow evenly through a conductor in a closed circuit. In 1801 he was summoned to Paris by Napoleon who had a special medal struck in Volta's honor. The world honored Volta by naming the unit of electric potential — the volt after him. Source: IEEE Global History Network. http://ethw.org/Milestones:Volta\%27s_Electrical_Battery_Invention,_1799. (C) Copyright 2013 IEEE All Rights Reserved

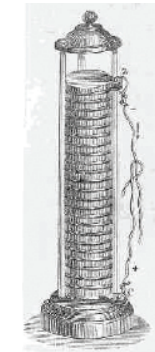

Using XSD beamline 2-BM-A,B at the APS, the investigators examined two non-graphitic, high-capacity anode materials. One was a copper-tin alloy $\left(\mathrm{Cu}_{6} \mathrm{Sn}_{5}\right)$ electrodeposited onto copper foam; the other was a silicon ( $\mathrm{Si}$ )-based laminate. Both were studied ex situ and in situ before and after cycling to determine changes in three-dimensional structure and other properties.

Metal foams are a promising substrate for rechargeable Li-ion battery electrodes for several reasons, includ-

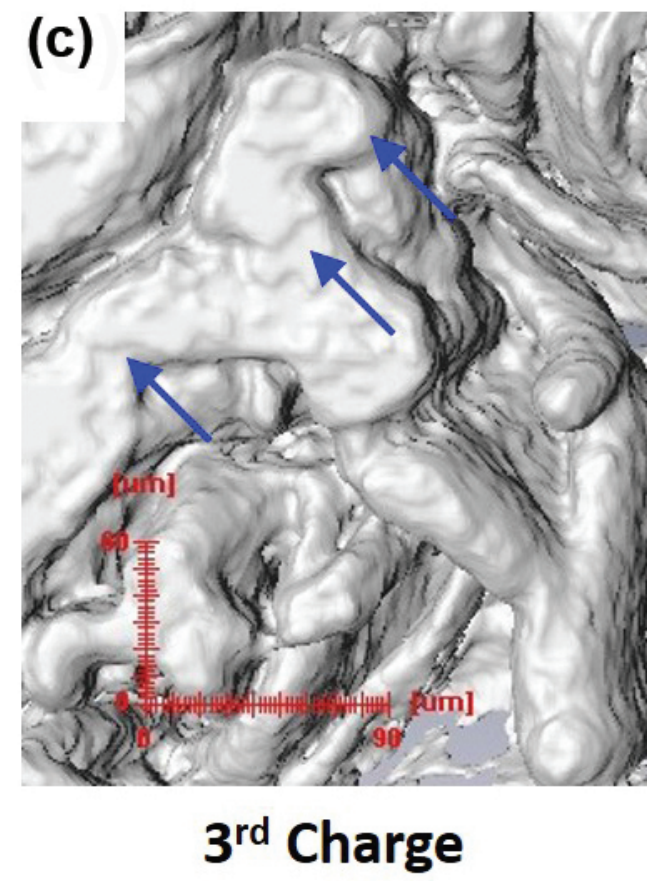

ing the facts that they can provide void volume to accommodate electrode expansion, their porosity supports highpower systems, and the deposition of electrochemically active materials is a good method to produce economically viable electrodes.

The fine three-dimensional structure of the $\mathrm{Cu}$ foam in the $\mathrm{Cu}_{6} \mathrm{Sn}_{5} / \mathrm{Cu}$ foam electrode model, as well as the overlying copper-tin anode, was clearly discernible with high detail on microtomographic images (Fig. 1). Upon cy- cling in coin cells, these electrodes displayed stable lithiation/delithiation for 20-30 cycles before beginning to decay.

Utilizing filtered white beam, synchrotron MicroCT is able to visualize the alterations in the $\mathrm{Cu}_{6} \mathrm{Sn}_{5}$ layer by showing how the surface area-to-volume ratio (SA/V) changes with cycling. The SA/V in these electrodes showed an increase of about $10-15 \%$ over cycling, without any changes in thickness. The current experiments represent the first time it has been possible to observe such structural and electrochemical phenomena in this way and with this detail.

The Si laminate electrodes were investigated using monochromatic 50keV x-ray energy and a suitable sample-to-detector distance as to enable phase contrast imaging. With quantitative phase retrieval, the separate components of the laminate electrodes, including Si particles, carbonaceous material, and voids, were readily distinguished from one another.

These anodes were also cycled inside coin cells and examined, showing a considerably different response than the foam electrodes.

After cycling, large Si particles were seen to break down into smaller pieces and the amount of carbonaceous material and void space increased. This was accompanied by an increased SA/V ratio, growth in the solid-electrolyte interphase (SEI) layer, and decreased porosity. Most significantly, the overall thickness of the laminate electrode increased by about $267 \%$ compared to the foam electrode, which showed no increase in thickness.

The researchers note that these in situ full-field microtomography studies, the first to be published on high-capacity Li-ion anodes operando, promise an exciting direction for further research and the design of new types of materials for rechargeable Li-ion batteries.
As the techniques demonstrated here are improved and expanded, they can provide more detailed and precise data on the various physical characteristics of potential battery materials for better modeling and simulations.

The MicroCT methods used in this work also reveal phenomena that could lead to electrode breakdown and failure (such as the thickening observed in the Si laminate) that might be overlooked by other imaging modalities.

Synchrotron MicroCT should prove to be an invaluable addition to the scientific toolbox as the search for fresh approaches to developing and perfecting the next generation of rechargeable batteries continues. - Mark Wolverton

See: Fikile R. Brushett ${ }^{1 *}$, Lynn Trahey ${ }^{2 * *}$, Xianghui Xiao ${ }^{2}$, and John T. Vaughey" ${ }^{2}$ "Full-Field Synchrotron Tomography of Nongraphitic Foam and Laminate Anodes for Lithium-Ion Batteries," Appl. Mater. Interfaces 6, 4524 (2014).

DOI: 10.1021/am5003124

Author affiliations: ${ }^{1}$ Massachusetts Institute of Technology, ${ }^{2}$ Argonne $\mathrm{Na}$ tional Laboratory

Correspondence: * brushett@mit.edu, ** trahey@anl.gov

Support from the Vehicle Technologies Program, Hybrid and Electric Systems at the U.S. Department of Energy (DOE) Office of Energy Efficiency and Renewable Energy, is gratefully acknowledged. F.R.B. was supported by a Director's Postdoctoral Fellowship. This research used resources of the Advanced Photon Source, a U.S. DOE Office of Science User Facility operated for the DOE Office of Science by Argonne National Laboratory under Contract No. DE-AC0206CH11357.

2-BM-A,B • XSD • Physics, life sciences, geoscience, materials science - Tomography, phase contrast imaging • 10-170 keV, 11-35 $\mathrm{keV} \cdot$ On-site • Accepting general users • 


\section{HYDROGEN ADSORPTION ON Shape-Shifting Platinum Nanoparticles}

he adsorption of hydrogen gas onto a solid material is a technologically-important phenomenon, showing promise, for instance, as a safe and efficient means of hydrogen storage. In the case of platinum, the adsorbed hydrogen resides in a thin layer on its surface. Considerable attention has been devoted to increasing the efficiency of hydrogen adsorption, including the use of solid nanoparticles (typically $1 \mathrm{~nm}$ to $100 \mathrm{~nm}$ across). Recent theoretical and experimental results indicate that decreasing nanoparticle size below the nanometer level enhances adsorption. Now, research carried out at the APS by scientists from the University of Central Florida, UOP LLC (Honeywell), and Ruhr-University Bochum (Germany) confirms earlier theoretical work linking nanoparticle morphology with hydrogen coverage. Since the pressures and temperatures used in this research mimic industrial conditions, these results may well lead to improvements in hydrogen storage, hydrogenation reactions, and electrocatalysis.

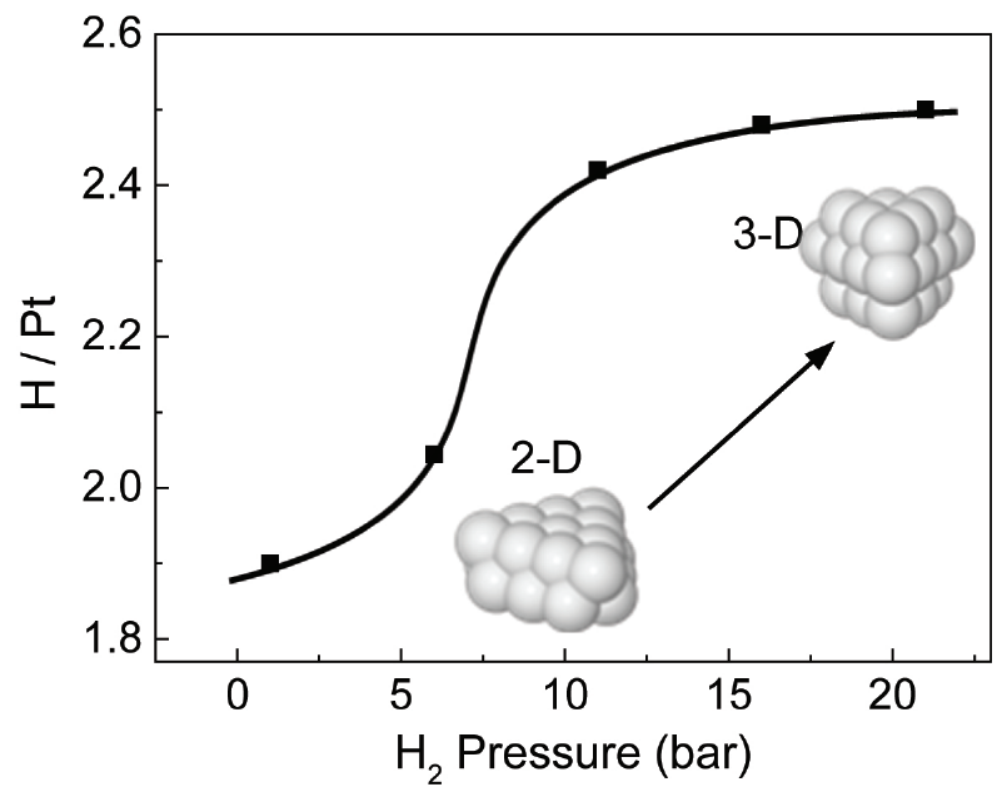

Fig. 1. The number of hydrogen atoms per surface $\mathrm{Pt}$ atom $(\mathrm{H} / \mathrm{Pt})$ determined from XANES measurements on 22 atom $\mathrm{Pt} / \mathrm{\gamma}-\mathrm{Al}_{2} \mathrm{O}_{3}$ nanoparticles in $\mathrm{H}_{2}$ pressures from 1 21 bar. The inset shows the initial biplanar 2-D shape of the Pt22 particles and a possible model 3-D shape at higher pressures after a shape transformation.

10-ID-B • MR-CAT • Materials science, environmental science, chemistry $\cdot X$-ray absorption fine structure, time-resolved $x$-ray absorption fine structure, micro $x$-ray absorption fine structure, microfluorescence (hard $x$ ray) • 4.3-27 keV, 4.3-32 keV, 15-90 keV • On-site $\cdot$ Accepting general users • 
In this research, the degree of hydrogen adsorption on sub-nanometersized platinum $(\mathrm{Pt})$ particles deposited on aluminum oxide $\left(\mathrm{Al}_{2} \mathrm{O}_{3}\right)$ was measured at room temperature as hydrogen pressure ranged from one to twentyone bar. X-ray absorption measurements performed at MR-CAT beamline 10-ID-B of the APS showed that the hydrogen to platinum ratio $(\mathrm{H} / \mathrm{Pt})$ increased with increasing pressure, which was accompanied by a change in nanoparticle shape from a raft-like two-dimensional (2-D) structure into a three-dimensional (3-D) form.

The platinum nanoparticles used here were both shape- and size-selected, with an average diameter of 0.8 $\mathrm{nm}( \pm 0.2 \mathrm{~nm})$. The nanoparticles were deposited onto a high-surface-area aluminum oxide support $\left(\mathrm{Pt} / \gamma-\mathrm{Al}_{2} \mathrm{O}_{3}\right.$, where $\gamma$ denotes the crystalline phase of the $\mathrm{Al}_{2} \mathrm{O}_{3}$ ). The supported platinum nanoparticles possessed a raft-like shape (see 2-D inset, Fig. 1).

Two x-ray absorption techniques were used to probe the $\mathrm{Pt} / \gamma-\mathrm{Al}_{2} \mathrm{O}_{3}$ sample: x-ray absorption near-edge structure (XANES) and extended $x$-ray absorption fine structure (EXAFS). In both techniques, $\mathrm{x}$-rays were used to excite core electrons in the platinum atoms. The XANES technique was used to determine the sample's hydrogen to platinum ratio $(\mathrm{H} / \mathrm{Pt})$ as the pressure of the hydrogen gas was steadily increased. The XANES measurements showed that as $\mathrm{H}_{2}$ pressure increased, the adsorption of hydrogen onto the surface of the platinum nanoparticles also increased. The average $\mathrm{H} / \mathrm{Pt}$ ratio rose from $\sim 1.9$ at a pressure of 1 bar, to a maximum ratio of $\sim 2.5$ at 21 bar. However, this rise in the $\mathrm{H} / \mathrm{Pt}$ ratio as a function of pressure was non-linear, as indicated by Fig. 1.

The other x-ray technique, EXAFS, was employed to determine any structural changes in the platinum nanoparticles as hydrogen gas pressure rose. These structural changes were deduced from two parameters: the first nearest neighbor (NN) coordination number, and the atomic bond lengths. The platinum-to-platinum (Pt-Pt) coordination number indicates the average number of neighboring platinum atoms forming a Pt-Pt bond within the nanoparticles. On the other hand, the
Pt-O coordination number indicates the average number of oxygen atoms neighboring a platinum atom, representing the Pt-O bonds formed between a platinum atom at the nanoparticle-support interface and oxygen atoms in the support material.

Figure 2a shows how the first NN coordination number for the platinum atoms rose with increasing hydrogen adsorption (due to increasing $\mathrm{H}_{2}$ pressure). By contrast, the coordination number for $\mathrm{Pt}-\mathrm{O}$ pairs (not shown) decreased as the hydrogen pressure and coverage grew larger, indicating a rupture of the interfacial connection between the platinum nanoparticles and their $\mathrm{Al}_{2} \mathrm{O}_{3}$ support.

Figure $2 b$ shows a gradual increase in the Pt-Pt bond lengths with increasing hydrogen coverage (due to increased pressure) from 1 through 16 bar, with a sudden decrease at 21 bar, likely associated with the decrease in the contact area of the nanoparticles with the support. All these EXAFS results - the increase in platinum coordination number and bond length, along with the separation of the platinum nanoparticles from the $\mathrm{Al}_{2} \mathrm{O}_{3}$ support - indicate a transition in nanoparticle structure from a two-dimensional biplanar form into a three-dimensional configuration. Figure 2a indicates one possible scenario (shown in red) for this 2-D-to-3-D transition based upon theoretical considerations. However, further research will be required to determine the actual three-dimensional shape of these platinum nanoparticles.

These findings confirm the results of other studies involving platinum nanoparticles, namely that the use of smaller-sized particles (in this case, sub-nanometer particles) leads to an increase in hydrogen adsorption.

- Philip Koth and William A. Atkins

See: Hemma Mistry ${ }^{1}$, Farzad Behafarid $^{1}$, Simon R. Bare ${ }^{2}$, and B. Roldan

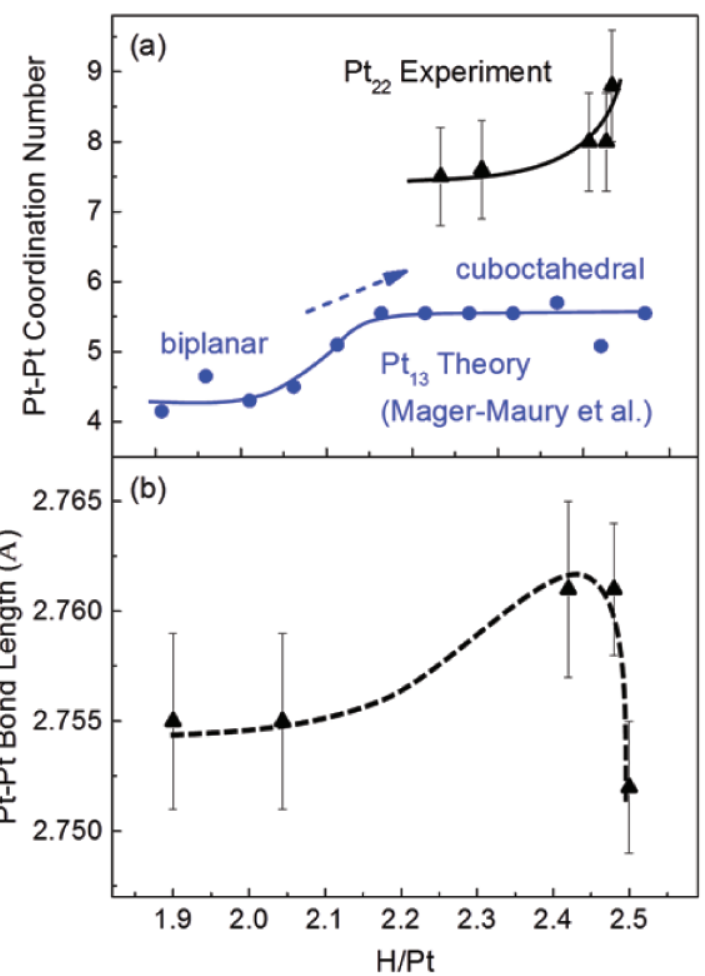

Fig. 2. EXAFS data revealing structural changes in $\mathrm{Pt}_{22}$ nanoparticles supported on $\gamma-\mathrm{Al}_{2} \mathrm{O}_{3}$ with increasing surface hydrogen coverage. a) Average $\mathrm{Pt}-\mathrm{Pt} 1$ st nearest neighbor coordination number acquired from EXAFS data of $\mathrm{Pt}_{22}$ nanoparticles (black triangles) as a function of the $\mathrm{H} / \mathrm{Pt}$ ratio at the particle surface. Blue circles show theoretical predictions for $\mathrm{Pt}_{13}$ nanoparticles from Mager-Maurey et al. b) Average $\mathrm{Pt}-\mathrm{Pt}$ bond length in $\mathrm{Pt}_{22}$ nanoparticles as a function of $\mathrm{H} / \mathrm{Pt}$.

Cuenya 1,3*, "Pressure-Dependent Effect of Hydrogen Adsorption on Structural and Electronic Properties of $\mathrm{Pt} / \gamma-\mathrm{Al}_{2} \mathrm{O}_{3}$ Nanoparticles," Chem. Cat. Chem. 6, 348 (2014).

DOI: $10.1002 /$ cctc. 201300783

Author affiliations: ${ }^{1}$ University of Central Florida, ${ }^{2}$ UOP LLC (Honeywell), ${ }^{3}$ RuhrUniversity Bochum

Correspondence:

*Beatriz.Roldan@rub.de

This work was funded by the U.S. Department of Energy (DOE) Office of Science Basic Energy Sciences under Contract No. DE-FG02-08ER15995. MR-CAT operations are supported by the DOE and the MR-CAT member institutions. This research used resources of the Advanced Photon Source, a DOE Office of Science User Facility operated for the DOE Office of Science by Argonne National Laboratory under Contract No. DEAC02-06CH11357. 


\section{Atomic-Level Clustering Responses of Metallic Alloy Liquids to Changing Temperatures}

Ithough the general tendency of most liquids is to expand in volume when heated
to higher temperatures, atomic clusters inside some liquids may partially contract
in response to heat influxes. This behavior is prevalent among many metallic alloy liquids, researchers in this study found. On the basis of data obtained at the XSD 6-ID beamline of the APS and molecular dynamics simulations, the researchers developed a qualitative understanding as to why some alloys exhibit such contractions while others do not. Structural studies of metallic liquids such as these are crucial to understanding the processes of melting, solidification, and glass formation.

Metallic alloy liquids possess a surprising amount of short- and medium-range order (SRO and MRO), which may extend over several interatomic distances. This behavior is due to attractive bonds and may be quantified in terms of the liquid's pair correlation function, which estimates average interatomic distances based on x-ray scattering data. Such data show a tendency for the atoms to cluster (SRO), with packing of these clusters extending over several atomic distances (MRO). The basic clusters contain a central atom and its nearest neighbors, with a second shell containing next nearest neighbors, and so on; the number of neighbors of the central atom inside each shell is the shell's coordination number.

The researchers from Washington University in St. Louis studied a total of 30 binary, ternary, and quaternary alloy liquids at the 6-ID-D beamline using the Washington University-Beamline Electrostatic Levitation facility, which permits $x$ ray scattering experiments on liquid drops (approximately $2.5 \mathrm{~mm}$ in diameter) under high vacuum while they remain levitated. Eighteen of the alloy liquids held at equilibrium or in a supercooled state clearly exhibited contractions of the nearest neighbor atomic distances as their temperature was increased, although the liquids expanded in overall volume.

The researchers found that the coordination number of each shell decreases with increasing temperature, but that only the first coordination shell may decrease in volume as this happens. They theorized that the decrease in the liquid's first coordination number is the fundamental reason for the anomalous contractions. Since atoms have only a fixed number of valence electrons to share in forming bonds with their neighbors, a smaller coordination number means more shared electrons for each bond and therefore stronger chemical bonds with the central atom. Stronger bonds tend to be shorter in length, thereby decreasing nearest neighbor separations, which would show up as a contraction in the first coordination shell.

To explain why some alloy liquids show first-shell contractions while others do not, the researchers noted that atomic packing inside the first shell is influenced by the relative sizes of the atoms, their chemical interactions, and their relative concentrations, so that both solute- and solvent-based clusters are possible. This means that in the alloy $A_{1-x} B_{x}$, for example, the $A A, B B$, and $A B$ bonds would need to be considered in terms of a total pair distribution function (PDF) that is a weighted sum of the $A A, B B$, and $A B$ partial distribution functions. This weighted sum determines the position of the first peak in the PDF, which gives a measure of the nearest neighbor atomic distance. Depending on the relative contributions of the $A A$, $B B$, and $A B$ bonds (which all try to shrink in length, producing a sort of tugof-war), the first coordination shell may appear to contract or expand with increasing temperature.

Experimentally, it is impossible to determine the partial pair correlation functions of an alloy liquid from the results of a single x-ray scattering experiment. Therefore, the researchers demonstrated the utility of their concept by studying the temperature dependences of the partial and total PDFs for $\mathrm{Cu}_{64} \mathrm{Zr}_{36}$ and $\mathrm{Cu}_{46} \mathrm{Zr}_{54}$ alloy liquids using molecular dynamics simulations. The results were consistent with their scattering data, as were comparisons involving several other alloy liquids.

The development of a more quantitative model will require detailed temperature-dependent structural information about metallic alloy liquids, which is lacking at the present time.

- Vic Comello

See: A.K. Gangopadhyay, M.E. Blodgett, M.L. Johnson, J. McKnight, V. Wessels, A.J. Vogt, N.A. Mauro, J.C. Bendert, R. Soklaski, L. Yang, and K.F. Kelton*, "Anomalous thermal contraction of the first coordination shell in metallic alloy liquids," J. Chem. Phys. 140, 044505 (2014).

DOI: $10.1063 / 1.4861666$

Author affiliation:

Washington University in St. Louis Correspondence: * kfk@wustl.edu

The work was partially supported by the $\mathrm{Na}$ tional Aeronautics and Space Administration under Grant Nos. NNX07AK27G and NNX10AU19G and by the National Science Foundation under Grant Nos. DMR-0856199 and DMR-12-06707. This research used resources of the Advanced Photon Source, a U.S. Department of Energy (DOE) Office of Science User Facility operated for the DOE Office of Science by Argonne National Laboratory under Contract No. DEAC02-06CH11357.

6-ID-D • XSD • Physics, materials science • Magnetic $\mathrm{x}$-ray scattering, high-energy $\mathrm{x}$-ray diffraction, powder diffraction, pair distribution function • 50-100 keV, 70-130 keV • On-site • Accepting general users • 


\section{Graphene Goes Organic}

T

he all-carbon material graphene has interesting and useful electronic and optical properties. Electronic and optoelectronic components based on carbon and carbon-containing organic semiconductors have come to the fore recently as researchers look for the natural successor to silicon, which is ubiquitous in current microelectronics. Graphene exists as single sheets of carbon atoms, with each atom at the vertex of a hexagon and the hexagons connected in such a way as to resemble chicken wire fencing or a honeycomb. The material was the subject of the 2010 Nobel Prize in Physics and has become the focus of countless research groups around the world intrigued by its unique properties. It is one hundred times stronger than steel, weight for weight, it is a highly efficient conductor of heat and electricity, and is almost transparent. But in order to fully exploit graphene, scientists must find ways to stabilize and modify the surface of the atomic layers. Now, utilizing information obtained by research at the APS, scientists have found they can add an organic coating that is exceptionally stable up to $260^{\circ} \mathrm{C}$ and provides the chemical hooks for connecting graphene to other components as well as enhancing graphene's properties.

Investigations of the electronic properties of graphene have led to the observation of a bipolar transistor effect, ballistic transport of electrical charges and large quantum oscillations that all point to possible applications in novel devices. These researchers from Northwestern University, have demonstrated how they can coat a graphene layer supported on silicon carbide with the organic compound perylenetetracarboxylic diimide (PTCDI). This compound and its chemical cousins are well known to chemists for their ability to self-assemble spontaneously into structured systems, which means fabricating devices is a lot easier than using complex sequences of synthetic reactions. Moreover, once self-assembled, PTCDI can be further modified, or functionalized, by chemically connecting other groups to its nanostructure.

One possible application of PTCDI that emerged from theoretical studies and experiment was the possibility of combining PTCDI with a graphene layer so that the repertoire and utility of the carbon material might be extended. Others have tried, but the thermal stability of prototype hybrid materials was somewhat limited; those made with 3,4,9,10-perylenetetracarboxylic dianhydride (PTCDA) break down at a temperature of $38^{\circ} \mathrm{C}$, lower than the PTCDI hybrid. The Northwestern University re- searchers employed scanning tunneling microscopy (STM) using a home-built room temperature UHV STM system, and $x$-ray reflectivity (XRR) performed at the DND-CAT 5-ID-B,C,D x-ray beamline at the Advanced Photon Source to characterize so-called adlayers of PTCDI on epitaxial graphene. This form of graphene is made by sublimating, or evaporating, silicon atoms from silicon carbide in vacuum, resulting in a carbon-rich surface that adopts the structure of graphene.

The addition of PTCDI to graphene in this way to make a stable hybrid material opens many new possibilities. Pristine, pure graphene, although interesting, has two important limitations that have precluded its more rapid development and exploitation in electronic devices. First, unlike the conventional semiconductors of electronic devices it has no band gap; there is no energy difference when electrons jump from a low-energy state to an active excited state. Secondly, it is chemically rather inert and so has been difficult to modify and conjoin with other components. The team, having found a way to modify graphene, might now be able to adjust the material's intrinsic electronic and physical properties indirectly through chemical tweaks to the PTCDI coating and make useful connections.

An additional feature of the PTCDI layer revealed by the STM experiments is that the organic compound easily traverses any defects in the underlying graphene layer and also forms a herringbone pattern of molecules. This pattern had been predicted theoretically but had never been observed experimentally with PTCDI as revealed by the pattern of brighter and darker stripes in the STM images. This herringbone pattern is very different from that observed with this and other organic molecules used to form layers on graphite and other types of mixed graphene layers where parallel rows were seen. The team suggests that the herringbone pattern is a manifestation of underlying graphene corrugations and could be further exploited to create specific patterned areas on the hybrid material. - David Bradley

See: Hunter J. Karmel, John J. Garramone, Jonathan D. Emery, Sumit Kewalramani, Michael J. Bedzyk, and Mark C. Hersam*, "Self-assembled organic monolayers on epitaxial graphene with enhanced structural and thermal stability," Chem. Commun. 50, 8852 (2014). DOI: 10.1039/c4cc02761b Author affiliation:

Correspondence:

Northwestern University

*m-hersam@northwestern.edu

This work was supported by the National Science Foundation-Materials Research Science \& Engineering Center (DMR-1121262), the U.S. Department of Energy (DOE) (DEFG02-09ER16109), the Office of Naval Research (N00014-11-1-0463), the Air Force Office of Scientific Research (FA9550-11-10275), and the Keck Foundation. DND-CAT is supported by Northwestern University, E.I. DuPont de Nemours \& Co., and The Dow Chemical Company. This research used resources of the Advanced Photon Source, a U.S. DOE Office of Science User Facility operated for the DOE Office of Science by Argonne National Laboratory under Contract No. DE-AC02-06CH11357.

5-ID-B,C,D • DND-CAT • Materials science, polymer science $\cdot$ Powder diffraction, x-ray standing waves, x-ray optics development/ techniques, small-angle $x$-ray scattering, surface diffraction, $x$-ray reflectivity, wide-angle $\mathrm{x}$-ray scattering $\bullet$ 6-17.5 keV • On-site $\cdot$ Accepting general users $\cdot$ 


\section{Metals And Oxides Work TOGether to Make BetTer Catalysts}

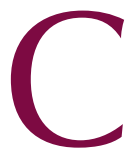

arbon monoxide (CO) is a poisonous gas generated in a variety of industrial processes and is abundant in fossil fuel feedstock (synthesis gas). Copperbased catalysts are often used to oxidize $\mathrm{CO}$, converting the environmental pollutant and protecting fuel cell catalysts from deactivation. Powdered mixtures of copper oxide ( $\mathrm{CuO}$ ) supported on oxides (i.e., $\mathrm{CeO}_{\mathrm{x}}$ ) are popular catalysts for oxidizing $\mathrm{CO}$ in industrial processes. However, because the powder has a heterogeneous structure, it is difficult to decipher the precise catalytic mechanism and the relative roles of $\mathrm{CuO}$ and $\mathrm{CeO}_{2}$ in the reaction. To unravel these mysteries, researchers utilized a novel experimental setup at the U.S. Department of Energy's Advanced Photon Source for a study of nanorods composed of $\mathrm{CeO}_{2}$ and dispersed with very small $\mathrm{CuO}$ nanoparticles. They watched as the $\mathrm{CuO} / \mathrm{CeO}_{2}$ nanorod oxidized $\mathrm{CO}$, gaining new mechanistic insights and understanding of the structure-function chemistry that may help produce better catalysts in the future.

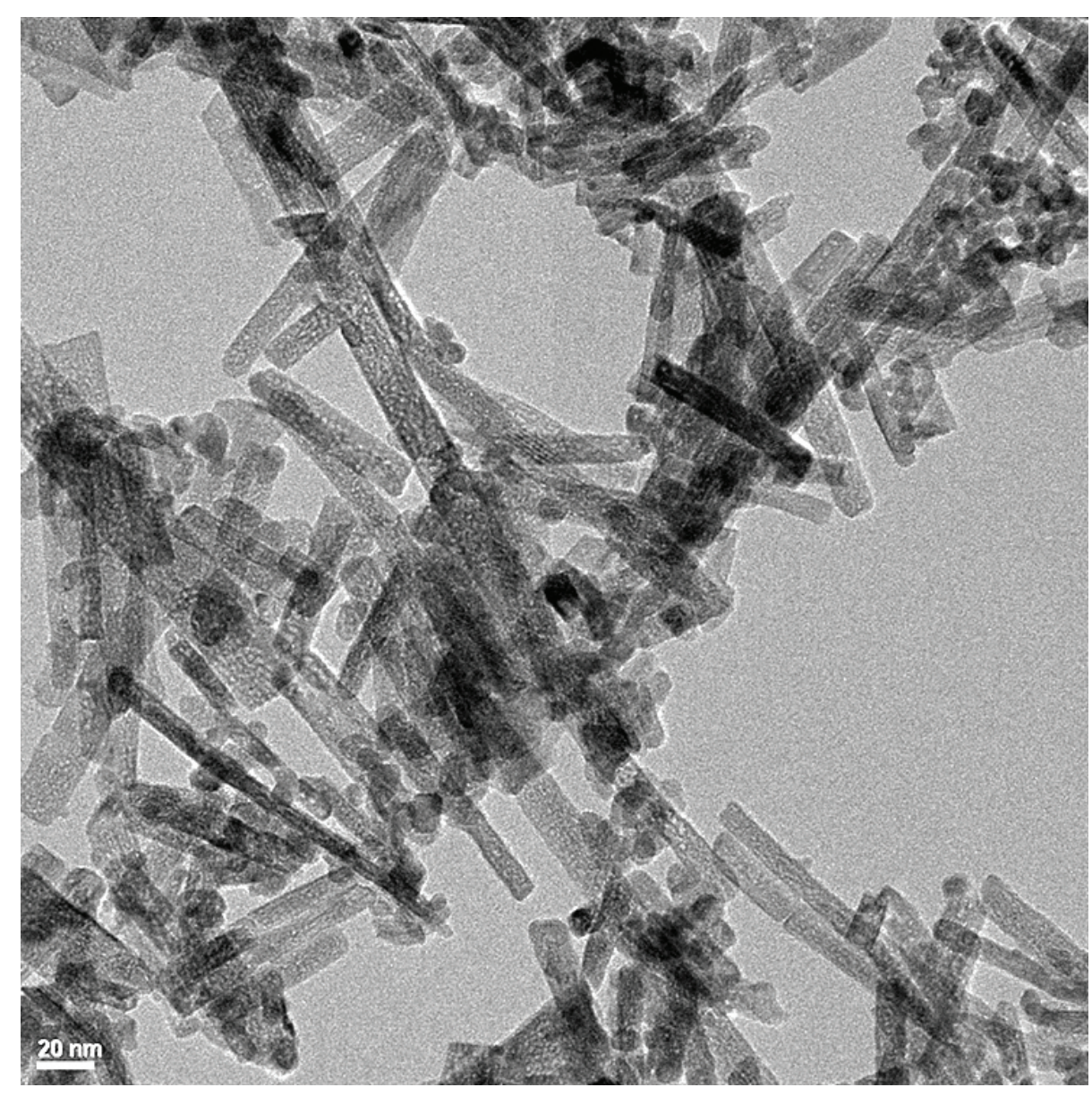

Fig. 1. Transmission electron microscopy image of $\mathrm{Cu}-\mathrm{CeO}_{2}$ nanorod catalysts.
To make the catalyst, the researchers from Brookhaven National Laboratory, Argonne, Consejo Superior de Investigaciones Científicas (Spain), and Peking University (Republic of China) prepared $\mathrm{CeO}_{2}$ support nanorods using wet chemical methods derived from the literature. Then, $\mathrm{CuO}$ was finely dispersed on the support to achieve $\mathrm{a}_{\mathrm{CeO}}$ nanorod with $5 \%$ copper by weight.

The researchers analyzed the structure of the $\mathrm{CuO} / \mathrm{CeO}_{2}$ nanorods using transmission electron microscopy and scanning transmission electron microscopy. The nanorods were $50 \mathrm{~nm}$ to $100 \mathrm{~nm}$ in length and $11 \mathrm{~nm}$ in diameter (Fig. 1).

Complex chemical reactions can involve myriad reaction intermediates and a catalyst with a continuously evolving structure. To identify and monitor all the moving parts in the $\mathrm{CuO} / \mathrm{CeO}_{2}$ catalyzed oxidation of $\mathrm{CO}$, the researchers combined, for the first time, diffuse reflectance infrared Fourier transform spectroscopy (DRIFTS) and fluorescence time-resolved x-ray absorption near-edge spectroscopy (XANES). The combination was made possible by adding extra windows to a Harrick in situ reaction cell (Fig. 2), allowing infrared radiation in and out while simultaneously allowing x-ray photons in and out (fluorescence). This set-up facilitated the simultaneous collection of fluorescence data 


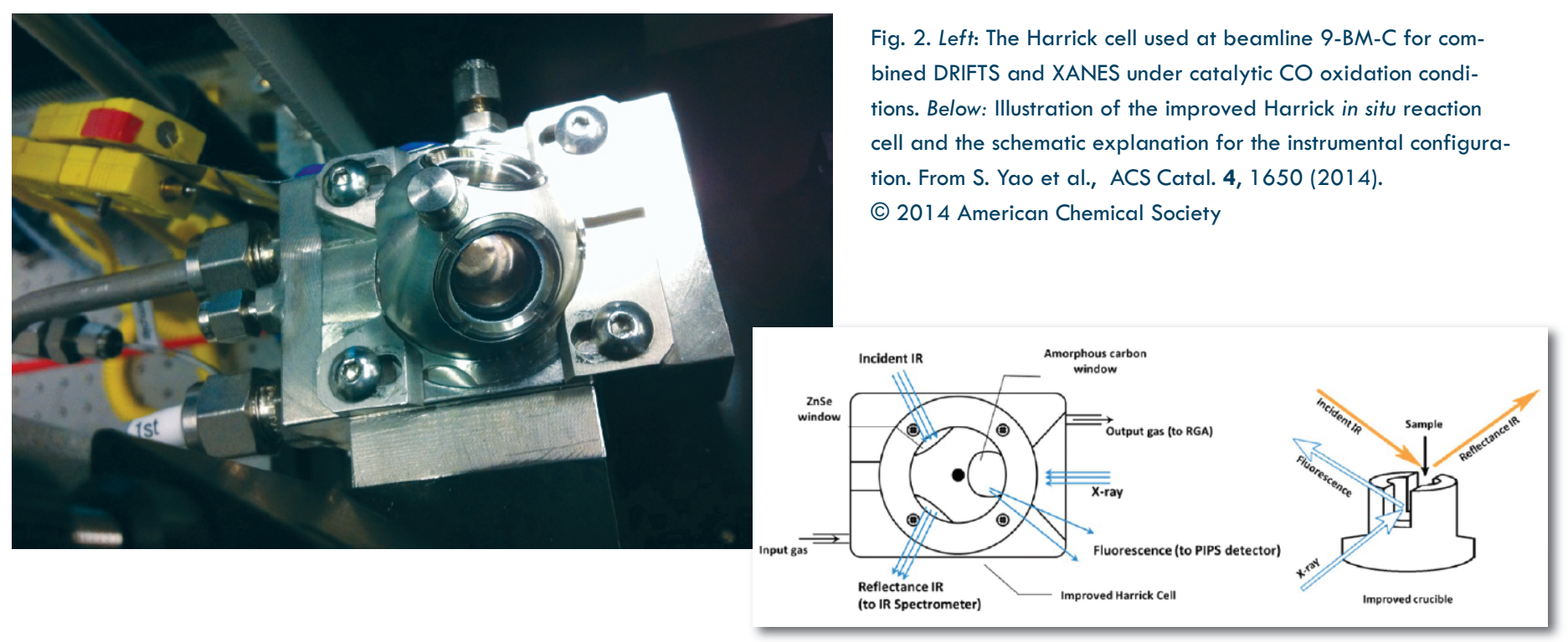

and x-ray data while the reaction was under catalytic conditions.

To perform the experiment, the researchers placed the $\mathrm{CuO} / \mathrm{CeO}_{2}$ nanorods in the reaction cell at the XSD 9-BM-B, $C$ beamline at APS under a steady stream of $\mathrm{CO}$ and $\mathrm{O}_{2}$ while collecting DRIFTS and XANES data. Some experiments were also performed at the X7B beamline of the National Synchrotron Light Source at Brookhaven National Laboratory.

The DRIFTS data provided chemical information about the catalyst's surface and the reaction intermediates. Meanwhile, fluorescence XANES detected chemical signatures and allowed the researchers to identify the structures that prevailed within the DRIFTSdetermined surfaces.

By collecting data sets simultaneously while the reaction proceeded, the team observed where and how chemical species changed during the $\mathrm{CO}$ oxidation process. They studied the reaction under a variety of temperature and pressure conditions, to see how these variables altered the chemical reaction, which could provide valuable information on optimal catalytic conditions.

The team compared the chemistry observed with the $\mathrm{CuO} / \mathrm{CeO}_{2}$ nanorod to that of plain $\mathrm{CuO}$. This provided a means to explore the role of the $\mathrm{CeO}_{2}$ as a support and further study the $\mathrm{Cu}$ Ce interaction. This comparison reinforced the importance of the dispersion of $\mathrm{CuO}$ on $\mathrm{CeO}_{2}$ and the interaction between $\mathrm{CuO}$ and $\mathrm{CeO}_{2}$ to this reaction.

Based on their results, the researchers proposed a four-step mechanism for the $\mathrm{CO}$ oxidation reaction over a $\mathrm{CuO} / \mathrm{CeO}_{2}$ nanorod catalyst. Typically, copper oxide is considered the most active component while cerium oxide is considered an inactive support. However, steps three and four of the reaction scheme directly involve cerium in the redox chemistry.

This is the key point, according to the researchers: $\mathrm{CeO}_{2}$ is not an idle bystander. Cerium and copper work together to catalyze the oxidation reaction, an observation that will be critical to improving catalytic performance.

The researchers also observed that the concentration of copper (I) on the surface of the nanorod corresponded with catalytic efficiency, offering another potential strategy for improving catalytic activity.

Using their new multi-technique approach, the team plans to study catalysts with different shapes, such as nanocubes and nanospheres, to assess what aspects of structure affect chemical reactivity.

$$
\text { - Erika Gebel Berg }
$$

See: Siyu Yao ${ }^{1,4}$, Kumudu Mudiyanselage ${ }^{1}$, Wenqian $\mathrm{Xu}^{1}$, Aaron C. Johnston-Peck ${ }^{1}$, Jonathan C. Hanson ${ }^{1}$, Tianpin $\mathrm{Wu}^{2}$, Dario Stacchiola ${ }^{1}$, José A. Rodriguez ${ }^{1}$, Haiyan Zhao ${ }^{2}$, Kevin A.
Beyer $^{2}$, Karena W. Chapman², Peter J. Chupas $^{2}$, Arturo Martínez-Arias ${ }^{3}$, Rui $\mathrm{Si}^{1}$, Trudy B. Bolin², Wenjian Liu ${ }^{4}$, and Sanjaya D. Senanayake ${ }^{1 *}$, "Unraveling the Dynamic Nature of a $\mathrm{CuO} / \mathrm{CeO}_{2}$ Catalyst for $\mathrm{CO}$ Oxidation in Operando: A Combined Study of XANES (Fluorescence) and DRIFTS," ACS Catal. 4, 1650 (2014). DOI: 10.1021/cs500148e Author affiliations: ${ }^{1}$ Brookhaven National Laboratory, ${ }^{2}$ Argonne National Laboratory, ${ }^{3}$ Consejo Superior de Investigaciones Científicas (ICP-CSIC), ${ }^{4}$ Peking University

Correspondence: * ssenanay@bnl.gov

Work performed at Brookhaven National Laboratory was supported by the U.S. Department of Energy (DOE) Office of ScienceBasic Energy Sciences, and Catalysis Science Program under Contract no. DE-AC02$98 \mathrm{CH} 10886$. Financial support from the China Scholarship Council (File No.2012060 10107 ) is gratefully acknowledged. Financial support from MINECO (Plan Nacional Project No. CTQ2012-32928) and EU COST CM1104 action is also acknowledged. This research used resources of the National Synchrotron Light Source, which is a DOE Office of Science User Facility, and the Advanced Photon Source, a U.S. DOE Office of Science User Facility operated for the DOE Office of Science by Argonne National Laboratory under Contract No. DE-AC0206CH11357.

9-BM-B,C • XSD - Materials science, chemistry, environmental science $\cdot \mathrm{X}$-ray absorption fine structure $\cdot 2.1-24 \mathrm{keV} \cdot$ On-site Accepting general users • 


\section{Who Shrunk the Catalyst?}

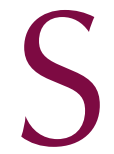

maller is better for a metal catalyst, but smaller also means more compact in terms of the metal's atomic structure. Chemical engineers often fabricate metal catalysts in the form of nanoparticles in order to increase their reactive surface area. However, the small size can lead to structural changes in how the metal atoms assemble together. A new suite of $\mathrm{x}$-ray experiments on platinum $(\mathrm{Pt})$ nanoparticles gives the most detailed picture yet of the consequences of going small. Measurements taken at three different $\mathrm{x}$-ray beamlines at the APS confirm that atom-spacing becomes smaller as the size of a nanoparticle decreases, but the spacing expands back to near normal when gas molecules adsorb on the surface (Fig. 1). Because this contraction-relaxation affects the way reactants behave on the metal surface, the results of these experiments may help in designing better catalysts.

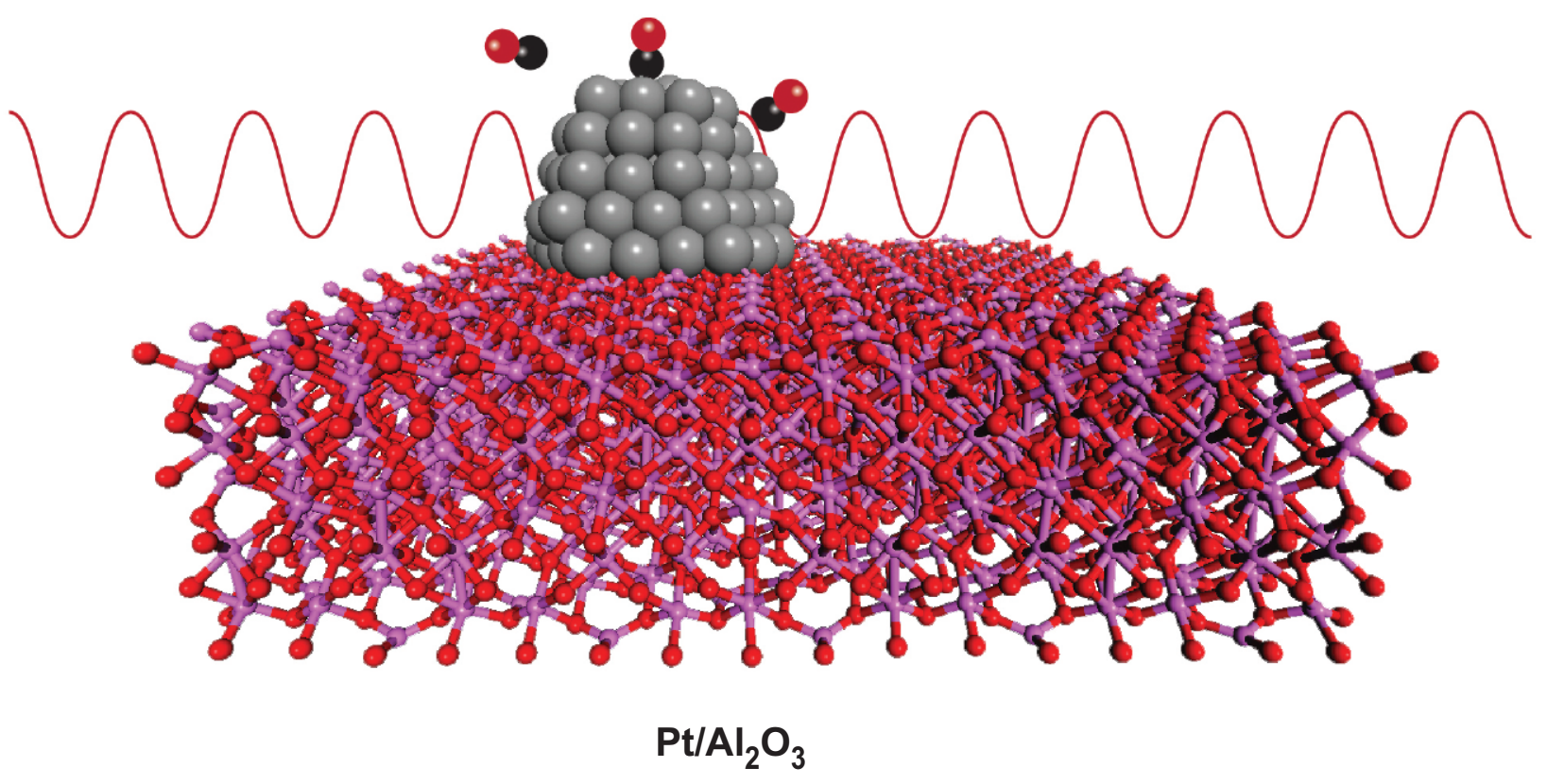

Fig. 1. Combined experimental and theoretical studies of Pt nanoparticles on alumina $\left(\mathrm{Al}_{2} \mathrm{O}_{3}\right)$ characterize precisely the contract and expansion of the lattice parameter caused by different chemical environments.

Platinum, nickel, palladium, and similar metals are used as catalysts in the petroleum and pharmaceutical industries to accelerate organic synthesis, as well as in car exhaust systems to remove toxic fumes. These metals are able to drive reactions by trapping reactants on their surface. Therefore, for a given amount of metal, reaction rates can be increased if the material is divided up into nanoparticles that have a high surface-to-volume ratio.

Earlier x-ray studies suggested that metal nanoparticles are slightly smaller in size than one would expect from the atom-spacing found in normal chunks of metal. One possible explanation for this contraction is that atoms forming the outer surface are pulled inward because they have no neighbors pulling from the other side. Previous experiments have found evidence for this model, but scientists would still like to understand precisely how the surface atoms behave in different circumstances.

Scientists from the Argonne and Brookhaven national laboratories, the
University of Alabama in Huntsville, Kansas State University, and Purdue University performed both $\mathrm{x}$-ray scattering and spectroscopy measurements on Pt nanoparticles.

The team began by growing $\mathrm{Pt}$ nanoparticles on powder grains of alumina $\left(\mathrm{Al}_{2} \mathrm{O}_{3}\right)$. A powder substrate like this is often used for industrial catalysts. However, the researchers used a special atomic layer deposition process that controlled the size of the $\mathrm{Pt}$ nanoparticles by varying the number of deposition cycles. Three separate sam- 
ples were produced with nanoparticle widths of 1,2 , and $3 \mathrm{~nm}$, as measured through small-angle $x$-ray scattering (SAXS) at the XSD 12-ID-B x-ray beamline at the APS.

The prepared samples were next placed in the XSD beamline 11-ID-B, where total scattering measurements revealed typical atom separations as represented by the pair distribution function (PDF, Fig. 2, left).

The sample chamber was initially filled with helium gas, which doesn't absorb on platinum. In this "bare surface" case, the atomic separations for 1-nm wide Pt particles were on average 1.4\% smaller than those in normal bulk metal. Similar contraction was observed - but to a lesser degree - for the bigger nanoparticles in the other two samples.

However, during catalysis, the nanoparticle surfaces will not be bare. To explore this, the team first injected hydrogen gas $\left(\mathrm{H}_{2}\right)$ into the sample chamber. The hydrogen adsorbed on the platinum, exemplifying the typical reduction step in a catalytic cycle. Afterwards, the team purged the system and introduced carbon monoxide (CO), which represents the complementary oxidation step in a catalytic cycle.

For both gas exposures, x-ray scattering measurements showed that the adsorption caused the nanoparticles to expand from their contracted bare surface state. However, the amount of expansion was not the same in the two cases. Nanoparticles with $\mathrm{CO}$ on their surfaces had slightly larger interatomic separations than nanoparticles with $\mathrm{H}_{2}$ adsorption.

To understand the chemical bond behavior during this contraction and expansion, the researchers performed $x$ ray absorption spectroscopy (XAS) at the 10-BM-A,B beamline of the MRCAT at the APS (Fig. 2, right).

The nanoparticles exhibited different absorption spectra, depending on whether the surrounding gas was helium, hydrogen or carbon monoxide. The largest spectral differences occurred for the 1-nm wide sample, which suggests that smaller nanoparticles form stronger bonds with the adsorbed gas molecules.
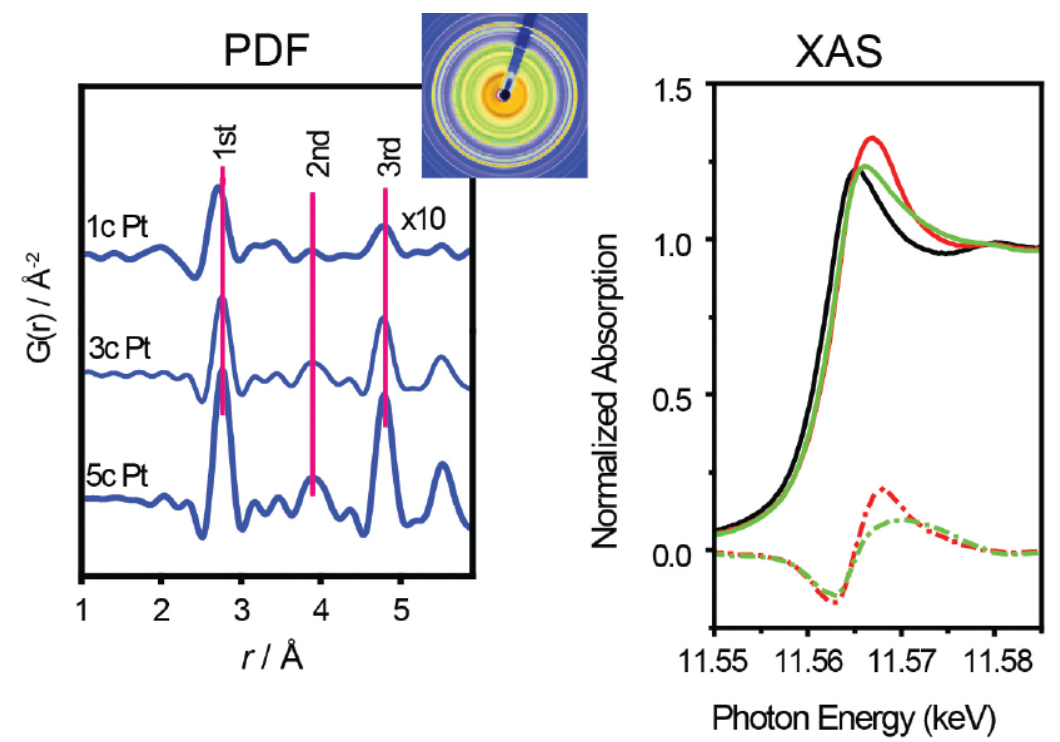

Fig. 2. Left: The PDF, which is derived from x-ray scattering data, has peaks that correspond to atomic separations in Pt nanoparticles that are 1-nm wide (labeled 1c Pt), 2-nm wide (labeled 3c Pt) and 3-nm wide (labeled 5c Pt). The two-dimensional diffraction image (upper right inset) represents the data used to construct the PDF. Right: X-ray absorption spectroscopy measurements show differences in the chemical bond structure when 1 -nm-wide Pt nanoparticles are exposed to the following gases: helium (black), hydrogen (solid green), and carbon monoxide (solid red). Also displayed are the spectral differences between hydrogen-helium (dashed green) and carbon monoxide-helium (dashed red).

Such quantitative information could be useful in designing catalysts, since engineers want a material that can hold reactants on its surface, but not too strongly. Finding the right balance may simply be a question of sizing up nanoparticles. - Michael Schirber

See: Yu Lei ${ }^{2}$, Haiyan Zhao ${ }^{1}$, Rosa Diaz Rivas $^{3}$, Sungsik Lee ${ }^{1}$, Bin Liu ${ }^{4}$, Junling $\mathrm{Lu}^{1}$, Eric Stach ${ }^{3}$, Randall E. Winans ${ }^{1}$, Karena W. Chapman ${ }^{1}$, Jeffrey P. Greeley $^{5}$, Jeffrey T. Miller ${ }^{1}$, Peter J. Chupas ${ }^{1}$, and Jeffrey W. Elam ${ }^{1 *}$, "Adsorbate-Induced Structural Changes in 1-3 nm Platinum Nanoparticles," J. Am. Chem. Soc. 136, 9320 (2014).

DOI: $10.1021 / \mathrm{ja} 4126998$

Author affiliations: ${ }^{1}$ Argonne National Laboratory, ${ }^{2}$ University of Alabama in Huntsville, ${ }^{3}$ Brookhaven National Laboratory, ${ }^{4}$ Kansas State University, ${ }^{5}$ Purdue University

Correspondence: * jelam@anl.gov

This material is based upon work supported as part of the Institute for Atom-efficient Chemical Transformations, an Energy Frontier Research Center funded by the U.S. De- partment of Energy (DOE) Office of ScienceBasic Energy Sciences. MR-CAT operations are supported by the DOE and the MR-CAT member institutions. Y.L. gratefully acknowledges the start-up support by the University of Alabama in Huntsville. B.L. also thanks the start-up support by the Kansas State University. This research used resources of the Advanced Photon Source, a U.S. DOE Office of Science User Facility operated for the DOE Office of Science by Argonne National Laboratory under Contract No. DEAC02-06CH11357.

10-BM-A,B - MR-CAT • Materials science, chemistry, environmental science, physics $\cdot X$ ray absorption fine structure, $x$-ray lithography, tomography • 3-200 keV, 4-32 keV • On-site •

11-ID-B • XSD • Chemistry, environmental science, materials science - Pair distribution function • $58.66 \mathrm{keV}, 86.7 \mathrm{keV} \cdot$ On-site • Accepting general users •

12-ID-B • XSD • Chemistry, materials science, life sciences, polymer science, physics $\cdot$ Small-angle $x$-ray scattering, grazing incidence small-angle scattering, wide-angle $x$-ray scattering, grazing incidence diffraction • 7.9-14 keV • On-site • Accepting general users ${ }^{\bullet}$ 


\section{INVESTIGATING THE TIES THAT BIND: CATALYSTS WITH PAIRED-Up Metals}

M

etals often work in pairs in enzymes, nature's chemical catalysts, accelerating tardy reactions to make life possible. Now, researchers using the APS have investigated the bonds that form between pairs of metals including cobalt ( $\mathrm{Co}$ ), iron ( $\mathrm{Fe})$, and manganese (Mn). Their findings could help us to better understand how bimetallic enzymes work and how to copy their chemistry in industrial catalysts for making pharmaceuticals and agrochemicals more efficiently, trapping greenhouse gases such as carbon dioxide from the atmosphere, or degrading toxic chemicals. Bimetallic clusters may also have useful magnetic properties for applications in electronics and computing, such as magnetic data storage.

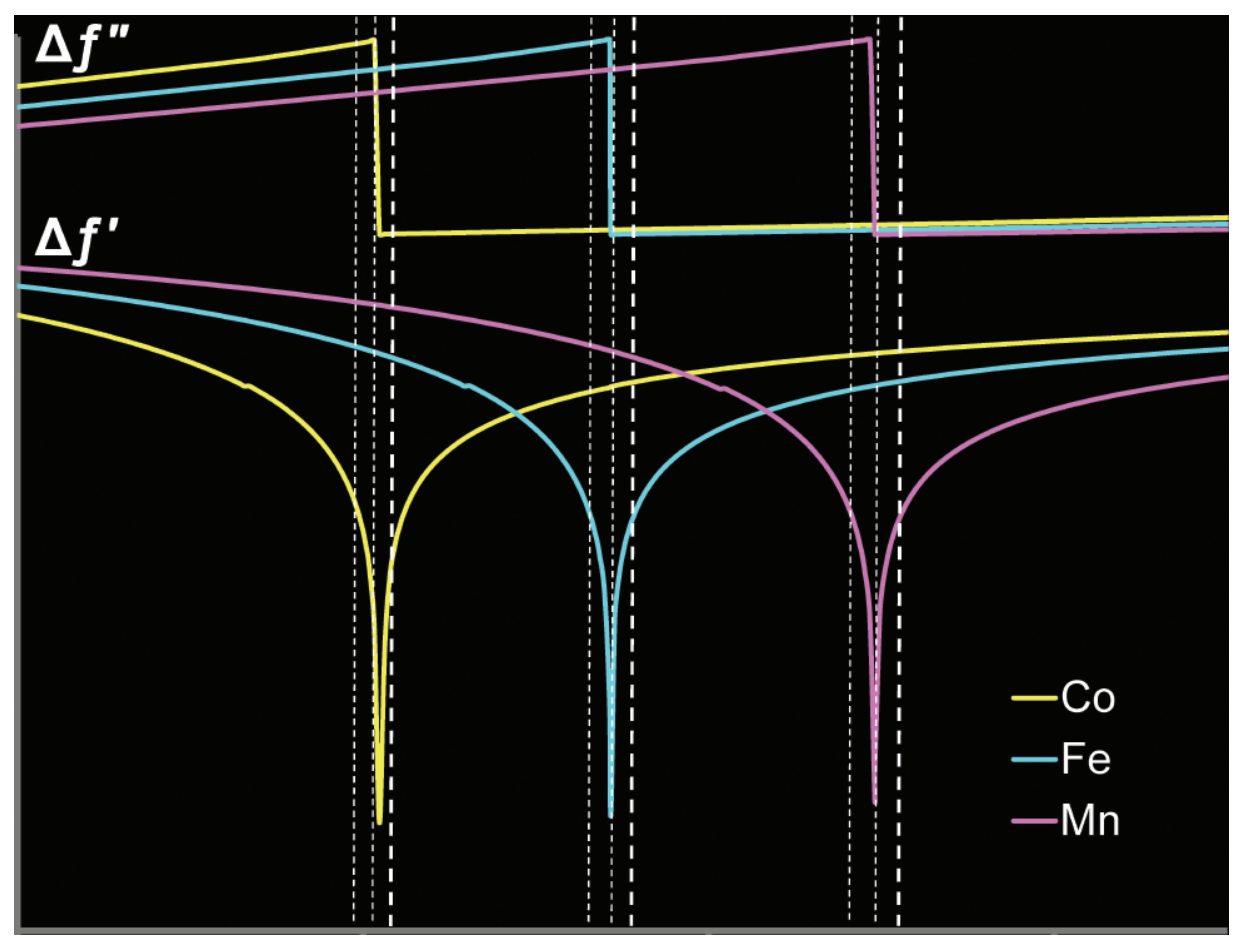

Fig. 1. Taking advantage of changes in $\mathrm{x}$-ray anomalous scattering factors near different metal K-edge energies.

The researchers from the University of Minnesota, The University of Chicago, and the Max Planck Institut für Chemische Energiekonversion (Germany) employed a raft of techniques to investigate the properties of pairs of transition metal atoms locked together using a molecule with bite.

The team points out that metal clusters are commonly used by nature as cofactors in various enzymes. For instance, molybdenum (Mo) and iron team up in a multimetallic cluster in nitrogenase enzymes, which allow some organisms to "fix" nitrogen gas from the atmosphere. Nitrogen-fixing nodules on members of the legume family carry these organisms and provide a source of nitrogen that allows the plants to grow. Some species are now known to have a VFe (vanadium-iron) unit at the core of their nitrogen-fixing enzymes. Finding ways to simulate it artificially is an important focus of future low-energy, artificial fertilizer production.

Nickel (Ni) and iron form a bimetallic pair, NiFe, in some hydrogenase enzymes found in anaerobic sulfur-reduc- ing enzymes that use hydrogen as an energy source. These enzymes are of particular interest for the possible development of clean and zero-carbon energy supplies. Other enzymes containing bimetallic clusters include the $\mathrm{Ni}-[3 \mathrm{Fe}-4 \mathrm{~S}]$ carbon monoxide dehydrogenases, and class Ic ribonucleotide reductases and many others involved in diverse aspects of the metabolism of countless organisms.

Intriguingly, most of these bimetallic pairs bring together metal atoms from different parts of the transition 


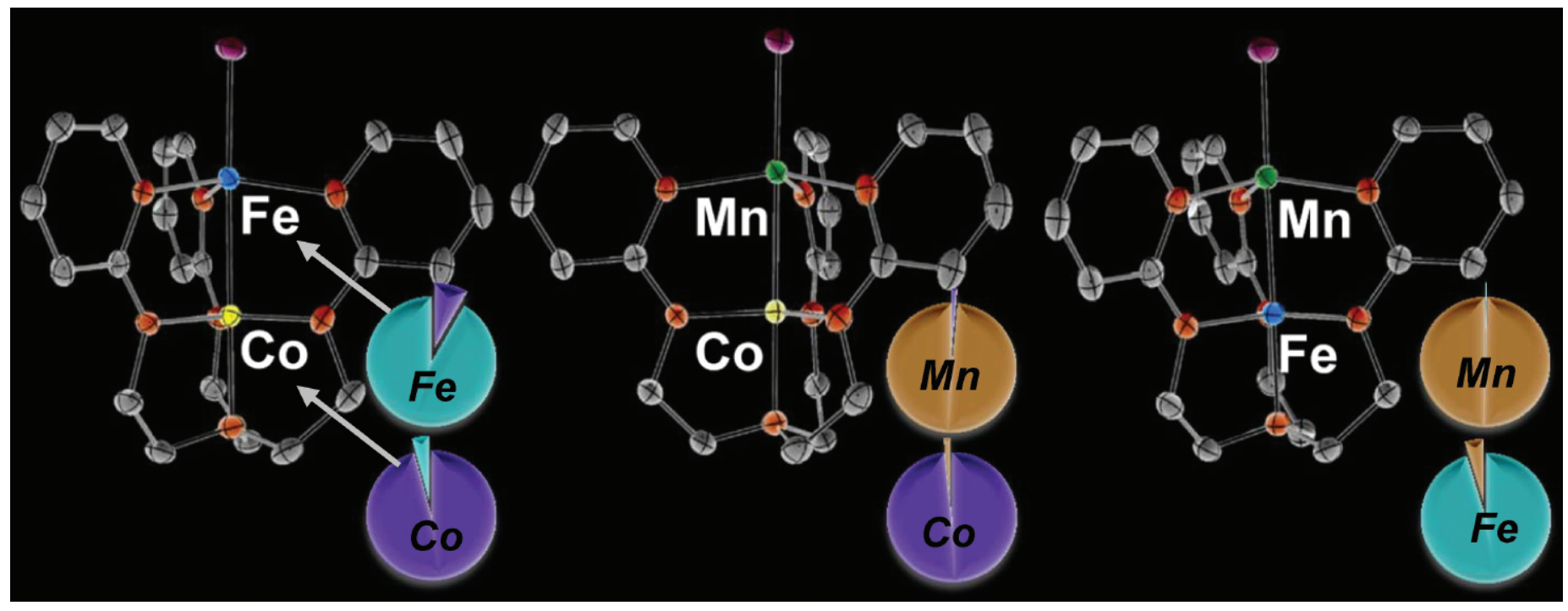

Fig. 2. X-ray structures of the heterobimetallics where the purity of the metal compositions at each site is shown using pie charts.

metal block in the periodic table. From this perspective, the class Ic ribonucleotide reductases are more interesting because they use metallic pairings of neighboring metals that are challenging to differentiate. These enzymes are involved in the synthesis of the genetic molecule DNA, deoxyribonucleic acid, on which all life depends, RNA viruses excepted. They are unique enzymes in that their metal pairing involves atoms that are close together in the periodic table, manganese and iron. These two similar metals lock together to tune the chemical properties of the enzyme precisely, something that class I, as opposed to class Ic RNRs, do with clusters of iron atoms rather than two different metals. Understanding why this might be so will provide new insights into these enzymes and perhaps point the way to new applications.

In the class I RNRs, the cluster is $\mathrm{FeFe}$, with both iron atoms in the II oxidation state abutting a tyrosine amino acid group in the enzyme structure. When an oxygen molecule touches the active center in this enzyme, a tyrosyl radical is formed and each atom in the $\mathrm{Fe}(\mathrm{II}) \mathrm{Fe}(\mathrm{II})$ is oxidized to the three state to form $\mathrm{Fe}(\mathrm{III}) \mathrm{Fe}$ (III) chemically bonded to the tyrosyl radical. Class Ic RNRs lack this tyrosine amino acid next to the metal center, so they have evolved to exploit iron's near neighbor, manganese. In this case, instead of $\mathrm{Fe}(\mathrm{II})$ $\mathrm{Fe}(\mathrm{II})$ being converted to $\mathrm{Fe}$ (III) $\mathrm{Fe}$ (III) by reaction with an oxygen molecule, an $\mathrm{Fe}(\mathrm{III}) \mathrm{Mn}(\mathrm{IV})$ species is formed.
To understand the details, the team has constructed a molecule with bite the multidentate molecule, N,N,N-tris(2(2-pyridylamino)ethyl)amine - that can grab on to the bimetallic clusters to allow them to investigate the bonds between the metal atoms without the complications of a whole enzyme protein structure being present.

The team used various techniques, including x-ray anomalous scattering studies, spectroscopy, and theoretical calculations to study the interactions between two metals. A basic problem that the team faced was identifying which metal atom is cobalt, iron, or manganese, as these metals "look" the same by standard X-ray studies. X-ray anomalous studies, conducted at the ChemMatCARS 15-ID-B,C,D beamline at the APS, resolved cobalt from iron from manganese by using diffraction data collected at the different metals' Kedge energies (Fig. 1). Three heterobimetallic complexes, CoMn, CoFe, and FeMn, were studied using this technique (Fig. 2). The team found that each complex behaves very differently from FeFe complexes perhaps because of the short iron to iron bonds.

These findings do not rationalize entirely the behavior of bimetallic metal clusters in different enzymes, but this and future work is closing in on an explanation. - David Bradley

See: Stephen J. Tereniak ${ }^{1}$, Rebecca K. Carlson ${ }^{1}$, Laura J. Clouston ${ }^{1}$, Victor G. Young, Jr. ${ }^{1}$, Eckhard Bill ${ }^{2 *}$, Rémi Mau- rice $^{1}$, Yu-Sheng Chen ${ }^{3}$, Hyun Jung $\mathrm{Kim}^{1}$, Laura Gagliardi ${ }^{1 * *}$, and Connie C. $\mathrm{Lu}^{1 * * *}$, "Role of the Metal in the Bonding and Properties of Bimetallic Complexes Involving Manganese, Iron, and Cobalt," J. Am. Chem. Soc. 136, 1842 (2014). DOI: 10.1021/ja409016w Author affiliations: ${ }^{1}$ University of Minnesota, ${ }^{2}$ Max Planck Institut für Chemische Energiekonversion, ${ }^{3}$ The University of Chicago Correspondence: * ebill@gwdg.de, ** gagliard@umn.edu, *** clu@umn.edu

The synthetic work was supported by the U.S. National Science Foundation (NSF) (CHE-1254621) and the Alfred Sloan Foundation (C.C.L.). The computational results (R.K.C., R.M., H.J.K., L.G.) are based on work supported by the NSF (CHE-1212575). ChemMatCARS Sector 15 is principally supported by the Divisions of Chemistry (CHE) and Materials Research (DMR), National Science Foundation, under grant number NSF/CHE-1346572. This research used resources of the Advanced Photon Source, a U.S. Department of Energy (DOE) Office of Science User Facility operated for the DOE Office of Science by Argonne National Laboratory under Contract No. DE-AC0206CH11357.

15-ID-B,C,D • ChemMatCARS • Materials science, chemistry - Single-crystal diffraction, anomalous and resonant scattering (hard $x$ ray), wide-angle $x$-ray scattering, microdiffraction, liquid surface diffraction, small-angle $x$-ray scattering, ultra-small-angle $x$-ray scattering, high-pressure diamond anvil cell $\bullet$ 6-32 $\mathrm{keV}, 10-70 \mathrm{keV} \cdot$ On-site $\cdot$ Accepting general users • 


\section{Single-Atom Gold Catalysts for Low-Temperature, Low-Cost Hydrogen Production}

$\mathrm{H}$

ydrogen gas $\left(\mathrm{H}_{2}\right)$ is used in a variety of commercial applications, including fertilizer production and as fuel for automotive and industrial fuel cells. Most $\mathrm{H}_{2}$ is extracted from hydrocarbons, especially via steam reforming of natural gas and the gasification of coal. These extraction processes produce a hydrogen/carbon monoxide $\left(\mathrm{H}_{2} / \mathrm{CO}\right)$ mix referred to as "synthesis gas." A long-established catalytic reaction called the "water-gas shift" removes $\mathrm{CO}$ from synthesis gas by combining $\mathrm{CO}$ with water to form additional hydrogen plus $\mathrm{CO}_{2}$. Now, a research team has unveiled a promising catalyst for low-temperature and low-cost water-gas shift processing. X-ray measurements performed at the APS were used to probe the distribution of various forms of the catalyst under realworld operating conditions. The new catalyst holds considerable potential for producing less-expensive alternative fuels, and for removing $\mathrm{CO}$ impurities from hydrogen for fuel cells. But perhaps the most far-reaching implication of this research is that current catalytic systems employing nanoparticles of precious metals may be overusing these expensive and rare materials, since catalysis is actually taking place at the scale of single atoms. This finding could lead to more efficient utilization of precious metals in the catalysts of the future. Catalysts with single-precious-metal-centers may also find use in other fuel processing reactions, and in the production of chemicals.
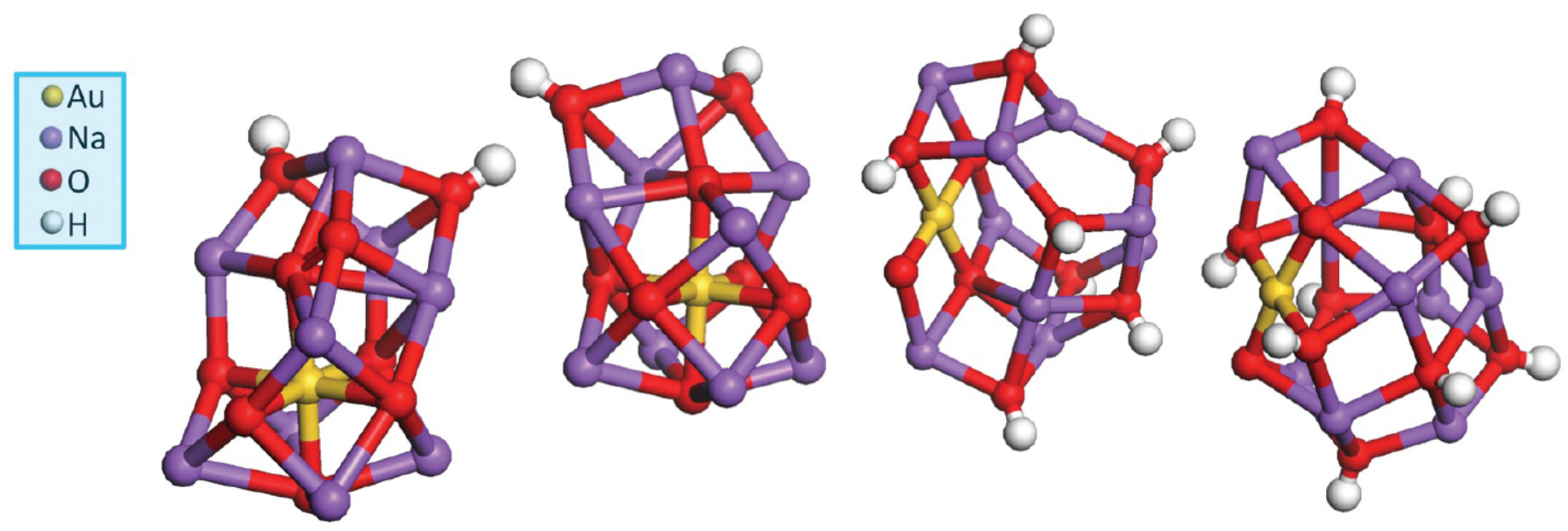

The researchers from Tufts University, the University of WisconsinMadison, Louisiana State University, Oak Ridge National Laboratory, Argonne, and the University of Sydney (Australia) found that each catalytic site is composed of a single gold atom attached to peripheral sodium or potassium ions through oxygen bonds. The gold-centric clusters can be adsorbed on a variety of supports, including zeolites and mesoporous silica, and are stable over a range of catalytic temperatures.

Although many catalysts can perform the water-gas shift reaction, each has certain limitations. Copper oxide, for instance, is widely used by industry

Fig. 1. Four possible structures of atomic-gold catalysts stabilized with alkali (in this case sodium) ions. The clusters shown are derived from computational modeling used to determine the most energetically favored, and hence the most stable, arrangements involving the atomic-gold catalyst with sodium ions. Note that each gold ( $\mathrm{Au}$ ) atom only bonds directly with oxygen $(\mathrm{O})$ atoms. Hydrogen $(\mathrm{H})$ and sodium $(\mathrm{Na})$ atoms are also shown. The $\mathrm{OH}$ groups on $\mathrm{Na}$ ions play an essential role in the water-gas shift reaction and are easy to populate by splitting water molecules. Both the gold and sodium ions are stabilized in the cluster structure.

to catalyze the water-gas shift. However, copper is pyrophoric, meaning it can spontaneously ignite in air at relatively low temperatures unless properly protected. This limits copper's usefulness with automotive fuel cells. Although there are non-pyrophoric catalysts, they typically have other drawbacks, such as requiring higher operating temperatures than gold, or using excessive amounts of expensive and/or scarce carrier materials (i.e., the materials supporting the catalyst).

The new gold-based catalyst avoids these problems. It is non-pyrophoric, and it provides a sustainable solution to CO removal: by incorporating just a single gold atom within each catalytic site, utilization of the precious metal is maximized. Moreover, the alkali metals used in the gold-centric catalyst are abundant and inexpensive, as 
are the silica-based and other non-exotic carriers. Figure 1 illustrates four computationally-derived catalytic clusters that best fit the actual structures of the catalytic sites produced in this research.

This single-gold-atom catalyst is based on a similar platinum catalyst developed previously by the same Tufts University team that led this research. The original platinum-based catalyst features a single platinum atom combined with oxygen, hydroxide, and sodium/potassium. The researchers substituted gold for platinum since gold has a lower activation energy, Ea, which yields a lower catalytic temperature $\left(<200^{\circ} \mathrm{C}\left[\sim 400^{\circ} \mathrm{F}\right]\right)$. To the surprise of the researchers, the substitution succeeded, with gold performing well in place of platinum.

To gauge the effects of adding alkali ions to the atomic-gold catalyst, different samples were prepared, with some containing potassium or sodium ions, and some not. Each sample was subsequently dispersed into a silicabased carrier. Electron microscopy and various $x$-ray techniques were then used to probe the distribution of the catalysts within the carrier. Electron micrographs (Fig. 2) indicate that the atomicgold catalysts containing alkali ions were thoroughly distributed throughout their carriers, with no clumping observed.

X-ray absorption spectroscopy (XAS) was likewise used to probe the distributions of the atomic-gold catalysts within the silica-based carriers. The $x$-ray measurements were performed at XSD x-ray beamline 12-BM$B$ of the APS. The XAS data on the atomic-gold catalysts were collected while their carrier materials were subjected to conditions mimicking an actual water-gas shift conversion, including prolonged exposure to a $\mathrm{CO} / \mathrm{H}_{2} \mathrm{O}$ mixture at catalytic temperatures (Fig 3). The XAS results confirmed the excellent distribution of the alkali-stabilized gold catalysts within the carriers, with no observed metallic aggregation, including the complete absence of nanoparticle formation. Conversely, both electron microscopy and XAS revealed that the atomic-gold catalysts lacking alkali ions did not disperse well within the supports, but instead formed nanoparticles that suppressed their catalytic activity.

The new alkali-stabilized atomic gold catalysts, dispersed within silica carriers, demonstrated stability in excess of four days (100 h) under catalytic conditions. Moreover, they performed as well as conventional gold catalysts deposited on more-costly and less-abundant metal-oxide carriers.

$$
\text { - Philip Koth }
$$

See: Ming Yang ${ }^{1}$, Sha $\mathrm{Li}^{2}$, Yuan Wang $^{1}$, Jeffrey A. Herron ${ }^{2}$, Ye $\mathrm{Xu}^{3}$, Lawrence F. Allard ${ }^{4}$, Sungsik Lee $^{5}$, Jun Huang ${ }^{6}$, Manos Mavrikakis ${ }^{2}$, and Maria FlytzaniStephanopoulos ${ }^{1 *}$, "Catalytically active $\mathrm{Au}-\mathrm{O}(\mathrm{OH})_{x}$ species stabilized by alkali ions on zeolites and mesoporous oxides," Science 346(6216), 1498 (19 December 2014).

DOI: $0.1126 /$ science. 1260526 Author affiliations: ${ }^{1}$ Tufts University, ${ }^{2}$ University of WisconsinMadison, ${ }^{3}$ Louisiana State University, ${ }^{4}$ Oak Ridge National Laboratory, ${ }^{5}$ Argonne National Laboratory, ${ }^{6}$ University of Sydney Correspondence:

*maria.flytzani-

stephanopoulos@tufts.edu

The financial support by the U.S. Department of Energy-Basic Energy Sciences (DOE-BES) under grant DE-FG02-05ER15730 is gratefully acknowledged. This research used resources of the Advanced Photon Source, a U.S. DOE Office of Science User Facility operated for the DOE Office of Science by Argonne National Laboratory under Contract No. DE-AC02-06CH11357.

12-BM-B - XSD - Materials science, polymer science, chemistry, physics, environmental science - X-ray absorption fine structure, general diffraction, x-ray reflectivity, fluorescence spectroscopy, small-angle $x$-ray scattering, wide-angle $x$-ray scattering $\cdot$ 4.5-23 keV $\bullet$ On-site $\cdot$ Accepting general users

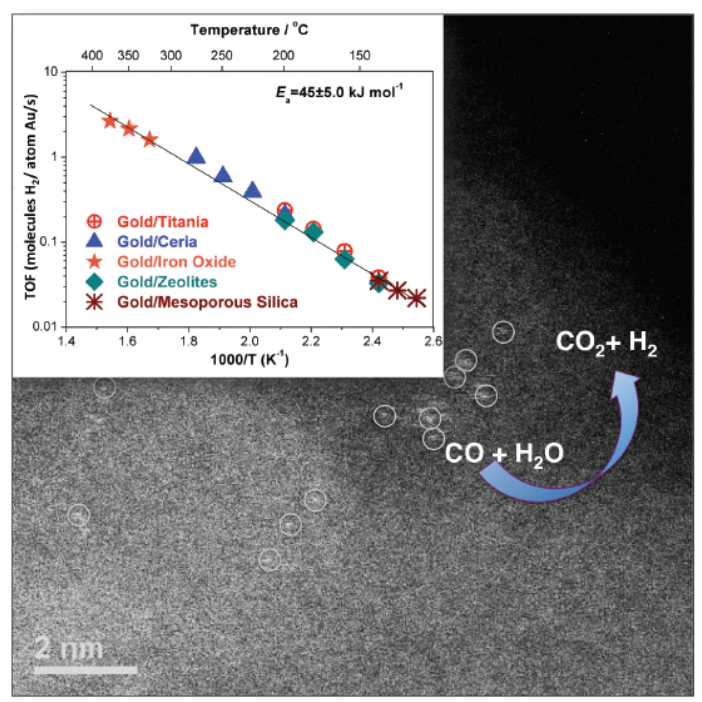

Fig. 2. Electron micrograph revealing individual atomicgold catalysts (gold atoms circled) anchored on a silicabased support. Inset: reaction rates for gold-based catalytic systems at various temperatures. For this study, the atomic-gold catalysts stabilized by alkali ions were dispersed on silica-based supports (zeolites and mesoporous silica). The plot shows low-temperature catalytic activity for the water-gas shift reaction for the new gold/zeolite and gold/mesoporous silica systems, similar to that of conventional "reducible oxide" supports of ceria, iron oxide, and titania.

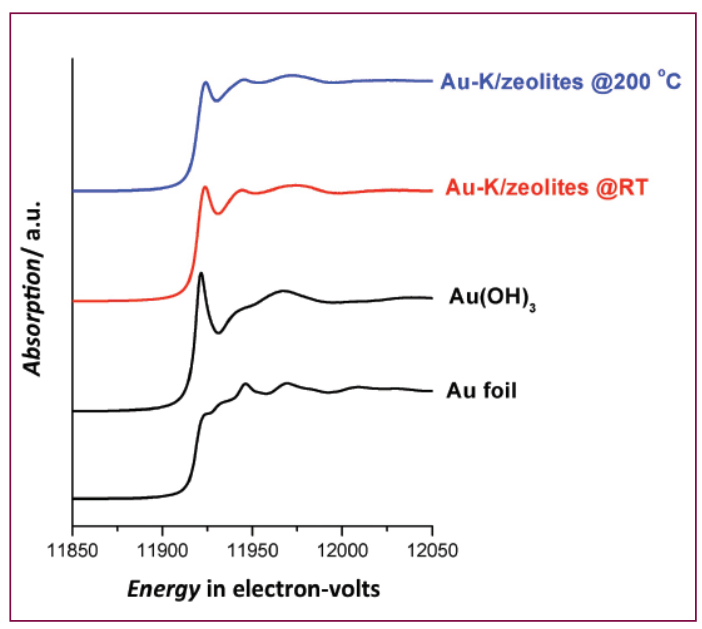

Fig. 3. X-ray absorption performed at the APS of the alkali-stabilized gold species within a zeolite carrier, at room temperature (RT) and at $200^{\circ} \mathrm{C}$ in a water-gas shift reaction atmosphere, with the references of metallic gold foil and gold hydroxide, $\mathrm{Au}(\mathrm{OH})_{3}$. The x-ray absorption plot, featuring a dramatic spike followed by a dip, indicates that the gold-oxygen bonds remained intact over a range of temperatures for the alkali-stabilized gold catalytic systems. 


\section{Just Say NO to Iron Porphyrins}

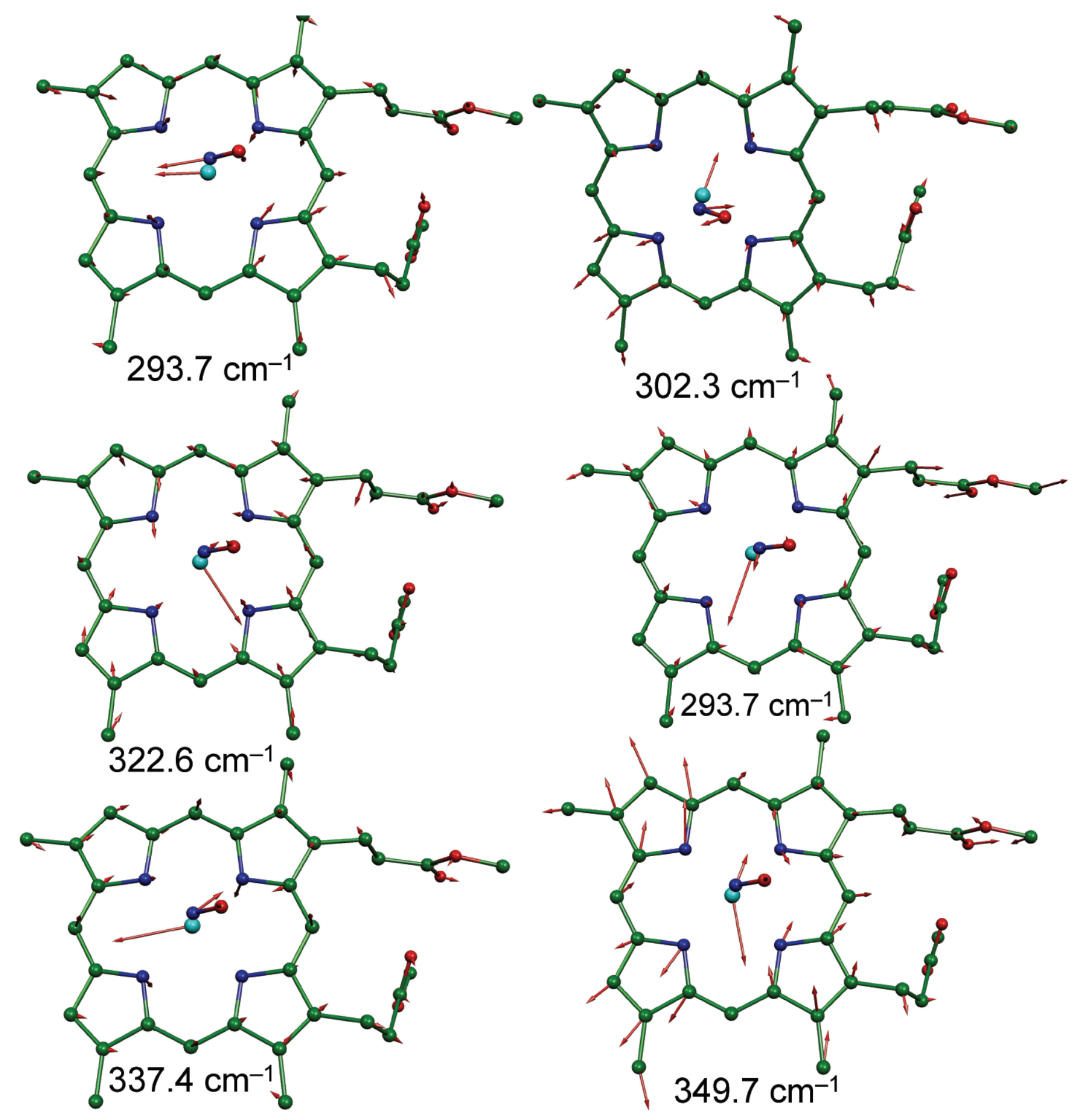

Fig. 1. This schematic diagram illustrated the effects of $\mathrm{NO}$ orientation on the in-plane iron vibrations. Note that the motion of iron is approximately parallel or perpendicular to the NO plane. Iron is colored light blue in the figures. Values are the DFT predicted frequencies. The red arrows show the eigenvectors of the dynamical matrix, indicating the direction and magnitude of motion associated with this mode. From J.W. Pavlik et al., Inorg. Chem. 53, 2582 (2014). @American Chemical Society. 
A small gas molecule, nitric oxide (NO), once thought to be nothing more than a poison turns out to be an important neurotransmitter involved in regulating our blood pressure, sexual arousal, and other biological functions. Understanding how it is made in our bodies by a group of enzymes that process the amino acid arginine could lead to new ways to controlling NO release and fixing problems such as high blood pressure and sexual dysfunction. Model compounds of the flat iron porphyrin systems found in the active site of several enzymes and the oxygen-carrying center in the blood protein hemoglobin reveal important clues about function and form. Given that the same iron-porphyrin system is so critical in the processing of the neurotransmitter NO, and the transport of the toxic gas carbon monoxide $(\mathrm{CO})$ as well as oxygen $\left(\mathrm{O}_{2}\right)$, research carried out at the APS could lead to important insights about how our bodies use and are harmed by small gas molecules.

Nitric oxide is a small molecule that exists as a gas, but in the body it is an important signaling molecule, or neurotransmitter. Its involvement in blood pressure regulation through the dilation of blood vessels makes it a vital key to many physical processes. Indeed, the role of NO is inextricably linked to heart function and the release of NO from the drug nitroglycerin is explained by this role.

The researchers in this study from the University of Notre Dame, Argonne, and Northeastern University hoped to explain how these iron porphyrin compounds in proteins can discriminate between the different small molecules $\mathrm{NO}, \mathrm{CO}$, and $\mathrm{O}_{2}$ - with which they interact, something that was not apparent previously.

They point out that studies using vibrational spectroscopy have not generally been fruitful in unraveling the properties of the active site and how different chemical bonding interactions between gas molecule and the ironporphyrin center give rise to the discriminatory behavior of the same group present in different proteins, whether enzyme or hemoglobin.

They utilized nuclear resonance vibrational spectroscopy (NRVS), a synchrotron-based measurement carried at the XSD 3-ID-B,C,D beamline at the APS, concentrating on oriented single crystals to get a clearer picture of the interactions beyond the insights that would be available through an $\mathrm{x}$-ray crystal structure determination alone.

The NRVS data they obtained allowed them to probe the low-frequency vibrations of chemical bonds between a nitric oxide molecule, or nitrosyl group, attached to one of two different ironporphyrin compounds acting as molecular models of the natural units found in the enzyme, a synthetic heme group.

By observing the spectra of the crystals of these compounds from two different directions at right angles to each other, the team teased apart the various vibration modes of the bonds connecting the iron atom to the NO group and to the flat porphyrin molecule.

Vibrational motions of the iron atom in the first, easily synthesized iron-porphyrin complex, were found to differ significantly along two directions parallel to the plane of the molecule, but rotated by $90^{\circ}$ in the plane.

A second, more sophisticated model compound offered a similar distinction. The team then used computational chemistry, in the form of density functional theory (DFT) to help them explain why this might be so (Fig. 1).

Fundamentally, it is the orientation of the bent bond between the iron atom at the porphyrin center and the attached $\mathrm{N}-\mathrm{O}$ group attached to it, the $\mathrm{Fe}-\mathrm{N}-\mathrm{O}$ bond, in other words, that give rise to the different spectra dependent on the angle at which they are measured. The orientation of the $\mathrm{Fe}-\mathrm{N}-\mathrm{O}$ unit controls the vibration of the iron atom within the plane of the porphyrin group.

The team suggests that this offaxis tilting of the $\mathrm{Fe}-\mathrm{N}-\mathrm{O}$ unit and its influence on bonding highlights how unusual the NO molecule is despite its small size and superficial simplicity.
The degree of vibration seen in different directions is related to the strength of the bond between the iron center and the NO group, as opposed to other small molecules, such as $\mathrm{CO}$ or $\mathrm{O}_{2}$, and given that the team used an iron-porphyrin group closely resembling that found in the NO enzyme, the finding provides a new clue as to the natural affinity of the enzyme for its target molecule, NO. - David Bradley

See: Jeffrey W. Pavlik ${ }^{1}$, Qian Peng ${ }^{1}$, Nathan J. Silvernail ${ }^{1}$, E. Ercan Alp², Michael Y. $\mathrm{Hu}^{2}$, Jiyong Zhao', J. Timothy Sage ${ }^{3 *}$, and W. Robert Scheidt ${ }^{1 * *}$, "Anisotropic Iron Motion in Nitrosyl Iron Porphyrinates: Natural and Synthetic Hemes," Inorg. Chem. 53, 2582 (2014). DOI: 10.1021/ic4028964

Author affiliations: ${ }^{1}$ University of Notre Dame, ${ }^{2}$ Argonne National Laboratory, ${ }^{3}$ Northeastern University

Correspondence: * jtsage@neu.edu, ** scheidt.1@nd.edu

This research was supported by the National Institutes of Health under Grant GM-38401 to W.R.S. and the National Science Foundation under CHE-1026369 to J.T.S. This research used resources of the Advanced Photon Source, a U.S. Department of Energy (DOE) Office of Science User Facility operated for the DOE Office of Science by Argonne National Laboratory under Contract No. DE-AC02-06CH11357.

3-ID-B,C,D • XSD • Physics, geoscience, life sciences, chemistry, materials science $\bullet \mathrm{Nu}$ clear resonant scattering, inelastic $x$-ray scattering, high-pressure diamond anvil cell $\cdot$ 7-27 keV, 14.41-14.42 keV • On-site • Accepting general users 


\section{STABILITY VS. ACTIVITY: Characterizing Strontium Ruthenate Crystals FOR ElECTROCHEMICAL APPLICATIONS}

$\mathrm{S}$ ummer's heat and humidity bring us to the controls for the air conditioning unit long before we receive a government alert to monitor our use of electricity. While we may lower the thermostat setting or switch off the unit once the ambient temperature drops after sunset, we may only give a passing thought to our area's power grid. It is even less likely that we'd wonder about the fuel cells powering the grid's backup system or the electrolysers that produce the hydrogen and oxygen fuel for those cells. Researchers from Argonne using the APS have characterized a new electrolyser catalyst with the potential to improve the production of hydrogen for input into fuel cells. Their study is the first to focus on methods for predicting how to synthesize well-defined oxide surfaces with the specific characteristics necessary for electrolysers, and for tailoring those characteristics to different engineering needs. Their results speak not only to the behavior of this specific material, but also offer guidelines for designing future oxide-surface electrocatalysts.

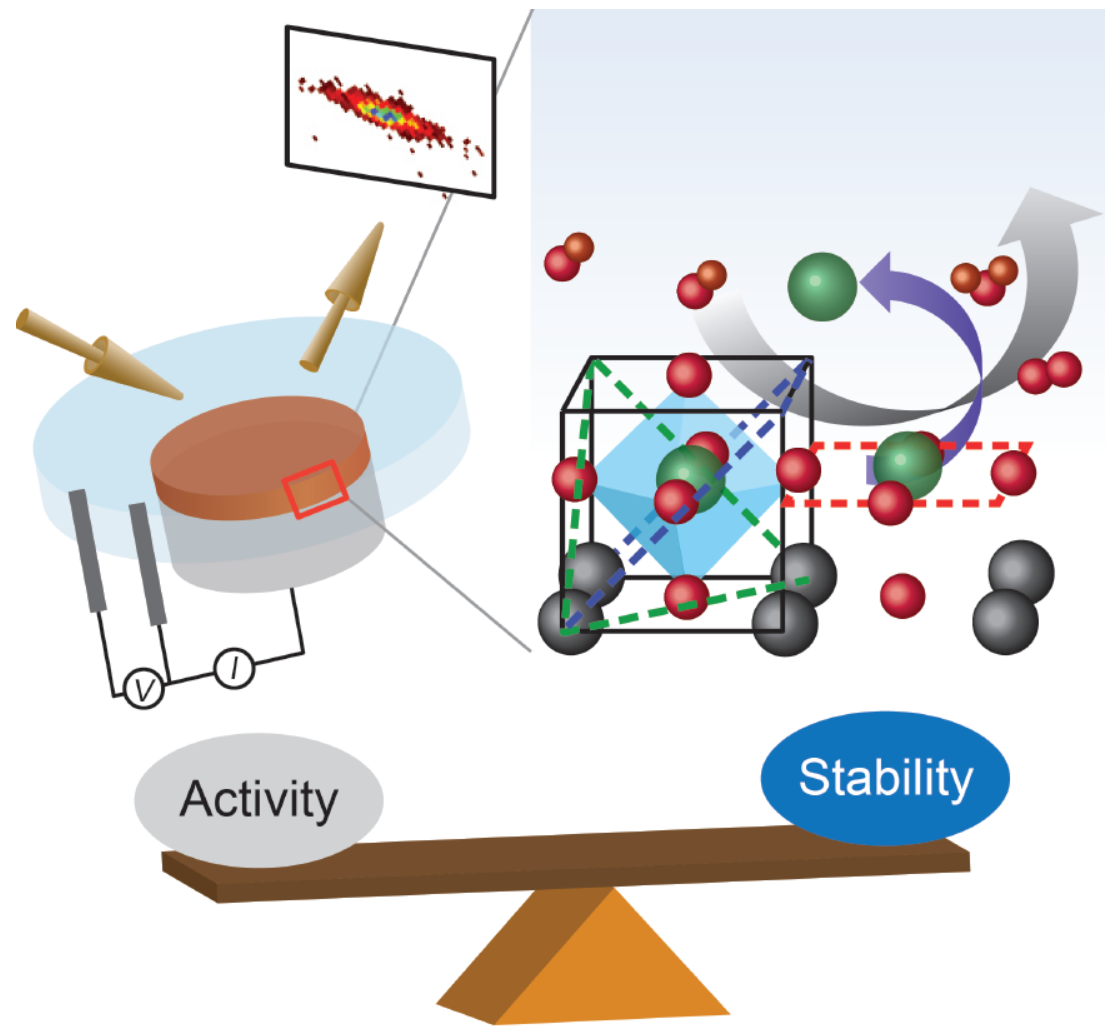

\section{Structure-function relation}

Fig. 1. Left: Schematic of in situ $x$-ray studies conducted at the APS for oxygen evolution reaction. A cylindrical sample, $\mathrm{SrRuO}_{3}$ (red) on $\mathrm{Nb}_{\mathrm{SrTiO}}$ (gray) is exposed to a thin electrolyte layer (blue, $0.1 \mathrm{M} \mathrm{KOH})$. Right: The model system $\left[\mathrm{SrRuO}_{3}(001)\right.$-red, (1 10$)$-blue, and (1 111 )green dashed line] produces oxygen and dissolves simultaneously during oxygen evolution reaction. Bottom: structure-function relation of $\mathrm{SrRuO}_{3}$ film.
Fuel cells rely on an electrolyser (a method of using a direct electric current to drive an otherwise non-spontaneous chemical reaction) to provide their hydrogen fuel. Electrolysers depend on active, stable catalyst materials to operate. In an alkaline-based electrolyser, the anode catalyst accelerates the oxygen evolution reaction, which generates molecular oxygen through a chemical reaction.

One choice for the electrolyser's anode catalyst material is a functional oxide. Since the oxide surface can degrade through a number of mechanisms, it is necessary to balance its stability and activity in order to produce cost-effective, active, stable catalysts, as shown in the figure. The results described in the team's paper provide a basis for predicting how to synthesize oxide surfaces with the desired activity and stability properties.

Utilizing the XSD x-ray beamlines 12-BM-B and 12-ID-C,D at the APS, the researchers investigated three orientations of a perovskite-type, singlecrystal film, $\mathrm{SrRuO}_{3}$.

The team began by growing films at the Argonne Materials Science Division, creating three orientations of a $\mathrm{SrRuO}_{3}$ crystal. Then they characterized and monitored changes

"Stability" cont'd on page 88 


\section{Peeling Back the Layers of Thin-Film Structure AND CheMistry}

$\mathrm{P}$

erovskites - any material with the same structure as calcium titanium oxide $\left(\mathrm{CaTiO}_{3}\right)$-continue to entice materials scientists with their ferroelectricity, ferromagnetism, catalytic activity, and oxygen-ion conductivity. In recent years, scientists realized that they could vastly improve the properties of perovskites by assembling them into thin films. The problem was that no one understood why thin films beat out bulk materials. Researchers gained new insight into thin-film superiority by probing the structure of perovskites utilizing APS high-brightness $\mathrm{x}$-rays and a groundbreaking approach to tease apart the thin-film structure and chemistry layer-by-layer.

As the researchers peeled back the layers, they found that, instead of having a uniform distribution of elements, there were drastic differences in composition between the thin-film layers. This observation may help researchers design thin-film perovskites with enhanced activity and stability.

Industrial applications for perovskites, which efficiently reduce oxygen, include the conversion of energy from fossil fuels to electricity, oxygen purification, and electrocatalysis. The research team from the Massachusetts Institute of Technology, Hebrew University (Israel), Argonne, and Oak Ridge National Laboratory studied LSCO thin films perovskites made from lanthanum, strontium, cobalt, and oxygen (LSCO) - as a model system for studying why thin films have greater reducing power than their bulk counterparts.

The researchers studied two 4-nm LSCO thin films at the XSD 33-ID-D,E beamline at the APS; one annealed thin film had been previously heated to $550^{\circ} \mathrm{C}$ for $1 \mathrm{~h}$ to simulate real-world industrial settings, while the other as-deposited thin film was left at ambient temperatures.

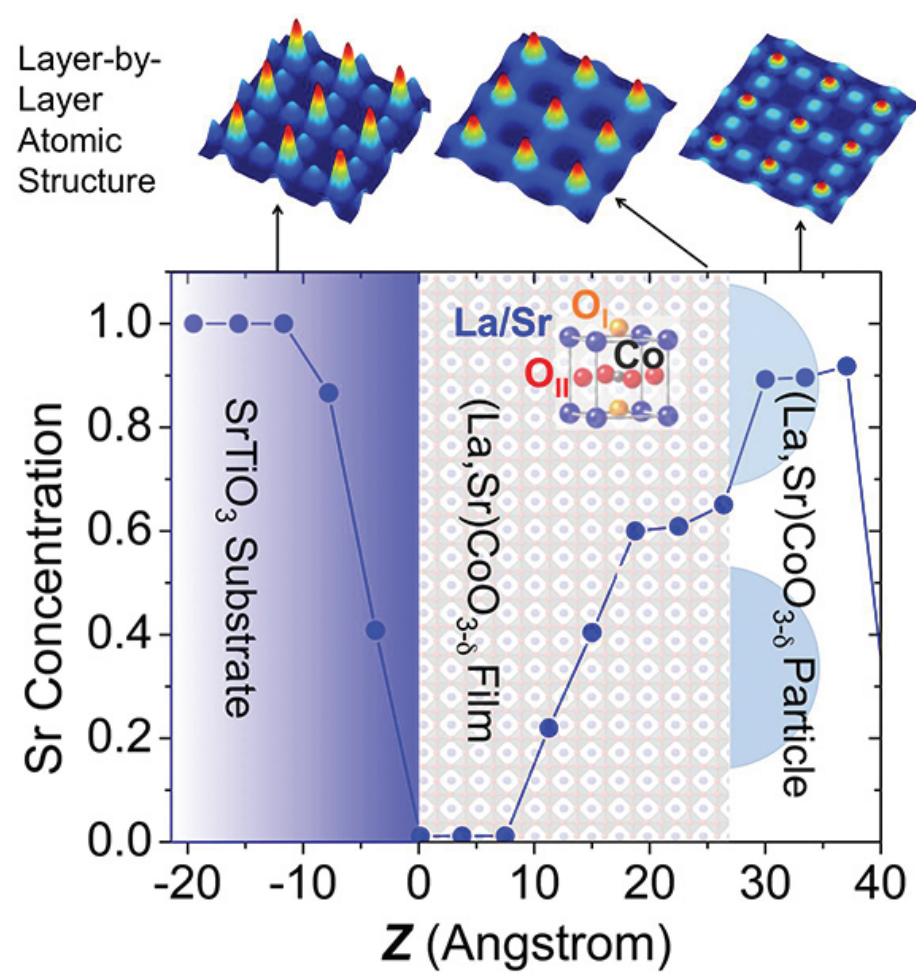

Fig. 1. The layer-by-layer analysis of the concentration of strontium within a 40-A thick (La, Sr) CoO thin film applied to a $\mathrm{SiTiO}_{3}$ substrate. Examples of 3-D electron density maps of layers within the thin film are shown (top) along with a crystal model inset.

greater number of electrons, allowing the researchers to differentiate elements at different sites within the LSCO thin films.

But COBRA alone does not give information about the distribution of elements that occupy the same atomic site within the layers. Therefore, the researchers applied a second method called "energy differential COBRA," namely, performing COBRA measurements along Bragg rods by varying the incident $\mathrm{x}$-ray energies around the strontium Kedge at each reciprocal space point. This approach provided the absolute strontium occupation fraction in a layer-by-layer fashion.

The end result of combining conventional COBRA with energy differential $\mathrm{CO}$ BRA was high-resolution

The researchers then collected diffraction intensities along 10 different reciprocal space objects (Bragg rods) defined by the substrate. They used Coherent Bragg Rod Analysis (COBRA) to determine the three-dimensional (3-D) atomic structure of each thin-film layer, with higher peaks in the map indicating an element with a (sub-angstrom) 3-D atomic images of the LSCO thin films that included information about elemental distribution (Fig. 1).

The 3-D atomic images clearly showed that strontium tended to cluster in the outer layers of the LSCO thin films, while lanthanum filled those positions in the deeper layers of the film.

"Peeling" cont'd on page 88 
"Stability" cont'd from page 86

in the films' morphology, thickness, and surface roughness using in situ x-ray scattering, finding that the films were well-defined and smooth.

Working at the Argonne Center for Nanoscale Materials, they applied high voltage $(>1.23 \mathrm{~V})$ to the films in alkaline environments; the films changed.

These changes allowed the team to determine the stability of each crystal orientation.

Unlike previous studies, this work was able to overcome the difficulties of monitoring the stability of high-surface area catalysts at the atomic thanks to the in situ x-ray scattering technique.

To understand the relationship between structure and function during the oxygen evolution reaction, the team compared results from a series of measurements: oxygen evolution reaction activity, the stability of the films, the change in the cation oxidation state, and the variations in structural properties. They concluded that the degree of stability - with the (001)-oriented film being the most stable - was inversely proportional to the activity: surfaces with more defects were more active but less stable. Put another way, the activity of the oxygen evolution reaction depends on the surface energy and the number of defects.

These results are the first published example of the structure-function relationship of single-crystal oxide surfaces in the electrochemical environment. In addition to characterizing the structure-function relationship of Sr$\mathrm{RuO}_{3}$, this research demonstrates a viable method - usable by other research teams and applicable to other functional oxides - of investigating this relationship in other materials to assess their feasibility as catalysts for the oxygen evolution reaction in electrolyzers.

Further, the researchers' results reveal the basis for a predictive ability to synthesize electrolyser oxide surfaces with characteristics tailored to electrochemical challenges. While $\mathrm{SrRuO}_{3}$ may not be supplying backup power to your town during the next heat wave, this research shows its promise for future electrolysers.

$$
\text { - Mary Alexandra Agner }
$$

See: Seo Hyoung Chang, Nemanja
Danilovic, Kee-Chul Chang, Ram Subbaraman, Arvydas P. Paulikas, Dillon D. Fong, Matthew J. Highland, Peter M. Baldo, Vojislav R. Stamenkovic, John W. Freeland, Jeffrey A. Eastman, and Nenad M. Markovic*, "Functional links between stability and reactivity of strontium ruthenate single crystals during oxygen evolution," Nat. Commun. 5, 4191 (2014).

DOI: 10.1038/ncomms5191

Author affiliation:

Argonne National Laboratory

Correspondence: *nmmarkovic@anl.gov

This work was supported by the U.S. Department of Energy (DOE)-Basic Energy Sciences, Materials Sciences and Engineering Division. This research used resources of the Advanced Photon Source and the Center for Nanoscale Materials, DOE Office of Science User Facilities operated for the DOE Office of Science by Argonne National Laboratory under Contract No. DE-AC02$06 \mathrm{CH} 11357$.

12-BM-B $\cdot X S D \cdot$ Materials science, polymer science, chemistry, physics, environmental science - X-ray absorption fine structure, general diffraction, $x$-ray reflectivity, fluorescence spectroscopy, small-angle $\mathrm{x}$-ray scattering • 4.5-23 keV • On-site - Accepting general users •

12-ID-C,D • XSD • Chemistry, physics, materials science $\cdot$ Small-angle $x$-ray scattering, grazing incidence small-angle scattering, wide-angle $x$-ray scattering, surface diffraction - 4.5-40 keV • On-site - Accepting general users •

"Peeling" cont'd from page 87

Strontium is almost entirely absent in the thin-film layers closest to the substrate.

The researchers suspect that surface strontium segregation observed in the LSCO thin films may explain why they outperform bulk materials. Lanthanum and strontium have different charges, such that if a layer has more strontium, it must also have less oxygen, or more oxygen vacancies. A dearth of oxygen in a thin-film outer layer, where strontium was found to be plentiful, means that the material may have more opportunities to react with oxygen at its surface, explaining the enhanced performance.
The structure and chemistry of the annealed and as-deposited thin films were similar, suggesting that heat itself does not alter the material's structure or activity. In future experiments, the researchers will study thin films subjected to harsher real-world conditions. They also aim to use the insights gained at the APS to design better perovskite materials in the future. - Erika Gebel Berg

See: Zhenxing Feng ${ }^{1}$, Yizhak Yacoby ${ }^{2}$, Wesley T. Hong ${ }^{1}$, Hua Zhou ${ }^{3}$, Michael D. Biegalski ${ }^{4}$, Hans M. Christen ${ }^{4}$, and Yang Shao-Horn ${ }^{1 *}$, "Revealing the atomic structure and strontium distribution in nanometer-thick $\mathrm{La}_{0.8} \mathrm{Sr}_{0.2} \mathrm{CoO}_{3-\delta}$ grown on (001)-oriented $\mathrm{SrTiO}_{3}$," Energ. Environ. Sci. 7 (3), 1166 (2014).

DOI: 10.1039/C3EE43164A

Author affiliations: ${ }^{1}$ Massachusetts Institute of Technology, ${ }^{2}$ Hebrew University, ${ }^{3}$ Argonne National Laboratory, ${ }^{4}$ Oak Ridge National Laboratory Correspondence: * shaohorn@mit.edu

This work was supported in part by the U.S. Department of Energy (DOE, SISGR DESC0002633) and King Abdullah University of Science and Technology. The authors thank the King Fahd University of Petroleum (KFUPM) and Minerals in Dharam, Saudi Arabia, for funding the research reported in this paper through the Center for Clean Water and Clean Energy at the Massachusetts Institute of Technology and KFUPM. The PLD preparation performed was conducted at the Center for Nanophase Materials Sciences, which is sponsored at Oak Ridge National Laboratory by the Scientific User Facilities Division, Office of Basic Energy Sciences, U.S. DOE. This research was supported by the Israel Science Foundation under grant no. 1005/11. This research used resources of the Advanced Photon Source, a DOE Office of Science User Facility operated for the DOE Office of Science by Argonne National Laboratory under Contract No. DE-AC02-06CH11357.

33-ID-D,E - XSD • Materials science, physics, chemistry, geoscience, environmental science - Anomalous and resonant scattering (hard xray), diffuse x-ray scattering, general diffraction, surface diffraction, surface diffraction (UHV), x-ray standing waves, $x$-ray reflectivity • 4-40 keV, 6-25 keV • On-site • Accepting general users • 


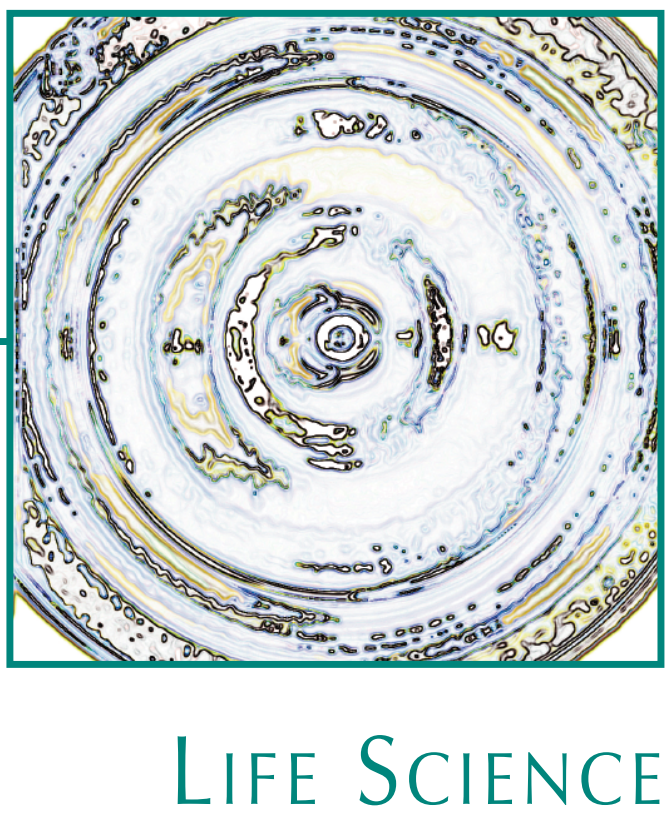




\section{A Novel Mode of Action for an OSteoporosis DruG}

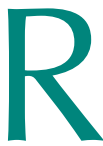

aloxifene is a U.S. Food and Drug Administration-approved treatment for decreasing fracture risk in osteoporosis. While raloxifene is as effective at reducing fracture risk as other current treatments, this works only partially by suppressing bone loss. With the use of wide- and smallangle x-ray scattering (WAXS and SAXS, respectively), researchers carried out experiments at the APS that revealed an additional mechanism underlying raloxifene action, providing an explanation for how this drug can achieve equivalent clinical benefit. These data, together with complementary techniques, help define a novel mechanism by which raloxifene increases inherent bone toughness.
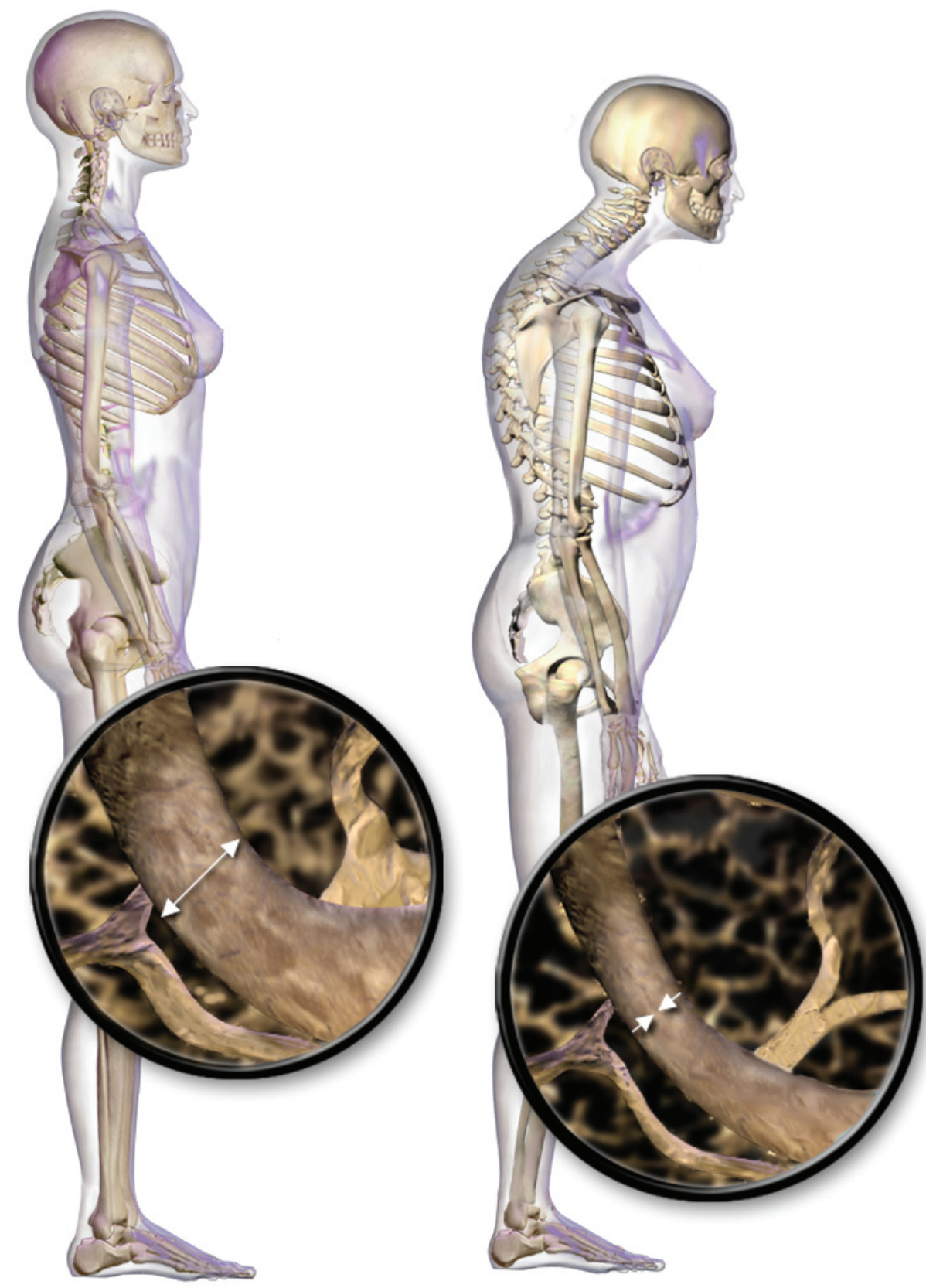

In osteoporosis, the bone mineral density is reduced, bone microarchitecture deteriorates, and the amount and variety of proteins in bone are altered. Image: "Blausen 0686 Osteoporosis 01 " by BruceBlaus - Own work. Licensed under Creative Commons Attribution 3.0 via Wikimedia Commons http://commons.wikimedia.org/wiki/File:Blausen_0686_Osteoporosis_01.png\#mediaviewer/File:Bla usen_0686_Osteoporosis_01.png 
Osteoporosis ("porous bones" from the Greek: обтоúv/ostoun, "bone;" and то́pos/poros, "pore") is a progressive bone disease that is characterized by a decrease in bone mass and density. According to the National Osteoporosis Foundation (http://nof.org/), approximately 54 million Americans have osteoporosis, or low bone mass placing them at increased risk for osteoporosis. Studies suggest that approximately 1 in 2 women and up to 1 in 4 men age 50 and older will break a bone due to osteoporosis.

All current drugs for treatment of this disease act upon living cells within the bone matrix to either decrease bone resorption, a process by which the mineral components of bone are broken down and released into the bloodstream, or to increase net bone formation during remodeling, a process by which bone is also broken down but then reforms bone. In either case, treatment results in an overall increase in bone density, and therefore a reduction in fracture risk.

While raloxifene is known to mildly suppress bone loss, it has always been somewhat paradoxical that raloxifene suppresses bone loss less than other osteoporosis therapies, yet reduces fracture risk to about the same level.

To uncover the density-independent mechanism by which raloxifene (marketed as Evista ${ }^{\circledR}$ by Eli Lilly and Company) increases bone toughness, researchers in this study from the Indiana University School of Medicine; Purdue University; Indiana University-Purdue University at Indianapolis; the University of California, San Diego; Northwestern University; and Argonne assessed the effect of the drug on devitalized bone cleared of living cells that normally mediate resorption and remodeling. In these bone samples, raloxifene prolonged the loading that the bone could bear before fracturing, indicating that the drug was acting upon the physical properties of the bone itself. Using ultra-short-echo-time nuclear magnetic resonance, researchers found that raloxifene-mediated water retention within the bone matrix is associated with the observed increase in toughness.

In order to elucidate the mechanism underlying this association, re-

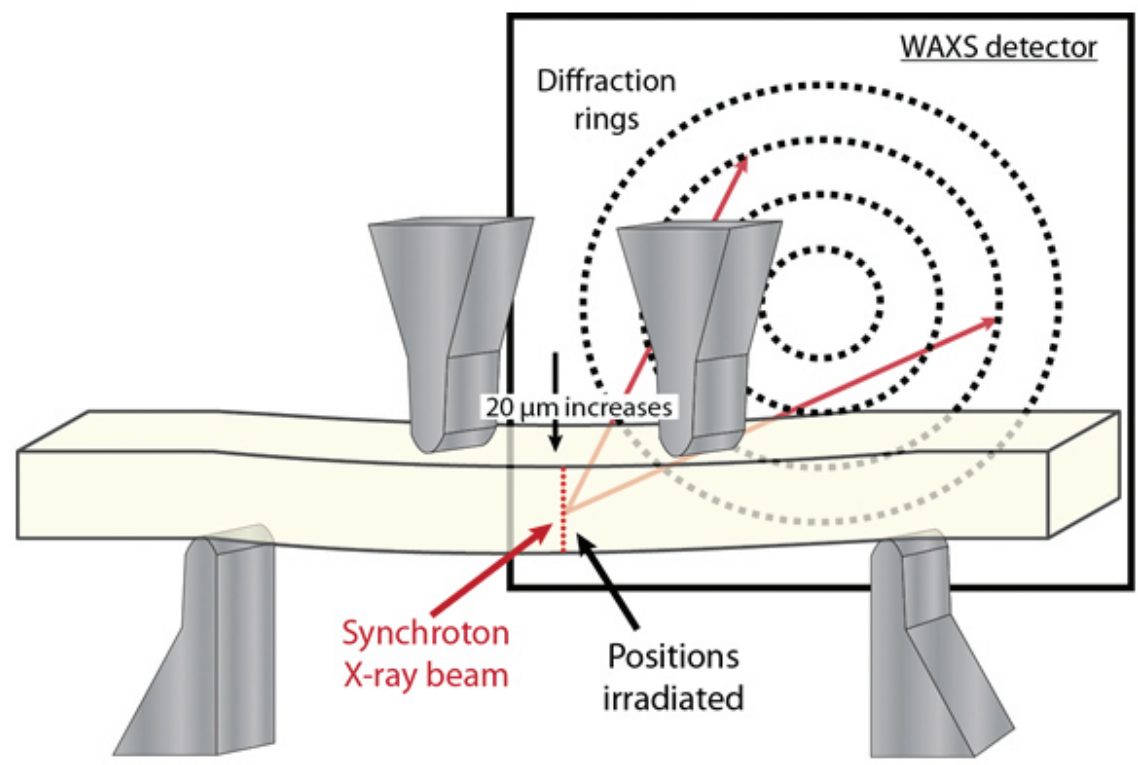

Fig. 1. Schematic of the mechanical testing apparatus utilized during collection of WAXS diffraction data at the APS. Bone beams were subjected to 4-point bending, and after each displacement of 20 $\mathrm{mm}$ (black arrow), 20 x-ray scattering measurements were taken (red dashed line). Diffraction patterns collected by the detector (indicated) allow for quantification of strain experienced by hydroxyapatite crystals and mineralized collagen within the bone. Adapted from M.A. Gallant et al., Bone $61,191(2014)$.

searchers collected WAXS and SAXS diffraction patterns of carbonated hydroxyapatite crystals (cAp), the mineral component of bone that had been subjected to four-point bending. These data, collected at the XSD 1-ID-B, C,E $x$-ray beamline at the APS (Fig. 1) allowed the researchers to measure mechanical strains on cAp crystals at a resolution of $1 \mu \mathrm{m}$ and showed that raloxifene increased the amount of physical deformation, or strain, that occurred at the collagen-mineral interface before fracture.

This increased strain between cAp and collagen reduces stresses and may be caused by water-mediated slipping between these components at their interface, increasing the amount of energy the bone can absorb prior to fracture.

This work uncovers an entirely novel mechanism of action for raloxifene and paves the way for a new class of drugs to treat osteoporosis, therapies that do not act by altering celIular activity or bone remodeling, but act by directly changing the physical properties of the bone matrix constituents. - Emma Nichols

See: Maxime A. Gallant ${ }^{1}$, Drew M. Brown ${ }^{1}$, Max Hammond ${ }^{2}$, Joseph M.
Wallace ${ }^{3}$, Jiang $\mathrm{Du}^{4}$, Alix C. DeymierBlack $^{5}$, Jonathan D. Almer ${ }^{6}$, Stuart R. Stock $^{5}$, Matthew R. Allen ${ }^{1}$, and David B. Burr ${ }^{1,3 *}$, "Bone cell-independent benefits of raloxifene on the skeleton: A novel mechanism for improving bone material properties," Bone 61, 191 (2014). DOI: 10.1016/j.bone.2014.01.009 Author affiliations: ${ }^{1}$ Indiana University School of Medicine; ${ }^{2}$ Purdue University; ${ }^{3}$ Indiana University-Purdue University at Indianapolis; 'University of California, San Diego; ${ }^{5}$ Northwestern University; ${ }^{6}$ Argonne National Laboratory Correspondence: * dburr@iupui.edu

This work was supported by National Institutes of Health grants to D.B.B. and M.R.A. Raloxifene was provided by Eli Lilly and Co. under a Material Transfer Agreement. This research used resources of the Advanced Photon Source, a U.S. Department of Energy (DOE) Office of Science User Facility operated for the DOE Office of Science by Argonne National Laboratory under Contract No. DE-AC02-06CH11357.

1-ID-B,C,E - XSD - Materials science, physics, chemistry - High-energy $x$-ray diffraction, tomography, small-angle $x$-ray scattering, fluorescence spectroscopy, pair distribution function, phase contrast imaging • 50-90 keV, 50-150 keV • On-site • Accepting general users • 


\section{Protein-Lipid InTERACtions Dictate Cellular Fates}

$\mathrm{T}$

he immune system is the judge, jury, and executioner of the body, deciding whether a cell lives or dies. When a cell approaches its natural end (apoptosis), the immune system targets that cell for removal. Defects in this process can lead to disease, including cancer. $T$ cell immunoglobulin and mucin-domain-containing molecule 4 (Tim4), a protein displayed on the surface of immune cells, binds to specific lipids in cellular membranes. That interaction helps the immune system differentiate between apoptotic and healthy cells, but the details have remained fuzzy. To better understand how the immune system picks out apoptotic cells, researchers from The University of Chicago and the University of Illinois at Chicago collaborated to perform $\mathrm{x}$-ray reflectivity measurements on interactions between Tim4 and lipids at the APS, as well as additional experiments. Their structural insights may spur the development of novel anticancer medications or drugs for immune system disorders. 
Previous studies identified a binding site in Tim4 for phosphatidylserine (PS) lipids, a component of the cellular membrane. However, that fact alone cannot explain how Tim4 identifies apoptotic cells since even healthy cells can be decorated with PS lipids.

The researchers suspected the selectivity may come from Tim4's ability to detect the concentration of PS on the cell surface; apoptotic cells cannot regulate their cellular lipids and end up with an uptick in PS on their surfaces.

To test this hypothesis, the research team needed an innovative strategy, since crystallographic structural studies of protein-lipid interactions are notoriously challenging due to the dynamic nature of lipids. They settled on a three-pronged approach that included interfacial x-ray scattering, molecular dynamics simulations, and membrane binding assays.

The crystal structure of Tim4 is known, but scientists lacked data on how Tim4 interacts with PS in a membrane. To determine how Tim4 orients itself relative to a lipid membrane, the researchers performed $\mathrm{x}$-ray reflectivity experiments at the ChemMatCARS 15ID-B, C,D beamline at the APS.

They constructed an imitation membrane for experimental purposes by spreading a lipid film containing PS at the surface of a buffer solution. The lipids formed a monolayer at the buffer surface, the mock membrane, with hydrophobic tails pointing into the air and hydrophilic head groups pointing into the buffer. They added Tim4 into the buffer and collected reflectivity measurements as the protein bound to the lipids. The reflectivity data provide information on molecular thickness electron density - perpendicular to the air-buffer interface. Using molecular modeling, the researchers fit Tim4 crystal structures into the reflectivitybased electron density to determine

< Fig. 1. Screen shot of a molecular dynamics simulation showing Tim4 (yellow) binding a lipid bilayer.
Tim4's orientation relative to the lipid monolayer.

The next step was to use an allatom molecular dynamics simulation to check whether the Tim4 orientation with the monolayer is compatible in the context of a physiological lipid bilayer. Using the protein orientation obtained from x-ray reflectivity as the initial configuration, the protein is found to remain stably bound to the membrane throughout the simulation (Fig. 1), confirming the orientation determined with the $\mathrm{x}$ ray reflectivity experiments.

Confident that they'd hit on the correct orientation, the researchers analyzed their structural models, finding that in addition to the known strong binding site, Tim4 appears to bind PS weakly at four additional sites via ionic interactions. To confirm that these sites contribute to binding between Tim4 and $\mathrm{PS}$, the researchers mutated basic residues in the four potential binding sites to alanine. Three of these mutations led to significantly weaker binding between Tim4 and PS, while the fourth mutation led to stronger binding, supporting the existence of other factors contributing to the strength and stability of binding.

As a final test, the researchers measured binding affinity of Tim4 with liposomes containing different concentrations of PS. They repeated the experiments with two other Tim proteins, Tim1 and Tim3, which are structurally similar but functionally distinct. The researchers observed that Tim4 was much more sensitive to the density of PS than was Tim1 or Tim3. The binding curve they observed suggests that Tim4 binds only moderately with cells having an intermediate PS surface density, while binding tightly to those cells with high PS surface densities. This offers a potential mechanism for how Tim4 identifies apoptotic cells; the PS concentration tunes Tim4's binding affinity, and thus whether Tim4 alerts the immune system to an apoptotic cell. - Erika Gebel Berg
See: Gregory T. Tietjen ${ }^{1}$, Zhiliang Gong $^{1}$, Chiu-Hao Chen ${ }^{2}$, Ernesto Vargas $^{1}$, James E. Crooks ${ }^{1}$, Kathleen D. Caob $^{1}$, Charles T.R. Heffern ${ }^{1}$, J. Michael Henderson ${ }^{1}$, Mati Meron ${ }^{1}$, Binhua Linc ${ }^{1}$, Benoît Roux ${ }^{1}$, Mark L. Schlossman ${ }^{2}$, Theodore L. Steck ${ }^{1}, \mathrm{Ka}$ Yee C. Lee ${ }^{1 * *}$, and Erin J. Adamsa ${ }^{1 *}$, "Molecular mechanism for differential recognition of membrane phosphatidylserine by the immune regulatory receptor Tim4," Proc. Natl. Acad. Sci. USA 111 (15), E1463 (2014). DOI: 10.1073/pnas.1320174111 Author affiliations: ${ }^{1}$ The University of Chicago, ${ }^{2}$ University of Illinois at Chicago

Correspondence:

* ejadams@uchicago.edu, **kayeelee@uchicago.edu

This research was supported by National Institutes of Health Grant R01Al073922 (to E.J.A.) and also through resources provided by the Computation Institute and the Biological Sciences Division of The University of Chicago and Argonne National Laboratory under Grant S10 RR029030-01. Funding support was also provided by the National Science Foundation (NSF) through Grants MCB-0920316 (to K.Y.C.L), CHE-0910825 (to M.L.S), and MCB-0920261 (to B.R.). K.Y.C.L. acknowledges support from The University of Chicago Materials Research Science and Engineering Center (DMR 0820054). ChemMatCARS is principally supported by the Divisions of Chemistry (CHE) and Materials Research (DMR), NSF, under grant number NSF/CHE-1346572. This research used resources of the Advanced Photon Source, a U.S. Department of Energy (DOE) Office of Science User Facility operated for the DOE Office of Science by Argonne National Laboratory under contract no. DE-AC02-06CH11357.

15-ID-B,C,D • ChemMatCARS • Materials science, chemistry - Single-crystal diffraction, anomalous and resonant scattering (hard $x$ ray), wide-angle $x$-ray scattering, microdiffraction, liquid surface diffraction, small-angle $x$-ray scattering, ultra-small-angle $x$-ray scattering, high-pressure diamond anvil cell $\bullet$ 6-32 $\mathrm{keV}, 10-70 \mathrm{keV} \cdot$ On-site $\cdot$ Accepting general users • 


\section{COMBATING AN INFECTIOUS INVADER}

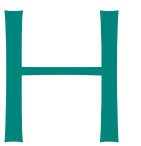

ow does one kill an infectious invader that is not technically alive? That question has vexed researchers studying prions-aggregates of aberrantly folded protein that propagate by inducing properly folded proteins to convert into misfolded, disease-associated forms-since these infectious agents were identified as the source of a range of devastating neurological diseases such as transmissible spongiform encephalopathies (TSEs), which include Creutzfeldt-Jakob disease and "mad cow disease." Prions contain no genetic material, so their structure determines their biological activity. That structure, made rigid and insoluble through aberrant protein folding, also makes prions difficult to combat. Researchers used two U.S. Department of Energy synchrotron light sources including the APS to pry out new structural information about prions, yielding insights into the fundamental mechanisms of prion at a level of complexity that has not been observed in short-peptide amyloids used as prion model systems.
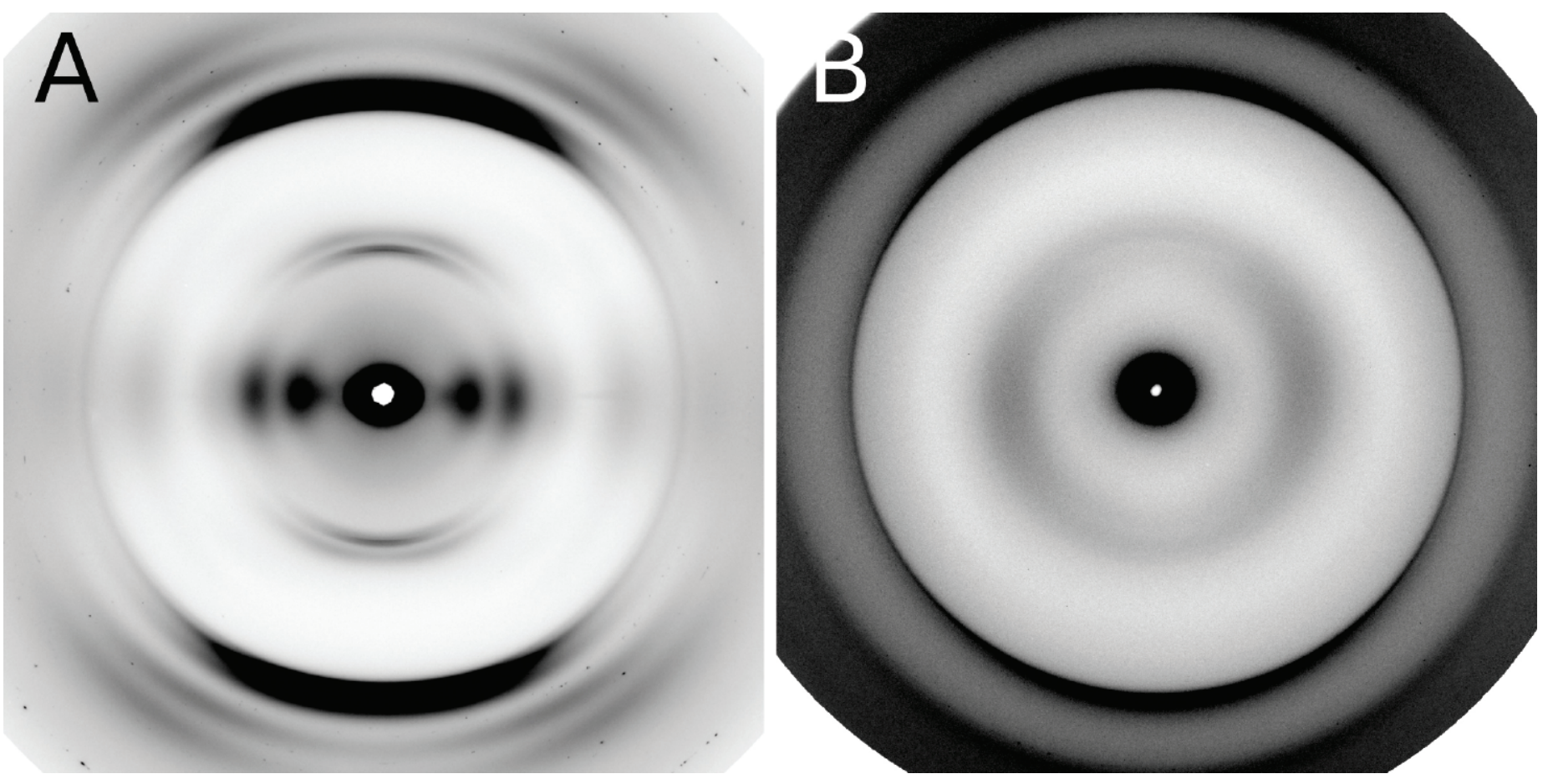

Fig. 1. X-ray fiber diffraction patterns of fungal prion HET-s(218-289). The pattern on the left represents the wild-type with two-rung $\beta$-solenoid diffraction while the pattern on the right represents a mutant version of HET-s with stacked $\beta$-sheets that are not in the $\beta$-solenoid structure. 
Unlike viruses, bacteria, fungi, and parasites, prions do not spread disease through the transfer of genetic material. They propagate through the insidious conversion of properly folded proteins into deviant forms, which consist of aggregates of amyloids - long, unbranched fibrils assembled into cross- $\beta$ architecture consisting of one or more tiers of $\beta$-strands running perpendicular to the fiber axis, forming $\beta$ sheets that run the length of the fibril. Notable examples of prion-caused diseases are the aforementioned TSEs, which involve amyloids of the prion protein PrP. Non-PrP amyloids have been implicated in diseases such as Parkinson's and Alzheimer's as well as type II diabetes.

Researchers from Vanderbilt University used site-directed mutagenesis together with $\mathrm{x}$-ray fiber diffraction studies to determine the relative importance of various structural features that facilitate deviant folding in a prion model system, the fungal prion HET-s. Their analysis suggests a redundancy of features that enable robust folding and stability even in the face of significant changes to prions' sequence of constituent amino acids.

Prions' insolubility and rigid structure make them hard to denature with chemical agents. A possible method to thwart prions is to make them unstable or prevent them from misfolding. In order to develop compounds that exploit these possible mechanisms, it is essential to determine the structural features that give prions the ability to propagate their structures.

To figure out how important each structural element of prions is to the ability to consistently misfold, researchers have looked to a prion model system, the fungal prion HET-s(218289). Fungal prions are not only incapable of causing diseases, they are in many cases thought to be adaptive. HET-s works well as a model system owing to its highly ordered structure that readily folds into a complex amyloid with two tiers of $\beta$-strands.

The researchers used site-directed mutagenesis of amino acid residues to alter the flexible loop sequences, buried polar interactions, salt bridges, and asparagine ladders found in HET$\mathrm{s}(218-289)$ in order to probe the rela-
Diseases caused by prions are known as spongiform diseases, because the brain tissue in infected individuals is filled with holes, giving it a sponge-like appearance. Although prions are found throughout the brain, the symptoms of spongiform diseases vary according to the regions they are most concentrated in. There are currently no effective treatments for spongiform diseases and all are fatal. Source (text and graphic): "Prions: On the Trail of Killer Proteins," Genetic Science Learning Center at the University of Utah, http://learn.genetics.utah.edu/content/molecules/prions/ (C) 2014 University of Utah.

tive importance of these features for the formation of $\beta$-solenoid structure. They also investigated the cumulative effects of multiple mutations.

To determine whether the modified structures contained cross- $\beta$ fibers, the researchers studied the samples with $x$ ray diffraction (Fig. 1) at the Bio-CAT beamline 18-ID-D at the APS and at beamline 4-2 at the Stanford Synchrotron Radiation Lightsource at the SLAC National Accelerator Laboratory.

As a final step, mutants that formed $\beta$-solenoids were subjected to assays examining the protein denaturation and the kinetics of fibril formation to assess the effects of mutations on the biophysical properties of the $\beta$-solenoid structure. The $\beta$-solenoid architecture of HET-s(218-289) was found to be surprisingly robust because of the redundancy of important biophysical features. Several mutations significantly impacted fibril nucleation, the rate of fibril formation, and fibril stability, but x-ray fiber diffraction revealed that the $\beta$-solenoid structure formed in most cases.

To effectively wipe out the $\beta$-solenoid structure required completely rearranging or reversing the long flexible loop connecting the two rungs of the $\beta$ solenoid or removing both asparagine ladders and all three salt bridges.

In addition, comparing the sequence alignment of HET-s(218-289) to one of its homologs, FgHET-s(218-289) suggests that of these three features, the long flexible loop and the asparagine ladders were most necessary for $\beta$-solenoid formation.

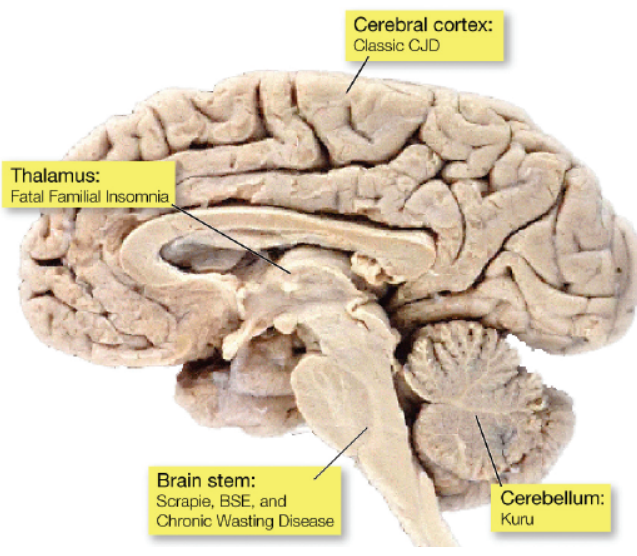

The investigation of the fungal prion HET-s(218-289) provides insights into the fundamental mechanisms of prion assembly and propagation of its infectious fold, which is made robust by a complex and diverse array of interand intramolecular structural features. This level of complexity has not been observed in short-peptide amyloids that have been used as prion model systems. - Chris Palmer

See: William Wan and Gerald Stubbs*, "Fungal prion HET-s as a model for structural complexity and self-propagation in prions," Proc. Natl. Acad. Sci. USA 111 (14), 5201 (2014).

DOI: 10.1073/pnas.1322933111

Author affiliation: Vanderbilt University Correspondence:

* gerald.stubbs@vanderbilt.edu

This work was supported by National Institutes of Health (NIH) Grants AG002132 (to Principal Investigator G.S.; Program Director Stanley Prusiner) and F31-AG040947 (to W.W.). Bio-CAT is supported by grant 9 P41 GM103622 from the National Institute of General Medical Sciences of the NIH. This research used resources of the Advanced Photon Source, a U.S. Department of Energy (DOE) Office of Science User Facility operated for the DOE Office of Science by Argonne National Laboratory under Contract No. DE-AC02-06CH11357.

18-ID-D • Bio-CAT • Life sciences $•$ Fiber diffraction, microdiffraction, small-angle $x$-ray scattering, time-resolved $\mathrm{x}$-ray scattering 3.5-35 keV • On-site - Accepting general users • 


\section{UnRAVELING ProteIn Folding}

- or many proteins, the ability to change shape is essential for their proper functioning within cells. One longstanding question concerns the process proteins follow when shifting from an unfolded to a three-dimensional globular form. Most previous studies have supported the idea that when an unfolded protein is exposed to native conditions (i.e., to an environment favoring its typically fully-folded, physiological form) a continuous unfolded-to-semi-folded collapse ensues. However, other studies suggest that there is an energetic bottleneck to this step that renders it an all-or-none transition. To resolve the issue, researchers from the University of Massachusetts Medical School and the Illinois Institute of Technology probed the folding stages of cytochrome c, an archetype for protein folding behavior. Its microsecond-scale folding dynamics were unambiguously characterized with Förster resonance energy transfer (FRET) complimented by smallangle x-ray scattering (SAXS) carried out at the APS. The SAXS and FRET measurements refuted the conventional view that cytochrome $c$ folding proceeds immediately and smoothly when exposed to native conditions; instead, subpopulations of unfolded and semi-folded protein were observed to coexist during brief intervals. These results provide fundamental insights for biochemistry, as well as for human diseases characterized by protein misfolding, including Parkinson's, Huntington's, Alzheimer's, and lysosomal storage diseases.

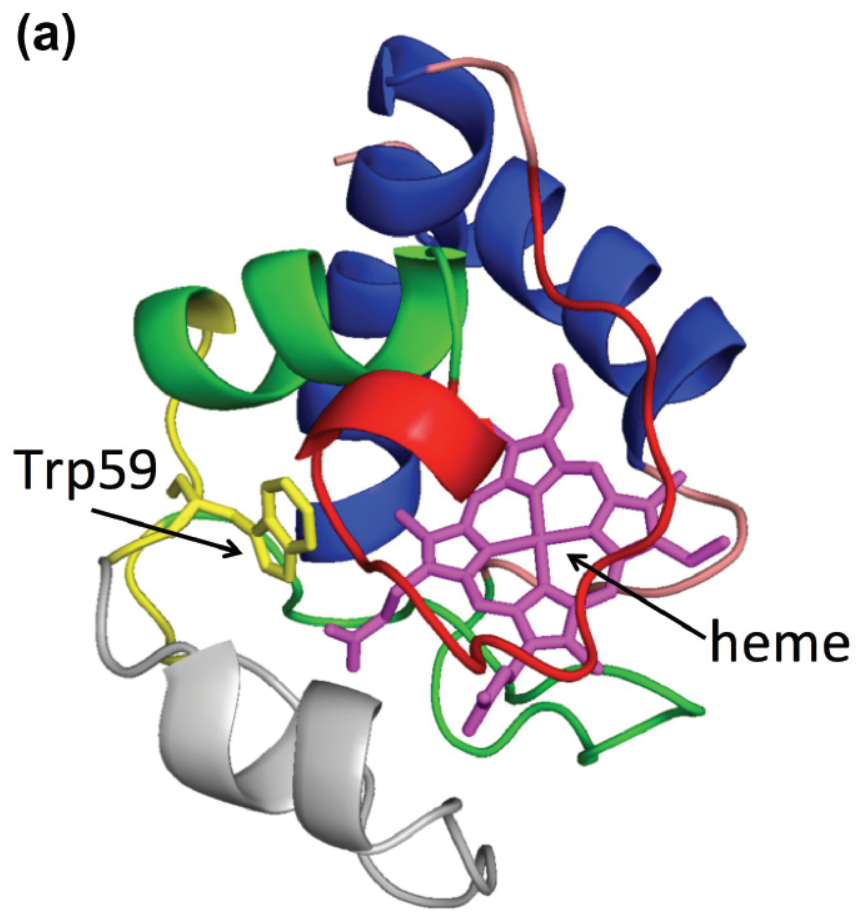

Fig. 1. (a) An illustration of the fully-folded globular protein cytochrome c. Different segments of the protein are color-coded, including key segments containing Trp59 (yellow) and heme (fuchsia). (b) An unfolded state of cytochrome c showing its worm-like chain structure. Note: The fully-folded and unfolded states are scaled to different lengths, i.e., the unfolded, chain-like state in panel (b) is much longer than the diameter of the protein's globular form in (a).

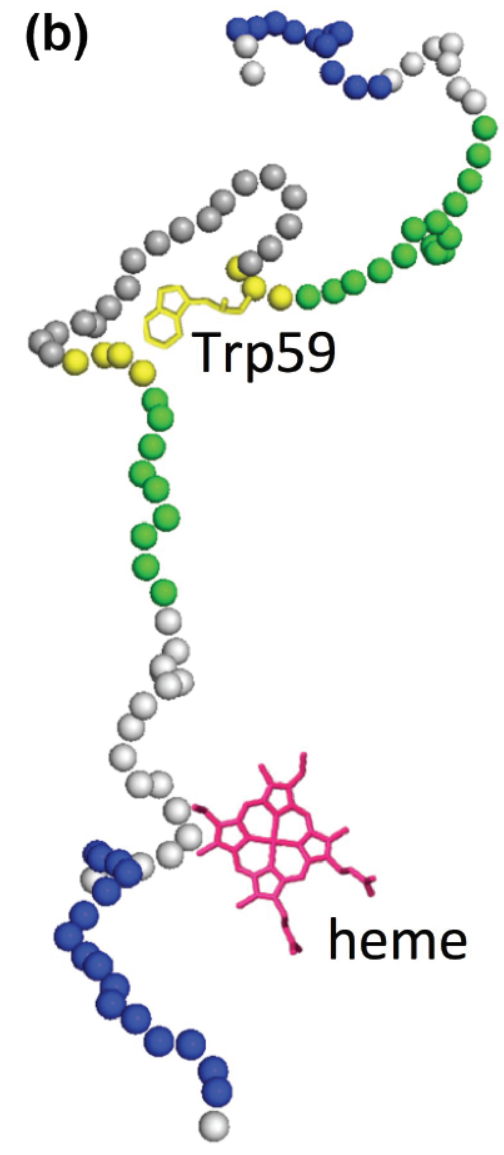




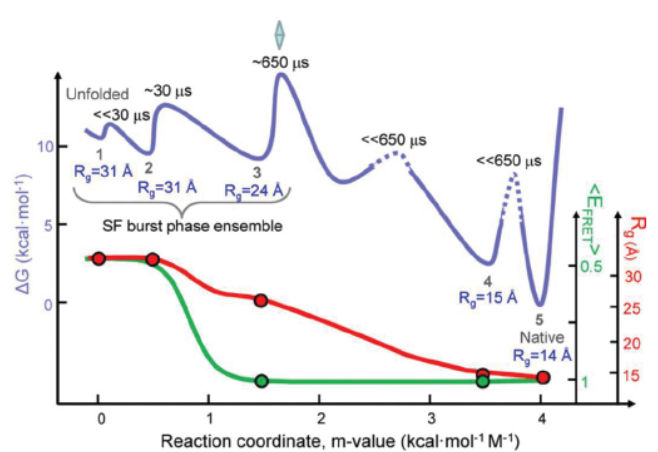

Fig. 2. Kinetic model for cytochrome c folding immediately following denaturant dilution (i.e., to an environment favoring compaction). The semi-quantitative reaction coordinate shows the approximate free energy $(\Delta G)$ and barrier heights for various states of protein folding. The left portion of the blue plot indicates the state that dominates at the barrier to the next folding step: well within the first 30 microseconds $(<<30 \mu \mathrm{s})$, unfolded state 1 dominates. At $\sim 30 \mu \mathrm{s}$, the most numerous protein structure is partially-folded state 2 . And $\sim 100-\mu$ s later, partially-folded state 3 dominates. (Previous state-of-the-art measurements, with $\sim 1$-msec resolution, lumped states 1, 2, and 3 together as indicated by "SF [stopped-flow] burst phase ensemble.") SAXS measurements give the radius of gyration, $R_{g}$, for each state. While state 3 was known previously, these experiments filled in knowledge of early folding dynamics, i.e., $<100 \mu$ s. The right portion of the blue plot shows that folding to the final, globular structure occurs very quickly $(<<650 \mu \mathrm{s})$ once the barrier after state 3 is overcome. Red and green plots at bottom show two reaction coordinates, $\mathrm{R}_{\mathrm{g}}$ and "average FRET efficiency," illustrating the global and pairwise distance changes, respectively.
In the laboratory, protein structure can be altered by varying the temperature, chemical environment, etc. For instance, the shapes of proteins suspended in solution can be changed by varying the concentration of a chemical denaturant. In this study, guanidinium hydrochloride $(\mathrm{GdnHCl})$ was used as a denaturant to uncoil cytochrome $c$, a small, globular, iron-containing protein (hemeprotein). Figure 1a shows its globule form, Fig. 1b an unfolded state.

Cytochrome $c$ molecules were initially suspended in a highly-concentrated solution of $\mathrm{GdnHCl}$, which relaxed some of the protein globules into a chain-like (unfolded) form. To start the folding process, the protein chains were suddenly exposed to a near-native state (i.e., to an environment with little denaturant) using microfluidics techniques that allowed extreme dilution of the $\mathrm{GdnHCl}$-concentrated solution in just 25-30 $\mu$ s.

Previous experiments capable of resolving early ( $<100 \mu \mathrm{s})$ folding dynamics were unable to differentiate between partially-folded and unfolded forms mixed together. By contrast, the FRET and SAXS techniques were capable of detecting the presence of both folded and unfolded subpopulations immediately following dilution of the $\mathrm{GdnHCl}$ denaturant.

FRET employed rapid laser pulses to excite the Trp59 chromophore (a molecular segment that gives the protein its color). Due to their close proximity (several nanometers) and similar electronic energy levels (the "resonance" in FRET), energy could pass from the Trp59 to the heme molecule (called the "donor" and "acceptor," respectively). This is a quantum mechanical, non-radiative process (i.e., no actual photon is transferred). Energy accepted by the heme molecule in this process was dis- sipated. However, sometimes the excited Trp59 molecule re-emitted the energy as a photon, which could then be detected by a sensor. The time between a laser pulse and Trp59 photon emission was measured. Because this interval varied according to the Trp59to-heme distance (which indicates protein shape) the proportions of any unfolded and partially-folded states could be calculated.

The FRET results revealed that following denaturant dilution, certain subpopulations dominated during particular time intervals: within the first $30 \mu$ s after dilution, the unfolded state (denoted as "state 1" for convenience) dominated, accompanied by a small proportion of partially-folded states. After $30 \mu \mathrm{s}$, a transition to a partially-folded "state 2" occurred, followed still later by dominance of partially-folded "state 3 ." These discrete jumps in subpopulation ratios, which occurred as the initiallyunfolded protein transitioned to the globular form, are referred to as "barrier-limited chain collapse."

The SAXS experiments, which were carried out at the Bio-CAT 18-ID$D$ beamline at the APS were performed as an independent check on the FRET results. SAXS measured a parameter called the radius of gyration $\left(R_{g}\right)$. This parameter quantified the spatial distribution of all of the molecular components of cytochrome $c$, thereby indicating the degree of folding. Figure 2 depicts folding dynamics as measured by a combination of the SAXS and FRET methods. The top curve in the figure gives a sense of the barrier limits seen at various stages of the folding process. Each phase in the dynamic folding process exhibits a particular $R_{g}$, which in turn indicates a particular structural state (a smaller $\mathrm{R}_{\mathrm{g}}$ implies greater protein compaction).
The SAXS and FRET results highlight the importance of employing techniques that can detect subpopulations in folding reactions. Moreover, firmly establishing the existence of barrier-limited protein folding will propel the search for the physical source of this behavior. — Philip Koth

See: Sagar V. Kathuria ${ }^{1}$, Can Kayatekin1, Raul Barrea², Elena Kondrashkina2, Rita Graceffa ${ }^{2}$, Liang Guo2, R. Paul Nobrega ${ }^{1}$, Srinivas Chakravarthy $^{2}$, C. Robert Matthews ${ }^{1}$, Thomas C. Irving ${ }^{2}$, and Osman Bilsel ${ }^{1 *}$, "Microsecond Barrier-Limited Chain Collapse Observed by Time-Resolved FRET and SAXS," J. Mol. Biol. 426, 9 (May 1, 2014). DOI: 10.1016/j.jmb.2014.02.020 Author affiliations: ${ }^{1}$ University of Massachusetts Medical School, ${ }^{2}$ Illinois Institute of Technology

Correspondence:

*osman.bilsel@umassmed.edu

This work was supported by National Institutes of Health (NIH) grants GM23303 and GM54836 and National Science Foundation grants MCB0327504 and MCB1121942, and by grants from the National Center for Research Resources (2P41RR008630-17) and the National Institute of General Medical Sciences (9 P41 GM103622-17) from the NIH.

Bio-CAT is supported by grant 9 P41 GM103622 from the National Institute of General Medical Sciences of the National Institutes of Health. This research used resources of the Advanced Photon Source, a U.S. Department of Energy (DOE) Office of Science User Facility operated for the DOE Office of Science by Argonne National Laboratory under Contract No. DE-AC0206CH11357.

18-ID-D • Bio-CAT • Life sciences • Fiber diffraction, microdiffraction, small-angle $x$-ray scattering, time-resolved $x$-ray scattering $\bullet 3.5$ $35 \mathrm{keV} \cdot$ On-site $\cdot$ Accepting general users $•$ 


\section{A Molecular Basis for Protease's Role in Preventing Alzheimer's Disease}

$\mathrm{P}$

roteases are specialized enzymes responsible for degrading damaged, misfolded, or unneeded proteins within the cell. The human mitochondrial presequence protease (hPreP) breaks down several distinct proteins, including beta amyloid $(A \beta)$ species known to aggregate and form the amyloid plaques associated with Alzheimer's disease. Using a combination of high-resolution $x$-ray crystallography and small angle $x$-ray scattering (SAXS) at the APS, researchers from The University of Chicago and the University of Illinois at Chicago were able to define the mechanism by which $\mathrm{hPreP}$ recognizes a diverse array of amyloid proteins. The findings reveal that hPreP uses a large catalytic chamber to capture "toxic" peptides on the basis of their size and charge distribution. Understanding the mechanism of hPreP substrate recognition and degradation paves the way for the development of small-molecule modulators of hPreP, which could ultimately serve as a new therapeutic approach in the treatment of Alzheimer's disease.

(a)

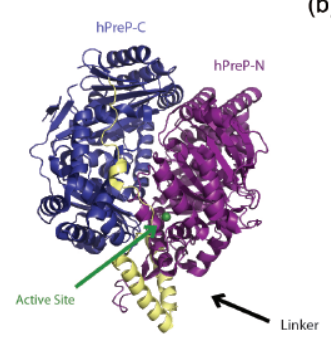

(b)

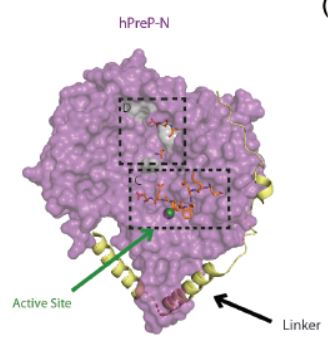

(c)

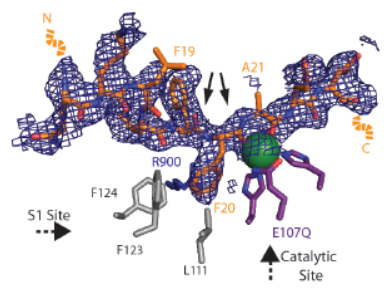

(d)

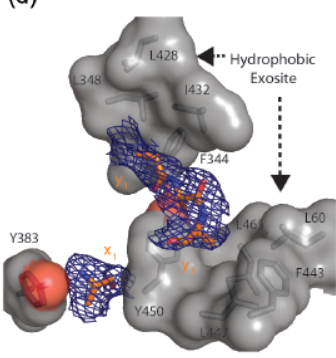

complementarity is a driving force for hPreP's ability to bind $A \beta$ in an orientation that permits specific cleavage of $A \beta$ within regions likely to form aggregates. Additional SAXS data show that in solution, hPreP exists in a mixture of "open" and "closed" conformations at $~ 1: 4$ ratio. Substrate binding promotes most of the hPreP to adopt the "closed" conformation, which induces the alignment of the catalytic residues in the proper orientation. This observation confirms that $\mathrm{hPreP}$ excludes molecules from the catalytic site on the basis of their size.

The flexibility of $\mathrm{hPreP}$ substrate recognition leaves unanswered the question of whether additional, currently unidentified proteins are targeted by $\mathrm{hPreP}$ for degradation. Identification of hPreP substrates will illuminate additional roles hPreP has in other important cellular pathways.

- Emma Nichols
Proteases are specialized enzymes responsible for degrading damaged, misfolded, or unneeded proteins within the cell. The human presequence protease (hPreP) is responsible for degrading "presequences," which are amino acid sequences that direct newly synthesized proteins to the mitochondria. Once inside the mitochondria, however, presequences are toxic, and can interfere with the mitochondria's ability to provide energy to the cell. In addition to degrading presequences, hPreP also degrades several $A \beta$ proteins. $A \beta$ peptides, when they accumulate, form the amyloid aggregates, or plaques, characteristic of Alzheimer's disease.

Diffraction data critical for defining the crystal structure of hPreP alone and bound to $A \beta$ were collected at the SBCCAT 19-ID-D beamline at the APS. SAXS data were collected at the BioCAT 18-ID-D beamline at the APS.

The data (Fig. 1) reveal how hPreP is able to accommodate distinct molecules within its catalytic site, and how
Fig. 1. (a) hPreP is comprised of an N-terminal domain (purple) and C-terminal domain (blue), which enclose the catalytic chamber and active-site (green) (b) hPreP captures $A \beta$ using an exosite (dashed box; D) and active site (dashed box; C) (c) Close-up of the hPreP active site (blue mesh), bound to $A \beta$ (gray) prior to cleavage between $A \beta$ residues $F 20$ and $A 21$. The $A \beta$ peptide is trapped in the catalytic site by the $\mathrm{S} 1$ site (indicated) (d) Closeup view of $A \beta$ (blue mesh) anchored in the hydrophobic hPreP exosite (shaded gray). Adapted from J.V. King et al., Structure 22, 996 (July 8, 2014).

this enzyme specifically recognizes amyloid proteins. The high-resolution diffraction patterns of hPreP alone and in complex with $A \beta$ provide an atomiclevel snapshot of the large $(\sim 13,000 \AA)$ catalytic chamber and the molecular interactions required for substrate capture and cleavage. Within this chamber, amino acid residues form a hydrophobic pocket, a basic pocket adjacent to the catalytic site, and a hydrophobic exosite distant from the catalytic site. This arrangement indicates that charge
See: John V. King ${ }^{1 \ddagger}$, Wenguang G. Liang $^{1}$, Kathryn P. Scherpelz ${ }^{1}$, Alexander B. Schilling ${ }^{2}$, Stephen C. Meredith ${ }^{1}$, and Wei-Jen Tang ${ }^{1 *}$, "Molecular Basis of Substrate Recognition and Degradation by Human Presequence Protease," Structure 22, 996 (July 8, 2014).

DOI: 10.1016/j.str.2014.05.003

Author affiliations: ${ }^{1}$ The University of Chicago, ${ }^{2}$ University of Illinois at Chicago łPresent address: University of California, San Francisco Correspondence:

*wtang@uchicago.edu

This research was supported by the National Institutes of Health Grant R01 GM81539 to W.J.T. Bio-CAT is supported by grant 9 P41 GM103622 from the National Institute of General Medical Sciences of the National Institutes of Health. This research used resources of the Advanced Photon Source, a U.S. Department of Energy (DOE) Office of Science User Facility operated for the DOE Office of Science by Argonne National Laboratory under contract no. DE-AC0206CH11357. 


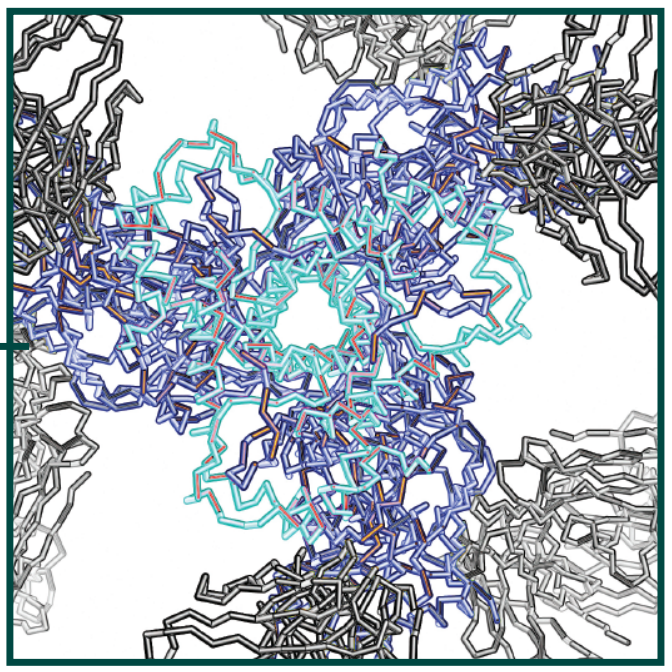

Structural Biology 


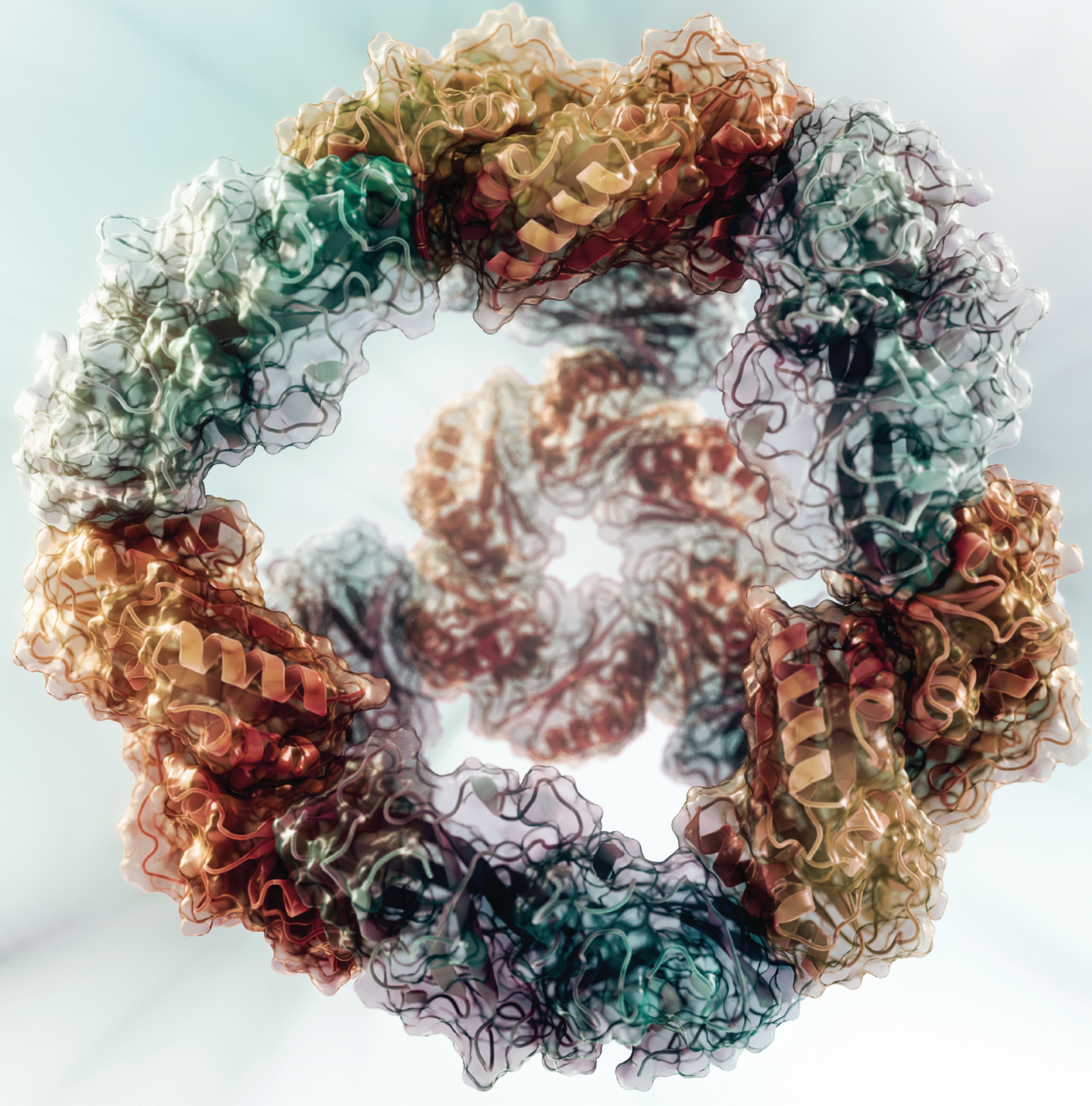




\section{On the Path toward Designer Proteins}

B

iologists would like to be able to design synthetic proteins that can spontaneously assemble themselves into a desired shape. In nature, proteins self-assemble all the time, but being able to control their final shape could help scientists create better vaccines or build tiny containers to carry drugs directly to cancer cells and other targets. Now researchers have created a computer program that could potentially find the right DNA sequence to make proteins form into whatever shape the user desires. The research team utilized high-brightness x-rays from the APS to check their computer models against actual protein structures.

The researchers from the University of Washington; the University of California, Los Angeles; and the Howard Hughes Medical Institute fed into the computer a desired shape, along with constraints on how the proteins could be moved. They started with two different building blocks, each a protein complex consisting of three different molecules. Each building block had patches that could bind to itself or to the other component through various forces, such as hydrogen bonding - in which a positively charge hydrogen atom sticks to a negatively charged atom - or the hydrophobic effect, where atoms stick to one another to avoid water. These protein-to-protein interfaces both provide the energy to drive the self-assembly, and define how the building blocks can be oriented to one another. By controlling which part of which protein would bind to another, the method encodes the final shape into the building blocks.

In the next step, the computer looked at the sequence of amino acids that encoded features of the interfaces between the building blocks, such as whether the molecules had hydrophobic side chains. The computer randomly mutated an amino acid sequence, then checked to see whether that made the interface more or less stable. If it was less stable, the computer discarded that mutation and tried a new one. If the mutation improved stability, the computer kept it and moved on to another spot. Once the computer had determined a complete set of DNA sequences, the team sent it to a laboratory that makes synthetic DNA, which then used E. coli to produce the designed proteins.

In the first part of the study, the computer docked several hundred thousand pairs of proteins to get the best shape. Then it performed several hundred thousand mutations to come up with about 100,000 potential pathways to a final design. After combing through those for the ones that seemed most promising, the team delivered 57 different design sequences to the synthetic DNA lab, five of which successfully selfassembled into protein nanocages capable of carrying a drug molecule.

To verify that the final products matched the initial design, the researchers turned to the NE-CAT 24-ID$C$ beamline of the APS where they performed protein crystallography studies. The high brightness of the APS x-ray beam allowed them to get high-resolution images, which is important when one is designing protein cages down to the atomic level. The actual structures turned out to be extremely similar to the models in the computer.

One potential use of this method is to build protein structures that very closely resemble actual viruses, without being infectious; that could lead to novel, more effective vaccines. The researchers say that by working with more than two building blocks, their program should be able to build much more complex shapes with a variety of biological properties. - Neil Savage

See: Neil P. King ${ }^{1}$, Jacob B. Bale ${ }^{1}$, William Sheffler ${ }^{1}$, Dan E. McNamara²,
Shane Gonen ${ }^{1,3}$, Tamir Gonen ${ }^{3}$, Todd O. Yeates ${ }^{2}$, and David Baker ${ }^{1 *}$, "Accurate design of co-assembling multi-component protein nanomaterials," Nature 51, 103 (5 June 2014). DOI: 10.1038/nature13404 Author affiliations: ${ }^{1}$ University of Washington; ${ }^{2}$ University of California, Los Angeles; ${ }^{3}$ Howard Hughes Medical Institute Correspondence: *dabaker@u.washington.edu

This work was supported by the Howard Hughes Medical Institute (T.G. and D.B.) and the Janelia Farm Research Campus visitor program (S.G.), the U.S. National Science Foundation (NSF) under CHE-1332907 (D.B. and T.O.Y.), grants from the International AIDS Vaccine Initiative, Defense Threat Reduction Agency (N00024-10-D6318/0024), Air Force Office of Scientific Research (FA950-12-10112), and U.S. Department of Energy (DOE) (DESC0005155) to D.B., a National Institutes of Health Biotechnology Training Program award to D.E.M. (T32GM067555), and a NSF graduate research fellowship to J.B.B. (DGE-0718124). T.O.Y. and D.E.M. also acknowledge support from the Biological and Environmental Research program of the DOE Office of Science. NE-CAT is supported by a grant from the National Institute of General Medical Sciences (P41 GM103403) from the National Institutes of Health. This research used resources of the Advanced Photon Source, a U.S. DOE Office of Science User Facility operated for the DOE Office of Science by Argonne National Laboratory under Contract No. DE-AC02$06 \mathrm{CH} 11357$.

24-ID-C • NE-CAT • Life sciences • Macromolecular crystallography, microdiffraction, single-wavelength anomalous dispersion, single-crystal diffraction, microbeam $\cdot 6.5-23 \mathrm{keV}$ - On-site, remote $\cdot$ Accepting general users $•$

$<$ Fig. 1. Two different building blocks (green and orange) were synthesized in bacteria and then self-assembled into this protein nanocage, based on the computer's design. 


\section{Playing Hide and SeEk With HIV}

\section{$\mathrm{T}$}

he human immunodeficiency virus type 1 (HIV-1) was responsible for 1.5 million deaths around the world in 2013. Although researchers have been able to develop anti-retroviral therapies that allow patients to live longer with the disease, it has been a struggle to develop an effective vaccine that prevents the spread of infection. Theoretically, the problem is one we think we know how to solve. HIV-1 has a single spike protein on its surface called Env that mediates viral fusion and entry into host cells. If we can prime the immune system to recognize Env and neutralize it, we should be able to stop HIV-1 in its tracks. However, up to this point, HIV-1 has used molecular trickery to evade our efforts to bolster anti-HIV-1 immunity. Now, new structural data collected at the APS by a research team from universities in the United States and South Africa, has revealed the basis for some of HIV-1's elusiveness. Their work maps sites of potential vulnerability identified in the sera of patients who have made neutralizing antibodies to HIV-1, opening the door to the design of potentially more effective vaccines against HIV.
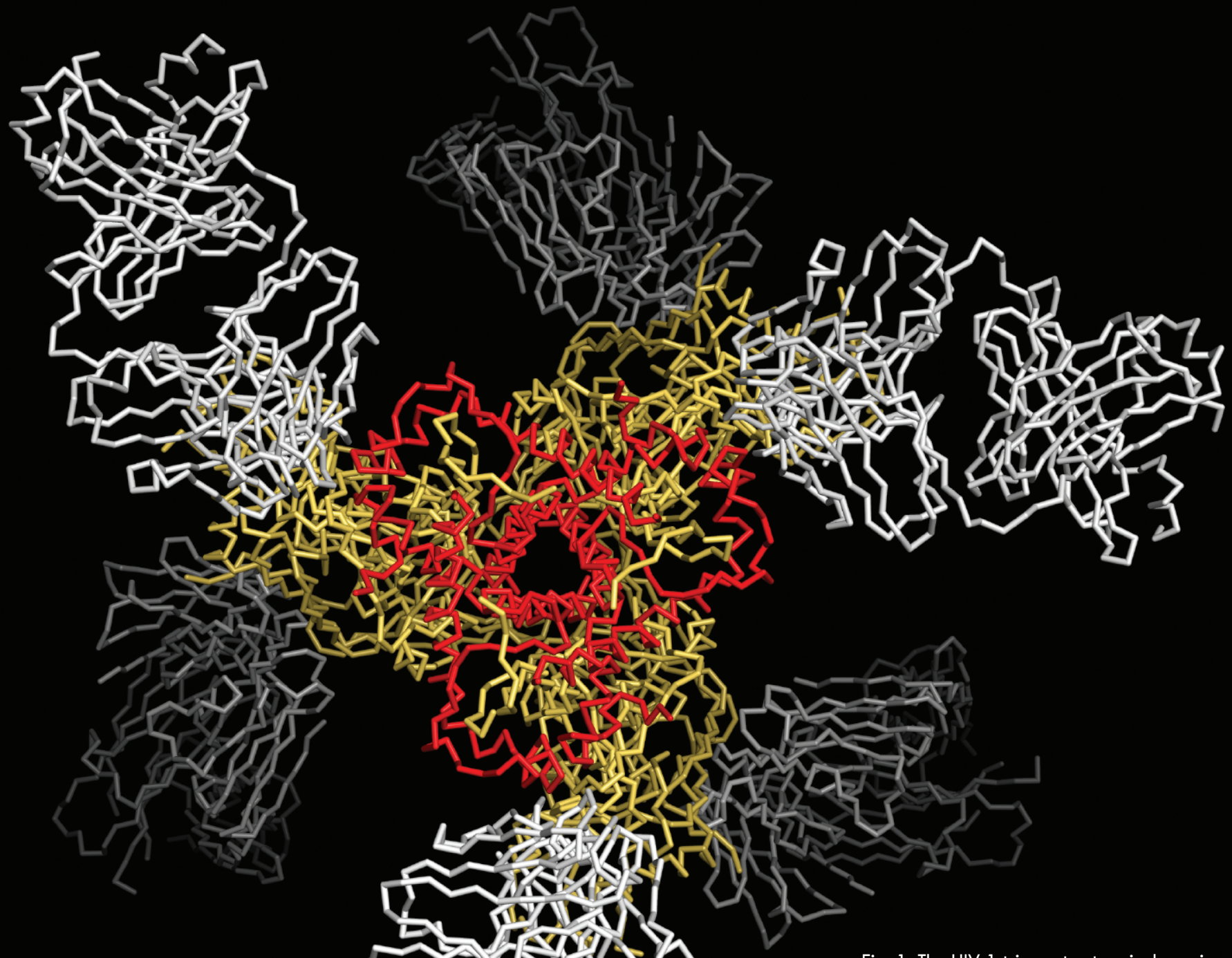

Fig. 1. The HIV-1 trimer structure is shown in $\mathrm{C}_{\alpha}$ representation with gp 120 s in yellow and gp4 $1 \mathrm{~s}$ in red. The bound neutralizing antibodies are shown in white. Image courtesy of Marie Pancera and Jonathan Stuckey, National Institutes of Health/National Institute of Allergy and Infectious Diseases/Vaccine Research Center 
One of the challenges in our understanding of HIV-1 has been in obtaining good structural information on the Env protein. This protein is a complex fusion machine composed of three copies of a subunit called gp120 (receptor binding domain) and three copies of a subunit called gp41 (fusion domain). These assemble on the surface of HIV-1 in a closed pre-fusion mature state that is activated upon receptor binding to undergo a dramatic conformational change in which the gp41 Env protein pierces the host cell and merges the viral membrane with the host cell membrane. This process allows the HIV-1 genome entry to the host cell cytoplasm.

Researchers believe that neutralizing antibodies that target the pre-fusion state of Env have the best chance of conferring lasting immunity. However, because the pre-fusion association between gp120 and gp41 is fragile and because the post-fusion state is more energetically favored, it has been difficult to crystallize the pre-fusion state. The researchers in this study from the National Institutes of Health, the National Institute for Communicable Diseases of the National Health Laboratory Service (South Africa), the University of North Carolina at Chapel Hill, the Duke University School of Medicine and the Center for HIVIAIDS Vaccine Immunology-Immunogen Discovery at Duke University, the University of the Witwatersrand (South Africa), the University of KwaZulu-Natal (South Africa), the Yale University School of Medicine, and the Weill Cornell Medical College of Cornell University used their own molecular trick to overcome this They were able to crystallize the gp120/gp41 complex by stabilizing it with two antibodies that recognize different parts of the structure.

The structural data obtained by utilizing the SER-CAT 22-ID-D beamline at the APS show a number of interesting features of the complex. The subunits are generally oriented into a propeller-like shape, and each molecule of gp41 in the structure forms a kind of collar through which the ends of gp120 extend towards the viral membrane (Fig. 1). The collar is formed by helices and loops of gp41 and is fastened with a molecular clasp.

Comparison to the structure of the post-fusion complex shows that gp 120 binding to the host cell causes the gp41 collar to rearrange and become a pair of extended helices that force a molecular spike through the host membrane and pull the two membranes together.

The team compared the HIV-1 fusion machine to other known viral fusion machines and found that the topology of the interaction is similar to the influenza, respiratory syncytial, and Ebola viruses. But what makes it so hard to recognize immunologically?

The answer lies, in part, in sugar modifications (glycosylations) that are added to the surface of the molecule after it is made. Modeling showed that glycosylation, which is less likely to cause an immune response, leaves only $3 \%$ of the protein surface free for antibodies to bind. Compared to influenza at $14 \%$ and respiratory syncytial virus at $48 \%$, that is precious little space for mounting an immune response. In addition, the exposed surface of HIV-1 exhibits extreme genetic variability that makes it even more difficult to target effectively with antibodies.

The final part of this study was to look at some immunological successes. Despite HIV-1's molecular trickery, some patients have developed neutralizing antibodies to HIV-1. The team looked at these to see where they bind and found that, in general, successful antibodies can accommodate HIV-1's variability in their recognition pattern and incorporate recognition of glycosylation as well.

The researchers hope that this new information will provide the edge they need to finally block HIV-1's spike.

$$
\text { - Sandy Field }
$$

See: Marie Pancera ${ }^{1}$, Tongqing Zhou ${ }^{1}$, Aliaksandr Druz ${ }^{1}$, Ivelin S. Georgiev ${ }^{1}$, Cinque Soto ${ }^{1}$, Jason Gorman ${ }^{1}$, Jinghe Huang ${ }^{1}$, Priyamvada Acharya ${ }^{1}$, Gwo-Yu Chuang ${ }^{1}$, Gilad Ofek ${ }^{1}$, Guillaume B.E. Stewart-Jones ${ }^{1}$, Jonathan Stuckey ${ }^{1}$, Robert T. Bailer ${ }^{1}$, M. Gordon Joyce ${ }^{1}$, Mark K. Louder ${ }^{1}$, Nancy Tumba ${ }^{2}$, Yongping Yang ${ }^{1}$, Baoshan Zhang ${ }^{1}$, Myron S. Cohen $^{3}$, Barton F. Haynes ${ }^{4}$, John R.
Mascola ${ }^{1}$, Lynn Morris ${ }^{2,5,6}$, James B. Munro $^{7}$, Scott C. Blanchard ${ }^{8}$, Walther Mothes $^{7}$, Mark Connors ${ }^{1}$, and Peter D. $\mathrm{Kwong}^{1 *}$, "Structure and immune recognition of trimeric pre-fusion HIV-1 Env," Nature 514, 455 (23 October 2014). DOI: $10.1038 /$ nature 13808

Author affiliations: ${ }^{1}$ National Institutes of Health, ${ }^{2}$ National Institute for Communicable Diseases of the National Health Laboratory Service, ${ }^{3}$ University of North Carolina at Chapel Hill, ${ }^{4}$ Duke University School of Medicine and the Center for HIVIAIDS Vaccine Immunology-Immunogen Discovery at Duke University, ${ }^{5}$ University of the Witwatersrand, ${ }^{6}$ University of KwaZulu-Natal, ${ }^{7}$ Yale University School of Medicine, ${ }^{8}$ Weill Cornell Medical College of Cornell University Correspondence: * pdkwong@nih.gov

Support for this work was provided by the Intramural Research Program of the Vaccine Research Center, National Institute of Allergy and Infectious Diseases (NIAID), National Institutes of Health (NIH); and by grants from the Division of AIDS, NIAID, NIH (R21Al100696, CHAVI-AI0678501, and CHAVIImmunogen Discovery-Al100645), from the National Institutes of General Medical Sciences (PO1-GM56550 and RO1-

GM098859), from the Irvington Fellows Program of the Cancer Research Program, and from the International AIDS Vaccine Initiative's (IAVI's) Neutralizing Antibody Consortium. IAVI's work is made possible by support from many donors including: the Bill \& Melinda Gates Foundation; the Ministry of Foreign Affairs of Denmark; Irish Aid; the Ministry of Finance of Japan; the Ministry of Foreign Affairs of the Netherlands; the Norwegian Agency for Development Cooperation; the UK Department for International Development; and the United States Agency for International Development. The full list of IAVI donors is available at http://www.iavi.org. The Southeast Regional Collaborative Access Team supporting institutions can be found at www.ser-cat.org/members.html. This research used resources of the Advanced Photon Source, a U.S. Department of Energy (DOE) Office of Science User Facility operated for the DOE Office of Science by Argonne National Laboratory under Contract No. DE-AC02-06CH11357.

22-ID-D • SER-CAT • Life sciences • Macromolecular crystallography, multi-wavelength anomalous dispersion, microbeam • $6-20 \mathrm{keV}$ - On-site, remote $\cdot$ Accepting general users • 


\section{Synthetic Peptides Use the Force to Influence Cell Survival}

$\mathrm{P}$ eptide amphiphiles (PAs) are an emerging class of molecules that can be designed for novel therapies in advanced medicine. They are designed with structural regions that allow them to spontaneously assemble into large complex structures like nanofibers (fibers with diameters of approximately $10 \mathrm{~nm}$ ). The researchers in this study investigated how positively charged PAs interact with cells when water-hating properties and hydrogen bonding (a force that holds the nanofibers together) are altered. Using the APS to collect data, they evaluated forces within the biological assemblies. Their research shows that when bonding between molecules is weak, it allows them to rapidly associate with lipids in the cell membrane, leading to cell breakdown and death. In contrast, stronger bonds between molecules favor cell survival. These findings will be important in guiding future work to design biological materials for various therapeutic uses.

Peptides that are amphiphilic contain water-loving (hydrophilic) and water-hating (hydrophobic) structural regions. Many of these peptides that exist in nature have positive charge (cationic) in the hydrophilic region, which contributes to various functions such as self-defense. It is their positively charged and water-hating regions that allow the peptides to associate so strongly with cell membranes that they can even lead to peptide breakdown. There are two classes of these peptides: cell penetrating peptides and natural host defense peptides; both are useful in many therapeutic areas, including cancer therapy, anti-microbials, and delivery of genes or drugs.

Self-assembling peptides are a class of designer peptide-based molecules that make use of interactions between molecules that cause them to spontaneously assemble into large complex molecular structures of different shapes. Interest in these peptides continues to increase due to their potential for applications in the biomedical field, where they serve as building blocks for numerous materials and devices. This technology aims to mimic what happens in nature, basically using forces and bonds between molecules to produce ordered biological assemblies of building blocks with biochemical functions. One example of this involves nanofibers that mimic components of the mammalian extracellular matrix.

PAs are a type of self-assembling peptide. Some studies have shown that changing the amino acid sequence and degree of hydrogen bonding in PAs can significantly alter their shape and ability to assemble into three-dimensional networks. Other studies have also shown that changes in hydrogen bonding and electrostatic forces in these biological assemblies affects the strength of the materials they form, and their ability to signal cells. However, the interactions between cell membranes and these materials are not well understood. With this in mind, the researchers from Northwestern University aimed to investigate how hydrogen bonding, the presence of structural water-hating regions, and the charge of PAs affect how they interact with cells.

The researchers used data from wide-angle $x$-ray diffraction studies beamline at the APS to show that forces between molecules, particularly hydrogen bonding, influence the cell's ability to survive. They determined that differences in attractive forces in biological assemblies are the key in determining cell membrane stability, and therefore whether the cell dies or survives.

The most significant finding was in relation to changes in hydrogen bondperformed at the BioCARS 14-BM-C ing among molecules. The results show that, when biological assemblies were fortified by strong hydrogen bond forces between molecules, cell survival was favored. However, when bonds between molecules were weak, biological assemblies were able to integrate into, and break down, the lipid layer of the cell membrane, and thereby stimulate cell death (Fig. 1). They also demonstrated that both the water-hating region and the positive charge of the PA were required to promote cell death. - Nicola Parry

See: Christina J. Newcomb, Shantanu Sur, Julia H. Ortony, One-Sun Lee, John B. Matson, Job Boekhoven, Jeong Min Yu, George C. Schatz, and Samuel I. Stupp*, "Cell death versus cell survival instructed by supramolecular cohesion of nanostructures," Nat. Commun. 5, 3321(2014).

DOI: $10.1038 /$ ncomms4321

Author affiliation:

Northwestern University Correspondence:

* s-stupp@northwestern.edu

This work was supported by the National Institutes of Health (NIH), National Institute of Dental and Craniofacial Research 2R01 DE015920-06, and the National Institute of Biomedical Imaging and Bioengineering 2R01EB003806-06A2. J.B. was supported by a Rubicon grant from the Netherlands Organisation for Scientific Research, and both O.-S.L. and G.C.S. were supported by National Science Foundation grant CHE1147335. Use of BioCARS was supported by the National Institute of General Medical Sciences of the NIH under grant number R24GM111072. This research used resources of the Advanced Photon Source, a U.S. Department of Energy (DOE) Office of Science User Facility operated for the DOE Office of Science by Argonne National Laboratory under Contract No. DE-AC02$06 \mathrm{CH} 11357$.

14-BM-C • BioCARS • Life sciences • Macromolecular crystallography, fiber diffraction, biohazards at the BSL2/3 level, subatomic $(<0.85 \AA)$ resolution, large unit cell crystallography $\bullet$ 8-14.9 $\mathrm{keV} \cdot$ On-site $\cdot$ Accepting general users • 


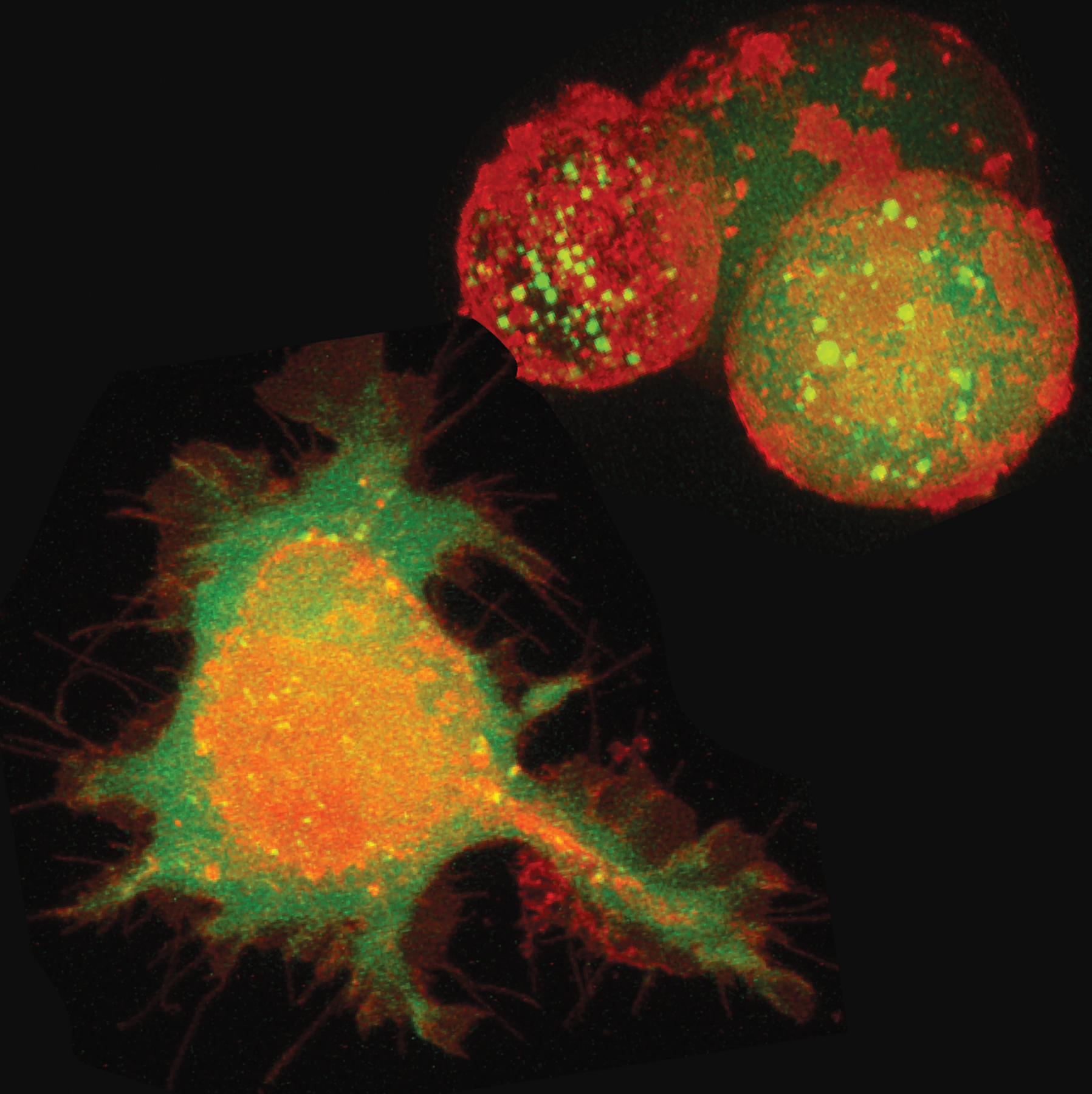

Fig. 1. An image of a cell growing on a strongly cohesive material that encourages cells to grow and spread (bottom), and a small group of dying cells in contact with the weakly cohesive material (top). The presence of the green dye inside the cell confirms that it is living, and the red dye stains the cell membrane for visualization. 


\section{How Ebola Silences Those Who Would SOUND THE Alarm}

$\mathrm{E}$

bola virus causes a deadly hemorrhagic disease with case fatality rates up to $90 \%$. This is due to the virus' ability to rapidly shut down innate immune responses and cause a severe immune reaction called a "cytokine storm." But how does it do this and why is it so effective? Researchers using two U.S. Department of Energy $x$-ray light sources, including the APS, have uncovered an important part of the answer. Their work has shown how an Ebola virus protein called VP24 blocks cellular responses to interferons, which exert a critical antiviral effect as part of the innate immune response. Ebola virus VP24 protein (eVP24) does this by blocking the movement of a critical signaling molecule into the nucleus where it normally activates the antiviral state in cells. Their structural and biochemical analysis of the interaction between eVP24 and the protein that acts as a shuttle to move cargo through the nuclear gate, KPNA5, reveals details of the interaction that might be exploited for designing pharmaceutical interventions to fight Ebola.

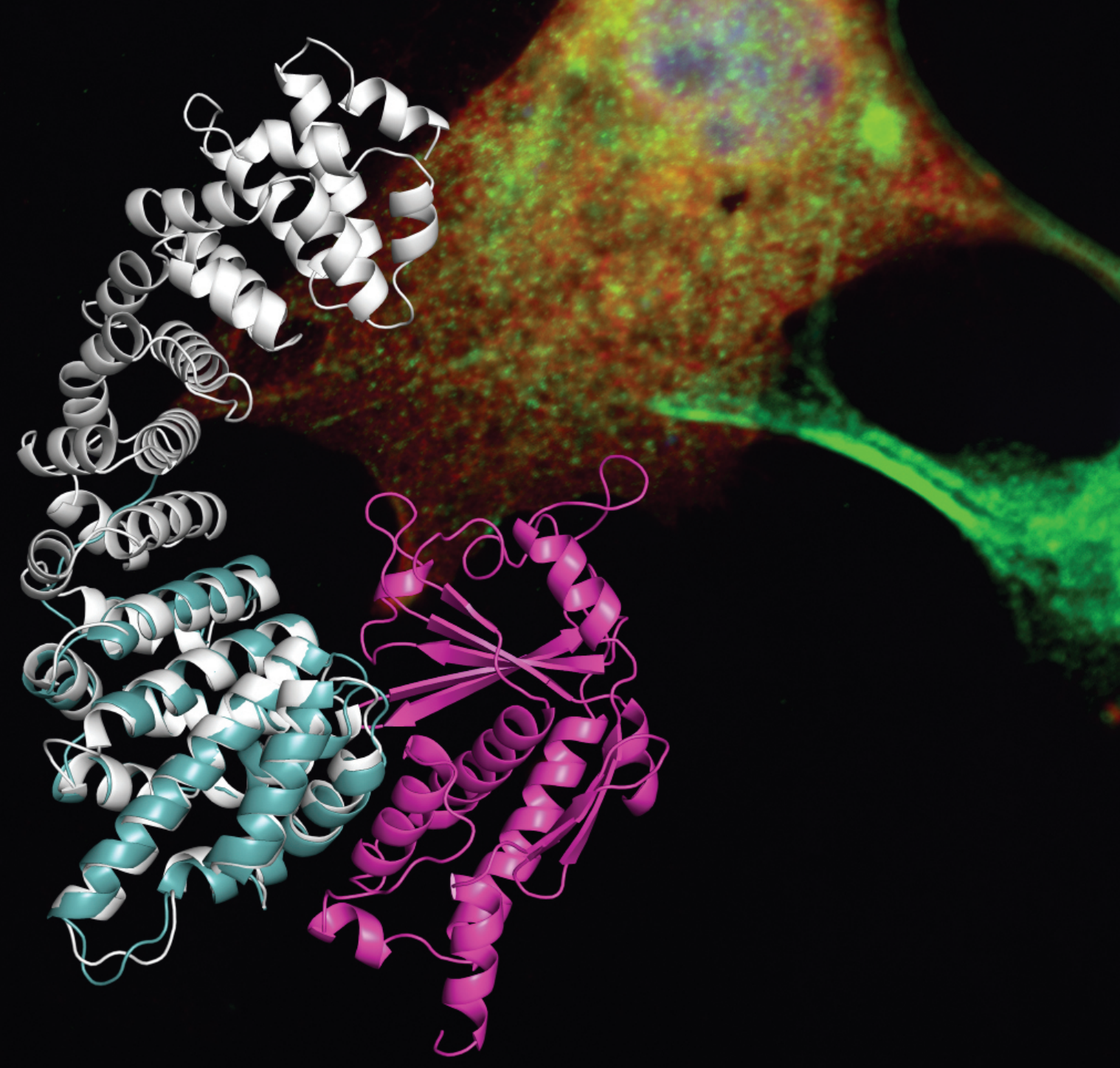


When the body encounters a virus, it sends out signals, activating a cascade of events that sound the alarm and produce an antiviral state in cells. This state is triggered by interferon (IFN), which activates the transcription factor STAT1. Once activated, STAT1 is able to access a special entrance into the nucleus through transporters via a non-classical pathway. The non-classical pathway is a secondary pathway that is distinct from the classical transport pathways through which most protein traffic enters the nucleus. STAT1's access to the non-classical pathway is facilitated by several nuclear transporters from the NPI-1 subfamily, including KPNA5, which was the protein used in this study. Once inside the nucleus, STAT1 activates a group of genes that gets the antiviral arsenal up and running.

The researchers in this study from Washington University School of Medicine in St. Louis, Icahn School of Medicine at Mount Sinai, the University of Texas Southwestern Medical Center at Dallas, and Washington University in St. Louis started their investigation on the basis of previous work. They knew that Ebola blocks the entry of STAT1 into the nucleus, effectively blocking antiviral gene transcription, and they knew that it did it by blocking STAT1's access to KPNA5 with eVP24. What they didn't know was the molecular details of the eVP24-KPNA5 interaction that might suggest a way to block eVP24 with pharmaceutical interventions. For this they needed structural information.

< Fig. 1. Effects of Ebola VP24 in cells and structural interaction with KPNA5. A cell expressing fluorescent Ebola VP24 (red) and labeled for STAT 1 (green) after interferon activation shows that VP24 effectively blocks STAT 1 entry into the nucleus (overlap of two proteins in yellow). Ribbon diagram shows structural interactions between eVP24 (magenta) and the C-terminal end of KPNA (cyan) (PDB: 4U2X). Full-length KPNA5 is overlaid into the image in white (PDB: 1BK5). Image courtesy of Daisy W. Leung (Washington University School of Medicine in St. Louis) and Christopher F. Basler (Icahn School of Medicine at Mount Sinai)
The team's first step was to define the interaction between KPNA5 and eVP24. They determined that eVP24 binds just to one end of KPNA5, allowing them to use this smaller region of the protein for crystallization. The crystal structure of eVP24 bound to the fragment of KPNA5 was obtained at the SBC-CAT beamline 19-ID-D at the APS and the Advanced Light Source beamline 4.2.2 at Lawrence Berkeley Laboratory. The structure revealed a number of important features of the interaction (Fig.1). First, the binding site for eVP24 on KPNA5 is unique and, while it overlaps the known site for STAT1, it does not resemble that for any other cargo that come in through the non-classical pathway. Also, while neither protein changes conformation much in the binding interaction, eVP24 contributes a large binding interface to the interaction involving several amino acids from various loops on the structure.

Biochemical analysis of eVP24 proteins with these amino acids mutated showed that the loss of single contacts only resulted in slightly impaired binding while loss of several contacts resulted in completely abolished binding. Interestingly, the specificity of the interaction is mediated mostly by eVP24 amino acids that are different from those in the same region in the related Marburg virus VP24 (mVP24) protein. In fact, despite significant protein sequence similarity to eVP24, mVP24 adopts a different structure in this region. This may explain why Marburg does not block interferon signaling using the same mechanism.

Further analysis of eVP24 proteins possessing mutations in the KPNA5 binding domain showed that eVP24 mutants with reduced KPNA5 binding also have reduced ability to block STAT1 nuclear transport and IFN-induced gene activation. This supports a model in which the eVP24 interaction with KPNA5 is critical to its ability to block innate immune system activation by IFN. Similar assays also show that eVP24 does not block the classical transport pathway. This is important because it allows Ebola to block progress to the antiviral state while at the same time keeping cellular machinery up and run- ning to churn out viral proteins needed to propagate its deadly progeny.

The research team hopes that this work will provide clues that will allow them to identify new or existing drugs that can block the interaction between eVP24 with KPNA5 while leaving STAT1 signaling intact. - Sandy Field

See: Wei Xu ${ }^{1}$, Megan R. Edwards ${ }^{2}$, Dominika M. Borek ${ }^{3}$, Alicia R. Feagins ${ }^{2}$, Anuradha Mittal ${ }^{4}$, Joshua B. Alinger ${ }^{1}$, Kayla N. Berry ${ }^{1}$, Benjamin Yen², Jennifer Hamilton ${ }^{2}$, Tom J. Brett ${ }^{1}$, Rohit V. $\mathrm{Pappu}^{4}$, Daisy W. Leung ${ }^{1}$, Christopher F. Basler ${ }^{2}$, and Gaya K. Amarasinghe ${ }^{1 *}$, "Ebola Virus VP24 Targets a Unique NLS Binding Site on Karyopherin Alpha 5 to Selectively Compete with Nuclear Import of Phosphorylated STAT1," Cell Host Microbe 16, 187 (August 13, 2014).

DOI: 10.1016/j.chom.2014.07.008 Author affiliations: ${ }^{1}$ Washington University School of Medicine in St. Louis, ${ }^{2}$ Icahn School of Medicine at Mount Sinai, ${ }^{3}$ University of Texas Southwestern Medical Center at Dallas, ${ }^{4}$ Washington University in St. Louis

Correspondence:

* gamarasinghe@path.wustl.edu

This work is supported in part by the U.S. National Science Foundation (MCB-1121867 to R.V.P.) and National Institutes of Health grants (J.A.B. and K.N.B. supported by T32GM07200; R01HL119813 to T.J.B; R01Al107056 to D.W.L.; R01Al059536 to C.F.B.; U19AI109945 [Basler-PI] and U19AI109664 [Basler-PI] to C.F.B. and G.K.A.; U19AI070489 [Holtzman-PI] and R01AI081914 to G.K.A). SBC-CAT is operated by UChicago Argonne, LLC, for the U.S. Department of Energy (DOE_ Office of Biological and Environmental Research under contract DE-AC02-06CH11357. This research used resources of the Advanced Photon Source, a U.S. DOE Office of Science User Facility operated for the DOE Office of Science by Argonne National Laboratory under Contract No. DE-AC0206CH11357.

19-ID-D • SBC-CAT • Life sciences • Macromolecular crystallography, multi-wavelength anomalous dispersion, subatomic $(<0.85 \AA)$ resolution, microbeam, ultra-low-temperature (15K), large unit cell crystallography, singlewavelength anomalous dispersion $\cdot 6.5-19.5$ $\mathrm{keV} \cdot$ On-site, remote, mail-in $\bullet$ Accepting general users • 


\section{Lights, Conformational Change...Action!}

$\mathrm{S}$

unlight is the basis for all life on Earth so it should come as no surprise that many organisms have developed complex systems for detecting the quality and quantity of light in their environment. Plants, fungi, and many species of bacteria use light-sensitive proteins called phytochromes to control biological functions that respond to changes in light. In the bacterial species Deinococcus radiodurans, a red/far-red-light absorbing phytochrome controls the production of light-protective pigments. The structure of the phytochrome in the resting state has been solved, but the question of how the signal from light gets transformed into a biological action has remained elusive. Now, in work that used the BioCARS beamline 14-ID-B at the APS, as well as beamlines at the European Synchrotron Radiation Facility (France) and the Swiss Light Source, researchers have collected structural data on the active state of the phytochrome sensory module that provide exciting new insights into how it transforms light into action. These findings fill in critical steps in our understanding of these complex sensory systems, manipulation of which may have many future applications in agriculture.

\section{Resting}

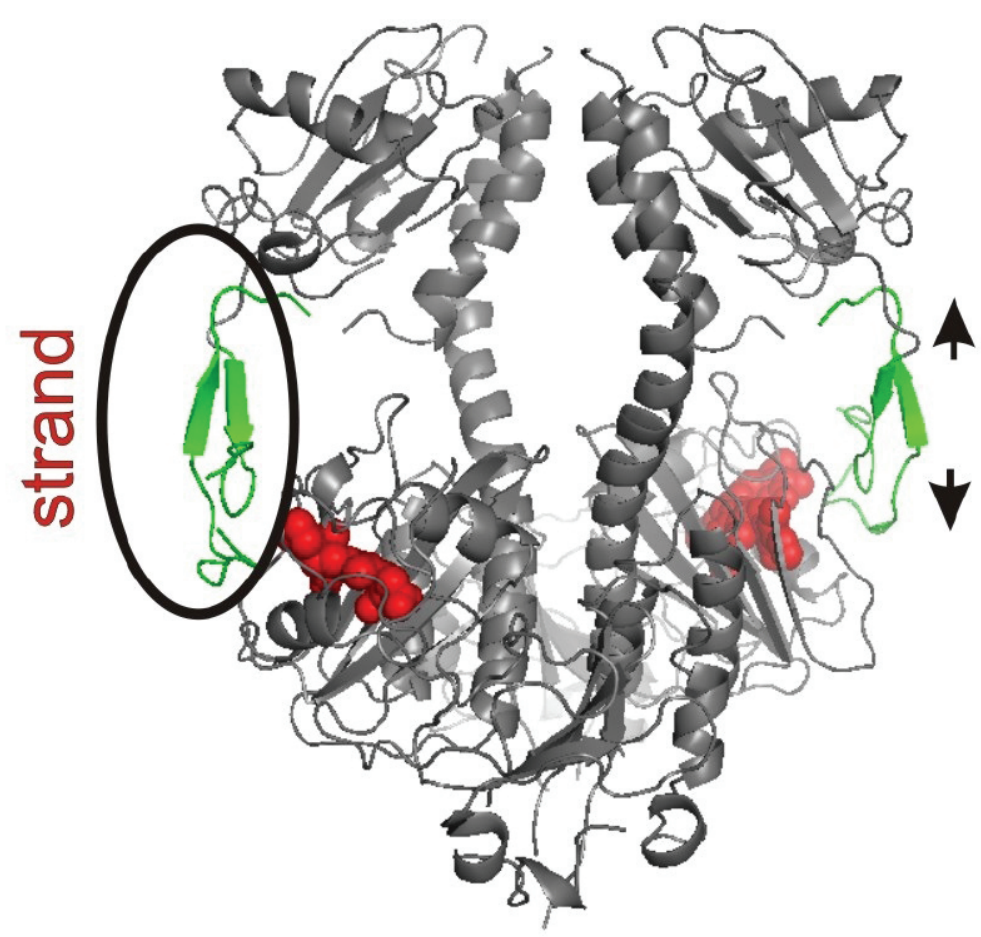

Fig. 1. Model of protein secondary structural changes in the photosensory module from D. radiodurans between the resting ( $\mathrm{Pr}$, this page) and active ( $\mathrm{Pfr}$, next page) states in response to light. Red-light absorption by the chromophore (red) in the $\mathrm{Pr}$ form of the phytochrome results in dramatic changes in both the "tongue" structure (green) and the overall interaction between the two copies of the protein in the dimer (gray). 
A research team from the University of Jyväskylä (Finland), the University of Gothenburg (Sweden), The University of Chicago, and the Paul Scherrer Institut (Switzerland) sought to learn more about the active-state structure of phytochrome systems by comparing structural data for the resting and the activated forms of the phytochrome from $D$. radiodurans. Their initial experiment involved utilizing the BioCARS beamline at the APS for timeresolved solution $\mathrm{x}$-ray scattering measurements of the phytochrome sensory module in the two states. To achieve this, they flashed a solution of the phytochrome with short bursts of red light to convert between the resting and the active state. In the interim between the flashes, they collected the x-ray scattering data. Analysis of the data revealed the build-up of large conformational changes between the two structures at the nanometer scale.

With this promising data in hand, the team sought higher resolution data to determine the exact nature of the molecular changes that were taking place between the two states. For this, they crystallized the phytochrome in both the resting, $\operatorname{Pr}$ (redabsorbing) and active, $\mathrm{Pfr}$ (far-red absorbing) state. Both structures showed that the phytochrome forms a dimer with two copies of the protein forming a single unit. In the resting state, the dimer is in a "closed" configuration and a highly evolutionarily conserved amino acid sequence forms a tongue-like structure (Fig. 1).

In the resting state, the tongue forms a $\beta$-sheet secondary protein structure. Absorption of red light by the Pr form causes dramatic changes in the protein. The two halves of the dimer adopt an "open" conformation, widening the gap between them by several nanometers. Remarkably, the tongue now converts to a completely different protein secondary structure and adopts a much shorter, $\alpha$-helical conformation. The new tongue is $2.5 \AA$ shorter and interacts with different conserved amino acids than the resting state tongue. This change is sufficient to explain how the active state of the protein may transmit changes to the next protein in the signaling cascade in response to the light.

The data obtained by the team provide the sequences of steps required to convert light into action. First, red light absorption causes changes in the chromophore at the atomic level, which are amplified and transmitted to the surrounding phytochrome. These changes result in dramatic rearrangement of the tongue, which shortens by $2.5 \AA$. Short-

anism have already been verified in publications by other groups. The team's next goal is to decipher how the structural signal is converted into a biochemical signal in the output domain of the phytochrome. This will require collecting more structural data on the $D$. radiodurans and other phytochromes.

$$
\text { - Sandy Field }
$$

See: Heikki Takala ${ }^{1,2}$, Alexander Björling ${ }^{2}$, Oskar Berntsson², Heli Lehtivuori $^{1}$, Stephan Niebling ${ }^{2}$, Maria Hoernke $^{2}$, Irina Kosheleva ${ }^{3}$, Robert Henning ${ }^{3}$, Andreas Menzel ${ }^{4}$, Janne A. Ihalainen $^{1 *}$, and Sebastian Westenhoff $2 * *$, "Signal amplification and transduction in phytochrome photosensors," Nature 509, 245 (8 May 2014). DOI: $10.1038 /$ nature13310 Author affiliations: ${ }^{1}$ University of Jyväskylä, 2University of Gothenburg, ${ }^{3}$ The University of Chicago, ${ }^{4}$ Paul Scherrer Institut Correspondence:

* janne.ihalainen@jyu.fi, **westenho@chem.gu.se

This research was supported by grants to S.W. by the Foundation of Strategic Research, Sweden, and the Swedish and European Research Councils, agreement number 279944; support to J.A.I. by Finnish Academy grant 138063; and to H.T. by the Finnish Cultural Foundation grant 0131067. Use of BioCARS was supported by the National Institute of General Medical Sciences of the National Institutes of Health under grant number R24GM111072. This

ening of the tongue causes a shift in the rigid dimer structure of the phytochrome and the dimer "opens" by several nanometers. The conformational signal is amplified from a small nudge at the atomic level to a dramatic protein rearrangement on the nanometer scale. Action!

According to the team, this mechanism of action has fallen on fruitful ground in the research community and different aspects of the structural mech- research used resources of

the Advanced Photon Source, a U.S.

Department of Energy (DOE) Office of Science User Facility operated for the DOE Office of Science by Argonne National Laboratory under Contract No. DE-AC0206CH11357.

14-ID-B • BioCARS • Life sciences, materials science, physics, chemistry - Time-resolved crystallography, time-resolved x-ray scattering, Laue crystallography, wide-angle $x$-ray scattering, biohazards at the BSL2/3 level, macromolecular crystallography $\cdot 7-19 \mathrm{keV}$. On site $\cdot$ Accepting general users $\bullet$ 


\section{A Tale of Two Glutamate Receptors}

\section{T}

he majority of excitatory brain signals are transmitted by the simple amino acid glutamate, which activates metabotropic and ionotrophic glutamate receptors (mGluR and iGluR, respectively) embedded in neuronal membranes. Activation of mGluRs kicks off a series of chemical cascades that can alter the function of neurons, while activation of iGluRs opens ion channels in the neuronal membrane that can trigger the neuron to fire action potentials. Disruptions in glutamate signaling are implicated in many neurological diseases including Alzheimer's, Parkinson's, schizophrenia, and depression. Not surprisingly, glutamate receptors are the target of a significant proportion of pharmacological therapies. The complexity of the glutamate receptors bears some of the credit for the large number of targeting compounds; in addition to binding glutamate, m MluRs and iGluRs have multiple binding sites for other molecules that can modulate receptor function. Knowing these receptors' precise physical structure can facilitate the design of drugs to treat specific diseases. Studies carried out at the APS by two separate groups of researchers havelprovided the first $x$ ray crystal structures of an mGluR called mGlu1 and the NMDA receptor, one of three iGluRs. Comparisons of the new structures to those of closely related glutamate receptors have contributed to a deeper understanding of the relationship between structure and function in both classes of receptor.

NMDA receptors are heterotetramers mainly composed of two copies each of GluN1 and GluN2 subunits, which activate upon concurrent binding of glycine to GluN1 and L-glutamate to GluN2. Researchers from Cold Spring Harbor Laboratory used the GM/CA-XSD 23-ID-B and -D beamlines at the APS, and the BL41XU beamline at the SPring8 synchrotron $x$-ray facility in Japan to capture the $\mathrm{x}$-ray crystallographic structure of the GluN1a-GluN2B NMDA receptor in the presence of glycine, L-glutamate, and ifenprodil, an inhibitory allosteric modulator (i.e., a compound that binds to a secondary site) [1]. The structure was initially resolved at $5.7 \AA$. Mutations to select amino acids residues facilitated a series of cross links to improve the resolution to better than $4 \AA$. The resulting structure had the shape of a bouquet of flowers where the blooming flowers and stems correspond to the extracellular domains, which bind neurotransmitters and modulators and control ion channel opening, and the transmem-

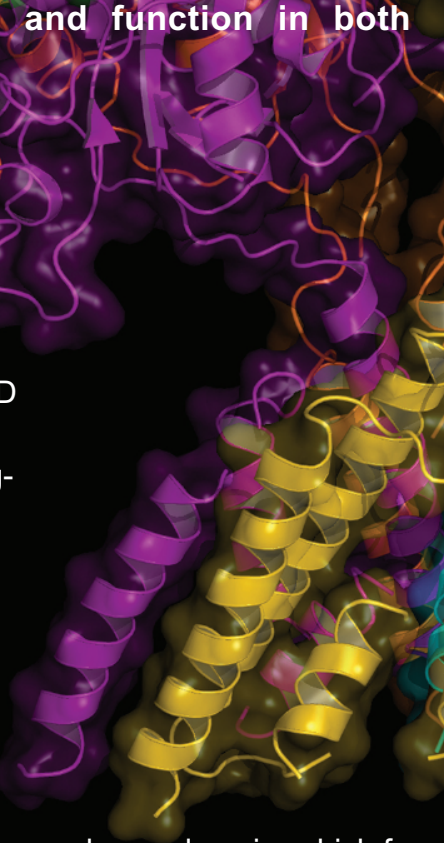

brane domain, which forms the heterotetrameric ion channel, respectively (Fig. 1). The overall shape of the NMDA receptor is distinct from that of the "Y"-shaped homotetrameric GIuA2AMPA receptor - the only other iGluR whose structure has been solved - despite both receptors having a tetrameric arrangement. This difference

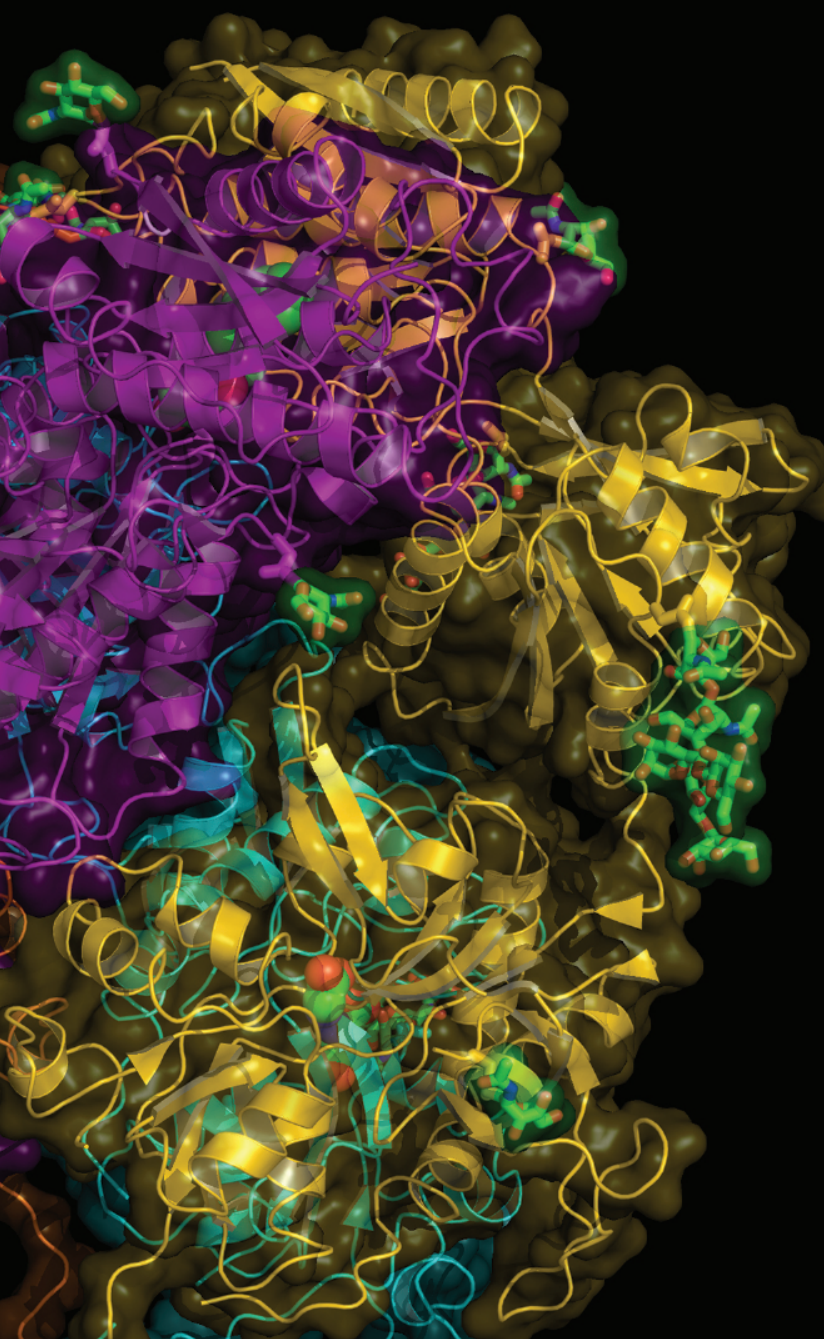

Fig. 1. Structure of NMDA receptor. The crystal structure of the NMDA receptor is shown bound by L-glutamate (large molecular structure, middle left), glycine (large molecular structure, middle right), and two ifenprodil molecules (large molecular structures buried behind top portion of purple subunit). The GluN1 aa (orange), GluN1 a $\beta$ (yellow), GluN2Ba (cyan), and GluN2B $\beta$ (magenta) subunits form NMDA's heterotetrameric structure. 
Like the NMDA receptor, the mGlu1 receptor is a promising therapeutic target. Because its glutamate-binding site is so selective for glutamate, the most accessible target for drug candidates are allosteric receptors on the extracellular portion of mGlu1's seven-transmembrane (7TM) domain.

To gain a better understanding of the geometry of those allosteric binding sites, researchers from The Scripps Research Institute, Vanderbilt University, and Monash University (Australia) likewise used the GM/CA-XSD beamline 23-ID-D at the APS to determine the crystal structure of the human mGlu1 receptor 7TM domain (residues 581-860) with an inhibitory modulator, FITM, bound to the allosteric site (Fig. 2) [2]. The original resolution of $4.0 \AA$ was improved to $2.8 \AA$ by integrating data collected from 14 crystals.

The crystal structure revealed that FITM fits tightly into a long, narrow, boomerang-shaped pocket. Examination of contact residues in the binding pocket revealed only four residues of mGlu1 that differ from mGlu5, a closely related receptor with a lower affinity for FITM. Mutating those four residues to become their corresponding amino acid in mGlu5, the researchers demonstrated that residue Thr815 was critical for FITM binding. Furthermore, comparing FITM binding properties with those of less active compounds indicates that polar interactions within the binding crevice are important for the regulation of binding at this site. Overall, the mGlu1 crystal structure provides a deeper understanding of its molecular recognition mechanism and facilitates the discovery of allosteric modulators.

\section{- Chris Plamer}

[1] See: Erkan Karakas and Hiro Furukawa*, "Crystal structure of heterotetrameric NMDA receptor ion channel," Science 344(6187), 992 (30 May 2014). DOI: $10.1126 /$ science. 1251915 Author affiliation: Cold Spring Harbor Laboratory

Correspondence: * furukawa@cshl.edu

This work was supported by the National Institutes of Health (MH085926 to H.F.), and a Mirus Research Award, a Robertson Research Fund of Cold Spring Harbor Laboratory, and support frfom the National Institute of Mental Health, all to H.F.

[2] See: Huixian $\mathrm{Wu}^{1}$, Chong Wang ${ }^{1}$, Karen J. Gregory2,3, Gye Won Han', Hyekyung P. Cho ${ }^{2}$, Yan Xia ${ }^{2}$, Colleen M. Niswender ${ }^{2}$, Vsevolod Katritch ${ }^{1}$, Jens Meiler $^{2}$, Vadim Cherezov ${ }^{1}$, P. Jeffrey Conn $^{2}$, and Raymond C. Stevens ${ }^{1 *}$, "Structure of a Class C GPCR Metabotropic Glutamate Receptor 1 Bound to an Allosteric Modulator," Science 344, 58 (4 April 2014).

\section{DOI: 10.1126/science.1249489}

Author affiliations: ${ }^{1}$ The Scripps Research Institute, ${ }^{2}$ Vanderbilt University Medical Center, ${ }^{3}$ Monash University Correspondence: * stevens@usc.edu

Supported by National Institute of General Medical Sciences PSI:Biology grant U54 GM094618 for biological studies and structure production (target GPCR-68) (V.K., V.C., and R.C.S.); National Institutes of Health (NIH) Common Fund in Structural Biology grant P50 GM073197 for technology devel- opment (V.C. and R.C.S.); NIH grants R01 NS031373 (P.J.C.), R01 MH062646 (P.J.C.), and R21 NS078262 (C.M.N.); a basic research grant from the International Rett Syndrome Foundation (C.M.N.); an NHMRC (Australia) Overseas Biomedical postdoctoral fellowship (K.J.G.); and a NARSAD Maltz Young Investigator award (K.J.G.).

Both studies used resources of the Advanced Photon Source, a U.S. Department of Energy (DOE) Office of Science User Facility operated for the DOE Office of Science by Argonne National Laboratory under Contract No. DE-AC02-06CH11357.

23-ID-B • GM/CA-XSD • Life sciences • Macromolecular crystallography, microbeam, large unit cell crystallography, subatomic $(<0.85 \AA$ ) resolution, multi-wavelength anomalous dispersion, single-wavelength anomalous dispersion - 3.5-20 keV • On-site, remote $\cdot$ Accepting general users •

23-ID-D • GM/CA-XSD • Life sciences • Macromolecular crystallography, microbeam, large unit cell crystallography, subatomic $(<0.85 \AA$ ) resolution, multi-wavelength anomalous dispersion, single-wavelength anomalous dispersion • 5-20 keV • On-site, remote - Accepting general users • 


\section{How to Remove Tangles in Your DNA}

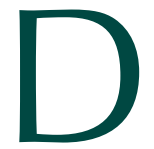

NA damage is a fact of life. On any given day, an organism's DNA will suffer between 10,000 and $1,000,000$ breaks or other damage. These problems are repaired by enzymes in our cells that fix the breaks, remove errors, and maintain the integrity of the genome. One of these DNA repair enzymes acts as a kind of molecular scissors to cut DNA at damage points and resolve tangles that can form when things go wrong. This must be done with great specificity in order to restore the DNA code to its previous state and not generate mutations. Researchers utilizing the APS and the Pohang Accelerator Laboratory in South Korea solved the structure of one of these molecular scissor proteins, called Mus81-Eme1, in a complex with DNA. Their analysis reveals the elegant way the enzyme recognizes, positions, and cuts the DNA, and then secures the two ends to avoid a new tangle and furthers our understanding of the mechanisms of DNA repair, with positive implications for fields such as reproduction, cancer, and aging.

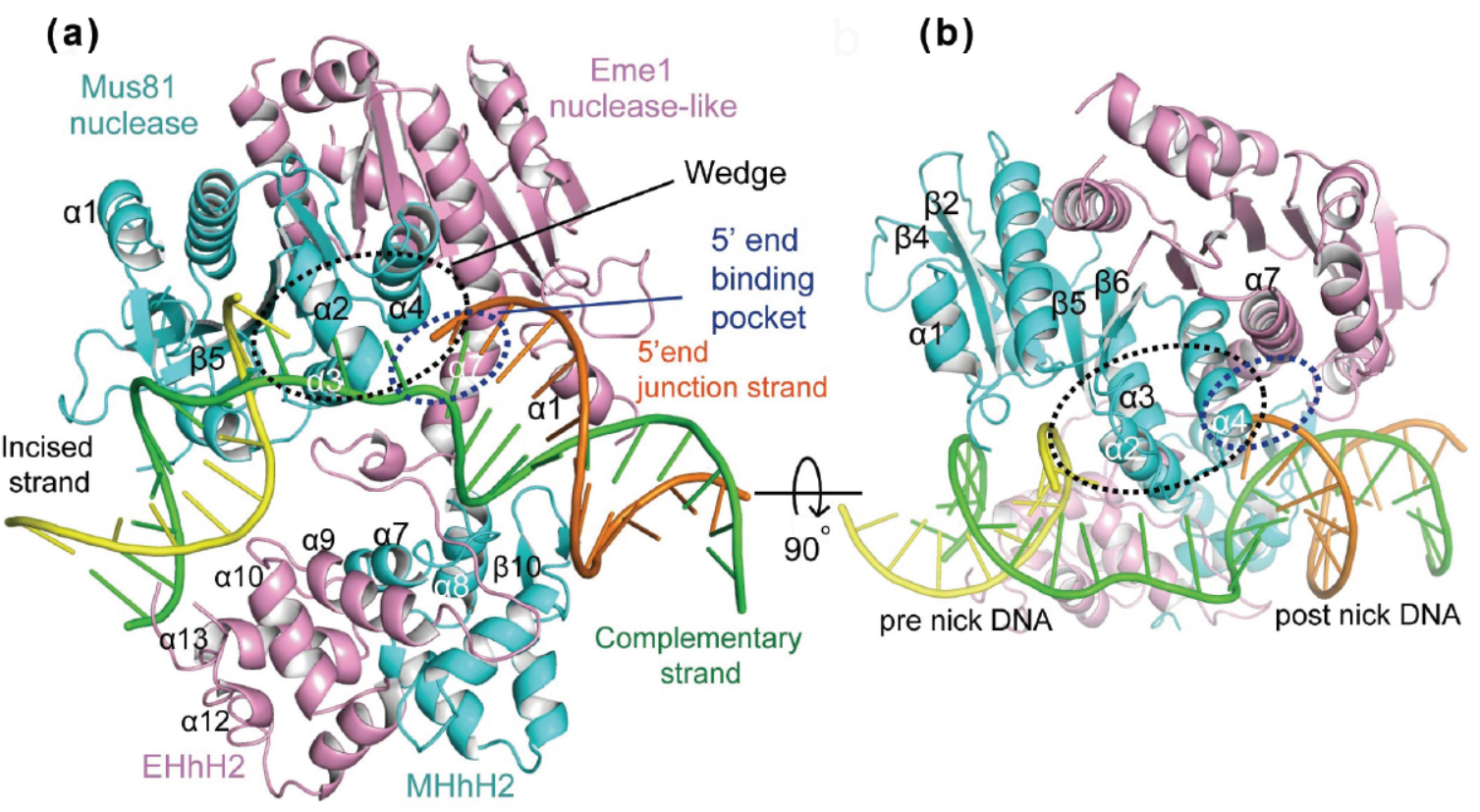

Fig. 1. a) A complex structure containing Mus81 (cyan) Eme 1 (pink) bound to a 3' flap DNA. The DNA strand to be cut is shown in yellow, the $5^{\prime}$ end (orange) is shown in the $5^{\prime}$ binding pocket (blue dotted circle) and the complementary DNA strand is shown in green. The wedge is shown as a black dotted circle. b) A $90^{\circ}$ rotated view of 1 a. On the right and left of the wedge, the $5^{\prime}$ junction of the $5^{\prime}$ end junction strand and the $3^{\prime}$ end of the strand to be cut are placed at the $5^{\prime}$ end binding pocket and the active site, respectively.

19-ID-D • SBC-CAT $・$ Life sciences $・$ Macromolecular crystallography, multi-wavelength anomalous dispersion, subatomic $(<0.85 \AA)$ resolution, microbeam, ultra-low-temperature (15K), large unit cell crystallography, singlewavelength anomalous dispersion • 6.5-19.5 $\mathrm{keV} \cdot$ On-site, remote, mail-in $\bullet$ Accepting general users • 
Most genes that have been found to affect lifespan are involved in DNA repair. If breaks and other DNA damage are not repaired, disease can result from adverse effects on normal gene expression and cell division functions that are critical to life.

One type of enzyme involved in DNA repair is a molecular scissors called an endonuclease. Mus81-Eme1 is one of a family of structure-selective endonucleases responsible for resolution of complex DNA overlaps that are the result of DNA damage. These overlaps, or flaps, come in two different flavors, $5^{\prime}$ and $3^{\prime}$ (pronounced "5-prime" and "3-prime" in DNA parlance) depending on the direction of the DNA break that occurred.

The researchers from the Pohang University of Science and Technology (South Korea) and Argonne were interested in why the Mus81-Eme1 endonuclease specifically recognizes only the $3^{\prime}$ flaps and not the $5^{\prime}$ flaps. To study this, they crystallized Mus81-Eme1 in complex with small pieces of DNA with either $3^{\prime}$ or $5^{\prime}$ flaps and collected diffraction data for the crystals at the SBC-CAT beamline 19-ID-D at the APS, and at the Pohang Accelerator Laboratory.

As one might expect for an enzyme that performs an important basic DNA repair function, Mus81-Eme1 works with elegance and precision. The team compared structures for Mus81Eme1 in complex with one $5^{\prime}$ flap DNA and two $3^{\prime}$ flap DNAs. The $5^{\prime}$ flap structure showed that the DNA could bind to Mus81-Eme1 but the binding did not result in cutting of the DNA. Analysis of the $3^{\prime}$ flap structures revealed why.

When Mus81-Eme1 binds to the $3^{\prime}$ flap DNAs it undergoes a dramatic shape change and convertst from a compact structure to a more open structure (Fig. 1), unmasking a protein domain that acts as a wedge to keep the two cut ends separated. The $5^{\prime}$ end that is not being cut is held in place by a specialized binding pocket that keeps the end away from the action.

Next, Mus81-Eme1 bends the DNA to position the $3^{\prime}$ end right in the active site. Biochemically, the $5^{\prime}$ flaps don't work because the free $3^{\prime}$ ends don't fit properly into the binding pocket and so the DNA doesn't bend to place the $5^{\prime}$ end into the active site.

The team validated their model in two ways. First, they confirmed reduced enzyme activity in mutant versions of Mus81-Eme1 based on structure model predictions. Second, in experiments in which they labeled both ends of the DNA with fluorescent tags, the addition of Mus81-Eme1 resulted in the two ends of the DNA moving closer together, supporting the bending formation in the structure.

What's next? Because endonucleases like Mus81-Eme1 are responsible for repair of some of the most complex types of DNA damage, when these are not repaired due to a malfunction of an endonuclease, serious developmental and genetic disease can occur. The next step for the team will be to study the structures of other members of this endonuclease family, including one involved in a disease called Fanconi anemia, in order to provide a frame-work for curing these diseases.

$$
\text { - Sandy Field }
$$

See: Gwang Hyeon Gwon ${ }^{1}$, Aera Jo ${ }^{1}$, Kyuwon Baek ${ }^{1}$, Kyeong Sik Jin ${ }^{1}$, Yaoyao Fu${ }^{1}$, Jong-Bong Lee ${ }^{1}$, YoungChang $\mathrm{Kim}^{2}$, and Yunje Cho ${ }^{1 *}$, "Crystal structures of the structureselective nuclease Mus81-Eme1 bound to flap DNA substrates," EMBO J. 33 (9), 937 (2014).

DOI: 10.1002/embj.201487820

Author affiliations: ${ }^{1}$ Pohang University of Science and Technology, ${ }^{2}$ Argonne National Laboratory

Correspondence: * yunje@postech.ac.kr

This work was supported by grants from the National R\&D Program for Cancer Control, the Ministry for Health and Welfare (1020280), a National Research Foundation of Korea grant funded by the Korea government (MEST, No. 2012004028, No. 2012-054226, and No. 20120008833), a rising star program (POSTECH), and the BK21 program (Ministry of Education). SBCCAT is funded by the U.S. Department of Energy (DOE) Office of Biological and Environmental Research. This research used resources of the Advanced Photon Source, a U.S. DOE Office of Science User Facility operated for the DOE Office of Science by Argonne National Laboratory under Contract No. DE-AC02-06CH11357.

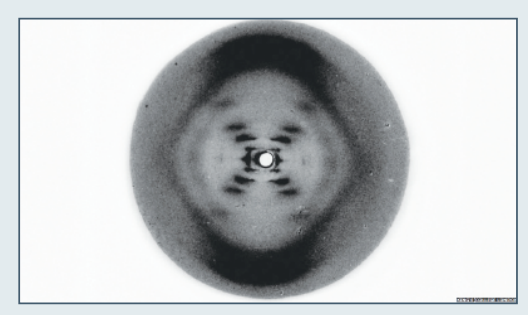

Rosalind Franklin was a brilliant x-ray crystallographer whose photograph of a fiber of DNA [above] was critical to James Watson and Francis Crick's discovery of the double helix. Her x-ray studies of DNA provided data that helped to bring into being the theoretical model constructed by Watson and Crick.

Her colleague at King's College, Maurice Wilkins, showed her photograph of the "B" form of DNA to Watson early in 1953 , when she was preparing to leave King's to start a new project at Birkbeck College, London. Watson describes his reaction in The Double Helix: "The instant I saw the picture my mouth fell open and my pulse began to race... the black cross of reflections which dominated the picture could arise only from a helical structure... mere inspection of the $\mathrm{x}$-ray picture gave several of the vital helical parameters." ... The laboratory notebooks in which Franklin set out her findings and noted her own thoughts about the structure... contain annotations made ten years after her untimely death by her colleague Aaron Klug, who published a paper in Nature in 1968 showing that she had been ahead of Watson and Crick in establishing key parameters of the structure.

DNA was only an interlude in Franklin's career. She had made important contributions to the study of carbon at the British Coal Utilisation Research Association; subsequently she used her skills to reveal the hollow centre of the tobacco mosaic virus particle, and to trace the helical form of its genetic material within this cylinder.

Excerpted from: "Codebreakers: Makers of Modern Genetics, The Rosalind Franklin papers," the Wellcome Library, http://wellcomelibrary.org 


\section{A Novel Mechanism of DNA Demethylation}

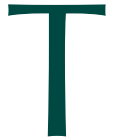

he Tet family of enzymes regulates gene expression through chemical modification (demethylation) of DNA, turning specific genes "on" for important cellular events during development and cell proliferation. To understand how the Tet enzymes interact with DNA and perform the chemical reactions required for gene regulation, researchers from the Emory University School of Medicine, New England Biolabs, the University of Georgia, and SER-CAT used x-ray crystallography at the SER-CAT 22-BM-D and 22ID-D beamlines of the APS to a resolution of $2.9 \AA$. to obtain high-resolution snapshots of the Naegleria Tet-like dioxygenase (NgTet1) in complex with 5-methylcytosine (5mC) DNA (Fig. 1). The findings show that Tet enzymes interact with DNA in a fashion distinct from other DNA modifying enzymes. Understanding how Tet enzymes interact with target DNA is important for understanding of their regulation and for potential development of inhibitors to modulate their activity in contexts ranging from brain development to cancer.

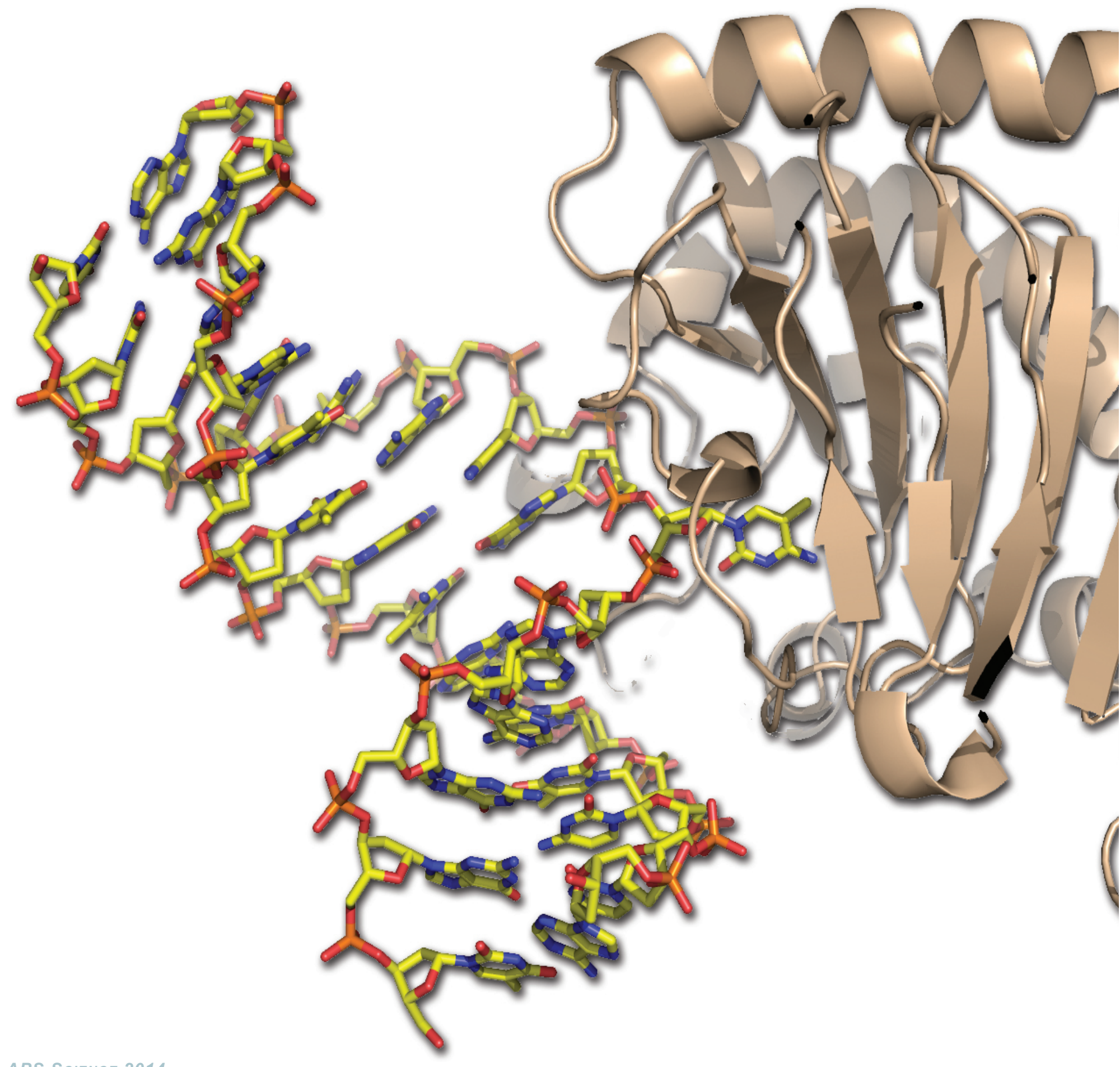


The important roles of the Tet family of enzymes have only recently emerged. These enzymes are dioxygenases, which bind to DNA with a chemically modified building block, a 5methylcytosine $(5 \mathrm{mC})$, and catalyze its conversion to three possible products, 5-hydroxymethylcytosine (5hmC), 5formylcytosine $(5 \mathrm{fC})$, and 5-carboxylcytosine $(5 \mathrm{caC})$ through three consecutive oxidation reactions. By carrying out this reaction, Tet-dioxygenase chemically changes DNA, turning the expression of genes from "off" to "on." Control of both the timing and level of gene expression is critically important during development, and in the control of cell growth and differentiation. Indeed, Tet is required for maintaining the developmental plasticity of stem cells, is involved in early embryonic development, brain development, and is implicated in some cancers.

The Tet-dioxgenases are present throughout the tree of life in a wide range of organisms. The free-living amoeboflagellate Naegleria gruberi possesses a Tet protein (NgTet1) remarkably similar to that present in humans. This protein was used in the current study to model Tet interactions with DNA, and the findings here can be applied to understanding the human Tet proteins.

The high-resolution crystal structures obtained at the SER-CAT beamlines reveled that $\mathrm{NgTet} 1$ interacts with the DNA through interactions with the basic surface of the protein (Fig. 1). This surface interacts with the negatively charged phosphate backbone of the DNA in the minor groove of the DNA helix. This interaction causes the DNA phosphate backbone surrounding

$<$ Fig. 1. The structure of the Naegleria gruberi Tet protein ( $\mathrm{NgTet} 1$, beige) in complex with DNA containing a $5 \mathrm{mC}$ cytosine 5' to a Guanine ( $5 \mathrm{mCpG}$ dinucleotide). Molecular components of DNA are indicated: carbon (yellow), phosphate (orange), oxygen (red) and nitrogen (blue). NgTetl binding to the DNA minor groove bends the DNA towards the major groove, flipping out the $5 \mathrm{mC}$ base into the active site of the protein. the $5 \mathrm{mC}$ to kink, flipping out the $5 \mathrm{mC}$ base into the active site of $\mathrm{NgTet1}$.

Once in the active site, this base undergoes chemical reactions that result in the demethylation of the DNA. The current work describes the first detailed molecular structure of a Tet enzyme and describes the unique way that this enzyme induces base flipping, which is distinct from the mode employed by other DNA repair and modifying enzymes.

Understanding the regulation and structural bases of Tet activity provides a platform for researchers to develop inhibitors that modulate Tet in contexts beneficial to human health.

\section{- Emma Nichols}

See: Hideharu Hashimoto ${ }^{1}$, June E. Pais $^{2}$, Xing Zhang ${ }^{1}$, Lana Saleh ${ }^{2}$, Zheng-Qing Fu ${ }^{3,4}$, Nan Dai ${ }^{2}$, Ivan R. Correâ, Jr. ${ }^{2}$, Yu Zheng ${ }^{2 * *}$, and Xiaodong Cheng $1^{*}$, "Structure of a Naegleria Tet-like dioxygenase in complex with 5-methylcytosine DNA," Nature 506, 391 (20 February 2014).

DOI: 10.1038/nature12905 Author affiliations: ${ }^{1}$ Emory University School of Medicine, ${ }^{2}$ New England Biolabs, ${ }^{3}$ University of Georgia, ${ }^{4}$ SER-CAT Correspondence:

*xcheng@emory.edu,

This work was supported by grants from the National Institutes of Health GM049245 to X.C. (who is a Georgia Research Alliance Eminent Scholar) and GM095209 and GM105132 to Y.Z. SER-CAT supporting institutions may be found at www.ser-cat.org/ members.html. This research used resources of the Advanced Photon Source, a U.S. Department of Energy (DOE) Office of Science User Facility operated for the DOE Office of Science by Argonne National Laboratory under Contract No. DE-AC02$06 \mathrm{CH} 11357$.

22-BM-D • SER-CAT • Life sciences • Macromolecular crystallography $\bullet 8-20 \mathrm{keV} \cdot \mathrm{On}$ site, remote $\cdot$ Accepting general users $\bullet$

22-ID-D • SER-CAT • Life sciences • Macromolecular crystallography, multi-wavelength anomalous dispersion, microbeam $\bullet 6-20 \mathrm{keV}$ - On-site, remote - Accepting general users • 


\section{Generating More Efficient and Stable BISPECIFIC ANTIBODIES}

$\mathrm{B}$

ispecific antibodies (BsAbs) are an emerging class of biological therapeutic agents designed to bind to two distinct regions on either the same or different targets, and therefore improve the body's efficiency in fighting disease. However, their development for clinical use has been slowed, particularly due to issues with their structure that allow mispairing between the heavy and light chain regions of the antibody. Researchers in this study designed a computer-assisted experimental approach, and using the LRL-CAT beamline 31-ID-D at the APS to collect x-ray diffraction data, they produced novel mutant antibodies that were more stable and had the desired structure to reduce this indiscriminate pairing. These findings will be important in guiding future work to design new agents for varied therapeutic use.

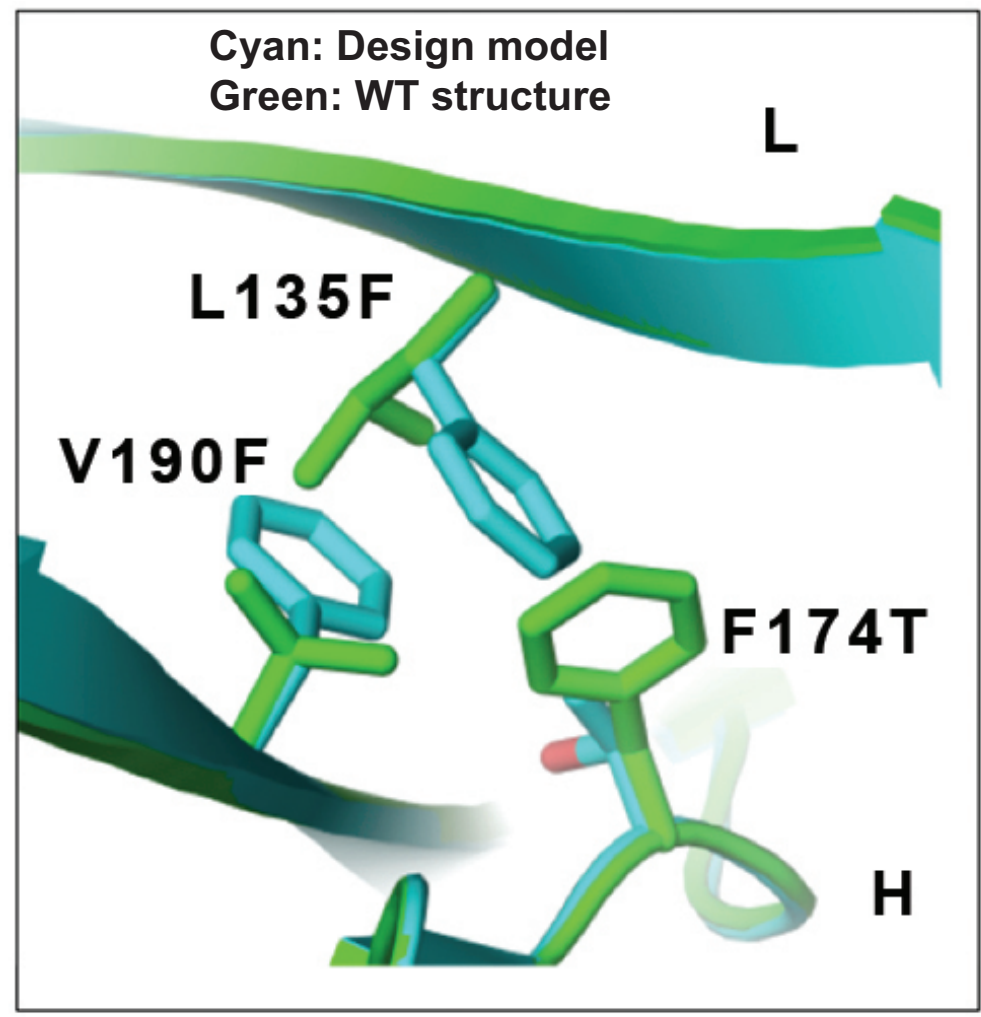

Fig. 1. This page and next: Computer-based redesign of amino acid packing at the interface between an antibody light chain (top, L) and an antibody heavy chain (bottom, H). The panel on this page compares the computer model with the starting antibody structure (wildtype, WT). The panel on the facing page shows experimental validation of the computer model via a high-resolution crystal structure solved at the APS. 
Monoclonal antibodies (MAbs) have become an increasingly important source of therapeutic agents in recent years. These are antibodies that can recognize a single target antigen. However, BsAbs recognize two different antigens at the same time and can therefore bridge two distinct targets. This produces opportunities for increased therapeutic impact.

Although BsAbs have been produced for clinical use using a variety of approaches, production methods have been hindered by problems of manufacturability and issues with their physical and chemical properties.

Antibodies, or immunoglobulins, are large Y-shaped proteins used by the immune system to attack foreign substances such as bacteria, viruses, and even cancer cells. The two basic structural units of an antibody are heavy chains and light chains. The antibody recognizes a specific part (antigen) of the foreign substance - the tip of the " $Y$ " contains a region that is specific for a particular part (epitope), on a target antigen, allowing them to bind together very precisely. The fragment antigen-binding (Fab fragment) is the region that binds to antigens, and contains one constant and one variable domain region of each of the heavy and light chain.

The "variable region" at the tip is made up of amino acids belonging to both light and heavy chains. This region also binds to antigen, but allows for variations in amino acid components, thereby enabling millions of antibodies with slightly different antigen-binding sites to exist. As a result of this, antibody heavy chains have evolved to bind light chains in a relatively nonspecific manner. This can be a major challenge with respect to generating BsAbs for clinical use, because this indiscriminate pairing leads to production of non-functional BsAbs. Specific pairing is essential for their optimal manufacturability and effectiveness.
Because Fab fragments can be produced in the laboratory, one solution to potentially produce a more specific BsAb and prevent mispairing of heavy and light chains involves the design of an antibody Fab with a fully orthogonal (perpendicularly arranged) structural interface where the heavy and light chain connect. Re-engineering a specific area of the antibody, known as the $\mathrm{CH} 3$ domain, plays a key role in efficient heavy chain pairing.

In this study, researchers from the the University of North Carolina at Chapel Hill and the Eli Lilly Biotechnology Center used a combination of computational and rational design methods to produce novel BsAbs with the desired structure. This computer-aided

The researchers identified mutant antibodies that were more stable and prevented mispairing of heavy and light chains, facilitating expression of correctly assembled BsAbs using a variety of parental mAbs containing the mutant Fab interface.

These results will be important in guiding future work to investigate whether this strategy can be applied successfully to all antibodies, and may hold the potential to generate powerful therapeutic agents for the effective treatment of complex diseases. - Nicola Parry

See: Steven M. Lewis ${ }^{1}$, Xiufeng $\mathrm{Wu}^{2}$, Anna Pustilnik ${ }^{2}$, Arlene Sereno ${ }^{2}$, Flora Huang ${ }^{2}$, Heather L. Rick ${ }^{1}$, Gurkan Guntas $^{1}$, Andrew Leaver-Fay ${ }^{1}$, Eric M. Smith², Carolyn $\mathrm{Ho}^{2}$, Christophe HansenEstruch$^{2}$, Aaron K. Chamberlain², Stephanie M. Truhlar $^{2}$, Elaine M. Conner ${ }^{1}$, Shane Atwell ${ }^{2}$, Brian Kuhlman $^{1 *}$, and Stephen J. Demarest $^{2 * *}$, "Generation of bispecific IgG antibodies by structure-based design of an orthogonal Fab interface," Nat. Biotechnol. 32(2), 191 (February 2014). DOI: 10.1038/nbt.2797 Author affiliations: ${ }^{1}$ University of North Carolina at Chapel Hill, ${ }^{2}$ Eli Lilly Biotechnology Center Correspondence:

*bkuhlman@email.unc.edu, ** demarestsj@lilly.com

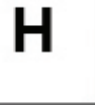

approach involved choosing a suitable antibody to act as a protein "scaffold" from which new mutant Fabs were engineered following specific changes to the structure of the original scaffold. The researchers inserted mutations into the $\mathrm{CH} 3$ domains of various parental mAbs to allow expression of correctly paired BsAbs.

Using x-ray diffraction data sets collected from the 31-ID-D beamline, they were then able to validate the three dimensional crystal structures of the newly produced proteins (Fig. 1).
Program. Use of the LRL-CAT beamline was provided by Eli Lilly Company, which operates the facility. This research used resources of the Advanced Photon Source, a U.S. Department of Energy (DOE) Office of Science User Facility operated for the DOE Office of Science by Argonne National Laboratory under Contract No. DEAC02-06CH11357.

31-ID-D • LRL-CAT • Life sciences • Macromolecular crystallography, single-wavelength anomalous dispersion, single-crystal diffraction $\cdot 4.7-28 \mathrm{keV} \cdot$ Mail-in • Accepting general users • 


\section{The Viral Secrets of Replication AND IMMUNE SYSTEM EVASION}

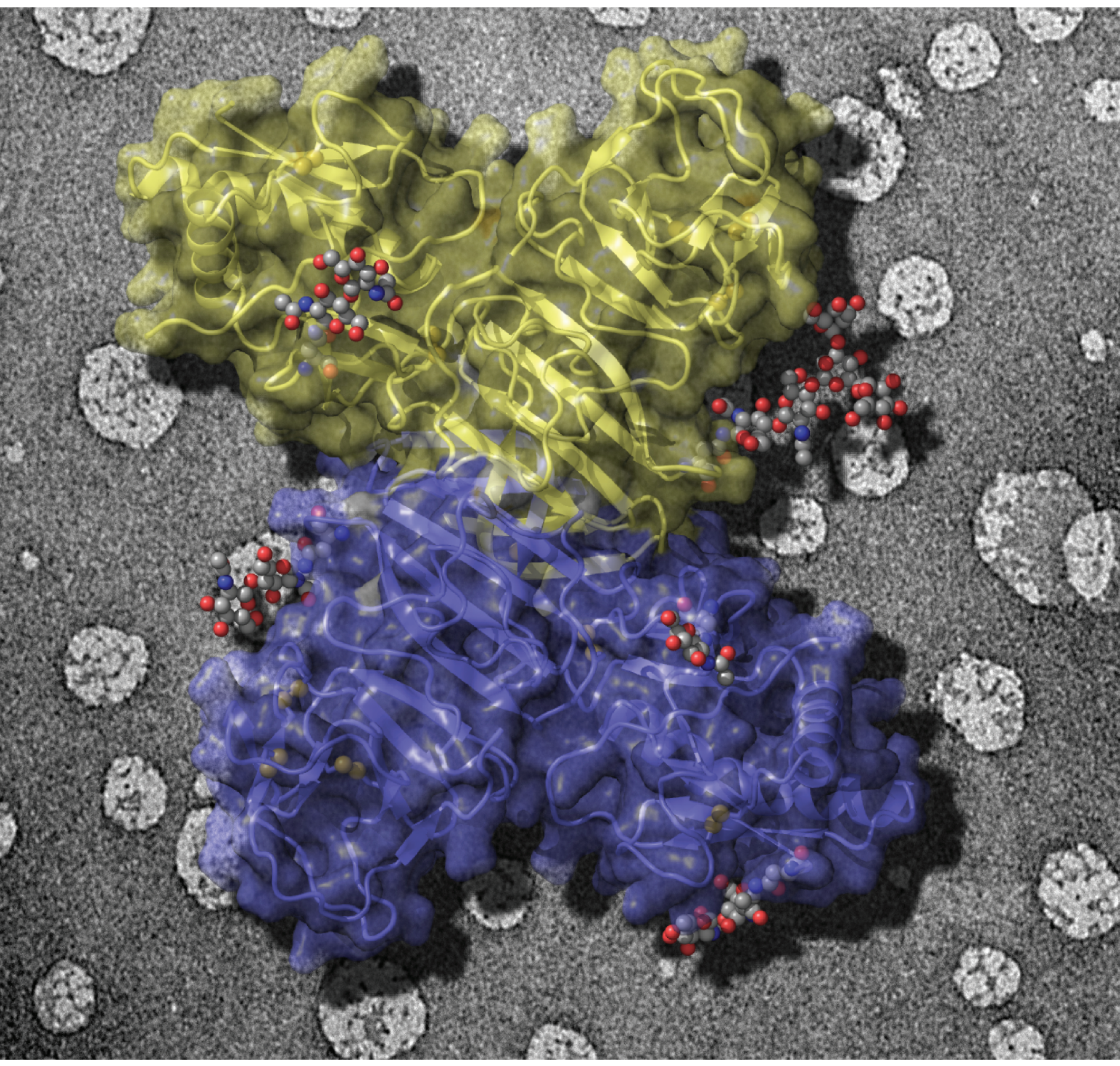

Fig. 1. The external face of the flavivirus nonstructural protein 1 (NS1) dimer (subunits in blue and yellow) is exposed on infected cell surfaces where it can interact with the immune system. NS1 contains sugar moieties (grey balls). NSI's external face is also exposed in secreted NS1 particles present in patient sera. The background image shows artificial spherical membrane particles coated with the NS1 protein. Image: David Akey and Somnath Dutta, both University of Michigan 
A mosquito or tick bite may trigger more than an itchy welt; these bloodsuckers can transmit flaviviruses, which cause dengue fever and West Nile fever, among other illnesses. No vaccine exists that can curb their spread. The dengue virus alone may infect more than four hundred million people each year. Scientists trying to decipher how flaviviruses replicate inside a host's cells and evade detection by the immune system have honed in on a flavivirus protein called nonstructural protein 1 (NS1). Evidence suggests that NS1 is involved in both the replication of the viral genome and the manipulation of the host immune system that allows the viruses to spread from cell to cell. However, the structure of this enticing molecule had long remained a mystery because NS1 proved difficult to produce in the laboratory and its sequence doesn't match that of any protein with a known structure. But a research team from the University of Michigan, GM/CA-XSD, and Purdue University achieved a breakthrough that allowed them to make NS1 in the lab. They then proceeded to solve the protein's three-dimensional structure with x-ray diffraction data collected at the APS (Fig. 1). The molecular structure reveals the parts of NS1 involved in the replication and spread of the flavivirus, providing targets that may help speed the development of vaccines and antiviral medications.

Scientists have been trying to make NS1 crystals for 15 to 20 years, with little success. The problem was that NS1 has a hydrophobic surface, which encourages the molecule to stick to membranes and not let go. Several years of research by members of this team were required to determine the appropriate protocol for production of NS1. The final "a-ha" moment came when they treated broken cells from the expression culture with a mild detergent, gently convincing the membranes to release NS1. After that, crystallization was relatively straightforward.

The researchers next visited the GM/CA-XSD beamline 23-ID-D at the APS and proceeded to collect data on 18 NS1 crystals, ending up with more than 100 data points per diffraction spot. Due to the lack of structural homologues, they relied on anomalous scattering from NS1's native sulfurs in 12 cysteines and 5 methionines to elucidate the structure. The researchers solved the structure of NS1 from West Nile virus, but found that crystals from the dengue virus NS1 yielded an identical structure. As the primary sequence suggested, the structure of NS1 is unlike any other known protein structure.

Previous research indicated that, early in infection, NS1 forms a dimer inside a host's cell and interacts with the viral replication complex on the opposite surface of the endoplasmic reticulum membrane. To explore NS1's role in viral replication, the team mixed the protein with liposomes, as a proxy for endoplasmic reticulum membranes. NS1 coated the liposomes and broke the vesicles apart, forming smaller lipoprotein nanoparticles. This observation suggested that NS1 may organize the viral replication complex through membrane remodeling. Using the structure as a guide, the researchers identified the greasy face of NS1 most likely to interact with the membrane-bound replication complex.

In addition to its intracellular functions, NS1 carries a secretion tag that sends the protein outside the cell and into the bloodstream. While NS1 is a dimer inside of cells, evidence from electron microscopy shows that NS1 forms a hexamer in the extracellular space. The NS1 hexamer interacts with immune system components, suggesting that the function of extracellular NS1 is to trick the immune system into ignoring the infection. Previous studies showed that human antibodies, immune system proteins, recognize 108 different pieces of the NS1 protein. To identify the parts of NS1 involved in immune system manipulation, the researchers mapped these 108 epitopes onto the structure of hexameric NS1. The epitopes clustered into a few "hotspots," all on a face of the protein opposite to the one used in membrane remodeling. One hotspot resembled a human protein involved in the defense against flaviviruses. This suggests that NS1 may thwart the immune system via protein mimicry, though more research is needed to tease out the details.

- Erika Gebel Berg

See: David L. Akey ${ }^{1 *}$, W. Clay Brown ${ }^{1 *}$, Somnath Dutta ${ }^{1}$, Jamie Konwerski ${ }^{1}$, Joyce Jose ${ }^{2}$, Thomas J. Jurkiw ${ }^{1}$, James DelProposto ${ }^{1}$, Craig M. Ogata ${ }^{3}$, Georgios Skiniotis ${ }^{1}$, Richard J. Kuhn², and Janet L. Smith ${ }^{1 *}$, "Flavivirus NS1 Structures Reveal Surfaces for Associations with Membranes and the Immune System," Science 343, 881 (21 February 2014).

DOI: 10.1126/science.1247749

Author affiliations: ${ }^{1}$ University of Michigan, ${ }^{2}$ Purdue University, ${ }^{3} \mathrm{GM} / \mathrm{CA}-\mathrm{XSD}$, Correspondence:

* janetsmith@umich.edu

This work was supported by a grant from the National Institutes of Health (P01AI055672) to R.J.K. and J.L.S., the Martha L. Ludwig Professorship of Protein Structure and Function to J.L.S., the Pew Scholar Program in Biomedical Sciences to G.S., and a Perrigo Undergraduate Summer Fellowship to T.J.J. GM/CA-XSD has been funded in whole or in part with Federal funds from the National Cancer Institute (ACB-12002) and the National Institute of General Medical Sciences (AGM-12006).

23-ID-D • GM/CA-XSD • Life sciences • Macromolecular crystallography, microbeam, large unit cell crystallography, subatomic $(<0.85 \AA)$ resolution, multi-wavelength anomalous dispersion, single-wavelength anomalous dispersion $\cdot 5-20 \mathrm{keV} \cdot$ On-site, remote -Accepting general users • 


\section{A Common Form of Protein Modification THAT IS ABUNDANT IN BACTERIA}

A nimals can rapidly adapt to changes in the environment through subtle changes to the structure of their bodies' proteins. This process is referred to as "post-translational modification," which can impact the function, structure, stability, and location of proteins involved in essential cellular processes, including translation, transcription and metabolism. Such modifications give organisms the flexibility to adapt rapidly to changes in their environment. One ubiquitous form of post-translational modifications is acetylation, the donation of an acetyl functional group from acetyl coenzyme A to a lysine residue. X-ray crystallography studies at the APS helped reveal that acetylation of Escherichia coli proteins can also be non-enzymatic, specific, and occurs at both active sites and co-factor binding sites. Additional experiments demonstrated that acetylation is likely capable of modifying the function of important enzymes, a finding that could have implications for the engineering of bacteria for specific applications in a variety of fields such as energy production, medicine, and environmental remediation.

Acetylation can have a variety of impacts on cellular processes, including altering protein size, DNA binding affinity, and enzymatic activity. While acetylation has been well documented in eukaryotes, only in the past few years have researchers obtained genetic, mass spectrometric, chemical, and structural evidence of acetylation of thousands of lysine residues in hundreds of $E$. coli proteins, suggesting that acetylation is every bit as critical and widespread in bacteria as it is in eukaryotes. Multiple threads of evidence also indicated that the molecule acetyl phosphate can function as an acetyl donor in bacteria, along with the better known acetyl coenzyme A.

One type of post-translational modification is $\mathrm{Ne}$-lysine acetylation, in which an enzyme catalyzes the donation of an acetyl group from acetylcoenzyme A (acCoA) to the $\varepsilon$-amino group of a lysine residue within a protein. This is known to be very common in eukaryotes, but has only recently been shown to occur in bacteria. However, the prevalence of acetylation and its mechanism(s) had not yet been examined in detail.

The research team in this study, which included scientists from Northwestern University Feinberg School of Medicine; Loyola University; the Buck Institute for Research on Aging; North- western University; and the University of California, San Francisco had previously discovered an E. coli mutant with markedly different acetylation patterns compared to wild type. The mutant accumulates a molecule called acetyl phosphate $(\mathrm{acP})$, leading the scientists to hypothesize that acP regulates acetylation. In the current study, increased acetylation in the presence of glucose was observed in the $E$. coli acP-accumulating mutants. In contrast, acetylation decreased, but was not eliminated, in other mutants that could not synthesize acP, results that confirmed the team's hypothesis.

Mass spectrometry and Western immunoblot analysis revealed thousands of acetylation sites on hundreds of lysine proteins across a handful of $E$. coli strains. Most of the acetylated proteins contained one or two acetylated lysines, but some contained as many as 24 . These experiments also verified the existence of both acP-dependent and acP-independent acetylation. Other mass spectrometry experiments indicated that acP can act directly as an acetyl donor without the need of an enzyme such as lysine acetyltransferase. Because acP is a small molecule it can access active sites that most enzymes cannot, leading the researchers to speculate that acP could have a role in acetylating, and thereby inhibiting, mol- ecules such as RNA polymerase.

Searching through the gene function database PANTHER revealed that the most common functions of the observed acetylated lysines were metabolic in nature. AcP-driven acetylation was also found to target proteins involved in translation and transcription. Using the LS-CAT 21-ID-D, 21-ID-F, and 21-ID-G beamlines at the APS, the researchers derived $x$-ray crystal structures of two of these proteins, triosephosphate isomerase (TpiA) and glyceraldehyde-3-phosphate dehydrogenase (GapA), both of which are involved in glycolysis [Fig.1(a) and (b)]. Comparing the three-dimensional structures before and after briefly bathing the proteins in acP confirmed that acP can bind lysine in the absence of an enzyme and can do so at both active sites and co-factor binding sites.

Given the geometry of acP binding sites observed in the crystal structures, as well as previously reported effects of acetylation in other organisms, the research team inferred that acetylation would likely inhibit the enzymatic activity of both TpiA and GapA.

The finding that acetylation of bacterial proteins involved in crucial cellular processes is commonplace, and likely carries functional consequences, opens avenues for custom engineering microbes used in a variety of fields. The 
research team plans to investigate how acetylation impacts protein function, as well as determine how the process is regulated within living bacteria.

- Chris Palmer

See: Misty L. Kuhn ${ }^{1}$, Bozena Zemaitaitis ${ }^{2}$, Linda I. $\mathrm{Hu}^{2}$, Alexandria Sahu $^{3}$, Dylan Sorensen ${ }^{3}$, George Minasov ${ }^{1}$, Bruno P. Lima ${ }^{2 \ddagger}$, Michael Scholle$^{4}$, Milan Mrksich ${ }^{4}$, Wayne F. Anderson ${ }^{1}$, Bradford W. Gibson ${ }^{3,5}$, Birgit Schilling ${ }^{3}$, and Alan J. Wolfe ${ }^{2 *}$, "Structural, Kinetic and Proteomic Characterization of Acetyl Phosphate-Dependent Bacterial Protein Acetylation," PLoS One 9(4), e94816 (2014).

DOI: 10.1371/journal.pone.0094816 Author affiliations: ${ }^{1}$ Northwestern University Feinberg School of Medicine, ${ }^{2}$ Loyola University Chicago, ${ }^{3}$ Buck Institute for Research on Aging, ${ }^{4}$ Northwestern University, ${ }^{5}$ University of California, San Francisco ¥Present address: University of California, Los Angeles Correspondence: *awolfe@luc.edu

This work was supported by grants from the National Institutes of Health (NIH), National Institute of Allergy and Infectious Diseases (NIAID), and National Institute of Diabetes and Digestive and Kidney Diseases (NIDDK); including R24 DK085610 (NIDDK, to B.W.G.), R01 GM066130 (National Institute of General Medical Sciences, to A.J.W.), Department of Health and Human Services Contracts HHSN272200700058C and HHSN272201200026C (NIAID, NIH, Department of Health and Human Services to W.F.A.) and U54CA151880 (National Cancer Institute, to M.M.). The use of LS-CAT Sector 21 was supported by the Michigan Economic Development Corporation and Michigan Technology Tri-Corridor (Grant 085P100817). This research used resources of the Advanced Photon Source, a U.S. Department of Energy (DOE) Office of Science User Facility operated for the DOE Office of Science by Argonne National Laboratory under Contract No. DE-AC02-06CH11357.

21-ID-D • LS-CAT • Life sciences • Macromolecular crystallography, microfluorescence (hard x-ray), nanofluorescence imaging, nanotomography $\cdot 6.5-20 \mathrm{keV} \cdot$ On-site, remote, mail-in - Accepting general users •

21-ID-F/21-ID-G • LS-CAT • Life sciences • Macromolecular crystallography • $12.7 \mathrm{keV}$ • On-site, remote, mail-in - Accepting general users •

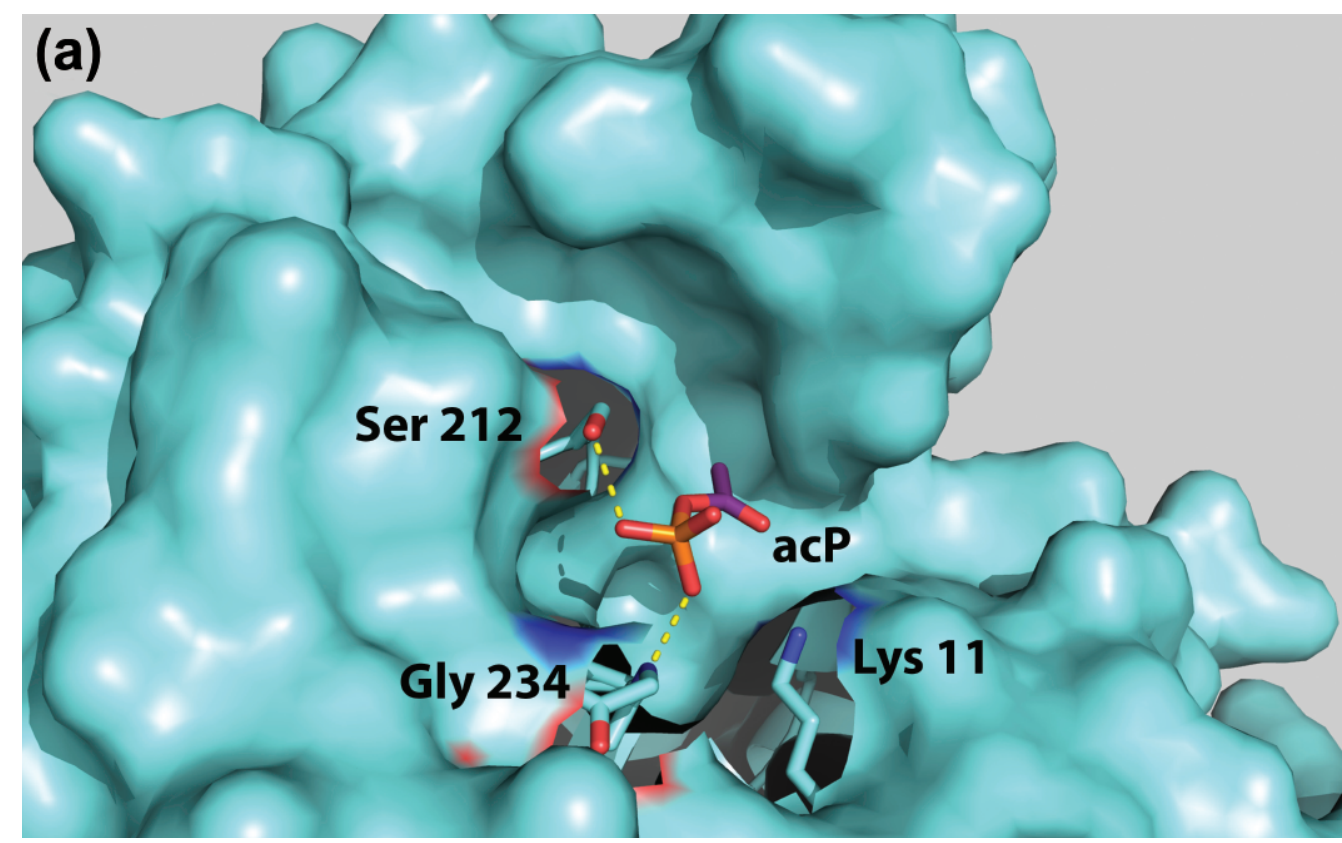

Fig. 1. Three-dimensional structures of glycolytic enzymes reveal that acetyl-phosphate (acP) can acetylate bacterial proteins non-enzymatically. (a) above: Surface representation of E. coli triosephosphate isomerase (TpiA) with acP bound in the active site. Polar contacts between acP and Ser 212 and the main-chain nitrogen of Gly 234 are indicated with yellow dashes. The catalytic Lys 11 is also shown. (b) below: Glyceraldehye 3-phosphate dehydrogenase (GapA) structure with selected acetylated lysine residues 249 and 257. Residues in the local environment of acetylated lysines are labeled. Modified lysine residues became non-enzymatically acetylated after quickly soaking crystals with acP.

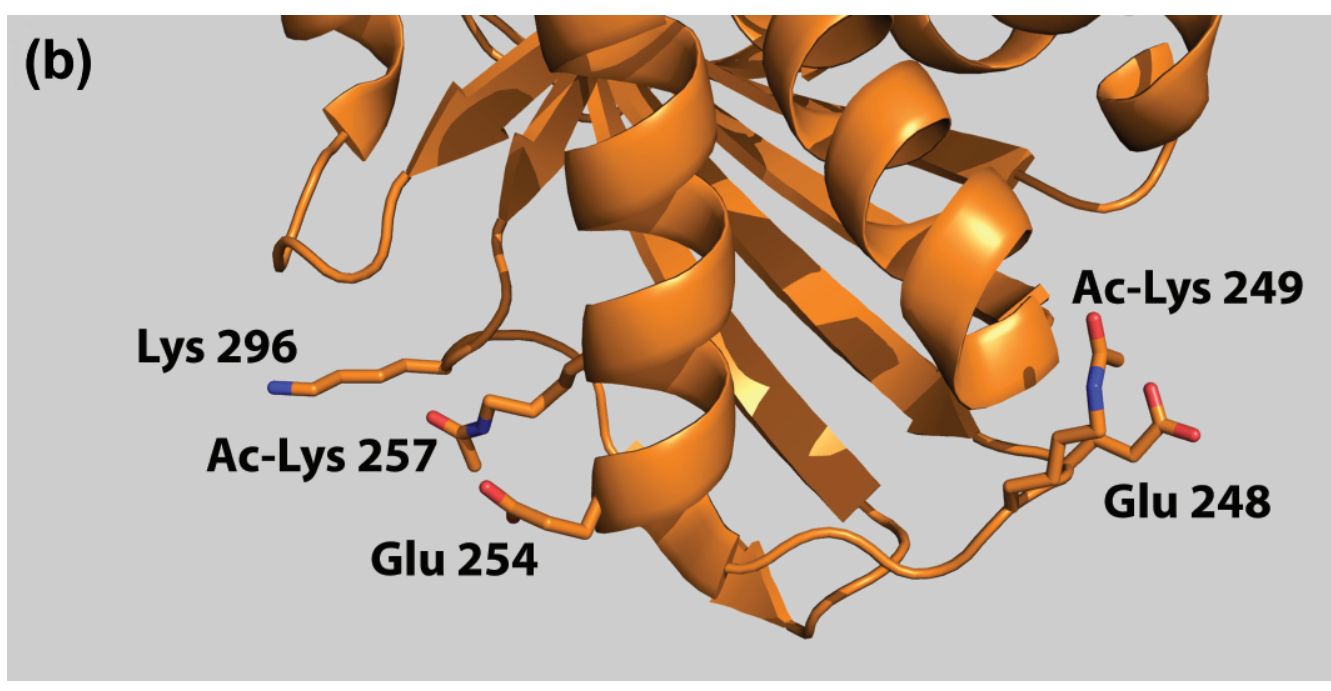




\section{Targeting Certain Cardiovascular Diseases}

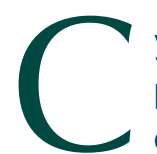

yclic guanosine monophosphate (cGMP) and cyclic adenosine monophosphate (cAMP) are important intracellular modulators of essential physiological processes which bind and activate the enzymes protein kinase $G(P K G)$ and $A(P K A)$, respectively. The interaction between cGMP and a form of PKG known as PKG I is particularly important in the cardiovascular system because existing drug therapies such as organic nitrates and phosphodiesterase inhibitors are ultimately designed to sustain the activity of PKG by elevating cellular cGMP. Therefore developing compounds to target PKG would be useful to treat certain cardiovascular diseases. But this has been difficult, particularly due to a lack of knowledge about its structure and poor understanding of its selective activation by cGMP. The researchers in this study showed that a specific region of the structure of PKG I, known as the C-terminal cyclic nucleotide binding domain (CNB-B), is important for selective cGMP binding, and that two amino acids in particular are key to this specificity. They also used the APS to examine the crystal structure of CNB-B with and without bound cGMP. In this way, they showed important structural changes in the cyclic nucleotide pocket of PKG I upon binding of cGMP, namely its capping by another amino acid which stabilizes the active site region. These findings will be important in guiding future work to design new drugs for treating hypertensive diseases such as arterial and pulmonary hypertension, heart failure and erectile dysfunction.

\section{Binding Affinities for Cyclic Nucleotides}

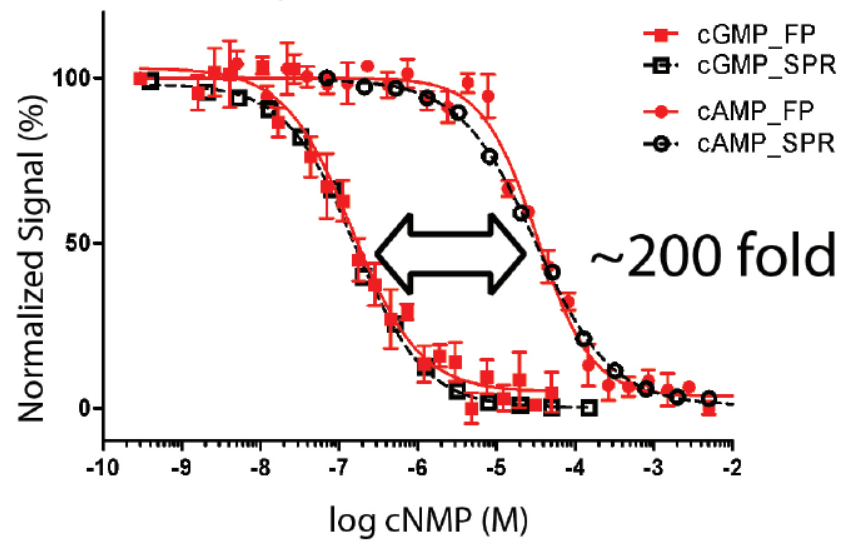

\begin{tabular}{|c|c|c|c|}
\hline \multirow{2}{*}{ PKG I CNB-B } & \multicolumn{2}{|r|}{ cGMP } & CAMP \\
\hline & FP & SPR & SPR CAMP \\
\hline $\mathrm{EC} 50 \pm \operatorname{SEM}(\mathrm{n})$ & $215 \pm 13 \mathrm{nM}(8)$ & $181 \pm 8 \mathrm{nM}(4)$ & $52 \pm 8 \mu \mathrm{M}(5) \quad 36 \pm 3 \mu \mathrm{M}(3)$ \\
\hline
\end{tabular}

cGMP Pocket (PKG I CNB-B)

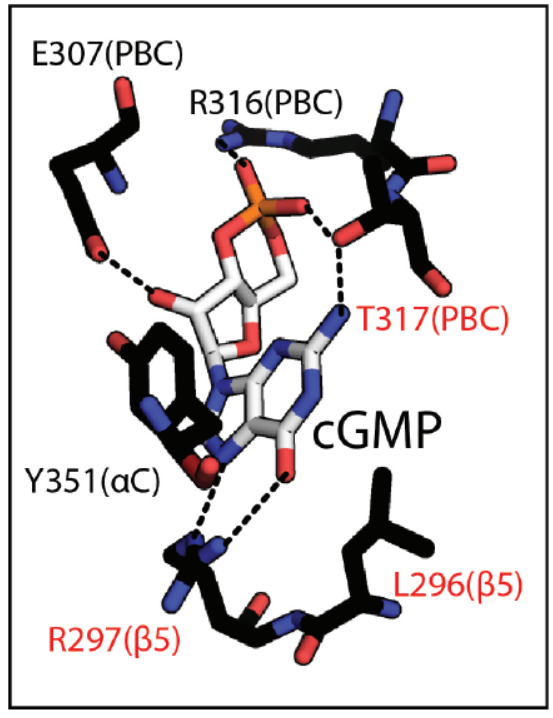

Fig. 1. Left panel, this page: Affinity measurements of PKG I CNB-B for cGMP and cAMP. PKG I CNB-B provides 200-fold selectivity for cGMP over cAMP. Competition fluorescence polarization (FP) curves are shown in red, and competition surface plasmon resonance (SPR) curves are shown in black. Error bars denote standard error of the mean SEM. Middle panel (this page) and right panel (facing page): Detailed interactions at cGMP pocket of PKG I CNB-B (middle panel) and cAMP pocket of PKA I CNB-B (right panel). The individual CGMP or CAMP interacting residues are shown, with the following color theme: side chain carbon, black; oxygen, red; nitrogen, blue. Key amino acid contacts are shown as dotted lines. 
The intercellul; ar modulators cGMP and CAMP are important secondary messengers - signaling molecules released by cells to trigger certain physiological responses. The enzymes PKG and PKA are activated after binding to cGMP and CAMP, and regulate many important physiological processes such as glycogen and steroid metabolism, vascular and smooth muscle tone, and memory formation. In the heart, for example, cAMP acts to increase the strength of contraction, while cGMP acts to weaken contractions. Although these opposing processes are kept in balance in the cell, selective binding of cGMP and cAMP is critical to keep the 2 pathways separated. However, the exact mechanism of this selectivity has so far been poorly understood.

\section{cAMP Pocket (PKA I CNB-B)}

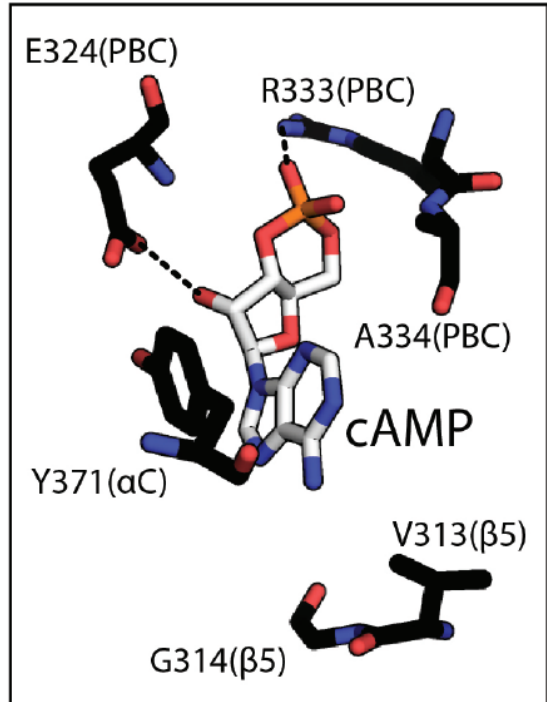

PKG is a key intermediary in a signaling pathway involving cGMP and nitric oxide, a compound produced in cellular reactions in the cardiovascular system, and the physiological importance of their interaction is highlighted by the abnormalities found in mice that lack the gene for certain forms of PKG I. These mice have high blood pressure and abnormal smooth muscle contraction, and they die young. People with similar mutations also suffer from aneurysms and dissection of the aorta.

Since PKG is also involved in the mechanism of action of drugs that increase cGMP in cells, either by stimulating its production (example: organic nitrate) or by preventing its degradation (example: sildenafil citrate), there is a strong interest in using compounds that activate PKG in particular. However, this has been difficult due to the lack of information about the structure of PKG and poor understanding of how it is specifically activated by cGMP.

In this study, researchers from the Baylor College of Medicine; the University of Kassel (Germany); Rice University; the University of California, San Diego; McMaster University (Canada); Rigaku Americas; and Lawrence Berkeley National Laboratory performed studies to further investigate the biochemical nature of the cGMP specificity of PKG, as well as its crystal and NMR structures.

The team discovered that the key to selectivity for cGMP binding lies in a specific part of the regulatory region of the structure of PKG I known as CNBB. Two particular amino acids (leucine and arginine at positions 296 and 297, respectively) were found to be essential for cGMP selectivity. Moreover these amino acids are replaced with valine and glycine in the corresponding region in PKA and no such interactions are seen between CAMP, further explaining the cyclic nucleotide selectivity mechanism (Fig. 1). Using diffraction data sets collected from the LRL-CAT beamline 31-ID-D of the APS, they were also able to determine the three-dimensional crystal structures of CNB-B with and without bound CGMP.

As a result of this study, the researchers were able to suggest a mechanism for the specific activation of PKG I by cGMP. By comparing the crystal structures of CNB-B with and without bound cGMP, they found structural changes in the C-terminal helix of CNB$\mathrm{B}$, which bridges the enzyme's N-terminal regulatory domain with its $\mathrm{C}$-terminal catalytic domain. Binding of cGMP resulted in its capping by the amino acid tyrosine at position 351, and subsequent stabilization of the structure of the C-terminal region.

These results will be important in guiding future work to investigate this activation mechanism in more detail, and may play an important role in the development of therapeutic agents for the treatment of hypertensive diseases. - Nicola Parry

See: Gilbert Y. Huang ${ }^{1}$, Jeong Joo Kim $^{1}$, Albert S. Reger ${ }^{1}$, Robin Lorenz ${ }^{2}$, Eui-Whan Moon ${ }^{1}$, Chi Zhao ${ }^{3}$, Darren E. Casteel $^{4}$, Daniela Bertinetti ${ }^{2}$, Bryan VanSchouwen ${ }^{5}$, Rajeevan Selvaratnam $^{5}$, James W. Pflugrath ${ }^{6}$, Banumathi Sankaran ${ }^{7}$, Giuseppe Melacini ${ }^{5}$, Friedrich W. Herberg ${ }^{2}$, and Choel $\mathrm{Kim}^{1 *}$, "Structural Basis for Cyclic-Nucleotide Selectivity and cGMP-Selective Activation of PKG I," Structure 22, 116 (January 7, 2014).

DOI: 10.1016/j.str.2013.09.021

Author affiliations: ${ }^{1}$ Baylor College of Medicine; ${ }^{2}$ University of Kassel; ${ }^{3}$ Rice University; ${ }^{4}$ University of California, San Diego; ${ }^{5}$ McMaster University; ${ }^{6}$ Rigaku Americas; ${ }^{7}$ Lawrence Berkeley National Laboratory Correspondence: * ckim@bcm.edu

C.K. is funded by National Institutes of Health grant R01 GM090161 and a BCM seed grant. G.Y.H. is supported by the Houston Area Molecular Biophysics Program, National Institute of General Medical Science grant no. T32GM008280. G.M. is funded by the Canadian Institute of Health Research. F.W.H. is supported in part by European Union grant "Affinomics" (Contract 222635) and the Federal Ministry of Education and Research Project "NoPain" (FKZ0316177F). This research used resources of the Advanced Photon Source, a U.S. Department of Energy (DOE) Office of Science User Facility operated for the DOE Office of Science by Argonne National Laboratory under Contract No. DE-AC02-06CH11357.

31-ID-D • LRL-CAT • Life sciences • Macromolecular crystallography, single-wavelength anomalous dispersion, single-crystal diffraction • 4.7-28 keV • Mail-in • Accepting general users $\cdot$ 


\section{An Engineered Protein for EXtracting URANIUM FROM SEAWATER}

$\mathrm{B}$

y many estimates, the worldwide reserves of uranium - between 5 and 6 million tons - could be exhausted by the end of this century. However, nearly 1,000 times as much of this key element used in nuclear energy production is thought to be dissolved in the world's oceans. Efforts to extract uranyl, the predominant aerobic form of uranium, from seawater date back many decades. So far, though, all solutions have been cost prohibitive. Last year, researchers used a computational algorithm and high-brightness $\mathrm{x}$ rays from the APS to guide the engineering of a protein to selectively bind uranyl with a very high affinity. Because the protein can be expressed in a common bacteria species, it can be used to mine ocean-based uranium cheaply and with far less environmental consequences than land-based deposits.

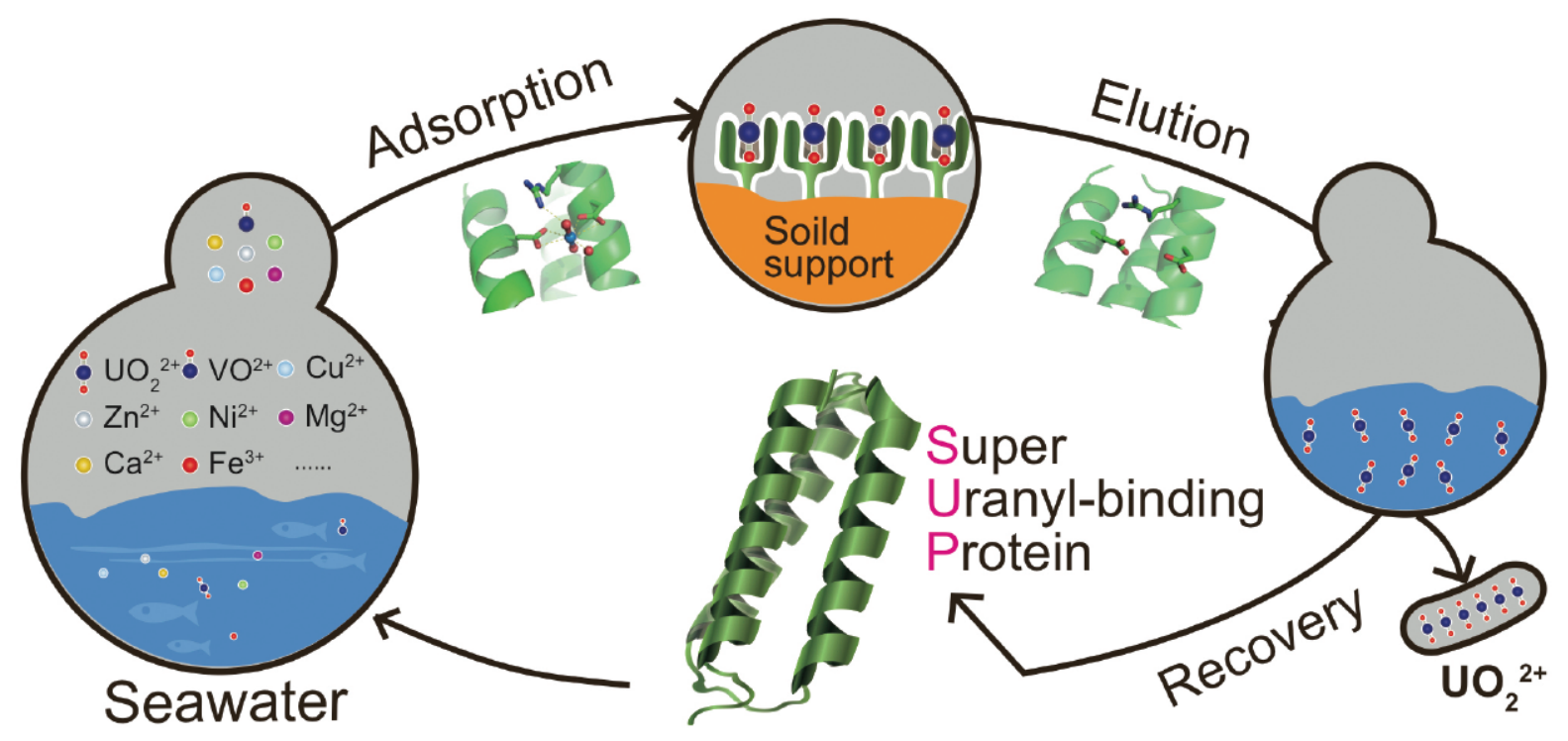

Fig. 1. Uranyl sequestration strategy. Immobilization of the computationally designed super uranylbinding protein on a solid support selectively adsorbs uranyl from synthetic seawater. After uranyl is removed by washing with a solvent, SUP is recovered for continued use. 
The oceans of the world contain enormous amounts of precious metals, though at miniscule concentrations. To tap these dispersed, yet highly lucrative, resources, scientists have devised numerous extraction systems. A common approach is constructing an inorganic binding surface that selects for the size and charge of the metal ion of interest. However, this general strategy is not suitable for the selective enrichment of metals such as uranyl that are similar in size and charge to other ions found in seawater.

While inorganic filters have been either too expensive to make and deploy or not sufficiently selective, nature freely produces proteins that can recognize metal ions in the femtomolar range. Researchers from The University of Chicago, Peking University (China), and Argonne developed a computational screening algorithm called URANTEIN to search the Protein Data Bank for proteins containing pockets, or binding sites, that could accommodate uranyl. Included in the search were proteins that could be readily modified to display amino acid residues that would optimally bind and hold uranyl. This initial search turned up more than 5000 candidate proteins. Further selections considering stability, potential steric clashes, and accessibility of the binding site led to 10 suitable candidates. Of these, 9 expressed well in a biological system, and 4 of those had sufficiently low binding affinities.

One of the candidates, a small protein with an unknown function from Methanobacterium thermoautotrophicum, an anaerobe isolated from sewage sludge in Urbana, Illinois, was particularly stable and useful. Several rounds of amino acid mutations brought the binding affinity down from $37 \mathrm{~nm}$ to $7.4 \mathrm{fM}$. Next, the team demonstrated that the uranyl binding selectivity of the protein - dubbed super uranyl-binding protein (SUP) was significantly higher than that of 17 metal ions that are commonly targeted for seawater extraction, as shown in Fig. 1.

The high-resolution crystal structure of SUP - captured using the LSCAT 21-ID-F x-ray beamline and the NE-CAT 24-ID-C x-ray beamline, both at the APS - revealed a binding-site geometry similar to that predicted by the URANTEIN model. The crystal structure also helped identify amino acid residues of SUP involved in functions such as binding and holding uranyl, creating a negative charge environment to hold uranyl in place, contributing to the high selectivity, and stabilizing the binding site.

To test the efficiency of SUP, the researchers treated water containing 158 parts per billion of uranyl with SUP fused to a maltose binding protein immobilized on resin. This setup resulted in sequestration of more than $95 \%$ of the uranyl. Next they tested the efficiency of uranyl enrichment in synthetic seawater, which was composed of many of the metal ions present in real seawater. The amount of uranyl sequestered depended significantly on the concentration of the SUP-maltose fusion protein; when fusion protein concentration was equivalent to that of uranyl, $17 \%$ of the uranyl was extracted; an excess of SUP (6,000 times that of uranyl) resulted in enrichment exceeding $90 \%$.

The team genetically expressed SUP on the surface of Escherichia coli and found that this bacterial system could extract more than $60 \%$ of the uranyl in synthetic seawater. This is the first known demonstration of a bacterial system used to sequester uranyl.

In summary, a computational approach guided the rational design of a protein that can be used to extract uranyl from seawater. The efficiency of uranyl extraction exceeded $60 \%$ when SUP was expressed in E. coli and $90 \%$ when bound to resin. In the future, similar cost-effective protein engineering strategies can be extended to the min- ing of other precious metals or in the remediation of polluted environmental sites. - Chris Palmer

See: Lu Zhou ${ }^{1}$, Mike Bosscher ${ }^{1}$, Changsheng Zhang ${ }^{2}$, Salih Özçubukçu ${ }^{1}$, Liang Zhang ${ }^{1}$, Wen Zhang ${ }^{1}$, Charles J. Li ${ }^{1}$, Jianzhao Liu ${ }^{1}$, Mark P. Jensen ${ }^{3}$, Luhua Lai ${ }^{2 *}$, and Chuan $\mathrm{He}^{1 * *}$, "A protein engineered to bind uranyl selectively and with femtomolar affinity," Nat. Chem. 6, 236

(March 2014).

DOI: 10.1038/NCHEM.1856

Author affiliations: ${ }^{1}$ The University of Chicago, ${ }^{2}$ Peking University, ${ }^{3}$ Argonne National Laboratory

Correspondence:

** chuanhe@uchicago.edu; *Ihlai@pku.edu.cn

This work was supported by the Division of Chemical Sciences, Geosciences, and Biosciences-Basic Energy Sciences of the U.S Department of Energy (DOE), under Contract no. DE-FG02-07ER15865 to C.H., and at Argonne (M.J.) under Contract no. DEAC02-06CH11357, the Dreyfus Foundation Postdoctoral Program in Environmental Chemistry to S.O., the Ministry of Science and Technology of China (2009CB918500) and the National Natural Science Foundation of China $(21173013,11021463)$ to L.L. Use of LS-CAT was supported by the Michigan Economic Development Corporation and the Michigan Technology Tri-Corridor (Grant 085P1000817). The NE-CAT beamlines are supported by a grant from the National Institute of General Medical Sciences (P41 GM103403) from the National Institutes of Health. This research used resources of the Advanced Photon Source and the Center for Nanoscale Materials, DOE Office of Science User Facilities operated for the DOE Office of Science by Argonne National Laboratory under Contract No. DE-AC02-06CH11357.

21-ID-F • LS-CAT • Life sciences • Macromolecular crystallography $12.7 \mathrm{keV} \cdot$ On-site, remote, mail-in • Accepting general users •

24-ID-C • NE-CAT • Life sciences • Macromolecular crystallography, microdiffraction, single-wavelength anomalous dispersion, single-crystal diffraction, microbeam $\cdot 6.5-23 \mathrm{keV}$ - On-site, remote $\cdot$ Accepting general users $•$ 


\section{Finding Novel Antibiotic Targets}

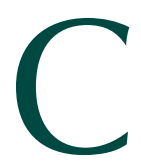

ooks know that getting the water-based and the oil-based components of a dish to agree can take some work. They just don't get along. Cells have exactly the same problem. Getting oily lipids from where they are made to where they need to be in a watery cellular environment can be a daunting task, but not if you are a skilled biochemist, as cells are. One of the ways that cells have developed to solve the oil-water problem is to design a protein that shuttles lipids between biosynthetic enzymes. These proteins, called acyl carrier proteins (ACPs), are found in all domains of life, from bacteria to humans. One particularly interesting version is found in bacteria where ACPs are used in the biosynthesis of membrane lipids that form the bacterial endotoxin called lipid A. Lipid $A$ is an activator of the human immune system and, because it is essential for bacterial survival, represents a possible novel target for antibiotic therapy. Recent work by researchers from the Duke University Medical Center has elucidated the structure of a bacterial ACP interacting with one of the key Lipid A biosynthetic enzymes, LpxD. Their work, conducted at the APS, reveals new information about the catalytic interactions between ACP and LpxD and a surprising new role for ACP in product release that may offer just the target they were seeking. trigger release of the new acylated product [Fig. 1(e)] and then finally the holo-ACP [Fig. 1(f)]. These unprecedented structural snapshots provide insight into movements within ACP and uncover key molecular interactions at the protein-protein interface. Molecules that could block acyl-ACP from binding to LpxD would potentially serve as novel antibiotics because the LpxD reaction is essential to bacterial life.

The next step is to determine the structure of LpxD in complex with the acylated lipid product, which will complete the "molecular landscape" of the active site and facilitate the design of novel antibiotics to treat bacterial infections. - Sandy Field

See: Ali Masoudi ${ }^{\star}$, Christian R.H. Raetz, Pei Zhou, and Charles W. Pem-
The researchers crystallized three different versions of the LpxD-ACP complex in order to get structural data on what was going on at each step of the catalytic interaction. The methods for purifying and crystallizing $L p x D$ were already known, their advance was in their ability to make different versions of the ACP and to manipulate the catalytic process to "stall" it at different stages. Central to this was the ability to make ACP with its $4^{\prime}$-phosphopantetheine (4'-PPT) prosthetic arm, which is responsible for binding to the oily cargo, with the 14-carbon acyl chain already loaded (acyl-ACP) and with it unloaded (holo-ACP) [Fig. 1(a) and 1(f)]. Then, they mixed these ACP versions with either catalytically active LpxD or a mutant of LpxD that was not catalytically active. Biochemical tricks to slow catalysis also helped to stall the enzyme in intermediate states.

Three structures were obtained at the SER-CAT 22-BM-D and 22-ID-D beamlines at the APS. Each structure showed that three LpxD molecules combine to form a trimer and that one molecule of ACP binds to each of these through a C-terminal ACP recognition domain (ARD). Aspects of the intact acyl-ACP structure [pre-catalysis, Fig. 1(b)] allowed them to elucidate the basis for the strict 14-carbon acyl chain (a) (b)

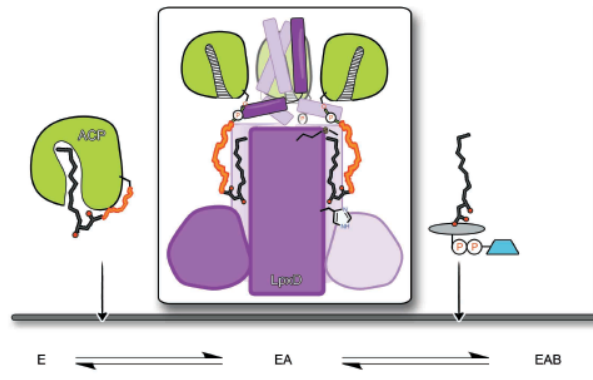

(e)

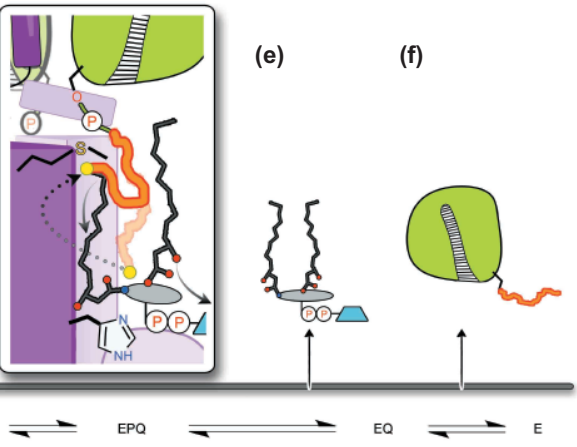

Fig. 1. Molecular model of ordered reaction mechanism between ACP and LpxD. (a) acyl-ACP is shown in green with 4 ' -phosphopantetheine (4' -PPT) group in orange and lipid "cargo" in black. (b) Three acyl-ACPs bind to LpxD trimer (purple/white). (c) Uridine diphosphate 3-O-( $\beta-O H-C 14)-\alpha-D-$ glucosamine (UDP-acyl-GIcN), which will accept acyl group from ACP, binds next. (d) Acyl group is transferred to UDP-acyl-GIcN, 4' -PPT blocks release (transparent orange), then moves away (orange). (e) UDP-diacyl-GIcN is released. (f) holo-ACP is released.

specificity of LpxD. The structure shows that one end of the acyl chain packs against an amino acid, methionine 290, which acts as a "ruler" that keeps the allowable chain length at 14 carbons.

The second step structure, in which the acyl group had been delivered to the recipient molecule but neither had left the complex, showed that the $4^{\prime}$-PPT prosthetic arm covers the exit site of the enzyme and keeps ACP and the product from leaving [Fig. 1(d)], transparent orange). Interestingly, in the final snapshot of catalysis, the 4' -PPT group moves a significant distance up and out of the way [Fig. 1(d), orange] to ble IV, "Chasing acyl carrier protein through a catalytic cycle of lipid A production," Nature 505, 422 (16 January 2014). DOI: 10.1038/nature 12679 Author affiliation:

Duke University Medical Center Correspondence: ali.masoudi@duke.edu

This work was supported by National Institutes of Health grants GM-51310 and Al055588 awarded to C.R.H.R. and P.Z. SER-CAT supporting institutions may be found at www.ser-cat.org/members.html. This research used resources of the Advanced Photon Source, a U.S. Department of Energy (DOE) Office of Science User Facility operated for the DOE Office of Science by Argonne National Laboratory under Contract No. DE-AC02-06CH11357. 


\section{NolR-Mediated Regulation of Symbiotic Root Nodule-Dependent Genes}

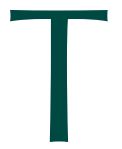

he NolR protein regulates the complex network of gene expression that guides formation of Rhizobia root nodules, a process critical for establishing the symbiotic relationship between nitrogen-fixing bacteria and legume plants. To explain the structural basis for NolR's ability to bind to genes, specifically variant regulatory sequences with genes, researchers used high-resolution $\mathrm{x}$-ray crystallography of NoIR alone and in complex with varying DNA sequences. The $x$-ray diffraction data, collected at the SBC-CAT 19-ID-D beamline, at a wavelength of $0.979 \AA$, shows how NolR uses a conformational switch to accommodate variation in the DNA sequences to which it binds. Understanding the molecular basis for the control of nodulation is important for potential applications that may improve nitrogen fixation in the soil, a process on which agriculture depends heavily.

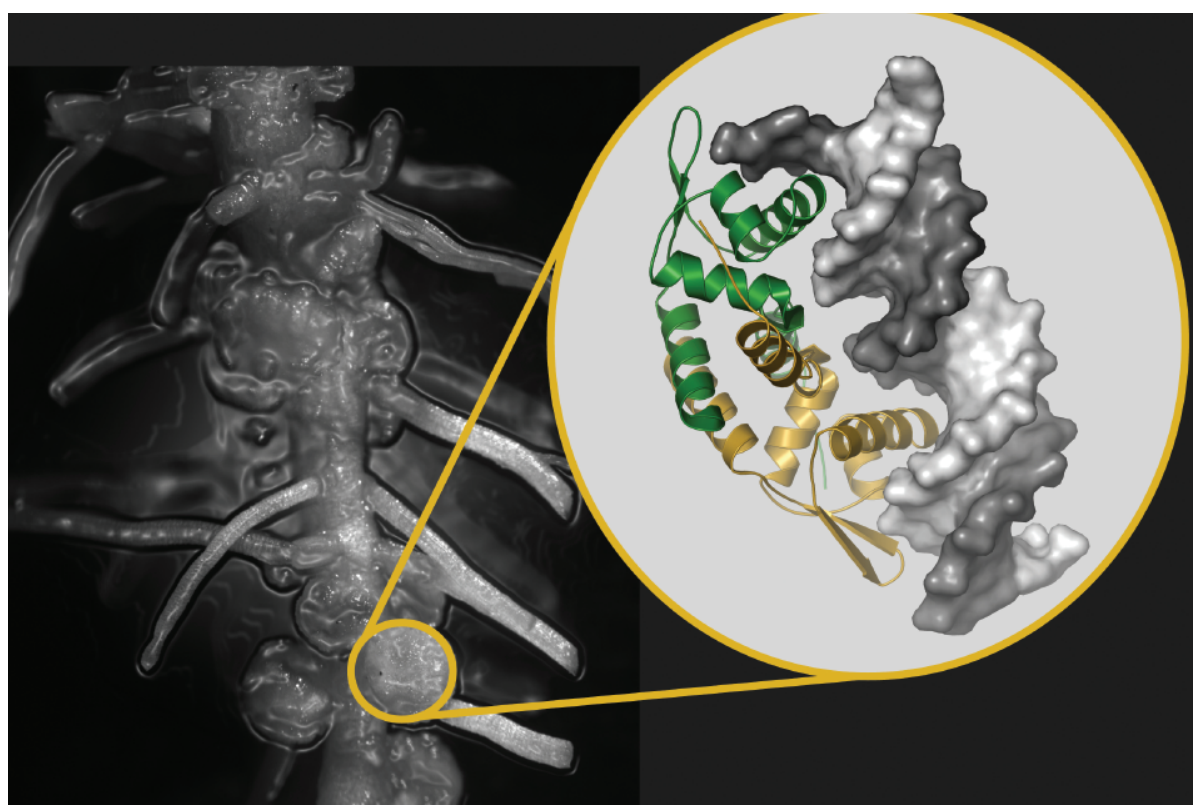

Fig. 1. Symbiosis between legume plants and rhizobial microbes is initiated by formation of Rhizobia nitrogen-fixing root nodules (left, nodule indicated by yellow circle). The global regulatory protein NoIR (right, shown in green and gold) controls gene expression patterns in Rhizobia by binding to nodulation-dependent regulatory elements (DNA in gray).

Bacteria permeate and are central to the function of every ecosystem on earth. Plants, including those used in agriculture, rely on soil-dwelling microorganisms for nitrogen, a nutrient whose availability is often growth limiting. While nitrogen is abundant in the atmosphere in the form $\mathrm{N}_{2}$, this form is unreactive and cannot be taken up by plants as a molecular building block until it is "fixed," or converted into ammonia $\left(\mathrm{NH}_{3}\right)$, a process plants are unable to complete independently. In the case of legume plants, rhizobial bacteria form root nodules (Fig. 1), which fix ni- trogen so efficiently that legumes such as soybeans are commonly used to enrich nitrogen-deficient soil. Indeed, the amount of nitrogen fixed by rhizobial bacteria surpasses that present in commercially available fertilizers.

A complex network of gene expression governs the switch from the free-living form of Rhizobia to the nodule form. To initiate this process, genes required for nodulation must be turned on and others that interfere with the process turned off by regulatory proteins. These proteins bind DNA upstream of the genes they control, modulating their transcription and expression. NolR is an important regulatory protein responsible for controlling expression of number of different nodulation-dependent genes during initiation of symbiosis. Prior to this work, however, it remained unclear how this single protein interacts with regulatory elements of varying sequences. Often, DNA sequences to which regulatory proteins bind are strictly defined, and interactions are disrupted by any change in DNA sequence.

Using x-ray crystallography at 19ID-D to solve the structure of both free NoIR and NolR bound to 22 base-pair DNA operator sequences comprised of nucleotides AT or AA, the researchers from Washington University in St. Louis and the United States Department of Agriculture showed that NoIR's ability to tolerate DNA base changes is reliant upon conformational switching of a single amino acid, glutamine 56 .

Unraveling the nature of the molecular networks involved in root nodule formation, including those genes controlled by regulators other than NolR, is critical for potential technological applications such as improving the efficiency of nitrogen fixation in existing systems or engineering non-legume crops to form symbiotic associations, eliminating the need for chemical fertilizers.

- Emma Nichols

See: Soon Goo Lee ${ }^{1}$, Hari B. Krishnan², and Joseph M. Jez ${ }^{1 *}$, "Structural basis for regulation of rhizobial nodulation and symbiosis gene expression by the regulatory protein NolR," Proc. Natl. Acad. Sci. USA, 111(17), 6509 (2014). DOI: 10.1073/pnas.1402243111 Author affiliations: ${ }^{1}$ Washington University in St. Louis, ${ }^{2}$ United States Department of Agriculture

Corresponding author: * jjez@wustl.edu

The SBC-CAT is a national user facility operated by The University of Chicago for the Department of Energy (DOE) Office of Biological and Environmental Research (DEAC02-06CH11357). This research used resources of the Advanced Photon Source, a U.S. DOE Office of Science User Facility operated for the DOE Office of Science by Argonne National Laboratory under Contract No. DE-AC02-06CH11357. 


\section{A New Perspective on Cancer Treatment}

"R as" is the name of a family of proteins found in all cell types. It is recruited to cell membranes and acts as a molecular switch, converting signals from the cell surface to the nucleus and other parts of the cell. Ras proteins are abnormal in most human cancers due to activating mutations in the Ras genes themselves or changes in upstream signaling components. While switching off activated Ras represents a rational therapeutic strategy for the treatment of such cancers, this has proven to be more difficult than previously thought, because the Ras protein lacks pockets on its surface for adequate binding of inhibitor compounds. Different therapeutic approaches using new methods to target Ras are therefore necessary for these cancers. With this in mind, researchers in this study characterized unique small molecules that bind to a pocket in the complex between Ras and the guanine nucleotide exchange factor (GEF) Son of Sevenless (SOS). They used x-ray crystallography at LS-CAT beamline 21-ID-F to evaluate this interaction. The results of this research will be important in guiding future studies in the search for new approaches for the therapeutic modulation of Ras signaling in cancer.

The Ras proteins control cell-signaling pathways. Ras is switched on by incoming signals in the cell, and then turns on other proteins that regulate numerous aspects of normal cell growth. Ras binds to the guanine nucleotides guanosine triphosphate (GTP) and guanosine diphosphate (GDP). In its "off" state it binds GDP, while in its "on" state it binds GTP. It is switched on by exchange of GDP for GTP, and this reaction is controlled by GEFs.

Over-activation of Ras can result from situations including alterations in upstream signaling components or mutations at specific sites on the Ras gene that favor GTP binding. Abnormal activation of Ras in this way has been shown to be involved in the deregulation of cell signals guiding growth and survival in about $30 \%$ of all human cancers.

However, although it therefore seems logical that Ras inhibitors would represent a good therapeutic approach to inhibit cancer growth, survival, and spread, such compounds have proven difficult to develop. This is due to the absence of suitable inhibitor binding pockets on the surface of the Ras pro- to a unique and important pocket in the Ras:SOS:Ras complex (Fig. 1). They evaluated the crystal structures of the complex to further investigate this pocket that may be used as a new approach to regulate Ras signaling. The pocket was formed by the CDC25 domain of SOS (the part responsible for stimulating Ras nucleotide exchange) near the region of Ras known as Switch II, which undergoes a shape change during the exchange. The researchers also discovered that these small molecules increase the rate of nucleotide exchange and levels of GTP-bound Ras, yet also disrupt downstream Ras signaling processes in the cell.

$$
\text { - Nicola Parry }
$$

See: Michael C. Burns, Qi Sun, R. Nathan Daniels, DeMarco Camper, J. Phillip Kennedy, Jason Phan, Edward T. Olejniczak, Taekyu Lee, Alex G. Waterson, Olivia W. Rossanese, and Stephen W. Fesik*, "Approach for targeting Ras with small molecules that activate SOS-mediated nucleotide exchange," Proc. Natl. Acad. Sci. 111(9), 3401 (March 4, 2014). DOI: 10.1073/pnas.1315798111 Author affiliation: Vanderbilt University School of Medicine Correspondence:

* stephen.fesik@vanderbilt.edu

This work supported by the Ann Melly Scholarship in Oncology (to M.C.B.), Public Health Service Award T32 GM07347 from the National Institute of General Medical Studies for the Vanderbilt Medical-Scientist Training Program (to M.C.B.), National Institutes of Health (NIH) Grants 5DP1OD006933 (NIH Director's Pioneer Award; to S.W.F) and 5P50A095103-09 (National Cancer Institute Specialized Program of Research Excellence in gastrointestinal cancer; to R. J. Coffey), and a Lustgarten Foundation grant (to S.W.F.). Use of LS-CAT was supported by the Michigan Economic Development Corporation and the Michigan Technology Tri-Corridor (Grant 085P1000817). This research used resources of the Advanced Photon Source, a U.S. Department of Energy (DOE) Office of Science User Facility operated for the DOE Office of Science by Argonne National Laboratory under Contract No. DEAC02-06CH11357. 


\section{A Structural Basis for the Evolution of BACterial ANTIBIOTIC Resistance}

A ntibiotics represent a tremendous advance in modern medicine, and are central to treatment of bacterial infections. Since their introduction overuse and misuse have contributed to an "arms race" in which bacteria develop resistance to even the most powerful antibiotics. Vancomycin is an antibiotic often used as a last-line treatment for bacterial infections caused by Staphylococcus and Enterococcus species. Vancomycin disrupts bacterial cell wall synthesis though high-affinity binding to cell wall peptidoglycan precursors, rendering them unsuitable for use by the cell and ultimately causing the bacteria to die. Bacteria can develop vancomycin resistance through acquisition of genes that synthesize cell wall building blocks with lower affinity for vancomycin along with genes for peptidases that ensure the concomitant depletion of vancomycinsensitive precursors. Researchers used high-resolution x-ray crystallography at the APS to describe the resistance D,D-peptidase VanXY, which has the unusual ability to selectively hydrolyze, or break apart, vancomycin-sensitive dipeptide and pentapeptide peptidoglycan precursors. This research reveals the mechanism of this bisubstrate selectivity and supports the development of drugs to be used to treat increasingly prevalent vancomycin resistant bacterial infections.

In Gram-positive bacteria such as Staphylococcus, the antibiotic vancomycin binds tightly to cell wall peptidoglycan precursors, interfering with proper cell wall synthesis, causing the bacteria to die. Acquisition of resistance genes permits synthesis of cell wall components that, while still able to serve as building blocks, are structurally distinct enough to bind vancomycin with lower affinity. Along with this ability, the bacteria must be able to selectively degrade vancomycin-sensitive cell wall precursors still being synthesized by the cell.

In one type of resistance, two distinct enzymes, VanX and VanY, degrade dipeptide and pentapeptide cell wall precursors, respectively. These enzymes are D,D-peptidases which break apart these peptides by hydrolyzing the bonds between terminal alanine amino acids, D-Ala-D-Ala. In a second type of resistance, a single enzyme, Van $\mathrm{XY}$, has evolved to selectively hydrolyze both the sensitive dipeptide and pentapeptide precursors.

In this work, researchers from the Institut Pasteur (France), the University of Toronto (Canada), and the Center for Structural Genomics of Infectious Diseases used LS-CAT beamlines 21-ID-D and 21-ID-G at the APS to understand how VanXY can act upon both a small dipeptide and a bulky pentapeptide.

The researchers grew crystals of VanXY alone, in complex with intermediate analogs, and in complex with DAla-D-Ala substrate and D-Ala hydrolyzed product. Diffraction data were collected at the 21-ID-D and -G beamlines, and the presence of zinc, a metal known to be critical for the function of VanXY, was confirmed using X-ray fluorescence at the 21-ID-D beamline.

The three-dimensional structures obtained revealed how this enzyme is able to differentiate between vancomycin resistant and sensitive cell wall components and identified a cap structure unique to VanXY. The structures also permitted researchers to compare Van XY with potential evolutionary precursors VanX and VanY, and form a better understanding of how bacteria evolve new tools against the action of vancomycin.

Understanding the relationship between the structure of this resistance peptidase and its function allows researchers to develop new antibiotics or new drugs that can resensitize the bacteria to vancomycin. - Emma Nichols

See: Djalal Meziane-Cherif ${ }^{1}$, Peter J. Stogios $^{2,3}$, Elena Evdokimova ${ }^{2,3}$, Alexei Savchenko ${ }^{2,3 * *}$, and Patrice Cour- valin ${ }^{1 *}$, "Structural basis for the evolution of vancomycin resistance D,D-peptidases," Proc. Natl. Acad. Sci. USA 111(16), 5872 (April 22, 2014). DOI: 10.1073/pnas. 1402259111 Author affiliations: ${ }^{1}$ Institut Pasteur, ${ }^{2}$ University of Toronto, ${ }^{3}$ Center for Structural Genomics of Infectious Diseases Correspondence: * pcourval@pasteur.fr,
** alexei.savchenko@utoronto.ca

This project has been funded in whole or in part with federal funds from the National Institute of Allergy and Infectious Diseases, National Institutes of Health, Department of Health and Human Services (Contracts HHSN272200700058C and HHSN27220$1200026 \mathrm{C}$ ) and by an unrestricted grant from Reckitt Benckiser. This research used resources of the Advanced Photon Source, a U.S. Department of Energy (DOE) Office of Science User Facility operated for the DOE Office of Science by Argonne National Laboratory under Contract No. DE-AC02$06 \mathrm{CH} 11357$.

21-ID-D • XSD • Life sciences • Macromolecular crystallography, microfluorescence (hard $\mathrm{x}$-ray), nanofluorescence imaging, nanotomography $\cdot 6.5-20 \mathrm{keV} \cdot$ On-site, remote, mail-in - Accepting general users •

21-ID-G • LS-CAT • Life sciences • Macromolecular crystallography $\bullet 12.7 \mathrm{keV} \cdot$ Onsite, remote, mail-in - Accepting general users • 


\section{HiJaCking the Sulfur-Delivery Machinery fOR BACTERIAL THIOSUGAR FORMATION}

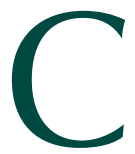

arbohydrates are abundant in nature and play many critical functional and structural roles in biology. They are generally composed of carbon, hydrogen, and oxygen. However, some sugars, called "thiosugars," also contain function groups derived from reduced sulfur. These unusual thiosugars play an important role in determining the biological activities of the parent molecules. Yet, while sulfur itself is an essential element widely found in living organisms, many questions remain about the natural formation of thiosugars due to both their scarcity in primary metabolism and a general lack of knowledge regarding the mechanism of sulfur delivery and incorporation. Bacterial natural products, in particular those with antibiotic activity, are rich sources of thiosugars, making them a research focus for the study of thiosugar formation in secondary metabolism. With this in mind, researchers working at the APS investigated the production of a thiosugar component of an antibiotic produced by the bacterium Amycolatopsis orientalis. The results of their study, the first to completely characterize thiosugar formation in a biological system, have important implications for understanding the mechanism of thiosugar formation and its application to the development of sulfur-containing pharmaceuticals.

The researchers from the University of Texas at Austin, Cornell University, Academia Sinica (China), and National Cheng-Kung University (Taiwan) studied the formation of the 2-thiosugar component of an antibiotic known as BE-7585A, which is produced by $A$. orientalis, subsp. vinearia BA-07585 strain. They identified a new enzyme, BexX, which is structurally similar to thiazole synthase (ThiG), an important enzyme responsible for incorporation of sulfur into thiamine (vitamin B1). The researchers therefore hypothesized that BexX might play a similar role in the formation of BE-7585A where it may catalyze the modification of sugar precursor glucose-6-phosphate (G6P) to produce 2-thioglucose, a sulfur-containing sugar.

On closer inspection, however, the researchers were unable to find a gene encoding a sulfur-carrier protein directly associated with BE-7585A formation in $A$. orientalis. After sequencing the entire bacterial genome, they only found genes that encode the four sulfur-carrier proteins ThiS, MoaD, CysO, and MoaD2 involved in the production of primary metabolites. Furthermore, genes encoding the activating enzymes required by ThiS, MoaD and CysO were missing. In fact, in the entire genome they only found one activating enzyme, named MoeZ, capable of activating all of the sulfur-carrier proteins.

Using $x$-ray diffraction data collected at the NE-CAT 24-ID-C beamline at the APS and the A1 beamline at the Cornell High Energy Synchrotron Source to solve the crystal structure of BexX and the sulfur-carrier proteins (Fig. 1), the researchers demonstrated that BexX can selectively distinguish between sulfur-carrier proteins. Thus, despite the mechanistic similarity of 2thiosugar formation in BE-7585A with thiamine production, BexX cannot use the sulfur-carrier protein, ThiS, from the thiamine pathway. Instead, sulfur insertion is achieved by an efficient strategy that recruits sulfur-carrier systems from other biosynthetic pathways in primary metabolism.

According to the researchers, this study also demonstrates the relaxed receptor specificity of the $A$. orientalis sulfur-delivery system. The researchers further speculate that the sulfur-delivery components of primary metabolism may, in general, play important roles in the production of rare sulfur-containing compounds associated with secondary metabolism. - Nicola Parry

See: Eita Sasaki ${ }^{1}$, Xuan Zhang $^{2}$, He G. Sun ${ }^{1}$ Mei-Yeh Jade Lu ${ }^{3}$, Tsung-lin Liu ${ }^{3,4}$, Albert $\mathrm{Ou}^{3}$, Jeng-yi $\mathrm{Li}^{3}$, Yu-hsiang Chen $^{3}$, Steven E. Ealick ${ }^{2}$, and Hungwen Liu 1*, "Co-opting sulphur-carrier proteins from primary metabolic path-

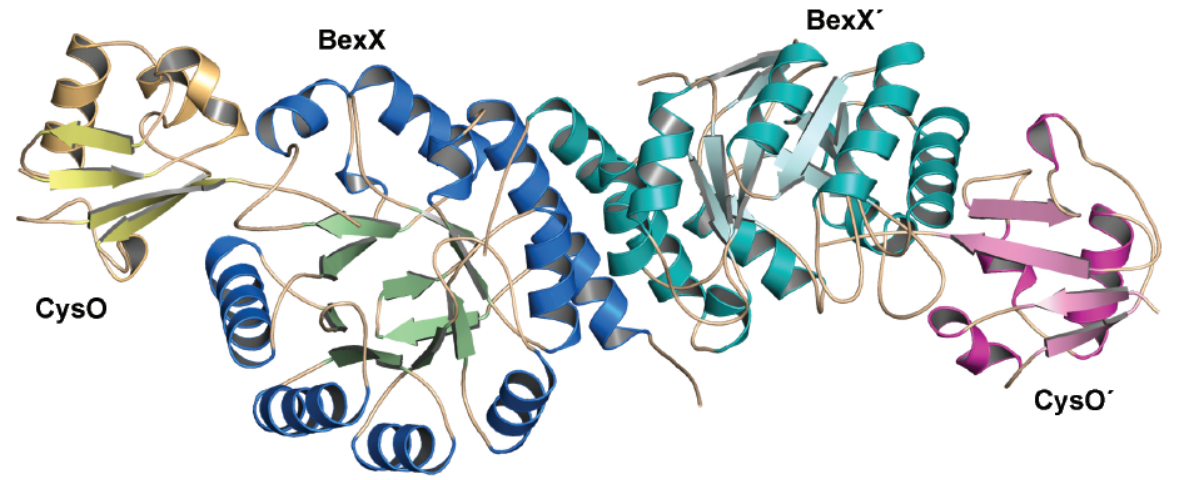

Fig. 1. A ribbon diagram of the crystal structure of $\mathrm{BexX}-\mathrm{Cys} \mathrm{O}$.

ways for 2-thiosugar biosynthesis," $\mathrm{Na}$ ture 510, 427 (19 June 2014).

DOI: 10.1038/nature13256

Author affiliations: ${ }^{1}$ University of Texas at Austin, ${ }^{2}$ Cornell University, ${ }^{3}$ Academia Sinica, ${ }^{4}$ National Cheng-Kung University

Correspondence:

*h.w.liu@mail.utexas.edu

This work was supported in part by Academia Sinica and by grants from the Nationa Institutes of Health (GM035906 to H.-W.L. and DK67081 to S.E.E.) and the Welch Foundation (F-1511 to H.-W.L.). This research used resources of the Advanced Photon Source, a U.S. Department of Energy (DOE) Office of Science User Facility operated for the DOE Office of Science by Argonne National Laboratory under Contract No. DE-AC02-06CH11357. 


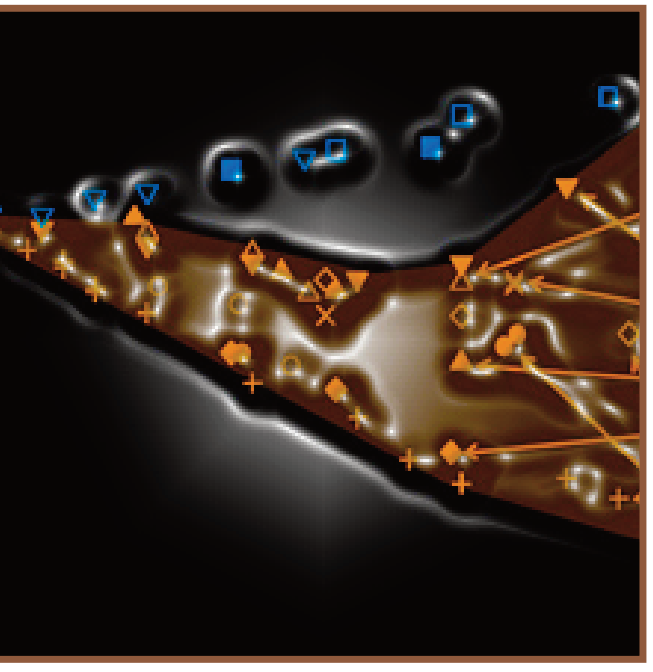

ENVIRONMENTAL, GEOLOGICAL \& Planetary SCience 


\section{STARDUST ON. EARTH}

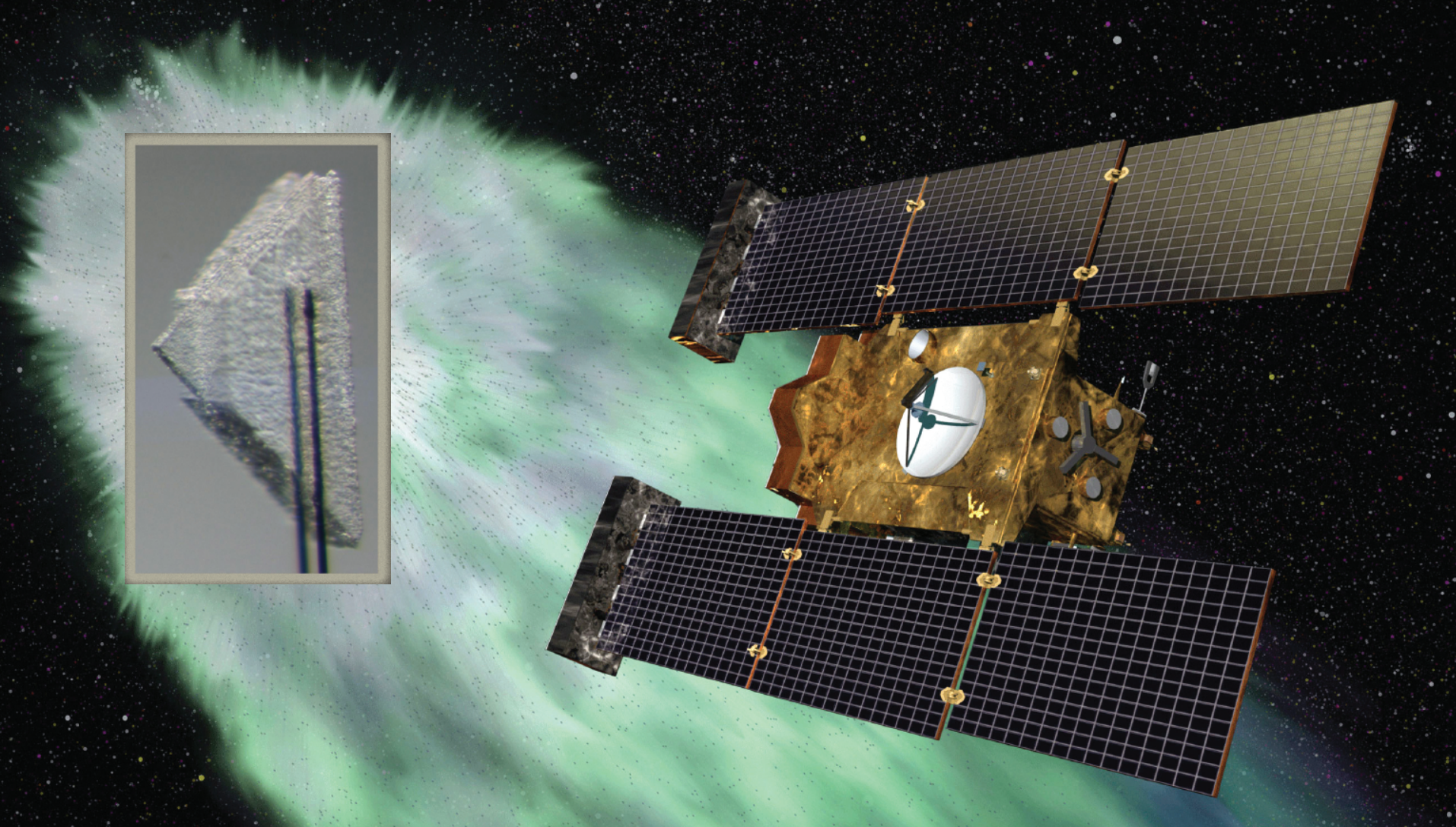

$\mathrm{T}$

iny rocks with a likely origin in the local interstellar medium have been returned to Earth and analyzed for the first time. Since the seven particles are diverse in elemental composition, crystallinity, and physical structure, they likely did not all originate in the same environment. The Stardust Interstellar Dust Collector (SIDC) on the Stardust spacecraft was the first mission to return solid samples from beyond the Moon. The two goals of the mission were to collect solid samples from comet 81P/Wild 2, and to collect interstellar dust (ISD) particles streaming into the heliosphere. Research by a large international collaboration, including studies carried out at the APS, identified the seven ISD particles collected by the SIDC. Previously, ISD was known only from astronomical observations of the interstellar medium and remote optical and spectroscopic data from instruments aboard the Ulysses and Galileo spacecraft.

Main image: Artist's conception of the Stardudt craft, courtesy of NASA/JPL.

Inset: Fig. 1. This image depicts a "picokeystone" - a tiny wedge of aerogel mounted on micromachined "pickle forks". The researchers extracted tracks from the aerogel tiles in such picokeystones using tools borrowed from microbiology labs. 
Solid particles were collected in ultra-low density aerogel tiles and aluminum foil strips. Collection on the foil was unplanned, but when the foil was returned to Earth, impact craters were identified by scanning electron microscopy. The collectors were exposed for a total of 195 days in 2000 and 2002 , and the sample capsule parachuted back to Earth in January 2006. By volume, the aerogel is $99.8 \%$ empty space so that the high-speed particles survive capture better than in any other medium. As it slows in the aerogel, each particle creates a long track and stops hundreds of microns to millimeters deep. Thousands of citizen-science volunteers searched optical micrographs of the aerogel to identify the tiny tracks.

Seventy-one tracks were identified; the most promising were extracted (Fig. 1) and analyzed with infrared and x-ray microprobes at multiple facilities, including the U.S. Department of Energy's (DOE's) X-ray Science Division 2-1D-D x-ray microprobe at the APS; the Advanced Light Source at Lawrence Berkeley National Laboratory (LBNL); the National Synchrotron Light Source at Brookhaven National Laboratory; and at the National Center for Electron Microscopy; as well as the European Synchrotron Radiation Facility (ESRF, France).

Tracks and particles identified as ISDs were consistent with interstellar particles in the following ways: morphology, trajectory, chemical analysis of the tracks; and mass distribution, structure, and composition of the particles. According to the terms of the Interstellar Preliminary Examination, analyses were limited to those that were non-destructive and non-invasive to preserve them as much as possible for future investigators. Most of the particles were secondary ejecta from impacts with the solar panels and the sample-return capsule.

Based on their elemental composition and impact features, seven particles were identified as likely interstellar, three collected in the aerogel and four in the foil. The identification of a particle as interstellar is a process of elimination of other possibilities; statistical analyses of their features revealed that they are much more likely to be inter- stellar than from any other origin. The particles are compositionally different from each other suggesting different sources.

One is a single silicate with a mantle-core structure, and the others are complex aggregates of various micrometer- to nanometer-size phases, such as oxides, metal, and sulfides in addition to silicates. The ISD from the aerogel were much more massive than expected, but the particles from the foil were closer to the average size of ISD inferred from astronomical observations. The ratio of smaller particles to larger particles was higher than expected. Models predicted that ISD impacts would be very high speed, but of the three particles that were captured by the aerogel, two arrived at much slower speed, suggesting that some interstellar dust particles have much lower density than expected.

While the preponderance of evidence supports an interstellar origin for the seven particles, alternative origins cannot be eliminated entirely until oxygen isotope analyses can be performed. ${ }^{18} \mathrm{O} /{ }^{17} \mathrm{O}$ values equal 5.2 in the solar system, but about 4 in the local galaxy. Future studies will include isotopic analyses. - Dana Desonie

See: Andrew J. Westphal ${ }^{1 *}$, Rhonda M. Stroud $^{2}$, Hans A. Bechtel ${ }^{3}$, Frank E. Brenker $^{4}$, Anna L. Butterworth ${ }^{1}$, George J. Flynn $^{5}$, David R. Frank ${ }^{6}$, Zack Gainsforth ${ }^{1}$, Jon K. Hillier ${ }^{7}$, Frank Postberg ${ }^{7}$, Alexandre S. Simionovici ${ }^{8}$, Veerle J. Sterken ${ }^{9,10,11,12}$, Larry R. Nittler ${ }^{13}$, Carlton Allen ${ }^{6}$, David Anderson ${ }^{1}$, Asna Ansari ${ }^{14}$, Saša Bajt ${ }^{15}$, Ron K. Bastien $^{6}$, Nabil Bassim², John Bridges ${ }^{16}$, Donald E. Brownlee ${ }^{17}$, Mark Burchell ${ }^{18}$, Manfred Burghammer ${ }^{19}$, Hitesh Changela ${ }^{20}$, Peter Cloetens ${ }^{21}$, Andrew M. Davis ${ }^{22}$, Ryan Doll23, Christine Floss ${ }^{23}$, Eberhard Grün ${ }^{11}$, Philipp R. Heck ${ }^{14}$, Peter Hoppe ${ }^{24}$, Bruce Hudson ${ }^{25}$, Joachim Huth ${ }^{24}$, Anton Kears$\mathrm{ley}^{26}$, Ashley J. King ${ }^{22}$, Barry Lai ${ }^{27}$, Jan Leitner $^{24}$, Laurence Lemelle ${ }^{28}$, Ariel Leonard ${ }^{23}$, Hugues Leroux ${ }^{29}$, Robert Lettieri ${ }^{1}$, William Marchant ${ }^{1}$, Ryan Ogliore ${ }^{30}$, Wei Jia Ong ${ }^{23}$, Mark C. Price ${ }^{18}$, Scott A. Sandford ${ }^{31}$, JuanAngel Sans Tresseras ${ }^{21}$, Sylvia Schmitz ${ }^{4}$, Tom Schoonjans ${ }^{19}$, Kate Schreiber ${ }^{23}$, Geert Silversmit ${ }^{19}$, Vicente A. Solé ${ }^{21}$, Ralf Srama ${ }^{9}$, Frank Stadermann ${ }^{23 \dagger}$, Thomas Stephan ${ }^{22}$, Julien Stodolna ${ }^{1}$, Stephen Sutton ${ }^{27}$, Mario Trieloff $^{7}$, Peter Tsou ${ }^{32}$, Tolek Tyliszczak ${ }^{3}$, Bart Vekemans ${ }^{19}$, Laszlo Vincze ${ }^{19}$, Joshua Von Korff ${ }^{1}$, Naomi Wordsworth ${ }^{33}$, Daniel Zevin $^{1}$, Michael E. Zolensky ${ }^{6}$, 30,714 Star- dust@home dusters ${ }^{34}$, "Evidence for interstellar origin of seven dust particles collected by the Stardust spacecraft," Science 345(6198), 786 (15 August 2014). DOI: 10.1126/science. 1252496

Author affiliatons: ${ }^{1}$ University of California at Berkeley, ${ }^{2}$ Research Laboratory, ${ }^{3}$ Lawrence Berkeley Laboratory, ${ }^{4}$ Goethe University Frankfurt, ${ }^{5}$ State University of New York at Plattsburgh, ${ }^{6}$ NASA Johnson Space Center, ${ }^{7}$ University of Heidelberg, ${ }^{8}$ Observatoire des Sciences de l'Univers de Grenoble, ${ }^{9}$ University of Stuttgart, ${ }^{10} \mathrm{TU}$ Braunschweig, ${ }^{11} \mathrm{Max}$ Planck Institut für Kernphysik, ${ }^{12}$ International Space Sciences Institute, ${ }^{13}$ Carnegie Institution of Washington, ${ }^{14}$ Field Museum of Natural History, ${ }^{15}$ Deutsches

Elektronen-Synchrotron, ${ }^{16}$ University of Leicester, ${ }^{17}$ University of Washington, ${ }^{18} \mathrm{Uni}-$ versity of Kent, ${ }^{19}$ University of Ghent, ${ }^{20}$ University of New Mexico, ${ }^{21}$ European Synchrotron Radiation Facility, ${ }^{22}$ University of Chicago, ${ }^{23}$ Washington University in St. Louis, ${ }^{24}$ Max-Planck-Institut für Chemie, ${ }^{25} 615$ William Street, Ontario, Canada, ${ }^{26}$ Natural History Museum, London, ${ }^{27} \mathrm{Ar}-$ gonne National Laboratory, ${ }^{28}$ Ecole Normale Superieure de Lyon, ${ }^{29}$ University Lille 1 , ${ }^{30}$ University of Hawai'i at Manoa, ${ }^{31}$ NASA Ames Research Center, ${ }^{32}$ Jet Propulsion Laboratory, ${ }^{33}$ Wexbury, Farthing Green Lane, Stoke Poges, South Buckinghamshire, UK, ${ }^{34}$ Worldwide List of individual dusters is at http://stardustathome.

ssl.berkeley.edu/sciencedusters ${ }^{\dagger}$ Deceased. Correspondence:

*westphal@ssl.berkeley.edu

We are deeply grateful to the Stardust@-home dusters, whose tremendous efforts were critically important to the success of this project. The ISPE consortium gratefully acknowledges the NASA Discovery Program for Stardust, the fourth NASA Discovery mission. NASA grants supported the following authors: NNX09AC36G - A.J.W., A.L.B., Z.G., R.L., D.Z., W.M., and J.V.K.; NNX09AC63G - C.F., R.D., A.L., W.J.O., K.S., and F.J.S.; NNH11AQ61I - R.M.S., H.C.G., and N.D.B.; NNX11AC21G - A.M.D., A.J.K., and T.S.; NNX11AE15G - G.J.F. M.T. and F.P. acknowledge support by Klaus Tschira foundation. A.A. and P.R.H. were supported by the Tawani Foundation. M.J.B. and M.C.P. are supported by Science and Technology Facilities Council (UK). F.E.B., J.K.H., P.H., J.L., F.P., S.S., R.S., and M.T. were supported by funding of the German Science Foundation (DFG) within SPP1385: the first ten million years of the solar system - a planetary materials approach. The ESRF ID13 measurements were performed in the framework of ESRF LTP EC337, with financial support by the Funds for Scientific Research (FWO), Flanders, Belgium (contract nr. G.0395.11, G.0257.12N and Big Science program G.0C12.13). G. Silversmit was postdoctoral fellow of the FWO during the ISPE investigations. This research used resources of the Advanced Photon Source, a U.S. Department of Energy (DOE) Office of Science User Facility operated for the DOE Office of Science by Argonne National Laboratory under Contract No. DE-AC02-06CH11357. 


\section{SMOOTH FlOW IN MOLTEN Rock}

$M$

ost liquids flow with greater resistance when squeezed. But certain

molten silicate rocks actually do the opposite: They become less viscous as the pressure rises. A detailed explanation of these silicate melts has been lacking, but now $x$-ray scattering measurements taken at the APS have characterized the role of connected molecular units, or "polymers," in the fluid properties of the melts. With the help of complementary computer simulations, researchers have constructed an atomistic model in which polymer breakage is responsible for the viscosity drop at high pressure. This model may help explain certain geological phenomena, such as chemical evolution in the Earth's early magma ocean and the low-velocity zone seen in seismic data.

Fig. 1. (a) Viscosities of silicate melts vs. pressure in gigapascals (GPa). Blue symbols are for polymerized melts and gold symbols for depolymerized melts. Note the difference in response to pressure for polymerized versus depolymerized liquids. (b) Viscosities of silicate melts normalized with respect to their ambient pressure values. All depoymerized melts fall onto a single line, in which viscosity increases with pressure. Viscosities of polymerized melts, on the other hand, decrease with increasing pressure, many reaching turn-over within the pressure range displayed. Approximate values of $\mathrm{NBO} / \mathrm{T}$ for the polymerized melts are given on the right side of the figure.

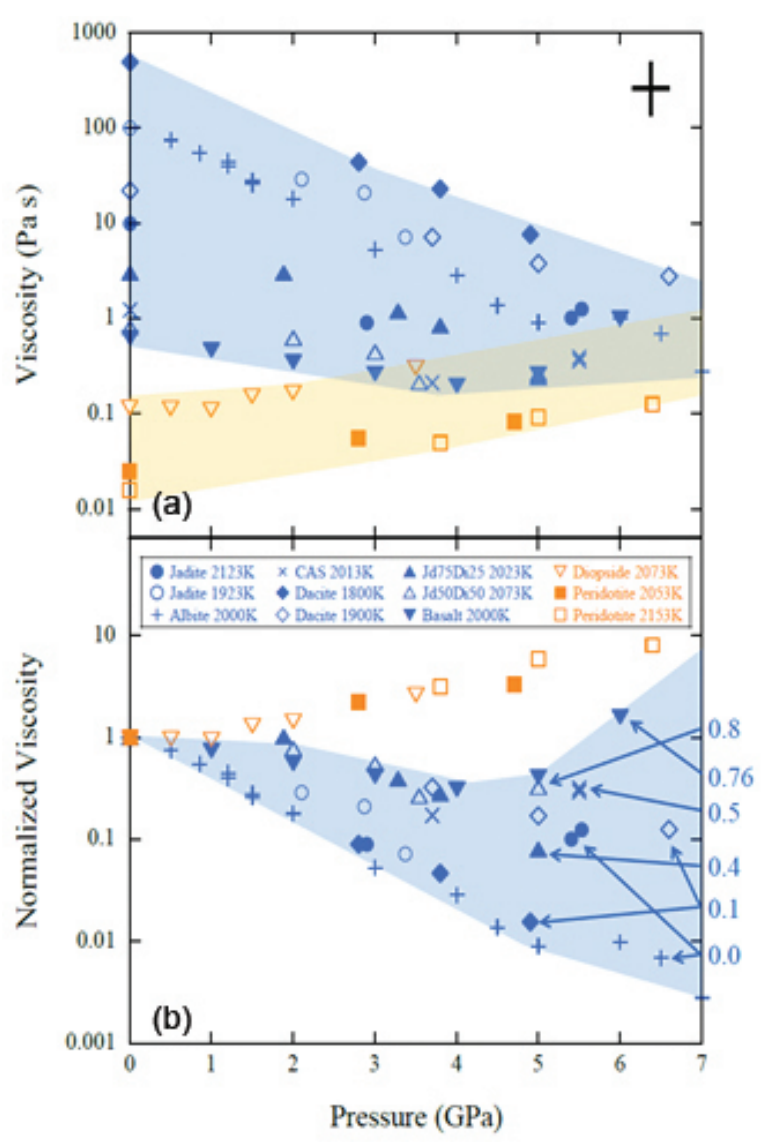

Pressure (GPa)
Silicate melts are commonly found underneath the Earth's surface as magma, or erupting as lava through a volcano. As their name suggests, these molten rocks contain "silica," or silicon dioxide. In most cases, the silicon and oxygen form tetrahedral-shaped clusters with one silicon atom in the center and four oxygen atoms at the corners. These tetrahedra can also link up to form chains, sheets, or three-dimensional networks, but this polymerization depends on the exact chemical makeup of the silicate (Fig. 1).

The silicate diopside, for example, contains calcium and magnesium atoms, which crowd around the siliconoxygen tetrahedra preventing polymerization. By contrast, the green mineral jadeite is a highly polymerized silicate whose make-up includes sodium and aluminum, the latter of which can form tetrahedra with oxygen atoms. Poly- 
merized silicate melts differ from depolymerized melts in that their viscosity decreases with rising pressure - at least up until a threshold pressure, after which point the viscosity begins to increase as in normal fluids.

Viscosity is due to diffusion of groups of atoms trying to move past each other. Clearly, large polymer units are major obstacles in a silicate melt, so the question is why increasing pressure reduces this friction.

In order to find the answer, scientists from The University of Chicago, Stony Brook University, and the Carnegie Institution of Washington performed a detailed $\mathrm{x}$-ray study of three types of silicate melts - a polymerized melt (jadeite), a depolymerized melt (diopside), and a 50-50 mixture of both.

This research counts as one of the first attempts to probe the atomic properties of silicate melts at subterranean pressures. The main hurdle in performing such experiments is that the materials utilized to contain the melt strongly scatter $\mathrm{x}$-rays. Extracting the relatively weak scattering signal from the light atoms in a silicate melt requires a highly collimated $\mathrm{x}$-ray beam.

For this study, the researchers chose the HP-CAT beamline 16-BM-D at the APS. The HP-CAT beamline allows the detection of $\mathrm{x}$-rays over a wide range of scattering angles.

During each run, millimeter-sized samples were exposed to temperatures up to $1800^{\circ} \mathrm{C}$ and pressures ranging from 0.2 to $4.9 \mathrm{GPa}$. To maintain stability under such extreme conditions, the experiments at 16-BM-D used a ParisEdinburgh press developed jointly by HP-CAT, GSECARS at Sector 13 of the APS, and the Consortium for Materials Properties Research in Earth Sciences.

The $x$-ray scattering data showed several peaks at particular scattering angles. From these peaks, the team could estimate the atomic ordering over short and intermediate distances.

In particular, they found the distance between neighboring tetrahedra decreased rapidly with increasing pressure in the case of polymerized melts. A similarly dramatic change was observed in the angle between two tetrahedra, which implied that the tetrahedra folded toward each other as pressure mounted. By contrast, the angle and

P (GPa, approx.)

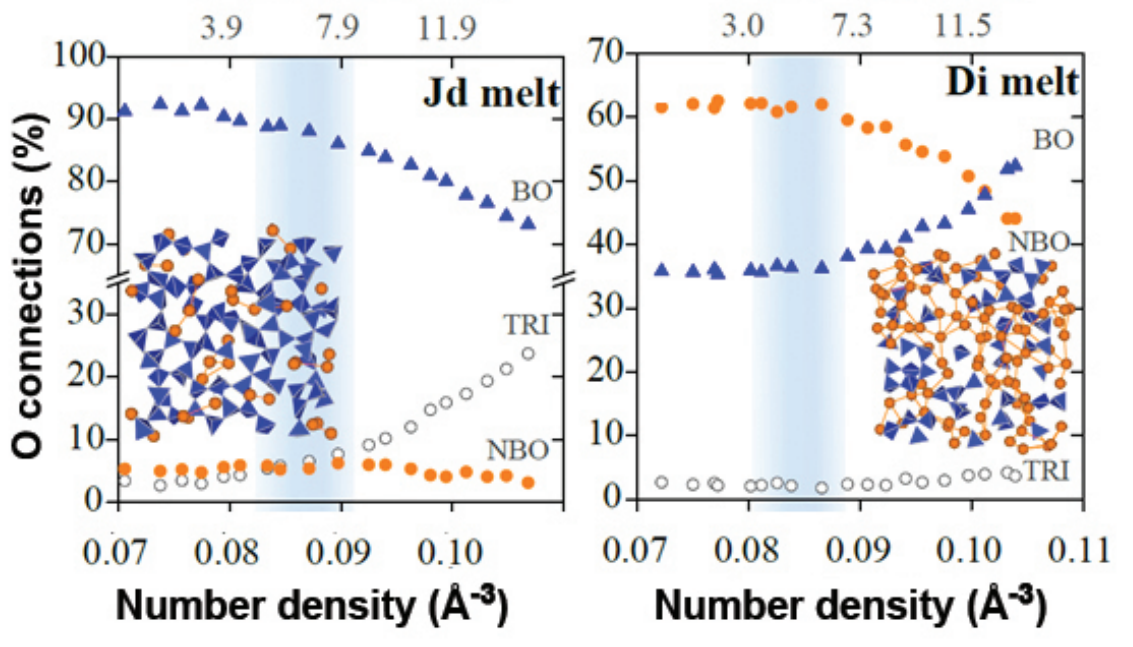

Fig. 2. Left: Molecular dynamics (MD) simulation of structural parameters for jadeite (Jd) melt, showing decrease in the fraction of bridging oxygens $(B O)$ with the increase in number density (or increase in pressure). This $B O$ decrease is offset by an increase in tri-clusters (TRI), which are oxygen atoms shared by three separate molecular units. The population of non-bridging oxygens (NBO) remains virtually unchanged with pressure. Inset shows a snapshot of the Jd melt, with $\mathrm{SiO}_{4}$ (dark-blue tetrahedra), $\mathrm{AlO}_{4}$ (light-blue tetrahedra), and sodium atoms (orange circles). Right: MD simulation of diopside ( $\mathrm{Di})$ melt. Note increasing $\mathrm{BO}$ and decreasing NBO populations with increasing number density/pressure (or number density). Inset is a snapshot of the melt structure, with with $\mathrm{SiO}_{4}$ (blue tetrahedra) and calcicum/magnesium atoms (orange circles).

atomic separations for depolymerized melts only showed a weak pressure-dependence.

To help make sense of this data, the team performed molecular dynamics simulations that tracked what fraction of oxygen atoms are acting as links, or "bridges," between two tetrahedra. These computations showed that polymerized melts begin with roughly $90 \%$ bridging oxygens, forming an interconnected network resembling a sponge (Fig. 2). As the pressure increases, the tetrahedral links try to bend but are obstructed by other atoms (in jadeite's case, sodium). So instead, the links break under the pressure, resulting in less bridging oxygens and lower viscosity. At a certain pressure, the polymerized melt becomes largely depolymerized, and the viscosity starts to increase with pressure.

\section{- Michael Schirber}

See: Yanbin Wang ${ }^{1 *}$, Tatsuya Sakamaki $^{1, \ddagger}$, Lawrie B. Skinner ${ }^{2}$, Zhicheng Jing ${ }^{1, \neq \ddagger}$, Tony $\mathrm{Yu}^{1}$, Yoshio Kono ${ }^{3}$, Changyong Park ${ }^{3}$, Guoyin Shen ${ }^{3}$, Mark L. Rivers ${ }^{1}$, and Stephen R. Sutton ${ }^{1}$, "Atomistic insight into viscosity and density of silicate melts under pressure," Nat. Comm. 5, 3241 (30 January 2014).
DOI: $10.1038 /$ ncomms4241

Author affiliations: ${ }^{1}$ The University of Chicago, ${ }^{2}$ Stony Brook University, ${ }^{3}$ Carnegie Institution of Washington Present addresses: †Tohoku University, $¥ \ddagger$ Case Western Reserve University Correspondence:

*wang@cars.uchicago.edu

Lava photo: https://www.flickr.com/photos/gnuckx/5350361507/

Funding was provided by the U.S. National Science Foundation (NSF) EAR-0968456 and 1214376. HP-CAT operations are supported by the U.S. Department of Energy (DOE) National Nuclear Security Administration under Award no. DE-NA0001974 and DOE Basic Energy Sciences under Award no. DE-FG02-99ER45775, with partial instrumentation funding by the NSF. GeoSoilEnviroCARS is supported by the NSF-Earth Sciences (EAR-1128799) and DOE-GeoSciences (DE-FG02-94ER14466). This research used resources of the Advanced Photon Source, a U.S. DOE Office of Science User Facility operated for the DOE Office of Science by Argonne National Laboratory under Contract No. DE-AC02$06 \mathrm{CH} 11357$.

16-BM-D • HP-CAT • Materials science, geoscience, chemistry, physics $•$ Powder angular dispersive $\mathrm{x}$-ray diffraction, $\mathrm{x}$-ray absorption near-edge structure, single-crystal diffraction, high-pressure diamond anvil cell $\bullet$ 6-70 keV • On-site $\cdot$ Accepting general users • 


\section{Respiration of BACteria In ANOXIC Aquifers}

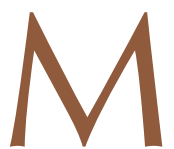

icrobes living deep underground must "breathe" without oxygen. Many microorganisms survive by reducing ferric iron ( $\mathrm{Fe}^{\mathrm{III}}$ ), sulfur $\left(\mathrm{S}^{0}\right)$, sulfate, and other substrates. Research carried out at the APS produced results that explore how dissimilar metal-reducing bacteria that have the ability to respire both iron and sulfur may gain an ecological advantage under fluctuating environmental conditions. Their findings shed new light on the coupled biogeochemical cycling of iron and sulfur, critical processes that in part determine the fate and transport of environmental contaminants (particularly in aquifers), the atmospheric flux of carbon from the subsurface, and even the geochemical evolution of the early Earth.

Sulfur (white-yellow powder, on top) may be far more essential than previously thought in helping microbes harvest energy from iron minerals (clockwise from top) yellow goethite, red hematite, orange lepidocrocite) and produce sulfur-iron minerals, like mackinawite (black). Photo: Mark Lopez, Argonne 
Dissimilatory metal-reducing bacteria (DMRB) are able to breathe using insoluble minerals as electron acceptors; the best studied of these involves the reductive dissolution of $\mathrm{Fe}^{\text {III }}$ minerals such as goethite. Many DMRB can also respire elemental sulfur, yet while $\mathrm{S}^{0}$ is abundant in marine sediments, it is much less prevalent in the freshwater sediments found in aquifers, but DMRB that can reduce both $\mathrm{Fe}^{\mathrm{III}}$ and $\mathrm{S}^{0}$ are common in these aquifers. Researchers from Argonne, The University of Chicago, the Illinois Institute of Technology, and the Georgia Institute of Technology sought to understand why such organisms would incur the cost of maintaining separate genetic pathways for $\mathrm{Fe}^{\mathrm{Ill}}$ and $\mathrm{S}^{0}$ reduction in the apparent absence of available $S^{0}$ to respire.

Using geochemical modeling, the researchers calculated the amount of thermodynamic energy available for microbial respiration from both $\mathrm{Fe}^{\mathrm{III}}$ and $\mathrm{S}^{0}$ reactions. The calculations revealed that under acidic conditions the microbes favor $\mathrm{Fe}^{\mathrm{III}}$, but that as $\mathrm{pH}$ increases $\mathrm{S}^{0}$ becomes more energetically favorable. These results were confirmed in laboratory experiments where a DMRB, Shewanella oneidensis, was found to reduce iron when conditions were acidic, but sulfur when $\mathrm{pH}$ was 9 and above. The $\mathrm{S}^{0}$ originated as sulfate, which is common in deep aquifers. Sulfate-reducing bacteria breathe in the sulfate, and exhale sulfide, which then reacts with iron minerals to form $\mathrm{S}^{0}$ and reduced iron. The $\mathrm{S}^{0}$ is then immediately used by Shewanella, resulting in the observed scarcity of sulfur in the aquifer (Fig 1.).

For analyzing the varied sulfur species, the authors used synchrotron measurement by $\mathrm{x}$-ray absorption nearedge structure (XANES) spectroscopy at the GSECARS 13-ID-E x-ray beamline at the APS.

The researchers hypothesize that DMRB with the genetic apparatus to enable them to respire both $\mathrm{Fe}^{\mathrm{III}}$ and $\mathrm{S}^{0}$ would gain a competitive advantage over those that could respire only one. They suggest that these separate pathways likely evolved on the early Earth, where parts of the primordial ocean may have been alkaline. These pathways were conserved since they allowed DMRB to generate energy over the wide $\mathrm{pH}$ ranges found on an evolving Earth. Although the direct reduction of Fe ${ }^{\text {Ill }}$ likely predominates in younger sediments, which tend to be slightly acidic, the reduction of $\mathrm{Fe}^{\mathrm{III}}$ through a $\mathrm{S}^{0}$ intermediate may be an important pathway in older, deeper aquifers, which tend to be more alkaline.

Understanding the activity of DMRB in the environment is critical to mitigating human problems such as the contamination of groundwater with heavy metals and the storage of excess atmospheric carbon in the subsurface. The goal of carbon capture and sequestration is to scrub excess carbon dioxide $\left(\mathrm{CO}_{2}\right)$, which contributes to global warming, from the atmosphere and store it in geological formations. One possible technique would be to compress and inject supercritical $\mathrm{CO}_{2}$ into a deep aquifer where it would react with reduced iron to form the mineral siderite, thus sequestering the carbon. Because DMRB are the primary drivers of the production of reduced iron in the subsurface, the creation of conditions favoring their growth is essential. Removing pollutants from aquifers is important because about one-fifth of the world's people depend on groundwater for drinking and many more depend on it for agriculture.

\section{- Dana Desonie}

See: Theodore M. Flynn ${ }^{1,2}$, Edward J. O'Loughlin ${ }^{1}$, Bhoopesh Mishra ${ }^{1,3}$, Thomas J. DiChristina ${ }^{4}$, and Kenneth M. Kemner" ${ }^{1 *}$, "Sulfur-mediated electron shuttling during bacterial iron reduction," Science 344(6187), 1039 (30 May 2014). DOI: $10.1126 /$ science. 1252066 Author affiliations: ${ }^{1}$ Argonne National Laboratory, ${ }^{2}$ The University of Chicago, ${ }^{3}$ Illinois Institute of Technology, ${ }^{4}$ Georgia Institute of Technology

Correspondence: * kemner@anl.gov

This research is part of the Subsurface Science Scientific Focus Area at Argonne Na-

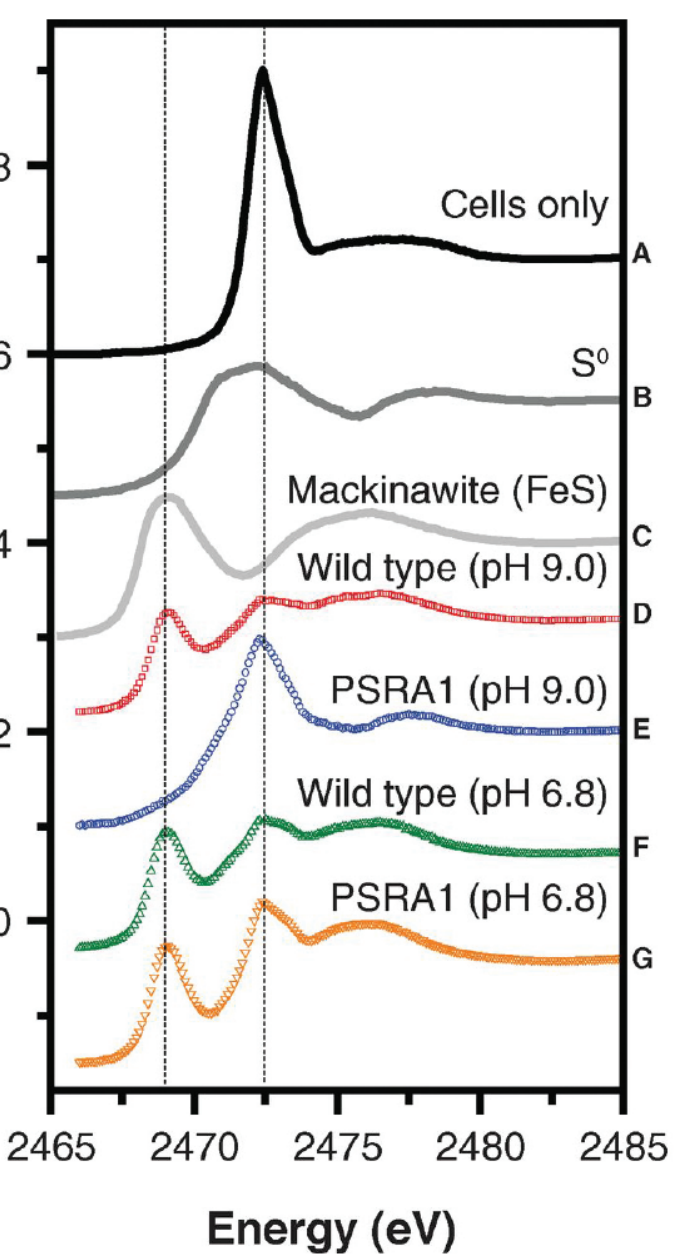

Energy (eV)

tional Laboratory supported by the Subsurface Biogeochemical Research Program, U.S. Department of Energy (DOE) Office of Science-Biological and Environmental Research, under DOE contract DE-AC02$06 \mathrm{CH} 11357$. T.F. was supported in part by an Argonne Director's Fellowship and the National Institute of Allergy and Infectious Diseases, National Institutes of Health, Department of Health and Human Services (contract no. HHSN272200900040C). T.D. was supported by the U.S. National Science Foundation (Molecular and Cellular Biosciences grant no. 1021735). GSECARS is supported by the National Science Foundation-Earth Sciences (EAR-1128799) and DOE-GeoSciences (DE-FG02-94ER14466). This research used resources of the Advanced Photon Source, a U.S. DOE Office of Science User Facility operated for the DOE Office of Science by Argonne National Laboratory under Contract No. DE-AC0206CH11357.

13-ID-E • GSECARS • Geoscience, environmental science - Microfluorescence (hard Xray), micro $x$-ray absorption fine structure, microdiffraction $\cdot 2.4-28 \mathrm{keV} \cdot$ On-site $\cdot$ Accepting general users $•$ 


\section{The EARTH'S DeEP LOWER MANTLE is Less Stable than We ThOught}

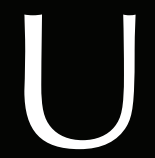

nderstanding what our Earth is made of is crucial to understanding our globe, how seismic waves travel through it, and the life cycle of any given mineral. But exploring what lies under the Earth's crust isn't simple - indeed, it can be just as difficult as determining what makes up other planets. Short of burrowing down some 1800 miles into the Earth, scientists must rely on work in the lab to understand the behavior of minerals at the intense heat and pressures experienced in such inaccessible regions.

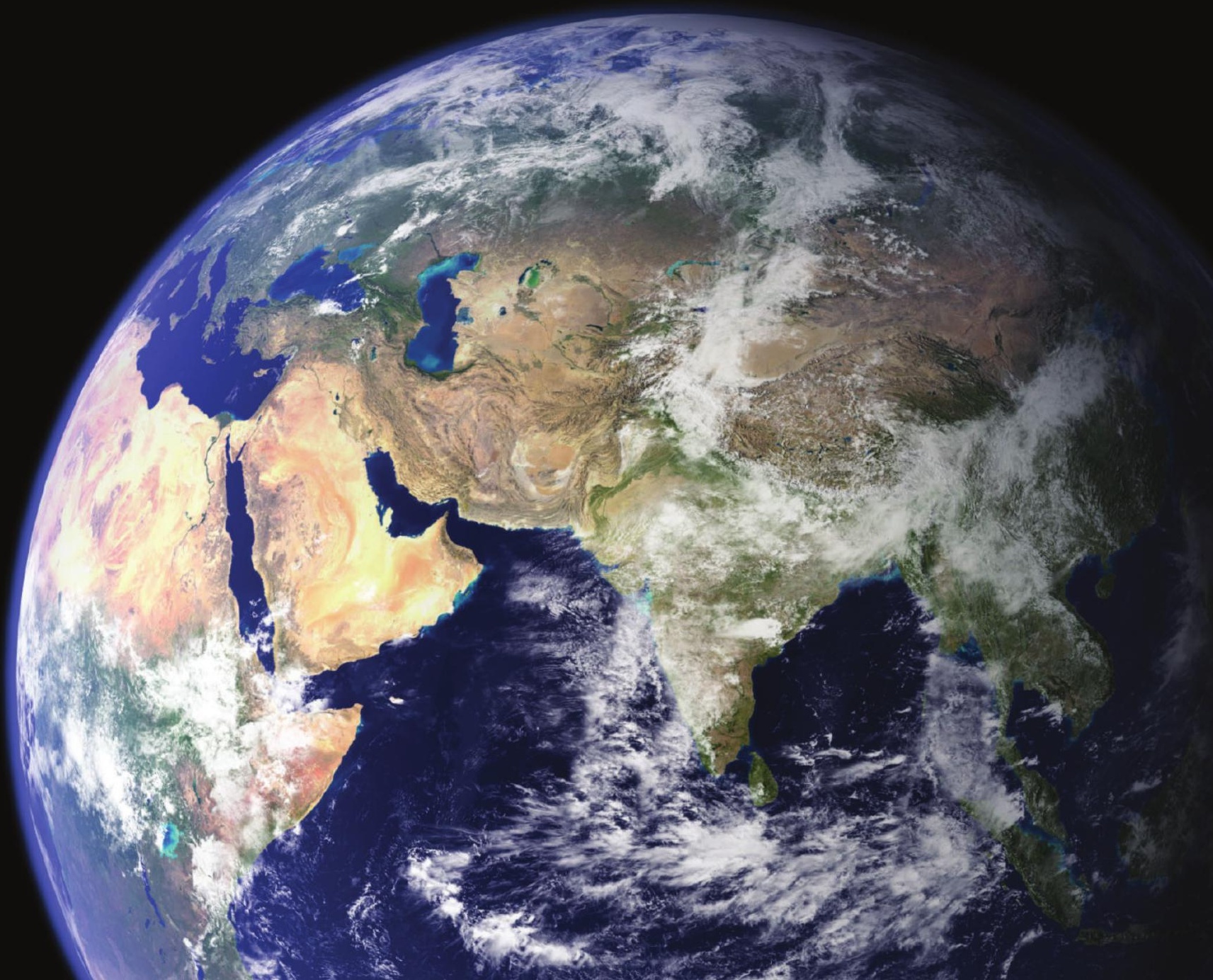


Recent work at the APS shows that the mineral perovskite, which had been thought to be the dominant mineral in the entire lower mantle stretching from around 400 miles to 1800 miles down - is unstable at the lowest depths. When studied under high temperatures and high pressures in the lab to simulate conditions at the deep lower mantle, perovskite separates into two distinct atomic structures, or phases. Including new minerals in our understanding of the Earth formed by scientists from the Center for High Pressure Science and Technology Advanced Research (China), the Carnegie Institution of Washington, Stanford University, SLAC National Accelerator Laboratory, the University of Minnesota, and Argonne, the team simulated deep lower mantle conditions to tease out just what was happening in this region.

The researchers squeezed pervoskite between diamond anvil cells to bring the samples to the appropriate

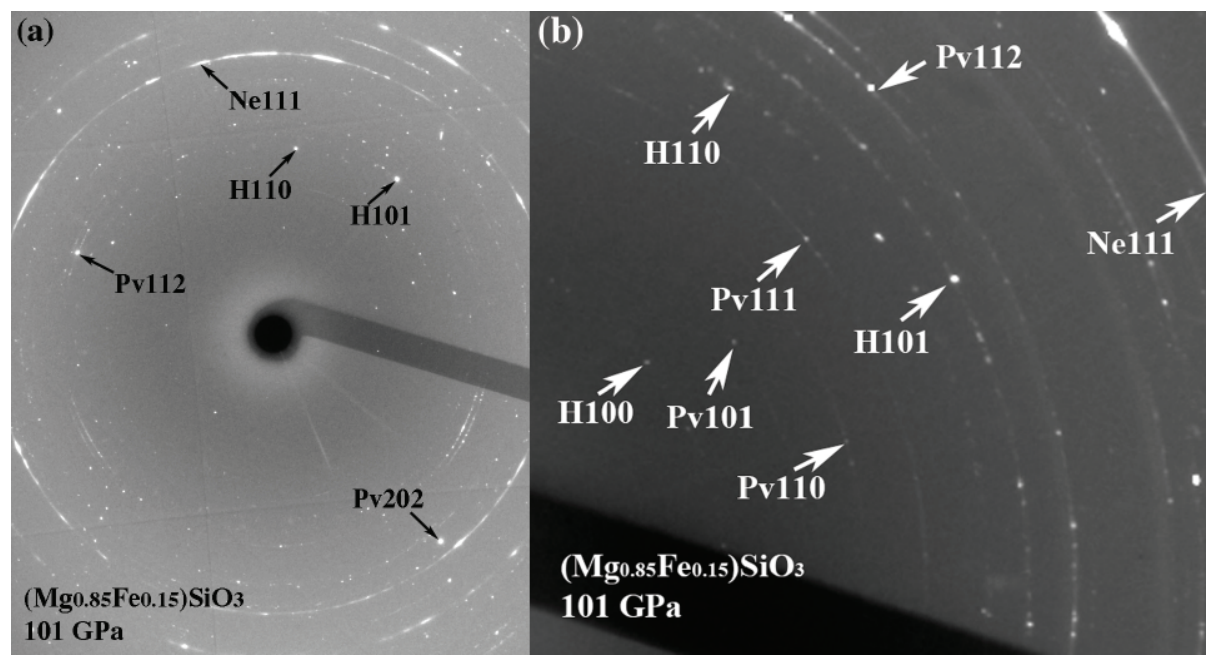

Fig. 1. X-ray diffraction images show the atomic structure of perovskite after it has experienced pressures and temperatures like those of Earth's deep lower mantle. Two distinct phases - perovskite and the $\mathrm{H}$-phase - are visible.

could lead to a serious readjustment of current theories.

The lower mantle may contain major amounts of previously unidentified phases of perovskite. All Earth models need to be reconsidered to take this into account.

Indeed, such geodynamic models have already been changed quite a bit in the last 10 years. The prevailing theory in the 20th century was that perovskite dominated the lower mantle. The perovskite was not thought to change structure until the bottommost part of the mantle, where the pressures help it transition into an iron-poor mineral called postperovskite.

However, data showing how seismic waves move through the Earth at about 1200 miles deep doesn't perfectly jibe with this theory. The waves travel in puzzling ways that simply can't be explained if they're traveling through perovskite and post-perovskite.

For the current research, per- pressure. Simultaneously, the samples were heated with lasers to between $3500^{\circ}$ and $3800^{\circ} \mathrm{F}$.

The team then determined the atomic structure of the samples via synchrotron x-ray diffraction using the GSECARS 13-ID-C,D beamline, the HP-CAT 16-ID-B beamline, and the XSD 34-ID-E beamline, all at the APS.

The scientists found that the pervoskite had undergone an unexpected change.

Surprisingly, the perovskite was unstable. It split into two phases, one without iron and one that was iron-rich, with a hexagonal structure, called the $\mathrm{H}$-phase (Fig. 1). This $\mathrm{H}$-phase may well help explain the enigmatic ways that seismic waves travel through the deep lower mantle.

The team will next try to tease out more detail about the mineral's structure and determine if it jibes with seismic observation. - Karen Fox
See: Li Zhang ${ }^{1,2 *}$, Yue Meng ${ }^{2}$, Wenge Yang $^{1,2}$, Lin Wang ${ }^{1,2}$, Wendy L. Mao ${ }^{3,4}$, Qiao-Shi Zeng ${ }^{3}$, Jong Seok Jeong ${ }^{5}$, Andrew J. Wagner ${ }^{5}$, K. Andre Mkhoyan ${ }^{5}$, Wenjun Liu ${ }^{6}$, Ruqing $\mathrm{Xu}^{6}$, Ho-kwang $\mathrm{Mao}^{1,2}$, "Disproportionation of $(\mathrm{Mg}, \mathrm{Fe}) \mathrm{SiO}_{3}$ perovskite in Earth's deep lower mantle," Science 344(6186), 877 (23 May 2014).

DOI: 10.1126/science.1250274

Author affiliations: ${ }^{1}$ Center for High Pressure Science and Technology Advanced Research, ${ }^{2}$ Carnegie Institution of Washington, ${ }^{3}$ Stanford University, ${ }^{4}$ SLAC National Accelerator Laboratory, 5University of Minnesota, ${ }^{6}$ Argonne National Laboratory Correspondence:

*zhangli@hpstar.ac.cn

Photo of the Pacific region of Earth on the facing page: Wikimedia Commons: http://earthobservatory.nasa.gov/IOTD/view. php?id $=4620$

The research is supported by National Science Foundation (NSF) grants EAR0911492, EAR-1119504, EAR-1141929, and EAR-1345112. HP-CAT operations are supported by the U.S. Department of Energy (DOE) National Nuclear Security Administration under award DE-NA0001974 and DOEBasic Energy Sciences under award DE-FG02-99ER45775, with partial instrumentation funding by the NSF. GeoSoilEnviro- CARS is supported by NSF-Earth Sciences (EAR 1128799) and DOE-GeoSciences (DE-FG02-94ER14466). This research used resources of the Advanced Photon Source, a U.S. DOE Office of Science User Facility operated for the DOE Office of Science by Argonne National Laboratory under Contract No. DE-AC0206CH11357.

13-ID-C,D • GSECARS • Geoscience, environmental science $\cdot$ Microdiffraction, $x$-ray absorption fine structure, microfluorescence (hard x-ray), high-pressure diamond anvil cell, high-pressure multi-anvil press $\cdot 4-45 \mathrm{keV}$ • On-site $\cdot$ Accepting general users $•$

16-ID-B • HP-CAT - Materials science, geoscience, chemistry, physics - Microdiffraction, single-crystal diffraction, high-pressure diamond anvil cell • 14-42 keV • On-site • Accepting general users $\bullet$

34-ID-E - XSD - Materials science, physics, environmental science, geoscience $-\mathrm{Mi}$ crodiffraction, Laue crystallography, microbeam $\cdot 7-30 \mathrm{keV} \cdot$ On-site - Accepting general users 


\section{A Meteorite Tells Tales of the EARTH'S DEEP MANTLE}

A meteorite is playing a role in helping scientists characterize an important mineral in Earth's mantle. This mantle mineral is $\mathrm{MgSiO}_{3}$ in its perovskite crystal form, which scientists theorize constitutes approximately $38 \%$ of Earth's mantle by volume. Through examination of the meteorite at the APS, scientists were able to study the crystal chemistry of this mineral, offering a window into conditions near the core-mantle boundary as well as heat and mass transfer throughout Earth's mantle. Additionally, the team was able to detect sufficient quantities of the $\mathrm{MgSiO}_{3}$-perovskite to meet the requirements of the International Mineralogical Association for naming a new mineral. This scientific body has approved the team's choice of "bridgmanite" in honor of Percy W. Bridgman, a 1946 Nobel Laureate in Physics.

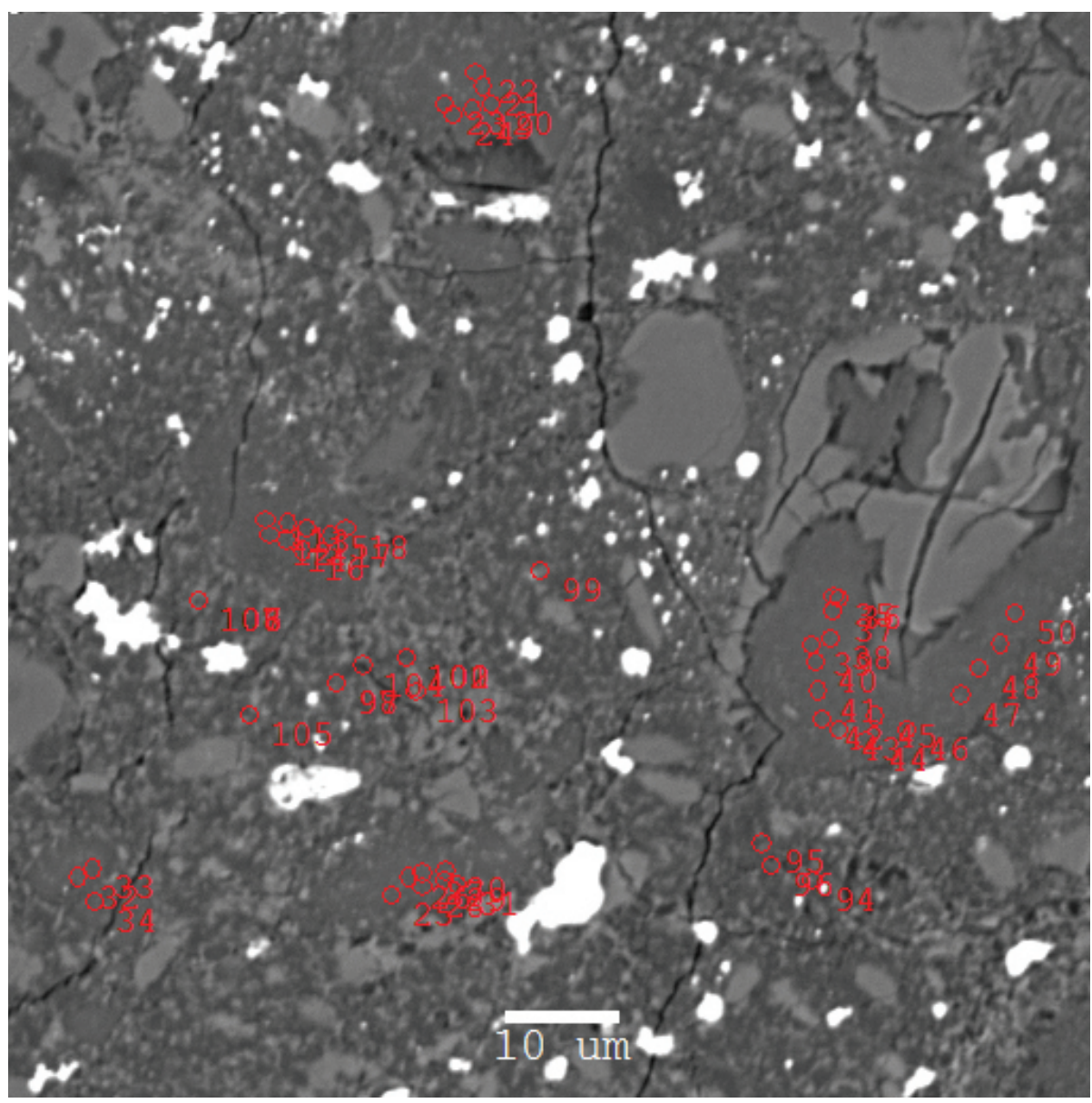

Fig. 1. Regions containing bridgmanite in mixture with akimotoite (another high-pressure silicate phase). The red circles indicate points of microchemical analysis where bridgmanite and akimotoite were found. As one can see, bridgmanite and akimotoite occur only in the dark gray regions. The brighter grey regions are ringwoodite (silicate-spinel) and the very bright regions are iron-sulfide (troillite). The aggregates of bridgmanite and akimotoite formed by transformation of low pressure silicate crystals (the mineral enstatite) that were trapped in shock-generated melt. This melt is now the fine-grained checquered matrix surrounding the aggregates of bridgmanite plus akimotoite, ringwoodite, and troillite.
Meteorites offer an opportunity to peer into Earth's depths for multiple reasons. The minerals they contain are more easily accessible than mantle minerals, by virtue of being found on Earth's surface as opposed to deep within our planet. Also, meteorites may preserve these minerals in a way that rocks subject to heat-related movement (convection) cannot.

Bridgmanite doesn't survive the trip via convection to Earth's upper mantle because it vitrifies - turning into a glass-like substance - at temperatures around $37^{\circ} \mathrm{C}\left(98^{\circ} \mathrm{F}\right)$. Bridgmanite is only stable in its crystalline form at high pressure and temperature, but remains stable down to a depth of approximately $2700 \mathrm{~km}$, near the Earth coremantle boundary.

Most meteorites experience heavy shock melting from collisions in space prior to their passage through the Earth's atmosphere. During shock melting, pressures and temperatures can be high enough to produce bridgmanite, but - equally important - shock melting returns the newly-formed bridgmanite to ambient conditions quickly enough to keep it from deteriorating.

The meteorite containing the team's bridgmanite is not a new one. The Tenham meteorite fell in South Gregory, Queensland, Australia, in 1879. It was approximately $51 \mathrm{~kg}$ before breaking up on entering the Earth's atmosphere. The Tenham meteorite may be classified as an "ordinary chon- 
drite" - a common type of meteorite theorized to be from one of three asteroid parent bodies - but this is not the first time it has been used to identify a new mineral nor name said mineral: in 1969 it was used as the first natural occurrence of ringwoodite.

Because chondrites are pieces of undifferentiated, or unmelted, parent bodies, their composition provides information about conditions in the early solar system. Since the Tenham meteorite must have collided with another body in order to produce the shock-melt veins within it, it tells scientists about the conditions for forming bridgmanite, which constrains those formation conditions here on Earth.

Researchers in this study from the University of Nevada, Las Vegas; the California Institute of Technology; and The University of Chicago searched the shock-melt veins of Tenham and other meteorites using microfocused synchrotron x-ray beams at the GSECARS 13-ID-C,D beamline at the APS. They chose microfocused synchrotron $\mathrm{x}$-ray diffraction over transmission electron microscopy because the beams from the latter turn bridgmanite to glass during testing. The researchers always found bridgmanite in the presence of the rare silicate mineral akimotoite and never as isolated crystals within the shock-melted portion of the Tenham meteorite (Fig. 1).

The pairing of bridgmanite and akimotoite sets limits for the formation of bridgmanite based on the known pressure and temperature limits for akimotoite. The lack of isolated crystals indicates that the conditions of bridgmanite formation were not of high enough pressure to crystallize it out of the liquid shock melt. The pressure and temperature values calculated from the team's results agree with previously-published data from both microscopy and computer modeling.

\section{- Mary Alexandra Agner}

See: Oliver Tschauner ${ }^{1 *}$, Chi Ma², John R. Beckett ${ }^{2}$, Clemens Prescher ${ }^{3}$, Vitali B. Prakapenka ${ }^{3}$, and George R. Rossman" $^{2}$, "Discovery of bridgmanite, the most abundant mineral in Earth, in a shocked meteorite," Science 346 (6213), 1100 (28 November 2014). DOI:

\subsection{6/science. 1259369}

Author affiliations: ${ }^{1}$ University of Nevada, Las Vegas; ${ }^{2}$ California Institute of Technology; ${ }^{3}$ The University of Chicago Correspondence:

*olivert@physics.unlv.edu

This work was supported by U.S. Department of Energy (DOE) award DESC0005278, NASA grant NNX12AH63G, and U.S. National Science Foundation (NSF) grants EAR-1128799, DE-FG02-94ER14466, EAR-0318518, and DMR-0080065.

GeoSoilEnviroCARS is supported by NSFEarth Sciences (EAR-1128799) and DOEGeoSciences (DE-FG02-94ER14466). This research used resources of the Advanced Photon Source, a U.S. DOE Office of Science User Facility operated for the DOE Office of Science by Argonne National Laboratory under Contract No. DE-AC02$06 \mathrm{CH} 11357$.

13-ID-C,D • GSECARS • Geoscience, environmental science - Surface diffraction, $x$-ray standing waves, microdiffraction, $x$-ray $a b-$ sorption fine structure, resonant inelastic $x$-ray scattering, high-pressure diamond anvil cell, high-pressure multi-anvil press $\bullet 4-45 \mathrm{keV}, 10$ $75 \mathrm{keV} \cdot$ On-site $\cdot$ Accepting general users •
"The Tenham Meteorite. Interesting specimens," from the Queensland Times, published at Ipswich, Queensland, August, 1911 , as quoted in The Mineralogical Magazine and Journal of the Mineralogical Society, No. 156, March, 1937 Vol. XXIV, "The Tenham (Queensland) meteoritic shower of 1879," by By L.J. Spencer, C.B.E., B.Sc., M.A., Se.D., F.R.S., Formerly Keeper of Minerals in the British Museum: "One night in February, 1869, Mr. M. Hammond, owner of Tenham Station, was camped with his brothers, whilst mustering cattle, near the junction of Cooper and Kyabra Creeks in southwest Queensland. The darkness was suddenly illuminated as if by a lightning flash, a noise like a rushing motor-car was heard, and upon looking up the brothers beheld a brilliant meteoric shower passing from west to east. Soon afterwards the locality in which the meteorites fell was found, and from time to time a number of specimens were collected, the largest weighing $130 \mathrm{lb}$. Twelve months ago Mr. Hammond told Mr. R. A. Wearne, B.A., Principal of the Ipswich Technical College, about these meteorites, and upon being urged to give publicity to such an important discovery, he promised to bring them to Brisbane at the first opportunity. On the 8th of July ultimo Mr. Hammond fulfilled his promise and brought a number of the meteoric fragments with him to Brisbane. A photo of the meteorites was taken at the Wiley Studio, and the collection was then handed to Mr. Wearne for distribution. Mr. Wearne then interviewed Mr. MacDonald of the University Department of Mines and Mr. Dunstan, Government Geologist, and arranged to have analyses made of these most interesting extra-terrestrial rocks. The complete analyses are not yet to hand, but preliminary tests show that the Tenham meteorites are of the stony variety, but contain a somewhat large percentage of nickel and iron. Small stony like enclosures can be seen between the bright metallic patches upon a polished surface. The specific gravity of the specimens averages about $3 \cdot 5$."

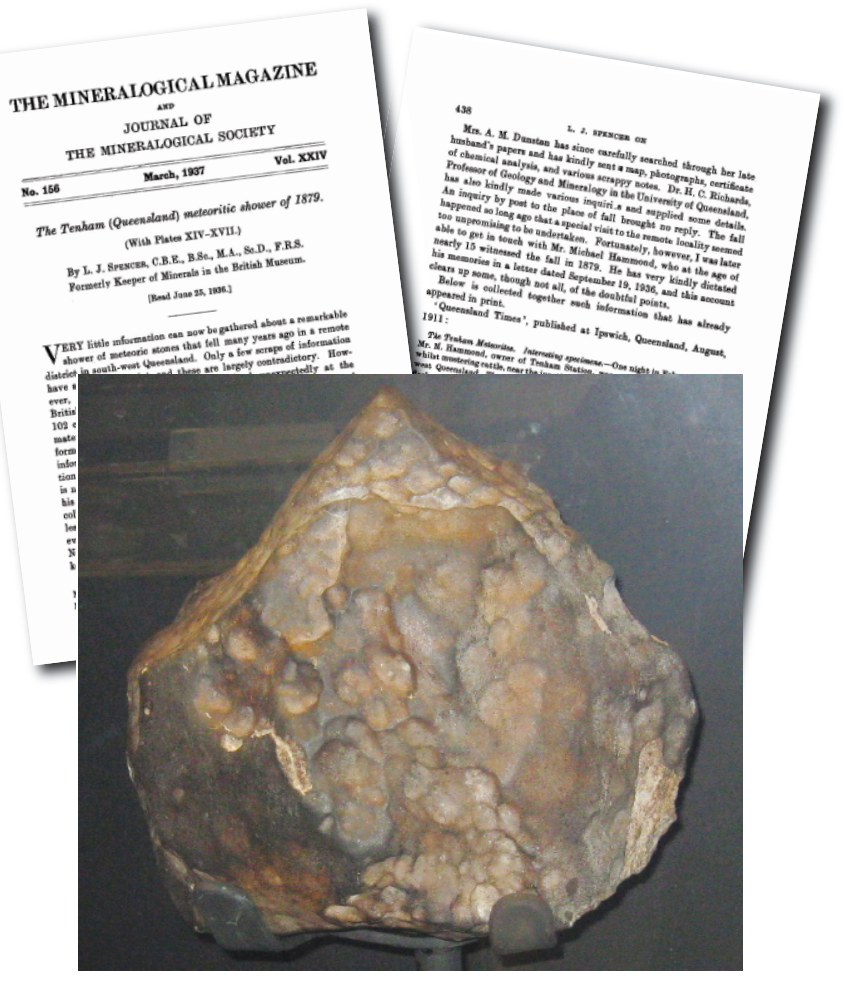

The Tenham Meteorite, on display in the Natural History Museum, London. Photo: Chemical Engineer - Own work. Licensed under CC BY-SA 3.0 via Wikimedia Commons http://commons.wikimedia.org/wiki/File:Tenham_Meteorite.JPG\#/media/File:Tenham_Meteorite.JPG 


\section{Hidden Carbon in the Earth's Core?}

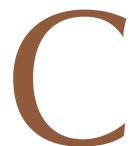

ertain seismic waves move slower than expected through the Earth's core, causing researchers to rethink our ideas about the composition of the innermost region of our planet. One possibility is that the core contains a large amount of carbon. New research on a form of iron-carbide, $\mathrm{Fe}_{7} \mathrm{C}_{3}$, shows that it may have the required low seismic wave velocity at high pressure. The experiments, performed at two APS beamlines, provide a first-ever estimate of the speed of seismic waves in this iron-carbide at core conditions and suggest that the iron-carbide's anomalous velocity behavior is due to a change in the electron spin configuration of iron in the material. The results could imply that the Earth's core is rich in $\mathrm{Fe}_{7} \mathrm{C}_{3}$, which might explain where some of Earth's supposedly "missing carbon" is hiding.

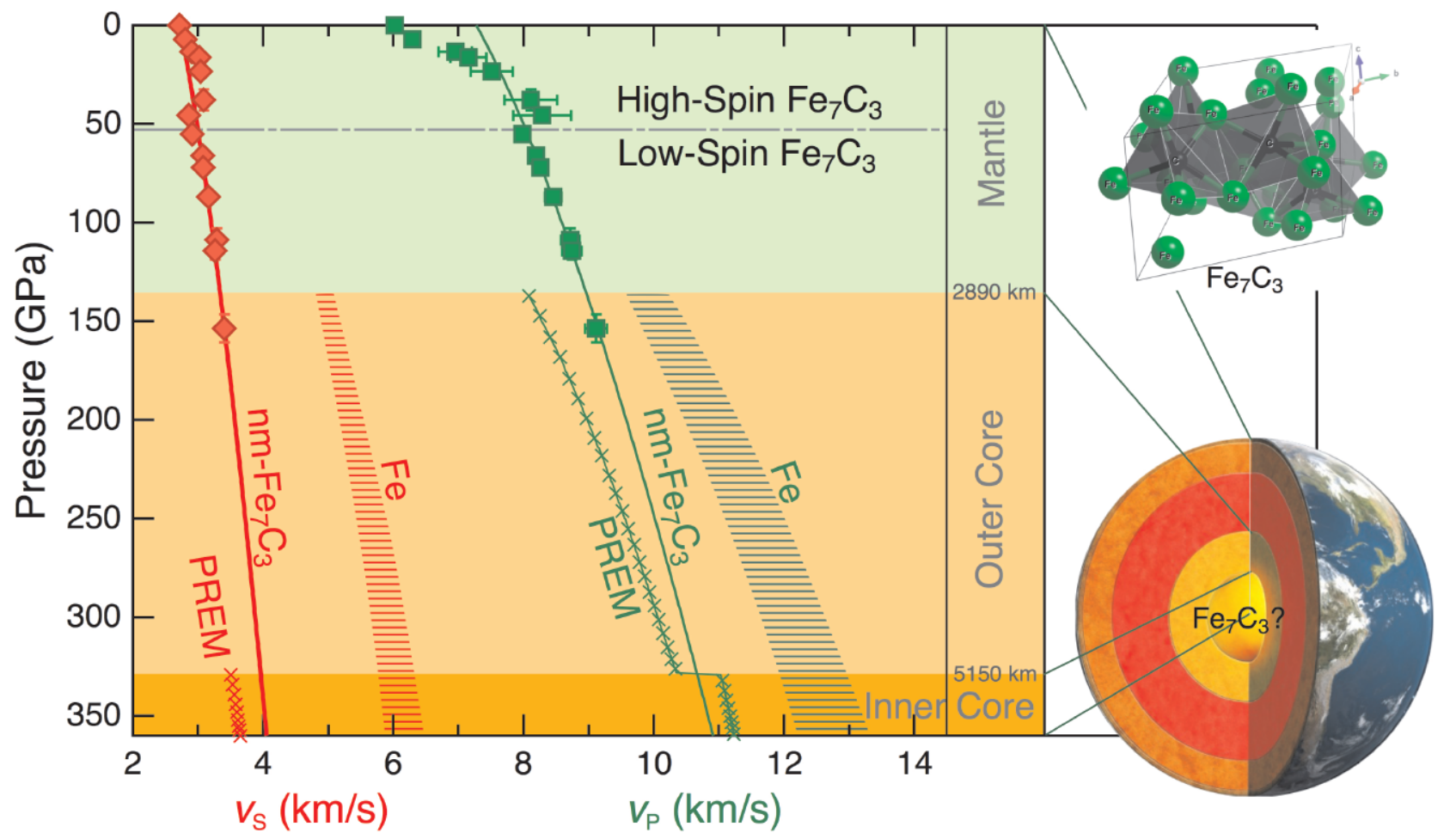

Fig. 1. Observations at the APS have provided the first-ever estimates of the seismic wave velocities of $\mathrm{Fe}_{7} \mathrm{C}_{3}$ at high pressure. The $\mathrm{S}$-wave velocity $\left(v_{\mathrm{s}}\right)$ data points are represented by red diamonds, while the $\mathrm{P}$-wave velocity $\left(v_{\mathrm{p}}\right)$ data points are green squares. The transition from high-spin to low-spin $\mathrm{Fe}_{7} \mathrm{C}_{3}$ occurs around $50 \mathrm{GPa}$. The solid red and green lines show the extrapolations towards inner core pressures (330 GPa) at room temperature. For comparison, the dashed line regions show the seismic wave estimates for pure iron. It's clear that the iron-carbide estimates come closer than the pure iron estimates to the empirically-defined values, shown as crosses, which come from preliminary reference Earth model, or PREM. Illustration: Bin Chen, University of Hawaii at Manoa 
Earthquakes produce seismic waves of two varieties: compression waves ( $\mathrm{P}$-waves) and shear waves (Swaves). Observations of waves traversing the center of the Earth have revealed that S-waves arrive much later (with respect to the P-waves) than would be expected for a solid pure-iron core. A potential explanation is that iron in the core is mixed with lighter elements - such as carbon, silicon, or oxygen. Geologists are currently studying different iron alloy candidates to find one that might explain the seismic wave observations.

As far as carbon is concerned, past work looked at the iron-carbide, $\mathrm{Fe}_{3} \mathrm{C}$, but recent studies showed that a different iron-carbide, $\mathrm{Fe}_{7} \mathrm{C}_{3}$, is more stable at high pressure. A group of researchers from the University of Michigan, the University of Illinois at UrbanaChampaign, the University of Hawaii at Manoa, the California Institute of Technology, the Carnegie Institution of Washington, and Argonne performed the high-pressure experiments on $\mathrm{Fe}_{7} \mathrm{C}_{3}$.

The $\mathrm{Fe}_{7} \mathrm{C}_{3}$ samples were synthesized at the University of Michigan and at the GSECARS facility at the APS. The team used the iron isotope 57 because it is a Mössbauer isotope. The nuclear resonance, excited with 14.4keV $\mathrm{x}$-rays, provided unique information about the valence, spin state, and geometric arrangement of atoms around the iron atom. The team loaded their samples into a diamond anvil cell to apply pressure up to a maximum of $154 \mathrm{GPa}$ and measured the effects of high pressure on $\mathrm{Fe}_{7} \mathrm{C}_{3}$ using nuclear resonant inelastic $x$-ray scattering (NRIXS), which was performed at the XSD beamline 3-ID-B,C,D. This beamline offers tightly-focused synchrotron radiation (with a beam-waist of less than $10 \mu \mathrm{m}$ ) and a small energy bandwidth (1 meV) at the ${ }^{57} \mathrm{Fe}$ resonance of $\sim 14 \mathrm{keV}$.

The NRIXS data provided a measurement of the phonon density-ofstates, from which the researchers derived the seismic wave velocities for P-waves and S-waves. At low pressures, both velocities increased with pressure, as is usually the case, but near $50 \mathrm{GPa}$, the S-wave velocity dropped abruptly from 3.1 to $2.8 \mathrm{~km} / \mathrm{s}$. Beyond this dip (toward higher pressures) both seismic wave velocities increased, but at a slower rate than before the dip.

The velocity dip corresponds to a spin-pairing transition in $\mathrm{Fe}_{7} \mathrm{C}_{3}$ near 50 GPa. In this transition, the electrons in the $3 d$ orbital of iron atoms pair up in a way that lowers the total spin momentum of the atoms. The team explored this transition using $x$-ray emission spectroscopy at the HP-CAT 16-ID-D beamline, as well as synchrotron Mössbauer spectroscopy at the 3-ID-B,C,D beamline. The observations suggested that the paired electrons exert less resistance to shearing, and this "shear softening" may explain why the S-wave velocity remains low above the transition.

When the team extrapolated their results to higher pressure, they found that the S-wave velocity of $\mathrm{Fe}_{7} \mathrm{C}_{3}$ at $330 \mathrm{GPa}$ and room temperature was only $14 \%$ above the empirically inferred S-wave velocity in the core (Fig. 1). The discrepancy is small enough that it could be explained by a modest temperature-dependence in the seismic wave velocity of $\mathrm{Fe}_{7} \mathrm{C}_{3}$. By comparison, pure iron has an S-wave velocity that is $65 \%$ above observed values, which is hard to account for without invoking a dramatic temperature-dependence.

One way, therefore, to explain the low S-wave velocity in the core is to assume that it is principally made up of $\mathrm{Fe}_{7} \mathrm{C}_{3}$. If such were the case, then the core would contain $60 \times 10^{20} \mathrm{~kg}$ of carbon, which is roughly 10 times the current core estimate. That amount of carbon would make the core the largest reservoir of carbon on Earth. Moreover, this could help explain why the carbon abundance is so low - relative to silicon - on the Earth's surface. According to this hypothesis, roughly twothirds of the planet's carbon inventory is locked in the core - trapped there by its interaction with iron during the formation of the planet.

$$
\text { - Michael Schirber }
$$

See: Bin Chen ${ }^{1,2,3 *}$, Zeyu $\mathrm{Li}^{1}$, Dongzhou Zhang ${ }^{4}$, Jiachao Liu ${ }^{1}$,
Michael Y. $\mathrm{Hu}^{5}$, Jiyong Zhao ${ }^{5}$, Wenli $\mathrm{Bi}^{2,5}$, E. Ercan Alp ${ }^{5}$, Yuming Xiao ${ }^{6}$, Paul $\mathrm{Chow}^{6}$, and Jie $\mathrm{Li}^{1 * *}$, "Hidden carbon in Earth's inner core revealed by shear softening in dense $\mathrm{Fe}_{7} \mathrm{C}_{3}$," Proc. Natl. Acad. Sci. USA 111(50), 17755 (December 16, 2014).

DOI: 10.1073/pnas.1411154111 Author affiliations: ${ }^{1}$ University of Michigan, ${ }^{2}$ University of Illinois at UrbanaChampaign, ${ }^{3}$ University of Hawaii at Manoa, ${ }^{4}$ California Institute of Technology, ${ }^{5}$ Argonne National Laboratory, ${ }^{6}$ Carnegie Institution of Washington Correspondence:

*binchen@hawaii.edu, ** jackieli@umich.edu

This study was supported in part by GSECARS, which is supported by the National Science Foundation (NSF)-Earth Sciences (EAR-1128799) and the U.S. Department of Energy (DOE)-GeoSciences (DE-FG0294ER14466). The authors acknowledge support from Grants NSF EAR-1219891, NSF EAR-1023729, NSF INSPIRE AST-1344133, and Carnegie/DOE Alliance Center CI JL 2009-05246. HP-CAT operations are supported by the DOE-National Nuclear Security Administration under Award No. DENA0001974 and DOE-Basic Energy Sciences under Award No. DE-FG02-99ER45775, with partial instrumentation funding by the NSF. B.C. acknowledges support from COMPRES and the University of Hawaii. School of Ocean and Earth Science and Technology contribution no. 9228, and the Hawaii Institute of Geophysics and Planetology contribution no. 2057. This research used resources of the Advanced Photon Source, a U.S. DOE Office of Science User Facility operated for the DOE Office of Science by Argonne National Laboratory under Contract No. DE-AC02-06CH11357.

3-ID-B,C,D • XSD • Physics, geoscience, life sciences, chemistry, materials science $\cdot \mathrm{Nu}$ clear resonant scattering, inelastic $x$-ray scattering, high-pressure diamond anvil cell $\bullet$ 7-27 $\mathrm{keV}, 14.41-14.42 \mathrm{keV} \cdot$ On-site $\cdot$ Accepting general users $•$

16-ID-D • HP-CAT • Materials science, geoscience, chemistry, physics $\bullet$ Nuclear resonant scattering, inelastic $x$-ray scattering (1-eV resolution), x-ray Raman scattering, x-ray emission spectroscopy, high-pressure diamond anvil cell • 5-35 keV, 14.41-14.42 keV • Onsite $\cdot$ Accepting general users $•$ 


\section{Testing Crystal Candidates for EARTH'S INNER CORE}

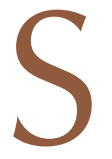

cientists have uncovered much about the Earth's solid inner core, but many mysteries still surround our planet's deep interior. The major element in the core is iron, but the exact crystal form of this element continues to be debated. Also uncertain is the identity and quantity of light elements that are likely alloyed with iron in the core. Laboratory experiments on candidate core materials have generally focused on a specific crystal form of iron called hexagonal close-packed (hcp), but recent theoretical evidence shows that pure hcp iron may not explain some seismic observations. A new study looks at an alternative possibility: body-centered cubic (bcc) iron and its alloy with silicon. Using high-brightness $x$-rays from the APS, researchers measured for the first time the sound speed of bcc iron at high temperature and pressure simultaneously. When compared with other observations, the new results suggest that bcc iron alloy could be a core constituent.

Fig. 1. Representative HERIX spectra of bcc iron (bcc-Fe) and iron-silicon alloy (bcc$\mathrm{Fe}_{0.85} \mathrm{Si}_{0.15}$ ). Each row provides data at a specific scattering angle, which corresponds to different values of the momentum transfer $(Q)$. The columns from left to right correspond to: bcc-Fe at $7 \mathrm{GPa}$ and $500 \mathrm{~K}$; bcc-Fe at 11 $\mathrm{GPa}$ and $700 \mathrm{~K}$; bcc- $\mathrm{Fe}_{0.85} \mathrm{Si}_{0.15}$ at $9 \mathrm{GPa}$ and $300 \mathrm{~K}$. The location of the longitudinal acoustic phonon peak (LA) allows for the sound speed to be determined.

Much of our knowledge about the Earth's core has come from studying seismic waves that pass through the center of the planet. A puzzling aspect about these waves is that they travel about $3 \%$ to $4 \%$ faster in the direction along the Earth's rotation axis than in the plane of the equator. The current understanding of this seismic wave anisotropy is that crystals in the inner core are preferentially aligned in such a way that the core materials display elastic anisotropy. This elasticity affects the sound speed at which seismic waves propagate. However, the elasticity of hcp iron crystals - which are thought to be the most stable iron phase in the extreme environment of the core - is fairly constant (or isotropic) with respect to direction. By contrast, bcc iron crystals exhibit a large elastic anisotropy (as high as $30 \%$ ), so one plausible hypothesis is that a fraction of the core is made up of bcc iron, with the rest being perhaps hcp iron.

In order to validate this possibility, geologists need more data on bcc iron. Previously, ultrasonic studies of bcc iron have measured its sound speed and other properties at high temperature, but not at high pressure. Scientists from the University of Texas at Austin, Argonne, and the University of Illinois at Urbana-Champaign investigated bcc iron simultaneously at high temperatures and pressures. They also performed high-pressure, room-temperature measurements on a bcc iron alloy containing $8 \%$ silicon by weight. Using an externally-heated diamond anvil cell, the team was able to heat samples to temperatures as high as $700 \mathrm{~K}\left(330^{\circ}\right.$ $\mathrm{C})$, while squeezing them to pressures as high as $11 \mathrm{GPa}$.

To probe the iron under these extreme conditions, the researchers used synchrotron radiation from the high-energy resolution inelastic $x$-ray scattering (HERIX) instrument at the XSD 3-ID$B, C, D$ beamline at the APS and recorded the results. The HERIX data showed peaks (Fig. 1) that correspond to $x$-ray photons interacting with iron crystal phonons (i.e., quantized sound waves). Analysis of the HERIX spectra allowed the researchers to derive the sound speed at different temperatures and pressures. They also measured the density of their samples using in situ $x$ ray diffraction measurements.

By combining their data, the researchers showed that the sound speed of bcc iron decreases by about $1 \%$ as the temperature increases from $300 \mathrm{~K}$ to $700 \mathrm{~K}$. A similar drop in sound speed was recently found for hcp iron. If this velocity reduction continues at higher temperatures like those in the core, then the sound speed of pure iron may be too low to explain seismic wave observations. One way to offset the temperature effect would be to include more light elements, like silicon. The research team showed that the sound speed of their iron-silicon alloy is $1 \%$ $2 \%$ higher than pure bcc iron.

Of course, these measurements were all taken at temperatures and pressures that are far below those in the core (5000 K and $350 \mathrm{GPas})$. But the authors argue that trends in their data and the data from previous studies imply that the sound speed and elastic anisotropy of bcc iron alloy could help explain seismic wave observations.

\section{- Michael Schirber}

See: Jin Liu ${ }^{1 *}$, Jung-Fu Lin ${ }^{1}$, Ahmet Alatas" ${ }^{2}$, Wenli $\mathrm{Bi}^{2,3}$, "Sound velocities of bcc-Fe and $\mathrm{Fe}_{0.85} \mathrm{Si}_{0.15}$ alloy at high pressure and temperature," Phys. Earth Planet. In. 233, 24 (2014).

DOI: 10.1016/j.pepi.2014.05.008 Author affiliations: ${ }^{1}$ University of Texas at Austin, ${ }^{2}$ Argonne National Laboratory, ${ }^{3}$ University of Illinois at UrbanaChampaign Correspondence: * jinliu@utexas.edu

J.-F. Lin acknowledges support from the U.S. National Science Foundation (EAR-1053446 and EAR-1056670) and the Carnegie/U.S. Department of Energy (DOE) Alliance Center. This research used resources of the Advanced Photon Source, a U.S. DOE Office of Science User Facility operated for the DOE Office of Science by Argonne National Laboratory under Contract No. DE-AC02-06CH11357.

3-ID-B,C,D • XSD • Physics, geoscience, life sciences, chemistry, materials science $\cdot \mathrm{Nu}$ clear resonant scattering, inelastic x-ray scattering, high-pressure diamond anvil cell $\bullet$ 7-27 $\mathrm{keV}, 14.41-14.42 \mathrm{keV} \cdot$ On-site $\cdot$ Accepting general users $\bullet$ 


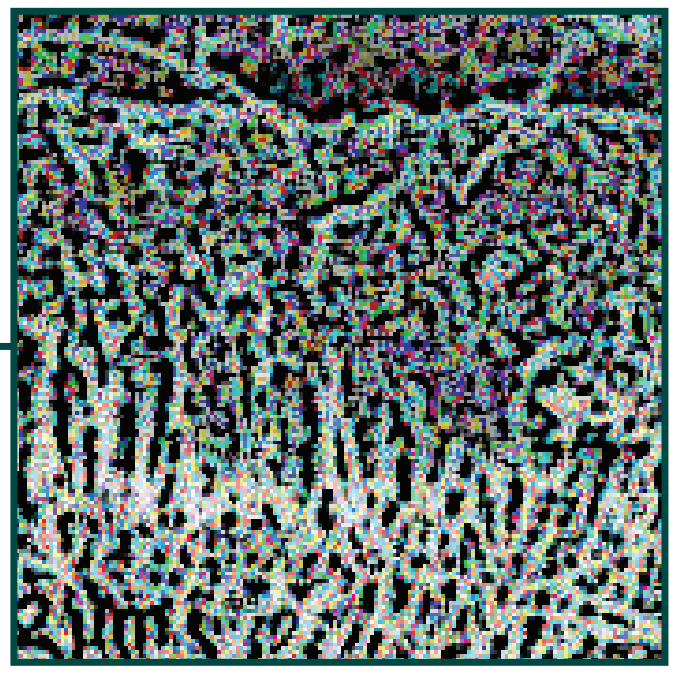

Nanoscience 


\section{Self-Assembly of Layered Membranes}

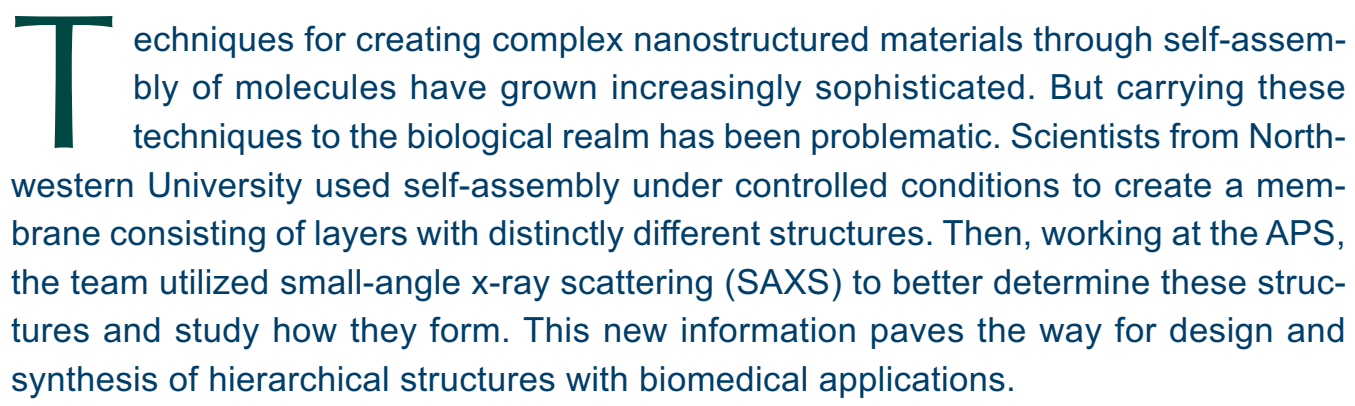

a)

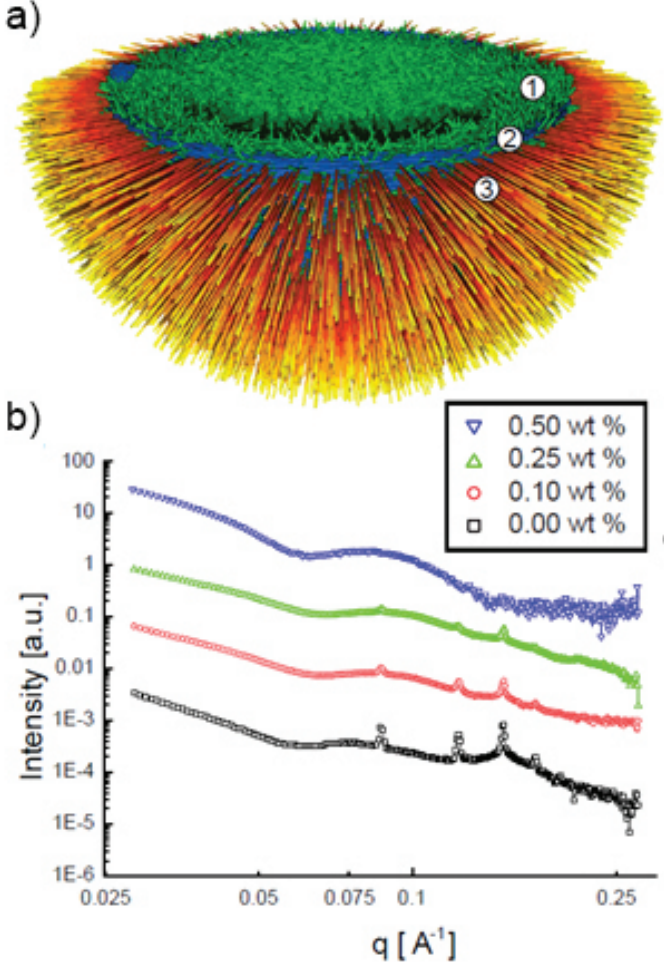

c)

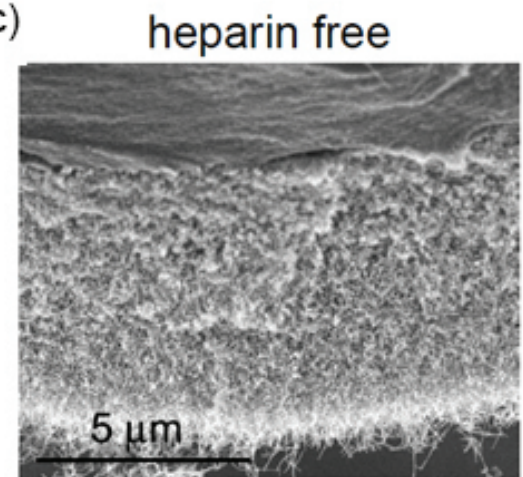

d)

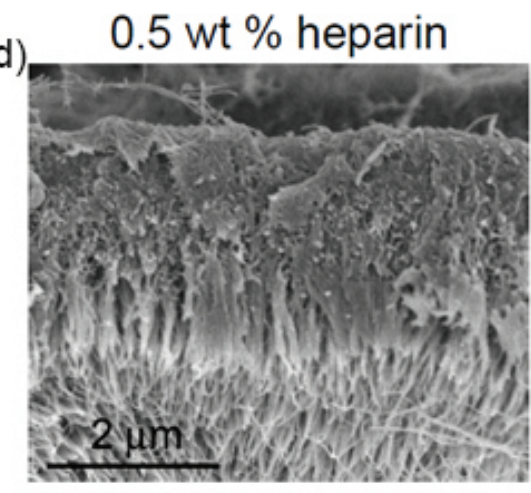

Fig. 1. a) Schematic representation of one hemisphere in a closed sac with a hierarchically structured membrane of three zones, (1) a gel phase, (2)parallel nanofibers in the plane of the membrane, and (3) nanofibers perpendicular to the plane of the membrane. b) Small-angle x-ray scattering patterns of HA/HBPA sacs self-assembled with heparin. Curves were shifted vertically for visual convenience. c) and d) Scanning electron micrographs of membrane cross-sections of HA/HBPA sacs self-assembled with c) $0 \mathrm{wt} \%$ heparin, and d) $0.5 \mathrm{wt} \%$ heparin.

Peptide amphiphiles (PAs) are chains of amino acids tipped with other molecules so that one end is hydrophilic (mixes well with water) and the other hydrophobic (not fond of water). In aqueous solution, PAs form long, thin nanofibers as the amino acid chains bind to adjacent chains to form $\beta$-sheets. The Northwestern University scientists had previously found that when an aqueous solution containing positively-charged PAs was put into contact with an aqueous solution of negatively charged hyaluronic acid (HA - a large biological molecule that occurs in connective and other tissues), a dense, fibrous layer formed within milliseconds, creating a barrier that kept the two solutions from mixing.

More precisely, the researchers found that the fibrous layer prevents aggregated PAs from migrating to the HA side, but allows HA molecules to slowly insinuate themselves through the barrier to the PA side, on a timescale of minutes or longer.

The result was a three-zone membrane structure: a gel-like layer on the HA side, a fibrous mat consisting of PA nanofibers lying in the plane of the interface between the solutions, and a coating of fibers directed perpendicularly away from the interface and formed by electrostatically bound complexes of PA and HA (Fig. 1).

The team's interest in these membranes hinged on possible biomedical uses in which the peptide sequence forming the nanofibers would have a chosen biological activity. In one example, they incorporated a heparin-binding sequence to promote angiogenesis (the 
formation of new blood vessels), so that the membrane might assist with tissue repair. For the three-zone structure to form, the researchers found that the HA solution had to contain heparin in a certain concentration range. Scanning electron microscopy clearly showed linear structure crossing the membrane that formed when heparin was present at $0.5 \%$ by weight (Fig. $2 a$ ), in contrast to the more homogeneous appearance of the membrane created in the absence of heparin (Fig. 2b).

The scientists turned to SAXS at the DND-CAT beamline 5-ID-B,C,D at the APS. The studies provide insight about the precise structure of the three-zone membranes and a better understanding of the dynamics of their formation.

The heparin-free membranes produced well-defined Bragg peaks, while the three-zone membranes did not. Moreover, membranes that arose in the presence of smaller heparin concentrations showed larger Bragg peaks than those produced when the heparin concentration was higher, indicating a competition between two structures whose outcome depended on heparin levels.

A time-series of SAXS measurements on a heparin-free experiment showed that the Bragg peaks began to form a few minutes after the two solutions were brought into contact and reached full strength after about 45 minutes.

Interpreting the SAXS findings in the light of their previous experiments and the known properties of PAs and $\mathrm{HA}$, the scientists explain the differences between the two types of membrane as the result of different kinds of aggregation. In the absence of heparin, the PA and HA come together in nanospherical aggregates that pack together in a cubic arrangement, over a period of some tens of minutes, to form a membrane that generates well-defined Bragg peaks.

When heparin is present, by contrast, it binds strongly with the PA and alters its interaction with $\mathrm{AH}$ molecules. In this case, a barrier of nanofibers ly- (a)

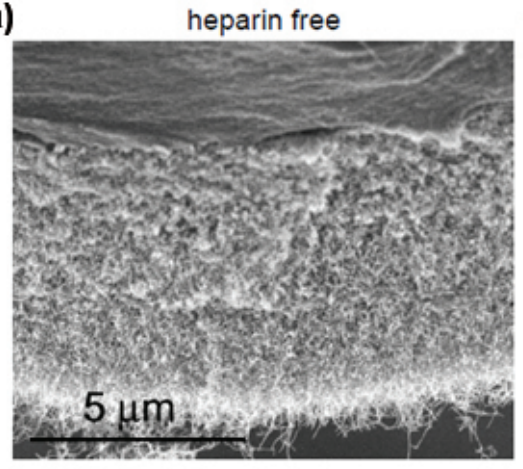

(b)

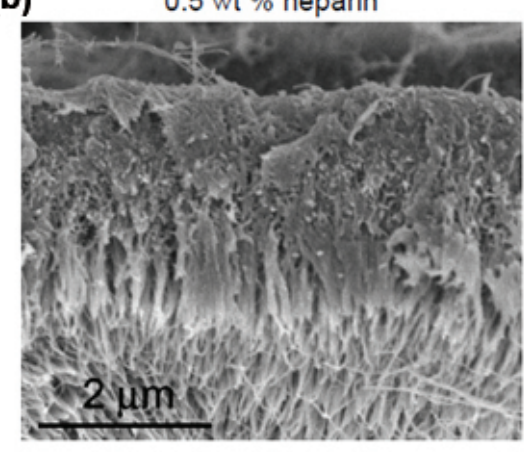

(c)

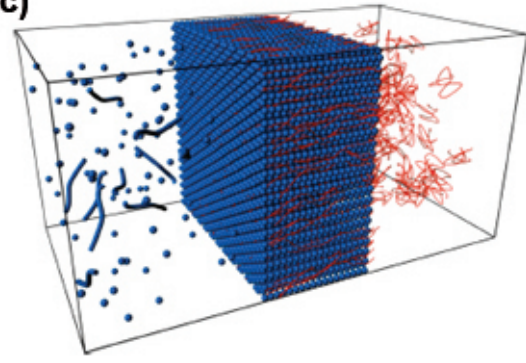

(d)

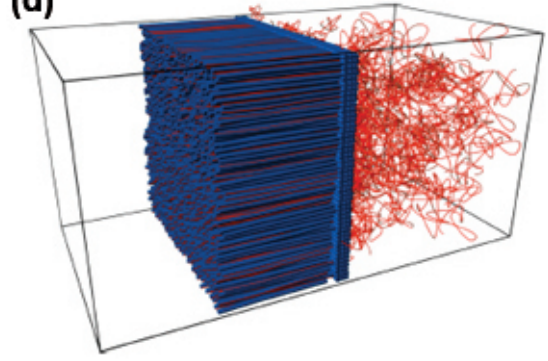

Fig. 2. Scanning electron micrographs show the homogeneous membrane that forms in the absence of heparin (a), while in the presence of heparin is a fibrous structure forms transverse to the membrane (b). (c) and (d): Schematic representation of the nanostructured membrane formed by peptide amphiphiles (blue) and polyelectrolytes (red) in the case of weak aggregation (c) or strong aggregation (d) at the interface.

ing parallel to the solution interface forms immediately, then acts as a diffusion barrier through which HA slowly passes. As it emerges on the other side, it binds to PA to form nanofibers that grow perpendicular to the interface. This ordered nanofiber array produces no Bragg peaks.

The increased understanding and control of these processes derived from this research could make it possible to build bioactive membranes with a variety of structures and purposes.

\section{- David Lindley}

See: Ronit Bitton, Lesley R. Chow, R. Helen Zha, Yuri S. Velichko, E. Thomas Pashuck, and Samuel I. Stupp*, "Electrostatic Control of Structure in Self-Assembled Membranes," Small 10 500 (2014).

DOI: 10.1002/smll.201300254

Author affiliation:

Correspondence:

Northwestern University

*s-stupp@northwestern.edu
This work was supported by the National Institute of Biomedical Imaging and Bioengineering at the National Institutes of Health (5R01EB003806-05) and the U.S. National Science Foundation Materials Research Science and Engineering Centers program (DMR-0520513). R.B. acknowledges support from the Ben Gurion University of the Negev in the form of a postdoctoral fellowship. The DND-CAT is supported by E.I. DuPont de Nemours \& Co., The Dow Chemical Company and Northwestern University. This research used resources of the Advanced Photon Source, a U.S. Department of Energy (DOE) Office of Science User Facility operated for the DOE Office of Science by Argonne National Laboratory under Contract No. DE-AC02-06CH11357.

5-ID-B,C,D - DND-CAT - Materials science, polymer science $\cdot$ Powder diffraction, $x$-ray standing waves, x-ray optics development/ techniques, small-angle $x$-ray scattering, surface diffraction, $x$-ray reflectivity, wide-angle x-ray scattering $\bullet$ 6-17.5 keV $\bullet$ On-site $\cdot$ Accepting general users $\bullet$ 


\section{Polymer Nanostructures Grow Bigger WHEN AGITATED}

M

acromolecular structures that self-assemble in solution have a number of uses, such as drug delivery nanocapsules and nanoscale vessels for chemical reactions. Often, these nanocarriers form in a suitably chosen mixture of solvents to create well-defined structures with desired properties, and then are transferred to a purely aqueous environment for application. Researchers from the University of Delaware wondered whether conditions of actual use would affect the stability of nanocarriers over periods of days to weeks. Using diagnostic techniques including small-angle x-ray scattering (SAXS) at the APS, they found that agitation of a solution containing spherical, polymer-based structures caused the size of the carriers to grow significantly, potentially affecting their performance and utility.
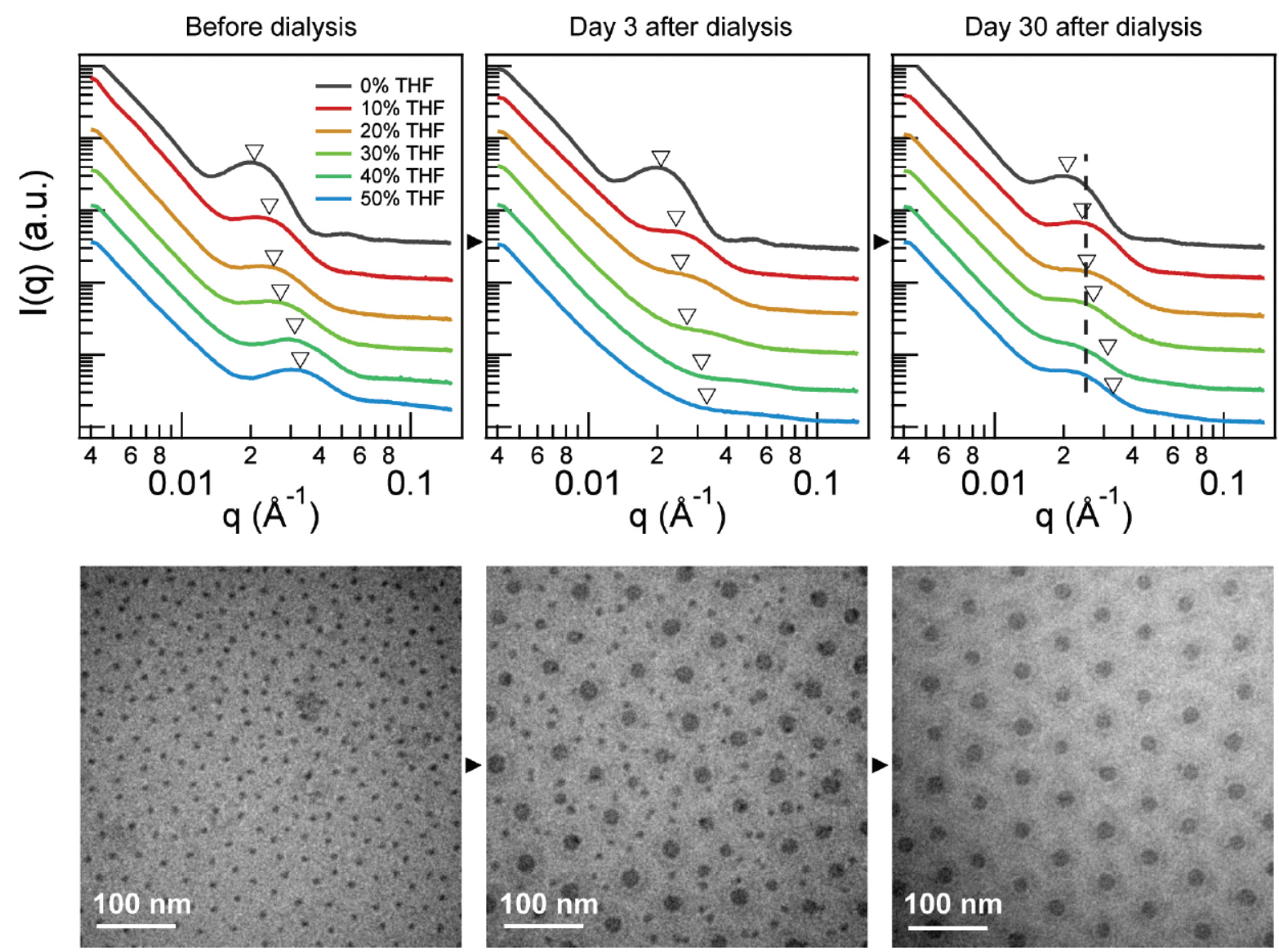

Fig. 1. Top: SAXS data shows evolution in size distribution of micelle populations. Before THF was removed (left), the SAXS peaks (triangles) show a monodisperse micelle population with radius that decreases with THF concentration. Three days after THF removal (center), the micelle populations that formed in low THF concentrations showed no change, but in other cases, the SAXS peak disappeared, indicating a range of micelle sizes. Thirty days after THF removal (right), reappearance of SAXS peaks at smaller wavenumbers than the original peaks indicated the emergence of a new monodisperse population of larger micelles. Bottom: Cryo-TEM images of micelle populations made at $43 \%$ THF concentration at the same time as the SAXS results above. An initially monodisperse population (left) evolves through the emergence of larger micelles of a single size (center), which eventually form a new monodisperse population (right). 
The team built nanocarriers from the block polymer poly(butadiene-bethylene oxide), or PB-PEO. Dissolved in water, the PB-PEO self-assembles into spherical nanostructures, or micelles, with a core made of the hydrophobic PB surrounded by a corona of the hydrophilic PEO. If the micelles were being made for drug delivery, molecules of the drug would have been added to the water, and the micelles would form around the drug molecules. But the purpose of these experiments was to study the micelles alone.

A dynamic light-scattering (DLS) technique showed that, in pure water, the micelles had a radius of approximately $35 \mathrm{~nm}$. This radius was near the equilibrium micelle size resulting from the thermodynamic balance between the hydrophobic portion of the polymer and the water. To mirror common solution processing conditions, the researchers then added a second solvent, tetrahydrofuran (THF), in various concentrations ranging up to $50 \%$ by volume. The greater the amount of THF, the smaller the micelles became, down to a radius of less than $15 \mathrm{~nm}$, as a result of the changed thermodynamic balance in the modified solvent mixture.

After the THF was carefully removed using dialysis, leaving the micelles once again in pure water, the micelle sizes remained constant provided that the solutions were not agitated (Fig. 1). But the researchers found that stirring the water fairly gently caused the smaller micelles - those that formed in solutions with THF concentrations greater than $10 \%$ and hence were furthest from their equilibrium sizes in pure water - to grow, reaching a new stable radius of approximately $25 \mathrm{~nm}$ after three weeks. The DLS technique revealed little about the nature of this evolution, so the team turned to other methods.

Working at XSD beamline 12-ID$C, D$, they conducted SAXS measurements at a wavelength of $0.073 \mathrm{~nm}$, on samples modified with various THF concentrations, at different times after the THF had been removed. In all cases, the SAXS plots initially had a prominent peak, indicative of a micelle population with a single characteristic size. For micelle samples that had not been treated with THF, that peak remained unchanged. In the case of samples to which more than $10 \%$ THF had been added and later removed, however, the SAXS peak largely subsided after just three days of stirring, suggesting that the micelle distribution now was polydisperse. Later still, at 30 days, peaks reappeared in the SAXS plots, but at a position corresponding to the enlarged size of the micelles revealed by DLS.

The SAXS measurements suggested that the micelle population had started at one size, evolved into a mixture of sizes, then reformed at a single, larger size. To further understand this transformation, the researchers used cryogenic-transmission electron microscopy (cryo-TEM) to study frozen samples of a few micelles, along with small-angle neutron scattering conducted at the National Institute of Standards and Technology Center for Neutron Research and at the Oak Ridge National Laboratory High Flux Isotope Reactor to study the micelle populations. Both methods indicated that the micelle population evolved bimodally, with larger micelles appearing among the smaller ones. Surprisingly, the volume of the larger micelles was up to 8fold larger than that of the smaller micelles in some cases, and no intermediate-sized micelles were noted during the growth process. Over a period of weeks, the larger micelle population became dominant, while the smaller micelles vanished.

The researchers noted that if pairs of micelles occasionally fused and then intermittently combined with additional micelles, there should have been a range of micelle sizes evident during the evolution process. They argue instead that an evolving bimodal population is often characteristic of a mechanism of cooperative self-assembly involving nucleation and growth. Because agitation evidently was essential to the growth of the micelles, they suggest that the introduction of an air-water interface into the solution may provide a nucleation site for the aggregation of the hydrophobic components of the micelles. Such nucleation and growth processes are well known to affect the stability of protein biopharmaceuticals during storage and handling, yet have not previously been documented in polymer assemblies.

The researchers say that their findings point out a need for further study of micelle stability in realistic conditions, where solutions are inevitably subjected to various kinds of disturbance.

- David Lindley

See: Elizabeth G. Kelley, Ryan P. Murphy, Jonathan E. Seppala, Thomas P. Smart, Sarah D. Hann, Millicent O. Sullivan*, and Thomas H. Epps, III** "Size evolution of highly amphiphilic macromolecular solution assemblies via a distinct bimodal pathway," Nat. Commun. 5, 3599 (7 April 2014). DOI: 10.1038/ncomms4599 (2014) Author affiliation: University of Delaware Correspondence: * msullivan@udel.edu, ** thepps@udel.edu

This research was supported by an Institutional Development Award from the National Institute of General Medical Sciences of the National Institutes of Health Grant, P20GM 103541. E.G.K. also acknowledges support from a Department of Defense, Air Force Office of Scientific Research, National Defense Science and Engineering Graduate Fellowship, 32 CFR 168a. This research used resources of the Advanced Photon Source, a U.S. Department of Energy (DOE) Office of Science User Facility operated for the DOE Office of Science by Argonne National Laboratory under Contract No. DE-AC02$06 \mathrm{CH} 11357$.

12-ID-C,D • XSD • Chemistry, physics, materials science - Small-angle $x$-ray scattering, grazing incidence small-angle scattering, wide-angle $x$-ray scattering, surface diffraction - 4.5-40 keV • On-site - Accepting general users • 


\section{Building Nanocrystals to Order With DNA}

$\mathrm{T}$

he formation of crystals is one of nature's most highly-organized and systematic processes, resulting in objects of singular beauty and exquisite form. However, certain crystallization processes can be hard to control when one wants to build crystals to order for specific purposes. A group of Northwestern University researchers working at the APS has developed a novel way to engineer the crystallization of nanoparticles, creating highquality Wulff equilibrium structures using a set of well-established design rules that culminate nearly 20 years of research. In the future, the researchers will use their experimental design rules and theoretical models to predict formation of nanoparticle microcrystals of other shapes and synthesize them for specific purposes, including practical applications in electronics, photonics, catalysis, and materials science. Just as the ability to reliably grow atomic crystals has revolutionized many industries, the ability to precisely fashion nanoparticle crystals promises to be just as game-changing.

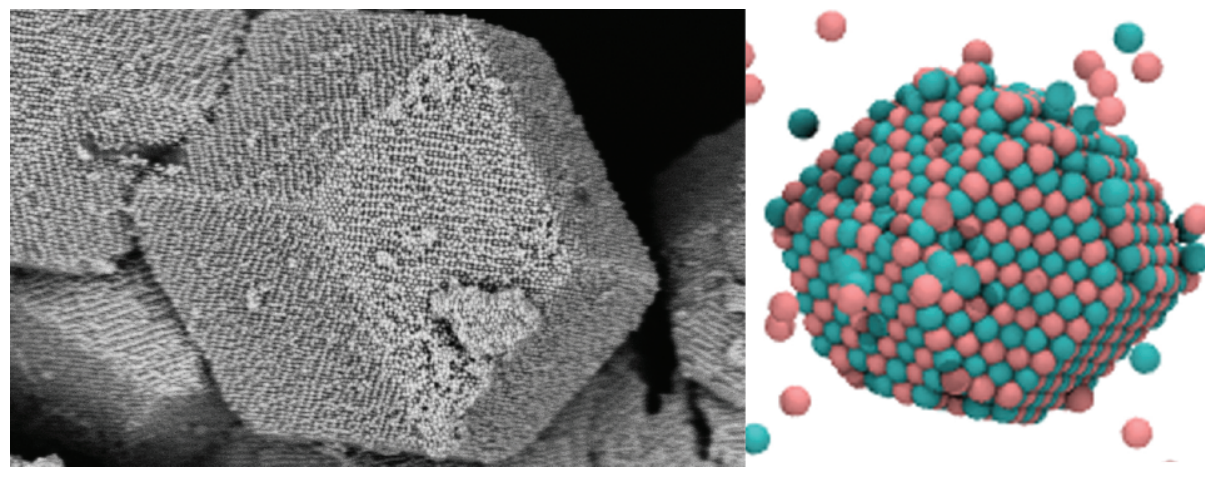

Fig. 1. Electron microscopy image (left) of faceted rhombic dodecahedra microcrystals comprised of gold nanoparticles assembled by the DNA design rules. The observed crystal shape is consistent with the structure predicted by MD simulations (right).

The experimenters began with gold nanoparticles surface-functionalized with a high density of highly oriented DNA strands (namely, spherical nucleic acids) and DNA linker strands that were partially complementary to the DNA strands on the surface of the particles. By heating the solution and then cooling it very slowly through the system's melting temperature (a known property for a particular DNA system) over a period of two to three days - a methodology often employed in crystallizing atoms and molecules - the researchers were able to deliberately anneal the nanoparticles into the thermodynamically favored products of micron-sized single crystalline nanoparticle assemblies with rhombic dodecahedron shapes or "crystal habit." The crystal structures were confirmed with small-angle $x$-ray scattering at the DND-CAT beamline 5-ID-B,C,D of the APS. Transmission electron microscopy and scanning electron mi- croscopy imaging were also performed. (Fig.1)

The theoretical models and molecular dynamics (MD) simulations were used to validate the structures of the experimentally obtained nanoparticle microcrystals. These confirmed that the surface energy of the system is a crucial factor in determining crystal shape. In this system, the observed Wulff polyhedra emerge from the original mixture of nanoparticles because, using a broken-bond approximation, the formation of such shapes requires breaking the smallest number of particle-to-particle interactions and exposes the lowest surface energy facets. Indeed, the experimental results and the simulations were highly consistent, showing that the approximations that describe surface energies and crystal growth in atomic systems can be applied to describe nanoparticle systems with DNA links. - Mark Wolverton
See: Evelyn Auyeung, Ting I.N.G. Li, Andrew J. Senesi, Abrin L. Sch-mucker, Bridget C. Pals, Monica Olvera de la Cruz, and Chad A. Mirkin", "DNA-mediated nanoparticle crystallization into Wulff polyhedra," Nature 505, 73 (2 January 2014). DOI: 10.1038/nature12739

Author affiliation:

Northwestern University Correspondence:

*chadnano@northwestern.edu

C.A.M. and M.O.d.I.C. acknowledge support from the following awards: the Air Force Office of Scientific Research Multidisciplinary University Research Initiative FA9550-11-10275, the National Science Foundation Materials Research Science and Engineering Center program DMR-1121262 at the Materials Research Center of NorthwesternUniversity, and the Non-equilibrium Energy Research Center, an Energy Frontier Research Center funded by the U.S. Department of Energy (DOE) Office of

Science-Basic Energy Sciences under Award DE-SC0000989. E.A. acknowledges a National Defense Science and Engineering Graduate Fellowship (number 32 CFR 168a). T.L. acknowledges a Ryan Fellowship from Northwestern University. DND-CAT is supported by E.I. DuPont de Nemours \& Co., The Dow Chemical Company and Northwestern University. This research used resources of the Advanced Photon Source, a U.S. DOE Office of Science User Facility operated for the DOE Office of Science by Argonne National Laboratory under Contract No. DEAC02-06CH11357.

5-ID-B,C,D - DND-CAT - Materials science, polymer science $\cdot$ Powder diffraction, x-ray standing waves, $x$-ray optics development/ techniques, small-angle $x$-ray scattering, surface diffraction, $x$-ray reflectivity, wide-angle $\mathrm{x}$-ray scattering • 6-17.5 keV • On-site $\cdot$ Accepting general users $•$ 


\section{Laying Out Nanoparticles According to Plan}

A

chunk of gold is just a chunk of gold, shiny and ductile and familiar. But break that gold into tiny, nano-sized bits, or nanoparticles, and it takes on new and different properties. In order to use those special nano properties, we must be able to easily manipulate and arrange the particles. Researchers used the APS to show that templates etched into a substrate could guide nanoparticles into patterns of parallel lines. In principle, the technique could be used to make any conceivable pattern. For a lighttrapping nanoparticle such as gold, such templates could make it possible to build nano-scale versions of lenses, electronics, and solar cells.

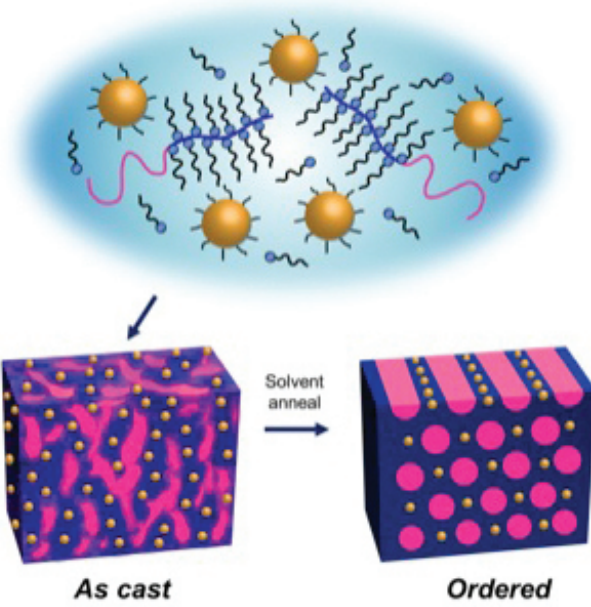

Fig. 1. The gold nanoparticles are mixed into the polymer/small molecule solution (top). After the solution is spread over the templates, the supermolecules formed by the polymers and small molecules help the nanoparticles align with the templates, forming an ordered array (bottom). Graphic: Ting Xu, University of California, Berkeley, from J. Kao et al., Nat. Commun. 5, 4053 (2014), (C) 2014 Macmillan Publishers Limited

Previous attempts to assemble nanoparticles into three-dimensional (3D) structures with long-range order had been challenging, especially in thin films containing more than two layers. Researchers from the University of California, Berkeley; Argonne; the University of Massachusetts; MIT; and Lawrence Berkeley National Laboratory showed that their patterned substrates could jump the 3-D hurdle. They made multi-layer nanoparticles structures macroscopically aligned as thick as 200 $\mathrm{nm}$ using two different types of template, one made of saw-toothed sapphire and the other made of lithographically patterned silicon.

The process first required the researchers to mix small molecules into a polymer solution so that they stuck to block copolymer molecules and formed supermolecules. The small molecules mediate the interactions between nanoparticles and the polymer, and are a key factor in making the technique work. The supermolecules were mixed with nanoparticles, and the mix was then spread over a silicon or sapphire template and allowed to dry quickly. The researchers found that with this technique, not only could they make three-dimensional arrays of nanoparticles; they could also control the orientation of the nanoparticles, making sure they all faced in the same direction (Fig. 1).

The team used the XSD 8-ID-E beamline at the APS to quantify the structure and alignment of the nanoparticles. The beamline was chosen primarily for its geometry, which allowed the researchers to know the incident angle of the $\mathrm{x}$-rays very precisely and characterize the position of the nanoparticles to a precision of less than $1 \mathrm{~nm}$.

The grazing incidence small-angle x-ray scattering (GISAXS) pattern, taken at an incident angle between the $\mathrm{x}$-ray direction and the direction of the one-dimensional nanoparticle chains, showed multiple orders of scattering in the in-plane direction. As the sample rotated and the incident angle increased, the GIXAS peaks weakened and broadened, showing that the nanoparticles were highly aligned in the in-plane direction over broad areas of the sample. In this context, "broad" means on the order of centimeters, which is huge for a nanoscale phenomenon.

The beamline geometry also enabled the researchers to look at the nanoparticle super structure at any depth, allowing them to see if the orientation of particles in the middle was the same as that of particles on the edge.
With a technique to align nanoparticles over broad areas, the researchers intend to explore how they can mix and match different types of nanoparticles for different effects such as energy transfer or exciton diffusion.

They will also start creating different template shapes, starting with a circular template for a nanoscale lens. Macroscopic lenses depend on altering the thickness of the lensing material in order to bend the light to a greater or lesser degree. But nanoscale lenses could work in a different way, using the choice of nanoparticles with specific properties to bend the light in the desired manner. Simply controlling the orientation of the nanoparticles can also control their refractive index.

- Kim Krieger

See: Joseph Kao ${ }^{1}$, Seong-Jun Jeong ${ }^{1}$, Zhang Jiang ${ }^{2}$, Dong Hyun Lee ${ }^{3 \ddagger}$, Karim Aissou ${ }^{4}$, Caroline A. Ross ${ }^{4}$, Thomas P. Russell ${ }^{3}$, and Ting $\mathrm{Xu}^{1,5 *}$, "Direct 3-D Nanoparticle Assemblies in Thin Films via Topographically Patterned Surfaces," Adv. Mater. 26, 2777 (2014).

DOI: 10.1002/adma.201305561

Author affiliations: ${ }^{1}$ University of California, Berkeley; ${ }^{2}$ Argonne National Laboratory; ${ }^{3}$ University of Massachusetts; ${ }^{4}$ Massachusetts Institute of Technology; 5 Lawrence Berkeley National Laboratory łPresent address: Dankook University Correspondence: * tingxu@berkeley.edu

This work was supported by the U.S. Department of Energy (DOE) Office of ScienceBasic Energy Sciences under Contract DE-AC02-05CH11231 through the "Organicinorganic Nanocomposites" program at Lawrence Berkeley National Laboratory (J.K., S.-J.J., and T.X.). C.A.R. and K.A. acknowledge the support of the Semiconductor Research Corporation, NSF DMR1246740 and TSMC. This research used resources of the Advanced Photon Source, a U.S. DOE Office of Science User Facility operated for the DOE Office of Science by Argonne National Laboratory under Contract No. DEAC02-06CH11357.

8-ID-E • XSD • Materials science, polymer science, physics - Grazing incidence small-angle scattering, x-ray photon correlation spectroscopy $\cdot 7.35-7.35 \mathrm{keV} \cdot$ On-site $\cdot$ Accepting general users $\bullet$ 


\section{ImProving NanosCale EleCtronics}

M

any electronic devices - batteries, solar cells, transistors - work better under engineered strain. Carefully squeezing a semiconductor can improve its electron mobility so that current flows through it more easily, making the device more efficient. Modern computer chips depend on this effect, but the individual devices can be as small as tens of nanometers, so it's important to understand exactly how strain can be applied at the smallest length scales. Previously, it had been difficult to study this without cutting the device open and altering the strain. Now, scientists have developed a new, non-destructive technique that lets them analyze spatial distortions inside the structure of a device using synchrotron $x$-rays from the APS, which could help them design even better devices.

The researchers from Argonne and IBM based their analysis on a technique, recently developed in the Argonne Materials Science Division, called Bragg projection ptychography (BPP), which creates structural maps from the diffraction patterns created when coherent $\mathrm{x}$-rays are scattered off a structure. They made their measurements at the Hard X-ray Nanoprobe beamline 26-ID-C operated jointly by the Center for Nanoscale Materials (CNM) and $X S D$. That beamline is one of the few worldwide that can focus hard $\mathrm{x}$-rays to a 35-nm spot size with the ability to change beam position by less than 5 $\mathrm{nm}$.

The team examined sample semiconductor devices provided by IBM Research that consisted of singlecrystal silicon on a buried oxide insulator and etched into parallel channels 60-nm wide and 100- $\mu \mathrm{m}$ long, each separated by $460 \mathrm{~nm}$. Silicon germanium was deposited between and on either side of those channels to impose stress on the silicon-on-insulator.

The researchers then scanned the $x$-ray beam over their device in a spiral pattern, recording coherent diffraction patterns and acquiring 16 spiral scans over 4 channel regions. Taking a spiral scan in overlapping steps of $13 \mathrm{~nm}$ gave them a resolution of $\sim 10 \mathrm{~nm}$, much smaller than the $30-\mathrm{nm}$ spot size of the $\mathrm{x}$-ray beam and enough to give them several points of reference across the 60-nm-wide silicon channel. $\mathrm{X}$-rays scattering off the atomic lattice of the materials created interference patterns, which were caused both by changes in the orientation of the lattice and the sharp boundaries between the materials.

Mapping distortions in the atomic lattices revealed both lattice strain the spacing of the atoms - and lattice tilt - the curvature of the structure (Fig. 1). This is the first time those two separate characteristics have been measured independently and quantitatively without sectioning, providing more detailed information than was previously available. The team compared their results to mechanical models of the nanostructure and found they were in good agreement.
This technique not only allows researchers to peer inside an intact structure, it can also be used to watch a device in operation, which can reveal how electronic behavior is related to mechanical properties of a structure. This ability could be useful for studying piezoelectric devices, which generate a current in response to mechanical stress or change shape in response to a current. It could also shed light on ferroelectric devices, which reverse their electrical polarization in response to an electric field.

A planned upgrade to the APS coule allow researchers to use a more intense and even smaller x-ray spot at the CNM/APS Hard X-ray Nanoprobe, making their measurements more precise. The increased understanding from these studies may lead to more efficient nanoscale engineered materials such as transistors that operate on lower voltages, batteries less likely to produce excess heat, and more efficient photovoltaics. - Neil Savage

See: Martin V. Holt ${ }^{1 *}$, Stephan O. Hruszkewycz ${ }^{1}$, Conal E. Murray ${ }^{2}$, Judson R. Holt ${ }^{2}$, Deborah M. Paskiewicz ${ }^{1}$, and Paul H. Fuoss", "Strain Imaging of Nanoscale Semiconductor Heterostructures with X-Ray Bragg Projection Ptychography," Phys. Rev. Lett. 112, 165502 (2014).

DOI: 10.1103/PhysRevLett.112.165502 Author affiliations: ${ }^{1}$ Argonne National Laboratory, ${ }^{2}$ IBM

Correspondence: * mvholt@anl.gov

The work performed at the Center for Nanoscale Materials and use of the Advanced Photon Source were both supported by the U. S. Department of Energy (DOE) Office of Science-Basic Energy Sciences, under Contract No. DE-AC02-06CH11357. S.O.H., D.M.P., and P.H.F. were supported by the U.S. DOE Office of Science-Basic Energy Sciences, Materials Sciences and Engineering Division.

26-ID-C • CNM/XSD • Physics, materials science $\cdot$ Nanofluorescence imaging, microdiffraction, nanotomography $\bullet 8-12 \mathrm{keV} \cdot$ On-site - Accepting general users • 


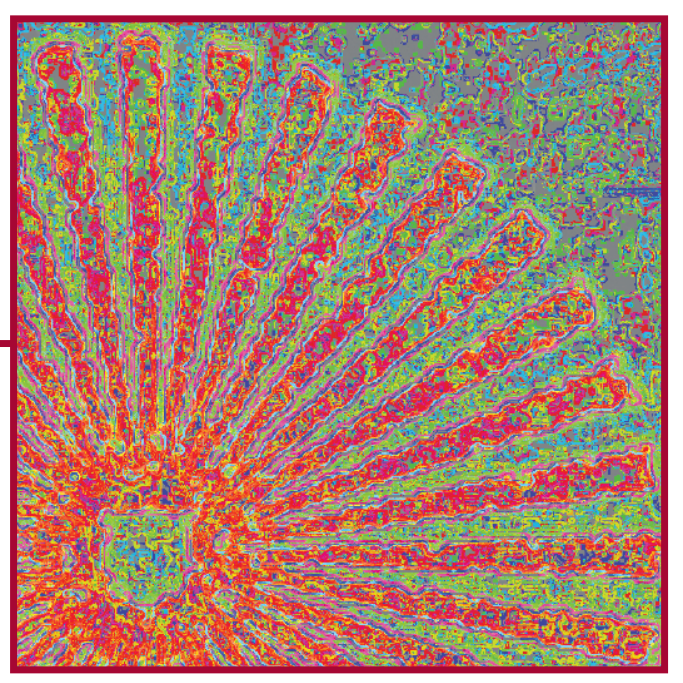

Novel X-ray TeChniques \& INSTRUMENTATION 


\section{New Light for Fluorescence Microscopy AT THE APS}

$\mathrm{T}$

he powerful blend of fluorescence microscopy with x-ray synchrotron imaging is nothing new. But as some recent experimental work has amply demonstrated, technical advances at the APS have greatly enhanced the utility of techniques such as synchrotron-based x-ray fluorescence microscopy (XFM). Through increased data acquisition speed, it is now possible to achieve both high spatial resolution and a large field of view at trace-element sensitivity. Two recent studies highlight the newly-enhanced capabilities of these imaging tools for tasks such as determining the elemental distribution and speciation in biological specimens.

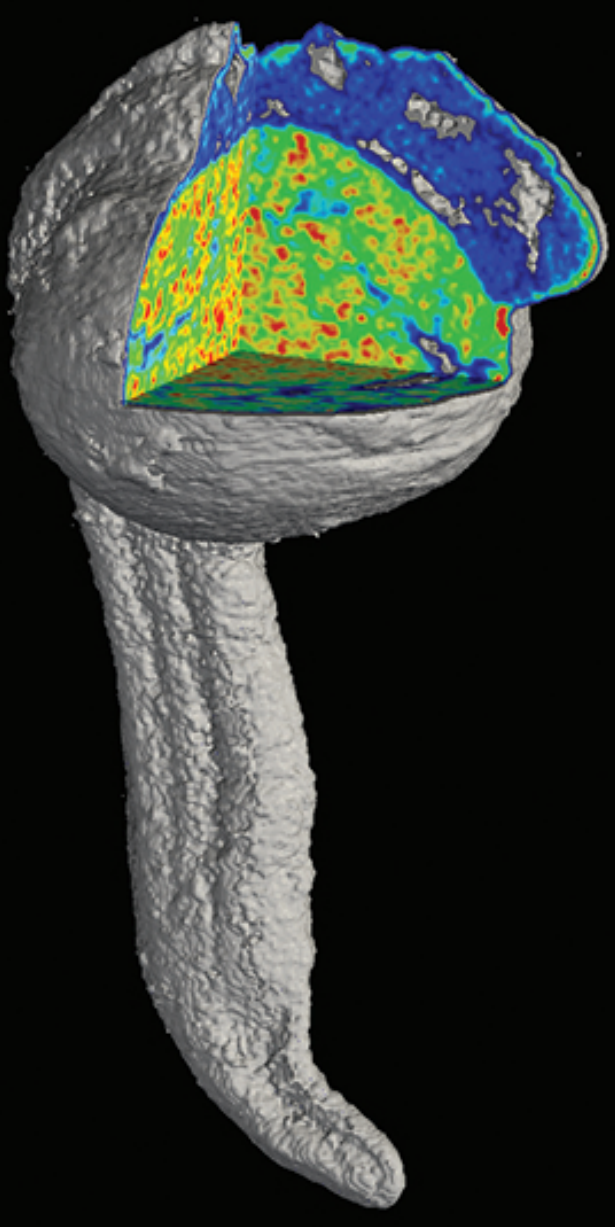

Fig. 1. A composite of three elemental slices and a three-dimensional view of the embryo.

In one study [1], investigators from the Georgia Institute of Technology, Argonne, Emory University, and Rowiak LaserLabSolutions $\mathrm{GmbH}$ (Germany) employed XFM to delineate elemental distribution of transition metals in the zebrafish embryo.

The ability to detect and quantify trace metal levels within intact biologi-

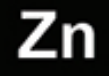

$\mathrm{Fe}$
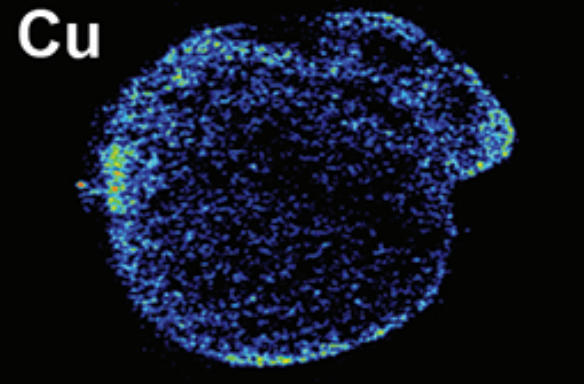
some forms more efficacious and beneficial than others.

The (bio)- chemistry of these elements is fragile and having to "prepare" the samples often leads to modification of the properties that we are trying to examine. By using both XFM and XAS, the researchers could examine the distribution and speciation of selenium and copper in intact tissues from animals exposed to some experimental treatments.

The team took advantage of the "fly-scanning" mode of XFM recently developed at the APS, which allows much faster imaging of wider sample areas. Before this, the sample image was built up point by point, stopping at each spot on the sample to collect a spectrum, now the sample keeps moving and the spectrum is collected rapidly on the fly. This allows imaging much larger areas of tissue than could be achieved previously.

The work at the XSD 2-ID-E beamline revealed a striking correlation between selenium and copper distribution in the kidneys of rats on a 5-ppm selenite diet (Fig. 2). While this phenomenon had been identified before in cell cultures, the current work using the XFM/XAS combination allowed this intriguing co-localization of elements to be studied in tissue specimens in vivo for the first time.

This team plans to further refine their experimental techniques to study the causes of this unusual co-distribution of elemental concentrations.

Both groups of researchers were enthusiastic about the results they were able to achieve with the fluorescence microscopy facilities at the APS, and are looking forward to future research. With further improved data acquisition times, it would be possible to perform larger-scale comparative studies of organisms.

There are many other selenium species that are important in the diet or as potential treatments for various diseases and similar work would hopefully yield useful information about their biochemistry. - Mark Wolverton

[1] See: Daisy Bourassa ${ }^{1}$, SophieCharlotte Gleber ${ }^{2}$, Stefan Vogt ${ }^{2}$, Hong
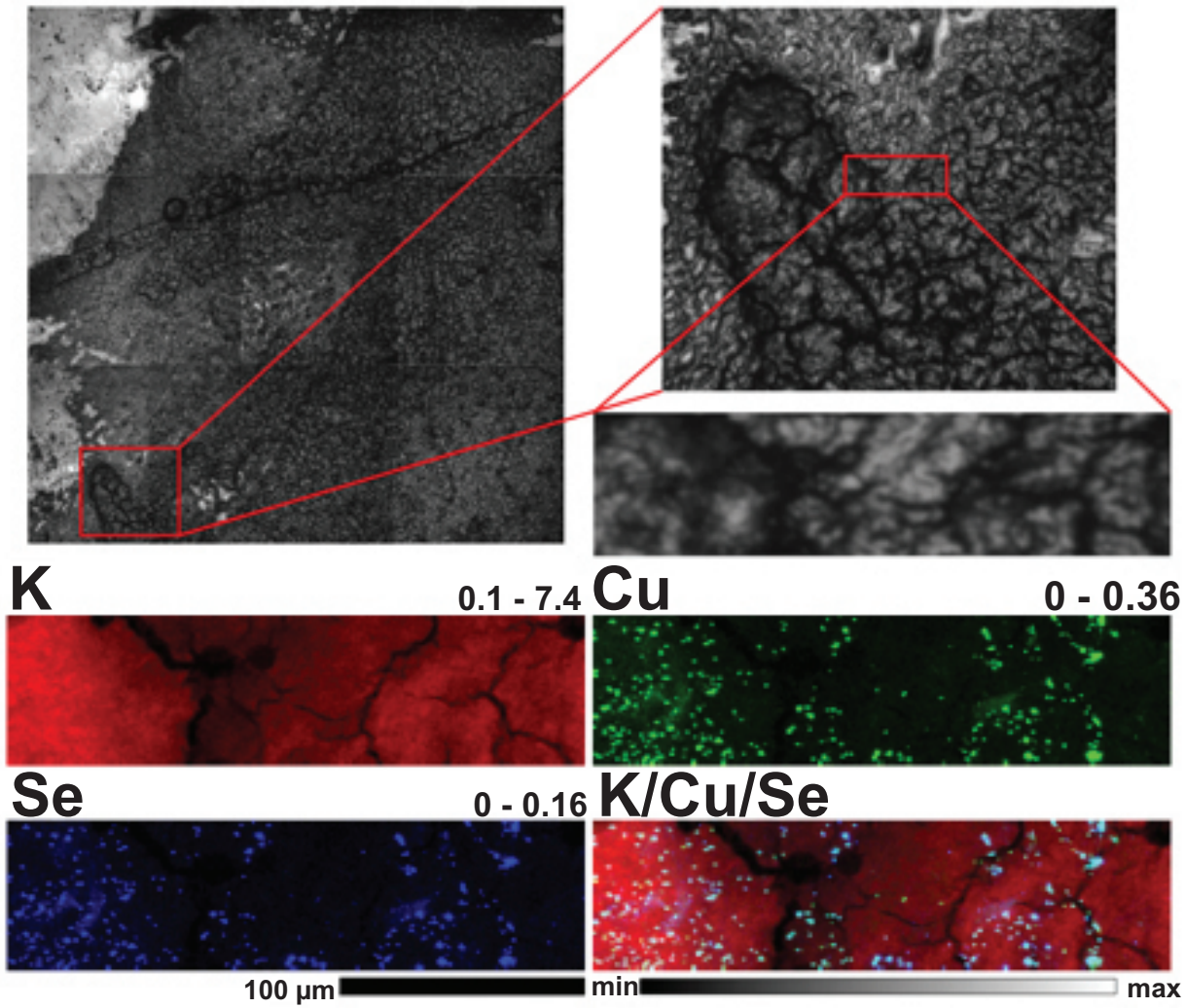

$\mathrm{K} / \mathrm{Cu} / \mathrm{Se}$

Fig. 2. Colocalization of copper and selenium in a kidney section of a rat fed a 5-ppm Se diet.

$\mathrm{Yi}^{3}$, Fabian Will ${ }^{4}$, Heiko Richter ${ }^{4}$, Chong Hyun Shin ${ }^{1}$, and Christoph J. Fahrni ${ }^{1 *}$, "3D imaging of transition metals in the zebrafish embryo by X-ray fluorescence microtomography," Metallomics 6, 1648 (2014). DOI: 10.1039/c4mt00121d Author affiliations: ${ }^{1}$ Georgia Institute of Technology, ${ }^{2}$ Argonne National Laboratory, ${ }^{3}$ Emory University, ${ }^{4}$ LLS Rowiak LaserLabSolutions $\mathrm{GmbH}$ Correspondence:

*fahrni@chemistry.gatech.edu

Financial support by the National Science Foundation (CHE-1306943 to CJF), the National Institutes of Health (K01DK081351 to $\mathrm{CHS}$ ), and the Vasser Woolley Foundation is gratefully acknowledged. This research used resources of the Advanced Photon Source, a U.S. Department of Energy (DOE) Office of Science User Facility operated for the DOE Office of Science by Argonne National Laboratory under Contract No. DE-AC02$06 \mathrm{CH} 11357$.

[2] See: Claire M. Weekley ${ }^{1}$, Anu Shanu ${ }^{2}$, Jade B. Aitken ${ }^{2}$, Stefan Vogt ${ }^{3}$, Paul K. Witting ${ }^{2}$, and Hugh H. Harris ${ }^{1 *}$, "XAS and XFM studies of selenium and copper speciation and distribution in the kidneys of selenite-supplemented rats,"
Metallomics 6, 1602 (2014).

DOI: $10.1039 / \mathrm{c} 4 \mathrm{mt} 00088 \mathrm{a}$

Author affiliations: ${ }^{1}$ The University of Adelaide, ${ }^{2}$ The University of Sydney,

${ }^{3}$ Argonne National Laboratory Correspondence:

*hugh.harris@adelaide.edu.au

Part of this research was undertaken at the X-ray Fluorescence Microprobe beamline at the Australian Synchrotron, Victoria, Australia. Portions of this research were carried out at the Stanford Synchrotron Radiation Lightsource. Travel funding provided by the International Synchrotron Access Program (ISAP) managed by the Australian Synchrotron and funded by the Australian Government, research funding from the Australian Research Council (DP0985807 to H.H.H.) and the Australian Synchrotron Postgraduate Award (C.M.W.). This research used resources of the Advanced Photon Source, a U.S. Department of Energy (DOE) Office of Science User Facility operated for the DOE Office of Science by Argonne National Laboratory under Contract No. DE-AC0206CH11357.

2-ID-E • XSD • Life sciences, environmental science, materials science - Microfluorescence (hard x-ray) • 7-10.5 keV, 11-17 keV • On-site $\cdot$ Accepting general users $•$ 


\section{Ptychography: Difficult To Pronounce, But Easy to Use for Beautiful Images}

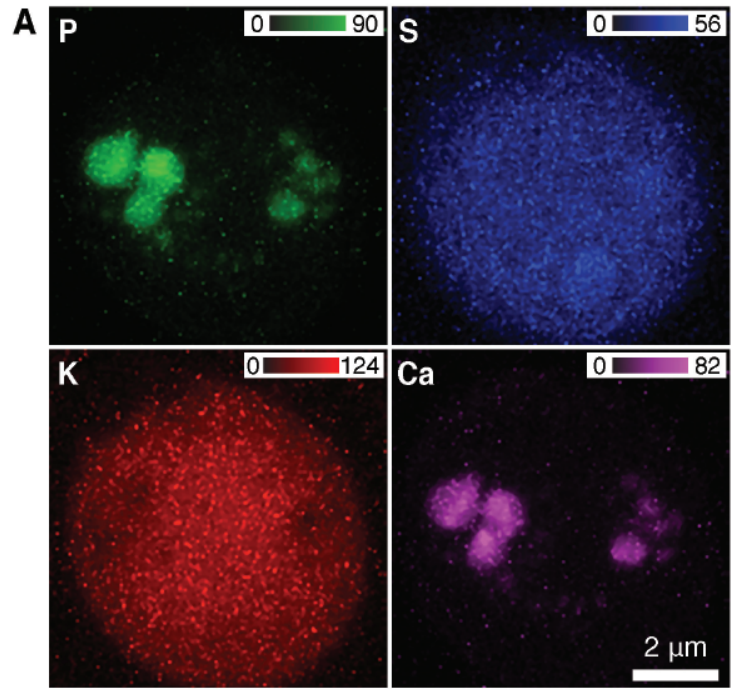

Fig. 1. Simultaneous $x$-ray fluorescence and ptychographic microscopy of the green alga Chlamydomonas reinhardtii. Left: X-ray fluorescence maps reveal the elemental composition of the alga. Right: The ptychographic reconstruction shows the biological ultra-structure in high resolution. The ptychograph reveals features not seen in the elemental maps and adds rich contextual information.

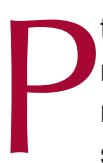

tychography is a coherent $x$-ray microscopy method that uses multiple overlapping illumination spots to reconstruct an image from far-field diffraction patterns. It is able to work at a resolution limited not by optics, but by the scattering strength of the sample, and it delivers a phase image that can have a contrast hundreds of times higher than absorption contrast for lighter atoms using highenergy $x$-rays. A team from Argonne and Northwestern University has worked together to achieve three separate advances in x-ray ptychography.

Northwestern University Ph.D. student Junjing Deng showed the use of two imaging methods at once to obtain complementary information on cells that were quickly frozen from the living state: ultrastructural information at sub-30-nm resolution by ptychography, and x-ray fluorescence measurements of trace element distributions at sub-100-nm resolution [1]. This puts elemental distributions into their proper biological context. Figure 1 shows an image of a green alga (Chlamydomonas reinhardtii) measured by Deng and demonstrates the significance of his work. The ptychographs reveal in amazing detail the organelles and membranes which do not fluoresce. Taken together the ptychographs aid identification of the cellular components and the quantified elemental concentrations may reveal the underlying cause of diseases and disorders in studies of mammalian cells.

In another advance, Deng and others have shown that data collection can be sped up by the use of continuous scanning [2], rather than having to repeat a stop-measure-move sequence for each illumination spot. The stop-measure-move sequence is notoriously inefficient because measurements cannot be made while the stage accelerates, moves, and then decelerates. Using the continuous scanning approach, the time required to complete one scan is reduced by about threefold at present, but with the planned high-brightness upgrade of the APS, the speedup can reach a factor of 100 or more.

Finally, Youssef Nashed of the Mathematics and Computer Science Division at Argonne and others have developed a ptychography computer code [3] that runs at the Argonne Leadership Computing Facility to speed up image reconstruction by more than a factor of 100 relative to previously-used code by employing graphical processing units on individual compute nodes and distributing the task over more than 100 nodes in the cluster. Nashed pioneered a new approach to partitioning the data across many compute nodes, which then communicated their results to each other during the reconstruction. The results from each node are then stitched together seamlessly as if the reconstruction was performed using a single node. The work of Nashed allows experimenters like Deng to see their images immediately, rather than waiting for days.

Together, these advances point the way toward high-speed imaging without lens limits to the resolution achieved, and this capability is central to the planned multi-bend achromat lattice upgrade of the APS.

Contact: cjacobsen@anl.gov, dvine@aps.anl.gov

\section{REFERENCES}

[1] J. Deng, D. Vine, S. Chen, Y.S.G. Nashed, Q. Jin, N.W. Philips, T. Peterka, R. Ross, S. Vogt, and C. Jacobsen, "Simultaneous cryo X-ray ptychographic and fluorescence microscopy of green algae," Proc. Natl. Acad. Sci. USA 112(8), 2314 (February 24, 2015).

[2] J. Deng, Y.S.G. Nashed, S. Chen, N.W. Phillips, T. Peterka, R. Ross, S. Vogt, C. Jacobsen, and D. Vine, "Continuous motion scan ptychography: characterization for increased speed in coherent x-ray imaging," Opt. Express 23(5), 5438 (2015).

[3] Y.S.G Nashed, D. Vine, T. Peterka, J. Deng, R. Ross, and C. Jacobsen, "Parallel ptychographic reconstruction," Opt. Express 22(26) 32082 (2014). 


\section{Data ExChange: a COMmOn Data Format For Tomographic Data Sets}
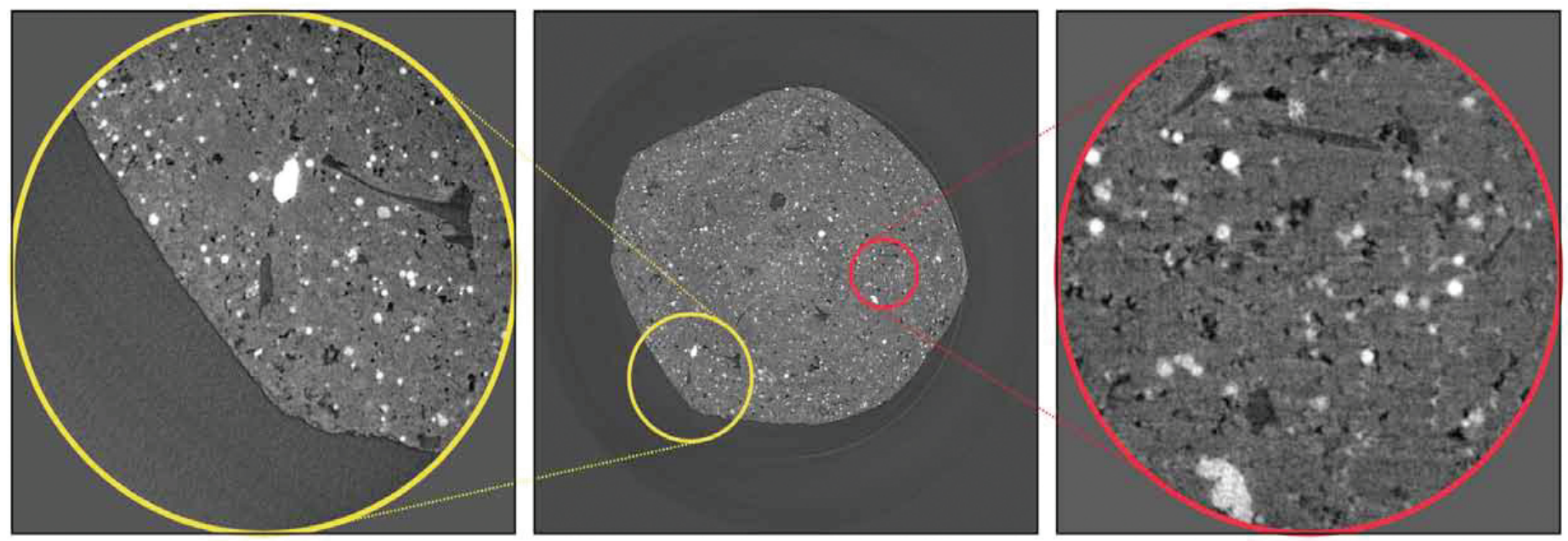

Fig. 1. Region-of-interest reconstruction with TomoPy of a shale sample. Porosity and micro-cracking in these rocks is vital for understanding the process of fracking. The middle image corresponds to the reconstruction using the full data set, and the left and right images are the different reconstructions that are obtained using a sub-sample of the full data to focus onto different locations within the sample. The Data Exchange data standard allows the raw experimental images, as well as provenance information and the reconstructed tomograph, to be included in a single file but yet be separated with simple utility programs.

\section{T}

he handling of datasets at scientific user facilities is becoming progressively more challenging as advances in sources and detectors drive increasingly aggressive data acquisition rates. These recent developments allow for the studies of multi-dimensional dynamic systems but they also limit the ability to share and process these data unless the originator of the data and the persons making use of that data are able to see and interpret the data equally and thus unequivocally agree on what these data mean. This understanding is driven by use of standardized data formats and by the ability to capture all experimental metadata required by the following data analysis.

The Scientific Data Exchange [1] is a generic data model that is designed to interface, or exchange data among different experimentation instruments, and to enable sharing of data analysis tools. Its implementation uses the Heirarchical Data Format (HDF5), a widely used and supported storage format for scientific data. The use of a specialized area detector [2] plug-in allows all tomography instruments of the
XSD Imaging Group to save data natively as Scientific Data Exchange.

Data Exchange [3] is an open-source software that provides different tomographic raw data importers to allow TomoPy [4] to be used at all major synchrotron facilities. TomoPy, which was developed at the APS, is a Python toolbox utilized to perform tomographic data processing and image reconstruction tasks, as shown in Fig. 1 [5].

Data Exchange also allows tomography users running experiments at any synchrotron facility to share data in a unified model.

Future plans include extending the Data Exchange module to support other techniques, starting with the ones being added to TomoPy including $x$-ray transmission tomography, $\mathrm{x}$-ray fluorescence microscopy, $x$-ray diffraction tomography, x-ray photon correlation spectroscopy, ptychography, and combinations of multimodal techniques.

Contact: Francesco De Carlo, decarlo@aps.anl.gov
The root of the HDF5 file

implements "exchange"

exchange

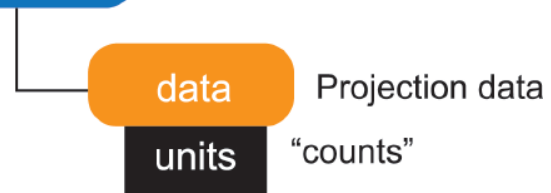

\section{REFERENCES}

[1] F. De Carlo et al., J. Synchrotron Rad. 21, 1224 (2014).

[2] Mark L. Rivers, AlP Conference Proceedings, 1234, (2010)

[3] https://github.com/data-exchange/data-exchange

[4] Doğa Gürsoy et al., J. Synchrotron Rad. 21, 1188 (2014).

[5] Doğa Gürsoy et al., SPIE Proceedings, 9212 (2014).

This research was supported by the U.S. Department of Energy Office of Science under Contract No. DE-AC02-06CH11357. 


\section{An IMPULSE fOr DYNAMIC COMPRESSION SCIENCE}

T

he IMPact system for ULtrafast Synchrotron Experiments (IMPULSE) gun was designed to perform dynamic compression experiments using advanced $\mathrm{x}$-ray imaging and diffraction methods at synchrotron sources such as the APS. Over the past three years, scientists from Los Alamos National Laboratory (LANL), National Security Technologies, Lawrence Livermore National Laboratory, and Argonne have successfully performed numerous experiments at the XSD beamline 32-ID-B,C of the APS to highlight the vast capabilities of IMPULSE. Their results provide novel information about material response at high strain rate loading, and have identified new experimental methods and detector systems needed to perform these high spatial resolution measurements on nanosecond timescales. This work helps to highlight the importance of dynamic compression platforms at other advanced light-source facilities such as the proposed LANL Matter-Radiation Interactions in Extremes (MaRIE) facility.

The IMPULSE efforts will be highlighted when the U.S. Department of Energy National Nuclear Security Administration-funded Dynamic Compression Sector 35-ID-B,C,D beamline at the APS becomes the first facility dedicated to dynamic compression science.

For over 50 years, shock wave or dynamic compression experiments have successfully examined material response at extreme environments (high temperatures and large strain rates) on very short time scales (picoseconds to microseconds). Such traditional experiments, including those involving the formation and propagation of cracks, phase transitions, and the elastic-plastic deformation of materials have resulted in the measurement of a wide array of properties in many materials under extreme conditions.

However, IMPULSE represents a new paradigm over these traditional diagnostic methods (which provide indirect information) because it is able to examine in situ, and in real time, the dynamic response of complex materials such as foams, powders, and high explosives, and complicated processes like phase transitions and detonation initiation zones (hot spots) in explosives (Fig. 1).

Three reasons lie at the heart of IMPULSE's success. First, its multiframe detector system is the first of its kind to create "shock movies" of x-ray imaging, and with an amazing spatial resolution of 1 to $2 \mu \mathrm{m}$.

Second, the improved design of the gun allows for better synchronization (bracketing) of the impact event with the detectors and the incident $x$ ray pulses, and then is able to detect the diffracted or transmitted $x$-rays from a single, 80-psec $x$-ray bunch.

Finally, IMPULSE has been utilized to educate and grow the user knowledge base needed to take advantage of these new, advanced diagnostics.

IMPULSE was designed specifically for use at synchrotron sources and consists of a launcher system to generate shock waves and a detection system for $\mathrm{x}$-ray imaging or diffraction measurements. The launch system, which consists of a gas breech, a barrel with a $12.6-\mathrm{mm}$ bore, a target chamber, and a mobile support structure can reach impact velocities of up to approximately $1-\mathrm{km} / \mathrm{sec}$. The system was designed to move easily into and out of the $\mathrm{x}$-ray beam, allowing for rotation of the target chamber during beam alignment. Further, the target chamber can withstand detonations of up to $750 \mathrm{mg}$ of TNT-equivalent explosives.

The novel, high-spatial-resolution, multi-frame imaging system uses $x$ rays that have passed through the target chamber - after entering from a side port - and through a series of shutters and slits, eventually being diffracted or transmitted through the target. The $x$-rays finally impinge on the scintillator within the detection system. Four intensified charge-coupled device detectors were optically multiplexed and triggered to capture images generated from any desired $x$-ray bunch from the synchrotron. In standard operating mode, $x$-ray bunches come every 153.4 nsec.

For its first dynamic $x$-ray imaging experiment, IMPULSE demonstrated in October 2011, at the APS 32-ID-B,C beamline, that it could create images of dynamic compression using a single 80-psec-width x-ray pulse. Since then, various other experiments have been conducted involving detonator performance, shock wave propagation through aerogels, high strain rate poly- 

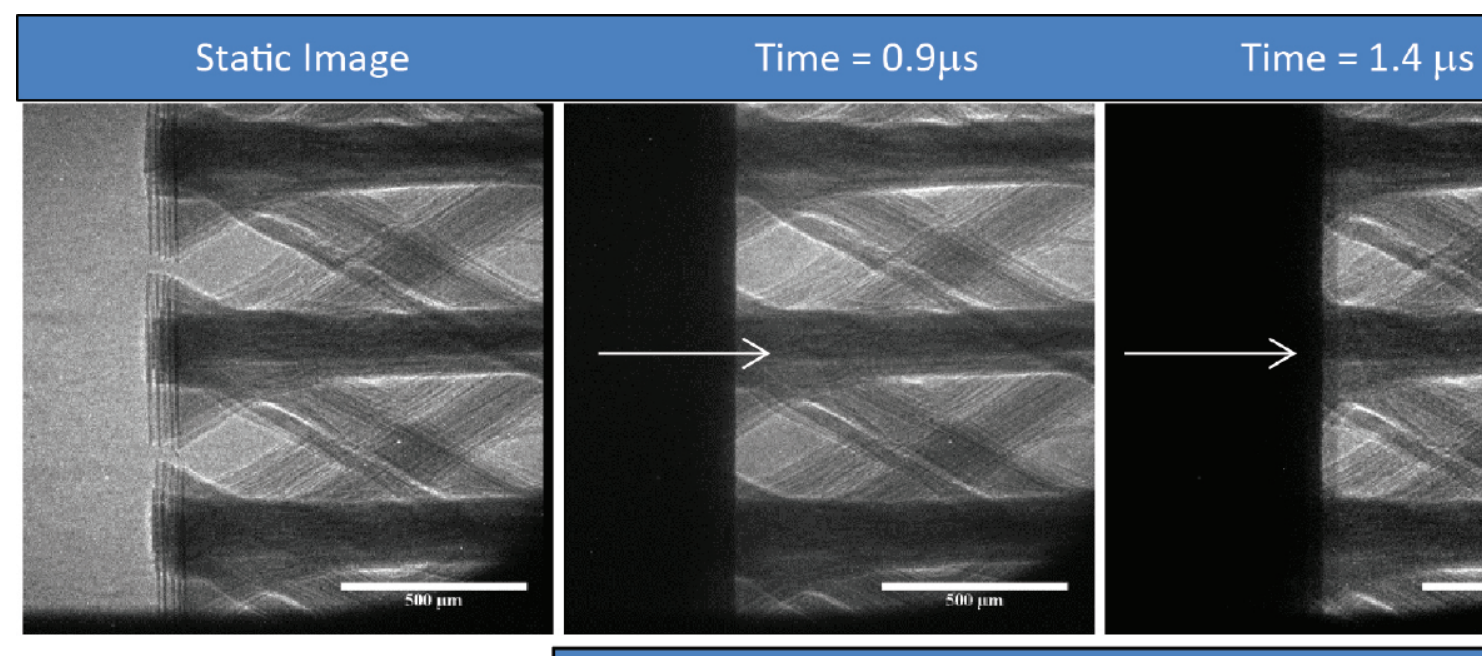

Fig. 1. X-ray phase contrast images of a micro-lattice foam impacted by a projectile. The images show the deformation of the lattice as a function of time. Images are approximately $1.4-\mathrm{mm}$ square. The projectile is observed entering the field-of-view from the left to the right. Times are approximate, with microseconds $(\mu \mathrm{s})$.

mer extrusion, compaction in idealized sphere systems to powders, spallation in metals and plastics, and many others. For instance, an experiment involving Laue $x$-ray diffraction showed the ability of IMPULSE to obtain highquality images on low-Z molecular crystals using a single $x$-ray bunch.

The ability of IMPULSE to provide in situ, nanosecond-resolution measurements at microscopic length scales is the key to its future success in helping to achieve a better understanding of condensed matter phenomena under extreme dynamic compression.

\section{- William A. Atkins}

See: Brian J. Jensen ${ }^{1 *}$, K.J. Ramos ${ }^{1}$, A.J. Iverson ${ }^{2}$, J. Bernier ${ }^{3}$, C.A. Carlson $^{2}$, J. D. Yeager ${ }^{1}$, K. Fezzaa ${ }^{4}$, and D.E. Hooks ${ }^{1}$, "Dynamic experiment using IMPULSE at the Advanced Photon Source," J. Phys. Conf. Ser. 500, 042001 (2014).
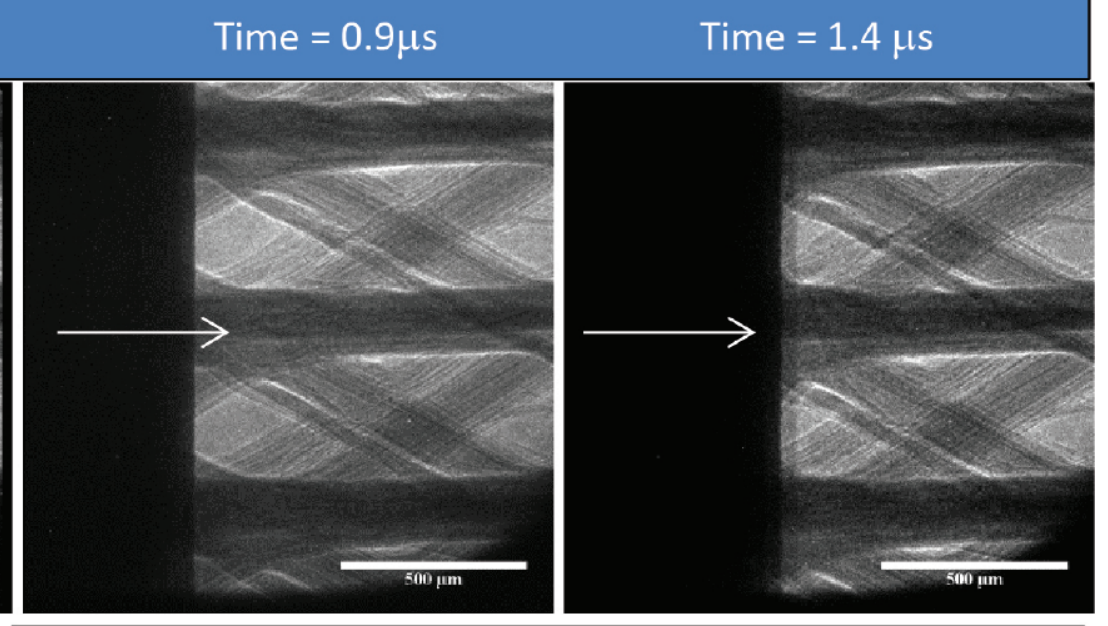

\section{Time $=1.8 \mu \mathrm{s}$}

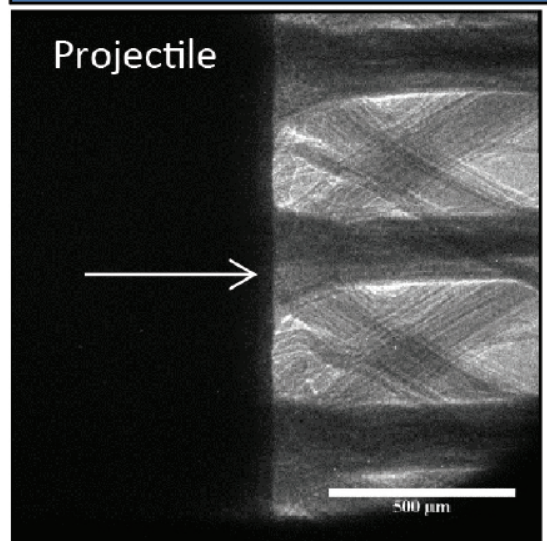

Time $=2.3 \mu \mathrm{s}$

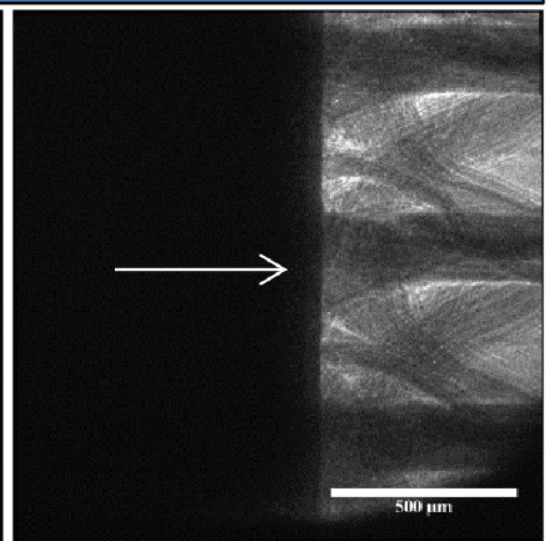

Author affiliations: ${ }^{1}$ Los Alamos National Laboratory, ${ }^{2}$ National Security Technologies, ${ }^{3}$ Lawrence Livermore National Laboratory, ${ }^{4}$ Argonne National Laboratory

Correspondence: * bjjensen@lanl.gov

This work was supported by LANL's MaRIE and Science Campaign programs and National Security Technologies Shock Wave Physics Related Diagnostics program. This research used resources of the Advanced Photon Source, a U.S. Department of Energy (DOE) Office of Science User Facility operated for the DOE Office of Science by Argonne National Laboratory under contract no. DE-AC02-06CH11357.

32-ID-B,C - Materials science, life sciences, geoscience - Phase contrast imaging, radiography, transmission x-ray microscopy, tomography $\cdot 7-40 \mathrm{keV} \cdot$ On-site $\cdot$ Accepting general users • 


\section{A NarRower SpeCtrum FOr a Wider View OF MATter}

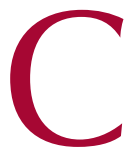

ondensed matter physicists, who study the physics of solids and liquids, often use the experimental technique inelastic $x-$ ray scattering (IXS), in which photons or neutrons of selected energy are bounced off a material to measure the energy loss and examine the collective vibrations of atoms in that material, to understand the dynamics of energy transfers that occur in picoseconds over distances of nanometers. But there are gaps in the resolutions of these techniques - distances of one nanometer to a few hundredths of a nanometer and time scales from a few picoseconds to approximately 100 psec. Unfortunately, a key physical phenomenon takes place within that gap: the liquid-glass transition. The details of how a material transforms from liquid to glass is one of the great mysteries of condensed matter physics, and understanding it could not only provide new theoretical insights, but also help pharmaceutical researchers make drugs that are more easily absorbed in the body. Now, researchers have developed a new version of IXS that bridges those gaps. Using the APS, they were able to enter previously inaccessible time and length scales, and then use that access to study the dynamics of liquid glycerol.

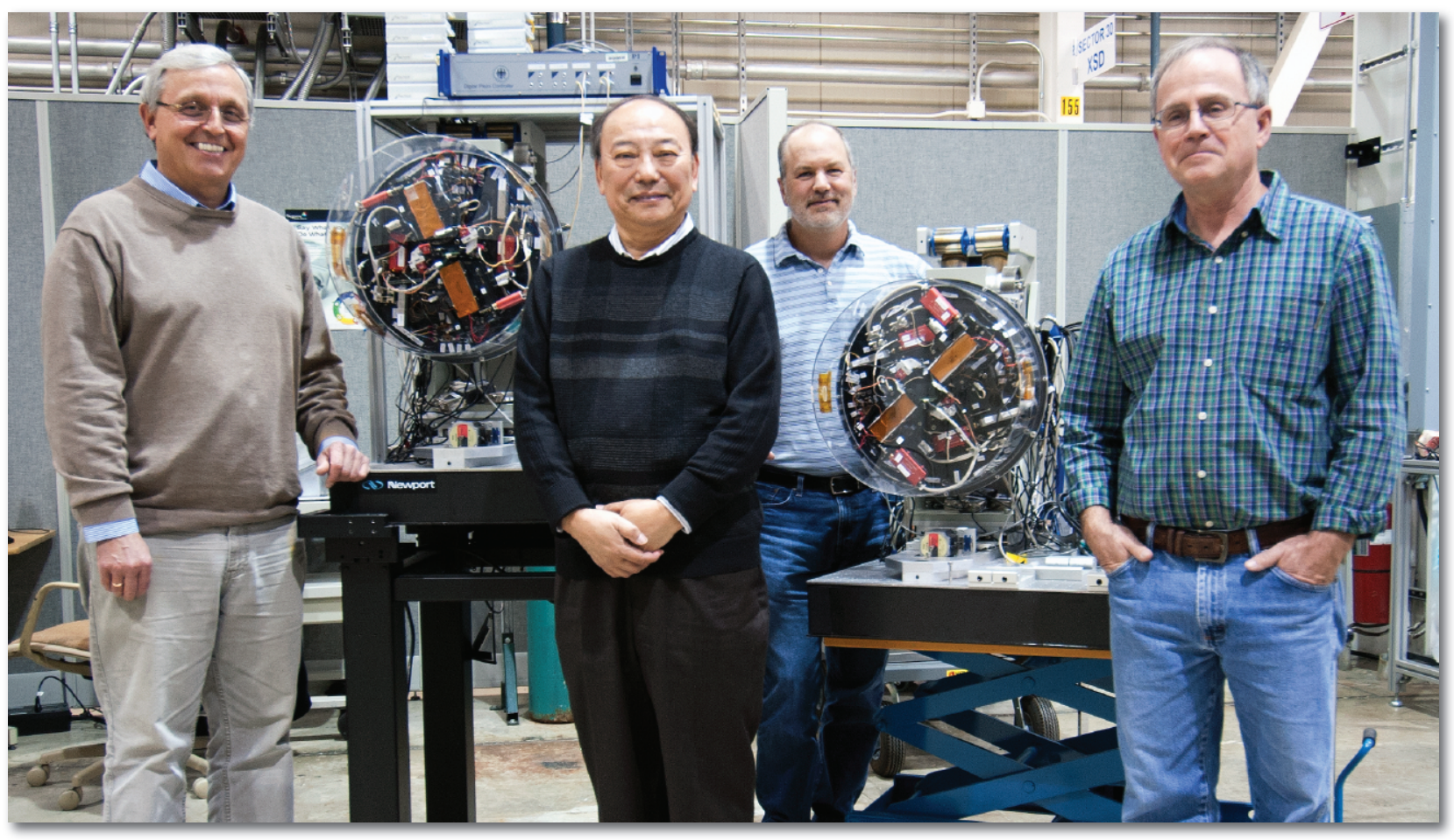

The IXS spectrometer development team. Left to right: Yuri Shvyd'ko (XSD), Deming Shu (AES), Kurt Goetze (AES), and Tim Roberts (XSD). 


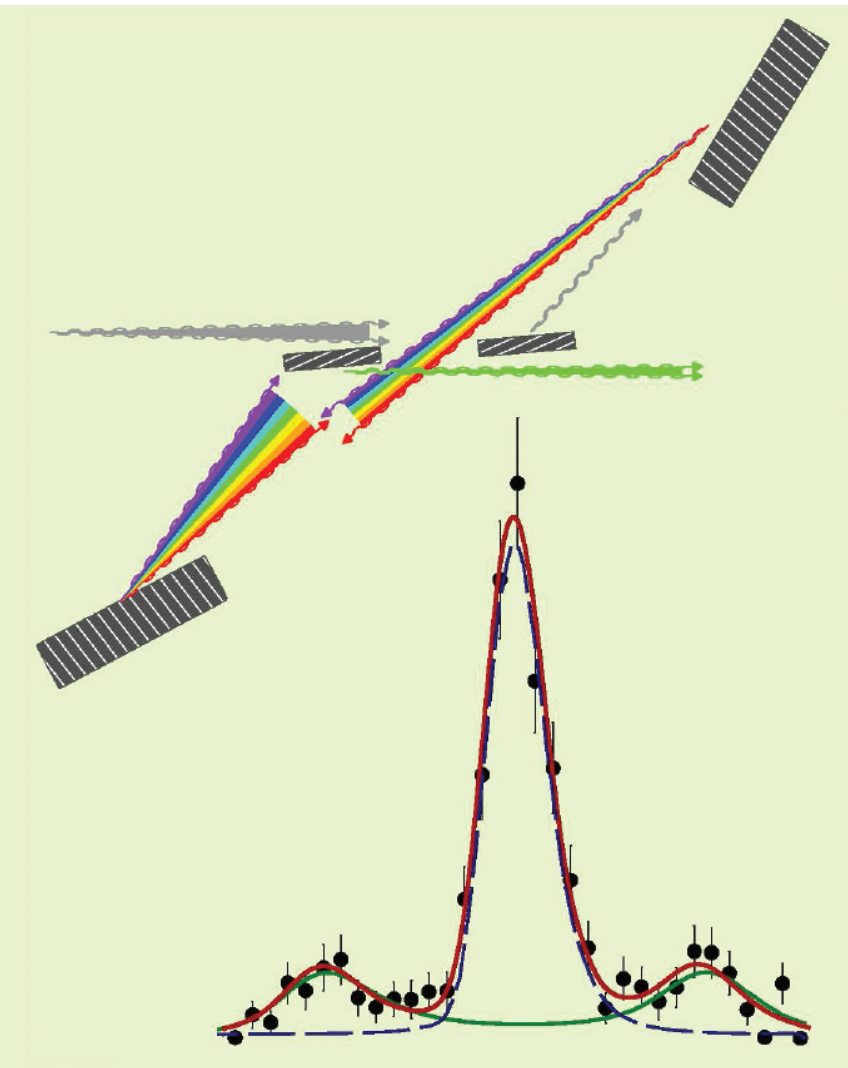

Fig. 1. Ultra-high-resolution dispersive optics of the new IXS spectrometer (top) and the IXS spectrum of liquid glycerol measured with this spectrometer (bottom). In the dispersive optics of the IXS spectrometer, an incoming beam of x-rays (double gray line) strikes a collimator crystal, which sends a virtually parallel beam (single gray line) toward a dispersion element (top right). That in turn spreads the beam into a rainbow and sends it to a second dispersion element (bottom left), which spreads it further and sends it to a wavelength selector, which passes only photons traveling within a narrow range of angles and wavelengths (green line). When measuring the IXS spectrum of a sample of liquid glycerol, the system records exceptionally narrow $620-\mu \mathrm{eV}$ spectral lines (solid circles).

The researchers from Argonne, the Diamond Light Source (UK), and the Deutsches Elektronen-Synchrotron (Germany) created an ultra-high-resolution inelastic $x$-ray scattering (IXS) spectrometer containing crystals with asymmetric, atomic-scale gratings that diffract x-rays. In one end of the set-up, the $x$-ray beam first strikes a collimator crystal, which ensures that the photons bouncing off it are close to parallel. The beam then hits a dispersion element, which spreads the photons into a "rainbow" depending on their wavelength. The light strikes another crystal, a wavelength selector, which only passes that portion of the light that lies within a very narrow angular window and is spectrally pure.

Using this basic setup, the researchers built a monochromator that consisted of a collimator, two dispersion elements, and a wavelength selector; taken together, they deliver $\mathrm{x}$-rays with a very narrow band of wavelengths to a sample of the material being studied.

The $\mathrm{x}$-rays from the beamline pass through the monochromator and other focusing optics to the sample, and then to an analyzer element. The analyzer is a variant of the monochromator, with an additional collimator of large acceptance angle to gather the scattered photons for the crystal optics. The analyzer can measure the wavelength shift, or energy loss, induced in the $\mathrm{x}$-rays by scattering from the sample. That tells scientists how the sample atoms' phonons transfer energy and momentum through the material.

XSD beamline 30-ID-B,C at the APS is dedicated to inelastic $x$-ray scat- tering studies. It is ideal for this work, because high-brightness $x$-rays can be generated in a well-directed beam to a small area in the 9-keV energy range required by the new spectrometer. The traditional version of inelastic scattering uses photons in the 20- to $25-\mathrm{keV}$ range, but the lower energies used in the new setup provide better momentum resolution.

The technique also has spectral resolution and contrast that are superior to traditional techniques. The researchers measured a spectral bandwidth of $620 \mu \mathrm{eV}$, a 3-fold improvement over what was previously available. Additionally, spectral contrast improved by an order of magnitude.

The technique was applied to a sample of liquid glycerol. The researchers were, indeed, able to make more precise measurements than had ever been made on such a sample before (Fig. 1).

The researchers call their concept a new paradigm, which could be developed into an even better spectrometer, for instance by adding more focusing optics and a position-sensitive detector, thus opening it to a wide range of measurement applications. Because this spectrometer uses a working energy of $9 \mathrm{keV}$, it is practical for most $\mathrm{x}$ ray synchrotron and free-electron laser facilities. - Neil Savage

See: Yuri Shvyd'ko ${ }^{1 *}$, Stanislav Stoupin ${ }^{1}$, Deming Shu ${ }^{1}$, Stephen P. Collins $^{2}$, Kiran Mundboth ${ }^{2}$, John Sutter ${ }^{2}$, and Martin Tolkiehn", "High-contrast sub-millivolt inelastic $X$-ray scattering for nano- and mesoscale science," Nat. Commun. 5, 4219 (2014).

DOI: $10.1038 /$ ncomms5219

Author affiliations: ${ }^{1}$ Argonne National Laboratory, ${ }^{2}$ Diamond Light Source Ltd., ${ }^{3}$ Deutsches Elektronen-

Synchrotron

Correspondence: *shvydko@aps.anl.gov

This research used resources of the Advanced Photon Source, a U.S. Department of Energy (DOE) Office of Science User Facility operated for the DOE Office of Science by Argonne National Laboratory under Contract No. DE-AC0206CH11357. 


\section{Two Good Things That Are Better Together: STM AND X-RAY SYNCHROTRON MICROSCOPY}

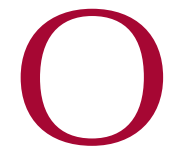

ver the past three decades, scanning tunneling microscopy (STM) has rapidly become a major component of the condensed matter physics toolbox. While STM can provide vast quantities of data about the electronic, structural, and magnetic properties of materials at atomic resolution, its Achilles heel is its inability to characterize elemental species. But a team from Argonne and Ohio University has found a way around this limitation by combining STM with the spectroscopic versatility of synchrotron x-rays, achieving chemical fingerprinting of individual nickel clusters on a copper surface at a resolution of $2 \mathrm{~nm}$ (Fig. 1), creating a powerful and versatile nanoscale imaging tool with exciting promise and potential for the materials and biological sciences.
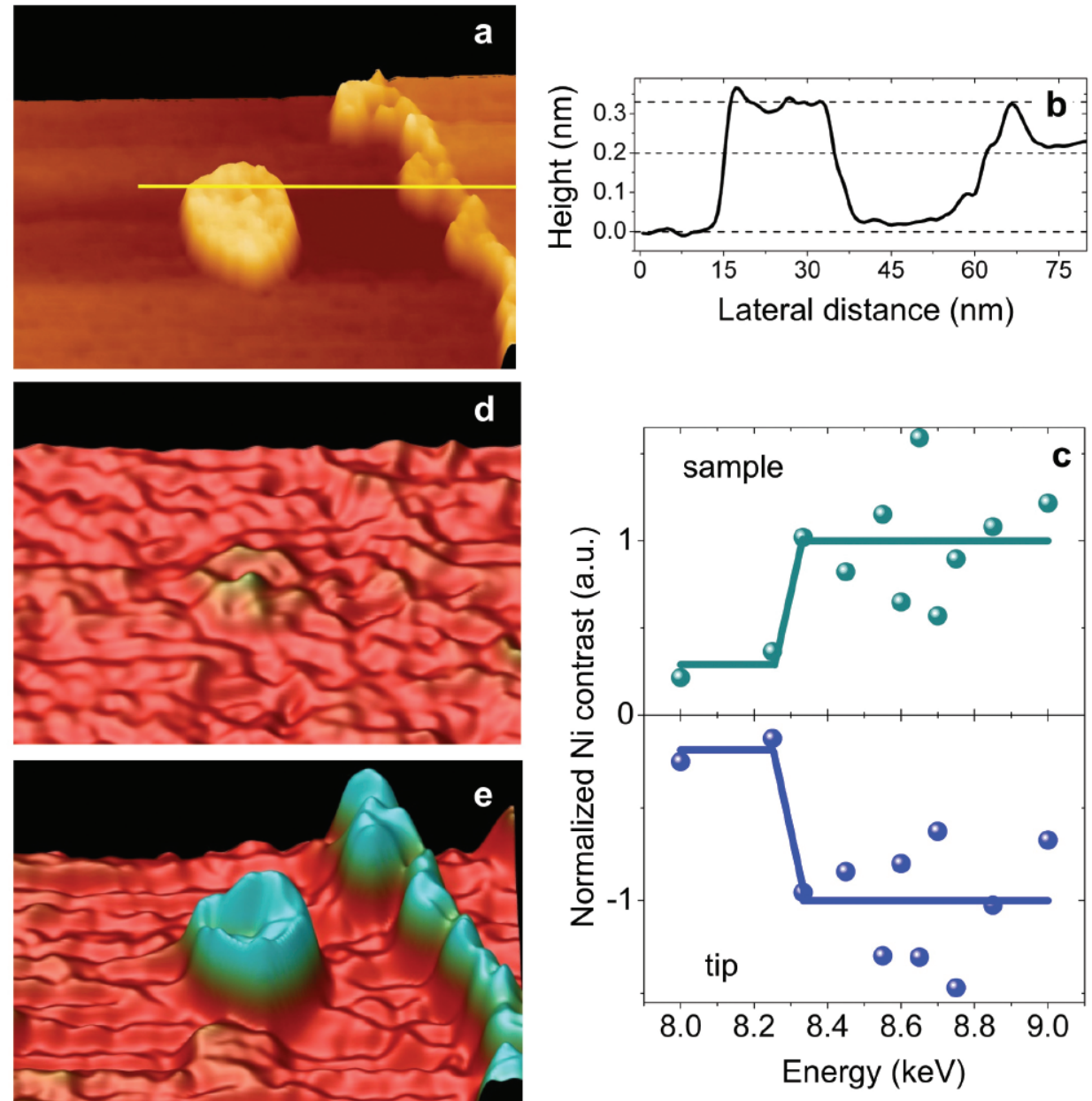

Fig. 1. Localized x-ray cross-section of a Ni island. (a) Perspective three-dimensional view of a STM topography scan $\left(110 \times 60 \mathrm{~nm}^{2},-1 \mathrm{~V}, 1 \mathrm{nA}\right)$. (b) Height profile of the line shown in the topography scan. (c) X-ray cross section of a single $\mathrm{Ni}$ island obtained from $\mathrm{I}^{\mathrm{x} \text {-ray }}{ }_{\text {sample }}$ (top) and $\mathrm{I}_{\text {tip }}^{\mathrm{x} \text {-ray }}$ (bottom). (d) Sample current $\mathrm{I}_{\text {sample }}^{\mathrm{x} \text {-ray }}$ does not provide chemical contrast, when the $\mathrm{x}$-ray energy $(E=8.25 \mathrm{keV})$ is below the $\mathrm{Ni}$ K-edge $(8.33 \mathrm{keV})$. (e) The $\mathrm{Ni}$ island on the $\mathrm{Cu}\left(\begin{array}{lll}1 & 1 & 1\end{array}\right)$ terrace and islands along the $\mathrm{Cu}$ step edge become clearly visible for photon energies above the Ni K-edge, here $\mathrm{E}=8.55 \mathrm{keV}$. Figures from N. Shirato et al., Nano. Lett. 14(1 1), 6499 (2014). (C 2014 American Chemical Society 


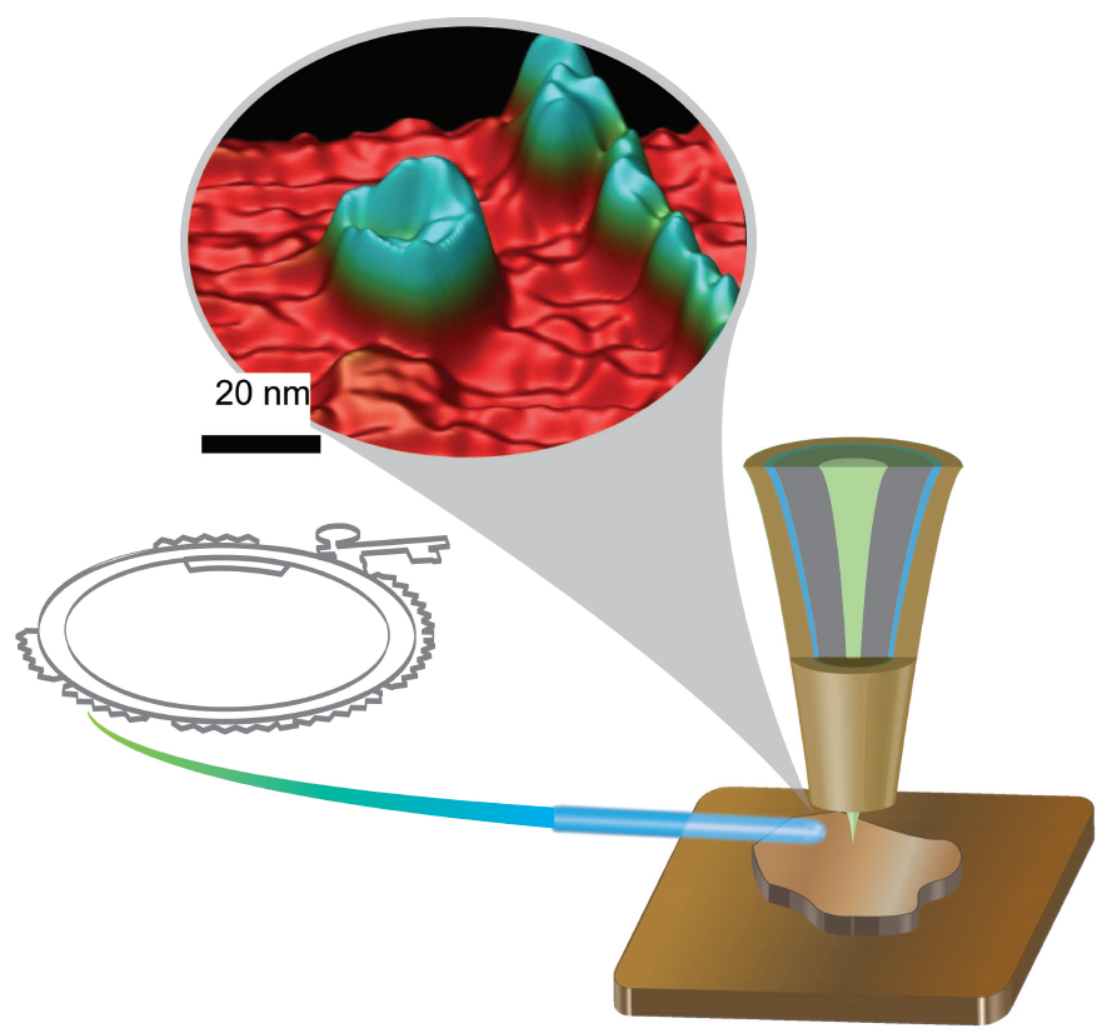

Fig. 2. A diagram of the imaging setup, featuring the STM "smart tip" and an image of Ni clusters (green) on the Cu surface (red).

Working at the CNM/XSD 26-ID-C beamline at the APS, the researchers took advantage of some new technological innovations developed by Argonne researchers.

However, the team had to overcome some experimental hurdles to combine STM with synchrotron x-rays. The resolution and sensitivity of STM can be adversely affected by photoejected electrons from the sample interfering with the measurement of tunneling effects. The Argonne researchers invented and patented a nanofabricated "smart tip" for the scanning tunneling microscope that sharply focuses detection of electrons solely to those collected at the scanning tip where it interacts with the sample, ignoring the background electrons from the sidewalls of the tip (Fig. 2). The various coatings for the smart tip were grown at the Center for Nanoscale Materials (CNM) at Argonne. Then, focused ion beam milling at the CNM Electron Microscopy Center at Argonne was used to expose the tip apex.

The team also developed a filter circuit that separates the chemical and magnetic data from the $x$-ray-induced currents and topographical data from conventional tunneling effects into two channels, allowing them to be recorded separately without mutual interference.

Using the markedly enhanced resolution and sensitivity made possible with these advances in synchrotron $\mathrm{x}$ ray tunneling microscopy (SX-STM), the Argonne/Ohio University experiment team analyzed nickel clusters deposited on a copper surface. Usually, $\mathrm{x}$-rays is based on photoionization cross sections, such measurements are averaged over a rather wide surface area and depth. But the new technique was able to image and obtain a photoionization cross section of a single nickel cluster on the sample surface with 2-nm resolution.

The team demonstrated a world record in the spatial resolution of chemical imaging using synchrotron x-ray scanning tunneling microscopy, with tremendous impact for many scientific areas including materials science, chemistry, and energy materials.

Both that remarkable resolution and the precise chemical fingerprinting of individual nickel nanoclusters were also clearly evident in the topographic images of the sample surface, even because chemical fingerprinting using down to the height of a single atom. The experimenters note that the thickness of individual clusters appears to have no effect on the contrast intensity of their chemical signature. They suggest that because tunneling is a local effect sensitive only to the topmost layer of materials, this phenomenon as observed topographically results from the tunneling of $x$-ray excited photoelectrons from states between the Fermi level and the work function.

While the current experiments were conducted at room temperature, the researchers anticipate achieving the same resolution of SX-STM at far lower temperatures.

Even in its present form, the techniques demonstrated here can revolutionize nanoscale imaging in realms far beyond materials science, including electronics and biology. By overcoming the inherent limitations of both STM and x-ray microscopy, this new work has also combined the strengths of each to create a powerful and versatile imaging tool with an exciting promise and potential. - Mark Wolverton

See: Nozomi Shirato ${ }^{1}$, Marvin Cummings ${ }^{1}$, Heath Kersell ${ }^{2}$, Yang Li ${ }^{2}$, Benjamin Stripe ${ }^{1}$, Daniel Rosenmann², Saw-Wai Hla ${ }^{1,2 * *}$, and Volker Rose ${ }^{1 *}$, "Elemental Fingerprinting of Materials with Sensitivity at the Atomic Limit," Nano Lett. 14(11), 6499 (2014). DOI: $10.1021 / \mathrm{nl} 5030613$

Author affiliations: ${ }^{1}$ Argonne National Laboratory, ${ }^{2}$ Ohio University Correspondence: * vrose@anl.gov, ** shla@anl.gov

This work was funded by the Office of Science Early Career Research Program through the Division of Scientific User Facilities-Basic Energy Sciences, U.S.

Department of Energy (DOE), through Grant no. SC70705. H.K. and Y.L acknowledge the support by the U.S. DOE Office of ScienceBasic Energy Sciences Grant DE-FG0202ER46012. Work at the Advanced Photon Source, the Center for Nanoscale Materials, and the Electron Microscopy Center was supported by the U.S. DOE Office of Science-Basic Energy Sciences, under Contract DE-AC02-06CH11357.

26-ID-C • CNM/XSD • Physics, materials science $\cdot$ Nanofluorescence imaging, microdiffraction, nanotomography $\bullet 8-12 \mathrm{keV} \cdot$ On-site -Accepting general users • 


\section{FABRICATING, TESTING, AND INSTALLING a KirkPatrick-Baez MirRor System at the APS}

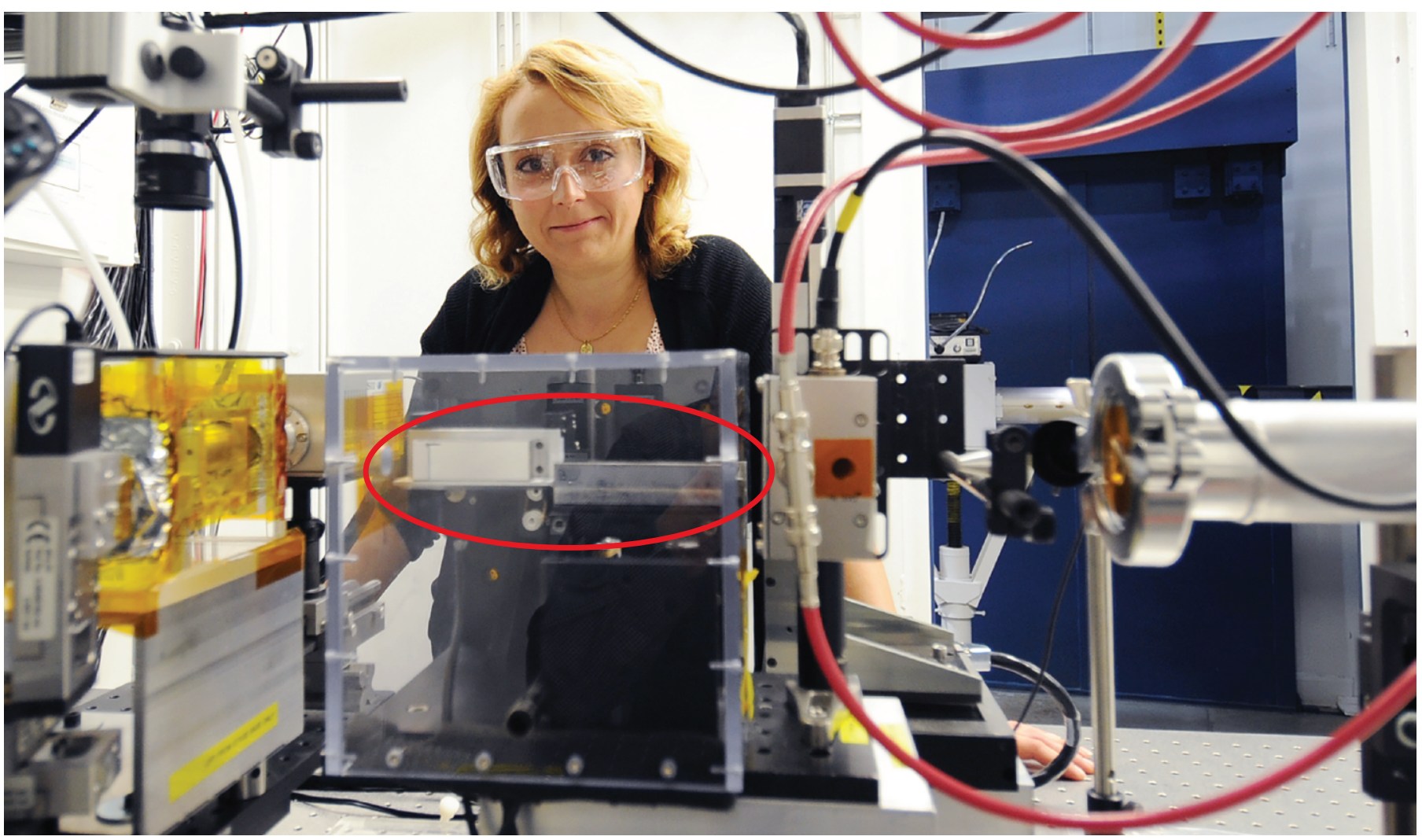

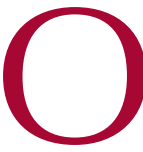

ne of the earliest methods developed for focusing $x$ rays is the Kirkpatrick-Baez (K-B) mirror system, which was eventually adapted to focus the hard x-rays generated in synchrotron facilities, thereby greatly increasing the illumination of micron- and sub-micronsized sample areas. In this article, we describe the first fixed-geometry (i.e., non-bendable) K-B mirror system that is entirely developed, fabricated, and tested by the XSD Optics Group in collaboration with the Microscopy Group in XSD and staff in the APS Engineering Support Division. The parameters for this new K-B mirror system were specified (by Lydia Finney, XSD) to focus the 8-BM-B beam to a 1- $\mu \mathrm{m}$ focal spot size for microfluorescence imaging. Prior to implementation and use at the host beamline, the mirror system was fully calibrated and tested at XSD beamline 1-BM-B,C, the new Optics and Detector Test Beamline (see "The Newly Reconfigured Beamline 1-BM," APS Science 2012, pg. 174). The focused beam achieved by the new system, measuring approximately $1 \mu \mathrm{m}^{2}$, has provided improved micro-fluorescence imaging at 8-BM-B. Fixed-geometry K-B mirrors offer great mechanical stability compared to bender-based K-B mirrors, especially for applications that do not require dynamical bending. They are also the only viable solution for applications requiring sub-100-nm focusing. The delivery and implementation at 8-BM-B constitutes a first complete technology transfer of a K-B mirror arrangement from design and fabrication in the Optics Group, to testing at 1-BM-B,C, and finally to successful commissioning at 8-BM-B, thereby demonstrating a new in-house capability and laying solid foundations to develop nanofocusing $\mathrm{K}$ $B$ mirror systems for the proposed APS MBA nanoprobes and beamlines.

Paul Kirkpatrick and Albert Baez (father of folk singer Joan Baez) described the first K-B mirror system in 1948. A typical contemporary system consists of two metal-coated silicon bars acting as x-ray mirrors, with one
Lydia Finney (XSD) in the 8-BM-B enclosure. The K-B mirrors (circled; horizontal focusing mirror on the left and verical on the right) made by the XSD Optics Group are an integral component of the microprobe at the 8$B M-B$ beamline.

mirror focusing in the vertical direction, the other horizontally. The metal coating provides the elliptical shape necessary to focus the x-rays, which glance off the mirrors at a very shallow angle (referred to as grazing-incidence reflection). Compared to techniques that diffract or refract $\mathrm{X}$-rays, $\mathrm{K}$-B mirrors are achromatic and permit $x$-ray focusing over a larger range of focal distances (the distance from the mirrors to the focal plane) and acceptance angles (the largest angle for which incident $x$-rays are efficiently gathered, which varies according to $\mathrm{x}$-ray energy).

The ideal elliptical shape of the mirrors in a particular system is determined by three quantities: the distance from the x-ray source to the mirrors; the distance from the mirrors to the focal plane; and the angle the x-rays graze 
off the mirrors. (Fig. 1 illustrates distances in the test beamline.) The elliptical shapes of the mirrors in the new system were calculated using the source-to-mirror and mirror-to-focus distances for beamline 8-BM-B. Although the 1-BM-B,C test beamline has different source/focal distances than 8-BM, the researchers calculated that the minor differences in their layouts would nonetheless permit successful performance evaluation at the test beamline.

The mirror fabrication process started with two flat silicon bars, each nearly an inch thick and wide $(20 \mathrm{~mm})$ and superpolished at one surface to be profiled to elliptical shape. The longer of the two bars, intended for vertical focusing, is slightly over 3 in. $(80 \mathrm{~mm})$ in length; the shorter bar, for horizontal focusing, is $\sim 2.4$ in. $(60 \mathrm{~mm}$ ) long. The required elliptical shape was achieved by depositing platinum on the bars via profile-coating, a technique originally developed at the APS. Although profilecoating is very precise, slight imperfections (or figure errors) inevitably arise, resulting in microscopic surface deviations. Measurement (done at the APS metrology laboratory) of the mirrors' surface figure errors before installation at beamline 8-BM-B were quite small: $0.67 \mathrm{~nm}$ rms and $0.31 \mathrm{~nm}$ rms. for the vertical and horizontal mirrors, respectively.

The finished mirrors were affixed to mounting stages (designed by D. Shu, AES) that feature stepping motors for very fine adjustments: one motor produces a linear motion perpendicular to the mirror's surface, while a second motor adjusts mirror pitch, thereby varying the grazing-incidence angle.

To measure system performance, monochromatic (18-keV) $\mathrm{x}$-rays were separated from the multi-wavelength beamline using a monochromator. Slits narrowed this monochromatic beam to a square measuring 180-by-180 $\mu^{2}$, which the K-B mirrors further focused to $\sim 1 \mu \mathrm{m}^{2}$.

The optimum focus was determined using two techniques. The first technique converted the focused $\mathrm{x}$-rays to a visible image, allowing the researchers to visually adjust mirror orientation. The second technique provided

quantitative measurements using a wire scanning method that passes an ultrafine tungsten wire stepwise through the x-ray beam. A detector measured the change in x-ray intensity as the wire moved through and partially absorbed the beam, revealing the beam's shape and intensity.

Using these methods, the grazing angle was adjusted to obtain a focal area of 1.3- $\mu \mathrm{m}$ vertical by $1.21-\mu \mathrm{m}$ horizontal; these values compare favorably to the best theoretically-calculated values for beamline 1-BM-B,C of $0.8-\mu \mathrm{m}$ vertically by $1.18-\mu \mathrm{m}$ horizontally. Optimum focal size occurred at a beam/mirror incidence angle of 2.97 $\operatorname{mrad}\left(0.17^{\circ} \mathrm{de}-\right.$

grees). Figure 2 shows focusing data for beamline 1-BM-B,C.

The focal size achieved at 1-BM$B, C$ was smaller than the value required for operations at 8-BM-B. Since calculations indicated similar focusing performance between the two beamlines, the $\mathrm{K}-\mathrm{B}$ mirror system was installed at beamline 8-BM-B. Subsequent microprobe experiments have demonstrated the desired image resolution.

$$
\text { - Philip Koth }
$$

See: Naresh Kujala*, Shashidhara Marathe, Deming Shu, Bing Shi, Jun Qian, Evan Maxey, Lydia Finney, Albert Macrander, and Lahsen Assoufid, "Kirkpatrick-Baez Mirrors to Focus Hard Xrays in Two Dimensions as Fabricated, Tested and Installed at the Advanced Photon Source," J. Synchrotron Rad. 21, 662 (2014).
DOI: 10.1107/S1600577514006493 ] Author affiliation:

Argonne National Laboratory Correspondence: *assoufid@aps.anl.gov

This research used resources of the Advanced Photon Source, a U.S. Department of Energy (DOE) Office of Science User Facility operated for the DOE Office of Science by Argonne National Laboratory under Contract No. DE-AC02-06CH11357.

1-BM-B,C $\cdot$ XSD - Materials science, physics - Optics testing, detector testing, topography, energy dispersive $\mathrm{x}$-ray diffraction, white Laue single-crystal diffraction - 6-30 keV, 50-120 $\mathrm{keV} \cdot$ On-site $\cdot$ Accepting general users $•$

8-BM-B - Chemistry, life sciences, environmental science, materials science $\cdot$ Microfluorescence (hard x-ray) • 5.5-20 keV, 9-18 keV - On-site 


\section{ID'ing Cells With the Help of a Computer}

X -ray fluorescence microscopy (XFM) is a powerful method for studying biological samples; it can be used, for instance, to measure how disease progresses within a group of cells, or how a particular population of cells affects whether a tumor is vulnerable to a particular drug. However, the quantity and complexity of the data produced using a highbrightness, high-resolution source for XFM, such as the APS, makes it extremely challenging for a human microscopist to sort through it manually. Now, researchers have developed a method that lets a computer locate and identify cell structures rapidly and without human supervision, allowing scientists to take full advantage of the $x$-ray data. logical sample through a small, focused $x$-ray spot. The x-rays cause the elements within the sample to emit characteristic fluorescence and with the help of a suitable detector, allow one to determine the location and concentration of, say, iron or zinc within the sample. The balance of metals in a cell can be critical indicators of disease progression; cancer patients, for instance, can lose $10 \%$ of the zinc from their cells.

Researchers from Argonne and Northwestern University developed a machine-learning algorithm to locate and identify different types of cells, even when some overlap. The algorithm starts by assuming that cells of a specific type have generally similar shape and size, and have the same signature concentration of elements. In this experiment, the researchers used red blood cells (characteristically high in iron), yeast cells (with a high concentration of zinc), and green algae (high in manganese). Both the blood and algae cells were approximately $18 \mu \mathrm{m}^{2}$ in area, while the yeast cells were $2.7 \mu \mathrm{m}^{2}$. The team tested the algorithm on XFM data taken at the XSD beamline 2ID-E at the APS. The x-ray beam, focused to a $0.8 \mu \mathrm{m} \times 0.8 \mu \mathrm{m}$ spot, was used to map elemental distributions of groups of cells typically $200 \mu \mathrm{m}$ in size.

The algorithm divided the whole image into foreground and background pixels, based on their intensities, then drew an ellipse around groups of pixels (a)

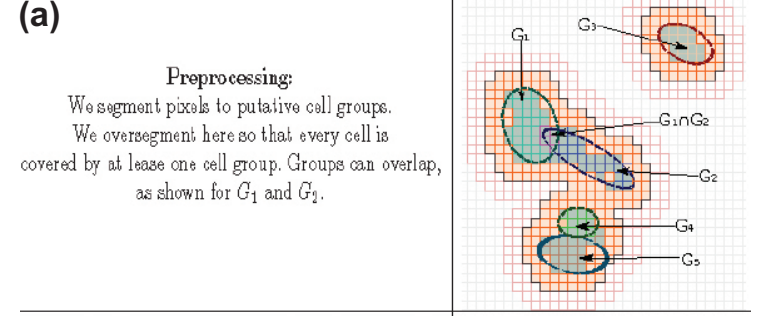

(b)

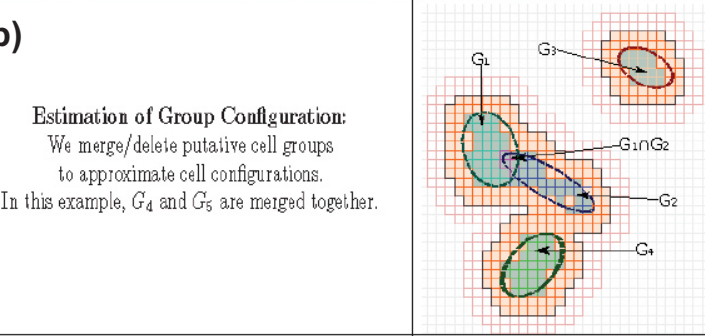

(c)

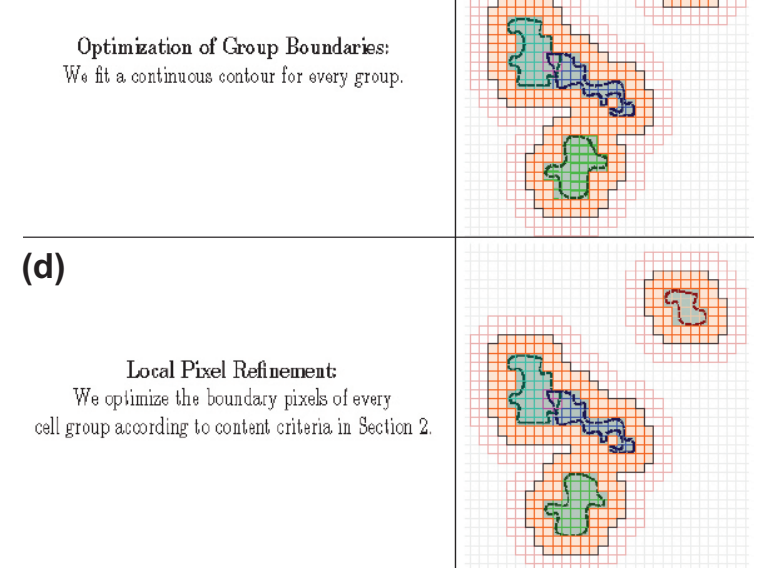

Fig. 1. In a series of steps, the algorithm first sorts pixels into putative cell groups (a), then merges or deletes groups to better fit the elemental content of cells (b). It optimizes the boundaries of the groups (c), and finally refines the shape by individual pixel (d). From S. Wang et al., J. Synchrotron Rad. 21, 568 (2014), (c) 2014 International Union of Crystallography

it thought should go together, based on the size and the elemental signature of the cell it sought (Fig. 1). In their initial experiment, the researchers aided this step by initializing the algorithm with a handful of regions of interest, but in the future those handdrawn regions can be replaced by other data or learned automatically.

The algorithm then looked at the background pixels to see if it could refine the locations and figure out if cells overlapped. It repeated these steps to make sure each region it identified matched well with the expected contents of the cell. The algorithm then examined these groups and determined whether deleting some or merging two together would make them fit better. Finally, it replaced the ellipse with a more realistic outline of the cell, and performed statistical tests to optimize the cell boundaries down to the level of individual pixels. To validate their approach, the researchers compared the results of applying the program to data from each of four different detectors and with different initial elemental content hypotheses, and found a good match.

The researchers believe this new algorithm will allow biologists to find and identify individual cells in three dimensions and in real time, making XFM an even more powerful research tool. - Neil Savage

See: Siwei Wang ${ }^{1 *}$, Jesse Ward ${ }^{1}$, Sven Leyffer ${ }^{1}$, Stefan M.Wild ${ }^{1}$, Chris Jacobsen ${ }^{1,2}$, and Stefan Vogt ${ }^{1}$, "Unsupervised cell identification on multidimensional X-ray fluorescence datasets," J. Synchrotron Rad. 21, 568 (2014).

DOI: $10.1107 / S 1600577514001416$

Author affiliations:

${ }^{1}$ Argonne National Laboratory,

${ }^{2}$ Northwestern University Correspondence:

*siweiw@gmail.com

This work was supported by the U.S. Department of Energy Office of Science, Advanced Scientific Computing Research, and Basic Energy Sciences (DEAC02-06CH11357).

2-ID-E • XSD • Life sciences, environmental science, materials science - Microfluorescence (hard x-ray) • 7-10.5 keV, 11-17 keV • On-site $\cdot$ Accepting general users $•$ 


\section{Wave Hello to Faster X-ray Pulses}

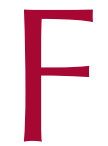

ast X-ray pulses from the APS can act as powerful x-ray camera flashes to image molecular motion. But some biological and chemical reactions - proteins refolding or molecular bonds shifting, for example - happen too fast even for the APS, which can produce 100 -psec bursts of $x$-rays at its speediest. A team of researchers is trying to slice those bursts into a series of much shorter pulses, using sound waves to deform a perfect crystal. Their results, obtained in tests at XSD beamline 7-ID-B,C,D at the APS, could broaden the kind of time-resolved science possible at this facility.

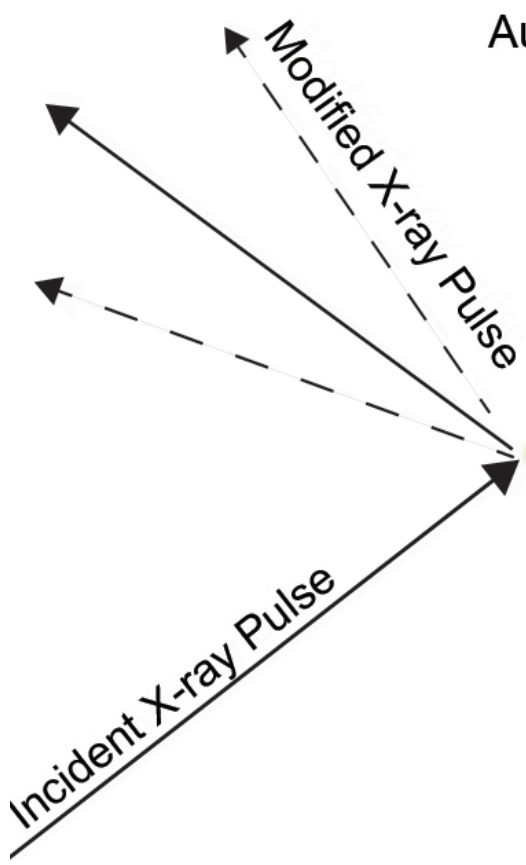

Au Film

Ge Crystal

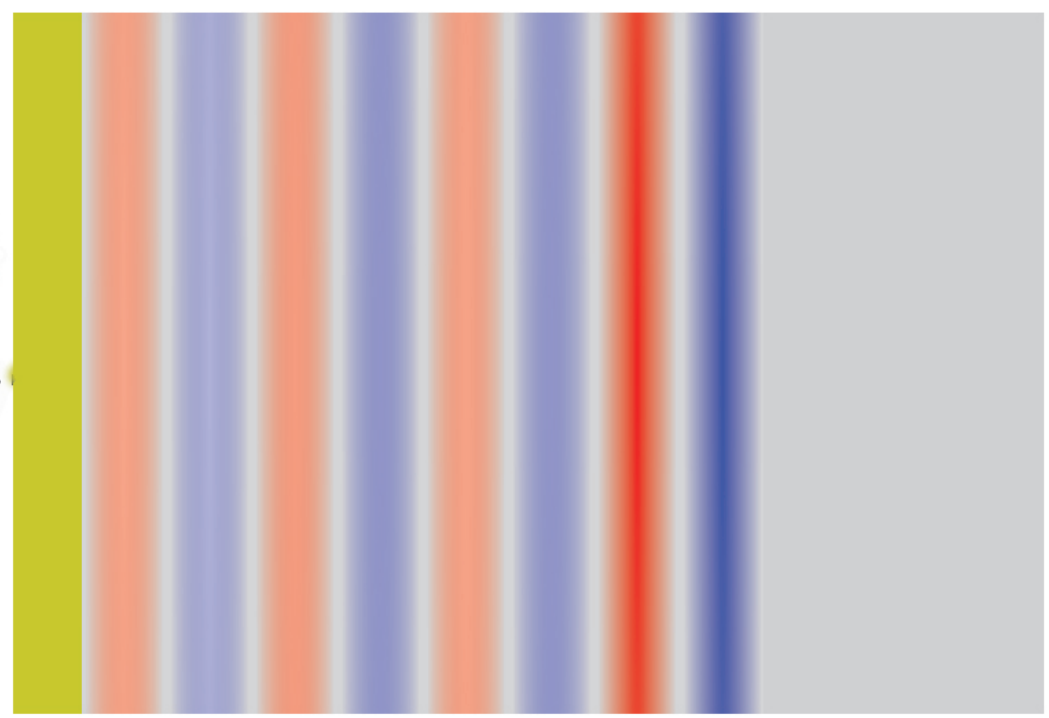

Ultrafast Sound Wave

Fig. 1. As the sound wave moves through the germanium, it deforms the crystalline structure and displaces atoms. By properly timing the sound wave, researchers can control the displacement of the atoms and so reflect the incident $x$-ray pulse at different angles (dotted and solid lines).

There are many synchrotron radiation facilities in the world, but very few match the APS in brightness or available beam time. One of its few drawbacks is that its pulses are not short enough to image ultrafast phenomena. There is another x-ray source in the U.S. that does have that capability, the Linac Coherent Light Source (LCLS) at the SLAC National Accelerator Laboratory, but its $\mathrm{x}$-ray pulses are so powerful that they can destroy the sample under examination, making it difficult to do repeatable experiments.

University of Delaware and Argonne researchers may now be able to tweak the APS to make its pulses as short as those at the LCLS by coating a perfect germanium crystal with a thin layer of gold and then illuminated it with an ultrafast optical laser source. The laser flash quickly heated the gold and caused it to expand, sending a pressure wave through the crystal. As the wave moved through the crystal it moved the germanium atoms into a slightly different configuration. After the wave passed, the atoms snapped back into their perfect crystal pattern.

$X$-rays are very sensitive to atomic positions. Atoms in one crystalline pattern reflect x-rays one way; atoms in a slightly different pattern will reflect the $x$-rays in a different direction (Fig. 1). If the sound wave can be precisely timed and controlled, the researchers hope to use the germanium crystal like a mirror that can shift back and forth, dividing a single 100-psec $x$-ray pulse into several 10-psec pulses.

Other researchers are trying ways to divide up an x-ray pulse. One, at the Advanced Light Source at Lawrence Berkeley National Laboratory, shoots a laser into an electron beam to produce $\mathrm{x}$-rays, but the process is inefficient. It slices out just $1000 \mathrm{x}$-ray photons per second, making a very weak beam.

At the European Synchrotron Radiation Facility (France) a team used a

"Wave" cont'd on page 170 


\section{X-RAY PTYCHOGRAPHY: IMAGING VIBRATIONS WITHOUT THE BLUR}

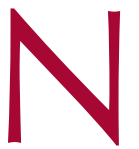

ature is ever on the move, and our ability to understand it in detail is often improved by "freezing" the motion of the dynamic systems under study using "snapshot" images. Standard imaging techniques have been hugely successful, but only when snapshot durations are much shorter than the time scales over which objects move. This requirement is becoming increasingly difficult to meet when imaging living systems and complex materials at the nanometer scale. While it may seem impossible to avoid blurred images when motions are much quicker than snapshot durations, there is actually a technique that promises to accomplish exactly that, a lensless imaging method under development called ptychography. Working at the APS, scientists were able to demonstrate experimentally that $\mathrm{x}$-ray ptychography could image a vibrating object whose frequency of vibration was one order magnitude faster than the measurement time. This was accomplished for two types of vibration and two amplitudes of vibration as well. The researchers speculate that this mode manipulation may prove very useful in designing custom mode shapes for use in high-resolution $\mathrm{x}$-ray or electron imaging where illuminations having very specific properties are required.

Static

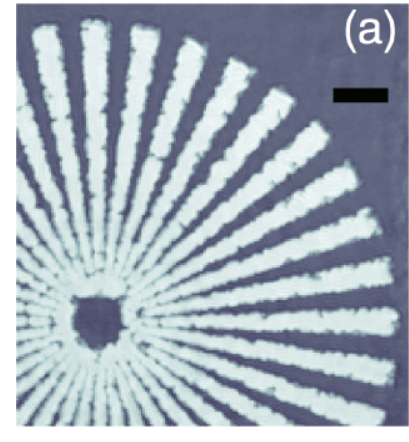

Square
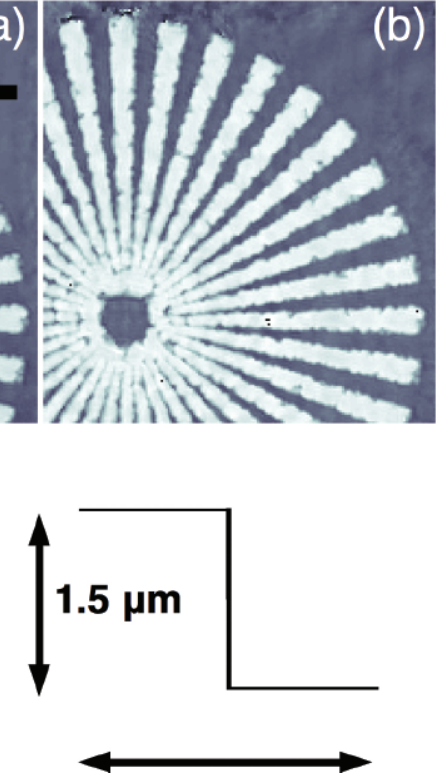

Fig. 1. (a) Reconstructed image of the sample when it was stationary. This can be compared to the reconstructions when the sample was moving horizontally with (b) square wave motion (i.e., occupying two discrete positions) and when it was moving with (c) sine wave motion (i.e., moving continuously). The motion in each case was approximately one order of magnitude faster than the measurement time. Reconstructions of the moving sample without using the mixed-state algorithm failed to provide an interpretable image (not shown). The black scale bar in (a) is $1 \mu \mathrm{m}$.
Ptychography is a form of coherent diffractive imaging in which a specimen is stepped through a localized "probe" consisting of coherent $x$-rays, generating a series of diffraction patterns at the plane of a detector. By stepping the specimen in such a way that the illuminated area at each position overlaps with its neighbors, redundancy is introduced into ptychographic data that can be exploited during the mathematical reconstruction of an image. Although it was known since the late 1960s that this type of data could, in principle, recover an image of a specimen from diffraction data having wavelength-limited resolution, it was many years before relatively fast and robust iterative computational methods were developed. The key task has been to provide a solution to the classical phase-retrieval problem. Longstanding crystallographic phase-retrieval methods are not applicable because the objects being studied are typically not structurally periodic and are much more complicated than crystal lattices.

In this experiment, the researchers from University College London (UK), Brookhaven National Laboratory, Argonne, and the Research Complex at Harwell (UK) imaged a lithographed

"Ptychography" con'td on page 170 


\section{Defining the Thermal Fatigue Limits of GlidCop ${ }^{\circledR}$ High-HeAt-LOAD X-RAy AbSORbers}

$\mathrm{H}$ ard x-ray, third-generation synchrotron facilities have commonly used alu-

minum oxide dispersionstrengthened copper alloy (GlidCop $®$ ) to fabricate $x$-ray absorbers, and the material will likely be used in the design of new $\mathrm{x}$-ray absorbers for the proposed multibend achromat lattice upgrade of the APS and upgrades of other x-ray light source facilities. GlidCop® Xray absorbers are used throughout the APS facility to aperture or completely stop the $\mathrm{x}$-ray beam and therefore are critical to proper operation of the front ends, beamlines, and storage ring. Although the design criteria limits used at the APS for x-ray absorbers have been successful in avoiding component failures over the present life of the facility, the aforementioned potential upgrade of the APS and other facilities may result in higher thermal loads on the $x$-ray absorbers. Efforts are being made to establish less conservative, more realistic design criteria based on the thermomechanically-induced fatigue limits of GlidCop® $[1,2]$.

For these studies, carried out by AES Division personnel, several tasks were performed in parallel and used as the basis for development of a thermal fatigue model for GlidCop $®$. GlidCop $®$ is a proprietary copper alloy, and consequently thermomechanical data and fatigue data available in the open literature are limited. Temperature- dependent true stress versus true strain data were obtained for GlidCop $®$ and were used in all transient non-linear finite element analysis (FEA) simulations. Temperature-dependent uniaxial mechanical fatigue data were also ob-

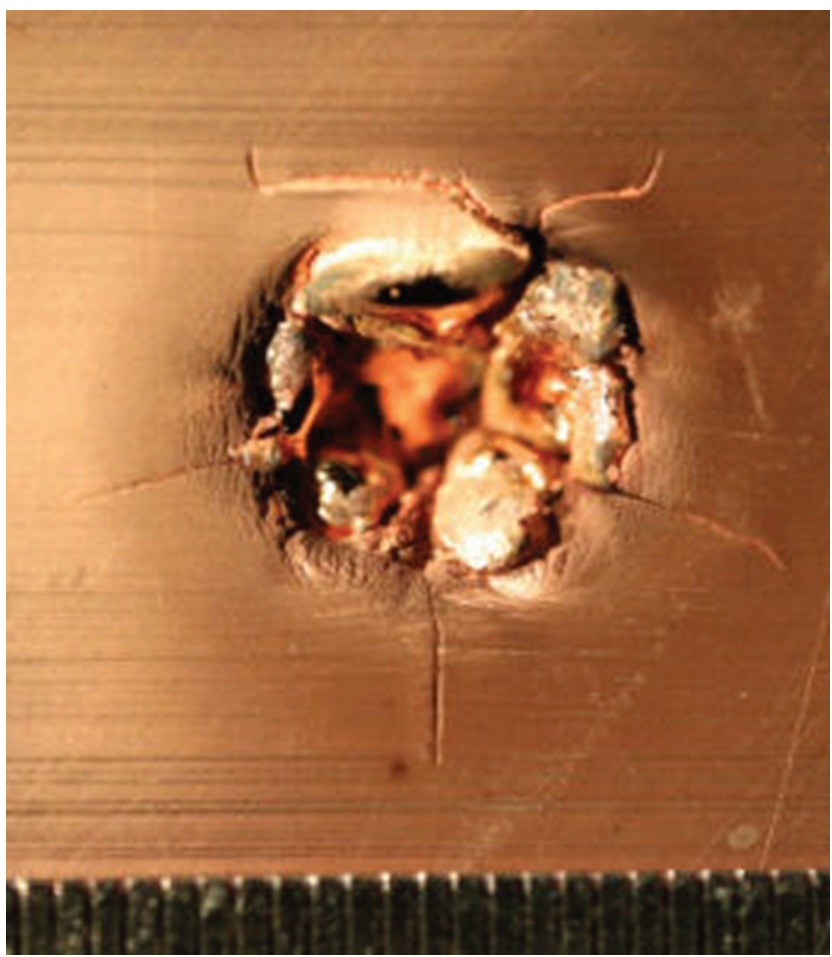

Fig. 1. GlidCop $\AA$ sample tested under worst-case-possible beam conditions ( 1 scale division $=500 \mu \mathrm{m}$ ).

1. Components can be designed with a maximum surface temperature of $375^{\circ} \mathrm{C}$ or to where the cooling water will begin to boil; whichever occurs first will be the limiting criteria

2. Components can be designed with a maximum surface temperature up to $405^{\circ} \mathrm{C}$, the creep temperature for GlidCop(B) AL-15, if transient non-linear analysis is performed to ensure that the number of cycles to failure exceeds 20,000 cycles using the thermal fatigue model below: $\frac{\Delta E_{t}}{2}=\left(.67-\frac{T_{m}}{2000}\right)\left(2 N_{f}\right)^{-.066}+\left(2.0+\frac{3900}{T_{m}}\right)\left(2 N_{f}\right)^{-.48} \quad \begin{aligned} & A z=\text { Total Strain Range (P. } \\ & T_{m}\end{aligned}$ $T_{m}=$ Mean Temp. $(K)=$ average or
$N_{f}=$ Number of Cycles to F allure

3. Components can be designed beyond the boiling point of the water if critical heat flux (CHF) analysis is performed to ensure that a dry-out condition can never be reached.

Note: A surface roughness of Ra $\leq 0.4 \mu \mathrm{m}$ shall be specified for the beam strike surface.

Fig. 2. Proposed new design criteria limits for GlidCop ${ }^{\circledR}$ x-ray absorbers.

tained and were used to develop a mechanical fatigue model.

Using $x$-ray power from the APS, 30 GlidCop® test samples were subjected to 10,000 cycles each of severe thermal loading under various beam power conditions. These samples were metallurgically examined for crack presence/geometry. The mechanical fatigue model was used as a base to develop a thermal fatigue model by matching observed damage with life cycle predictions. This process allowed "failure" to be defined and quantified based on thermal fatigue model predictions and observed damage to the samples.
"Failure" was found to be the start of shallow regions of surface grain drop-out, resulting from surface thermal compression ejecting weakly bound grains, with the possibility of small, shallow cracks less than $2 \mathrm{~mm}$ in surface length. This is consistent with the criteria adopted at the Japanese x-ray light source, SPring-8, based upon Japanese industrial standards [3].

A number of test samples were subjected to beam power conditions far beyond the "failure" limit quantified by the thermal fatigue model. The sample shown in Fig. 1 was tested at nearly six times this limit under the worstcase possible beam conditions using two in-line U33.0 undulators operating at maximum storage ring current with closed gaps. Although the damage is significant, with evidence of surface extrusion, severe radial cracking, melting, and evaporation, the maximum crack length was less than $10 \mathrm{~mm}$ and the maximum crack depth was less than $2 \mathrm{~mm}$. Considering that the minimum cooling wall thickness for any APS x-ray absorber design is $6.35 \mathrm{~mm}$, and modern designs use a 9-mm cooling wall thickness, the "failure" limit quantified by the thermal fatigue model is still quite conservative.

Based on the results of this study and the transient non-linear FEA performed on all of the existing APS frontend x-ray absorbers, new design criteria limits are proposed for GlidCop $\AA$ x-ray absorbers as summarized in Fig. 2. The new design criteria limits allow operation significantly beyond the limits imposed by the existing design criteria limits. Consequently, new x-ray absorber designs for the proposed APS Upgrade could be made more compact, saving on required beamline real estate, and at lower cost than previous

"Absorbers" cont'd on page 170 
"Wave" cont'd from page 167

laser-heated crystal to slice out x-ray photons, but with a different geometry than this team's technique. The European effort is also low efficiency, obtaining just 1 in 10,000 x-ray photons from the original source. These researchers, by contrast, can potentially get $20 \%$ to $30 \%$ of $x$-ray photons from the beam into each flash, which is 2 or 3 orders of magnitude more photons than other techniques.

The laser-heated pressure wave approach worked; they switched $\mathrm{x}$-rays into different, precisely determined angles. The next step is to check how fast the $x$-rays are switching direction by using a streak camera to map x-ray intensity over time.

The decision to use the XSD 7-ID$B, C, D$ beamline was primarily because of familiarity with how the beamline functioned. But the research could have been done at any APS beamline equipped with an ultrafast laser.

Commercial application of the technique could help computer chip manufacturers test chip integrity more quickly and accurately. But the most significant application could be for the APS itself, which will finally be able to accommodate researchers in the biological and chemical sciences who want to observe chemical bonds and biological molecules in action. - Kim Krieger

See: A. Loether ${ }^{1}$, Y. Gao ${ }^{1,2}$, Z. Chen ${ }^{1}$, M.F. DeCamp ${ }^{1}$, E.M. Dufresne ${ }^{2}$, D.A. Walko" , and H. Wen" ${ }^{2 *}$, "Transient crystalline superlattice generated by a photoacoustic transducer," Str. Dynamics 1, 024301 (2014).

DOI: 10.1063/1.4867494]

Author affiliations: ${ }^{1}$ University of Delaware, ${ }^{2}$ Argonne National Laboratory Correspondence: *wen@aps.anl.gov

This work was supported from the DOE-EPSCoR Grant No. DE-FG02-11ER46816 and used resources of the Advanced Photon Source, a U.S. Department of Energy (DOE) Office of Science User Facility operated for the DOE Office of Science by Argonne National Laboratory under Contract No. DEAC02-06CH11357.

7-ID-B,C,D • XSD • Materials science, atomic physics, chemistry $\bullet$ Time-resolved $x$-ray scattering, time-resolved $x$-ray absorption fine structure, phase contrast imaging $\cdot 6-21 \mathrm{keV} \cdot$ On-site $\cdot$ Accepting general users •
"Ptychography" con'td from page 168

1.5- $\mu$ m-thick tungsten Siemens star using coherent 9-keV $x$-rays from the XSD 34-ID-C beamline at the APS that had been passed through horizontal and vertical slits. X-ray diffraction pattern measurements were taken as the sample was moved along a line perpendicular to the x-ray beam.

To examine the effect of sample vibration, the sample was simultaneously vibrated using an analog signal generator. The sine-wave and square-wave vibrations that were introduced had a frequency of vibration of $1 \mathrm{~Hz}$, much faster than the scanning time of $6 \mathrm{sec}$ per position. Vibration amplitudes of $1 \mu \mathrm{m}$ and $1.5 \mu \mathrm{m}$ were employed.

The researchers used a "mixed state" iterative algorithm that allowed for simultaneous reconstruction of Siemens star dynamics (object modes) and the probing $\mathrm{x}$-ray wave fields (probe modes). This approach permitted the researchers to demonstrate experimentally not only how ptychography can be used image a quickly moving sample, but also how the mode shapes and coherence properties of the incident wave field can be manipulated and customized through use of vibration.

Overall image quality was high whether or not the sample was vibrating (Fig. 1). The estimated resolution of $49 \mathrm{~nm}$ was good enough to reveal small defects in the lithographic process used in making the Siemens star. Achieving this resolution with the vibrating sample required simultaneously reconstructing five probe modes along with the sample.

The researchers also studied the effect of vibration on the recovered probe modes, showing that the addition of different types of vibration changes the form of the modes. - Vic Comello

See: Jesse N. Clark ${ }^{1 * \ddagger}$, Xiaojing Huang $^{2}$, Ross J. Harder ${ }^{3}$, and Ian K. Robinson ${ }^{1,4}$, "Dynamic Imaging Using Ptychography," Phys. Rev. Lett. 112, 113901 (2014).

DOI: 10.1103/PhysRevLett.112.113901 Author affiliations: ${ }^{1}$ University College London, ${ }^{2}$ Brookhaven National Laboratory, ${ }^{3}$ Argonne National Laboratory, ${ }^{4}$ Research Complex at Harwell †Present address: SLAC National Accelerator Laboratory

\section{Correspondence:}

* jesclark@stanford.edu

This work was supported by an FP7 advanced grant from the European Research Council. X.H. is supported by the U.S. Department of Energy (DOE) Office of Science, Basic Energy Sciences under Contract No. DE-AC-02-98CH10886. The experimental work was carried out at the Advanced Photon Source Beamline 34-ID-C built with funds from the U.S. National Science Foundation under Grant No. DMR-9724294 and operated by the U.S. Department of Energy Office of Science under Contract No. DEAC02-06CH11357.

34-ID-C • XSD • Materials science, physics • Coherent $x$-ray scattering $\cdot 5-15 \mathrm{keV}, 7-25$ $\mathrm{keV} \cdot$ On-site $\cdot$ Accepting general users

"Absorbers" cont'd from page 169 designs because less material will be required. Details of these studies were recently presented at the Mechanical Engineering Design of Synchrotron Radiation Equipment and Instrumentation (MEDSI 2014) conference in Melbourne, Australia [4].

Contact: Jeff T. Collins (collins@aps.anl.gov), Jeremy Nudell (nudell@aps.anl.gov), Gary Navrotski (navrotski@aps.anl.gov), Zunping Liu (zpliu@anl.gov), Patric Den Hartog (denharto@aps.anl.gov)

\section{REFERENCES}

[1] V. Ravindranath, S. Sharma, B. Rusthoven, M. Gosz, L. Zhang, and J. Biasci (2006), Proceedings of the International Workshop on Mechanical Engineering Design of Synchrotron Radiation Equipment and Instrumentation 2006, Himeji, Hyogo, Japan.

[2] S. Takahashi, T. Mochizuki, M. Sano, A. Watanabe, and $\mathrm{H}$. Kitamura, "Fatigue life prediction for high-heat-load components made of GlidCop $₫$ by elastic-plastic analysis," J. Synchrotron Rad. 15, 144 (2008).

[3] JIS Z2279 (1992). Japanese Industrial Standard. Method of high-temperature lowcycle testing for metallic materials.

[4] J. Collins, J. Nudell, G. Navrotski, Z. Liu, and P. Den Hartog (2014), "Results from studies of thermomechanically-induced fatigue in GlidCop $®$," Proceedings of the $\mathrm{Me}$ chanical Engineering Design of Synchrotron Radiation Equipment and Instrumentation 2014 Conference, October 20-24, 2014, Melbourne, Australia.

This research was supported by the U.S. Department of Energy Office of Science under Contract No. DE-AC02-06CH11357. 


\section{SONICC AT GM/CA-XSD}

$\mathrm{S}$ econd-order nonlinear optical imaging of chiral crystals (SONICC) based on femtosecond laser scanning microscopy has been implemented at the GM/CA-XSD undulator beamline 23-ID-B for rapid protein crystal localization and centering.

The technique is based on infrared laser light impinging on non-centrosymmetric crystals of proteins, which may selectively yield a frequency-doubled, visible signal generated by the anharmonic response of the electron cloud of the protein in response to the laser field. One aim of this method is to locate small crystals grown in opaque crystallization media for centering in $\mathrm{x}$ ray beams of only a few microns or less in cross-section.

The optical system implemented at the beamline includes "trans" and "epi" detection of second harmonic generation (SHG) signals. In addition, scanning visible laser light across the sample and detecting two-photon excited UV fluorescence (TPE-UVF) provides complementary contrast based on the native fluorescence of proteins [3].

SONICC provides the opportunity to detect crystals of $<1 \mu \mathrm{m}$ on an edge even in opaque media, such as with membrane protein crystals grown in lipidic cubic phase (LCP). A collaboration between the group of Garth Simpson at Purdue University and GM/CAXSD, IMCA-CAT, and SBC-CAT previously showed no evidence of damage to protein crystals due to a SONICC laser [1]. A SONICC system is installed at beamline 23-ID-B [2], and efforts are under way to transition to SONICC and TPE-UVF as user capabilities, including implementation of laser-safety interlocks for the experimental hutch, and user-friendly software updates.

SHG signals vary greatly between different protein crystals, depending on the space group, intrinsic protein signal, and crystal size [4]. To date, testing with various G-protein-coupled receptors in the lipidic cubic phase has been successful, but not all crystal systems will necessarily show a readily-detectable signal. Systems that are favorable to SHG signals include lower-sym-

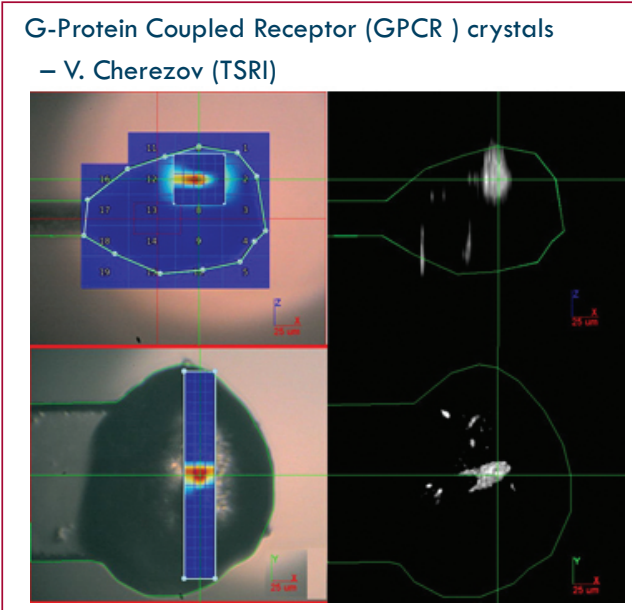

Intimin crystals - N. Noinaj, S. Buchanan (NIH)

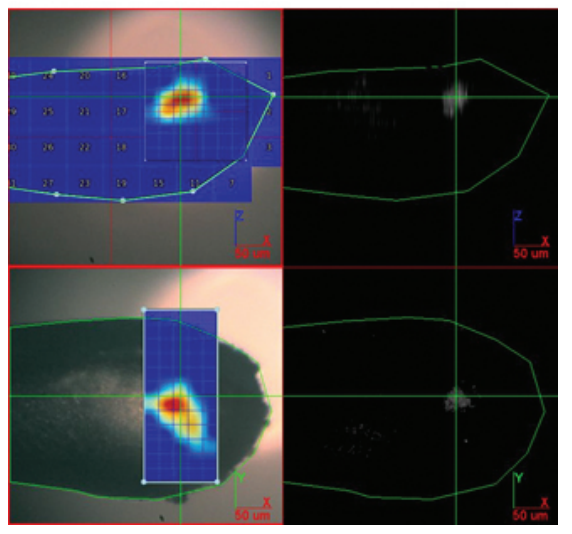

Left in both panels: Orthogonal views of smoothed, corrected Bragg candidates from diffraction raster in JBlulce. Right in both panels: Corresponding orthogonal views of SONICC images. Due to the limited depth of the focal plane for SHG, SONICC images must be taken at several depths in the sample. The top SONICC images show a sum of SHG signals acquired by rapidly translating the sample in 15- $\mu \mathrm{m}$ steps through the laser focal plane. The bottom SONICC image shows the SHG signal from one plane in the sample. Time for $x$-ray raster data collection was $\sim 5$ min., compared to $\sim 2 \mathrm{~min}$. for SONICC imaging.

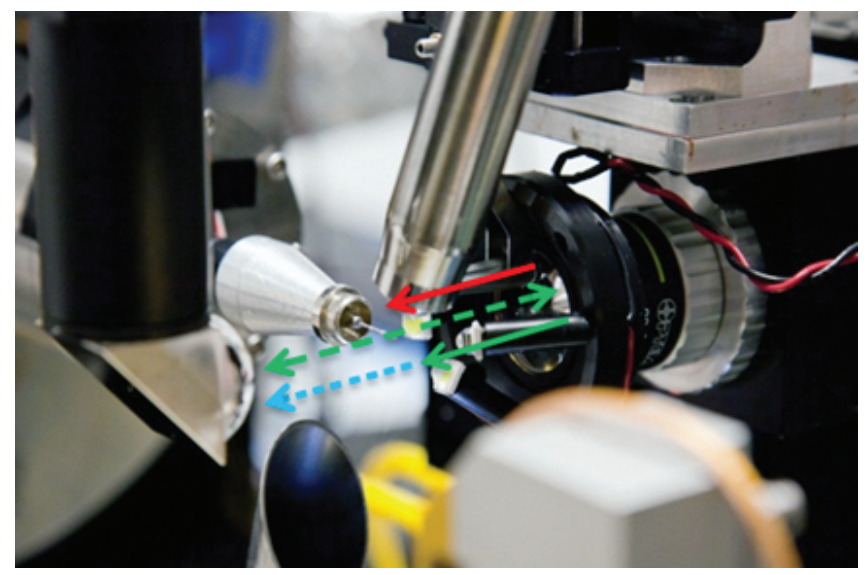

Close-up view of the upstream Nonlinear Optical Microscope in the SONICC system installed at beamline 23-ID-B, with solid arrows representing incident laser propagation (red: 1064 nm; green: 532 nm), and dashed arrows representing the measured signal (green: SHG at $532 \mathrm{~nm}$, blue: TPE-UVF).

metry space groups and low- background sample mounts, such as Hampton loops or MiTeGen UV-Vis loops. Examples of systems that are challenging for SONICC include: Examples of systems that are challenging for SONICC include high-symmetry space groups, prior exposure of samples to x-rays that generate spurious SHG signals [5], ice on a sample that scatters laser light, very thick samples that scatter laser light, and some salt crystals that give spurious large signals. If working with opaque media and unfamiliar with crystal properties, an initial x-ray diffractionraster control experiment can be useful for ascertaining the sensitivity of the system. Contact: Michael Becker mbecker@anl.gov

\section{REFERENCES}

[1] D.J. Kissick, M. Becker, A.M Mulichak, V. Cherezov, S.L. Ginell, K.P. Battaile, L.J. Keefe, R.F. Fischetti, and G.J. Simpson, Acta. Cryst. D 69, 843 (2013).

[2] J.T. Madden, S.J. Toth, C.M. Dettmar, J.A. Newman, R.A. Oglesbee, H.G. Hedderich, R.M. Everly, M. Becker, J.A. Ronau, V. Cherezov, M.E. Morrow, S. Xu, D. Ferguson, O. Makarov, C. Das, R. Fischetti, and G.J. Simpson, J. Synchrotron Rad. 20 , 531 (2013).

[3] J.T. Madden, E.L. DeWalt, and G.J. Simpson, Acta. Cryst. D 67, 839 (2011).

[4] L.M. Haupert, E.L. DeWalt, and G.J. Simpson, Acta. Cryst. D 68, 1513 (2012).

[5] C.M. Dettmar, J.A. Newman, S.J. Toth, M. Becker, R.F. Fischetti, and G.J. Simpson, Proc. Natl. Acad. Sci. 112(3), 696 (January 20, 2015)

GM/CA-XSD has been funded in whole or in part with Federal funds from the National Cancer Institute (ACB-12002) and the National Institute of General Medical Sciences (AGM-12006). 


\section{High-Performance Computing Software to ImProve Beamline AND ACCelerator EfFiciency}
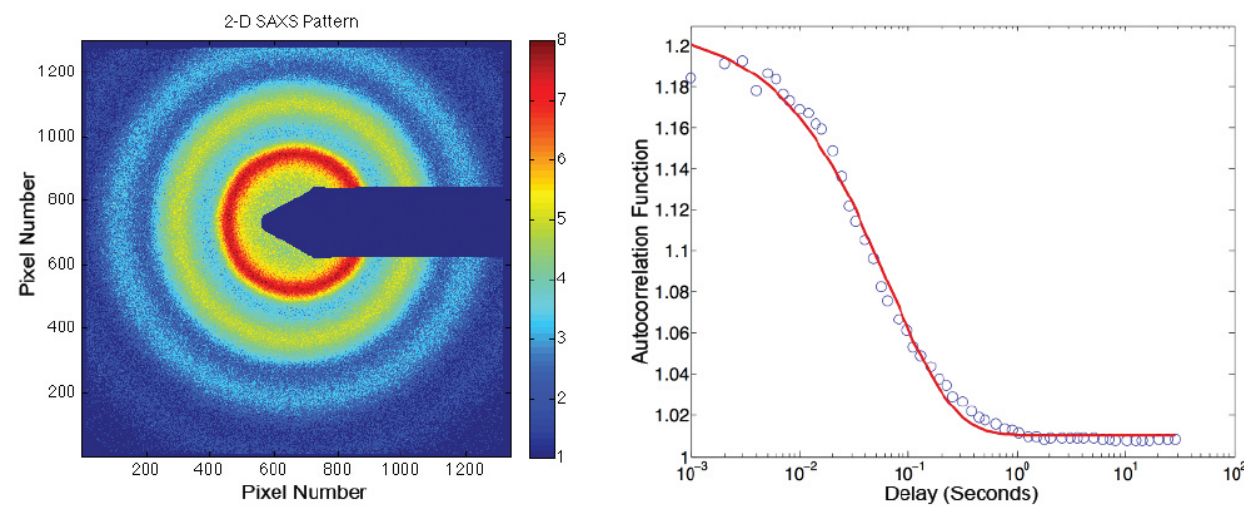

Fig. 1. Left: Small-angle x-ray scattering pattern (plotted on a logarithmic scale) from a concentrated colloidal suspension of silica spheres dispersed in ethylene glycol. The strong structure factor peak due to the spatial correlation of the colloids along with the form factor of the colloids is clearly seen. Right: Time autocorrelation function measured from a colloidal suspension in polymer. The correlation function measured over nearly five decades in time was computed using the HPC software in near real-time.

$\mathrm{S}$ oftware has become ubiquitous in the operation of synchrotron facilities such as the APS.

Through both improvements in existing software applications and the development of new software applications, the APS can realize efficiencies on par with conventional infrastructure improvements.

The AES Software Services Group (AES-SSG) is engaging the power of high-performance computing (HPC) by developing scientific software applications that have helped improve the efficiency of both APS beamlines and the APS accelerator.

\section{Big-Data Analysis for 8-ID-I}

Low latency between data acquisition and analysis is of critical importance to any experiment. The combination of a faster parallel algorithm and a data pipeline for connecting disparate components (detectors, computing resources, file formats) enables us to greatly enhance the operational efficiency of the x-ray photon correlation spectroscopy (XPCS) experiment facility at the APS (Fig. 1).

The workflow starts with raw data (120 MB/s) streaming directly from a detector, through an on-the-fly firmware discriminator, to the Hadoop Distributed File System (HDFS) in a structured HDF5 file. The user then triggers the
MapReduce-based parallel analysis on the 96-core APS computing cluster. Provenance information and reduced results are added to the original HDF5 file. Finally, the data pipeline triggers software for visualizing the data (Fig. 2).

The whole process is completed shortly after data acquisition — a significant improvement over the previous setup. The faster turnaround time helps scientists make near-real-time adjustments to their experiments. The system is in production use for almost $100 \%$ of users at the XSD 8-ID-I beamline. It performs approximately 50 times faster than a serial implementation.

\section{Experiment Planning for Microscopy}

Appropriate experiment planning is also of importance to APS experiments. uProbeX is a software tool that allows users and beamline staff to more easily plan and perform x-ray microscopy experiments on micro- and nano- fluorescence $\mathrm{x}$-ray microscopes at the APS (Fig. 3).

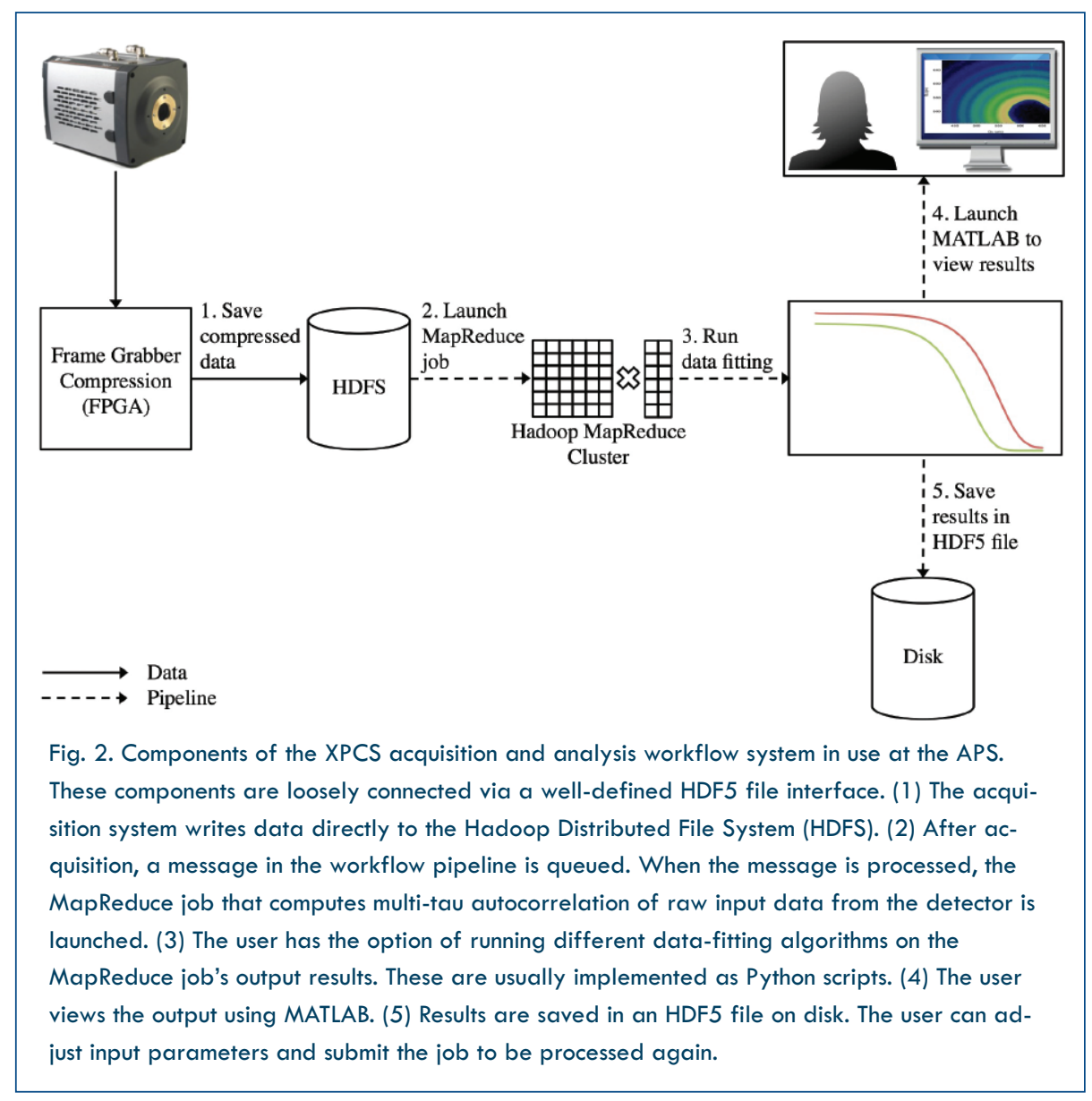


First, an overview image is collected on a light microscope as a mosaic of smaller images. uProbeX reads this mosaic and displays the image along with the coordinates on the light microscope. To calibrate the system, users select calibration points on a known visible light image, and enter corresponding $\mathrm{x}$-ray microscope coordinates. Users can then draw regions over which to perform x-ray microscope scans.

This open-source tool is used regularly at the XSD 2-ID-B, 2-ID-D, and 2ID-E fluorescence beamlines, and saves users $10 \%$ to $20 \%$ of their beam time in the case of conventional experiments, and up to $30 \%$ for more challenging experiments.

\section{Time-Correlated}

\section{Data Acquisition System}

In order to support the commissioning of the multi-bend achromat lattice (MBA) for the proposed APS Upgrade, current accelerator machine studies, and ongoing high availability of x-rays from APS accelerator operations, a system-wide data acquisition system is being developed and deployed that can provide continuous performance monitoring, postmortem fault analysis, and abnormal condition detection.

The system captures synchronously sampled, time-correlated data from several critical subsystems, such as turn-by-turn beam position monitor values, storage ring radio-frequency (rf) cavity field measurements, fast injection kickers, beam diagnostics, and power supply readbacks. Data is acquired from multiple subsystems and correlated within 3.6 $\mu$ s (turn-by-turn rate). This ensures scalability so that additional components may be easily added for monitoring by using commodity software message queue systems to aggregate data from sources and redistribute data to interested client applications.

Currently, two Data Acquisition "front-ends" are deployed that capture more than 2500 signals from the APS real-time feedback system and the storage ring if cavities. These have been used for studying alternative feedback algorithms for use by the APS Upgrade. The system allows continuous data capture for long periods of time at the
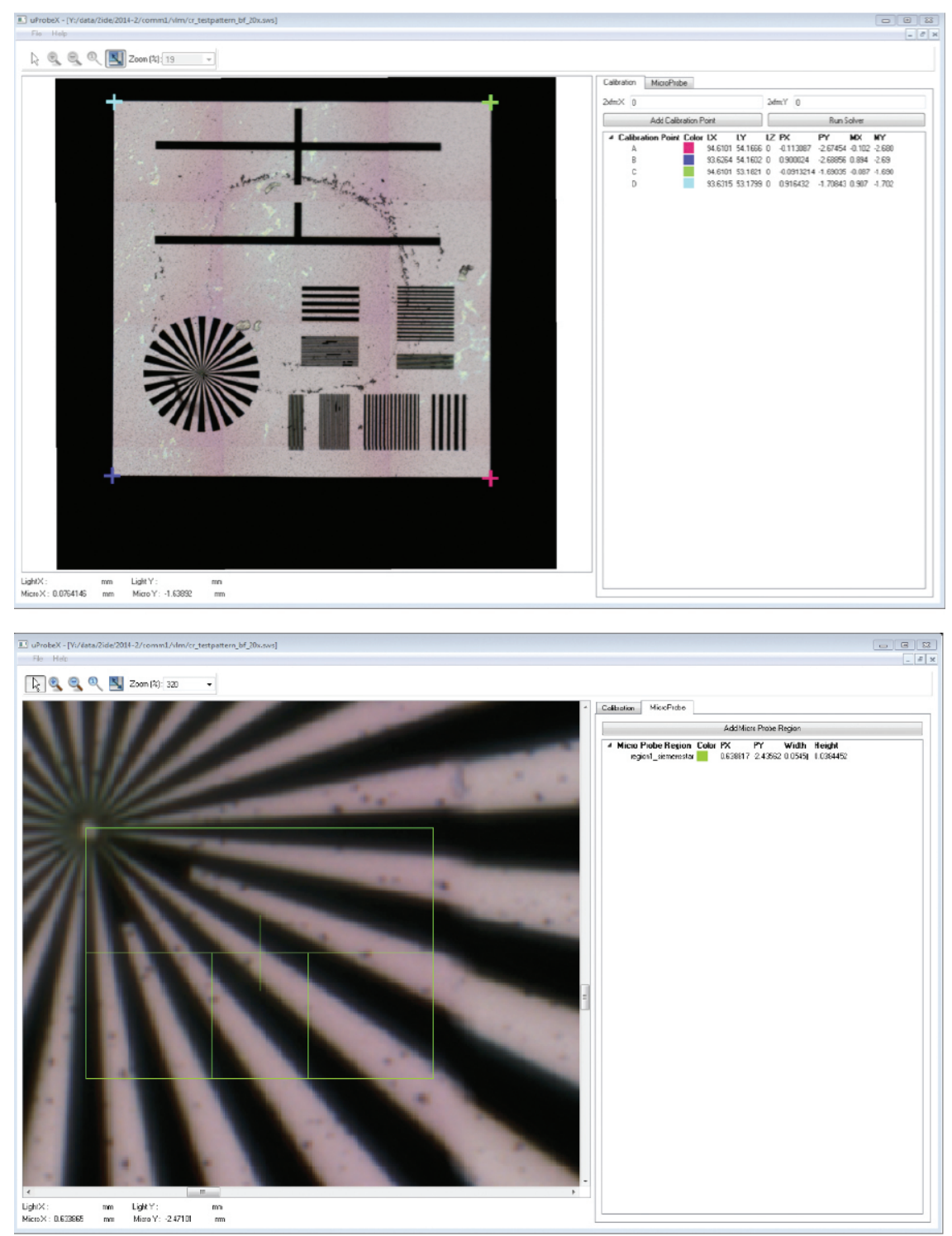

Fig. 3. Top: uProbeX in calibration mode. Four calibration points are selected by the user on a light-microscopy image, one at each corner of the calibrant sample. Positions of the points are imported from the light microscope. Bottom: uProbeX in scan mode. A region box, over which a scan is taken on the x-ray microscope, is drawn by the user.

full sample rate, a capability not previously available, giving researchers an understanding of accelerator performance over long periods of time.

Contact: Nicholas Schwarz

(nschwarz@aps.anl.gov), Ned Arnold

(nda@aps.anl.gov), Arthur Glowacki (aglowacki@aps.anl.gov), Sinisa Veseli

(sveseli@aps.anl.gov), Ke Yue

(kyue@aps.anl.gov)

The XPCS computing system was developed, and is supported and maintained by AES-SSG in collaboration with Suresh Narayanan (XSD Time Resolved Research Group) and Tim Madden (XSD Detectors Group) with funding from the U.S. Department of Energy (DOE) Office of Science under Contract No. DE-AC02-06CH11357.
The APS Information Technology Group maintains the high-performance computing environment used by the software. uProbeX was developed, and is supported and maintained by Arthur Glowacki and Ke Yue (AESSSG) in collaboration with Stefan Vogt, David Vine, and Sophie Charlotte Gleber (XSD Microscopy Group) with funding from the U.S. DOE Office of Science under Contract No. DE-AC02-06CH11357. The Timecorrelated Data Acquisition System was developed by Sinisa Veseli (AES-SSG) and Ned Arnold (AES Technical Support Services Group) in collaboration with John Carwardine (APS-U), Nick Sereno (ASD Diagnostics Group), and Vadim Sajaev (ASD Accelerator Operations and Physics Group) with funding from the U.S. DOE Office of Science under Contract No. DE-AC02-06CH11. 


\section{HYBRID: \\ Simulating Partially Coherent X-Ray Wavefronts Down Non-IDeal Beamline Components}

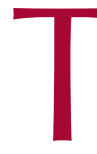
he future advent of new storage rings with ultra-low-emittance [1], which includes the proposed multi-bend achromat upgrade of the APS, will enable new fields of research as well as capabilities not feasible with existing light sources. These new capabilities will require beamlines that can preserve the $\mathrm{x}$-ray beam coherence. This in turn demands efficient tools capable of simulating the expected beamline performance including the effects of diffraction and imperfections on the optical elements so that experiment designers have near-realworld data from which to work. For this purpose, we have developed HYBRID $[2,3]$, a very fast simulation tool to evaluate beamline performance and determine the specifications required from the optical elements. HYBRID can simulate the performance of beamlines when illuminated with coherent, partially coherent, and incoherent x-rays.

HYBRID simulates the geometric effects of the optical components (focusing, defocusing, or collimating) and apertures (losses) by means of raytracing, where their diffraction contributions due to mirror sizes or apertures are calculated independently for each element using wavefront propagation. The results from the diffraction contribution of an element are integrated into the ray-tracing results by numerical convolution and ray re-sampling. An entire beamline can be simulated by iteratively applying this procedure. The HYBRID code uses SHADOW [4] as the ray-tracing program thanks to its popularity in the synchrotron radiation community. The wavefront propagation part uses the fast Fourier transformbased algorithm for its calculation speed. The HYBRID code is incorporated in the SHADOWVUI distribution $[3,4]$.

HYBRID was benchmarked against the multi-electron SRW "HYBRID" cont'd on page 177

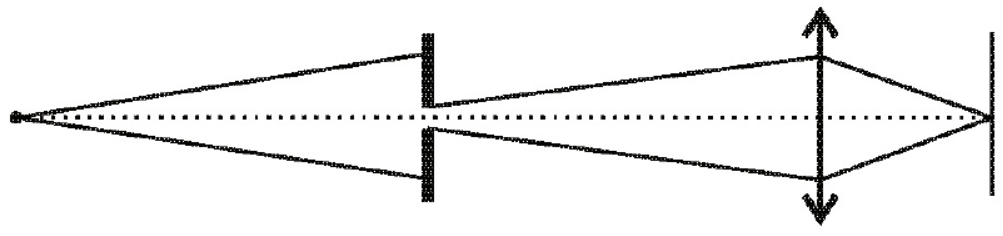

$\begin{array}{lll}\text { Source } & \text { BDA } & \text { HKB }\end{array}$

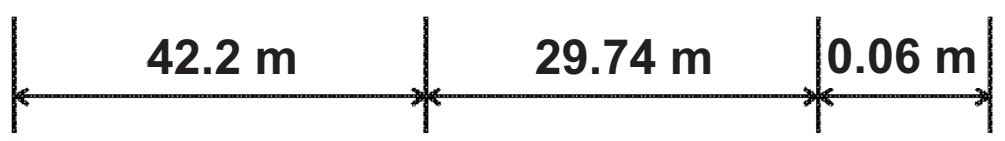

Fig 1. Schematic layout of the optical design of the ISN beamline in the horizontal plane.

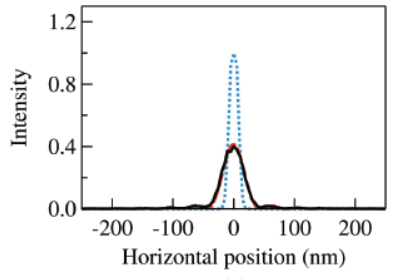

(a)

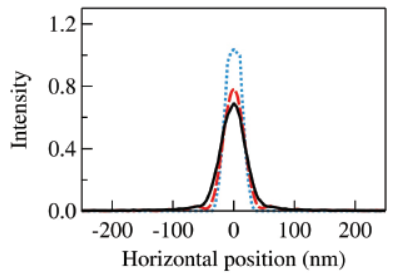

(c)
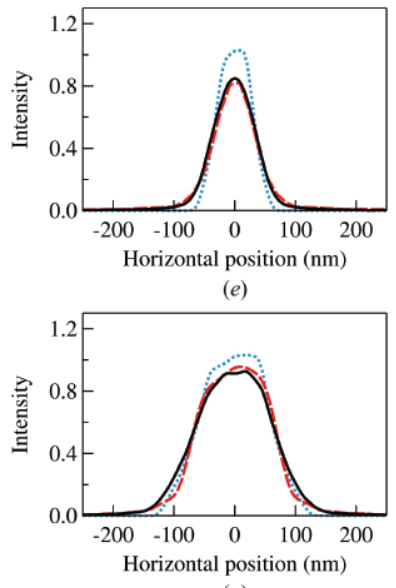

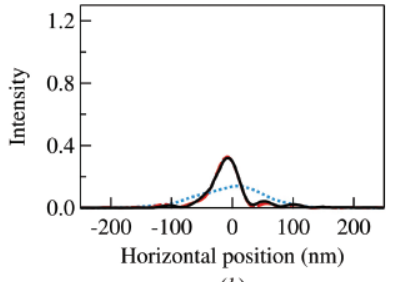

(b)

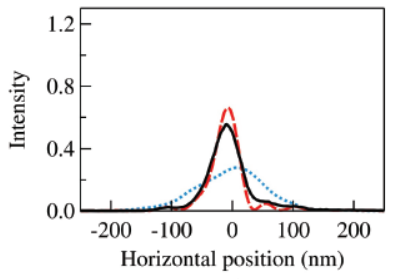

$(d)$
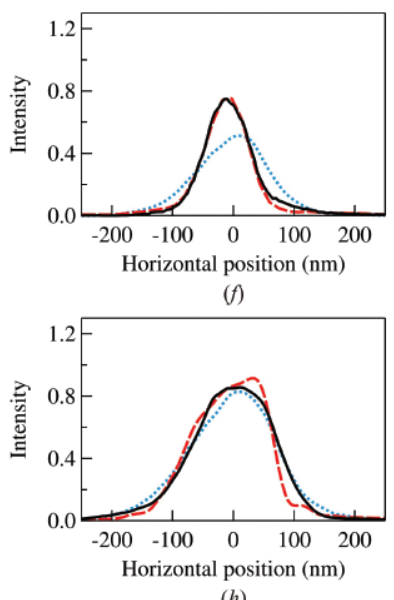

Fig. 2. Horizontal intensity profiles of the ISN beamline at the focal plane simulated using the Hybrid method (black solid lines), the multi-electron SRW (red dashed lines), and the ray-tracing by SHADOW (blue dotted lines) with different BDA sizes of $(a, b)$ $10.5 \mu \mathrm{m},(c, d) 21 \mu \mathrm{m},(e, f) 42 \mu \mathrm{m}$, and $(g, h) 84 \mu \mathrm{m}$. The traces at the left (right) were calculated for an ideal (with figure errors) elliptical cylinder mirror. 


\section{SEEING THE LIGHT AT 29-ID \\ with a New Transition-Edge Superconducting Detector}

$\mathrm{A}$ new transition-edge superconducting (TES) detector designed to overcome the challenges associated with measurements of collective electron behavior in correlated electron systems has been developed for the XSD Intermediate Energy X-ray (IEX) beamline 29-ID of the APS. The new detector was designed and built by the Quantum Devices Group at the National Institute of Standards and Technology (NIST) at Boulder, CO, for the IEX beamline and in collaboration with Peter Abbamonte, of the University of Illinois at UrbanaChampaign, and at the time of writing is being characterized as part of the IEX commissioning activities.

Electrons near the Fermi level determine the macroscopic properties of a material. These include electronic properties (is the material a metal, insulator, semiconductor, or superconductor); thermal properties; magnetism; and even physical properties such as color. Understanding the nature of these electrons, particularly their collective behavior, not only gives us a deeper understanding of the physics that govern the world around us but enables us to design materials with very specific properties for any number of applications.

One way to investigate these interactions is by utilizing $x$-rays via resonant soft $x$-ray scattering (RSXS,

Fig. 1), which marries $x$-ray spectroscopy and $\mathrm{x}$-ray diffraction and provides a direct probe of the real-space ordering of charge, spin, and orbital degrees of freedom carried by electrons near the Fermi level. This is done by tuning the incident $x$-ray beam to a specific absorption edge, exciting a corelevel electron into the conduction band, and then measuring the emitted $x$-rays that are produced as the system decays back into the ground state. By scanning the photon energy and polarization, we are able to probe the electronic structure with not only elemental specificity, but (utilizing the dipole selection rules) directly scatter from collective charge, orbital and spin ordered states. Traditionally, these $x$-rays are measured with

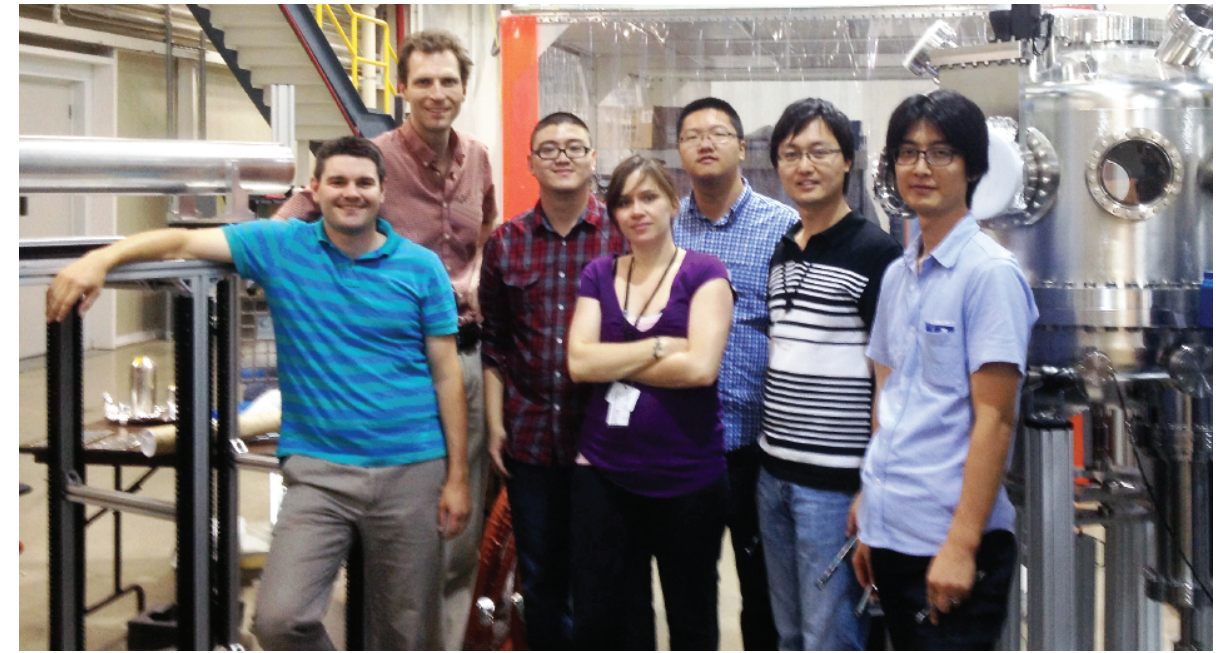

Left to right: Daniel Swetz, William Randy Doriese, Yifeng Wu, Fanny Rodolakis, Yifei Li, Hongping Yan, and Hideyuki Tatsun of NIST during installation of the TES detector. Photo: Jessica McChesney

non-energy-resolving detectors such as a photodiode or an area detector like a chargecoupled device. Even though there is a significant signal enhancement at resonance, this signal can sit on a broad fluorescence background.

In order to overcome these challenges, the new detector utilizes microcalorimetry to measure the energy of the emitted photon with $\mathrm{eV}$ resolution, by measuring the change in temperature of the detector. The quantum efficiency of the TES detector is ten times better than that of soft $x$-ray grating spectrometers. Each of the

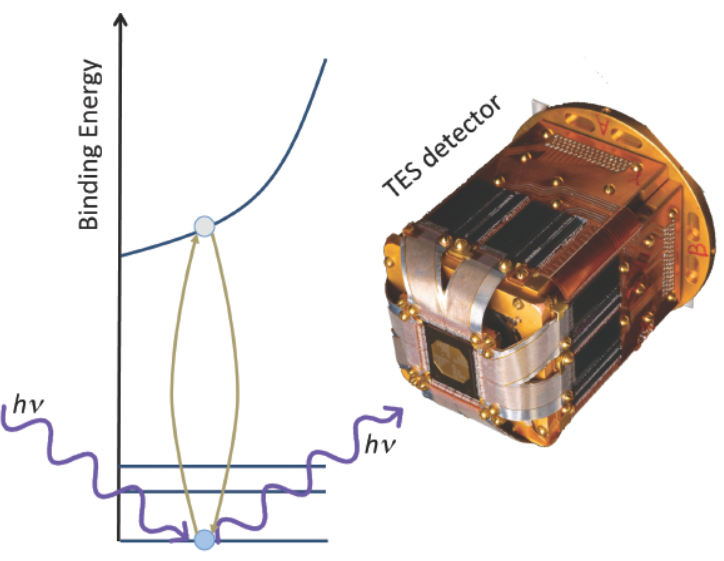

Fig. 1. In resonant soft $x$-ray scattering, an electron is photoexcited from a core level into the conduction band. Using the transition-edge sensor detector being developed at 29-ID, the photon emitted as the system decay back into the ground state ca be measured with $\sim 1 \mathrm{eV}$ resolution.

detector's 240, 105- $\mu \mathrm{m} \times \mathrm{85}$ $\mu \mathrm{m}$ pixels is made of a superconducting thin film (a 300-nm-thick copper/molybdenum bilayer) cooled to $100 \mathrm{mK}$ and held in its superconducting-to-normal-metal transition via a voltage bias. A 2- $\mu$ m-thick bismuth film on each pixel serves as an absorber and ensures high quantum efficiency. The energy of an incoming $x$-ray is converted entirely to heat in the absorber, causing a small increase in the temperature of the film but (due to the sharp superconducting phase transition) also causing a large increase in the film's resistance. The resulting pulsed de- crease in the film current is measured by a superconducting quantum interference device (SQUID) ammeter; the SQUID readout is multiplexed into only eight output amplifier channels. Each detector pixel can receive several hundred x-ray counts per second, and achieve an energy resolution of about 1 $\mathrm{eV}$ in the sub-keV energy range of interest.

As an imaging detector with energy resolution that was previously available only in a grating spectrometer, the TES-array detector represents a new "29-ID" cont'd on page 177 


\section{TAMING THE TSUNAMI: A New Approach for Tomographic Data}

\section{T} o gather data about the universe they study, scientists need tools to see in ways that go beyond the powers of human sight. The crude optical microscopes and x-ray tubes of the past could see only dimly and imperfectly compared to the modern instruments that scientists use to probe inorganic and organic matter from its outside veneer down to the level of individual atoms. The current generation of x-ray synchrotron facilities boasts a dazzling array of instruments, detectors, and techniques of exquisite sensitivity and capacities, and the next generation promises to increase those capabilities by orders of magnitude.

It all sounds like an indisputably positive state of affairs, but ironically, there is a down side. As our scientific tools become ever more sensitive and powerful, able to gather ever increasing amounts of raw data, the need to process and analyze all that data also increases exponentially. The ubiquitous digitization of scientific data does not help the problem: while digitization makes it easier than ever to collect and store information, it has also led to proliferation of different and not always compatible software and computing resources among different research facilities. As new synchrotron facilities come online, this dilemma will only increase.

To tame this ever-growing tsunami of data, a team of researchers at the APS is developing a collaborative framework for the analysis, processing, and reconstruction of tomographic datasets from diverse synchrotron sources. Based on the Python programming language, the TomoPy platform is intended to provide a common means for researchers to share, integrate, and use data collected at different facilities.

Along with the growing number of synchrotron $x$-ray sources inevitably comes a somewhat greater diversity in methods and techniques. Facilities naturally tend to rely upon and use data analysis tools and software they have developed with an eye to the specific
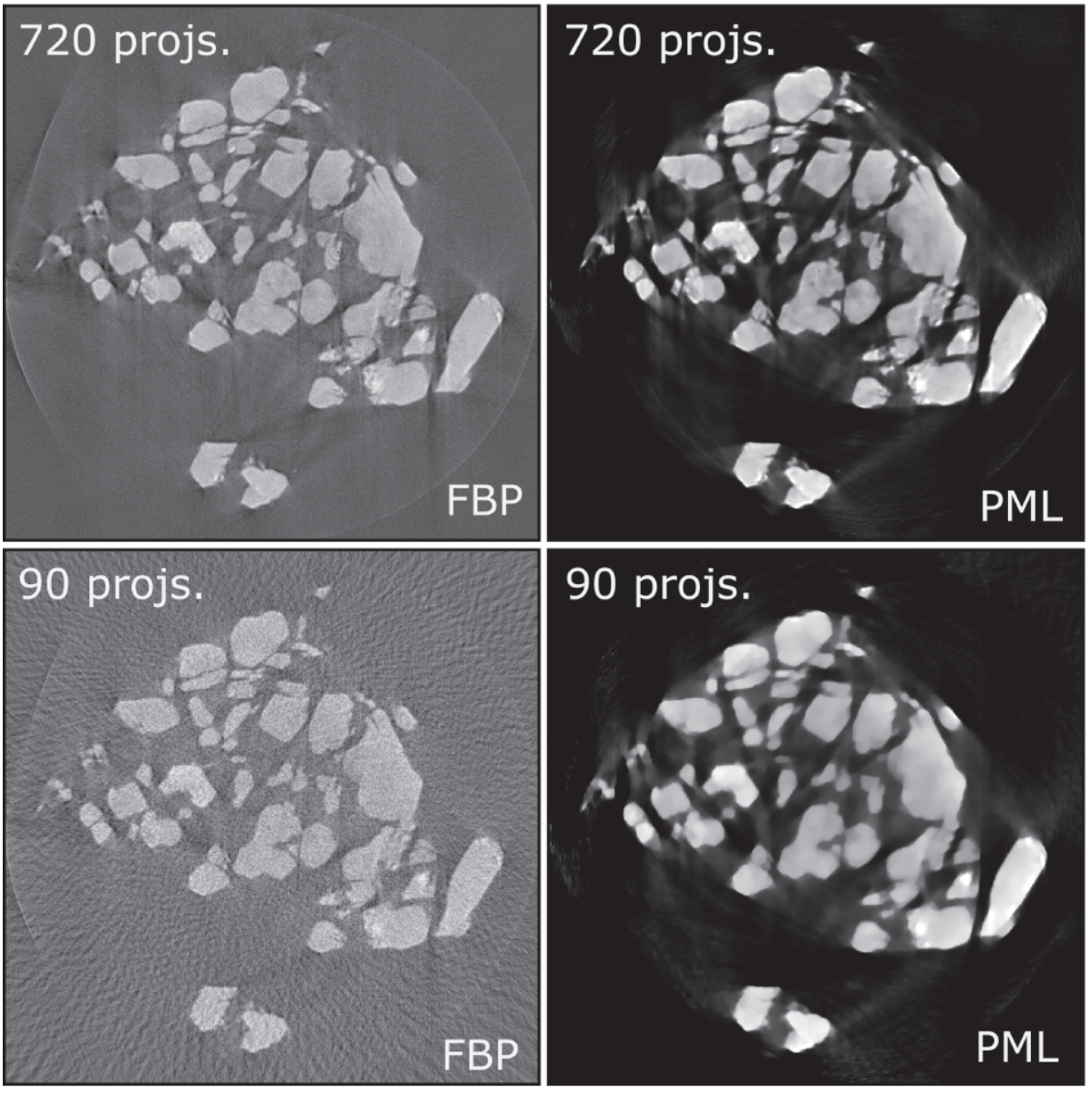

Fig. 1. Comparison of filtered back-projection (FBP) and penalized maximum likelihood (PML) reconstructions of a cathode electrode sample using 720 and 90 projections.

characteristics and capabilities of their own instruments. But these may not be quite fully compatible with software used at other locations when attempting to share or integrate datasets and results. There is also a growing need for the flexibility of doing data processing either in real time at the synchrotron site or at other times at off-site locales. TomoPy addresses all of these issues and others by utilizing a modular approach that manages to be both general enough to work for various sites and flexible enough to be adaptable for local site preferences and needs.

The team demonstrated the utility of the TomoPy model by showing how the process of tomographic data analysis can be divided into a series of sequential steps, grouping or "modularizing" them into a chain of related major tasks that can be branched off into sub- tasks specific to particular tomographic types, such as $\mathrm{x}$-ray transmission tomography, $x$-ray fluorescence microscopy, or x-ray diffraction tomography. As an example, the researchers examine how $x$-ray transmission data passes through the TomoPy framework from acquisition to final image display.

The first main module in the TomoPy model is pre-processing, involving matters such as data normalization of raw data (e.g., dark-field and whitefield adjustments, artifact removal, compensating for insufficient field of view). Next comes reconstruction, in which the raw corrected data is mapped into image space. TomoPy uses the Fourier-based Gridrec method by default for this task, chiefly for its computational speed. However, the TomoPy reconstruction module can also

"Tsunami" cont'd on page 177 
"HYBRID" cont'd from page 174 (Synchrotron Radiation Workshop) [5] and ray-tracing using the technical specifications for the In Situ Nanoprobe (ISN) [6] beamline, which was designed with the current APS parameters as one part of a proposed APS beamline upgrade. The modeled comparisons were carried out in the horizontal direction since the beam can be tuned from diffraction limited (fully coherent) to partially coherent by adjusting the size of an aperture (BDA). The design source of this beamline is a $2.4-\mathrm{m}$-long APS undulator $A$ with a period of $3.3 \mathrm{~cm}$ tuned to the first harmonic energy at 10 $\mathrm{keV}$. The total horizontal beam size and divergence used in the ray-tracing are $\Sigma_{x}=274 \mu \mathrm{m}$ and $\Sigma^{\prime}{ }_{x}=12.4 \mu \mathrm{rad}$. A schematic layout of the In Situ Nanoprobe beamline along the horizontal direction is shown in Fig. 1. The horizontal Kirkpatric-Baez mirror (HKB), which images the BDA at the sample position, is a $60-\mathrm{mm}$-long elliptical cylinder with a 2.5-mrad grazing angle. A r.m.s. figure error of $2.3 \mathrm{~nm}$ was assumed in the calculations.

Figure 2 presents the simulation results of the horizontal spot size at the focal plane using HYBRID (black solid lines), the multi-electron SRW (red dashed lines), and the SHADOW raytracing (blue dotted lines) with different BDA sizes. The figures on the left are without (and the figures on the right, with) the assumed mirror figure errors. When the size of the BDA is limited to $10.5 \mu \mathrm{m}$ [cf. Fig. 2(a,b)], the HKB is coherently illuminated and the beam size is dominated by diffraction. A very good agreement is found in this case between HYBRID and SRW. The ray-tracing calculation fails since it excludes the diffraction from both the finite size of the BDA and the acceptance of the HKB. In the partially coherent cases, BDA 21 and $42 \mu \mathrm{m}$ [cf. Fig. 2(c-f)] there is a satisfactory agreement between HYBRID and the SRW results. The SHADOW result is again deviating largely from the other two methods. For a BDA of $84 \mu \mathrm{m}$ [cf. Fig. 2( $g, h)$ ], the beam can be considered completely incoherent and in the geometrical optics regime. HYBRID and multi-electron SRW results are alike and are very similar to the SHADOW results.

The main advantage of HYBRID is its speed, making it an excellent tool for beamline design and optimization. Further improvement of HYBRID will include the implementation of better models to represent the source and its coherence properties.

Contact: Xianbo Shi (xshi@aps.anl.gov), Ruben Reininger (rreininger@aps.anl.gov)

\section{REFERENCES}

1. See Synchrotron Radiation News 27 (6) (2014).

2. X. Shi, R. Reininger, M. Sanchez del Rio, and L. Assoufid, J. Synchrotron Rad. 21 , 669 (2014).

3. X. Shi, M. Sanchez del Rio, and R. Reininger, Proc. SPIE, 9209, 920911 (2014).

4. http://www.esrf.eu/home/Instrumentation/software/data-analysis/xop2.4/shadowvui.html

5. N. Canestrari, O. Chubar, and R. Reininger, J. Synchrotron Rad. 21, 1110 (2014).

6. J. Maser et al., Metall. Mater. Trans. A 45, 85 (2014).

This research was supported by the U.S. Department of Energy Office of Science under Contract No. DE-AC02-06CH11357.

“29-ID" cont'd from page 175 paradigm in RSXS measurements. Not only will users be able to probe the electronic structure with elemental specificity and directly couple to specific orbitals or spin states via the dipole selection rules as with traditional RSXS measurements, but they can further discriminate resonant, elastic emission from the inelastic background, revealing electronic ordering in complex materials even when only a small fraction of the valence electrons are involved.

Contact: Jessica McChesney (jmcchesn@aps.anl.gov),

Peter Abbamonte (abbamont@illinois.edu),

Daniel Swetz (daniel.swetz@nist.gov)

This research used resources of the Advanced Photon Source, a U.S. Department of Energy (DOE) Office of Science User Facility operated for the DOE Office of Science by Argonne National Laboratory under Contract No. DE-AC02-06CH11357 and has been supported, in part, by DOE grant \# DEFG02-06ER46285 through the University of Illinois at Urbana-Champaign.
"Tsunami" cont'd from page 176 employ iterative model-based inversion techniques, which can be useful particularly in cases where few projections are available or the data is of low signal-to-noise ratio. The final post-processing module of TomoPy allows for whatever further processing or imaging steps may be needed or desired, including region segmentation or quantitative analysis of the images (Fig. 1).

The modular strategy of TomoPy allows processing methods that are common across different tomographic techniques to be efficiently shared and executed concurrently and in parallel by multiple processor cores, saving time and using available computer resources to their best capabilities. The TomoPy framework is open-source and designed to be platform- and data-format independent, and while presently implemented for CPU and GPU computing, can easily be integrated with large scale computing facilities.

As our scientific instruments continue to become ever more powerful, the TomoPy framework is intended to unify and streamline the analysis and sharing of the harvest of data they provide. By taking advantage of faster and more efficient computing technology, increasingly sophisticated software, and the collaboration of physicists and mathematicians to create new mathematical methods, the designers of TomoPy hope to enhance the ability of scientists to use their ever-improving tools to their ultimate capacities.

- Mark Wolverton

See: Doğa Gürsoy*, Francesco De Carlo, Xianghui Xiao, and Chris Jacobsen, "TomoPy: a framework for the analysis of synchrotron tomographic data," J. Synchrotron Rad. 21, 1188 (2014).

DOI: $10.1107 / S 1600577514013939$

Author affiliation:

Argonne National Laboratory Correspondence: * dgursoy@aps.anl.gov

This work is supported by the U.S. Department of Energy Office of Science under Contract No. DE-AC02-06CH11357. 


\section{State-OF-the-Art MX Detectors at the APS}

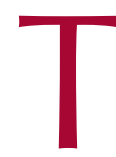
hree macromolecular crystallography (MX) beamlines at the APS have recently implemented new state-of-the art detectors.

At the GM/CA-XSD beamline 23-ID-D, a Pilatus3 6M with a high-efficiency, 1000- $\mu \mathrm{m}$ thick sensor (DECTRIS Ltd., Baden, Switzerland) was installed in January 2014.

At SER-CAT beamline 22-ID, an MX300HS charge-coupled device (CCD) detector (Rayonix, L.L.C.; Evanston, IL) was installed in February 2014.

BioCARS received an MX340-HS CCD detector (Rayonix, L.L.C.; Evanston, IL) at the end of February 2014; it was installed on their 14-ID-B beamline.

Two other beamlines at the APS already have Pilatus detectors: IMCA-CAT has a Pilatus $6 \mathrm{M}$ on their 17-ID-B beamline, and NECAT has a Pilatus-F 6M on their 24-ID-E beamline. The newest detectors employ advanced technologies providing even higher frame rates, count rates (Pilatus 3 ) and dynamic range.

These high-frame-rate detectors have enabled increased throughput and several new capabilities including shutterless data collection, rastering (grid search), and collection along a vector (helical). Many users now bring to the beamlines sample mounting loops that contain one or more microcrystals crystals grown in the lipidic cubic phase (LCP), which are difficult to visualize due to the high opacity of the LCP medium. Shutterless rastering will significantly reduce the time-from several minutes to a few seconds-needed to screen the sample mount, locate microcrystals, and ascertain their quality. The low electronic read-out noise and high dynamic range are ideal for studying the structure of membrane proteins and protein complexes, which tend to form small, weakly scattering crystals. The short read-out time allows for efficient employment of fine phi slicing to improve data quality by reducing the amount of background in a frame relative to the Bragg diffraction intensity. The combination of a high degree of automation and high-intensity, micron-sized beams allows users to rapidly screen many samples.

Contact: v-srajer@uchicago.edu (BioCARS), rfischetti@anl.gov (GM/CA-XSD), keefe@anl.gov (IMCA-CAT), chrzas@anl.gov (SER-CAT), rajashankar@anl.gov (NE-CAT)
$>$ Vukica Srajer, BioCARS Senior Scientist, and Robert Henning, BioCARS Operations Manager, with the Rayonix MX340-HS detector in the 14-ID-B enclosure.
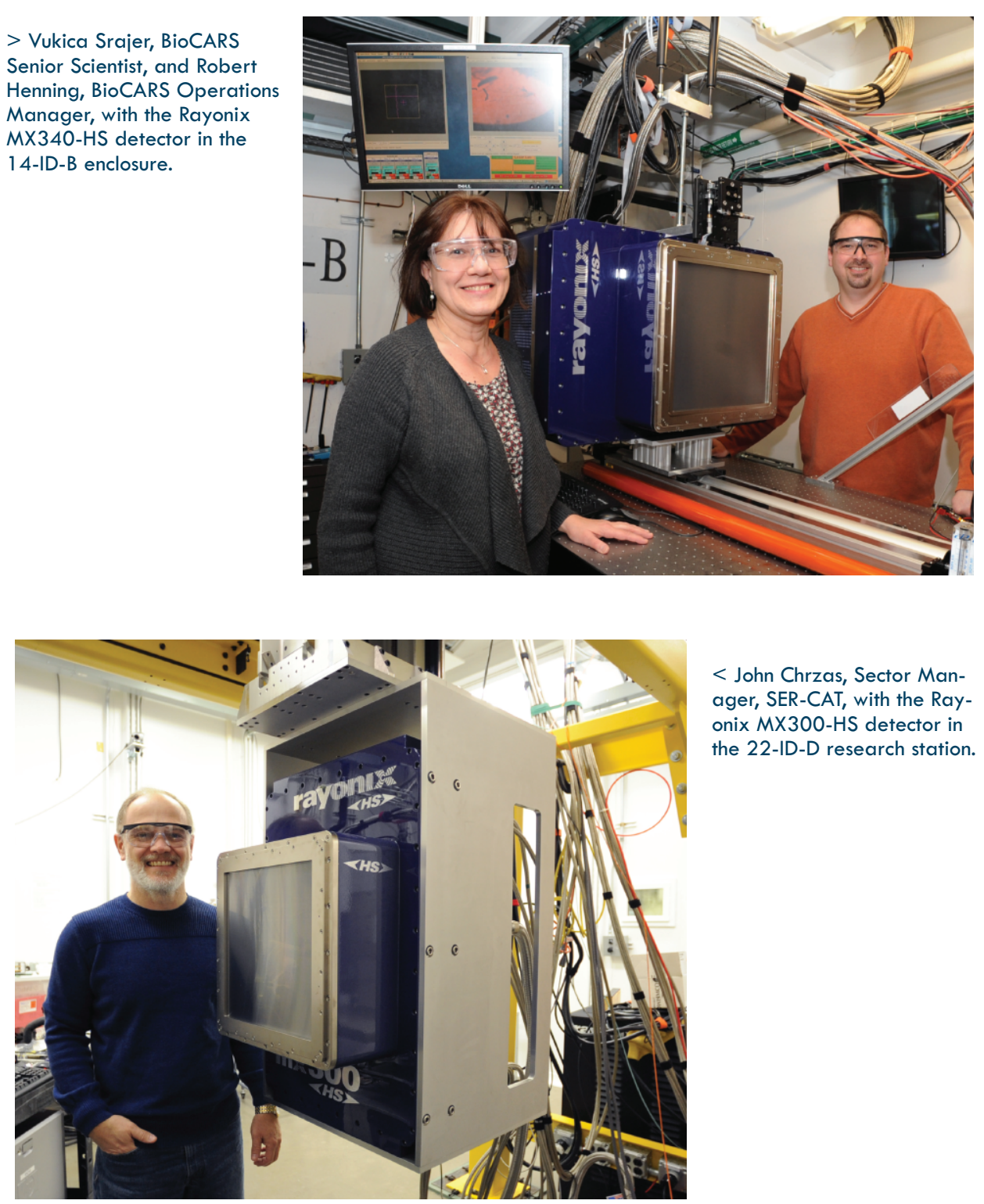

$<$ John Chrzas, Sector Manager, SER-CAT, with the Rayonix MX300-HS detector in the 22-ID-D research station.
$>$ Robert Fischetti, Associate Division Director and Group Leader, GM/CA, in the 23ID-D research station with the Pilatus3 6M detector.

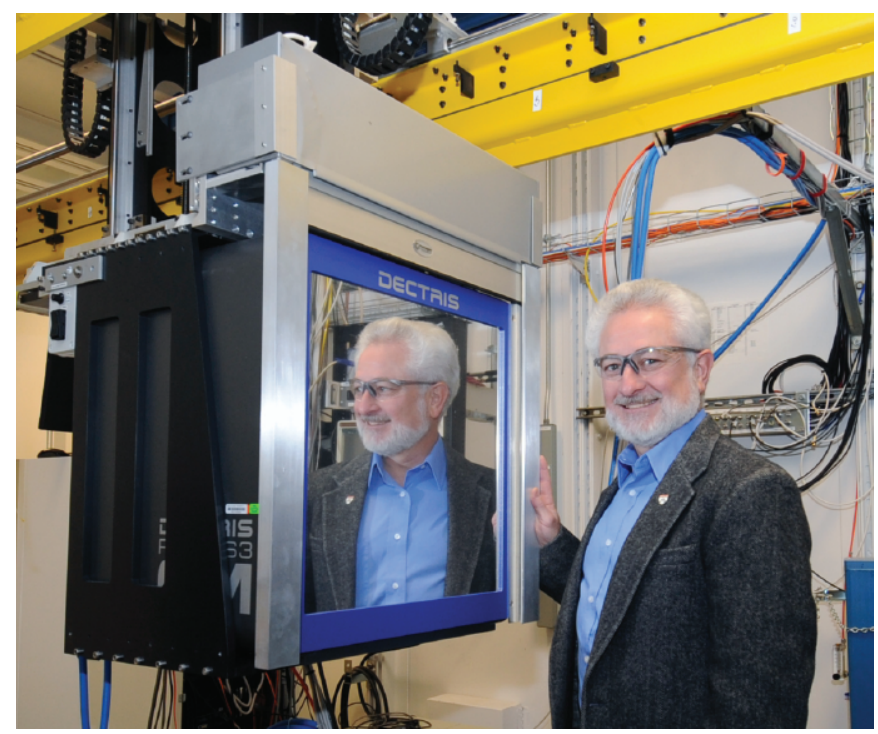




\section{A New TXM Instrument to Address Future Challenges in Nanoscience at the APS}

$\mathrm{A}$

transmission $x$-ray microscope (TXM) at Sector 32 of the APS was commissioned in 2014 and made available for general-user operations, replacing a former first-generation commercial system.

The new instrument, situated in the 32-ID-C research station, provides superior analytical imaging performance and in situ capabilities. It was developed to explore and better understand nanomaterials in the fields of energy storage, microelectronics, and nanoporous materials functions as well as the life, earth, and environmental sciences.

The analytical techniques currently available include absorption, Zerniketype phase contrast, and x-ray absorption near edge structure (XANES) tomography.

One of the key design features of the new TXM is

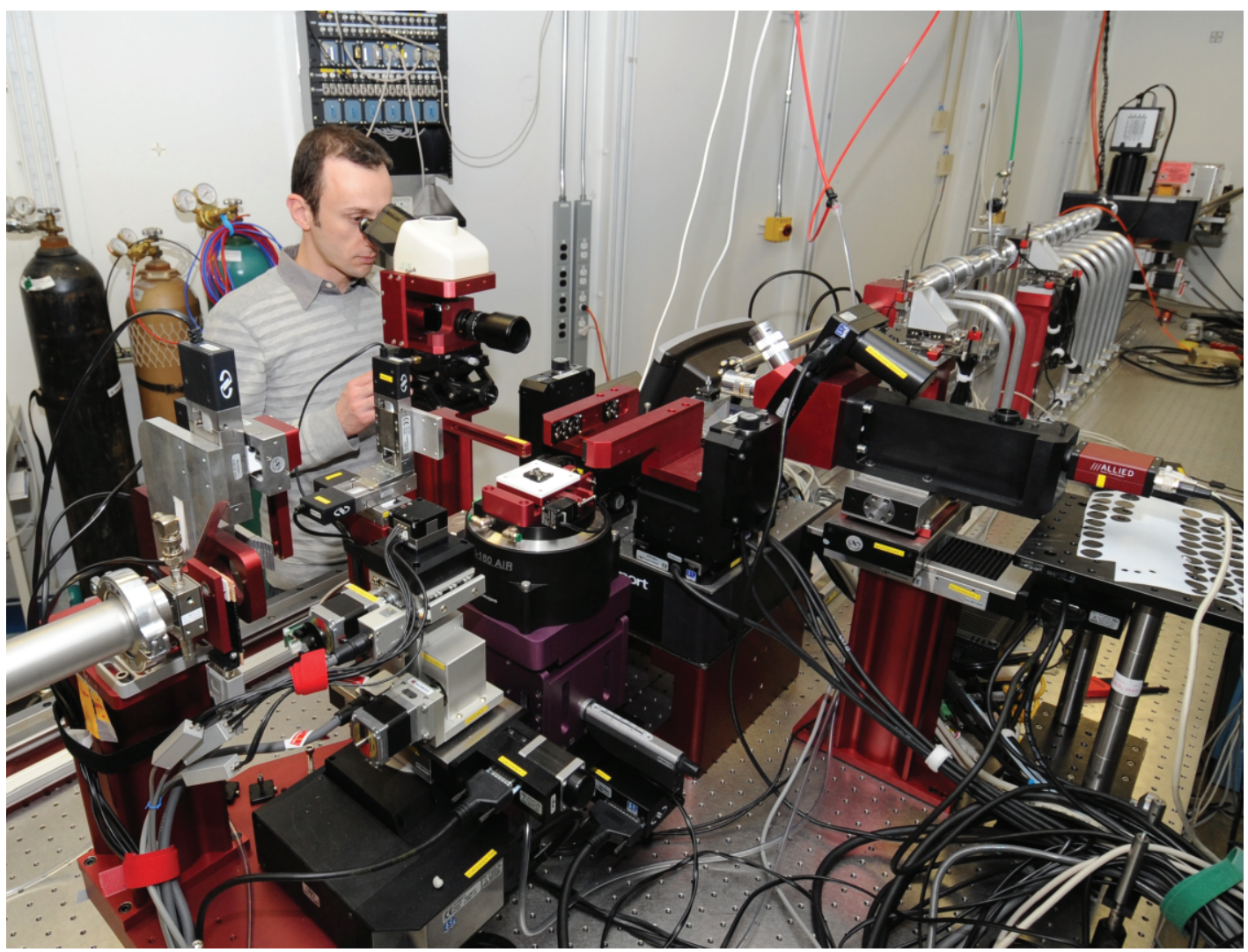

Advanced Photon Source physicist and beamline scientist Vincent De Andrade, who is in charge of the TXM in the 32ID-C research station, during commissioning of the instrument. The setup consists in a condenser optic (mono-capillary, not installed when this photo was taken) to focus $x$-rays at the sample location while defining the size of the illumination; a high-stability sample stage; and an objective lens (Fresnel zone plate) forming a magnified image of the sample on a charge-coupled device-based detector located between $3 \mathrm{~m}$ and $4 \mathrm{~m}$ downstream for magnification purposes. the ability to perform routine operations with a spatial resolution of 20 $\mathrm{nm}$, making this instrument the highest resolution hard $x$-ray full-field imaging system at the APS. The energy range has also been expanded. The TXM, equipped with an undulator and a fixed exit double-crystal monochromator (Si 111), now operates from $6 \mathrm{keV}$ to more than $25 \mathrm{keV}$, but the objective zone plates are optimized for $8 \mathrm{keV}$. The lowenergy limit will be pushed below 5 $\mathrm{keV}$, enabling, for instance, spectroscopy at the Fe K-edge.

The available set of optics provides fields of view ranging from $100 \mu \mathrm{m}$ to 20 $\mu \mathrm{m}$ with spatial resolutions of $60 \mathrm{~nm}$ and $20 \mathrm{~nm}$. The Fresnel zone plate geometry also offers working distances from 20 $\mathrm{mm}$ to $70 \mathrm{~mm}$, allowing the TXM to accommodate a variety of sample environments.
Electrical and high-pressure fluid feed-throughs were integrated into a high-accuracy, air-bearing rotary stage to facilitate a wide range of in situ environmental controls, including temperature, pressure, and chemical bath without affecting the three-dimensional nanocomputed tomography imaging performance.

The instrument is supported by several key R\&D activities at the APS, such as the fabrication of new zone plates in the framework of the proposed multibend achromat lattice upgrade of the APS. A more complete set of zone plates, which will provide a broader range of spatial resolution as well as a 16-nm $\Delta \mathrm{rn}$ zone plate, are in production. The TXM will directly benefit from these R\&D programs by achieving 10-nm spatial resolution and a higher efficiency at high energy (> $20 \mathrm{keV}$ ), which will enable tomography of denser materials within the next three years. Additional upgrades are planned for 2015.

The TXM is also the centerpiece for a program focusing on scientific software development of methods for merging multilength scale and multi-modality data. The outcome of this activity is the software framework called TomoPy (/www.aps.anl.gov/tomopy/) an opensource Python-based toolbox for the analysis of synchrotron tomographic data that has the goal of unifying the effort of different facilities and beamlines performing similar tasks (see page 176).

Contacts: Vincent De Andrade (vdeandrade@aps.anl.gov),

Francesco De Carlo (decarlo@aps.anl.gov) 


\section{A Revolver Undulator for Sector 35 of the APS}

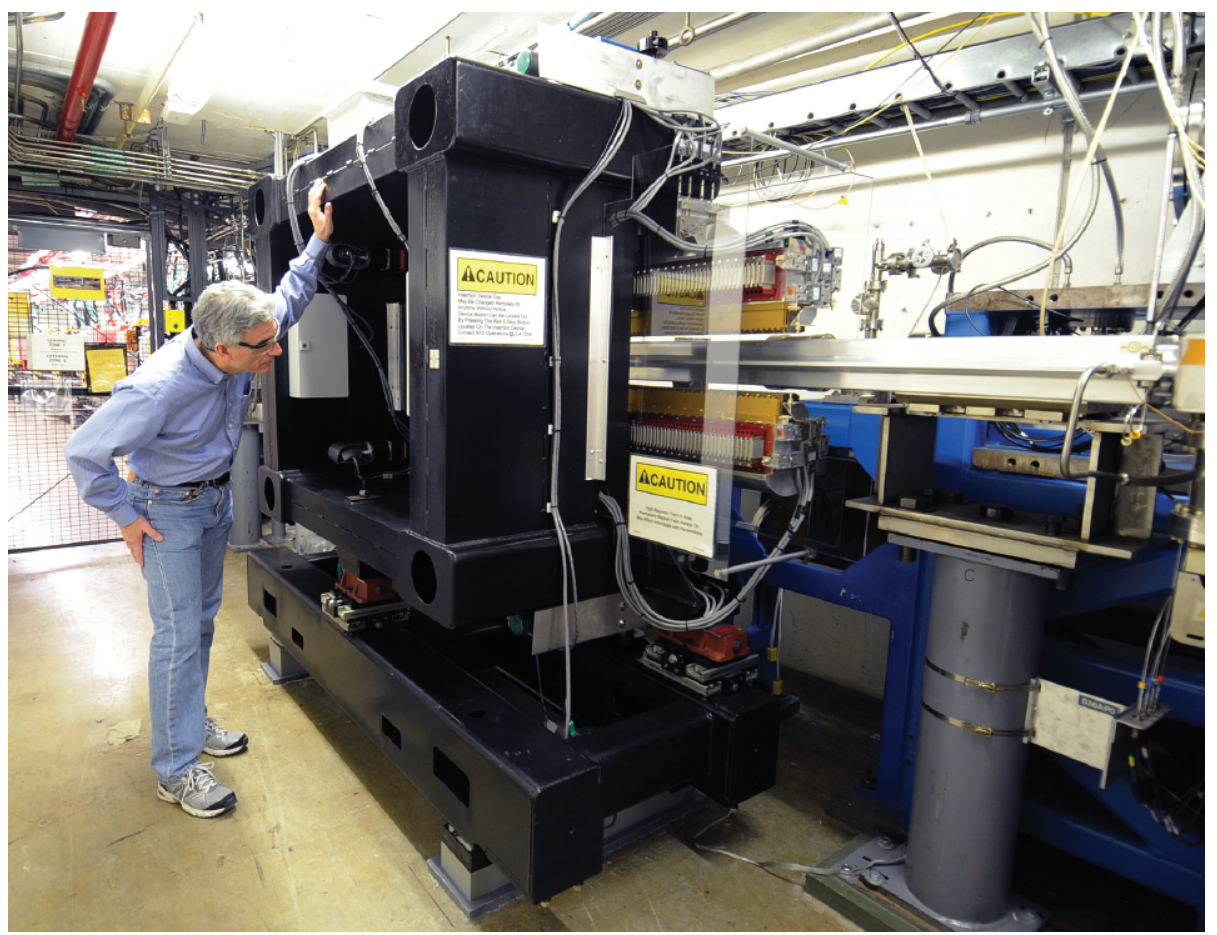

Kevin D'Amico, DCS Project Manager, with the newly installed prototype revolver undulator at Sector 35 in the APS storage ring.

A prototype revolver undulator was installed at Sector 35 of the APS during the January 2015 shutdown. This insertion device will provide the new Dynamic Compression Sector (DCS) with the benefits of two undulators in the space of one.

In a matter of minutes, the active undulator magnet structure can be switched between a $3.3-\mathrm{cm}$ period and a $2.7-\mathrm{cm}$ period. This arrangement allows higher $x$-ray brightness and flux over a broader energy range than could be achieved with a single conventional undulator.

The installation was the culmination of a multi-year Argonne Directed Research and Development (LDRD) program carried out by the Magnetic Devices Group in ASD to develop a robust revolver undulator platform for nearterm use at the APS. The platform is also expected to be further developed in conjunction with a future upgrade of the APS.

The demands for precise, fail-safe positioning of powerful undulator magnetic arrays, in close proximity to the vacuum chambers that house the electron proven gap separation mechanism of the

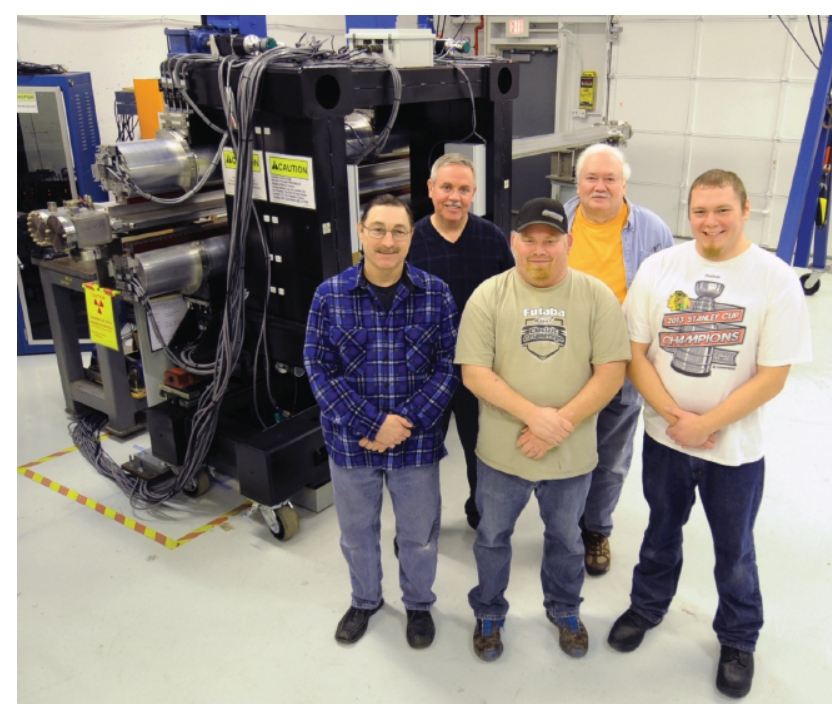

The prototype revolver undulator is the backdrop for the members of the ASD Magnetic Devices Group who assembled the new device. Left to right are: John TerHAAR, John Grimmer, Eric McCarthy, Mike Merritt, and Joe Gagliano, III. Martin Smith (not pictured) of the APS Engineering Support Division Controls Group did the controls hardware/software design and development.

beam, must be met by all of the APS undulators. But the revolver undulator must also precisely index the two sets of magnetic arrays, and be safeguarded against more complex failure modes. Toward this end, the revolver undulator uses the well- most-recent 33 APS undulators, and applies the same interlock and control scheme to the revolver mechanisms. The use of this gap separation mechanism also allows future repurposing of these mechanisms as revolver undulators.

The revolver undulator installed at Sector 35 is the final prototype of the revolver undulator LDRD program, and benefits from post-LDRD development primarily focused on control software. The first "production" revolver undulator is undergoing assembly at the APS, with installation at Sector 35 planned for May 2015. That device uses $3.0-\mathrm{cm}$ period and $2.7-\mathrm{cm}$ period magnet structures.

The new revolver undulator can accommodate undulator magnet structures up to 2.4-m long. Any combination of the APS-designed undulator magnet structures, from $1.72-\mathrm{cm}$ to $3.6-\mathrm{cm}$ periods can be used. All of the functions of the conventional undulator are retained, including gap tapering. The revolver undulator is compatible with all of the APS insertion device vacuum chambers, but requires the insertion device vacuum chamber support stands to be retrofitted to a new design.

Contact: Efim Gluskin
(gluskin@aps.anl.gov),
John Grimmer
(grimmer@aps.anl.gov)

The DCS Project is funded by the DOE/National Nuclear Security Administration (NNSA) and is being carried out by the APS DCS project team. Washington State University, with funding by NNSA, leads the effort to develop the DCS experimental program and build the instrumentation in collaboration with the APS, DOE/NNSA national laboratories (Los Alamos, Livermore, and Sandia); Department of Defense laboratories, including the Army Research Laboratory and the Naval Research Laboratory; and academic institutions. 
KIM OF ASD AWARded 2014 Wilson Prize for

AChievement IN Physics of Particle Accelerators • Kwang-Je

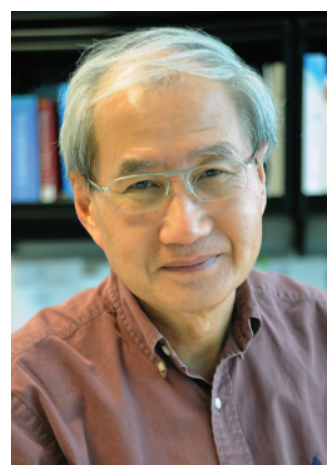

Kim of ASD was named the recipient of the 2014 Robert R. Wilson Prize for Achievement in the Physics of Particle Accelerators. Kim was cited for "his pioneering theoretical work in synchrotron radiation and free electron lasers [FELs] that laid the foundation for both third and fourth generation x-ray sources." The annual Wilson Prize was established in 1986 by friends of the late Robert R. Wilson, founding Director of Fermilab, and by the Division of Particles and Fields and the Division of Physics of Beams of the American Physical Society to recognize and encourage outstanding achievement in the physics of particle accelerators. As noted on the Wilson Prize website, Kim made pioneering theoretical contributions to the understanding of photocathode electron guns, synchrotron radiation, and FELs. In synchrotron radiation, he developed a method to compute the brightness of partially coherent beams. This has become more important as light sources have become brighter and moved closer to the diffraction limit. He advanced the method of analyzing high-gain x-ray FELs and self-amplified spontaneous emission from $1 D$ to $3 D$ so that a reliable prediction of the performance of practical devices can be made. Recently, he has revived the idea of an $\mathrm{x}$-ray $\mathrm{FEL}$ in oscillator configuration employing Bragg crystals as $x$-ray cavity mirrors, showing that it could be a source of fully coherent $\mathrm{x}$-ray pulses with extremely high spectral purity.

Contact: kwangje@aps.anl.gov
ACS Division of Energy and Fuels 2014 Distinguished Service Award to Winans

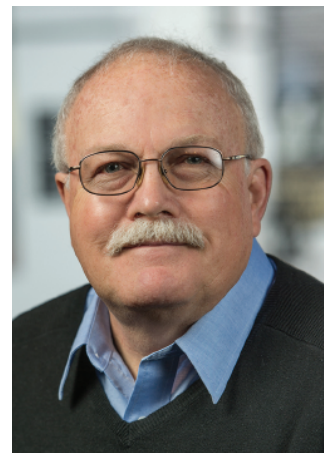

of XSD • The 2014 American Chemical Society Division of Energy \& Fuels Distinguished Service Award was presented to Randy Winans of XSD. Winans has spent his entire career at Argonne National Laboratory where he led programs that have had a great impact on energy and fuels research, including as head of the Heavy Hydrocarbon Group in the Argonne Chemistry Division and Director of the Basic Energy Sciences Synchrotron Radiation Center at the APS. He now heads the Chemical \& Materials Science Group in XSD. His major research accomplishments include developing and applying methods to understand the fundamental chemistry of complex disordered systems such as catalysts, coals, heavy petroleum, oil shale, and carbons. He combines chemistry with characterization techniques including mass spectrometry, nuclear magnetic resonance, and small-angle x-ray scattering and spectroscopy. Contact: rewinans@anl.gov

\section{John Freeland of XSD Named an American} PhySICAL SOCIETY FELLOW • John W. Freeland,

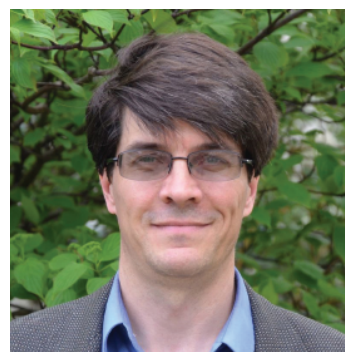
physicist with the XSD Magnetic Materials Group, has been named a Fellow of the American Physical Society by the Division of Condensed Matter Physics. He was cited "for development and use of new $\mathrm{x}$-ray techniques to understand chemical, structural, electronic, and magnetic features at oxide interfaces." Freeland's research interests include understanding the physics behind phase transitions in novel oxide systems (e.g., metal to insulator transitions, spin state transitions, multiferroics, etc.); emergent states at the interface between dissimilar complex oxides; and the effect of confinement on the ground-state of complex oxides. In 2014, Freeland became a co-principal investigator $(\mathrm{PI})$ of a DOE-funded team to explore how to efficiently control the states of matter in designer materials on ultrafast time scales. He is also a co-PI for the Indo-US Virtual Research Center funded by the Indo-US Science and Technology Forum to advance understanding of the physics of nickelates in both bulk and heterostructure form.

Contact: freeland@anl.gov
BORLAND OF the ASD NAMEd AN ARgONNE Distinguished Fellow • Michael Borland, Associate Division Di-

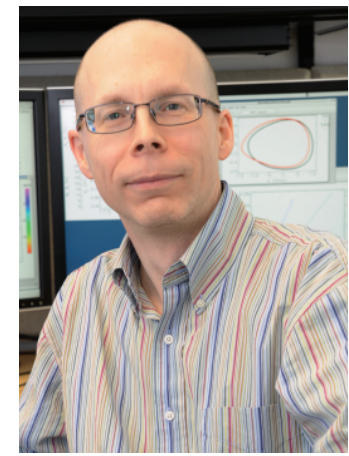
rector of ASD, is one of four Argonne scientists named as Argonne Distinguished Fellows for 2014, the Laboratory's highest scientific and engineering rank. The Argonne Distinguished Fellow title is comparable in stature to an endowed chair at a top-ranked university and recognizes exceptional contributions in a person's field. The rank is given for sustained outstanding scientific and engineering research and can also be associated with outstanding technical leadership of major, complex, high-priority projects. Borland's best-known work, named "ELEGANT," is an accelerator simulation code that provides physicists world-wide with a flexible tool to design and understand linear accelerators and storage rings. ELEGANT, the primary tool at the APS for accelerator design and simulation, was used to develop the present APS storage ring low-emittance lattice (the configuration of magnet strengths) and played an essential role in the development of top-up mode at the APS. ELEGANT is also used to design and understand free-electron lasers (FELs), including the world's first hard x-ray FEL, the Linac Coherent Light Source at the SLAC National Accelerator Laboratory. Contact: borland@aps.anl.gov 


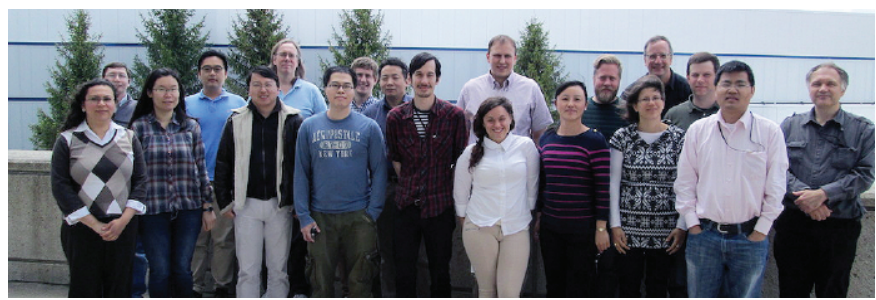

SAXS Software Packages Irena and NIKa 2014 Courses • May 1 9 (ABOVE) AND 15-16, SePTEMBER 18-19 - The APS small-angle scattering (SAXS) special interest group organized these hands-on courses specifically on the SAXS software Nika and Irena. The courses included installation of the Igor Pro, and Irena and Nika packages on users' computers; data reduction using Nika as an example of SAXS data including calibration, mask design, and necessary corrections; examples of transmission and grazing incidence data; data analysis using Irena (all steps necessary); presentation and hands-on education for the use of Irena tools (unified fit, size distribution, modeling, analytical models, smallangle diffraction, etc.); explanation of advanced support tools such as the scripting tool; and discussion and help with reduction of users' data. Contact: ilavsky@aps.anl.gov

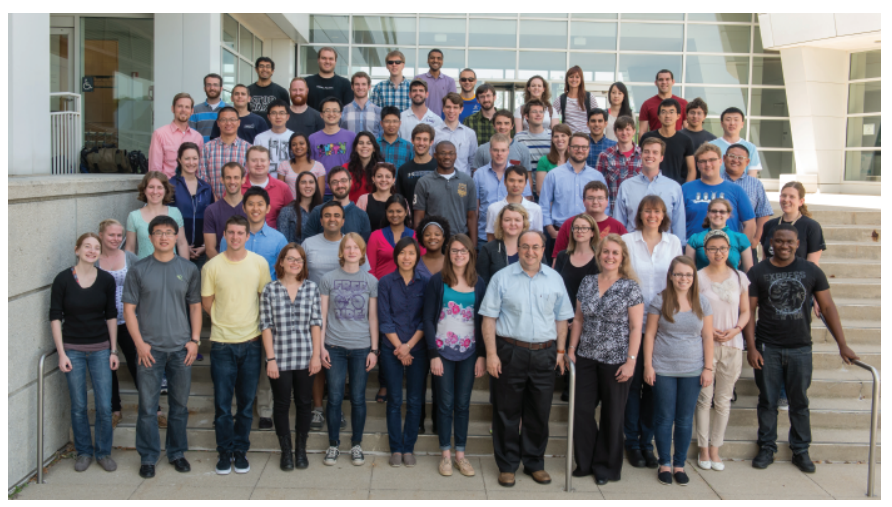

National School on Neutron and X-Ray Scattering • June 14-28 - The main purpose of the National School on Neutron and Xray Scattering is to educate graduate students on the utilization of major neutron and x-ray facilities. Lectures, presented by researchers from academia, industry, and national laboratories, include basic tutorials on the principles of scattering theory and the characteristics of the sources, as well as seminars on the application of scattering methods to a variety of scientific subjects. Students conduct short experiments at Argonne's APS and Oak Ridge's Spallation Neutron Source and High Flux Isotope Reactor facilities to provide hands-on experience for using synchrotron and neutron sources. The target audience is graduate students attending North American universities majoring in physics, chemistry, materials science, or related fields. The school is jointly conducted by Argonne's Communications, Education and Public Affairs Division; Materials Science Division; and the APS; and Oak Ridge National Laboratory's Biology and Soft Matter, Chemical and Engineering Materials, and Condensed Matter divisions. Contact: nxschool@dep.anl.gov

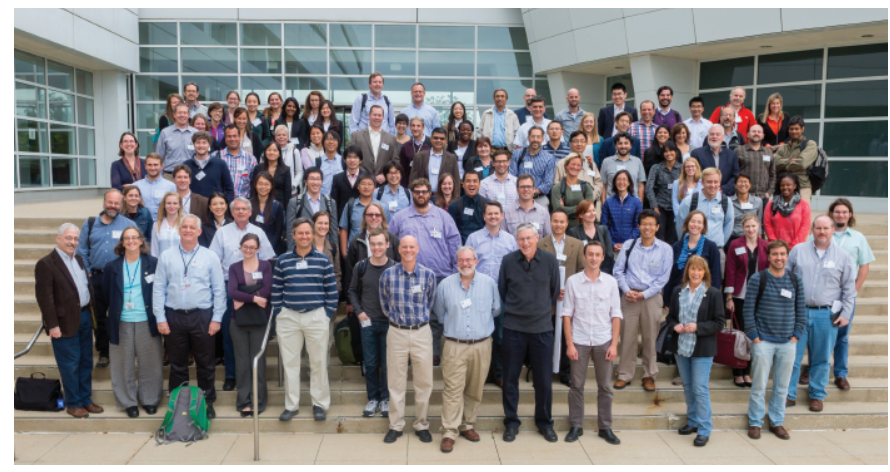

SynChrotron EnVIRONMENTAl SCIenCe VI - September 11 -12 • Hosted by GSECSARS, SES-VI brought together and promoted dialogue between synchrotron science experts and environmental science experts. Building on the recent excitement of science that would be enabled by diffraction limited $x$-ray sources, as well as by free-electron laser $\mathrm{x}$-ray sources, the emphasis of the meeting was on identification of new opportunities in environmental science afforded by new or soon-to-be-available x-ray sources. 110 individuals attended the conference, which included presentations from synchrotron scientists describing the projected qualities of these new sources (x-ray spot and/or illumination size, coherence/spectral qualities, timing capabilities, etc.). Also included were presentations from scientists who have used synchrotron radiation sources in their environmental science research describing their work and their vision for how these new sources will benefit their research in the future. Contact: lanzirotti@uchicago.edu, sutton@cars. uchicago.edu, vdeandrade@aps.anl.gov, kemner@anl.gov
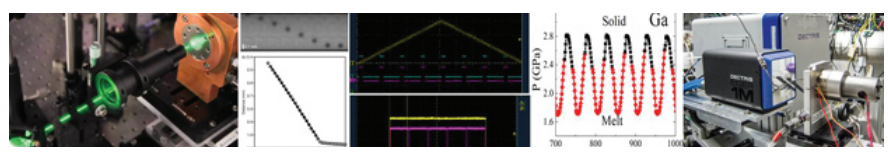

Workshop on High-Pressure TIME-Resolved SynCHROtron TeCHNIQUES 2014 • SEPTEMBER 25-27 • HP-CAT presented a two-anda-half day workshop dedicated to time-resolved x-ray scattering techniques. Ideas were exchanged through invited and contributed talks, discussion periods, and an evening poster session for a diverse group of researchers in high-pressure and time-resolved techniques, with a significant number of young researchers attending. The workshop purpose and goals were to introduce time-resolved apparatus and techniques currently available at HP-CAT and other synchrotron facilities; present the results of time-resolved high-pressure research carried out at HPCAT and other facilities; motivate students and early career researchers to pursue time-resolved research programs; demonstrate available facilities and software during hands-on practical sessions; identify scientific goals and associated technical challenges for future time-resolved studies; and anticipate the expanded scope of time-resolved high-pressure research that could be enabled by the proposed APS upgrade.

Contact: HPTRWorkshop2014@ hpcat.aps.anl.gov 


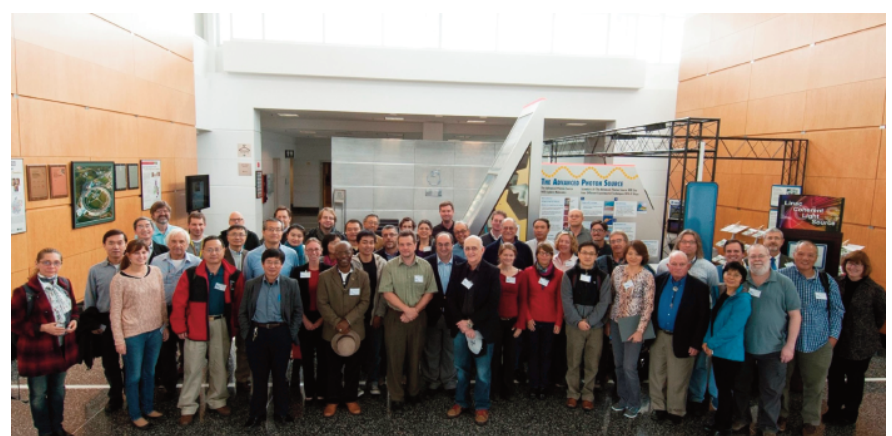

Mineral Physics Planning Workshop • October 10-12 • Hosted by GSECSARS, the purpose of the Chicago workshop was to identify the most important challenges and opportunities in the discipline, review the main science drivers, and formulate a strategic roadmap for the next decade. Particular emphasis was placed on the possible role emerging new technologies (e.g., experimental, cyberinfrastructure, etc.) play in furthering understanding of planetary interiors and high-pressure phenomena. Similar to the two previous long-range planning workshops, the goal of the Chicago workshop was to produce a written "Mineral Physics Long Range Vision Report," following the examples of the 2003 "Bass Report" and 2009 "Williams Report," which will then be distributed throughout the community and to the funding agencies.

Contact:dweidner@notes.cc.sunysb.edu,pdera@hawaii.edu

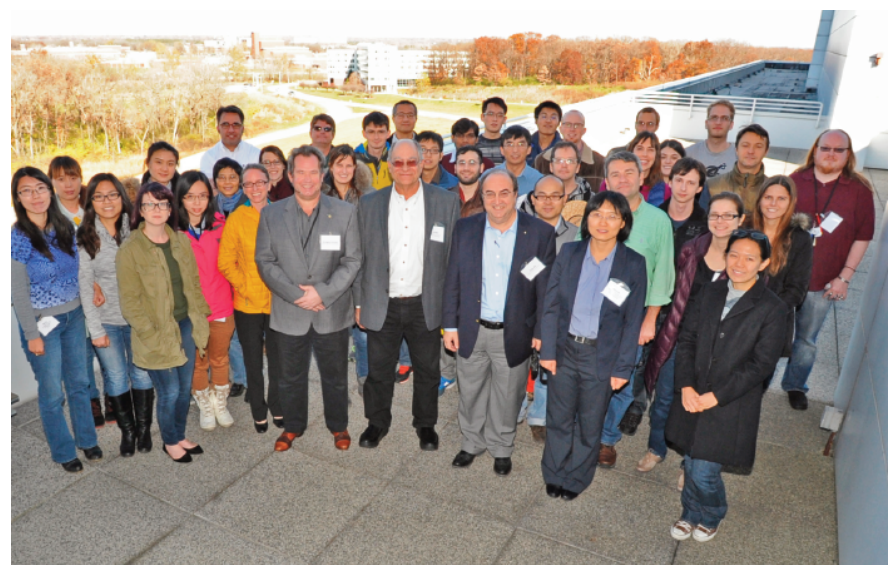

Nuclear Resonant Scatterring Workshop • November 7-9 • This workshop was organized as part of the COMPRES Education, Outreach and Infrastructure Development programs to promote the application of the state-of-the-art nuclear resonant scattering (NRS) techniques for characterizing the properties of materials under the high P-T conditions of planetary interiors. Two NRS techniques, synchrotron Mössbauer spectroscopy (SMS) and nuclear resonant inelastic x-ray scattering (NRIXS), were covered. Synchrotron Mössbauer spectroscopy provides information on magnetic properties and valence state in minerals containing Fe and other resonant isotopes (Sn, Eu, Dy, and Kr); NRIXS gives phonon density-of-state, and therefore information on vibration and elastic properties. The workshop offered an introduction to SMS and NRIXS techniques and beamline instrumentation; introduced CONUSS, PHOENIX, and SciPhon for data evaluation; provided hands-on training in the use of CONUSS, PHOENIX, and SciPhon; addressed common issues that users confront; and discussed recently added features to the new version of the software. Contact: wbi@aps.anl.gov

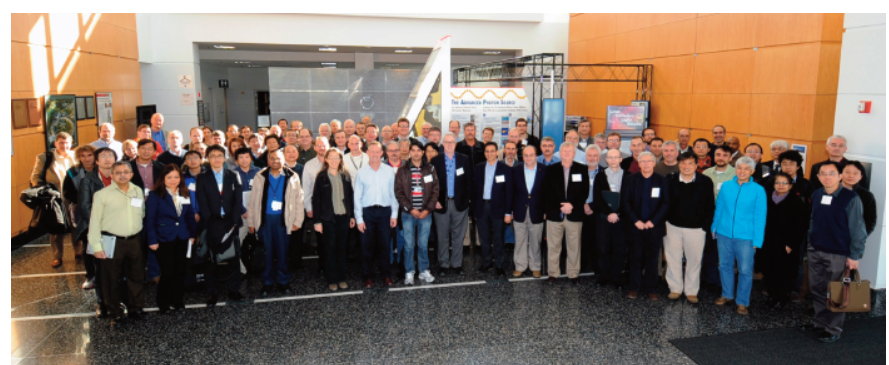

4th Diffraction Limited Storage Ring Workshop - November 19-21 - Synchrotron facilities around the world are developing plans for multi-bend achromat (MBA) storage rings that will push the performance of high-energy storage rings toward the diffraction limit in the hard x-ray regime. The very low electron-beam emittances made possible by these facilities will enable dramatic improvements in many areas of $x$-ray science, especially for experiments that directly use $x$-ray coherence. This workshop addressed both accelerator and beamline technical challenges associated with the design, construction, commissioning, and operation of fourth-generation storage rings enabled by MBA lattices.

Contact:dwilkin@aps.anl.gov

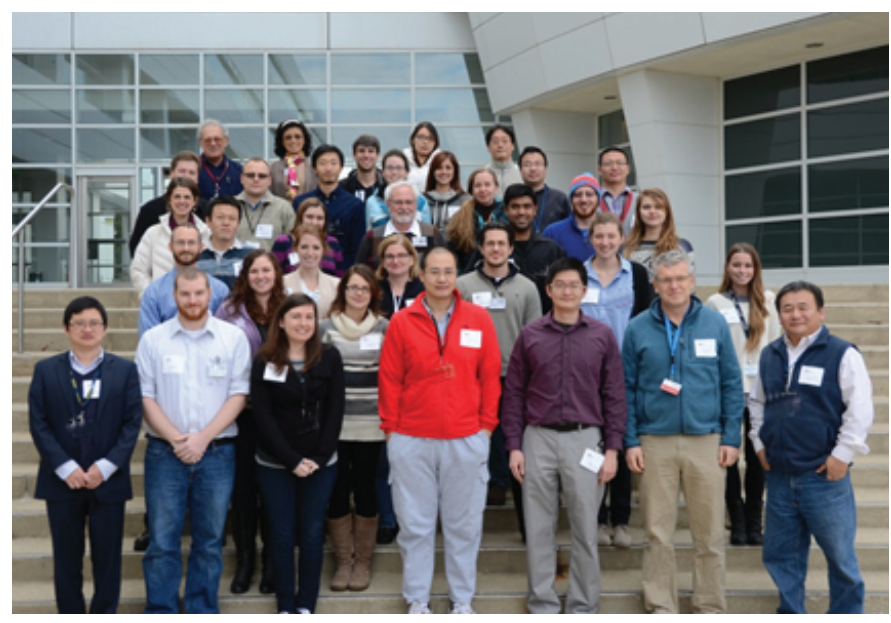

SCHOOL FOR SYNCHROTRON CRYSTALLOGRAPHY: INTRODUCTION AND EXPerimental Methods • December 3-6 • On December 3-6, 2014, ChemMatCARS hosted a three-day (including a 16-hour handon session) school for synchrotron crystallography. The school was attended by 26 participants from 6 countries, among them graduate students, postdocs, crystallographic staff, and faculty. The program included a series of lectures given by eight speakers who are leaders in the field of synchrotron-based crystallography, as well as 16 hours of hands-on sessions aimed at introducing the participants to synchrotron-based, single-crystal x-ray diffraction techniques. A primary goal of the school was to give students and postdocs a realistic inside look at synchrotron research early in their careers. The school was sponsored by Bruker and ChemMatCARS.

Contact: Charles.Campana@bruker-axs.com, yschen@cars.uchicago.edu,mpink@indiana.edu 


\section{X-RAY AVAILABILITY AND RELIABILITY}

In fiscal year $2014^{*}$ the APS $x$-ray source continued to function as a highly reliable delivery system for synchrotron $\mathrm{x}$-ray beams for research. Several factors support the overall growth in both the APS user community and the number of experiments carried out by that community. But there is a direct correlation between the number of $x$-ray hours available to users; the success of the APS experiment program; and the physicists, engineers, and technicians responsible for achieving and maintaining optimum $\mathrm{x}$-ray source performance. Below are definitions of important measures for the delivery of $\mathrm{x}$-ray beam to users (latest data shown graphically).

Storage Ring Reliability: A measure of the mean time between beam losses (faults), or MTBF, calculated by taking the delivered beam and dividing by the total number of faults. The APS targets, and routinely exceeds, $70 \mathrm{~h}$ MTBF. A fault is defined as complete unavailability of beam either via beam loss or removal of shutter permit not related to weather. A fault also occurs when beam has decayed to the point where stability and orbit can no longer be considered reliable. At the APS, this threshold is $50 \mathrm{~mA}$.

APS storage ring reliability (MTBF), fiscal years 1999-2014

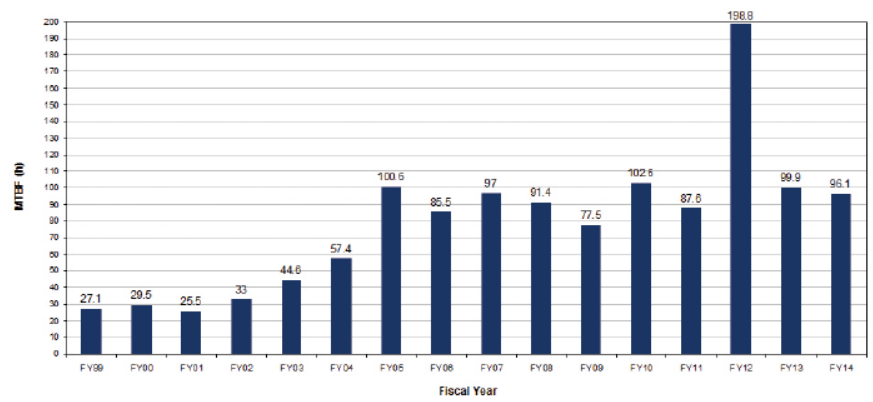

X-ray Availability: The number of hours that the beam is available to the users divided by the number of hours of scheduled beam delivery prior to the beginning of a run. The specific definition of available beam is that the APS Main Control Room has granted permission to the users to open their shutters, and there is more than 50 -mA stored beam in the storage ring.

APS x-ray availability, fiscal years 1999-2014

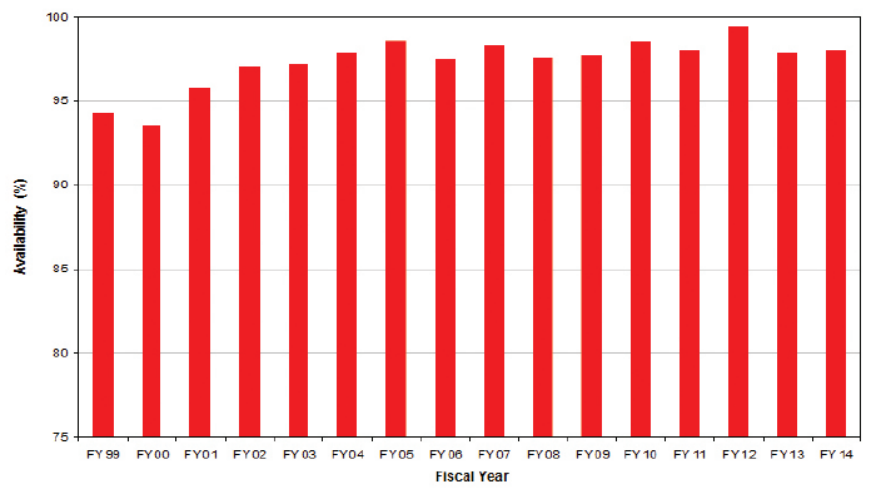

* While the highlights in, and title of, this report cover calendar year 2014, data on accelerator performance and user statistics are measured on the basis of fiscal years.

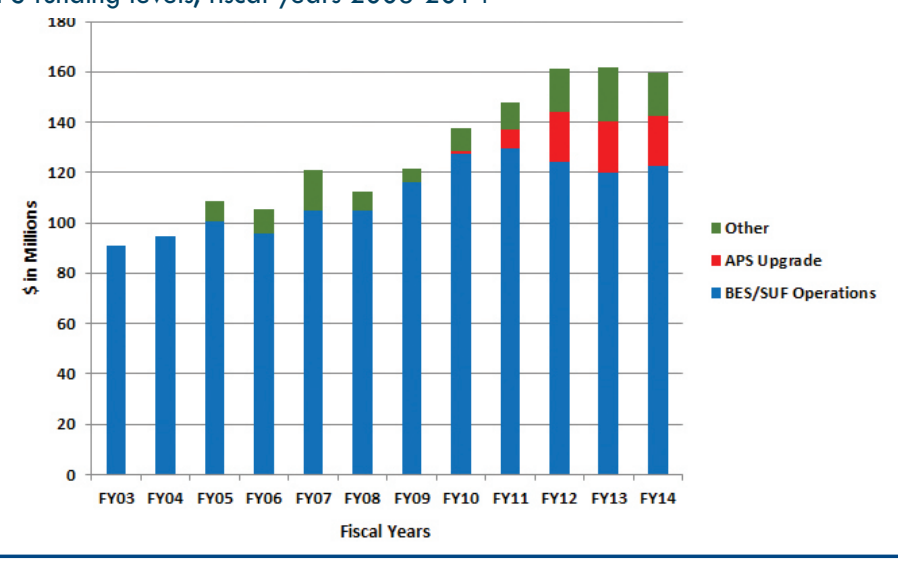

APS staffing levels, fiscal years 2003-2014

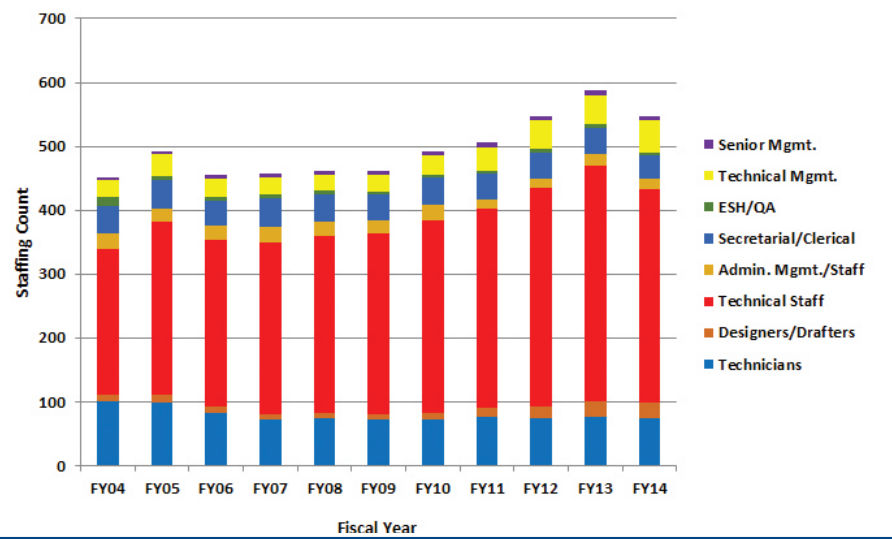

Number of APS publications, calendar years 1998-2013, as of 5.14

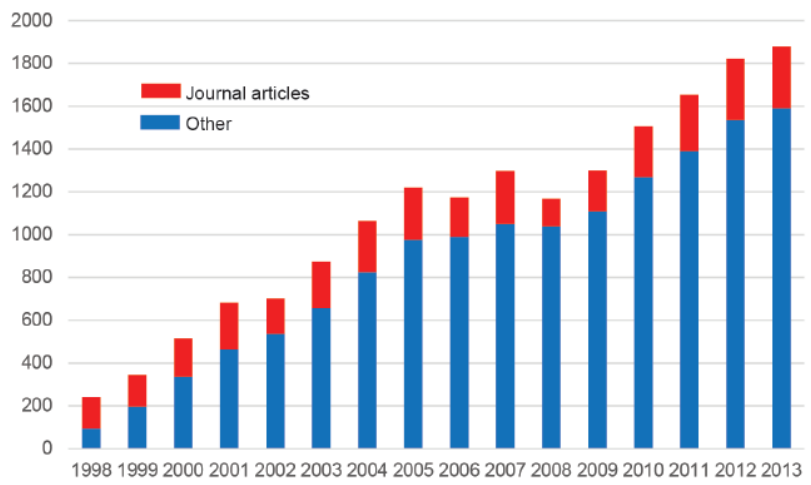

For lists of APS publications see http://www.aps.anl.gov/Science/Publications/

Deposits in Protein Data Bank from research at the APS,

calendar years 1998-2013, as of 5.15

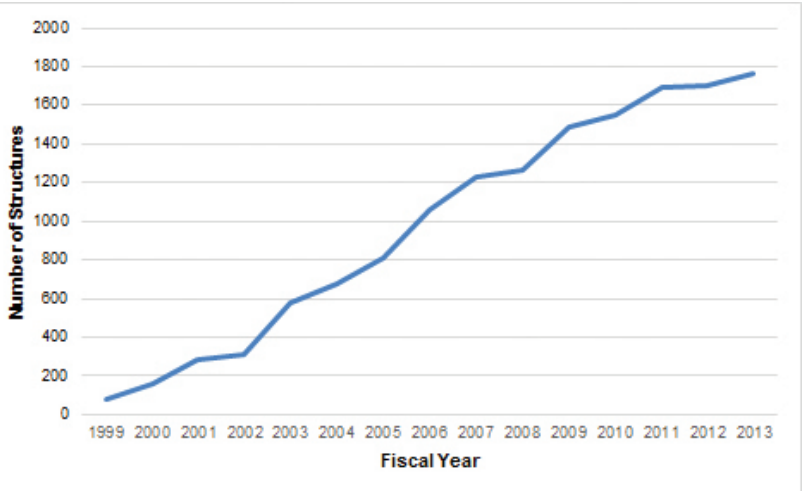


Number of experiments at the APS, fiscal years 1998-2014

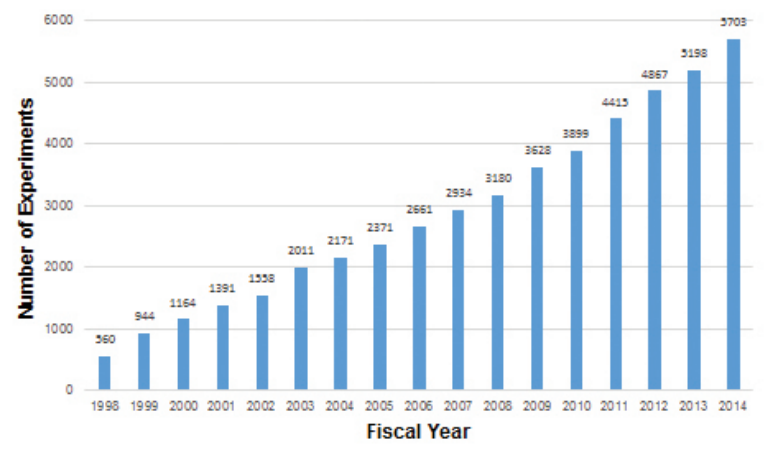

APS users by experiment subject, fiscal year 2014

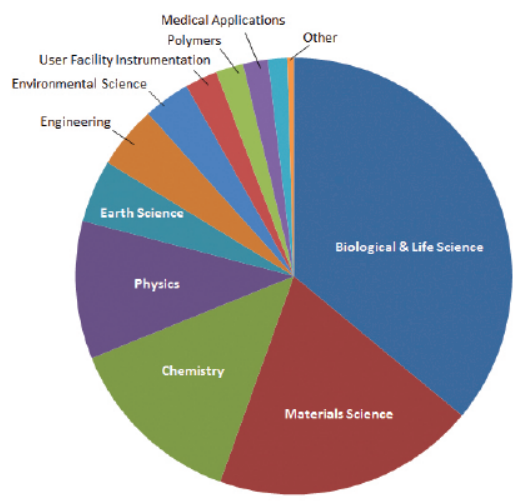

APS users by employer, fiscal year 2014

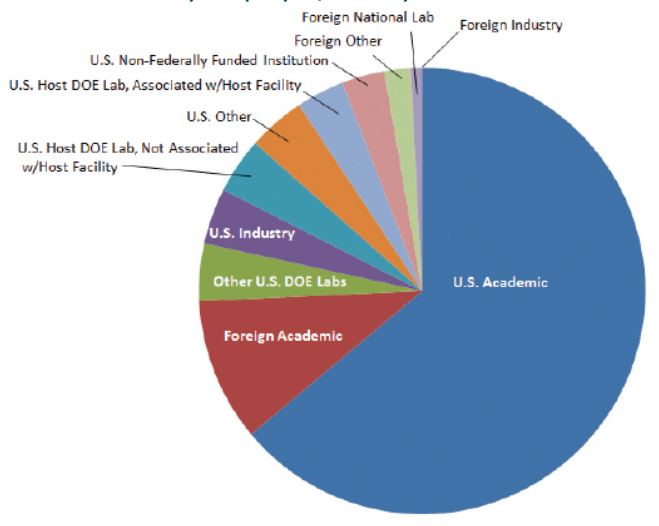

APS users by proprietary type, fiscal year 2014

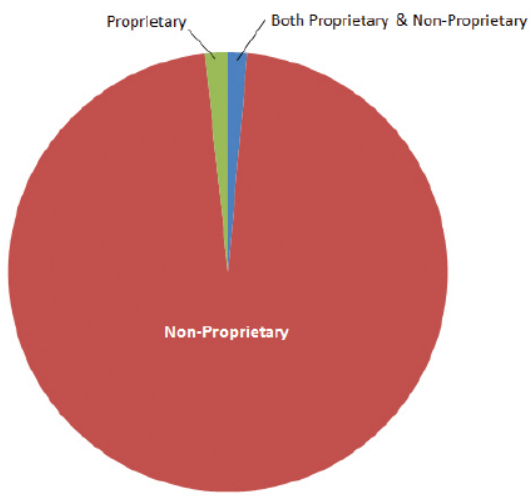

Number of unique on-site \& off-site APS users, fiscal years 1998-2013

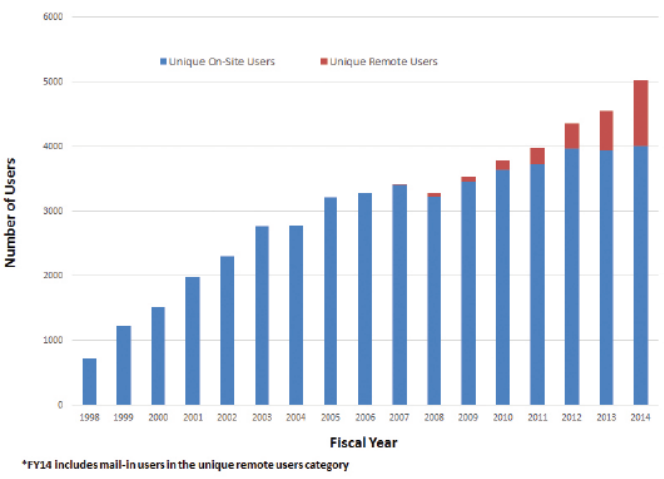

APS users by user type, fiscal year 2014

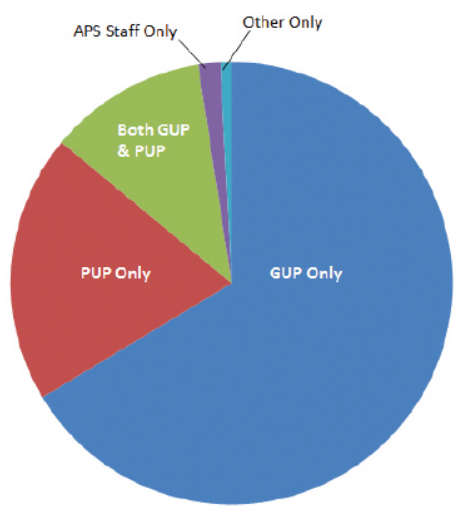

APS users by employment level, fiscal year 2014

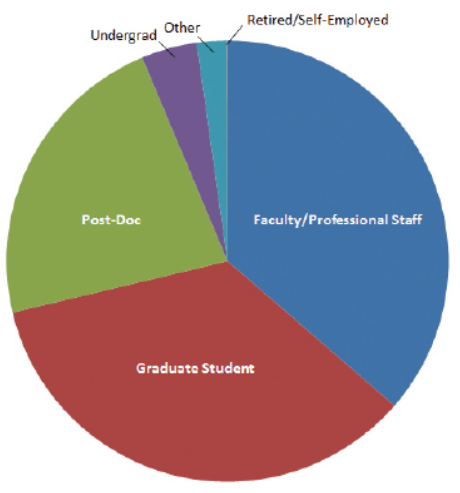

APS users by institutional geographic distribution, fiscal year 2014

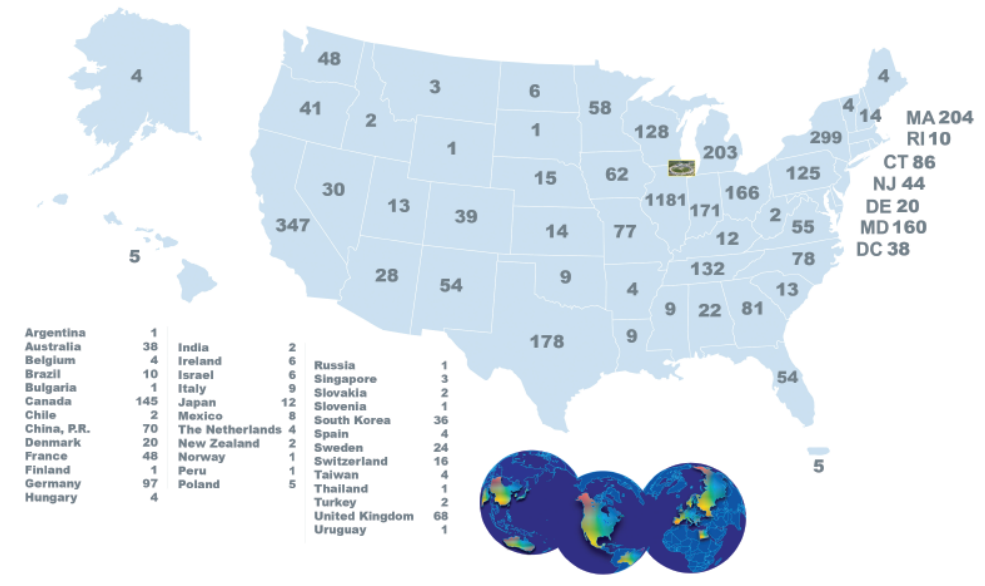




\section{Typical APS MaChine Parameters}

$\begin{array}{ll}\text { LiNAC } & \\ \text { Output energy } & 375 \mathrm{MeV} \\ \text { Maximum energy } & 450 \mathrm{MeV} \\ \text { Output beam charge } & 1-3 \mathrm{nC} \\ \text { Normalized emittance } & 10-20 \mathrm{~mm} \text {-mrad } \\ \text { Frequency } & 2.856 \mathrm{GHz} \\ \text { Modulator pulse rep rate } & 30 \mathrm{~Hz} \\ \text { Gun rep rate } & 2-12 \mathrm{~Hz} \\ \text { (1-6 pulses, 33.3 ms apart every } 0.5 \mathrm{~s}) & \\ \text { Beam pulse length } & 8-15 \mathrm{~ns} \\ \text { Bunch length } & 1-10 \mathrm{ps} \mathrm{FWHM}\end{array}$

\section{Particle Accumulator Ring}

Nominal energy

Maximum energy

Circumference

Cycle time

Fundamental radio frequency (RF1)

12th harmonic RF frequency (RF12)

RMS bunch length

(after compression)

\section{INJECTOR SYNCHROTRON (BOOSTER)}

Nominal extraction energy

Injection energy

Circumference

Lattice structure

Ramping rep rate

Natural emittance

Radio frequency

\section{Storage Ring System}

Nominal energy

Circumference

Number of sectors

Length available for insertion device

Nominal circulating current, multibunch

Natural emittance

RMS momentum spread

Effective emittance

Vertical emittance

Coupling

Revolution frequency

Radio frequency

Number of bunches

Time between bunches

RMS bunch length

RMS bunch length of $16 \mathrm{~mA}$ in hybrid mode
$375 \mathrm{MeV}$

$450 \mathrm{MeV}$

$30.66 \mathrm{~m}$

$500 \mathrm{~ms}$

$9.77 \mathrm{MHz}$

$117.3 \mathrm{MHz}$

$0.34 \mathrm{~ns}$

$7.0 \mathrm{GeV}$
$375 \mathrm{MeV}$
$368.0 \mathrm{~m}$
10 FODO cells/
quadrant
$2 \mathrm{~Hz}$
$69 \mathrm{~nm}-\mathrm{rad}$ (actual)
$92 \mathrm{~nm}-\mathrm{rad}$ (nominal)
$351.930 \mathrm{MHz}$

$7.0 \mathrm{GeV}$

$1104 \mathrm{~m}$

40

$5.0 \mathrm{~m}$

$100 \mathrm{~mA}$

$2.5 \mathrm{~nm}-\mathrm{rad}$

$0.096 \%$

$3.1 \mathrm{~nm}-\mathrm{rad}$

$0.040 \mathrm{~nm}-\mathrm{rad}$

$1.5 \%$

$271.554 \mathrm{kHz}$

$351.930 \mathrm{MHz}$

24 to 1296

153 to $2.8 \mathrm{~ns}$

40 ps to 22 ps

$50 \mathrm{ps}$

\section{APS Source Parameters}

\section{UNDULATOR A (28 INSERTION DEVICES [IDS])}

Period: $3.30 \mathrm{~cm}$

Length: $2.1 \mathrm{~m}$ in sectors 16, 21, 23, 24, 34; $2.3 \mathrm{~m}$ in Sector 6;

$$
2.4 \mathrm{~m} \text { in others }
$$

Minimum gap: $10.5 \mathrm{~mm}$

$\mathrm{B}_{\max } / \mathrm{K}_{\text {max }}: 0.892 \mathrm{~T} / 2.75$ (effective; at minimum gap)

Tuning range: $3.0-13.0 \mathrm{keV}$ (1st harmonic)

$$
\text { 3.0-45.0 keV (1st-5th harmonic) }
$$

On-axis brilliance at $7 \mathrm{keV}\left(\mathrm{ph} / \mathrm{s} / \mathrm{mrad}^{2} / \mathrm{mm}^{2} / 0.1 \% \mathrm{bw}\right)$ :

$4.1 \times 10^{19}(2.4 \mathrm{~m}), 4.0 \times 10^{19}(2.3 \mathrm{~m}), 3.3 \times 10^{19}(2.1 \mathrm{~m})$

Source size and divergence at $8 \mathrm{keV}$ :

$$
\begin{aligned}
& \Sigma_{\mathrm{x}}: 276 \mu \mathrm{m} \quad \Sigma_{\mathrm{y}}: 11 \mu \mathrm{m} \\
& \Sigma_{\mathrm{x}^{\prime}}: 12.7 \mu \mathrm{rad}(2.4 \mathrm{~m}), 12.8 \mu \mathrm{rad}(2.3 \mathrm{~m}), 12.9 \mu \mathrm{rad}(2.1 \mathrm{~m}) \\
& \Sigma_{\mathrm{y}^{\prime}}: 6.7 \mu \mathrm{rad}(2.4 \mathrm{~m}), 6.8 \mu \mathrm{rad}(2.3 \mathrm{~m}), 7.1 \mu \mathrm{rad}(2.1 \mathrm{~m})
\end{aligned}
$$

\subsection{0-CM UNDULATOR (3 IDS IN SECTORS 1, 11, 14)}

Period: $2.30 \mathrm{~cm}$

Length: $2.4 \mathrm{~m}$

Minimum gap: $10.5 \mathrm{~mm}$

$\mathrm{B}_{\text {max }} / \mathrm{K}_{\text {max }}: 0.558 \mathrm{~T} / 1.20$ (effective; at minimum gap)

Tuning range: $11.8-20.0 \mathrm{keV}$ (1st harmonic)

11.8-70.0 keV (1st-5th harmonic, non-contiguous) On-axis brilliance at $12 \mathrm{keV}\left(\mathrm{ph} / \mathrm{s} / \mathrm{mrad}^{2} / \mathrm{mm}^{2} / 0.1 \% \mathrm{bw}\right): 6.9 \times 10^{19}$ Source size and divergence at $12 \mathrm{keV}$ :

$$
\begin{array}{ll}
\Sigma_{\mathrm{x}}: 276 \mu \mathrm{m} & \Sigma_{\mathrm{y}}: 11 \mu \mathrm{m} \\
\Sigma_{\mathrm{x}}: 12.3 \mu \mathrm{rad} & \Sigma_{\mathrm{y}}: 5.9 \mu \mathrm{rad}
\end{array}
$$

\subsection{0-CM UNDULATOR (4 IDS IN SECTORS $3,12,14$ )}

Period: $2.70 \mathrm{~cm}$

Length: $2.1 \mathrm{~m}$ in Sector $12 ; 2.4 \mathrm{~m}$ in others

Minimum gap: $10.5 \mathrm{~mm}$

$\mathrm{B}_{\max } / \mathrm{K}_{\max }: 0.698 \mathrm{~T} / 1.76$ (effective; at minimum gap)

Tuning range: $6.7-16.0 \mathrm{keV}$ (1st harmonic)

6.7-60.0 keV (1st-5th harmonic, non-contiguous)

On-axis brilliance at $8.5 \mathrm{keV}\left(\mathrm{ph} / \mathrm{s} / \mathrm{mrad}^{2} / \mathrm{mm}^{2} / 0.1 \% \mathrm{bw}\right)$ :

$5.7 \times 10^{19}(2.4 \mathrm{~m}), 4.7 \times 10^{19}(2.1 \mathrm{~m})$

Source size and divergence at $8 \mathrm{keV}$ :

$$
\begin{aligned}
& \Sigma_{\mathrm{x}}: 276 \mu \mathrm{m} \quad \Sigma_{\mathrm{y}}: 11 \mu \mathrm{m} \\
& \Sigma_{\mathrm{x}^{\prime}}: 12.7 \mu \mathrm{rad}(2.4 \mathrm{~m}), 12.9 \mu \mathrm{rad}(2.1 \mathrm{~m}) \\
& \Sigma_{\mathrm{y}^{\prime}}: 6.7 \mu \mathrm{rad}(2.4 \mathrm{~m}), 7.1 \mu \mathrm{rad}(2.1 \mathrm{~m})
\end{aligned}
$$

\subsection{0-CM UNDULATOR}

\section{(8 IDs IN SECTORS 12, 13, 16, 21, 23, 30, 34)}

Period: $3.00 \mathrm{~cm}$

Length: $2.1 \mathrm{~m}$ in sectors $12,13,16,21,23,34 ; 2.4 \mathrm{~m}$ in Sector 30 Minimum gap: $10.5 \mathrm{~mm}$

$\mathrm{B}_{\text {max }} / \mathrm{K}_{\text {max }}: 0.787 \mathrm{~T} / 2.20$ (effective; at minimum gap)

Tuning range: $4.6-14.5 \mathrm{keV}$ (1st harmonic)

$$
\text { 4.6-50.0 keV (1st-5th harmonic) }
$$

On-axis brilliance at $8 \mathrm{keV}\left(\mathrm{ph} / \mathrm{s} / \mathrm{mrad}^{2} / \mathrm{mm}^{2} / 0.1 \% \mathrm{bw}\right)$ :

$4.8 \times 10^{19}(2.4 \mathrm{~m}), 3.9 \times 10^{19}(2.1 \mathrm{~m})$

Source size and divergence at $8 \mathrm{keV}$ :

$$
\begin{aligned}
& \Sigma_{\mathrm{x}}: 276 \mu \mathrm{m} \quad \Sigma_{\mathrm{y}}: 11 \mu \mathrm{m} \\
& \Sigma_{\mathrm{x}^{\prime}}: 12.7 \mu \mathrm{rad}(2.4 \mathrm{~m}), 12.9 \mu \mathrm{rad}(2.1 \mathrm{~m}) \\
& \Sigma_{\mathrm{y}^{\prime}}: 6.7 \mu \mathrm{rad}(2.4 \mathrm{~m}), 7.1 \mu \mathrm{rad}(2.1 \mathrm{~m})
\end{aligned}
$$




\section{APS Source Parameters}

\subsection{0-cM SMCo UndULATOR (SECTOR 4)}

Period: $3.50 \mathrm{~cm}$

Length: $2.4 \mathrm{~m}$

Minimum gap: $9.75 \mathrm{~mm}$

$\mathrm{B}_{\text {max }} / \mathrm{K}_{\text {max }}: 0.918 \mathrm{~T} / 3.00$ (effective; at minimum gap)

Tuning range: $2.4-12.5 \mathrm{keV}$ (1st harmonic)

$$
\text { 2.4-42.0 keV (1st-5th harmonic) }
$$

On-axis brilliance at $7 \mathrm{keV}\left(\mathrm{ph} / \mathrm{s} / \mathrm{mrad}^{2} / \mathrm{mm}^{2} / 0.1 \% \mathrm{bw}\right): 3.7 \times 10^{19}$

Source size and divergence at $8 \mathrm{keV}$ :

$$
\begin{array}{ll}
\Sigma_{\mathrm{x}}: 276 \mu \mathrm{m} & \Sigma_{\mathrm{y}}: 11 \mu \mathrm{m} \\
\Sigma_{\mathrm{x}^{\prime}}: 12.7 \mu \mathrm{rad} & \Sigma_{\mathrm{y}^{\prime}}: 6.7 \mu \mathrm{rad}
\end{array}
$$

\subsection{0-CM UndULATOR (SECTOR 13)}

Period: $3.60 \mathrm{~cm}$

Length: $2.1 \mathrm{~m}$

Minimum gap: $11.0 \mathrm{~mm}$

$\mathrm{B}_{\max } / \mathrm{K}_{\max }: 0.936 \mathrm{~T} / 3.15$ (effective; at minimum gap)

Tuning range: $2.2-11.8 \mathrm{keV}$ (1st harmonic)

$$
\text { 2.2-40.0 keV (1st-5th harmonic) }
$$

On-axis brilliance at $6.5 \mathrm{keV}\left(\mathrm{ph} / \mathrm{s} / \mathrm{mrad}^{2} / \mathrm{mm}^{2} / 0.1 \% \mathrm{bw}\right): 2.8 \times 10^{19}$

Source size and divergence at $8 \mathrm{keV}$ :

$$
\begin{array}{ll}
\Sigma_{\mathrm{x}}: 276 \mu \mathrm{m} & \Sigma_{\mathrm{y}}: 11 \mu \mathrm{m} \\
\Sigma_{\mathrm{x}^{\prime}}: 12.9 \mu \mathrm{rad} & \Sigma_{\mathrm{y}^{\prime}}: 7.1 \mu \mathrm{rad}
\end{array}
$$

\subsection{0-CM UndULATOR (Sector 2)}

Period: $5.50 \mathrm{~cm}$

Length: $2.4 \mathrm{~m}$

Minimum gap: $14.0 \mathrm{~mm}$

$\mathrm{B}_{\max } / \mathrm{K}_{\max }: 0.965 \mathrm{~T} / 4.96$ (effective; at minimum gap)

Tuning range: $0.64-7.0 \mathrm{keV}$ (1st harmonic)

0.64-25.0 keV (1st-5th harmonic)

On-axis brilliance at $4 \mathrm{keV}\left(\mathrm{ph} / \mathrm{s} / \mathrm{mrad}^{2} / \mathrm{mm}^{2} / 0.1 \% \mathrm{bw}\right): 1.7 \times 10^{19}$

Source size and divergence at $4 \mathrm{keV}$ :

$$
\begin{array}{ll}
\Sigma_{\mathrm{x}}: 276 \mu \mathrm{m} & \Sigma_{\mathrm{y}}: 11 \mu \mathrm{m} \\
\Sigma_{\mathrm{x}^{\prime}}: 13.9 \mu \mathrm{rad} & \Sigma_{\mathrm{y}^{\prime}}: 8.8 \mu \mathrm{rad}
\end{array}
$$

\section{IEX 12.5-CM QUASI-PERIOdIC PolaRIZING UndULATOR (SECTOR 29)}

Period: $12.5 \mathrm{~cm}$

Length: $4.8 \mathrm{~m}$

Circular polarization mode:

Max. currents: horizontal coils $34.4 \mathrm{~A}$, vertical coils $20.7 \mathrm{~A}$

$\mathrm{K}_{\text {max }}: 2.73$ (effective; at max. currents)

$\mathrm{B}_{\max }: 0.27 \mathrm{~T}$ (peak; at max. currents)

Tuning range: $0.44-3.5 \mathrm{keV}$ (1st harmonic)

On-axis brilliance at $1.8 \mathrm{keV}\left(\mathrm{ph} / \mathrm{s} / \mathrm{mrad}^{2} / \mathrm{mm}^{2} / 0.1 \% \mathrm{bw}\right): 1.4 \times 10^{19}$

Linear horizontal polarization mode:

Max. current: vertical coils $47.6 \mathrm{~A}$

$\mathrm{K}_{\max }: 5.39$ (effective; at max. current

$\mathrm{B}_{\max }: 0.54 \mathrm{~T}$ (peak; at max. current)

Tuning range: $0.24-3.5 \mathrm{keV}$ (1st harmonic) $0.24-11.0 \mathrm{keV}$ (1st-5th harmonic)

On-axis brilliance at $2.1 \mathrm{keV}\left(\mathrm{ph} / \mathrm{s} / \mathrm{mrad}^{2} / \mathrm{mm}^{2} / 0.1 \% \mathrm{bw}\right): 1.1 \times 10^{19}$ Linear vertical polarization mode:

Max. current: horizontal coils $50.3 \mathrm{~A}$

$\mathrm{K}_{\text {max }}: 3.86$ (effective; at max. current)

$\mathrm{B}_{\max }: 0.37 \mathrm{~T}$ (peak; at max. current)

Tuning range: $0.44-3.5 \mathrm{keV}$ (1st harmonic) $0.44-11.0 \mathrm{keV}$ (1st-5th harmonic)

On-axis brilliance at $2.1 \mathrm{keV}\left(\mathrm{ph} / \mathrm{s} / \mathrm{mrad}^{2} / \mathrm{mm}^{2} / 0.1 \% \mathrm{bw}\right): 1.1 \times 10^{19}$

Fast polarization switching not required

Source size and divergence at $2 \mathrm{keV}$ :

$$
\begin{array}{ll}
\Sigma_{\mathrm{x}}: 276 \mu \mathrm{m} & \Sigma_{\mathrm{y}}: 13 \mu \mathrm{m} \\
\Sigma_{\mathrm{x}^{\prime}}: 13.9 \mu \mathrm{rad} & \Sigma_{\mathrm{y}^{\prime}}: 8.8 \mu \mathrm{rad}
\end{array}
$$

\section{APS Source Parameters}

\section{8-CM CiRcularly Polarizing Undulator (SECTOR 4)}

Period: $12.8 \mathrm{~cm}$

Length: $2.1 \mathrm{~m}$

Circular polarization mode:

Max. currents: horizontal coils $1.34 \mathrm{kA}$, vertical coils $0.40 \mathrm{kA}$

$\mathrm{K}_{\max }: 2.85$ (effective; at max. currents)

$\mathrm{B}_{\max }: 0.30 \mathrm{~T}$ (peak; at max. currents)

Tuning range: $0.4-3.0 \mathrm{keV}$ (1st harmonic)

On-axis brilliance at $1.8 \mathrm{keV}\left(\mathrm{ph} / \mathrm{s} / \mathrm{mrad}^{2} / \mathrm{mm}^{2} / 0.1 \% \mathrm{bw}\right): 3.1 \times 10^{18}$

Linear horizontal polarization mode:

Max. current: vertical coils $0.40 \mathrm{kA}$

$\mathrm{K}_{\max }: 2.85$ (effective; at max. current)

$\mathrm{B}_{\text {max }}$ : $0.30 \mathrm{~T}$ (peak; at max. current)

Tuning range: $0.72-3.0 \mathrm{keV}$ (1st harmonic)

$0.72-10.0 \mathrm{keV}$ (1st-5th harmonic)

On-axis brilliance at $2.1 \mathrm{keV}\left(\mathrm{ph} / \mathrm{s} / \mathrm{mrad}^{2} / \mathrm{mm}^{2} / 0.1 \% \mathrm{bw}\right): 2.3 \times 10^{18}$

Linear vertical polarization mode:

Max. current: horizontal coils $1.60 \mathrm{kA}$

$\mathrm{K}_{\max }: 3.23$ (effective; at max. current)

$\mathrm{B}_{\max }: 0.34 \mathrm{~T}$ (peak; at max. current)

Tuning range: $0.58-3.0 \mathrm{keV}$ (1st harmonic)

$0.58-10.0 \mathrm{keV}$ (1st-5th harmonic)

On-axis brilliance at $2.1 \mathrm{keV}\left(\mathrm{ph} / \mathrm{s} / \mathrm{mrad}^{2} / \mathrm{mm}^{2} / 0.1 \% \mathrm{bw}\right): 2.3 \times 10^{18}$

Switching frequency (limited by storage ring operation): $0-0.5 \mathrm{~Hz}$

Switching rise time: $50 \mathrm{~ms}$

Source size and divergence at $2 \mathrm{keV}$ :

$$
\begin{array}{ll}
\Sigma_{\mathrm{x}}: 276 \mu \mathrm{m} & \Sigma_{\mathrm{y}}: 12 \mu \mathrm{m} \\
\Sigma_{\mathrm{x}}: 16.7 \mu \mathrm{rad} & \Sigma_{\mathrm{y}^{\prime}}: 12.7 \mu \mathrm{rad}
\end{array}
$$

\section{SCUO Superconducting UndULATOR (SECtOR 6)}

Period: $1.60 \mathrm{~cm}$

Length: $0.34 \mathrm{~m}$

Gap: $9.5 \mathrm{~mm}$ (fixed)

Max. current: $650 \mathrm{~A}$

$\mathrm{B}_{\max } / \mathrm{K}_{\max }: 0.774 \mathrm{~T} / 1.15$ (effective; at maximum current)

Tuning range: $17.5-26 \mathrm{keV}$ (1st harmonic)

17.5-100.0 keV (1st-5th harmonic, non-contiguous)

On-axis brilliance at $87.5 \mathrm{keV}\left(\mathrm{ph} / \mathrm{s} / \mathrm{mrad}^{2} / \mathrm{mm}^{2} / 0.1 \% \mathrm{bw}\right): 5.3 \times 10^{17}$

Source size and divergence at $87.5 \mathrm{keV}$ :

$$
\begin{array}{ll}
\Sigma_{\mathrm{x}}: 276 \mu \mathrm{m} & \Sigma_{\mathrm{y}}: 11 \mu \mathrm{m} \\
\Sigma_{\mathrm{x}}: 12.3 \mu \mathrm{rad} & \Sigma_{\mathrm{y}}: 5.8 \mu \mathrm{rad}
\end{array}
$$

\section{APS Bending MAgnet}

Critical energy: $19.51 \mathrm{keV}$

Energy range: $1-100 \mathrm{keV}$

On-axis brilliance at $16 \mathrm{keV}\left(\mathrm{ph} / \mathrm{s} / \mathrm{mrad}^{2} / \mathrm{mm}^{2} / 0.1 \% \mathrm{bw}\right): 5.4 \times 10^{15}$

On-axis angular flux density at $16 \mathrm{keV}\left(\mathrm{ph} / \mathrm{s} / \mathrm{mrad}^{2} / 0.1 \% \mathrm{bw}\right): 9.6 \times 10^{13}$

Horizontal angular flux density at $6 \mathrm{keV}(\mathrm{ph} / \mathrm{s} / \mathrm{mradh} / 0.1 \% \mathrm{bw}): 1.6 \times 10^{13}$

Source size and divergence at the critical energy:

$$
\begin{array}{ll}
\Sigma_{\mathrm{x}}: 92 \mu \mathrm{m} & \Sigma_{\mathrm{y}}: 31 \mu \mathrm{m} \\
\Sigma_{\mathrm{x}^{\prime}}: 6 \mu \mathrm{rad} & \Sigma_{\mathrm{y}^{\prime}}: 47 \mu \mathrm{rad}
\end{array}
$$

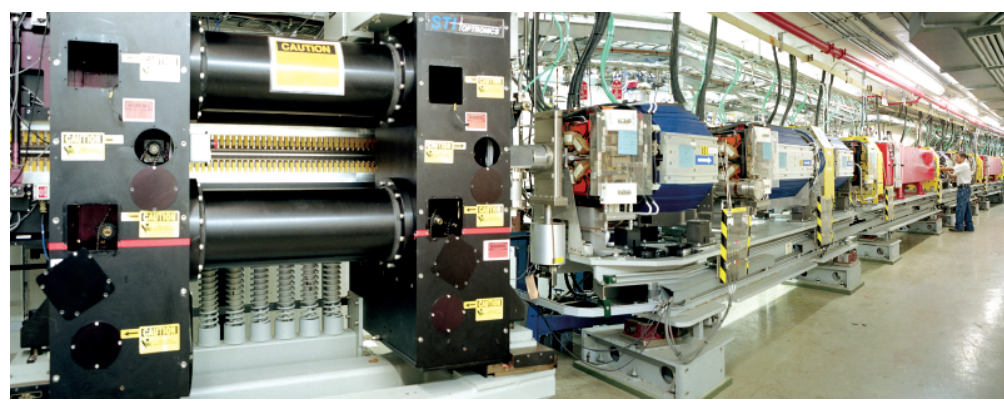




\section{ACKNOWLEDGMENTS}

\section{APS Science 2014 Editorial Board:}

Cele Abad-Zapatero (University of Illinois at Chicago), Mark A. Beno (ANL-XSD), Jonathan C. Lang (ANL-XSD), Dennis M. Mills, (ANL-PSC), John F. Maclean (ANL-AES), George Srajer (ANL-PSC), Stephen K. Streiffer (ANL-PSC), Linda Young (ANL-XSD), Alexander A. (Sasha) Zholents (ANL-ASD)

\section{The research highlights in this report were written by:}

Mary Alexandra Agner (marymary@gmail.com)

William Arthur Atkins (waarc@grics.net)

Erika Gebel Berg (erikagebel@gmail.com)

David Bradley (david@sciencebase.com)

Vic Comello (ANL-CEP, vcomello@anl.gov)

Dana Desonie (desonie@cox.net)

Sandy Field (sfield@fieldscientific.com)

Karen Fox (kfox@nasw.org)

Jenny Morber (jenny.morber.business@gmail.com)

Emma Nichols (emma@hittmedicalwriting.com)

Philip Koth (philkoth@comcast.net)

Kim Krieger (mskrieger@gmail.com)

David Lindley (dxlindley@gmail.com)

Chris Palmer (crpalmer2009@gmail.com)

Nicola Parry (nicola@parrymedicalwriting.com)

Neil Savage (neil@stefan.com)

Michael Schirber (mschirber@gmail.com)

Mark Wolverton (exetermw@earthlink.net)

Photography: Wes P. Agresta, Mark L. Lopez (both ANL-CEP), George Joch (ANL-CEP, ret.)

Aerial photograph of the APS: John Hill (Tigerhill Studio, http://www.tigerhillstudio.com)

Publications, contracts, rights and permissions, circulation: Jessie L. Skwarek (ANL-PSC)

Printing oversight: Gary R. Weidner (ANL-CEP)

CD production: Lorenza M. Salinas and Janet Barrett (both ANL-CEP)

Editorial, project coordination, design, photography: Richard B. Fenner (ANL-PSC)

Our thanks to the corresponding authors and others who assisted in the preparation of the research highlights, to the users and APS personnel who wrote articles for the report, and our apologies to anyone inadvertently left off this list. To all: your contributions are appreciated. 